\title{
Eine Analyse der Fußball- Weltmeisterschaft 1998 in Frankreich mit Hilfe einer sportartspezifischen Methode der Systematischen Spielbeobachtung
}

\author{
Dissertation \\ zur Erlangung des sozialwissenschaftlichen \\ Doktorgrades des Fachbereichs Sozialwissenschaften \\ der Georg-August-Universität zu Göttingen
}

\author{
Vorgelegt von \\ Ali Hussein Abdelrahman \\ aus \\ Zakazik - Ägypten
}

Göttingen 2004 
1. Gutachter:

Prof. Dr. Arnd Krüger

2. Gutachter:

PD.Dr. Wolfgang Buss

Tag der mündlichen Prüfung: 


\section{Inhaltsverzeichnis}

1. Einleitung 1

2. Charakterisierung des Sportspiels Fußball 9

2.1 Historische Entwicklung des modernen Fußballs 9

2.2 Merkmale des Spiels 10

2.3 Taktik 12

2.3.1 Hauptelemente der Taktik im Fußballsport 15

2.3.1.1 Differenzierung und Strukturierung taktischer Handlung 15

2.3.1.2 Taktische Leistungsfaktoren 19

2.4 Technik 21

2.4.1 Hauptelemente der Technik 24

2.4.1.1 Bewegung ohne Ball 24

2.4.1.2 Bewegung zum Ball 25

2.4.1.3 Bewegung mit Ball 25

3. Systematische Spielbeobachtung 27

3.1 Abgrenzung und Explikation der Systematischen Spielbeobachtung 29

3.2 Spielbeobachtung vs. Spielerbeobachtung 32

3.3 Spielbeobachtung als Spielanalyse 33

3.4 Ziele der Spielerbeobachtung 35

3.5 Methoden der Spielbeobachtung 39

3.6 Qualitative und Quantitative Spielbeobachtung 41

3.7 Videosysteme in der Spielbeobachtung 45

3.8 Formen und Anwendungsfelder der Spielanalyse unter besonderer Berücksichtigung von Fußball 48

4. Methodik 56

4.1 Zur Begründung und Weiterentwicklung einer spezifischen Methodik der Spielbeobachtung im Fußball 56

$\begin{array}{lll}4.1 & \text { Objektivität } & 63\end{array}$

$\begin{array}{lll}4.1 .2 & \text { Reliabilität } & 63\end{array}$

$\begin{array}{lll}4.1 .3 & \text { Validität } & 66\end{array}$

$\begin{array}{lll}\text { 4.1.4 Relevanz und Ökonomie } & 67\end{array}$ 
4.2 Untersuchungsplanung und -durchführung (Methode)

$\begin{array}{lll}\text { 4.2.1 Beobachtungseinheiten } & 68\end{array}$

$\begin{array}{lll}4.2 .2 & \text { Datenerhebung } & 76\end{array}$

$\begin{array}{lll}4.3 & \text { Datenauswertung } & 78\end{array}$

5. Untersuchungsergebnisse $\quad 79$

$\begin{array}{lll}5.1 & \text { Gütekriterien Prüferergebnisse } & 79\end{array}$

5.2 Darstellung der Kennziffern von technischen Fähigkeiten 81

5.3 Prozentuale Qualitätsanteile technischer Elemente 81

5.3.1 Achtelfinalisten 81

5.3.1.1 Dribblings 81

5.3.1.2 Körpertäuschung 85

$\begin{array}{lll}\text { 5.3.1.3 Torschuss } & 87\end{array}$

5.3.1.4 Kurze/ lange Pässe 90

$\begin{array}{lll}\text { 5.3.1.5 Ballkontrolle } & 95\end{array}$

$\begin{array}{lll}\text { 5.3.2 Viertelfinalisten } & 98\end{array}$

5.3.2.1 Dribblings 98

$\begin{array}{lll}\text { 5.3.2.2 Körpertäuschung } & 100\end{array}$

$\begin{array}{lll}\text { 5.3.2.3 Torschuss } & 102\end{array}$

5.3.2.4 Kurze/ lange Pässe 105

$\begin{array}{lll}\text { 5.3.2.5 } & \text { Ballkontrolle } & 109\end{array}$

5.3.3 Halbfinalisten 112

$\begin{array}{lll}\text { 5.3.3.1 Dribblings } & 112\end{array}$

$\begin{array}{lll}\text { 5.3.3.2 Körpertäuschung } & 114\end{array}$

$\begin{array}{lll}\text { 5.3.3.3 Torschuss } & 116\end{array}$

5.3.3.4 Kurze/ lange Pässe 119

$\begin{array}{lll}\text { 5.3.3.5 Ballkontrolle } & 123\end{array}$

5.4 Prozentuale Qualitätsanteile offensiv- taktischer Elemente 125

5.4.1 Achtelfinalisten 125

$\begin{array}{lll}\text { 5.4.1.1 } & \text { Freistöße } & 126\end{array}$

$\begin{array}{lll}5.4 .1 .2 & \text { Eckball } & 128\end{array}$

$\begin{array}{lll}\text { 5.4.1.3 Unterstützen } & 130\end{array}$

$\begin{array}{lll}\text { 5.4.1.4 Doppelpass } & 133\end{array}$

$\begin{array}{lll}\text { 5.4.1.5 Raumaufteilung } & 135\end{array}$ 
$\begin{array}{lll}\text { 5.4.1.6 Nachrücken } & 137\end{array}$

$\begin{array}{lll}\text { 5.4.1.7 In die Breite ziehen } & 140\end{array}$

$\begin{array}{lll}5.4 .2 & \text { Viertelfinalisten } & 142\end{array}$

$\begin{array}{lll}\text { 5.4.2.1 } & \text { Freistöße } & 142\end{array}$

$\begin{array}{lll}\text { 5.4.2.2 Eckball } & 144\end{array}$

$\begin{array}{lll}\text { 5.4.2.3 Unterstützen } & 146\end{array}$

5.4.2.4 Doppelpass 148

$\begin{array}{lll}\text { 5.4.2.5 } & \text { Raumaufteilung } & 150\end{array}$

$\begin{array}{lll}\text { 5.4.2.6 Nachrücken } & 152\end{array}$

$\begin{array}{lll}\text { 5.4.2.7 In die Breite ziehen } & 154\end{array}$

$\begin{array}{lll}5.4 .3 & \text { Halbfinalisten } & 156\end{array}$

5.4.3.1 Freistöße 156

$\begin{array}{lll}\text { 5.4.3.2 Eckball } & 158\end{array}$

$\begin{array}{lll}\text { 5.4.3.3 Unterstützen } & 160\end{array}$

$\begin{array}{lll}\text { 5.4.3.4 Doppelpass } & 162\end{array}$

$\begin{array}{lll}\text { 5.4.3.5 } & \text { Raumaufteilung } & 164\end{array}$

$\begin{array}{lll}\text { 5.4.3.6 Nachrücken } & 166\end{array}$

$\begin{array}{lll}\text { 5.4.3.7 In die Breite ziehen } & 168\end{array}$

5.5 Prozentuale Qualitätsanteile defensiv-taktischer Elemente 170

$\begin{array}{lll}5.5 .1 & \text { Achtelfinalisten } & 170\end{array}$

$\begin{array}{lll}\text { 5.5.1.1 Pressing hinten } & 170\end{array}$

$\begin{array}{lll}\text { 5.5.1.2 } & \text { Pressing Mitte } & 172\end{array}$

$\begin{array}{lll}\text { 5.5.1.3 Pressing vorne } & 174\end{array}$

$\begin{array}{lll}\text { 5.5.1.4 Raumaufteilung } & 176\end{array}$

$\begin{array}{lll}5.5 .1 .5 & \text { Zurückkommen } & 179\end{array}$

$\begin{array}{ll}\text { 5.5.1.6 Unterstützen } & 181\end{array}$

$\begin{array}{lll}\text { 5.5.1.7 Rückraum sichern } & 183\end{array}$

$\begin{array}{lll}\text { 5.5.1.8 Abseitsfalle } & 185\end{array}$

$\begin{array}{lll}5.5 .2 & \text { Viertelfinalisten } & 186\end{array}$

$\begin{array}{lll}\text { 5.5.2.1 } & \text { Pressing hinten } & 186\end{array}$

$\begin{array}{lll}\text { 5.5.2.2 } & 189\end{array}$

$\begin{array}{lll}\text { 5.5.2.3 Pressing vorne } & 191\end{array}$

$\begin{array}{lll}\text { 5.5.2.4 Raumaufteilung } & 193\end{array}$

$\begin{array}{lll}\text { 5.5.2.5 Zurückkommen } & 195\end{array}$ 
$\begin{array}{lll}\text { 5.5.2.6 Unterstützen } & 198\end{array}$

$\begin{array}{lll}\text { 5.5.2.7 } & \text { Rückraum sichern } & 199\end{array}$

$\begin{array}{lll}\text { 5.5.2.8 Abseitsfalle } & 201\end{array}$

5.5.3 Halbfinalisten 203

5.5.3.1 Pressing hinten 203

5.5.3.2 Pressing Mitte 205

$\begin{array}{lll}\text { 5.5.3.3 Pressing vorne } & 207\end{array}$

5.5.3.4 Raumaufteilung 209

$\begin{array}{lll}\text { 5.5.3.5 Zurückkommen } & 212\end{array}$

5.5.3.6 Unterstützen 214

5.5.3.7 Rückraum sichern 216

$\begin{array}{lll}\text { 5.5.3.8 Abseitsfalle } & 218\end{array}$

5.6 Qualitätsquotienten technischer Elemente 220

5.6.1 Achtelfinalisten $\quad 220$

5.6.2 Viertelfinalisten 225

5.6.3 Halbfinalisten 229

5.7 Qualitätsquotienten offensiv taktischer Elemente 232

5.7.1 Achtelfinalisten 232

$\begin{array}{lll}5.7 .2 \text { Viertelfinalisten } & 237\end{array}$

5.7.3 Halbfinalisten 240

5.8 Qualitätsquotienten defensiv taktischer Elemente 244

5.8.1 Achtelfinalisten 244

5.8.2 Viertelfinalisten 248

5.8.3 Halbfinalisten 252

5.9 Individuelle und mannschaftliche Fähigkeiten 256

5.9.1 Achtelfinalisten $\quad 257$

5.9.2 Viertelfinalisten $\quad 262$

5.9.3 Halbfinalisten 264

5.10 Technische, offensiv- und defensiv-taktische Fähigkeiten 266

$\begin{array}{ll}5.10 .1 \text { Achtelfinalisten } & 267\end{array}$

5.10.2 Viertelfinalisten 273

5.10.3 Halbfinalisten 276 
6. Analyse der Mannschaftsergebnisse

6.1 Technische, taktische- offensiv und defensiv - taktische Fähigkeiten von Halb-, Viertel- und Achtelfinalisten

6.2 Technische, offensiv - taktische und defensiv - taktische Fähigkeiten von Halbfinalisten

6.3 Individuelle und mannschaftliche Fähigkeiten von Halb-, Viertelund Achtelfinalisten

6.4 Individuelle und mannschaftliche Fähigkeiten von Halbfinalisten

6.5 Bewertung der Qualitätsquotienten

7. Zusammenfassung und Ausblick

Empfehlungen

300

Literaturverzeichnis

302

Abbildungsverzeichnis

Tabellenverzeichnis

318

Anhang

319

Danksagung

Eidesstattliche Erklärung

326

Lebenslauf

327 


\section{Einleitung}

Eine Urform des Fußballspiels, das chinesische T'SU CHU, ist inzwischen ca. 4000 Jahre alt. Ein Sportspiel, das eine derartige lange Tradition hat und das sich bis heute einer hohen Gunst bei Spielern und Zuschauern erfreut, hat seine eigene Anziehungskraft. Fußball bewegt die Massen und übt nach wie vor eine Faszination ohne gleichen aus. Er ist weltweit die absolute Sportart Nummer eins. Das wurde erst kürzlich wieder auch unter statistischen Gesichtspunkten deutlich. Wenn nämlich alle vier Jahre die Weltmeisterschaft stattfindet, werden bei Summierung der Einschaltquoten im Verlauf des Turniers etwa 15 Milliarden Fernsehzuschauer erreicht. (vgl. BAUER 2001)

Ein Fußballspiel kann nun unter den verschiedensten Gesichtpunkten betrachtet, ausgewertet und kommentiert werden. Zur Veranschaulichung und zum besseren Verständnis soll einleitend kurz auf unterschiedliche Sichtweisen von Journalisten, Trainern und Sportwissenschaftlern eingegangen werden.

Die Hör- und Sehmedien widmen dem Fußball spezielle Sendeplätze und die Zeitschriften Beilagen neben den spezialisierten Sportzeitschriften. Bei einem Sieg „ihrer“" Mannschaft gewähren die politischen, gesellschaftlichen und wirtschaftlichen Führer der Mannschaft auch Anerkennung und Unterstützung. Diese Fürsorge erreicht ihren Gipfel bei den allen vier Jahren stattfindenden Weltmeisterschaften. Insofern greifen Journalisten primär gesellschaftlich relevante Aspekte auf.

In den letzten Jahren wurde im Bereich der körperlichen Vorbereitung und des Techniktrainings sowie bei den Taktikschulungen ein Fortschritt erzielt, welcher zu Recht als großer Aufschwung gesehen wird. Doch dem Fortschritt entsprechend nehmen auch die Ansprüche der Trainer an die Spieler zu, die dann auf deren Schultern lasten (IBRAHIM 1992) und in der Härte des Spiels zum Ausdruck kommen. Auf der anderen Seite spornen Belohnungen und Zusagen sowie gesellschaftliche Auszeichnung die Spieler zum harten Kämpfen an. Solche Ausdrucksformen sind vorläufige Endpunkte einer langen Entwicklung. Insofern sehen Trainer primär die Leistungsresultate. 
Demgegenüber geht es Sportwissenschaftlern um eine exakte Analyse und Erklärung des Spiels und seiner Bedingungen. Weltmeisterschaften sind dazu ein willkommener Anlass, auch um eine Bilanz bezüglich des Entwicklungsstandes in der jeweiligen Sportart zu ziehen. Die Weltmeisterschaft, auf internationaler Ebene und nationale Meisterschaften setzen die aktuellen Leistungsmaßstäbe und zeigen Perspektiven auf. Daraus ergibt sich ebenso eine ständige Herausforderung für Trainer und Spieler aller Leistungsklassen. Für gewöhnlich werden dabei von Fachleuten subjektive Einschätzungen zu konditionellen, technischen und taktischen Fragestellungen gegeben. Nicht selten kommen aber die sog. „Experten“ zu dem (vor) schnelles Urteil, dass sich, einmal abgesehen von einigen „Kleinigkeiten“, im Verlauf der letzten Jahre kaum etwas verändert habe, d.h. die Entwicklung weitgehend stagniere (LOY 1995). Ohne die Bedeutung der subjektiven Eindrucksanalyse durch Fachleute bei einer derartigen Standortbestimmung schmälern zu wollen, sei darauf verweisen, dass einer rein qualitativen Betrachtung bestimmte Entwicklungen oftmals verbogen bleiben. Die Aufdeckung solcher verborgenen Entwicklungen liegt dabei v.a. im Interesse der (Sport-)Wissenschaft.

schmälern zu wollen, sei darauf verweisen, dass einer rein qualitativen Betrachtung bestimmte Entwicklungen oftmals verbogen bleiben. Die Problematik der qualitativen Spielbeobachtung hat in jüngster Zeit HANSEN (2003) umfassend behandelt. Die Aufdeckung solcher verborgenen Entwicklungen liegt dabei v.a. im Interesse der (Sport-)Wissenschaft. So konnten in jüngerer Zeit WILLIAMS/ LEE/ REILLY (1999) durch Videoanalysen nachweisen, dass die Spielmuster sich zwischen 1991 und 1998 im Fußball veränderten. Verglichen mit ihren Vorgängern, weisen moderne Fußballteams folgende Merkmale auf:

- sie sind effektiver im Schaffen von Standardsituationen und im daraus resultierenden Ausnutzen von Torchancen;

- sie können den Ballbesitz länger aufrechterhalten, insbesondere, wenn der Ball in der Abwehr und im Mittelfeld erobert wurde,

- $\quad$ sie sind erfolgreicher bei Distanzschüssen, sowie

- $\quad$ variabler und kreativer in ihrem Angriffsspiel.

Der wissenschaftliche Fortschritt wird nun als wichtige Aufgabe betrachtet, um zu einem gehobenen Niveau des Sports im Allgemeinen und des Fußballs im Besonde- 
ren zu gelangen. Obwohl es viele Sportarten gibt, so hat doch jede Sportart ihre Eigenheiten, durch welche sie sich von den übrigen unterscheidet, vor allem durch die Art der technischen oder taktischen Merkmale (vgl. ALLAWY 1993).

Besonders bei Olympischen Spiele und Weltmeisterschaften wird häufig klar, dass im Hochleistungssport ein exzellentes Niveau durch die Merkmalskomplexe psychologischer Bedingungen, der physiologischen Kapazität, der Technik und Taktik zu erreichen ist (vgl. ABDELMAKSUD 1979).

Die technischen Fertigkeiten ermöglichen es dem Spieler erst, seine Position für das Angriffs- und Verteidigungsspiel einzunehmen. Von dem Augenblick an, in dem ein Spieler einer Mannschaft den Ball in seine Gewalt bringt, wird diese Mannschaft die angreifende Mannschaft, weil sie nun versucht, ein Tor zu erzielen. Wenn die Mannschaft den Ball verliert, so wird sie die verteidigende Mannschaft, ohne Rücksicht auf die Positionen der Spieler auf dem Spielfeld. Im Verlauf des Wettkampfes wechseln sich Angriff und Verteidigung bei den beiden Mannschaften ab, eben bestimmt durch den Ballbesitz. Sie sind die wesentliche Bestimmungsstücke des Spiels (vgl. ABO EL-MAGD/ISMAIL/ SCHAALAN 1998). Bevor allerdings taktische Maßnahmen ergriffen werden können, müssen grundlegende Techniken beherrscht werden (vgl. MORSEY 1975). Daher ist auch der Entwicklung technischer Fähigkeiten stets besondere Aufmerksamkeit zu schenken. Über die (begrenzten) Möglichkeiten einer rein subjektiven Betrachtung hinaus verhelfen objektive Analysen zu vertiefenden Einblicken in den Ablauf von Bewegungen.

Die individuelle und Gruppenbezogene Leistung in den Sportspielen allgemein und Fußball im Besonderen wird wesentlich durch die konditionellen, technischen, aber auch taktischen, intellegenzbezogenen und sozialen Fähigkeiten bestimmt. Zu den konditionellen Fähigkeiten sind u. a. Kraft, Schnelligkeit, Ausdauer, zu den technischen Fähigkeiten u. a. das An- und Mitnehmen des Balles in der Bewegung, Dribbling, Kopfball, zu den taktischen Fähigkeiten u. a. das Freilaufen, das Verhalten in Angriff und Abwehr sowie zu den sozialen Fähigkeiten u. a. das Durchsetzungsvermögen, die Kooperationsfähigkeit und das Zusammengehörigkeitgefühl zu zählen. $\mathrm{Zu}$ den Intelligenzbezogenen Fähigkeiten gehören u. a. Antizipationsvermögen, Spielgedächtnis, Spielphantasie, geistige Anpassungs-, Umstellungs- und Lernfähigkeit (vgl. BAUER/ UEBERLE 1984, 65). 
Die Taktik ist die Koordination der Einzelaktionen mit der Mannschaft als Gesamtes. Sie beruht auf den Prinzipien und Methoden des Spiels und der Vorgehensweise des Gegners mit dem Ziel, das bestmögliche Ergebnis zu erreichen (vgl. MOAWAD 1980; KISK/ ELBOSATY 2000).

Das Fußballspiel kann als eine Aufeinanderfolge von individualtaktischen Teilhandlungen verstanden werden. Sie bilden die Basis von Interaktionsketten, welche ihrerseits wieder gruppen- und mannschaftstaktische Handlungen bestimmten. Individualtaktische Handlungen sind somit auch die Grundlage des kollektiven Verhaltens. Sie wiederum sind geprägt durch eine Vielzahl von Teilfunktionen oder Teilfertigkeiten aus unterschiedlichen Klassen. In einer empirisch- analytischen Taktikdiagnostik geht es letztlich um die Identifikation dieser leistungsrelevanten Fähigkeiten, um ihre qualitative und quantitative Ausprägung. Auf dieser Basis können dann auch Aussagen zur Effektivität von Spielhandlungen getroffen werden (vgl. AUGUSTIN 1997).

Im Fußballspiel besteht ein komplexer Zusammenhang zwischen Fähigkeiten und Fertigkeiten. Bei der in der vorliegenden Arbeit soll auf diesen Zusammenhang nicht eingegangen werden, da nur das Endprodukt des sportlichen Entwicklungsprozesses auf dem Spielfeld beobachtet wird und insofern die für den Trainer im Trainingsprozess wichtigen Zusammenhang nicht eingegangen werden braucht. Für den Zusammenhang von Fähigkeiten und Fertigkeiten sei in unserem Zusammenhang auf SCHNABEL u.a. $(1994,122$ - 158) verwiesen, auch wenn im Zusammenhang mit der hier vorliegenden Arbeit eine stärkere Orientierung an PÖHLMANN u. a. (1987, 128 140) erfolgte.

Die Beherrschung der Angriffstaktik wird für einen Spieler als Grundpfeiler zur Erfüllung seiner Aufgabe angesehen. Der Spieler muss sein Laufen und seine Bewegungen auf dem Spielfeld mit seiner Fähigkeit zur Ausführung von geschickten Angriffen verbinden. Die Fähigkeit des Spielers, geschickte Angriffe durchzuführen, hilft ihm bei der Bewältigung einer jeden Situation, welcher der Spieler sich im Wettkampf gegenübersieht. Die Angriffstaktik zielt auf die Überwindung der Verteidigungstaktik der gegnerischen Mannschaft. Dazu müssen die Spieler der angreifenden Mannschaft alle Methoden des Angriffs beherrschen, vom Einzelangriff bis zum gemeinschaftlichen Angriff. Die Umsetzung der Absicht in der Mannschaft ist jedoch schwierig und 
wird zu den wichtigen Faktoren gezählt, um einen Wettkampf zu gewinnen (vgl. YEHYA 1983).

Indem die Mannschaft sich auf eine starke Verteidigung stützt, kann sie ohne zu zögern und mit guter Aussicht auf Erfolg auch einen Angriff unternehmen. Mit einer guten Verteidigung im Rücken hat jeder Spieler mehr Selbstvertrauen im Spiel. Die Verteidigung wird deshalb als Grundlage erachtet, auf der die Angriffsstrategie aufbauen kann. Sie bildet den Schutz, um die gegnerische Mannschaft davon abzuhalten, Tore zu erzielen und gibt dem Spieler Sicherheit bei der Abwehr von aufeinanderfolgenden Angriffen.

Aufbauend auf solchen grundlegenden Überlegungen soll in dieser Arbeit nun ein umfassender Ansatz entwickelt und erprobt werden, um eine möglichst objektive Charakterisierung der Entwicklungstendenzen im Weltfußball zu erreichen. Wenngleich im vorgehenden Abschnitt das Moment der Unwägbarkeiten im Fußballspiel hervorgehoben wurde, so sollte damit nicht der Eindruck erweckt werden, dass Bemühungen um eine Erfassung des Spielgeschehens in diesem Sportspiel überflüssig oder gänzlich unmöglich seien. Kein Fußballtrainer wäre so vermessen, Glück und Zufall einen Spielbestimmenden Einfluss zuzuschreiben, sondern vielmehr dem Können seiner Spieler oder dem Konzept, das er in einem Spiel verwirklicht haben möchte. So ist auch davon auszugehen, dass dem Fußballspiel, auch wenn es sich um ein vergleichsweise offenes Spiel handelt, klar erkennbare Spielstrukturen zugrunde liegen.

Um den momentan Leistungsstand einer Mannschaft zu ermitteln, bedient sich der Trainer nahezu ausschließlich subjektiver Verfahren. Gezielte Beobachtungen und vorhandenes Erfahrungswissen bilden die Basis bei dieser Vorgehensweise. Vielfach stellt sich jedoch heraus, dass dieser Ansatz wichtige Leistungskomponenten nur grob bzw. unvollständig erfasst. Grund ist die natürliche Begrenzung des visuellen Wahrnehmungsvermögens des Menschen. Gerade bei einer Sportart wie Fußball mit großem Spielfeld, hoher Spielerzahl, vielfältigen Aktionsmöglichkeiten, kann das gesamte Geschehen auf diese Weise nur lückenhaft wahrgenommen werden (vgl. BASYONI/ FADEL 1995; KOLLATH 1992). 
In einer derartigen Situation erwartet der Praktiker zu Recht Unterstützung von Seiten der Sportwissenschaft. Doch auch dabei bereitet die Durchdringung der komplexen Struktur des Fußballspiels derzeit noch Probleme. Bestehende Ansätze bedürfen einer ständigen Verbesserung; nur in wenigen Bereichen stehen dem Trainer bislang praktikable und hilfreiche Unterstützungsverfahren zu Verfügung (vgl. KOLLATH 1992).

Umfassende Analysen beziehen nun vielfältige Faktoren ein, um differenzierte Teilaspekte in ein Gesamtbild zu integrieren. Die Ziele von solchen analytischen Systemen schließen die Studien über Bewegungen während Spiels, technische und taktische Elemente ein und versuchen im Anschluss daran eine statistische Kompilation (vgl. REILLY u.a 2003). Die Leistung von einzelnen Spielern kann im Detail erfasst werden und schließt die Anzahl von bestimmten Ereignissen, den Aktionen einzelner Spieler und den Standort dieser Ereignisse ein. Computergestützte Methoden können dazu hilfreich sein (HUGHES u.a 1998; PATRIC/ MCKENNA 1988). Darauf aufbauend können die Ergebnisse zu einer gezielten Rückmeldung für die Praxis fruchtbar gemacht werden. Solche Rückmeldungen wurden schon bei international spielenden Teams nützlich (vgl. OLSEN/ LARSEN 1997).

Als ich nun die Weltmeisterschaften 1998 naiv beobachtet hatte, fand ich, dass die meisten Mannschaften darunter leiden, im Vergleich zu früheren Weltmeisterschaften weniger Tore zu erzielen. Dies lässt zwei Vermutungen zu: Entweder hat sich insgesamt der Angriff gegenüber früher verschlechtert oder die Verteidigung ist wesentlich effektiver geworden. Um diese Frage zu beantworten, müssen sowohl die Taktik des Angriffs als auch der Verteidigung beobachtet werden. Dabei ist es notwendig, diese in ihre Bestandteile aufzulösen sowie Kenntnis des Einflusses dieser Bestandteile auf das Spielergebnis zu gewinnen. Als wichtigste Voraussetzungen dazu sind folgende Faktoren hilfreich:

- Kenntnis der wichtigsten Einzelangriffe,

- Kenntnis der wichtigsten Gemeinschaftsangriffe,

- Kenntnis der Gemeinschaftsverteidigung. 
Das Ziel der Wettkampfsleistungsdiagnostik besteht vor allem darin, die Richtungen und die Dynamik der inneren Beanspruchung in enger Wechselwirkung zu sportmethodisch vorgegebenen Belastungen zu erfassen und die Übereinstimmung zwischen der trainingsmethodischen Zielstellung und ihrem aktuellen Ergebnis sowie der Technik und Taktik qualitativ und quantitativ zu überprüfen. Dabei gilt es, verschiedenen Spielsysteme mit Hilfe wissenschaftlicher Beobachtungsverfahren (wie quantitativen und qualitativen Spielbeobachtungsverfahrens) anhand von Videoaufzeichnungen zu analysieren und die Frage nach wesentlichen Unterscheidungsmerkmalen zu beantworten. Herangezogen werden dazu 16 Spiele (ab Achtelfinale) der FußballWeltmeisterschaft 1998.

Mit den Videoanalysen sind konditionelle Fähigkeiten nicht zu analysieren, da die Kamera normalerweise den Ball verfolgt und somit die Laufwege der nicht unmittelbar in Ballnähe befindlichen Spieler nicht erfasst werden. Es soll daher insbesondere um die Art der Beziehung zwischen technischen und taktischen Fähigkeiten einerseits sowie dem Ergebnis des Spiels andererseits gehen, denn zweifellos haben Technik und Taktik positive Auswirkungen auf das Spielergebnis. Anders gesprochen, ist für diese Untersuchung von Interesse, wie sich diese beiden Faktoren auf das Spielergebnis auswirken. Es geht also um die Frage, inwiefern sich Gewinner und Verlierer anhand bestimmter Merkmale unterscheiden lassen.

Dies ist aber eben erst über den schon angesprochenen „Umweg“ sinnvoll zu beantworten, sodass folgende drei Fragen im Zentrum der Arbeit stehen sollen:

1. Gibt es Unterschiede in der Technik bei den untersuchten Mannschaften?

2. Gibt es Unterschiede in Angriff- und Verteidigungstaktik bei den Mannschaften?

3. lässt sich die Qualität des Spiels der Mannschaften systematisch erfassen?

Den notwendig zu klärenden Ausgangspunkt dazu bilden die Strukturmerkmale des Fußballspiels. In Kapitel 2 werden diese in charakterisierender Weise aufgearbeitet. Es schließt die begriffliche, strukturelle und inhaltliche Klärung und Abgrenzung technischer und taktischer Fähigkeiten ein. Darauf aufbauend sollte es möglich werden, die zentralen Fragen der Arbeit gezielt anzugehen. Einen zweiten wichtigen Aspekt stellt die Vorgehensweise der Analyse dar. Aber auch, wenn mit der Systematischen Spielbeobachtung ein geeignetes Verfahren vorzuliegen scheint, bestehen unterschiedliche Zugänge, sodass es notwendig wird, auch diese in einem eigenen 
Kapitel (3) darzustellen. Hieraus werden die zentralen spezifischen Fragen der Studie abgeleitet. Daran schließt sich die Darstellung der konkreten Methodik für die folgende Untersuchung an. Dabei werden zunächst die einzelnen Beobachtungseinheiten erläutert, die vor Untersuchungsbeginn festgelegt wurden. Diese Darstellung erfolgt anhand der einzelnen auf dem beidseitigen Auswertungsbogen erfassten Parameter, und anschließend wird die bei der Beobachtung eingesetzte technische Ausstattung vorgestellt. Es folgt eine Erläuterung der Datenerhebung, der Weiterverarbeitung und der Datenauswertung.

In Kapitel 4 wurde die Methodik, Turnierverlauf, und ausgewählte Elemente beschreiben. In Kapitel 5, 6 und 7 wurden als Darstellung zum technischen und taktischen Die Nationenergebnisse der sechzehn Mannschaften die im Achtelfinale bei der Weltmeisterschaft erreicht, zusammengefasst und analysiert. Schließlich wird hierbei ein eigenes Verfahren entwickelt, um die Qualität des Spiels zu charakterisieren und so die Methodik der Systematischen Spielbeobachtung weiter zu entwickeln. 


\section{Charakterisierung des Sportspiels Fußball}

\subsection{Historische Entwicklung des modernen Fußballs}

Das heutige Fußballspiel entwickelte sich vornehmlich in England aus einer Art "Raufball" (ohne feste Regeln), das im Mittelalter auf den Straßen Londons und anderer Städte mit einem kleinen Stoffball gespielt wurde. Es war nur Spiel ohne konventionelle Regelungen, bei dem im Vordergrund der Spaß stand und die Möglichkeit, sich mit Freunden und Bekannten die Zeit zu vertreiben. In der Folge entwickelten sich die verschiedenen Regeln vor allem in den Public Schools. Da jede Public School eigene Regeln hatte, konnten deren Absolventen auf der Universität kaum gemeinsam spielen. 1848 verfassten Studenten der Universität Cambridge erste verbindliche Fußballregeln.1857 wurde in Sheffield dann der erste Fußballklub gegründet. Die Gründung der FA (Football Association) 1863 bedeutete die endgültige Trennung vom traditionellen Rugby und die eigentliche Geburtsstunde des modernen Fußballs. Bis zur ersten offiziellen Weltmeisterschaft 1930 galten die jeweiligen Olympiasieger als inoffizielle Weltmeister. Doch anfangs fand das Olympische Fußballturnier nur wenig Beachtung. Bei den ersten drei Austragungen nahmen jeweils nur drei Mannschaften teil. Der Olympiasieger von 1896 (Dänemark) wird in den meisten Chroniken nicht einmal erwähnt. 1900 gewann England (mit dem Klubteam von Upton Park London), 1904 Kanada (gegen zwei US-amerikanische Teams) und bei den Zwischenspielen von 1906 besiegten die Dänen im Finale eine Auswahl von Spielern aus Griechenland, Frankreich und England. Das erste ernst zu nehmende Olympische Fußballturnier wurde 1908 in London von England gewonnen, und die Engländer wiederholten ihren Sieg bei den folgenden Spielen von 1912. Die Briten dominierten auch klar vor dem Ersten Weltkrieg den Weltfußball, der sich bis dahin vorwiegend auf dem europäischen Kontinent abspielte. Nach dem Ersten Weltkrieg holte sich Belgien den Titel im Skandalfinale von 1920 (die Tschechoslowaken verließen nach dem 0:2 den Platz und wurden disqualifiziert). Die Briten verließen zeitweise die Weltbühne des Fußballs auf ihre Art und Weise (engl. "Splendid Isolation"): sie traten aus dem Weltverband FIFA aus und nahmen von 1924 bis zum ersten WM-Auftritt Englands 1950 an keinem Weltturnier mehr teil. Als neue „Fußball-Großmacht“ etablierte sich in den 1920er Jahren Uruguay, dessen Strukturen im Sportwesen (insbesondere im Fußball) von britischen Einwanderern aufgebaut worden waren. Endlich fand 1924 ein richtiges Weltturnier statt, an dem mit Ägypten, den USA und Uruguay 
immerhin schon drei außereuropäische Teams und damit drei Kontinente beteiligt waren. Die „Usus“ gewannen die beiden letzten Olympischen Turniere vor der ersten offiziellen Weltmeisterschaft im eigenen Land. 1924 besiegten sie im Finale die völlig überraschend so weit vorgedrungenen Schweizer. Im Endspiel von 1928 trafen sie mit Argentinien auf den gleichen Gegner wie zwei Jahre später bei der WMPremiere. Die Idee, eine Fußballweltmeisterschaft zu organisieren, existierte zwar schon seit der Gründung der FIFA 1904, aber erst 1920 bekam sie mit der Wahl der Franzose Jules Rimet zum FIFA-Präsident einen mächtigen Verfechter. 1928 wurde die Durchführung der Fußball-WM im Vier-Jahresrhythmus beschlossen: das erste WM-Turnier sollte, wie schon gesagt, 1930 stattfinden (vgl. SCHEPART 2003).

\subsection{Merkmale des Spiels}

Das Fußballspiel ist wie jedes Spiel gekennzeichnet durch eine spezielle Spielidee; es hat eine nur inm eigene Struktur, durch die es sich von anderen Sportspielen unterscheidet; das Regelwerk gibt dem Spiel seine besondere Prägung. Die Summe der Eigenschaften macht es einzigartig.

Ein entscheidender Grund dafür, dass der Fußballsport so weit vorbereitet ist und vor allem auch von Jugendlichen so gerne betrieben wird, ist der einfache Spielgedanke: Tore ohne Benutzung der Hände schießen und Tore verhindern, das ist die ursprüngliche Idee des Spiels. Die geradezu simple Spielidee erlaubt es, dass unter vereinfachten Regelbestimmungen nahezu überall Fußball gespielt werden kann (vgl. BAUER 2001).

Jede Sportart hat charakteristische strukturelle Merkmale, die bezüglich der Spielidee miteinander wesensverwandt sind. Im Vergleich zu Sportspielen wie Basketball, Handball und Volleyball zeichnet sich Fußball durch folgende Merkmale aus (vgl. Bauer 2001):

- Das Handspiel ist im Fußball als Sonderrecht dem Torhüter in seinem Strafraum vorbehalten.

- Das Spielfeld ist - auch in Relation zur Anzahl der Spieler - größer als in den anderen Sportarten. Das Fußballfeld von $105 \mathrm{~m}$ x $68 \mathrm{~m}$ ist neunmal so groß wie das Handballspielfeld, 20-mal so groß wie das Basketballfeld und etwa 45-mal so groß wie das Volleyballfeld. Der für jeden Spieler anteilmäßig verfügbare Aktionsraum ist somit größer als in den anderen Spielen. 
- Die Anzahl der Spieler ist größer als in den vergleichbaren Spielen: Da beim Fußball 22 Spieler auf dem Feld sind - mehr als in den meisten anderen Sportspielen -, erhöht sich die Komplexität in Entscheidungssituationen.

- Die Trefferquote pro Spiel ist geringer als in anderen Sportspielen; bei dem häufig knappen Spielausgang steigt die Spannung für Spieler und Zuschauer deshalb besonders.

- Die Vorbereitung des Torschusses erfolgt durch einen systematischen Spielaufbau (Kombination), wobei die Mannschaften auch im Mittelfeld intensiv um Ball und Spielanteile kämpfen.

- Die Art der Ballkontakte ist im Fußball einzigartig. So gibt es die An- und Mitnahme des Balles mit dem Fuß, das Kopfballspiel, die Dribblings und Tacklings mit dem Fuß nur im Fußball.

- Die Ballkontaktzeit, d.h. die Zeit, in der die einzelnen Spieler den Ball in Besitz haben, ist - bezogen auf die Spieldauer und auf die Häufigkeit der Ballkontakte - auffallend kurz.

- Die spannenden Zweikämpfe zwischen den Gegenspielern sind bei den Mannschaften sehr häufig. Spielanalysen haben ergeben, dass im Spitzenfußball pro Spiel durchschnittlich etwa 250 Zweikämpfe stattfinden (BAUER 2001, 11).

Ein weiterer Grund für die Faszination des Fußballsports ist seine komplexe Leistungsstruktur. Sie begeistert Spieler und Zuschauer gleichermaßen. Die Leistung im Fußball setzt sich aus einer Vielzahl von Faktoren zusammen; Faktoren, die sich gegenseitig positiv oder negativ beeinflussen können und dadurch den Ausgang des Spiels so schwer berechenbar machen. In Abbildung 1 wird ein Überblick über dieses Bedingungsgeflecht gegeben. Schon beim einzelnen Spieler summieren sich psychische und physische Faktoren zur sogenannten individuellen Leistungsbereitschaft und Leistungsfähigkeit. Motivationale und konditionelle Fähigkeiten sind nur Schlagworte für eine sehr große Zahl einzelner Leistungsfaktoren. Da Fußball eine typische Mannschaftssportart ist, kommt es im Wettkampf immer darauf an, die Leistungspotentiale der einzelnen Spieler für die Mannschaftsleistung als Ganzes nutzbar zu machen (vgl. BAUER 1998). Da es Anliegen der Arbeit ist, beobachtbare Merkmale zu erfassen, seien nur die Komponenten Technik und Taktik explizit ausgeführt. 
Die individuellen Leistungsfaktoren

Geistige Fähigkei-
ten
${ }^{*}$ Aufmerksamkeit
*Wahrnehmung
*Mitdenken
*Antizipation
*Wissen

Temperamentsmerkmale *Durchsetzungsfähigkeit

*Ausgeglichenheit

*Selbstsicherheit

* Impulsivität

*Optimismus
Motive/Emotionen

${ }^{*}$ Angst vor Erfolg

${ }^{*}$ Freude auf Erfolg

*Leistungsstreben

*Geld/Prämien

*Anerkennung

$$
\text { Leistungsbereitschaft } \longrightarrow \text { Spielleistung } \longleftarrow \quad \text { Leistungsfähigkeit }
$$

\begin{tabular}{|l|}
\hline Kondition \\
${ }^{*}$ Kraft \\
${ }^{*}$ Ausdauer \\
${ }^{*}$ Flexibilität \\
${ }^{*}$ Schnelligkeit
\end{tabular}

Technik und Koordination

*Technik mit dem Ball

*Technik zum Ball

*Technik ohne Ball
Taktisches Handeln

*Einzel-Taktik

*Gruppen-Taktik

*Mannschafts-Taktik

*Spielsystem

Alter/Geschlecht

Abb. 1: Die individuellen Leistungsfaktoren und die sie beeinflussen Rahmenbedingungen steuern das Leistungsvermögen des einzelnen Spielers und damit der gesamten Mannschaft (modifiziert nach BAUER 1998).

\subsection{Taktik}

Der Begriff der Taktik hat seinen Ursprung nicht im Sportspiel. Im militärischen Bereich wird er seit Jahrhunderten verwendet und steht hier für geplantes, berechnendes Verhalten, welches nach Nutzenmaximierung und Verlustminimierung strebt und entsprechende Handlungsanweisungen und Entscheidungsalternativen entwickelt. In diesem Sinne wurde er in diesem Jahrhundert auch auf die Politik, die Diplomatie, die Wirtschaft (z.B. Verhandlungstaktik) und schließlich auch auf den Sport übertragen. Seitdem wurde der Taktikbegriff vor allem im Sportspiel „mit großer Selbstverständlichkeit" (BARTH 1994) benutzt und seine herausgehende Bedeutung in der Leistungsstruktur der Sportspiele nie bestritten (BARTH 1994; HAHN 1984; STIEHLER/ KONZAG/ DÖBLER 1988). 
In den Sportspielen versteht man unter Taktik das planmäßige, erfolgsorientierte, auf die eigene und die gegnerische Leistungsfähigkeit und auf die äußeren Umstände abgestellte Verhalten von einzelnen Spielern, Spielgruppen und Mannschaften. Allerdings gibt es bei der Definition des Begriffs unterschiedliche Auffassungen. Vor allem das Begriffspaar Strategie und Taktik wird von Sportspielforschern unterschiedlich dargestellt. So wird Taktik beispielsweise mitunter sehr weiträumig verstanden. Die Inhalte reichen dabei von einer langfristigen Saisonplanung über mögliche Vorgaben des Trainers, mit welcher Abwehrvariante agiert werden soll, bis hin zu konkreten Maßnahmen, die ein Sportler in einer Situation zur Erreichung des Handlungszieles zur Auswahl hat (vgl. HARRE 1971; SCHMIDT 1976; STÖBER 1985; LETZELTER 1985; BISANZ 1994).

Die Begriffe werden aber auch in anderer Weise abgegrenzt. Zur Strategie werden dabei die organisatorischen Aufgaben und übergreifenden Aspekte innerhalb einer Saison gezählt. Die kurzfristigen, Situationsbedingten Prozesse zur Problemlösung, die von den Athleten im Wettkampf bewältigt werden müssen, fallen demnach eindeutig in den Bereich der Taktik (vgl. BARTH 1980; KONZAG 1981; ROTH 1989). Einige Autoren (PETROV 1967; BARTH 1995) schlüsseln das Begriffspaar noch weiter auf. So geht PETROV in seinem Vorbereitungsmodell für Wettkämpfe mit wahrscheinlichen Gegnern von dem „Modellieren einer eigenen Strategie“ für den Wettkampf aus, die sich an den Informationen über die voraussichtlichen Gegner ausrichtet. Noch vor dem Wettkampf werden die strategischen Entscheidungen und taktischen Maßnahmen, die in dem Spiel zur Anwendung kommen sollen, theoretisch erörtert und im Training mit einem „imitierten“ Gegner einstudiert (vgl. GRAELLS 1999, 24).

Am sinnvollsten scheint die Trennung der Bereiche Strategie und Taktik durch die praktische Ausführung der Sportart. Zur Strategie gehört nach dieser Definition der Bereich der Saisonplanung und die Einbeziehung aller externen Faktoren in organisatorischer Hinsicht, wie zum Beispiel die Planung eines Trainingslagers oder die unmittelbare Vorbereitung auf einen Wettkampf. Der Begriff Taktik sollte angewendet werden, sobald die Sportart praktisch ausgeübt wird. 
Das Fußballspiel gehört wie jedes andere Sportspiel zu den Bereichen, in deren Anforderungsprofilen der Taktik eine wesentliche Bedeutung zugeschrieben wird. Die Sportwissenschaftler sind sich darüber einig, dass Sportspiele hinsichtlich ihrer Variabilität und Komplexität der situativen Bedingungen eindeutig höher einzustufen sind als Kampfsportdisziplinen.

Alle Aktionen im Verlauf des Fußballspiels müssen auf ein Ziel ausgerichtet werden. Dieses Ziel, der Erfolg der Mannschaft, kann nur durch das Zusammenwirken aller Spieler erreicht werden. Dazu sind die Aktivitäten der einzelnen Spieler aufeinander abzustimmen und es bedarf einer entsprechenden Taktik. Unter dem Begriff "Taktik" sei daher beim Fußballspiel ein planvolles Handeln, mit dem Ziel, unter den gegebenen Bedingungen ein bestmögliches Resultat zu erreichen, verstanden. In allen Mannschaftsspielen ist darüber hinaus offensichtlich, dass eine erfolgreiche Taktik sich aus Einzel- und Zusammenspiel zusammensetzt (vgl. KOLLATH 2000).

Das Ziel des Fußballspiels besteht darin, selbst Tore zu erzielen und Gegner am Torerfolg zu hindern. Demnach ist die Taktik auch danach einzuteilen, welche vorrangige Aufgabenstellung der Spieler, die Gruppe oder die gesamte Mannschaft in einer bestimmten Situation verfolgen.

Die Qualität des taktischen Handelns ist abhängig von der Schnelligkeit, Genauigkeit, der Zielgerichtetheit, Variabilität und der Stabilität dieser Prozesse. Da diese Prozesse handlungsbestimmend sind, werden wissenschaftliche Untersuchungen durchgeführt, um Hinweise über individuelle Stärken und Schwächen zu erhalten und die besondere Eignung für das individuelle Verhalten und Handeln zu ermitteln (vgl. SAND 1985; BINDING 1987).

Es liegen mehrere Untersuchungen vor, die sich mit den Modalitäten der Laufbewegung von Fußballspielern befasst haben (vgl. WHITEHEAD 1975; REILLY/ THOMAS 1976; WINKLER 1983, 1985; WITHERS 1982; MAYHEW/ WEGNER 1985; ECKBLOM 1986; OHASHI 1988; YAMANAKA 1988). Da die Methode der Datenerhebung, das Spielniveau, die Individual-, Gruppen; Mannschaftstaktik und Spielpositionen von Studie zu Studie differierten (vgl. BUTTLER/ KUHN/MARTIN 1997), soll 
zumindest eine Einführung in den allgemeinen Bereich der Taktik und der komplexen Sportspielleistung gegeben werden.

\subsubsection{Hauptelemente der Taktik}

\subsubsection{Differenzierung und Strukturierung taktischer Handlung}

In die Mannschafts- oder auch Spieltaktik fließen alle einzel- und gruppentaktischen Aktionen ein und verbinden sich zum 11 gegen 11. Somit umfasst die Mannschaftstaktik alle Maßnahmen in der Abwehr und im Angriff, die auf das Verhindern bzw. Erzielen von Toren ausgerichtet sind. In den Sportspielen wird der Kernbereich taktischer Handlungen, wie in Abbildung 2 verdeutlicht, in drei Bereiche gegliedert: die individualtaktischen Handlungen, die teilgruppentaktischen Handlungen, Mannschaftstaktik. In wichtigen Standardwerken der Sportspielforschung verfahren die jeweiligen Autoren nach diesem Modell (TROSSE 1979; BUDINGER/ HILLMANN / STRÖDTER 1980; BISANZI GERISCH 1981; WALDOWSKI 1982).

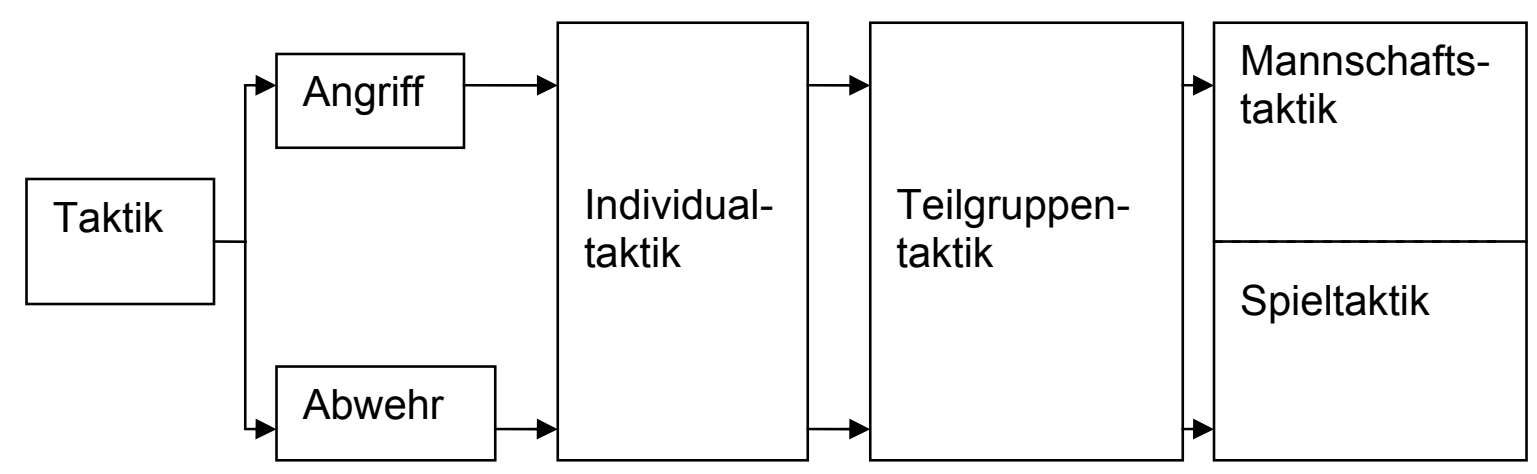

Abb. 2: Taktikbereiche im Sportspiel (modifiziert nach BISANZ/GERISCH 1981).

Die Individualtaktik befasst sich mit den Mitteln, die einzelne Spieler in bestimmten Situationen einsetzen. Diese Maßnahmen haben über die Einzelaktion hinaus auch Bedeutung für eine Gruppe und die gesamte Mannschaft. Sie betreffen den Torhüter, den Libero, den Abwehr-, Mittelfeld- und Angriffsspieler (nach KOLLATH 2000).

Daher werden alle Maßnahmen eines einzelnen Spielers in Abwehr- und Angriffssituationen zusammengefasst. Vor allen der Grundsituation 1:1, also ein Angreifer gegen einen Abwehrspieler, kommt hierbei eine besondere Bedeutung zu. Die Individualtaktik ist die Grundlage für die weitergreifenden und komplexeren Handlungen in 
den übrigen Bereichen der Taktik, insbesondere für die Effektivität der Mannschaftstaktik. Beispiele für die Handlungsselektionen, die zur Individualtaktik gerechnet werden, sind beim Fußballspiel beispielsweise Täuschung, Torschüsse, Ballkontrolle oder Dribbling eines einzelnen Angreifers.

Die Teilgruppentaktik umfasst das Handlungsfeld mehrerer Angreifer und Verteidiger. Die Spielsituationen 3:3 und 4:4 oder 5:5 stehen dabei im Mittelpunkt. Hierbei ist eine genaue Abstimmung der Aktionen untereinander notwendig, um die Handlung erfolgreich abzuschließen. Den teilgruppentaktischen Maßnahmen liegt immer das Ziel des Angreifers zugrunde, durch bestimmte Aktionen einen Verteidiger auszuspielen, um somit eine kurzzeitige Überzahlsituation zu erreichen, die zum Torerfolg führen kann.

Als Beispiele aus der Praxis können für das Angriffsspiel das Richtungswechsel beim Dribbling, das Flügelspiel bzw. der Flügelwechsel oder der Doppelpass exemplarisch genannt werden. In der Abwehr fällt das Übergeben oder Wechseln eines Gegenspielers in den Bereich der Teilgruppentaktik.

Bei der Mannschaftstaktik beziehen sich die Angriffs- und Abwehrmaßnahmen auf alle Spieler einer Mannschaft. Basis für diesen Bereich ist das Beherrschen des individual- und teilgruppentaktischen Repertoires. Beispiele aus dem Fußballsport für mannschaftstaktische Handlungen wären des Zurückkommen oder aber das Überzahlspiel.

Die Spieltaktik ist die Maßgabe taktischer Handlungen in allen drei genannten Bereichen, die genau auf den jeweiligen Gegner abgestimmt sind. Sie wird ausgehend von dem Leistungsvermögen jedes einzelnen Spielers festgelegt und richtet sich nicht nur an der gegnerischen Mannschaft aus, sondern bezieht auch äußere Umstände, wie zum Beispiel Bedeutung der Begegnung, den Tabellenstand, die Art des Spiels und mögliche Störfaktoren mit ein. In diesem Bereich steht vor allem ein Ziel im Vordergrund: Die Stärken der eigenen Mannschaft sollen wirksam eingesetzt und Schwächen des Gegners ausgenutzt werden. 
Abbildung 3 verdeutlicht schematisch die unterschiedlichen Gruppen von taktischen Handlungen. Individual-, Teilgruppen-, Mannschafts- und Spieltaktik gliedern sich vertikal. Auf der horizontalen Ebene sind die möglichen Grundsituationen für die angreifende und die abwehrende Mannschaft dargestellt.

Ein Kriterium bei der Einleitung von Verhaltensweisen und Handlungsselektionen ergibt sich aus der ambivalenten Zielsetzung der Mannschaftsspiele, zum einen selbst Tore oder Punkte zu erzielen, zum anderen die des Gegners zu verhindern. Demnach werden die Spielhandlungen in Angriffs- und Abwehrtaktik unterschieden. Darüber hinaus gibt es in jeder Sportart taktische Vorgaben für die Spieler (z.B. Manndeckung) und man kann besonders im Fußballspiel deutlich sehen, wie sich die einzelnen Elemente eines Sportspiels auf einander beziehen. So wird von den Trainern eine spezielle Taktik der Standardsituationen beim Fußball herausgegeben. 


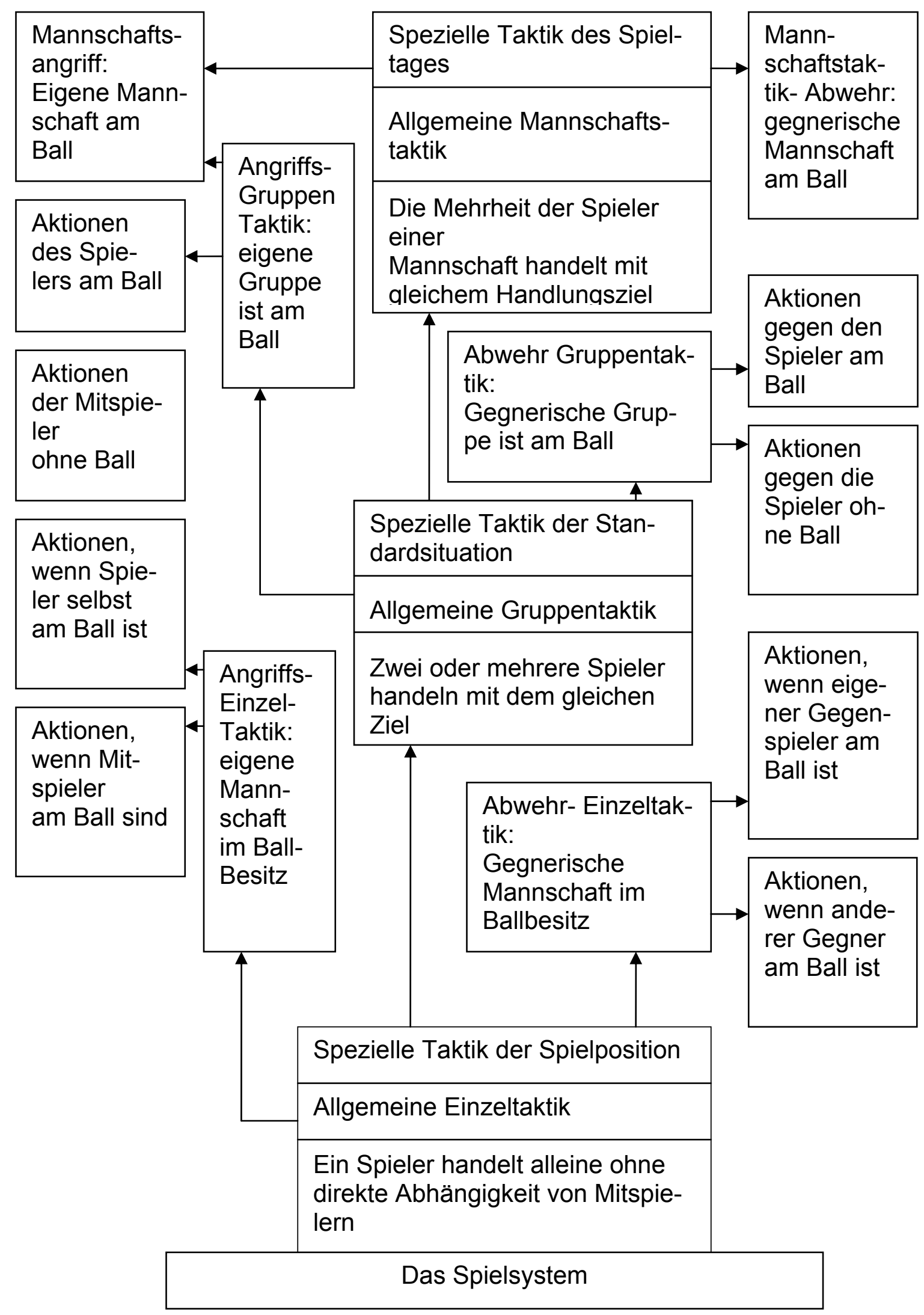

Abb. 3: Gruppen von taktischen Handlungen (modifiziert nach BAUER 2001) 


\subsubsection{Taktische Leistungsfaktoren}

In der Sportspielforschung steht eine Betrachtungsweise von Spielhandlung im Mittelpunkt, die als fähigkeitsorientiert bezeichnet werden kann (vgl. MECHLING/ BÖS 1984; ROTH 1983; FISCHER 1983; BÖS 2001). Bei diesen Ansätzen wird davon ausgegangen, dass Sportler über mehr oder weniger ausgeprägte komplexe Spielfähigkeiten (vgl. KUSNZOW u. a 1980; HOHMANN/ BRACK 1983) verfügen. Diese bilden eine entscheidende Grundlage für letztlich beobachtbare Leistungen. Viele Autoren haben sich mit der schematischen Darstellung der verschiedenen Erklärungsebenen befasst. Dabei wurden in einem mehrdimensionalen Konstrukt differenzierte Bedingungs- und Faktorengefüge entworfen. Die allgemeinen und speziellen Fähigkeiten, Fertigkeiten und Eigenschaften wirken in diesen Modellen leistungsbestimmend und greifen funktional, modifizierend und spezifizierend ineinander.

In Abbildungen 4 werden die drei wichtigen Kategorien Technik, Taktik und Kondition verdeutlicht. KRAUSPE (1981) und auch BRACK (1983) stellen jeweils die Taktik aufgrund des obersten Spielziels, Tore zu erzielen oder zu verhindern, in den Mittelpunkt. Technik und Kondition besitzen demnach keinen Selbstzweck und erfahren ihre Begründung erst mit der Durchführung taktischer Konzepte.

\begin{tabular}{|c|c|c|c|}
\hline \multicolumn{4}{|c|}{ Leistungsvoraussetzungen } \\
\hline \multicolumn{2}{|l|}{ Psychische } & \multicolumn{2}{|l|}{ physische } \\
\hline Intellektuelle & sensorische & $\begin{array}{l}\text { Psychomotorisch- } \\
\text { koordinative }\end{array}$ & $\begin{array}{l}\text { Anatomisch- phy- } \\
\text { siologische }\end{array}$ \\
\hline \multicolumn{4}{|c|}{ moralische Qualitäten } \\
\hline \multicolumn{2}{|c|}{ Technische Fertigkeit } & \multicolumn{2}{|c|}{ Konditionelle Fertigkeit } \\
\hline \multicolumn{4}{|c|}{ Taktische Leistungsfähigkeit } \\
\hline \multicolumn{4}{|c|}{ Spielfähigkeit } \\
\hline
\end{tabular}

Abb. 4: Hierarchisierung der Erklärungsebenen (nach KRAUSPE 1981). 
Damit geht es auch um verschiedene Erklärungsebenen. Wie in Abb. 5 veranschaulicht, wird hier die erste Erklärungsebene von HOHMANN (1985) in den Blick genommen.

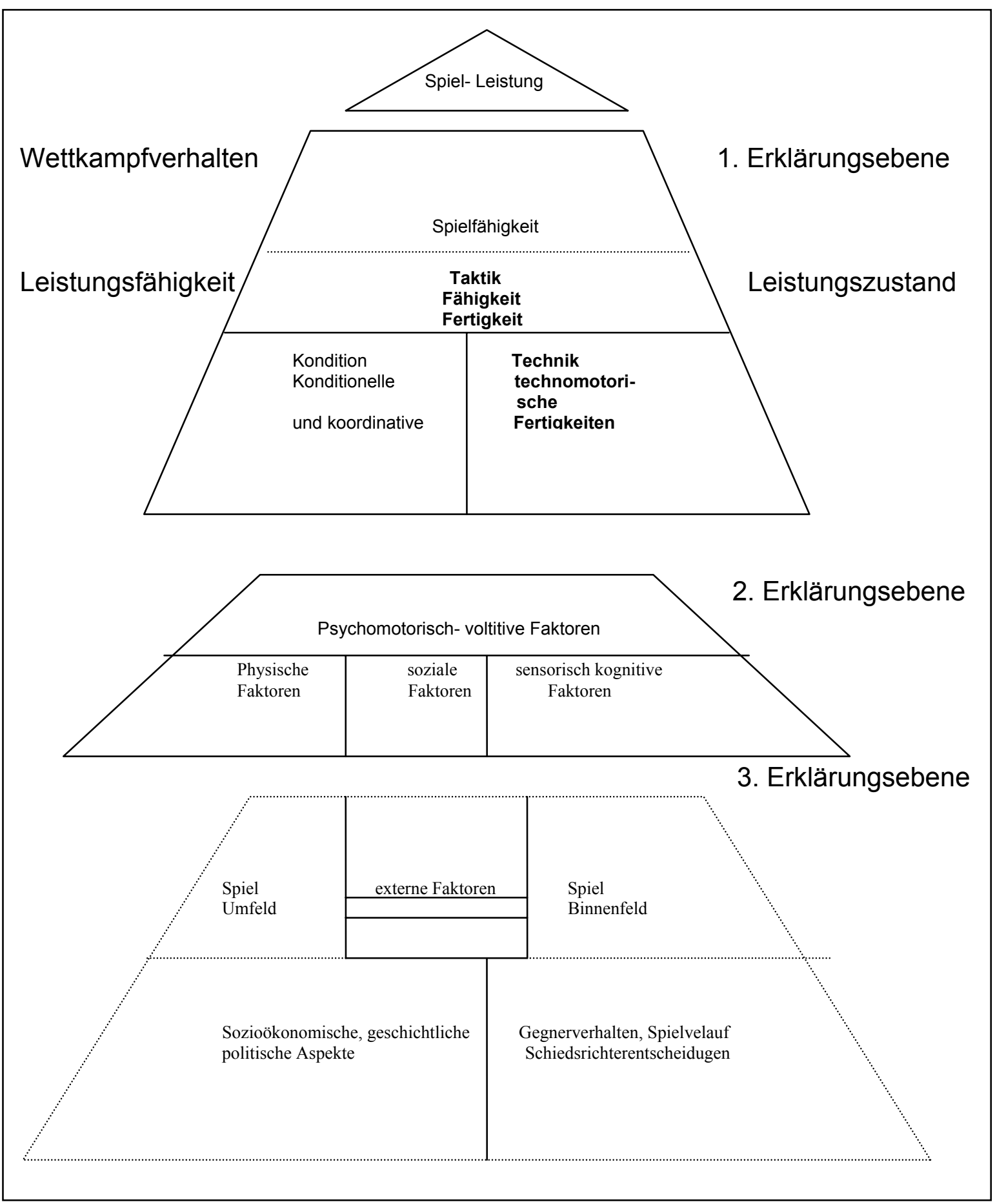

Abb. 5: Hierarchisierung der Erklärungsebenen (nach HOHMANN 1985). 
Auch für den Bereich der Taktik hat sich in der Sportwissenschaft eine Dreiteilung durchgesetzt. Die überwiegende Mehrzahl der Autoren, wie beispielsweise HARRE (1971) oder MATWEJEW (1981), unterscheiden zwischen taktischen Kenntnissen, Fertigkeiten und Fähigkeiten. Diese drei Bereiche sind gleichzeitig zentraler Inhalt des Sportspieltrainings. Zu den taktischen Kenntnissen gehören das Wissen über Spielregeln, Wettkampforganisation, angewendete taktische Grundregeln oder etwa die Prinzipien der Ökonomie und Vielseitigkeit. Mit diesen Kenntnissen ist ein Sportler den theoretischen Anforderungen eines Spiels gewachsen (BAUER 1973).

$\mathrm{Zu}$ den taktischen Fertigkeiten zählen die Autoren ausgebildete und automatisierte Handlungen als Antworten auf Anforderungen und Situationen. Diese Fertigkeiten beziehen sich auf die bereits erwähnten Bereiche taktischen Handelns wie Individualoder Mannschaftstaktik. Die Anwendung und Wiederholung dieser Fertigkeiten zeichnet sich vor allem durch Sicherheit und Genauigkeit aus. Taktische Fähigkeiten grenzen sich von den Fertigkeiten dadurch ab, dass sie situationsübergreifend wirksam sind. Sie erhalten deshalb eine besondere Bedeutung, da der Sportler hierbei nicht auf ein festgelegtes Handlungsmuster zurückgreifen kann, sondern sein sportliches Können der Situation entsprechen muss (vgl. LETZELTER 1980; MAHLO 1965; HARRE 1971).

\subsection{Technik}

Fußballtechnik kann in Anlehnung an die allgemeine Trainingslehre wie folgt definiert werden:

„Fußballtechnik ist die Summe der motorischen Handlungen, durch deren Einsatz ein Spieler die taktischen Aufgaben, die das Fußballspiel an inn stellt, erfolgreich lösen kann. Im Gegensatz hierzu steht die Meinung, Technik im Fußballspiel beinhalte all die Bewegungen, die unter Beachtung der Regeln zur Lösung einer bestimmten Aufgabe gewählt werden“ (BAUER 2001, 12; vgl. auch KOLLATH 2000).

Demnach spielt die Handlungsgenauigkeit für die Leistung im Sportspiel eine große Rolle. Zum einen ist sie das äußere Zeichen erfolgreicher technisch-taktischer Handlungen. Das beweisen die Analysen zur Leistungsentwicklung im Hochleistungssport (DÖBLER/ KRAUSPE 1989; SPÄTE 1992; JENTER/ TÜNNEMANN 1988). Kriterien, 
z.B. an biomechanischen Leitbildern orientierte, lassen aber aufgrund des ausgeprägten taktisch determinierten Wahlcharakters der Motorik keine zuverlässigen Schlüsse bezüglich der Handlungsqualität zu (vgl. ZIMMER 1985).

Den meisten Definitionen zur sportlichen Technik ist der technologische Aspekt gemeinsam, d.h. die Technik hat die Funktion eines Verfahrens zur Aufgabenbewältigung. Bei SCHNABEL (1991) ist die sportliche Technik ein "charakteristisches Verfahren zur Lösung von Bewegungsaufgaben, die im Rahmen sportlicher Handlungen zu lösen sind." Ähnliche Definitionen finden sich bei MARHOLD (1987) und aus der Sicht der Biomechanik bei HOCHMUTH (1974). Die sportliche Technik hat dabei (nach MARHOLD 1987, 22) folgende charakteristische Merkmale. Sie ist:

- den allgemeinen psychophysischen Voraussetzungen des Menschen und den mechanischen Umweltbedingungen angepasst,

- ein in der Praxis erprobtes Verfahren,

- von den Wettkampfbestimmungen abhängig,

- dem Ziel untergeordnet, die höchstmögliche sportliche Leistung zu erreichen und

- der jeweiligen taktischen Situation untergeordnet.

Die Technik im Sportspiel muss sich demnach der hohen Situationsvarianz unterordnen. Sie dient keinem Selbstzweck, sondern immer der Lösung einer taktischen Aufgabe. Die Lösung dieser Aufgabe ist ihr alleiniger Maßstab. Die Sportspieltechnik ist neben der bewegungstechnischen Realisierung auf engstem Raum und unter Gegnereinwirkung mit Handlungsentscheidungen unter Zeitdruck verbunden (vgl. KOTHE/STARK 1996). Aus diesem Grund werden im Gegensatz zu anderen Sportarten Technik und Taktik zum technisch- taktischen Leistungsfaktor zusammengefasst (vgl. STIEHLER/ KONZAG/ DÖBLER 1988).

Eine Technik im Sportspiel ist demnach ein spielcharakteristischer Bewegungsablauf „mit der Funktion der zweckmäßigsten Lösung einer Spielsituation zur Zielgerichteten Umsetzung einer taktischen Konzeption“ (vgl. STIEHLER/ KONZAG/ DÖBLER1988). Neben ihrer taktischen Determiniertheit weist sie noch andere Besonderheiten auf. Typisch ist unter besonderer Beachtung der Mannschaftsspiele Fußball 
- die hohe Ausführungsvariabilität der räumlichen und zeitlichen Parameter,

- Technikkombinationen bzw. Handlungsfolgen,

- die Beeinflussung der Technik durch die Zweikampfführung,

- die Verbindung mit Täuschungshandlungen

(vgl. STIEHLER/ KONZAG/ DÖBLER 1988; KÖTHE/ STARK 1996).

Die Technik-Analyse wird seit vielen Jahren praktiziert. Jedoch wird ihre Entwicklung zu einem systematischen Prozess, der wissenschaftliche Prinzipien und Methoden integriert, erst etwa seit einem halben Jahrhundert mit Publikation dokumentiert. Sie erscheint häufiger als Analyse der Technik (ELLIOTT 1999, ECKBLOM 1994), Analyse des Sporttechnik (BUNN 1972; HAY 1973), biomechanische Analyse der Technik (BARTLETT 1999), biomechanische Analyse der Bewegungen (ADRIAN/ COOPER 1995), Analyse der Sportfertigkeiten (CARR 1997), Untersuchungen der Sporttechnik (BOBER 1981), Analyse der menschlichen Bewegungen (HAY/ REID 1982) und mechanische Analyse der menschlicher Leistung (vgl. LUTTGENS/ HAMILTON 1997).

Im Fußballsport ist die Technik letztlich nicht Selbstzweck, sondern sie ist ein Mittel, um selbst Tore zu erzielen und Tore des Gegners zu verhindern. Die technischen Fertigkeiten des Spielers sind nur insofern von Bedeutung, als sie zum Erfolg der Mannschaft mit beitragen. Spieler, die lediglich technische Kabinettstückchen zeigen, aber nicht fähig sind, ihr Geschick im Umgang mit dem Ball und dem eigenen Körper in taktisch erfolgreiche Spielhandlungen umzusetzen, können zwar die Zuschauer gelegentlich begeistern, ihr Nutzen für die eigene Mannschaft bleibt aber häufig gering (vgl. KOLLATH 2000; BAUER 2001).

Das Fußballspiel zählt sicherlich zu den Sportarten, in denen der Technik eine dominierende Rolle zugesprochen werden kann. Ein großer Teil der Attraktivität dieses Mannschaftssports geht von den „Technikern“ aus, also von den Spielern, die über ein überdurchschnittliches Ballgefühl verfügen. Werden derartige Fähigkeiten einzelner Akteure in den Dienst der gesamten Mannschaft gestellt, so ist damit ein Grundstein für den Erfolg gelegt. 
Die Technik wird dann zu einer erfolgreichen Waffe im Kampf um den Sieg, wenn es der Spieler versteht, sie mit konditionellen Fähigkeiten so zu verknüpfen, dass daraus erfolgreiche taktische Handlungen entstehen. Diese setzen neben physischen Fähigkeiten auch wettkampfrelevante psychische Qualitäten voraus.

Das Hauptanliegen der wettkampfspraktischen Leistungsdiagnostik im Fußballspiel ist die Bestimmung der individuellen Spielleistung sowie die damit verbundene individuell Verlaufsbezogene Formenentwicklung (vgl. FERGER 1997).

\subsubsection{Hauptelemente der Technik}

Die Hauptelemente der Technik im Fußballsport lassen sich in drei Gruppen klassifizieren: Bewegung ohne Ball, Bewegung zum Ball und Bewegung mit Ball. Auch sie seien genauer ausgeführt und am Ende anhand von Grafiken in mögliche Ausprägungsformen zerlegt.

\subsubsection{Bewegung ohne Ball}

Obwohl jeder Fußballspieler bemüht ist, so oft wie möglich in Ballbesitz zu gelangen, bewegt er sich doch eine nicht unbeträchtliche Zeit im Spiel ohne Ball. Das geschieht, je nach Situation, in verschiedenen Bewegungsformen. Üblicherweise werden diese danach unterschieden, ob sich der Spieler mit hohem oder geringem Tempo bewegt. Eine grobe Unterteilung ist aus der folgenden Abbildung $6 \mathrm{zu}$ entnehmen

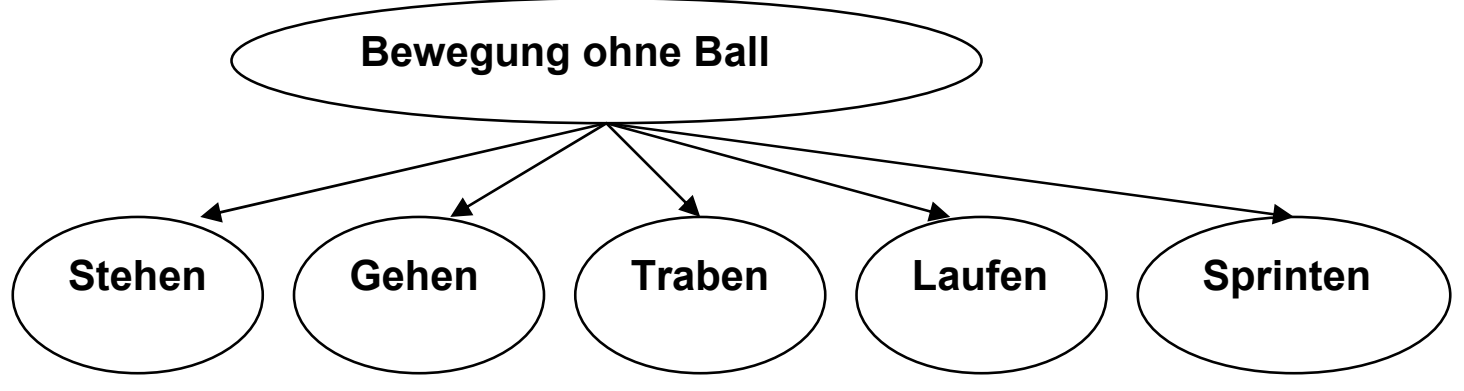

Abb. 6: Bewegung ohne Ball (modifiziert nach KOLLATH 2000). 


\subsubsection{Bewegung zum Ball}

Bei den Bewegungen zum Ball wird versucht, den Gegner gezielt zu stören, um selbst in Ballbesitz zu gelangen. Dabei handelt es sich im Wesentlichen um Techniken des Zweikampfes. Dieser Bereich wird im Training oft vernachlässigt. So findet man in allen Leistungsklassen viele Spieler, die in dieser Hinsicht unübersehbare Schwächen aufweisen. Ungeschickte Bewegung zum Ball ist entweder erfolglos, führt zu einem Foulspiel oder birgt gar die Gefahr eines Straf oder Freistosses in der Nähe die 16 Meter Bereiches (vgl. KOLLATH 2000; MOKHTAR 1998).

Daher ist es angebracht, sich mit der Technik der Bewegung zum Ball näher zu beschäftigen. Zwei Bedingungen müssen bei derartigen Aktionen erfüllt werden: Zum einen ist der Gegner erfolgreich zu stören, vom Ball zu trennen und zum anderen ist dabei regelgerecht vorzugehen. In Abbildung 7 werden die Bewegungen zum Ball dargestellt.

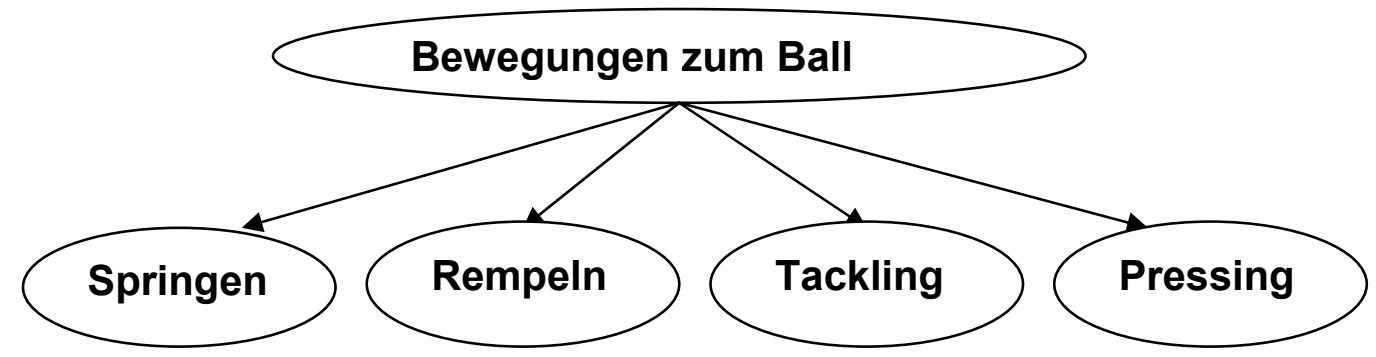

Abb. 7: Bewegung zum Ball (modifiziert nach MOKHTAR 1998).

\subsubsection{Bewegung mit Ball}

Der sicherlich wichtigste Teil der Fußballtechnik ist die Bewegung mit Ball. Die Beherrschung des Balles in den verschiedensten Spielsituationen ist ein zentrales Thema im Techniktraining eines jeden Fußballspielers. Hiermit beschäftigt sich nicht nur die Anfänger, sondern auch die Liga und internationale Spieler widmen einen Teil ihrer Trainingszeit der ständigen Verbesserung und Festigung ihrer Technik. In Abbildung 8 ist die Bewegung mit Ball systematisch gegliedert (vgl. ABO-ELMAGD/ ISMAIL/ SCHAALAN 1993). 


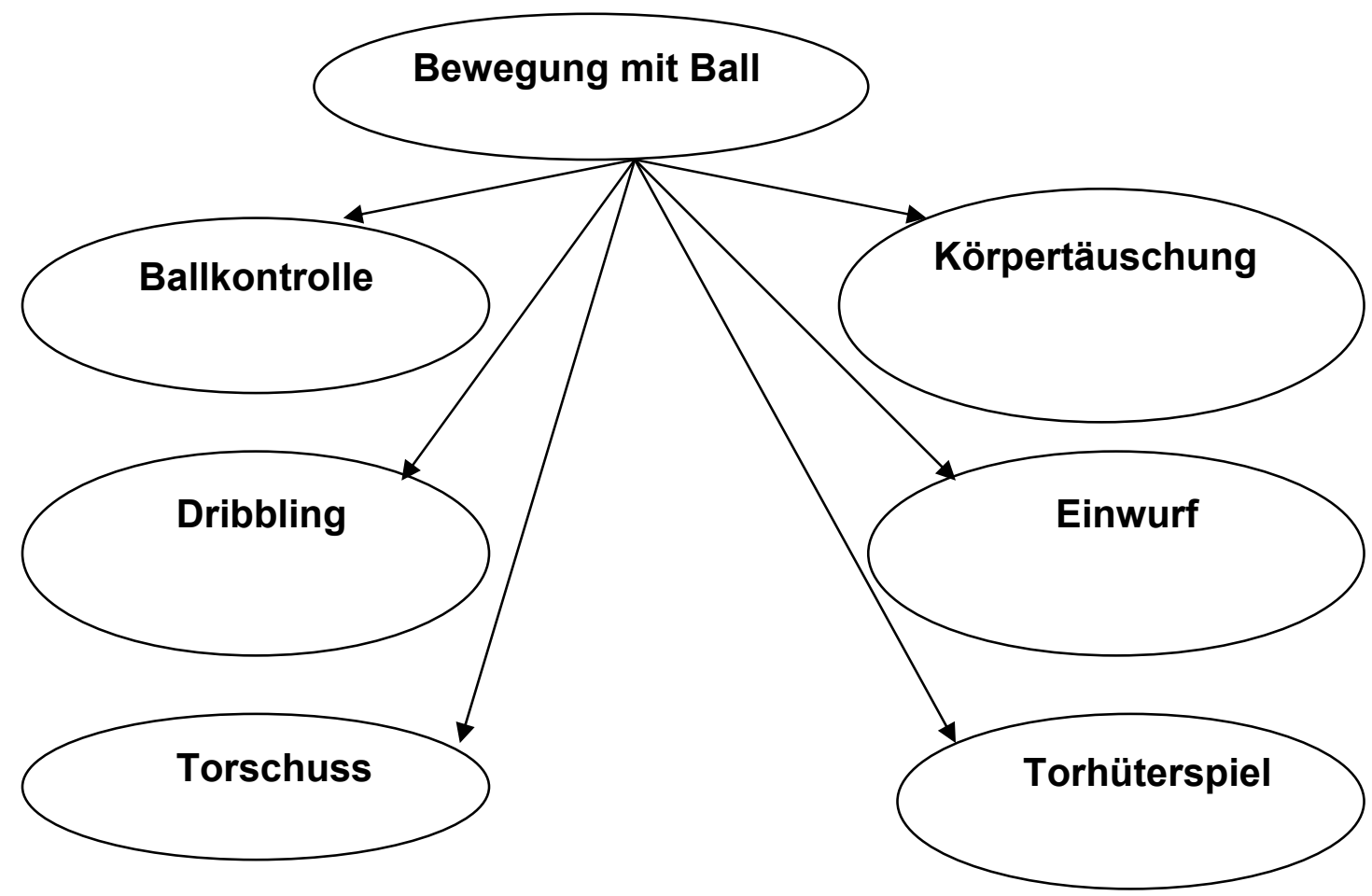

Abb. 8: Unterteilung der Bewegungen mit Ball (modifiziert nach MOKHTAR 1998). 


\section{Systematische Spielbeobachtung}

Mit der vorangegangenen Systematisierung und Differenzierung der einzelnen Elemente des Fußballspiels stellt sich nun die Frage, wie solche Elemente gemessen werden können. In der Sportwissenschaft haben sich verschiedene diagnostische Verfahren zur Analyse der Sportspiele etabliert. Mit Hilfe solcher Vorgehensweisen sollen Rückschlüsse auf die Wirksamkeit von Trainingsinhalten gewonnen werden. Für die Trainingssteuerung und Vorbereitung auf einen Wettkampf werden so die Auswahlentscheidungen der Trainer für bestimmte Maßnahmen erleichtert (vgl. WINKLER 1985).

Vor allem im Bereich der Sportmotorischen Tests wurden in den vergangenen Jahren viele Untersuchungen mit dem Ziel einer Optimierung von sportmotorischen Bewegungsabläufen durchgeführt. Während sich in Individualsportarten mit derartigen Tests gute Fortschritte erzielen lassen, stellen die Sportspiele mit ihrer Komplexität ein besonderes Problem dar. WINKLER/ FREIBICHLER (1991) fordern daher neben sportmotorischen Tests begleitend eine Systematische Spiel- und Wettkampfanalyse. Nicht immer entsprechen die gemessenen Leistungen eines Spielers seinem tatsächlichen Leistungsvermögen, da zu einer vollständigen Beurteilung alle Beobachtungsmerkmale herangezogen werden müssen.

Für WINKLER/ FREIBICHLER (1991) sind auch die in einem Spiel gewonnen „inneren" Informationen durch sportmedizinische Untersuchungen (wie Laktatwert- oder Herzfrequenzbestimmung), biomechanische oder psychologische Verfahren relativ wertlos, falls man nicht die dazugehörende „äußere“ Belstungstruktur erfasst hat: Laufweglängen und Anforderungsprofile an einen Athleten müssen ermittelt werden, um die „inneren“ und „äußeren“ Belastungen, Anforderungen oder Beanspruchungen in Beziehung setzen zu können (vgl. HOHMANN/ BRACK 1983). Für eine solche Diagnose kann man sich häufig der Aufzeichnung von Videobändern bedienen, um die Fehlerquelle zu verringern. Vorteilhaft erscheint dazu, mehr als nur einen Auswerter heranzuziehen und die Auswerter gründlich zu schulen. Dies trifft umso mehr zu, wenn es um die Bewertung und nicht nur um die Übertragung und Auswertung quantitativ bestimmter Daten geht (KRÜGER, 1985, 22). 
Die Computer- und Videotechnologie gehören heutzutage weiterhin zum Alltag vieler Menschen, so dass ihr Einsatz in einem so daten- und bildintensiven Feld wie der Spielbeobachtung eine Selbstverständlichkeit sein sollte (LAMES 1994).

Zudem ist das Spielverhalten beliebig häufig zu analysieren. Auf diese Weise können nach einander mehrere gleichzeitig stattfindende Spielhandlungen erfasst werden (vgl. HOHMANN/ ROMMEL 1994).

In Abbildung 9 sind die unterschiedlichen diagnostischen Verfahren, die in der Sportwissenschaft Anwendung finden, überblicksartig dargestellt. Die richtige Auswahl der Heranwachsenden für eine bestimmte Sportart muss beispielsweise nach Meinung von ELKASHEF (1987) Eigenschaften und Merkmale berücksichtigen, die in einer Sportart leistungsbestimmend sind. Hierzu müssen auch sportmotorische Tests, Spielbeobachtung, pädagogische Maßnahmen, biologische Untersuchungen und psychologische Tests durchgeführt werden (vgl. ELKASHEF 1987). An den Ausführungen wird deutlich, dass die Systematische Spielbeobachtung ein wichtiges diagnostisches Verfahren darstellt, um wichtige Interaktionen bzw. das Gesamtsystem aufzuarbeiten. Die Spielbeobachtung oder die Beobachtung ganz allgemein ist als eine (wenn auch wesentliche) Teilmethode zu kennzeichnen (SASS 1992).

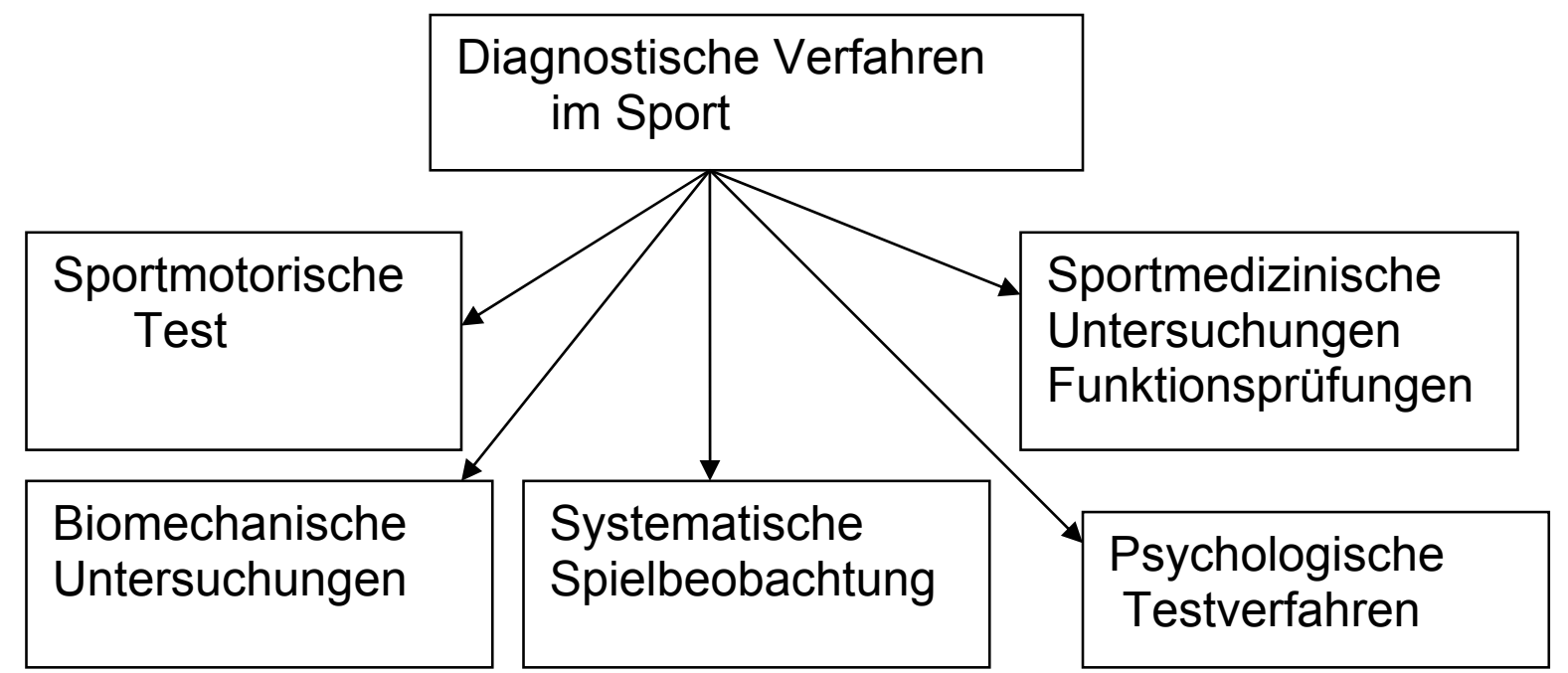

Abb.9: Diagnostische Verfahren der Sportwissenschaft (modifiziert nach CARL 1983). 


\subsection{Abgrenzung und Explikation der Systematischen Spielbeo- bachtung}

Die Systematische Spielbeobachtung bezieht sich auf die Wahrnehmung bestimmter Aspekte des Spiels (vgl. LAMES 1994; WINKLER 1984). In den 60er Jahren des vorigen Jahrhunderts wurden im deutschsprachigen Raum erste Anstrengungen unternommen, die Sportspiele zu beobachten. Dabei stand zunächst die subjektive und unsystematische Eindrucksanalyse von Experten im Vordergrund. Die subjektiven Eindrucksanalysen bezogen und beziehen sich noch heute auf Beobachtung von Trainern und Pädagogen. Die Merkmale sind dabei flexibel und erfolgen ohne eine systematische Fixierung. Die gewonnenen Ergebnisse resultieren ausschließlich aus subjektiven Eindrücken. Die Schwächen eines solchen Verfahrens liegen auf der Hand. Die Anwendung alltagsdiagnostischer Methoden (BÖS 1988) durch Trainer und Pädagogen ist vor allem aufgrund mangelnder Aufnahme- und Speicherkapazität, enormen Stress während des Beobachtungsvorganges sowie verschiedener möglicher Fehlerquellen, die durch nicht normierte Beurteilungsabläufe entstehen, immer fragwürdiger geworden (vgl. HAGEDORN 1979; WINKLER/ FREIBICHLER 1991).

Auch Fallanalysen galten daneben als ein Werkzeug für den Trainergebrauch, um die Spielerbedingungen während des Trainings oder das Zweikampfverhalten zu beschreiben, wobei sie auch heute noch als Methode angewandt wird, um ein geeignetes statistisches Verfahren zu finden. Die Fallanalyse ermöglicht, die Fähigkeiten der Spieler zu bestimmen und gezielt weiterzuentwickeln sowie den Vergleich mit Gegnern (vgl. HUSSEIN/ ABD EL-RAHMAN 1993).

Die Methoden der Spielbeobachtung wurden aber schnell mit dem sog. „Scouting“ zur Analyse des gegnerischen (insbesondere taktischen) Spielverhaltens weiterentwickelt. Das Scouting basiert darauf, eine genauere Beobachtungsform für den Trainer bereitzustellen, die es ihm ermöglicht, sowohl die eigene als auch die gegnerische Mannschaft während des Spiels zu beobachten. Dieses Verfahren wurde zum Auskundschaften von Beobachtungseinheiten wie zum Beispiel taktischen Varianten oder technischen Stärken einzelner Spieler auch subjektiven Eindrücken des Beobachters besonders verwendet (vgl. GRAELLS 1999). Dies kann auch heute Trainern 
dabei helfen, eine Spieltaktik zu entwickeln. Dazu benötigt man eine Gruppe von Personen, die die gesammelten Daten und Informationen notieren und analysieren, denn wegen der Geschwindigkeit des Spiels und der großen Anzahl von Spielern beim Fußball ist es schwierig, alles exakt und umfassend zu erfassen, was auf dem Spielfeld geschieht. Da eine Diagnose durch Gruppen nicht immer möglich ist, meint DESUKY (1994), dass es fruchtbringend sei, sich auf Bestandteile der Technik zu konzentrieren, um daraufhin gezielte Formen der Leistungssteigerung zu bestimmen.

Die genannten Methoden haben den Vorteil, dass durch das direkte Beobachten im Stadion schnell Lösungen gefunden werden können. Dabei können auch die Spieler beobachtet werden, die nicht am Ball sind, wohingegen man bei einer nachträglichen Analyse von Fernsehbildern darauf angewiesen ist, den Ausschnitt zu analysieren, den der Kameramann aufgenommen hat - also meist nur die Spieler in Ballbesitz. Allerdings weisen die Methoden auch Mängel auf, sodass der Ruf nach Alternativen laut wurde, um den Trainern mehr objektive Informationen über ihre Mannschaften sowie die gegnerischen Mannschaften zu geben (vgl. für Ägypten z.B. ELSAYED 1997), denn die Qualität der Analyse hängt letztlich von der Fachkompetenz des Beobachters ab. In Tabelle 2 werden die jeweiligen Eigenschaften der unterschiedlichen Verfahren dargestellt, wobei auf die Systematische Spielbeobachtung im Folgenden eingegangen wird.

\begin{tabular}{|l|l|l|}
\hline $\begin{array}{l}\text { Subjektive } \\
\text { Eindrucksanalysen }\end{array}$ & Scouting & $\begin{array}{l}\text { Systematische } \\
\text { Spielbeobachtung }\end{array}$ \\
\hline - flexible Merkmale & - festgelegte und fle- & - genau festgelegte \\
- ohne systematische & xible Merkmale & Merkmale \\
Fixierung & - teilweise schriftliche & - systematische \\
- subjektive & Fixierung & Fixierung \\
Eindrucke & - Eindrücke und & - Beobachtung \\
& Beobachtung & \\
\hline
\end{tabular}

Tab.1: Darstellung verschiedener Verfahren der Sportspielbeobachtung (modifiziert nach LAMES 1994). 
Die ersten Ansätze, typische Verhaltensweisen von Mannschaften und Spielern im Wettspiel systematisch zu beobachten und zu registrieren, entstanden in den 30er und 40er Jahren des 20. Jahrhunderts in den angelsächsischen Ländern (vgl. BORN 1983).

Obwohl erste Ansätze einer qualitativen Spielbeobachtung bis in die Mitte 1970er Jahre zurückverfolgt werden können (vgl. SCHMIDT 1979), liegen der deutschen Sportwissenschaft bislang im Gegensatz zu systematischen Verfahren nur relativ wenige Arbeiten vor (vgl. z.B. BREMER 1985; AUGUSTIN 1997; HEIN 1993). Gleichzeitig ist darauf hinzuweisen, dass es über eine methodologische Einordnung der qualitativen Spielbeobachtung bislang noch keine Einigung gibt. Nachfolgend soll daher mit LAMES/ HANSEN (1976) eine erste Begriffseingrenzung erfolgen:

„Qualitative Spielbeobachtung ist eine analytisch- interpretative Variante der Sportspielbeobachtung, die speziell für die Kopplung von Training und Wettkampf entwickelt worden ist. Das Verfahren findet durch eine quantitative Vorstrukturierung sowie eine qualitative Hauptanalyse statt. Ziel ist die Beschreibung und Analyse der Stärken und Schwächen der eigenen bzw. gegnerischen Mannschaft unter Berücksichtigung der qualitativen Methodologie“" (LAMES / HANSEN 1976, 34).

Seit den 80er Jahren treten mehr und mehr die beiden letztgenannten Beobachtungsformen in den Vordergrund. Film- und Videoaufzeichnungen erlauben dabei eine objektive Beobachtung (unabhängig vom jeweiligen Beobachter) und garantieren, dass den Beobachtern etwa durch Ermüdung keine Fehler unterlaufen.

Bisher findet die Systematische Spielbeobachtung allerdings nicht die Aufmerksamkeit, die ihr aufgrund ihres Potentials zur Erschließung von Leistungsreserven eigentlich zukommen sollte. Einer der Gründe hierfür ist, dass im Bereich des Sportspiels ein sinnvoller Einsatz der Systematischen Spielbeobachtung ohne eine fundierte Konzeption nicht möglich ist. Dies hängt im Wesentlichen mit der Leistungsstruktur der Sportspiele zusammen, in der Leistung nicht einfach per Bandmaß oder Stoppuhr ermittelt werden kann. Darüber hinaus liegen kaum Lehrbücher vor, die den aktuellen Stand der technischen und konzeptionellen Entwicklung der Systematischen Spielbeobachtung wiedergeben. 
Resümierend meint KRÖGER (1992) sogar, dass ohne Spielbeobachtung im Sportspiel kein Erfolg möglich sei („Ohne Beobachtung geht es nicht“, S. 58). Die Beobachtung taucht häufig als Bestandteil anderer Verfahren der Datenerhebung (z.B. Experiment) auf und erhält dort Exklusivität als spezifisches Datenerhebungsverfahren, wo andere Verfahren nicht greifen, so z.B. bei der Erfassung des Wettkampfgeschehens.

\subsection{Spielbeobachtung vs. Spielerbeobachtung}

Beobachtende Sportspielforschung befasst sich mit der alltagsnahen Analyse von Wettspielen und ist insoweit Feld- und nicht Laborforschung. Sie bedient sich der Spielerbeobachtung, wenn sie am Verhalten einzelner Spieler interessiert ist, und der Spielbeobachtung, wenn das Spiel selbst und/oder das Kollektivverhalten der Spieler erhoben werden soll. Letztere wird hauptsächlich für die trainingswissenschaftliche Leistungsdiagnostik eingesetzt; die Forschungsperspektive kann damit erweitert werden auf Erkenntnisse über Mannschaften (vgl. CZWALINA 1988).

Obwohl die Gütekriterien zunächst nur an die empirischen Methoden des Testens im engeren Sinne geknüpft worden sind, sind sie „in unterschiedlich starkem Maße auf alle Methoden der empirischen Sozialforschung“ anzuwenden; die Sinnhaftigkeit und Angemessenheit dieser Forderung für die Spielbeobachtung sowie ihre Zweckmäßigkeit sollen hier untersucht werden (CZWALINA 1992, 69).

Das Beobachten von Interaktionsverläufen (vgl. SCHMIDT/HAGEDORN 1972; HECKER 1974) hilft, um kollektives Verhalten von Ballspielmannschaften zu erschließen und daraus Maße (Normprofile) für derartiges Gruppenverhalten zu entwickeln. Mittels statistischer Methoden lassen sich Generalisierungen (vgl. HELLER/ ROSEMANN 1974) vornehmen, die zu einer Theorie der Sportspiele ausgebaut werden können. Angesichts solcher Forschungsabsichten wird es nötig sein, über eine lediglich dichotome Betrachtungsweise des Spielerverhaltens hinaus auch dessen Dimensionierung zu analysieren, also seinen Ausprägungsgrad unter dem „Aspekt des Mehr oder Weniger“ zu erfassen (HELLER/ ROSEMANN 1974). 
Aber auch wenn das Forschungsinteresse ausschließlich auf das Spiel gerichtet ist, kann man empirisch- analytisch das Spiel selbst nur über das Verhalten der Spieler erschließen. Spielerverhalten stellt quasi die operationalisierte Form des Spiels dar. Aus diesem Grund lassen sich die Erkenntnisse zur Spielerbeobachtung auch im Rahmen einer Spielbeobachtung verwenden.

\subsection{Spielbeobachtung als Spielanalyse}

In jüngerer Zeit wird an der Entwicklung eines zumindest für den professionellen Fußball interessanten „Medien-Integrations-System“ gearbeitet. Dieser Medienkomplex besteht aus Computer, angeschlossenem Videorecorder mit Monitor sowie Eingabe- und Steuerungsvorrichtungen nebst einem Programmsystem. Ein solches System verspricht folgende Vorteile: Daten lassen sich problemlos eingeben, effizient auswerten und grafisch veranschaulichen. Aufschlussreiche Handlungseinheiten wie Torszenen oder Standardsituationen können systematisch erfasst, separat überspielt und in unmittelbarer Folge abgerufen werden. Vor allem dieser gezielte Zugriff zu wichtigen Spielszenen ist für die Trainings- und Wettkampfsteuerung von hohem Wert und sollte bei der Kosten- Nutzen- Kalkulation ein wesentliches Argument sein (vgl. BISANZ/ GERISCH 1997).

Die folgenden Ausführungen sollen nun das Besondere an der als Spielanalyse verstandenen Systematischen Spielbeobachtung herausstellen. Dazu sei zunächst eine breit akzeptierte Klassifikation angeführt, die relevante Aspekte betont. Meist findet dies in der Gegenüberstellung von Gegensatzpaaren ihren Ausdruck (siehe im Überblick Abb. 10; vgl. auch HOHMANN 1985; LAMES 1991): 


\section{Systematische Spielbeobachtung}

\section{Andere Varianten} der Beobachtung
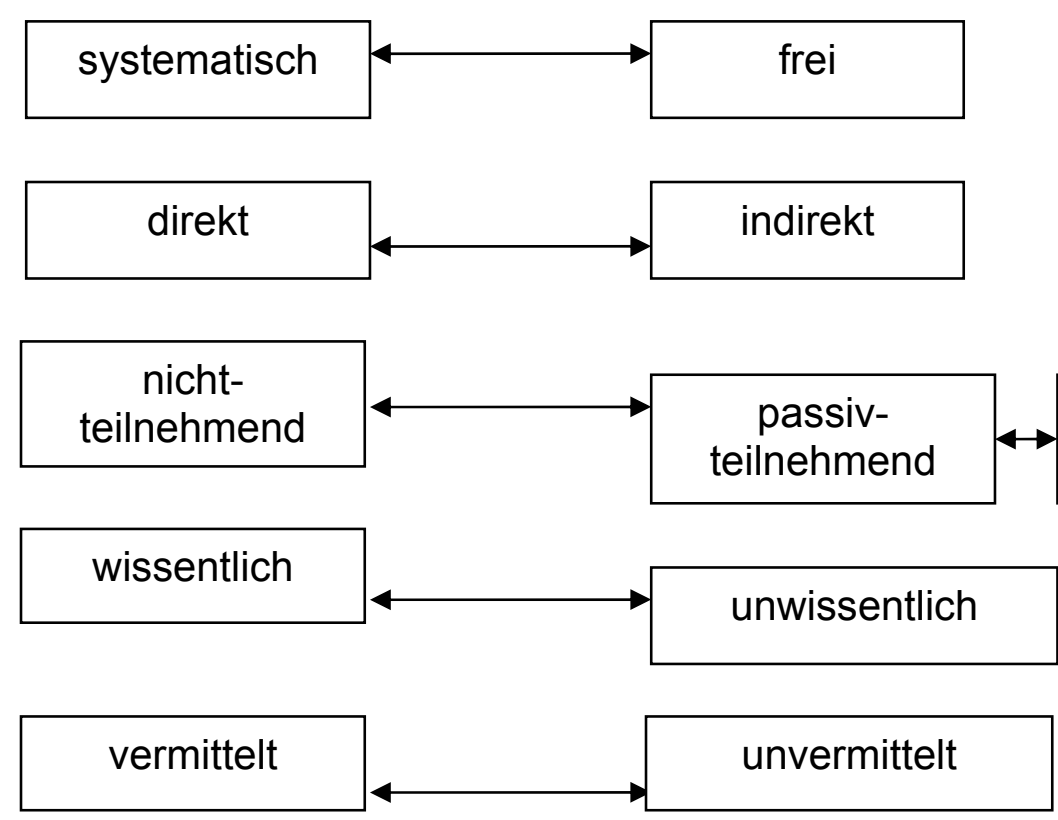

Abb.10: Varianten der Beobachtung (nach LAMES 1994).

Von einer nicht- teilnehmenden Beobachtung kann im strengen Sinne nur dann die Rede sein, wenn der Beobachter nicht in die Verhaltenssituation involviert ist, also lediglich Registrierapparaturen (Videorekorder, Kameras, Mikrofone....) installiert sind. In allen anderen Fällen sind anwesende Beobachter entweder aktiv- teilnehmend - der Beobachter tritt in Interaktion mit den Beobachteten - oder passiv- teilnehmend, wenn der Beobachter möglichst wenig in Erscheinung tritt (vgl. auch schon ATTESLANDER 1975). Die Variante des Teilnehmens ist grundsätzlich von dem Grad der Reaktivität, vom Grad des Einflusses der Beobachtung auf das Verhalten, zu trennen. So können Messapparaturen stark reaktiv sein - jeder Hobbyfotograf kennt den Kameraeffekt, während ein teilnehmender Beobachter unter Umständen auch kaum das Verhalten der Beobachteten beeinflusst (LAMES 1994, 36).

Die Systematische Spielbeobachtung bedient sich häufig der nicht - teilnehmenden Beobachtung, das Geschehen wird zunächst lediglich apparativ registriert, beispielsweise durch Mitschnitte auf Video, und erst anschließend ausgewertet. Passiv- teil- 
nehmend sind Kameraleute oder neutrale Beobachter. Ein vom Trainer bestellter Beobachter wäre aktiver einzustufen, und vollends die Grenze zur aktiv- teilnehmenden Beobachtung hätten Trainer und Assistenztrainer als Beobachter überschritten.

Mit obigem Verständnis richtet sich die Systematische Spielbeobachtung zunächst vor allem auf Spielerverhalten, das sich u.a. in sportspielmotorischen Fertigkeiten niederschlägt. Derart technisches Verhalten im Spiel lässt sich aber von einem Nichtteilnehmenden Beobachter am Rande des Spielfeldes sehr viel besser erkennen. Das resultiert vornehmlich aus der kurzen Aktionsdauer des Spielverhaltens, z.B. beim Torschuss oder Pass. Angesichts der Vielzahl solcher kurzfristigen Spieleraktionen bereitet das zusätzliche Protokollieren des Beobachteten erhebliche Schwierigkeiten für einen Beobachter, der mitspielt oder schiedsrichtert. Sie ist von ihm aufgrund mangelnder Gedächtnisleistung nach Spielende mit der nötigen Genauigkeit nicht zu leisten. Somit kommt für eine exakte Analyse nur die nicht-teilnehmende Spielerbeobachtung in Betracht.

„Bei dem Begriffspaar vermittelt / unvermittelt hat sich in den vergangenen Jahren die Auffassung durchgesetzt, dass es sich bei der Systematischen Spielbeobachtung im Sport immer um eine vermittelte Beobachtung handeln sollte. Um das Sportspielgeschehenen in seiner Komplexität systematisch und objektiv zu erfassen, ist eine technisch vermittelte Beobachtung durch eigene Wahrnehmungsprobleme, die Dichte der Interaktionen bei Sportspielen und die Vielzahl an Handlungsalternativen völlig überfordert “ (GRAELLS 1996, 43).

Bevor im Folgenden auf einzelne Methoden der Systematischen Spielbeobachtung eingegangen wird, seien nun genauer die angerissenen Ziele einer solchen Analyse aufgearbeitet.

\subsection{Ziele der Spielbeobachtung}

Warum befasst man sich überhaupt mit Spielbeobachtung und wozu kann sie dienen? Damit ist die Frage nach dem Begründungs- und Verwendungszusammenhang eines Projekts gestellt, das sich der Spielbeobachtung widmet. Die Bewegungsanalyse ist der erste grundlegende Schritt für die Beobachtung der 
Sportaktivitäten. Sie ist die wichtigste wissenschaftliche Basis bei der Erklärung und der Ausarbeitung von Programmen, sowohl im Lehr- und Ausbildungsbereich als auch beim praktischen Training (HOSNY 1974). Die Systematische Sportspielbeobachtung verfolgt das Ziel, eine exakte Analyse und Beurteilung des Spielgeschehens zu ermöglichen, um jene den Spielverlauf und den Spielausgang mitbestimmenden Strukturen zu erschließen. Während es im Fußball gelungen ist, entscheidende Strukturmerkmale sowie die wechselseitige Abhängigkeit von Aktionen und Interaktionen der Spieler zweier Mannschaften mit und ohne Ball festzuhalten, muss die Entwicklung der fußballspezifische Sportspielforschung vor allem unter forschungsökonomische und technologische Aspekten betrachtet werden (SCHMIDT 1991).

Dabei ist der Zusammenhang zwischen Begründung und Verwertung sehr eng zu sehen; beides bedingt sich wechselseitig. Diese Interdependenz machen die folgenden Punkte deutlich. Ihre Reihenfolge besagt nichts über deren Wichtigkeit. Eine Hierarchisierung oder Prioritätenliste ist damit nicht verbunden. Der Anwender von Spielbeobachtung bestimmt letztlich, unter welchem der nachstehenden Gesichtpunkte er sie einsetzt. Ist auch mit dem Gebrauch empirisch- analytischer Methoden stets eine quasi methodenimmanente Ideologie verbunden, so ist die Spielbeobachtung, lässt man diese prinzipielle „Beschränkung“ außer Acht, wie andere Methoden auch interessenoffen und damit vielseitig einsetzbar. Spielbeobachtung lässt sich nutzen u.a.

- zur Leistungsverbesserung von Spielern und Mannschaften in technischer und taktischer Hinsicht. Durch Beobachtung der eigenen wie der gegnerischen Spieler kann das Leistungsniveau beider Mannschaften ermittelt werden sowie die Stärken und Schwächen einzelner Spieler;

- zum Leistungsvergleich zwischen Training und Wettkampf. Erhebliche Differenzen zwischen Trainings- und Wettspielleistung eines Spielers sind meist auf die nicht voll kalkulierbare Störgröße Gegner und das veränderte psychische Klima („Ernsthaftigkeit“ von Punktspielen, Anwesenheit von Zuschauern u.a.) des Wettkampfes zurückzuführen. Andererseits kann beides auch auf einen trainingsschwachen Spieler als Leistungsstimulans wirken. Beim Vorliegen solcher Effekte ist die „Bedeutung der Wettkämpfe für die Entwicklung der Leistungsfähigkeit“ (HARRE 1971) durch den Trainer zu bedenken; 
- zur Objektivierung der Leistungsmessung;

- zur Kontrolle von Längsschnittuntersuchungen über Mannschaften, die in stets derselben Zusammensetzung gegen ebenfalls konstant homogene Mannschaften antreten (vgl. SÖLL 1973, 191);

- zur Diagnostik des technomotorischen Qualifikationsniveaus einzelner Spieler. Aufgrund der gewonnenen Diagnose können Lehrer und Trainer ihre Spieler in Bezug auf deren sportspielerischen Werdegang beraten. Damit wird eine gezielte Spieler- und Trainingsberatung ermöglicht (CZWALINA 1992);

- zur Entwicklung von Normprofilen als Leistungsstandard, der als numerische Bezugnorm gilt, zu der durch Spielbeobachtung erhobene Individualdaten eines speziellen Spielers in Relation gesetzt und damit bewertet werden können (FETZ 1973).

Durch die Entwicklung in Wissenschaft, Technologie und Kunst sei heute ein spezieller Bereich entstanden, der den Einfluss dieser wissenschaftlichen Beobachtungsmethoden begrenzt haben. Jetzt sei es die Aufgabe, die Beobachtung zuzuordnen, die ihren Fähigkeiten und Potentialen entsprechen. Durch diese Forderung soll ein möglichst hohes sportliches Niveau erreicht werden (vgl. ABO-ELELA/ ELROBY 1986).

BADAWY fordert, dass die Spielbeobachtung im Bereich der Trainingswissenschaft die Vielseitigkeit der verschiedenen Sportarten zu einer effektiven Kette verbinden muss, sodass gemeinsame Ziele im Hochleistungssport erreicht werden können. Das sportliche Training und die Leistungsmaximierung der Sportspielbeobachtung bis hin zu Meisterschaftsleistungen sind dabei als das Ergebnis vieler Bemühungen, komplexen Prozessen anzusehen, wobei der Trainingswissenschaft eine wichtige Aufgabe zukomme (BADAWY 1983). Die Spielbeobachtung ist den methodischen Kriterien (u.a. der Offenheit in der Erhebungs- und Interpretationssituation) eng an die begleitenden Trainings- und Wettkampfprozesse und damit prinzipiell an die notwendige Berücksichtigung von Kontextinformationen gebunden.

Jede Interessengruppe (wie Sportwissenschaftler oder Trainer) verfolgt, wie schon gesagt, bei der Anwendung oder dem Gedanken an eine Spielanalyse eine unterschiedliche Zielsetzung. Die Anwendbarkeit steht dabei im unmittelbaren Zusammenhang mit der gewollten zu beobachtenden Ausprägung. Die Anforderungen der 
Praxis und Sportwissenschaft an die Spielanalyse hängen letztlich von der Qualität des Beobachtungssystems ab (vgl. SCHMIDT 1992).

Auch die auf Erklärung gerichtete Sportwissenschaft verfolgt bei der Erarbeitung und Umsetzung bzw. Anwendung von Spielanalysesystemen und den unter diesen Vorstellungen konzipierten Beobachtungsmethoden vielschichtige Zielsetzungen (POMMERENKE 1997). Es geht dabei um die Analyse von Spielstrukturen und die Aufschlüsselung Leistungsbestimmender Faktoren sowie um eine globale und differenzierte Leistungserfassung bei Spielern und Mannschaften. Dazu zählen die qualitative Bewertung technischer, taktischer Handlungen und konditioneller Leistungen sowie die Quantifizierung einzelner Leistungen im konditionellen und im technischtaktischen Bereich (vgl. GERISCH 1989).

Eine qualitative Spielanalyse meint das Erfassen und Auswerten bestimmter, für unterschiedliche Zielsetzungen und Zielgruppen relevanter Spielstrukturen oder Spielszenen. Dazu gehört eine entsprechende Medienverarbeitung (TV/Video/Computer) für Informations- und Feedbackzwecke. Bei einer quantitativen Spielanalyse geht es um das numerische Erfassen von bestimmten, für unterschiedliche Zielsetzungen und Zielgruppen relevanten Ereignissen. Dazu gehört die entsprechende statistische Verarbeitung und (Ergebnis-) Darstellung für Informations- und Feedback- Zwecke.

Die einzelnen Handlungen eines Spielers oder die Ketten von Spielerhandlungen sind also sowohl unter qualitativem Aspekt sichtbar, wenn innerhalb einer Spielanalyse, zum Beispiel bei einem Torerfolg von Mannschaft A, nicht nur die Anzahl der verschiedenen taktischen Fehler von Mannschaft B erfasst und statistisch dargestellt werden (quantitativer Aspekt). Wenn die Suche nach den Ursachen der Fehler mit der Intention verbunden ist, diese Fehler per Video zu dokumentieren und für die Spielerschulung einzusetzen, handelt es sich um einen qualitativen Aspekt. Aber auch nur mit quantitativen Ergebnissen lassen sich Aussagen über qualitative Aspekte, zum Beispiel der Spielstärke einer Mannschaft, treffen.

In der Trainingspraxis können solche Erkenntnisse letztlich im Bereich der LeistungsTrainings- und Effektivitätskontrolle eingesetzt werden. Die Daten können außerdem der Vorbereitung auf einen Wettkampf, der Steuerung des Verlaufes oder der Dokumentation von sportlichen Wettkämpfen dienen (vgl. GRAELLS 1999). 


\subsection{Methoden der Spielbeobachtung}

Wie schon erwähnt, gibt es verschiedene Methoden, das Spiel zu analysieren. Trotz zahlreicher Unterschiede zwischen den verschiedenen Methoden weisen alle das gemeinsame Ziel der Beobachtung, Bewertung und Messung der Leistungsfähigkeit von Einzelpersonen auf, unabhängig davon, ob diese Methoden subjektive oder objektive Verfahren benutzen (ABD EL-MONEM/ HASANEN 1992).

In den Gegensatzpaaren wurden bereits die unterschiedlichen Möglichkeiten der übergeordneten Herangehensweise deutlich. Primär bedient sich die Sportspielforschung der Methoden empirischer Sozialforschung: Freie Spielbeobachtung, Aufnahmeprotokolle auf Formblättern, Beobachtungsbögen mit vorgegebenen Kategorien und Symbolen sowie Rastern für grafische Darstellungen, Tonbandnotizen mittels Diktiergerät, Videoaufzeichnungen, Computergestützte Aufzeichnungen. Die verschiedenen Formen der Spielbeobachtung unterscheiden sich hinsichtlich ihrer Systematik, Präzision, Praktikabilität und Ökonomie, insbesondere ihres Materialund Zeitaufwands sowie ihres Informationsgehalts. Die Entwicklung wird zeigen, inwieweit sich durch ein in den Sportspielen bereits angewandetes oder in der Erprobung befindliches Verfahren, die "Computergestützte Spielanalyse“ auch im Fußball Perspektiven eröffnen. Dieses Verfahren ermöglicht die unmittelbare Datenverwertung für die Sofortinformation sowie eine spätere detaillierte Datenaufbereitung und auswertung.

Die Aufbereitung der qualitativen Methodologie zeigt, dass sehr vielfältige, keinesfalls aber einheitliche Verfahren und Konzepte entwickelt wurden, die sich durch eine strenge Orientierung an Forschungszielen und den Bedingungen im Feld auszeichnen. Aufgrund dieser Feldorientierung sowie dem interpretativen Vorgehen lassen sich in der qualitativen Methodologie methodische Vorbilder für die Kopplung von Training und Wettkampf durch Spielbeobachtung finden (LAMES/ HANSEN 1975).

In Abbildung 11 werden die unterschiedlichen Untersuchungsformen und ihre Anwendungsbereiche veranschaulicht und systematisiert, wobei die computergestützte Spielbeobachtung in der Zukunft einen immer größeren Raum einnehmen wird. Mit dem zunehmenden Einsatz von Hard-Software werden EDV- Aufzeichnungen immer 
mehr Einzug halten (ALLENDORF/BRETTSCHNEIDER 1976; WIDMAIER/GAISSER 1984; HOHMANN 1985).

\section{Systematische Spielbeobachtung}

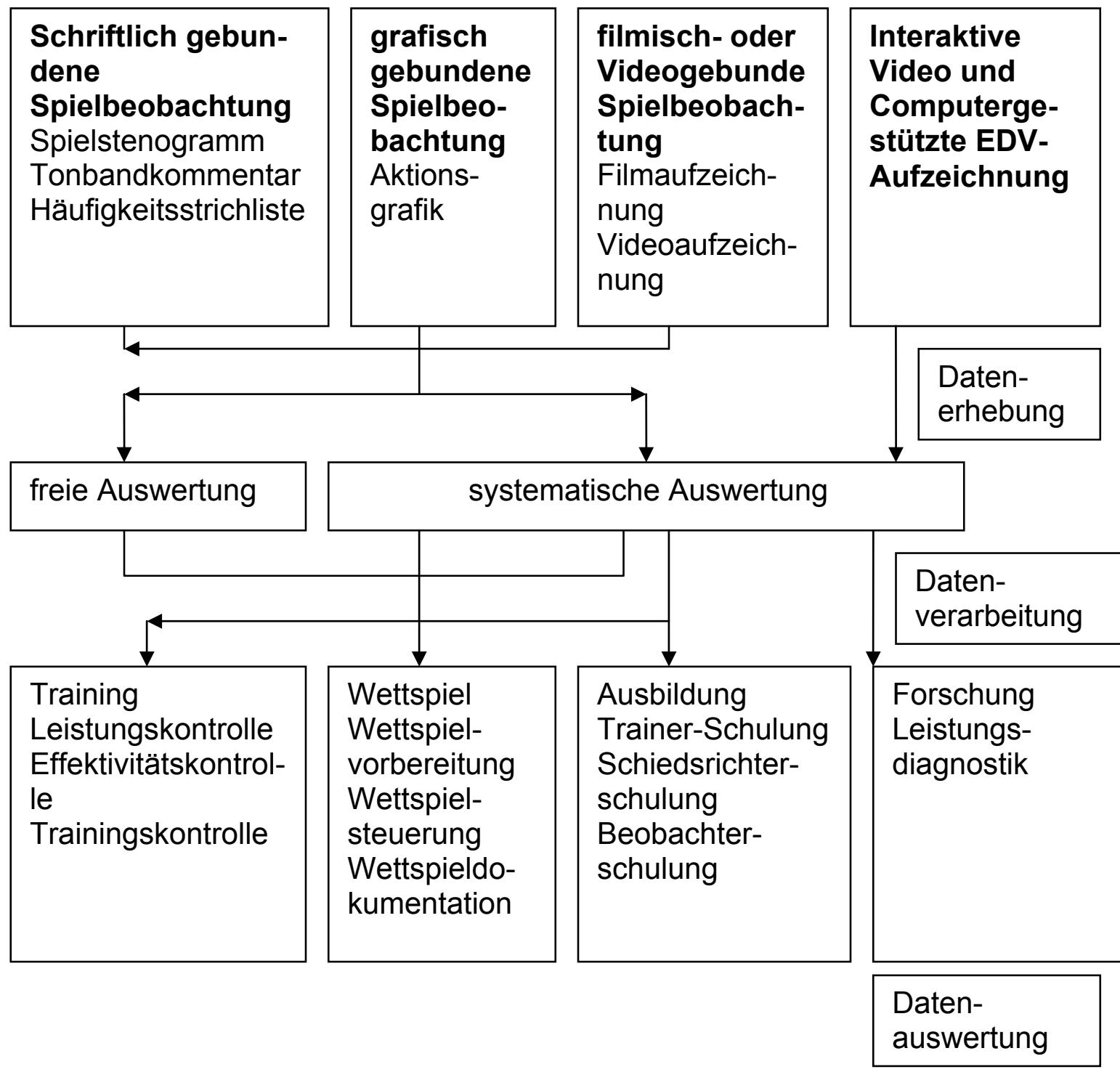

Abb.11: Formen und Anwendungsbereiche der systematischen Sportspielbeobachtungen (modifiziert nach HOHMANN 1985; vgl. auch HOHMANN/ ROMMEL 1994). 


\subsection{Qualitative und Quantitative Spielbeobachtung}

Eine Beobachtung entspricht so gut wie nie einer realitätsgerechten Abbildung des zu Beobachtenden. Verständlicherweise ist dies nicht einfach: Das Publikum bringt beispielsweise den Schlussfolgerungen von sog. WM-Analysten oder (vermeintlichen) Experten Vertrauen entgegen, auch wenn es die Entwicklung der Aussagen nicht nachvollziehen kann. Ein Wissenschaftler hingegen muss erst einmal ein Arsenal an Tabellen und Algorithmen vorweisen und sein Vorgehen begründen. Dabei ist u.a.

- der konzeptionelle Bezug offen zu legen;

- die Quelle der untersuchten Variablen zu nennen;

- eine Einbettung in die Praxisanwendung zu erbringen;

- die wissenschaftliche „Güte“ muss nachvollziehbar sein.

Viele für den Wettkampfsport verantwortliche Personen betonen die Notwendigkeit des Einsatzes exakter Methoden bei der Erlangung ausführlicher Informationen sowohl über ihre eigene als auch über die gegnerische Mannschaft. Diese Informationen liefern dem Trainer analytische Hinweise, die die eigene Mannschaft zum Erfolg führen kann.

Im Folgenden soll die unterschiedlichen Merkmale der qualitativen und der quantitativen Spielbeobachtung verdeutlicht werden. Diese Begriffe sind in vielen Wissenschaftsdisziplinen anzutreffen. Das Gegensatzpaar qualitativ - quantitativ tritt in vielerlei Zusammenhängen auf. Diese reichen von wissenschaftstheoretischen Grundpositionen bis zu Spielarten erhobener Daten bzw. Skalenqualitäten (bei der qualitativen Spielbeobachtung handelt es sich gewöhnlich um nominalskalierte Daten). Auch in der Sportspielforschung, hier vor allem im Bereich der Spielbeobachtung, wird zwischen diesen Ansätzen unterschieden.

Grundsätzlich unterscheidet man in (vgl. YASIN 1987):

\section{A. Qualitative Analyse}

1. Qualitative Analyse, die anstrebt, die Arten der Hauptbestandteile des Phänomens zu kennzeichnen.

2. Qualitative Analyse, die anstrebt, die Hauptbestandteile des Phänomens zu bewerten, indem es z.B. mit Vergleichen arbeitet. 
B. Quantitative Analyse, die genaue Quantitäten oder Prozentsätze der unterschiedlichen Bestandteile des Phänomens mittels des Messens und des Zählens anstrebt.

Bei der quantitativen Analyse geht es um langfristige und kurzfristige Informationen hinsichtlich kollektiver und individueller Analysen (Mannschafts- und Spieleranalyse):

- zur gezielten Trainings - und Wettkampfsteuerung im technisch -taktischen Bereich,

- zur Überprüfung der eigenen Trainingskonzeption,

- zur Überprüfung von Spielauffassungen und Spielkonzeptionen,

- zur Leistungsüberprüfung,

- zur Festlegung von Spielpositionen (Formationen),

- zur Gegnerbeobachtung und Gegnereinstellung,

- zur Überprüfung des Entscheidungsverhaltens der Spieler.

Laboranalysen werden durchgeführt, indem man die Aufgaben definiert, die Verantwortlichkeiten, den Grad der Kontrolle sowie Leistungsfähigkeit und Wissensstand. Fußball entzieht sich aber gerade der Standardisierung (Ausnahme: Ecken, Elfmeter), wodurch im Labor nicht das untersucht werden kann, was man vorgibt zu untersuchen (KRÜGER 1985).

Viele Trainer oder Sportwissenschaftler haben erkannt, dass quantitative (statistische) Informationen hilfreich sind, um z.B. das Augenmerk auf bestimmte Mängel zu richten. Bedeutender für die praktische Arbeit eines Trainers hinsichtlich der Verbesserung der Spielerleistungen ist jedoch die Untersuchung qualitativer Aspekte. „Zur Erstellung eines Analyseplanes ist es deshalb wichtig, festzuschreiben, welche Inhalte (Kategorien/Merkmale) Gegenstand der Untersuchung sein sollen“ (vgl. WINKLER 1997, 69).

Unter dem Begriff „quantitative Spielanalyse“ versteht man also das Beobachten und die numerische Erfassung von bestimmten Ereignissen, verbunden mit der entsprechenden statistischen Verarbeitung, tabellarischen/grafischen (Ergebnis-) Darstellung und fachlichen Interpretation. 
Beispiele von Ereignissen sind Torschüsse, gewonnene Zweikämpfe, Deckungsfehler, Fehler beim Zweikampf, Fehler beim Freilaufen, Fehler bei Standardsituationen. Wichtig ist, dass bei der Erstellung eines Analyseplanes ein Ereignis vom Trainer (oder Sportwissenschaftler) möglichst genau definiert wird (z.B. wann handelt es sich um einen Zweikampf? Wann um einen Deckungsfehler?), damit möglichst objektive Untersuchungsergebnisse gewonnen werden können (vgl. WINKLER 1997). Im Anschluss daran wird versucht Spielszenen hinsichtlich der Güte von Handlungen, des Verhaltens und Könnens einzelner Spieler als auch die Analyse der Spielstruktur einer Mannschaft vorzunehmen.

Als Forschungsparadigma versucht der qualitative Ansatz, das Geflecht der Wahrnehmungen und Interpretationen eines Individuums in seiner Umwelt wissenschaftlich zu rekonstruieren. Typische Merkmale der qualitativen Methodologie sind (LAMNEK 1989 zit. nach LAMES 1994, 42):

- Offenheit bezüglich des theoretischen Konzepts und der Erhebungssituation;

- der Zugriff auf die Wirklichkeit in einer sozialen Situation wird als kommunikativer Akt aufgefasst, und

- die Erschließung des Sinngehalts einer sozialen Situation geschieht bewusst interpretativ.

Methodische Anleihen für die qualitative Spielbeobachtung wurden vor allem bei der Qualitativen Inhaltsanalyse nach MAYRING (1995) gemacht. Die Qualitative Inhaltsanalyse stellt eine Methode dar, wie aus umfangreichem Textmaterial durch eine analytische Zerlegung in einzelne Arbeitsschritte, durch Regeln geleitet und intersubjektiv nachvollziehbar, Interpretationen gewonnen werden können. Dieses Verfahren wurde übertragen auf die Gewinnung von Interpretationen aus umfangreichem Bildmaterial, den Videoaufnahmen von Sportspielen, was den Kern der Qualitativen Spielbeobachtung ausmacht (ALAA 1985). 
Bei der Qualitativen Analyse wird Videoinformation durch Computergestützte, integrierte Videosteuerung und/oder als integrierter Videoschnitt verwendet. Auf der Basis objektiv gewonnener Daten (Datenbank) kann der Trainer das Spielverhalten hinsichtlich seiner Auffälligkeiten beurteilen. Die Datenbank hilft ihm dabei, Datenauffälligkeiten zu identifizieren bzw. lohnende Analysestellungen zu finden (HEIN/ PERL 1992).

Ihr Arbeitsziel ist die Begrenzung die verschiedenen Basisinhalte. Sie bezweckt die genaue Analyse in Werten und prozentualen Rechnungen der verschiedenen Basisinhalte der Erscheinungen durch Messen (MAHMOOD 1994).

Für LAMES (1994) liegt der wesentliche Punkt der systematischen, qualitativen Spielbeobachtung darin, dass sie in ein festgelegtes Beobachtungskonzept eingebunden ist und das Spielgeschehen kategorial beschreibt, um Hypothesen überprüfen zu können.

Probleme der systematischen Beobachtung sind evident. Beispielweise umschreibt (CZWALINA 1976, 22) eines mit „ungenügend gesicherte Interpretationsobjektivität. "Wer systematisch beobachten will, muss selektieren, abstrahieren, klassifizieren, systematisieren, relativieren“. In dieser Reduktion verkümmert der Gesamtzusammenhang, die Relationen verlieren sich. Der Vorwurf von BREMER $(1986,7)$, die Systematische Spielbeobachtung könne nur Aktionen mit und am Ball erfassen und ließe die taktische Dimension aus, trifft bei neuren Untersuchungen jedoch nicht mehr in dem Maße zu. Durch geeignete Beobachtungsmodelle und eine gezielte Merkmalsauswahl wurden diese Vorwürfe entschärft.

ESSER $(1983,4)$ hat schon aus wissenschaftstheoretischer Sicht zur Frage „Qualitative und quantitative Methoden- eine Scheinkontroverse“ Stellung bezogen. Er begründet die Fragestellung mit dem zunehmenden Unbehagen über das vorgebliche „Überhandnehmen einer zur Routine erstarrten „ quantifizierenden Sozialforschung“, die zudem durch „Irrelevanz, Ungültigkeit, Nicht- Beachtung der Kontextbestimmtheit sozialen Handelns und Farblosigkeit“ gekennzeichnet werden könne. Er stellt dem die Ansprüche der qualitativen Methoden gegenüber, die geprägt seien von unmittelbarer Nähe zum sozialen Geschehen. Ausgewählte Stärken und Schwächen beider 
Forschungsparadigmen sind ohne Anspruch auf Vollständigkeit in Tab.2 zusammengefasst. Daran wird deutlich, dass eine Berücksichtigung oder Mischung beider Untersuchungsmethoden erfolgversprechend sein kann.

\begin{tabular}{|l|l|}
\hline \multicolumn{1}{|c|}{ Quantifizierende Verfahren } & \multicolumn{1}{|c|}{ Qualitative Verfahren } \\
\hline Exakt und objektiv & Subjektiv bewertend \\
\hline $\begin{array}{l}\text { Vollständige Erfassung der Bausteine } \\
\text { taktischer Handlungen }\end{array}$ & $\begin{array}{l}\text { Arbeitet nach dem Verfahren des „event- } \\
\text { sampling“ }\end{array}$ \\
\hline $\begin{array}{l}\text { Statistisch verarbeitbar und damit hypo- } \\
\text { thesen- prüfend }\end{array}$ & $\begin{array}{l}\text { Keine Überprüfung von Hypothesen } \\
\text { möglich aufgrund fehlender Stichproben- } \\
\text { theorie }\end{array}$ \\
\hline $\begin{array}{l}\text { Stuative Aspekte nur in Rahmen der O- } \\
\text { perationalisierungsmöglichkeiten berück- } \\
\text { sichtigbar }\end{array}$ & $\begin{array}{l}\text { Berücksichtigung der situativen Gege- } \\
\text { benheiten }\end{array}$ \\
\hline $\begin{array}{l}\text { Wahrnehmungsaspekte bleiben außer } \\
\text { Betracht }\end{array}$ & $\begin{array}{l}\text { Berücksichtigung der subjektiven Sicht- } \\
\text { weise der Handelnden }\end{array}$ \\
\hline
\end{tabular}

Tab.2: Vor- und Nachteile der quantitativen und qualitativen Beobachtungsverfahren, (modifiziert nach ESSER 1983).

\subsection{Videosysteme in der Spielbeobachtung}

Mit der Erfindung des Videorekorders vor etwa 50 Jahren hielt diese neue Technologie nachfolgend auch im Sport schnell Einzug. Seit Mitte der 60er Jahre nutzen Trainer und Sportpädagogen die Möglichkeit der Aufzeichnung von Bewegungsablaufen aber auch von Sportspielszenen (ZIELINISKI 1986).

Ihre Attraktivität im Training besteht auch heute in relativ niedrigen Kosten, Zugänglichkeit und hohem Nutzen. So sind Sportspiele und insbesondere das Fußballspiel sorgfältig geplant. Die Trainer und ihre Spieler verbringen Tage, Wochen und Monate vor wichtigen Spielen mit der Analyse von alten Spielen und planen die Taktik für das bevorstehende Spiel. Die praktikabelste und vielleicht auch einzige Möglichkeit, diese Analyse durchzuführen, ist das wiederholte Anschauen verschiedener Spiele und der Versuch, die wichtigen Informationen zu gewinnen. 
Damit kam auch Dynamik in eine bis dahin belächelte Disziplin der Sportwissenschaft. Für die Systematische Spielbeobachtung für die wissenschaftliche Analyse, die bis dahin einzig auf einer schriftlich gebundenen Beobachtung wie des Scoutings basierte, ergaben sich neue Perspektiven, vor allem für die Optimierung der Trainingspraxis (DAUGS/ BLISCHKE/ MARSCHALL/ MÜLLER 1990). Damit man eine Fußballspiel-Aufnahme zeitsparend und effizient analysieren kann, muss auch der Experte dann zu Computer, Videorecorder und speziell entwickelten Systemen greifen, die das ganze Verfahren automatisch durchführen und die Ergebnisse speichern.

Video wird auch als ein den obigen Ausführungen entsprechendes Mittel für das Erhalten qualitativer Information über die Leistung verwendet. In Verbindung mit Computern können z.B. mit Hilfe der Wiederholungen von Spielszenen, Echtzeit, dreidimensionalen Simulationen oder Überlagerung von graphischen Vektordarstellungen Analysen erfolgen und gezielte Rückmeldungen gegeben werden. Weniger abstrakte und wichtige Information kann mit Hilfe der Videowiedergabetechnologie erhalten werden; zum Beispiel für den unmittelbaren Vergleich der Leistung von einem Athleten mit einem anderen.

Ein gutes Videoband ist also ein Schlüssel zu genauen Ergebnissen. Die Aufzeichnung erfolgt von einem Punkt aus mit einer klaren Sicht auf das Spielfeld (am besten von einem erhobenen Punkt wie einer überdachten Zuschauertribüne oder einem Hügel). Die Kamera soll während des ganzen Spieles aufnehmen und nicht abgeschaltet werden, wenn der Ball aus dem Spiel ist, wenn Spieler ausgewechselt werden oder während Verletzungspausen. Sie soll aber hauptsächlich der Bewegung des Balls folgen. Damit Informationen einer Partie nicht verloren gehen, muss das Video gleich kommentiert werden, beispielsweise auch, um Spieler zu identifizieren. Innerhalb der Systematischen Spielbeobachtung wurden verschiedenen Varianten der Videobeobachtung entwickelt. Eine interessante Variante für die Analyse basiert auf einem Zusammenschneiden von passend transformierten und graphisch verbesserten Bildfolgen (LIEBERMANN u.a. 2002).

Vor allem für den Bereich des Sportspiels stand dabei die Frage im Vordergrund, wie eine möglichst große Anzahl an Spielern auf den Aufzeichnungen zu sehen ist, um 
einen umfassenden Überblick über die teilgruppentaktischen und mannschaftstaktischen Handlungen zu erreichen (vgl. GRAELLS 1999).

Am Beispiel der Spielbeobachtung im Fußball lässt sich diese Problematik am deutlichsten aufzeigen. Die Fernsehbilder lenken das Auge des Zuschauers fast ausnahmslos auf den Ball. In diesem Umfeld befindet sich jedoch nur eine geringe Anzahl an Spielern, so dass taktische Analysen anhand von Fernsehbildern fast ausschließlich zu individualtaktischen Leistungen der unmittelbar in Ballnähe befindlichen Spieler möglich sind. Teilgruppen- oder mannschaftstaktische Handlungen können somit ebenso wenig beurteilt werden wie konditionelle Komponenten.

WINKLER (1985; WINKLER/FREIBICHLER 1991) hat diese Problematik aufgegriffen und umfassende Forderung an die Systematische Spielbeobachtung gestellt. Neben der exakten Festlegung der Analyseziele muss vor der Beobachtung die Wahl der Methodik und Technologie getroffen werden. Die wechselseitigen Abhängigkeiten von Zielen und Technologien sind in Abbildung 12 dargestellt und nachfolgend erklärt.

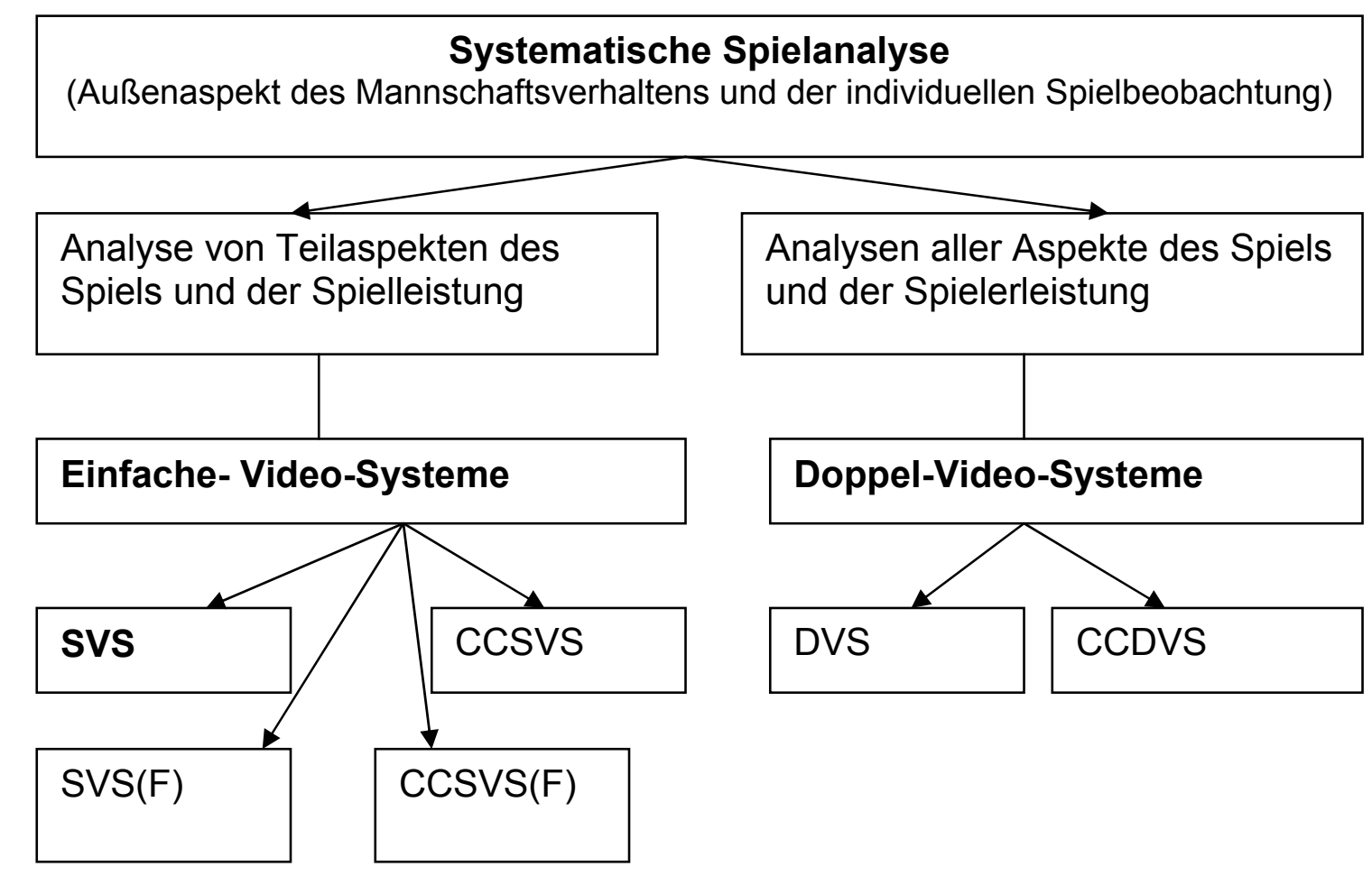

Abb.12: Systematische Spielanalyse (modifiziert nach WINKLER/FREIBICHLER 1991). 
Für die Analyse von Teilaspekten sind Einfach- Video- Systeme demnach ausreichend. Dabei wird in so genannte SVS (Single- Video- System) und CCSVS (Computer- Controller- Video- Systems) unterschieden. Zu den SVS gehören auch Fernsehaufnahmen wobei Kamera und Brennweiten ( $F$ ) frei gewählt und den jeweiligen Situationen angepasst sind. Für wissenschaftliche Untersuchungen bietet sich jedoch das Feststellen der Brennweiten an (SVS F). Dabei werden bei der Spielbeobachtung im Fußballspiel cirka zehn Spieler erfasst, durch Einsatz des High Definition Videosystems (HDVS) erhöht sich diese Zahl sogar auf bis zu 14 Spieler.

Sämtliche Aspekte eines Fußballspiels lassen sich nur mit einem Doppel-VideoSystem erfassen. Dabei werden zwei Videokameras auf einer Schiene nebeneinander montiert und auf einem Stativ angebracht. Beide Aufnahmegeräte können somit parallel geschwenkt werden, was allerdings durch den großen Spielfeldausschnitt, der hierbei erfasst wird, nur selten notwendig ist. Auch hier wird zwischen einem Dual-Video-System (DVS) und Computer-Controller-Dual-Video-System (CCDVS) unterschieden.

Eine entsprechende Software (FREIBICHLER 1983) steuert das Abspielen der gewünschten Bandschnitte mit den erforderlichen Geschwindigkeiten (Zeitlupe, Standbild). Außerdem ist der Anwender ohne großen Zeitaufwand in der Lage, mit zwei Videorecordern gewünschte Spielszenen zusammenzuschneiden. Die Einbindung von Tabellen und Grafiken in die Computerausdrücke ist mit Hilfe einer derartigen Software ebenfalls problemlos möglich. Bei der raschen Entwicklung auf dem Computermarkt ist die Grenze der Möglichkeiten einer derartigen Analyse noch lange nicht ausgeschöpft (vgl. GRAELLS 1999).

\subsection{Formen und Anwendungsfelder der Spielanalyse unter besonderer Berücksichtigung von Fußball}

Im Basketball hatte die Spielbeobachtung von jeher einem besonderen Stellenwert. Das Scouting als eine Form der schriftlich- gebundenen Beobachtung wurde in den USA bereits früh entwickelt und fand vielfach auch Anwendung im deutschen Basketball. Neben Fragestellungen zur Taktik (ALPHEIS 1979; KOZOSCA 1982; HAGEDORN/ LORENZ/ MESECK 1982), Technik (ERRAIS/ WEISZ 1980), Kondition 
(BÖS 1987) oder Pädagogik (KUGELMANN/ BERL 1979) ging HAGEDORN (1980) grundsätzlich der Frage nach, warum eine Computergestützte Spielbeobachtung im modernen Basketball erforderlich scheint. Die Komplexität eines Sportspiels und die große Anzahl an Einflussgrossen im Verlauf einer Begegnung machen die Einschätzung einer Sportspielleistung nur schwer möglich. Mit dem Einzug moderner Technologien in die Sportwissenschaft bietet sich auch eine Computergestützte Verarbeitung der gewonnen Daten einer über Spielbeobachtung an, um Leistungsbestimmende Faktoren zu erfassen und Rückschlüsse auf den Trainingsalltag ziehen zu können. Somit könnten Trainings- und Wettkampfleistungen genauer diagnostiziert werden. Eine Klärung von Zusammenhängen zwischen Training und Wettkampf erscheint ebenso einfacher. Zudem lassen sich technische Kriterien einer beobachteten Spielleistung aufschlüsseln und vor allem Kenntnisse über Handlungsstrategien und taktische Leistungen nachvollziehen.

Zahlreiche Publikationen zur Taktik im Volleyball befassen sich mit der Frage des Angriffsverhaltens (vgl. z.B. KRAKE 1988; HEUCHERT/ PIGORS/ THOSS 1988). FROHREICH (1992) unterteilt in seiner Betrachtung der Begegnungen bei der Weltmeisterschaft 1986 die Zuspiele der Angriffsspieler nach Höhe und Entfernung und schätzt anschließend anhand von Wertungskriterien die Wirksamkeit von angriffstaktischen Handlungen. In einer weiteren Arbeit beschäftigte sich FROHREICH (1992) mit der Entwicklung der Riegelabwehr von der Europameisterschaft 1983 bis hin zur Europameisterschaft 1991.

Im Handball griffen zahlreiche Autoren in ihren Beiträgen zur Spielbeobachtung taktische Fragestellung auf (SCHNELLER 1989, SCHUBERT 1986) die Position des Torwarts (z.B. SINGER 1983). KLEIN/KOCH/ ELLERBROCK (1983) analysierten beispielsweise die Leistungen der Torhüter bei der B- Weltmeisterschaft 1983. Dabei griffen die Autoren auf ein Bewertungssystem zurück, dass die Leistung eines Torhüters anhand gehaltener Bälle mit resultierendem Ballbesitz für die eigene oder gegnerische Mannschaft und nicht gehaltene Bälle unterteilt. 
In den letzten zwei Jahrzehnten, hat nach dem ersten Weltkongress zum Fußball 1987 ein enormes Wachstum der Forschung und Publikationstätigkeit stattgefunden, die sich direkt auf Fußball beziehen (vgl. REILLY / GILBOURNE 2003). Da die Leistungsdiagnostik bei Individualsportarten leichter ist, kam die Entwicklung demgegenüber aber erst langsam in Gang. Neben vielfältig interessierenden Aspekten wie anthropometrische Profile oder körperliche Vorbereitungen (vgl. REILLY / GILBOURNBE 2003), Mehrfachwahlentscheidungen (TEIPEL / GERISCH / SCHÄFER 1985, bei der WM 1982), psychologischer Situation von Torwart und Schütze bei Strafstößen (KUHN 1985) und der Analyse von Kommunikationsstrukturen unter den Spielern (RUTEMÖLLER 1994, bei der WM 1994 in den USA) hat auch die Erforschung des Spiels durch Methoden der Spielbeobachtung zugenommen und stellt eine große Herausforderung dar.

Im Fußball bieten in erster Linie die großen Turniere bei Welt- oder Europameisterschaften sowie herausragende Spiele in den Wettbewerben für Vereinsmannschaften auf europäischer Ebene Anlass für eine Untersuchung des taktischen Verhaltens in unterschiedlichsten Bereichen. Zuerst wurden genaue Beschreibungen von Journalisten vorgenommen. PONSONBY (1974), ein auf Fußball spezialisierter Sportjournalist, konzentrierte sich in seinen Ausführungen hauptsächlich auf das Endspiel der Fußballweltmeisterschaft 1974 (Deutschland - Holland). Die Datensammlung erfolgte durch eine schriftlich gebundene Beobachtung mit Hilfe vorgefertigter Beobachtungsbögen. Dabei registrierte er den Ballbesitz der angreifenden Mannschaft in Tornähe, d.h. einer Entfernung zum gegnerischen Tor von höchstens 30 Yards. Außerdem notierte er noch die Torschüsse, differenziert nach Schüssen innerhalb und außerhalb dieser 30-Yard-Zone, sowie die Ballkontakte der beiden dominierenden Spieler Beckenbauer und Cruyff. Zur Vervollständigung erfasste er auch Flanken, Diagonalpässe und Eckstöße. Auch in Deutschland setzte die Entwicklung u.a. durch Sportjournalisten ein.

KELBER / SETZEPFANDT (1973), beides Sportjournalisten, stellen sich die Aufgabe, vor dem Zusammentreffen der beiden Erstplazierten der Bundsliga in der Saison 1973/74 einen Leistungsvergleich vorzunehmen. Dazu wurden die Hin- und Rückspiele der beiden Kontrahenten herangezogen und folgende spielbestimmend Aktionen registriert: Torschüsse, Angriffsaktionen, die im Strafraum gestoppt werden, gelungene und missglückte Flanken, gewonnene und verlorene Zweikämpfe, Fouls (KUHN / MAIER 1978, 20). 
Auch BISANZ (1986) schilderte genau seine Eindrücke von der Fußball- Weltmeisterschaft 1986 in Mexiko und geht in seinem Beitrag vor allem auf das Angriffsverhalten der Teams ein.

Während in den bisher Untersuchungen (s.u.) die Leistungserfassung im Spiel 11 gegen 11 erfolgt, versuchte TIEGEL (1973) die technische Leistung im Spiel drei gegen zwei in einem 12 × 12 m großen Feld per Videoaufzeichnung und Expertenrating zu messen. Hierbei wurden Pässe, Dribblings und das Stellungsspiel anhand von dreistufigen Qualitätskategorien beurteilt.

KUHN / MAIER $(1975,21)$ berichten über den Versuch, durch die Registrierung von Angriffsaktionen exakte Daten zur Beurteilung der Stärken und Schwächen einer Mannschaft per Scouting-Report zu gewinnen. Sie verwendeten zwei Verfahren. Eines zur graphisch gebunden und eines zur schriftlich gebundenen Beobachtung:

a) Die Registrierung der Torschüsse mit Hilfe einer Schussskizze

Bei diesem Verfahren wurden mit Hilfe von Symbolen Torschüsse in einer Spielfeldskizze notiert. Die Symbole wurden an der Stelle der Skizze eingetragen, die der Position des Schützen auf dem Spielfeld entspricht. Dabei wurde registriert, welcher Spieler wann, wie (mit Kopf oder Fuß) und von wo auf das Tor geschossen hat, ob der Schuss das Tor traf oder ob er vorbeiging. Verfehlte der Ball das Tor nicht, wurde festgehalten, ob der Schuss zu einem Torerfolg führte, ob er vom Torwart abgewehrt wurde oder gegen Latte/ Pfosten prallte. Zu Interpretationszwecken konnten die Daten direkt aus der Skizze abgelesen oder in Tabellen übertragen werden. Die Skizze gab außerdem Aufschluss über die räumliche Verteilung der Torschüsse.

b) Die Registrierung der Ausgänge von Angriffsaktionen

Dieses Verfahren durch Formulare zur Spielcharakterisierung ist etwas aufwendiger als die Schussskizze, liefert jedoch auch mehr Informationen. Wurden bei der Schussskizze nur die Torschüsse registriert, so umfasst diese Methode auch die Regelwidrigkeiten, das individuelle Fehlverhalten (fehlerhafte An- und Mitnahme, verlorener Zweikampf, ...), Abwehrverhalten, Angriffsverhalten, Verhalten bei Eck- und Freistößen und unter „Sonstiges“ auch Aktionen, die nicht immer in einem unmittelbaren Zusammenhang mit dem Spielgeschehen stehen (z.B. Ball Abbruch, Halbzeitund Schlusspfiff). Jeder dieser Punkte wird noch weiter differenziert. Außerdem wird 
nach den wesentlichen Stärken und Schwächen der beobachteten Mannschaft und des Gegners gefragt. Es ist auch Raum für besondere Vorkommnisse wie Verletzungen, Spielerwechsel, Platzverweis usw. vorhanden. Der Beobachter wird zudem aufgefordert, sich zu Unterschieden zwischen der ersten und zweiten Spielhälfte zu äußern. Zur individuellen Spielercharakteristik werden zwei Beobachtungsbögen vorgelegt, einer für den Außen- und Innenverteidiger und einer für den Mittelstürmer. Die Bögen sind in ihrer Gesamtkonzeption identisch aufgebaut. Sie beginnen mit der Größe und Konstitution des beobachteten Spielers, fragen nach dem Spielertyp (passiv- aggressiv), nach dem Abwehr- und Angriffsverhalten, nach der Technik, nach auffallenden Besonderheiten und nach Unterschieden zwischen den beiden Spielhälften.

Auch bei dieser Methode werden die Ausgänge mit Hilfe von Symbolen registriert. WINKLER 1985 hatte dieses Verfahren in dem Europa- Pokalspiel Hamburger SVInter Mailand überprüft und sich dabei auch eines Video-Recorders bedient. Es ist allerdings auch möglich, dieses Verfahren direkt im Stadion anzuwenden.

Ziel der Untersuchung von JASCHOCK (1976) war die Ausarbeitung und Erprobung objektiver Messverfahren zur Leistungsdiagnostik in Amateurklassen, in der Bundsliga und bei der Fußball-Weltmeisterschaft 1974. Im Einzelnen ging es dabei um die Messung der Laufleistung und der technischen Fertigkeiten eines Spielers. Um die Laufleistung eines Spielers zu messen, hatten sie das Spielfeld in Segmente eingeteilt. Mehrere Beobachter tragen die Laufwege des beobachteten Spielers in Spielfeldskizzen ein. Nach jeweils fünf Minuten werden der größeren Übersichtlichkeit halber die Formblätter gewechselt.

Anhand dieser Skizzen wurden dann die Laufleistungen des Spielers berechnet und zur Interpretation in Fünf- Minuten- Blöcken in einem Säulendiagramm dargestellt. Zur Erläuterung der räumlichen Verteilung werden außerdem die absoluten Häufigkeiten und die Prozentanteile für das gesamte Spiel in einer Spielfeldskizze in die entsprechenden Segmente eingetragen. Zur objektiven Beurteilung der technischen Fertigkeiten registrierten die beiden Autoren die Zahl und die Qualität der Pässe. Sie unterscheiden dabei zwischen:

1. erfolgreichem Pass, der vom Mitspieler verwertet werden konnte, 
2. teilerfolgreichem Pass, der zwar den Mitspieler erreichte, jedoch noch verloren ging und

3. erfolglosem Pass, der den Mitspieler nicht erreichte.

Aus diesen Daten wurde ein Passerfolgsquotient errechnet. Dabei wurde die Zahl der erfolgreichen Pässe quadriert und durch die Gesamtzahl der Pässe dividiert. Es bleibt jedoch unerwähnt, wie ein teilerfolgreicher Pass berücksichtigt wird. Um auch noch den Einfluss der Kondition auf die Technikleistung zu berücksichtigen, wird die Spielzeit in vier Abschnitte unterteilt und für jedes Viertel der Passerfolgsquotient errechnet.

LEHNER (1976) stellte zwei Beobachtungsverfahren vor, die der Registrierung von Angriffsaktionen dienen sollten. Dabei wurden die individuell-technische Leistung und die kollektiv-taktische Leistung erfasst.

Als Beobachtungsverfahren wählt er das Spielstenogramm und die graphische Aufzeichnung. Bei dem Spielstenogramm werden alle Interaktionen während eines Angriffs mit Angabe der beteiligten Spieler, der Art und der Qualität der Aktion registriert. Damit das Verfahren bei einer Life-Beobachtung angewandt werden kann, verwendet der Beobachter Symbole für die Handlungen. Zusätzlich wird noch die Dauer der beobachteten Angriffsaktion festgehalten. Als weitere Hilfe für die Interpretation wird das Spiel in 18 Fünf-Minuten-Abschnitte eingeteilt. Bei der graphischen Aufzeichnung handelt es sich um die Registrierung des Ballweges in einer Spielfeldskizze. Um den Zusammenhang in der Skizze zu wahren, wird nicht nur der Ballweg bei der zu beobachtenden Mannschaft registriert, sondern auch beim Gegner. Zur Unterscheidung werden dabei verschiedene Markierungsmöglichkeiten benutzt (durchgehende und gestrichelte Linien). Zur Interpretation zählt der Autor die Häufigkeiten der beobachteten Kategorien aus und stellt Tabellen zusammen, die Aufschluss über die Spielhandlungen in jedem der 18 Zeitabschnitte geben (für die erste und die zweite Halbzeit und für die gesamte Spieldauer).

WINKLER (1985) beschäftigte eine Analyse eines UEFA-Cup-Spiels zwischen dem Hamburger SV und Inter Mailand. Er untersuchte verschiedene Belastungen, denen ein Spieler auf einem derartigen sportlichen Niveau ausgesetzt ist. Anhand einer systematischen Spielbeobachtung mittels Videoaufnahme geht der Autor auch auf taktische Faktoren wie Deckungsverhalten, Spielsysteme oder Flügelspiel ein. 
Auch in der sportwissenschaftlichen Literatur Ägyptens finden sich verschiedene Untersuchungen der Spielbeobachtung im Fußball: AHMED (1991) analysierte die grundlegenden technischen und taktischen Fertigkeiten der Nationalmannschaften, die bei der Afrikameisterschaft im Viertelfinale standen, um zu prüfen, ob diese mit dem Spielergebnis zusammenhingen. DESUKY (1994) analysierte die Ägyptische Fußball Liga anhand einer systematischen Spielbeobachtung mittels Videoaufnahmen. Der Autor ging dabei auch auf angriffstaktische Faktoren wie Raumaufteilung oder Flügelwechsel ein.

FERGER (1997) widmete sich neuerdings der Bedeutung der Videogestützten Spielanalyse für die individuelle Trainingssteuerung. Die Zielsetzung lag in einer möglichst individuellen Trainingssteuerung auf der Basis von kategorialen Trainingsprotokollierungen. Der Schwerpunkt dieser Studie liegt auf der Evaluierung der zeitlich verzögerten Wirkungszusammenhänge von individuellen Leistungsdaten und vorher absolvierten Trainingsbelastungen. Es wird versucht die Frage nach der Abhängigkeit der individuellen Formenentwicklung und verschiedenen Trainingsbelastungen im Verlauf des Trainingsprozesses zu beantworten. Dieser Ansatz bietet die Möglichkeit im laufenden Trainingsprozess zum richtigen Zeitpunkt intervenieren zu können. Dies ist der Versuch, die am Anfang erwähnten Defizite der Sportspielforschung zu minimieren, damit das Training nicht weiter dem Zufall überlassen bleibt.

KOLLATH/ KAISER (1997) entwickelten diesen Ansatz mittels Video-Digitalisierung zur Technikanalyse weiter. Sie weisen allerdings darauf hin, dass die theoretischen Grundlagen einerseits einer Weiterentwicklung bedürfen, indem Modelle geschaffen werden, die sowohl den mechanischen als auch den biologischen Bedingungen sportlicher Bewegungen Rechnung tragen. Andererseits sind in den Zielsetzungen der Analysen vermehrt die sportspieltypischen Gegebenheiten zu berücksichtigen, wobei übernommene Ansätze aus Nachbarwissenschaften entsprechend zu modifizieren sind. Gelingt dies, so erfährt vor allem die Sportpraxis hierdurch eine gewinnbringende Bereicherung. 
Die gilt aber nicht nur für die Technikanalyse, sondern gleichermaßen auch für die gesamte Spielbeobachtung. Im folgenden Kapitel wird daher etwas ausführlicher dargestellt, wie sich die Spielbeobachtung auf eine explizit wissenschaftliche Grundlage stellen lässt, d.h. wie sie zu begründen ist, aber auch in welche Richtungen sie weiterzuentwickeln und zu spezifizieren ist. Aufbauend auf diesen wie den vorangegangenen Überlegungen wird die genaue Vorgehensweise der eigenen Untersuchung entwickelt und dargestellt. 


\section{Methodik}

\subsection{Zur Begründung und Weiterentwicklung einer spezifischen Methodik der Spielbeobachtung im Fußball}

Etwas analysieren heißt, etwas möglichst genau kennen lernen wollen. Und das ist in der Regel damit verbunden, dass man aus sportwissenschaftlichen Sicht den Analysegegenstand in technische, taktische und leistungsphysiologische Elemente zerlegt, einzelne Beanspruchungsformen wie Ausdauer, Schnelligkeit und Kraft als Basiskomponenten herausgreift und in Zusammenhang mit anderen Komponenten diskutiert (SCHMIDT 1992).

Verschiedene Autoren, wie WEBER (1991) oder GERISCH (1989) gehen davon aus, dass die fußballspezifische Belastungsstruktur im Labor nicht adäquat simuliert werden kann. Zu diesem Zweck führen sie Felduntersuchungen durch, um genauere Aussagen darüber zu erhalten, wie zum Beispiel die Art der Energiebereitstellung in Abhängigkeit von der Belastungsdauer und - Intensität sowie der Spielsituation ausfällt (vgl. SCHMIDT/REICHELT 1992).

Ausgangspunkt ist die Bestimmung der Struktur der Sportspiele und zwar aus der Sicht der trainingswissenschaftlichen Leistungsdiagnostik. Dies führt zu deutlich anderen Konsequenzen als eine singuläre Betrachtungsweise (vgl. DIETRICH 1977; HAGEDORN 1977; SCHALLER 1980).

„Braucht der Sportspieltrainer die Spielanalyse?" fragte HAGEDORN $(1976,18)$, als es darum ging, die noch junge Disziplin der Spielbeobachtung innerhalb der Sportwissenschaft zu etablieren. SASS (1984), ALPHEIS (1984), CZWALINA (1980), HAGEDORN (1980) oder etwa BRETTSCHNEIDER/ THIRER (1984) seien an dieser Stelle als Autoren genannt, die sich grundlegend mit der Notwendigkeit einer systematischen Spielbeobachtung beschäftigten und eine Intensivierung dieses Forschungszweiges forderten, um zuverlässige und jederzeit überprüfbare Daten zur Entwicklung und Struktur der Sportspiele erheben zu können. Gleichzeitig sollen diese Erkenntnisse in den dynamischen Prozess der Trainings- und Wettkampfsteuerung einfließen.

Leistungsanalysen sind Grundbedingungen für die Trainings- und Wettkampfsteuerung. Darüber hinaus dienen sie u.a. der Motivierung, der Leistungskontrolle, der 
Überprüfung der Trainingseffektivität und der Talentsichtung. Aus dieser Zweckbestimmung leitet sich für die Trainer die zentrale Forderung nach unmittelbarem Praxisbezug ab, d.h. nach Praktikabilität und Verwendbarkeit von Informationen durch verschiedene Ansätze zur Objektivierung von Spielleistungen. Systematische Spielbeobachtung und entsprechende Tests scheinen fruchtbare Instrumente.

Spielbeobachtung bzw. Spielanalyse dient vorrangig der gezielten Trainings- und Wettkampfsteuerung. Unter diesem Gesichtpunkt richtet sich die Beobachtung zukünftiger Spielgegner grundsätzlich auf die Grundformation und des Spielsystem sowie auf typische Merkmale der Spieltaktik wie Spielgestaltung in Offensive und Defensive, Konterspiel, Abseitsfalle, Freistoss, Eckball etc. Weiterhin ist das Angriffsund Abwehrhalten bei Standardsituationen von Interesse. Umfassende Informationen erfordern über diese Beobachtungsschwerpunkte hinausgehende Detailbeobachtungen. Bei der Festsetzung der Prioritäten spielt die Spielauffassung des verantwortlichen Trainers eine maßgebliche Rolle. Eine globale Einschätzung der Mannschaftsleistung wird zum Beispiel durch eine differenzierte Beurteilung der Einzelspieler ergänzt, wobei sich ein besonderes Augenmerk auf die Spieler richtet, die als Leistungsträger und Spielerpersönlichkeiten ihrer Mannschaft Profil geben und in motivationaler und spielerischer Hinsicht Akzente setzen. Vor allem ihre Durchsetzungsfähigkeit in den Zweikämpfen (Schnelligkeit, Kraft, Ballgeschicklichkeit etc.) stufen Trainer als aussagestark ein. Weiterhin finden taktische Disziplin und spielgestaltende Initiative Beachtung.

Wir können die erforderlichen Informationen durch unterschiedliche Weisen der Analyse einholen, um die Wirksamkeit des Sportspiels zu analysieren und um die schwachen und starken Aspekte der Mannschaften herauszufiltern, die sich in Meisterschaften gegenüberstehen. Durch wiederholte schriftliche Codierung lässt sich das Spielerverhalten erfassen, sodass damit kleinste Details der Leistung erkennbar werden. Dies erfordert aber den Rückgriff auf geeignete Methoden (s.u.).

Bei der Spielbeobachtung stellt sich nun vorher das Problem, die vielfältigen Spielaktionen und die komplexen motorischen Beanspruchungen systematisch zu erfassen und zur Leistungsbewertung die zahlreichen in- und externen Einflussgrößen des rasch wechselnden Spielgeschehens angemessen zu berücksichtigen. Hinzu kommt 
die weite räumliche Ausdehnung des Spielfeldes, die beispielsweise bei Videoaufnahmen technische Schwierigkeiten aufwirft, und zwar durch Begrenzung des Aufnahmeradius und des Bildausschnitts sowie durch perspektivische Verzerrungen im Zusammenhang mit der Segmentierung (BISANZI GERISCH 1997).

Im Rahmen einer Fußball- Spielanalyse wollen wir daher als Ausgangspunkt unter „Beobachten“ ein gezieltes Wahrnehmen und Speichern von Informationen bei einem aktuellen Fußballspiel verstehen. Live-Beobachtungen ohne technische Hilfsmittel sind - insbesondere im Amateurbereich - immer noch unentbehrlich, falls sich ein Trainer einen groben Überblick verschaffen will über ein bestimmtes „gegnerisches“ Spielverhalten bzw. über bestimmte Spielerhandlungen; aufgrund des komplexen Spielgeschehens, der Informationsfülle und der begrenzten Speicherkapazität des menschlichen Gehirns kann so bei weitem nicht alles Wesentliche erfasst und gespeichert werden; oft gehen wichtige Informationen verloren. Deshalb werden von interessierten Trainern Spielaufnahme (Spieldokumentationen) in Form von TVMitschnitten oder - wenn möglich - eigene Camcorder-Aufzeichnungen für verschiedene Informationszwecke benutzt. Am wertvollsten für fachliche Auswertungen ist jedoch eine Kombination von eigenen Videoaufnahmen unter Zuhilfenahme von TVMitschnitten (WINKLER 1997, 22).

Nach der Spielaufnahme kann mit der kriterienbezogenen, kategorialen Untersuchung des Spiels begonnen werden (einschließlich einer Datenaufbereitung). Die statistischen Ergebnisdarstellungen in Form von numerischen oder grafischen Veranschaulichungen der „Fakten“ gehören ebenso wie die Zusammenschnitte relevanter Spielszenen zu diesem zweiten wichtigen Schritt einer Spielanalyse. Mit Hilfe von statistischen Daten und Grafiken, vor allem aber mit ausgewählten Videoszenen, kann man wesentliche Fakten, z.B. Konzeptfehler, Entscheidungsfehler oder technische Fehler bewusst machen (vgl. BORN 1996) und eine gezielte Videoschulung durchführen (vgl. WINKLER/ FREIBICHLER 1991, 25).

Bei Analysen im Fußballspielbereich werden von Sportwissenschaftlern oder Trainern oft unterschiedliche Begriffe und Definitionen verwandt, sodass es zunächst notwendig erscheint, eigene Auffassungen zu verdeutlichen und eine einheitliche Terminologie vorzuschlagen. Der Begriff „Match Analysis“ sollte z.B. auf keinen Fall 
mit dem Begriff „Spielbeobachtung“ gleichgesetzt werden. Außerdem sollte nochmals darauf hingewiesen werden, dass eine Spielanalyse nur dann als „systematisch“ bezeichnet werden, wenn folgende Bedingungen erfüllt sind (nach WINKLER 2000, 63):

- Die Beobachtungs- und Auswertungsziele sind eindeutig benannt (z.B. Ermittlung aller taktischer Fehler von jedem Spieler einer Mannschaft gemäß eines Fehlerkatalogs etc.).

- Die Beobachtungs- und Auswertungsmethoden sind eindeutig festgelegt, insbesondere die Verwendung technischer Hilfsmittel (Videoanlage; Computerhardware; Computersoftware).

- Es erfolgt eine Dokumentation der Ergebnisse: Tabellarische/grafische Darstellungsweise und Zusammenschnitt von Spielszenen (z.B. für eine Schulung der Spieler auf taktischen Gebiet).

- Es wird eine Interpretation/Bewertung der Ergebnisse bzw. Beurteilung der Spielerleistungen vorgenommen.

Mit Blick auf die Integrationsmöglichkeiten und anhand der bisherigen Ausführungen lässt sich zur Vorgehensweise der Systematischen Spielbeobachtung ein Modell von WINKLER (2000) anführen, das für eine systematische Analyse geeignet scheint (Abb. 12). 


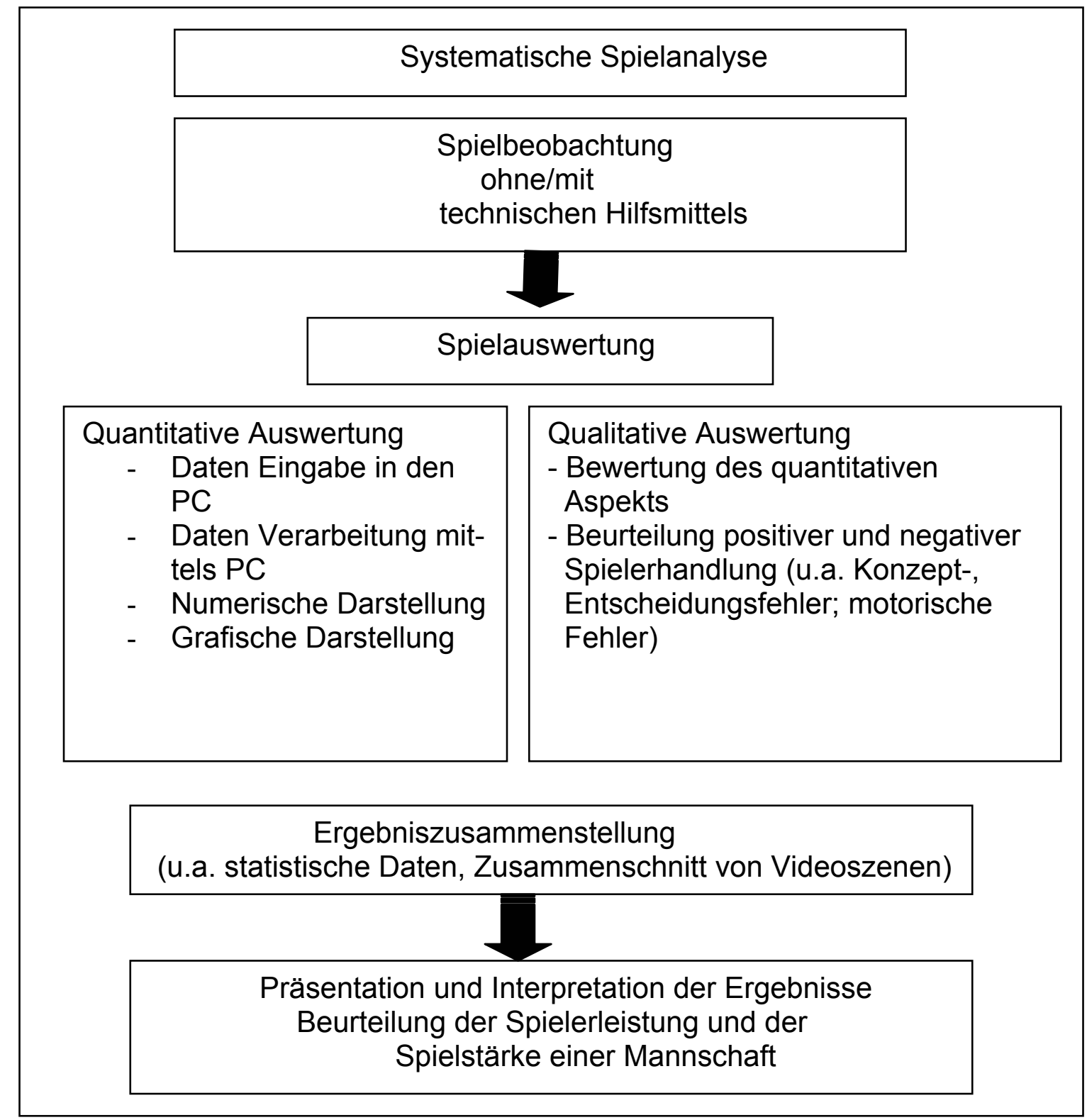

Abb.12: Prozessmodell einer "Systematischen Spielanalyse“ (modifiziert nach WINKLER 2000).

DUFOUR (1991) gibt nun anhand von zehn individuellen und kollektiven Parametern einen Überblick über die Entwicklung der Computerunterstützten Leistungserfassung im Sportspiel Fußball. Die hohe Leistungsfähigkeit der real- time Datenverarbeitung demonstrieren DUFOUR/ VERLINDEN (1991) anhand ihres selbst entwickelten Graphik- Tableaus, mit dessen Hilfe zwei Beobachter die Ergebnisse eines Spiels qualitativ und quantitativ beschreiben, erklären und in Hinblick auf ausgesuchte Parameter für den Trainer interpretieren. 
KUHN u.a. (1977) stellen die System- Komponenten einer real time- Analyse von Mannschaftsleistungen im Fußball vor, die sich auf „Spielaktionen mit Ball“ beschränkt, aber gleichzeitig eine lückenlose Analyse des Spielverlaufs anhand eines gut ausgearbeiteten Kategoriensystems gestattet. Das Problem dieses Ansatzes liegt eher in der Grundsatzfrage, ob der einzelne Spielbeobachter langfristig nicht überfordert ist, da er über einen längeren Zeitraum eine anspruchsvolle Zweihandkoordinationsaufgabe (gleichzeitige Beobachtung und Dateneingabe) zu erfüllen hat. Diese Problemeinschätzung teilen auch HEIN/ PERL (1992), denn trotz aller technologischen Entwicklung, die sie Beispielhaft an ihrem Software-Paket „TESSY“ erläutern, hängt auch ihrer Meinung nach die Güte der Spielbeobachtung letztlich von der Konstruktion eines geeigneten Kategoriensystems ab. HEIN/ PERL (1992; vgl. auch SCHMIDT 1991) legen deshalb besonderen Wert auf die Modellbildung. Dies bedeutet

- die exakte Zerlegung realer und komplexer Handlungen in prozessuale Teilstrukturen, die sich eindeutig Kategorien und Merkmalen zuordnen lassen,

- die Präsentation, die dem Benutzer eine adäquate und leicht verständliche Darstellungsform offerieren.

Bei der Konstruktion eines Beobachtungssystems zur Systematischen Spielbeobachtung (s.u.) nimmt der Begriff des Modells eine herausgehende Stellung ein. Der Konstruktionsvorgang ist als Vorgang der Modellbildung aufzufassen nach (LAMES 1994). In diesen erkenntnistheoretischen Rahmen lässt sich die "Allgemeine Modelltheorie“ von STACHOWIAK (1973) einordnen. Sein „Modellismus“ fasst alle Erkenntnisse als Erkenntnis in Modellen und durch Modelle auf. Abbildung 13 verdeutlicht, dass Modelle stets eine Abbildung von etwas sind, nämlich von den jeweiligen Originalen. Insofern sind auch ihre Attribute immer in Bezug sowohl zu den Modellen als auch zu ihren Originalen selbst zu betrachten. 


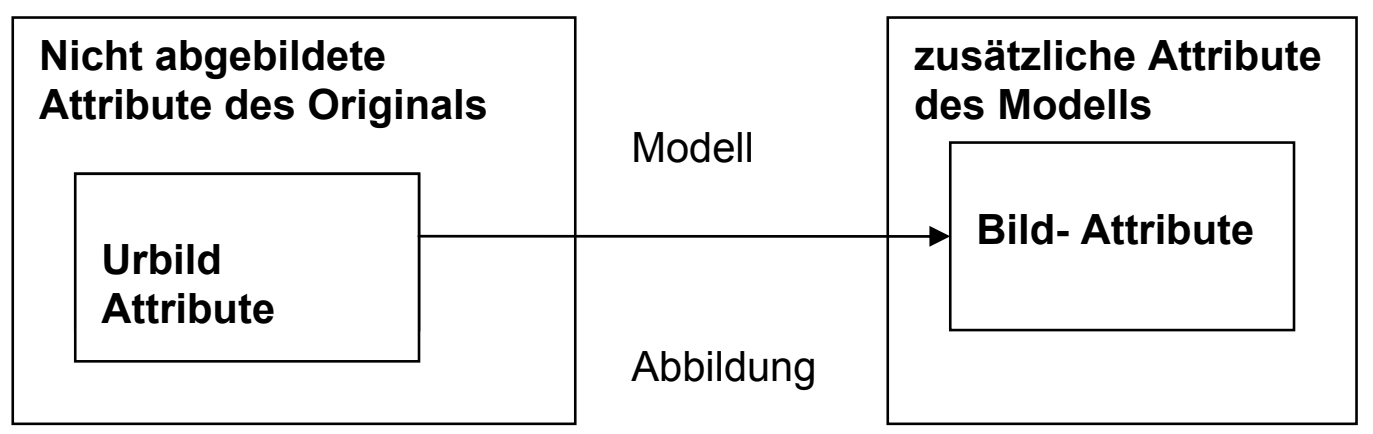

Abb.13: Veranschaulichung der Modellbildung als Abbildungsform (modifiziert nach STACHOWIAK 1973).

Die Rolle des Beobachters im Zusammenhang wissenschaftlicher Beobachtungsverfahren wird daher auch häufiger als diejenige eines Messinstruments bezeichnet. Analog dazu bringt der Beobachter einen Sachverhalt des beobachteten Realitätsausschnitts modellhaft ein. Nach einer kurzen Eingewöhnungszeit an diese „seelenlose“ Betrachtung durch den Beobachter erweist sich diese Perspektive als nützlich für ein besseres Verständnis und zur übergreifenden Erörterung der Qualität des Beobachtungsvorgangs. Insofern sind für das genannte übergreifende Modell von WINKLER (2000) zunächst auch Gütekriterien zu nennen.

Unter den Gütekriterien einer Messung sind Qualitätsmaßstäbe zu verstehen, die sich auf einzelne Aspekte der Messung beziehen. Insbesondere in der Testtheorie (vgl. LIENERT 1969) sind Gütekriterien entwickelt worden, die erfüllt sein müssen, um eine wissenschaftlich ausreichende Qualität des Tests zu garantiert. Diese Gütekriterien können auch in unterschiedlich starkem Maße auf alle Methoden der empirischen Sozialforschung, also auch auf die Spielbeobachtung, angewandt werden. Für diese kommen als Hauptgütekriterien in Betracht: Objektivität, Reliabilität und Validität, als Nebengütekriterien Ökonomie und Praktikabilität sowie Nützlichkeit. Die Gütekriterien sind abschließend also ebenso zu verdeutlichen. 


\subsubsection{Objektivität}

Unter Objektivität versteht man den Grad, in dem die Ergebnisse eines Tests unabhängig vom Untersucher sind (LIENERT 1969, 13). Da der Untersucher und das Messgerät an unterschiedlichen Stellen des Testvorganges eine Rolle spielen, kommen auch an mehreren Stellen Fragen der Objektivität zum tragen (KRÜGER 1985, 20).

Die Durchführungsobjektivität lässt sich ermitteln, indem zwei oder mehr Tester denselben Test an derselben Personenstichprobe durchführen und die Ergebnisse korreliert werden. Da dies häufig nicht möglich ist, ohne auf Reliabilitätsbestimmungsverfahren zurückgreifen und damit sich die dort beschriebenen Probleme einzuhandeln, sollen die Faktoren herausgestellt werden, die die Durchführungsobjektivität beeinflussen, damit man sein besonderes Augenmerk auf deren Vereinheitlichung in der Testsituation richten kann und deren quantitativen Einfluss versuchen kann zu bestimmen (KRÜGER 1985, 20).

BALLREICH $(1970,20)$ meint zwar, dass eine hohe Reliabilität die Überprüfung der Objektivität überflüssig mache, "da ein Test mit einer hohen Reliabilität per definitionem eine hohe Objektivität voraussetzt“. Er übersieht dabei jedoch, dass die hohe rechnerische Reliabilität auch durch sich gegenseitig ausgleichende Messfehler entstanden sein kann. Es kann daher nicht darauf verzichtet werden, auf eine möglichst große Objektivität zu achten und sie auch berechnen, da erst hohe Objektivität zusammen mit hoher Reliabilität die Situationsunabhängigkeit des Tests bezeugt (KRÜGER 1985, 20).

\subsubsection{Reliabilität}

Mit Reliabilität ist die Zuverlässigkeit, also die formale Messgenauigkeit eines Tests gemeint. In diesem Fall können die Forderungen der klassischen Testtheorie für den Bereich der Systematischen Spielbeobachtung nicht eingehalten werden. In der Diagnostik wird davon ausgegangen, dass sich die Beobachtungsergebnisse aus einen „wahren Testwert" und einem „Fehlerwert" additiv zusammensetzen (SINGER/UNGERER-RÖHRIG 1983). Deshalb wird durch Paralleltests, Testwiederholungen oder Testunterteilungen der Reliabilitäts- Koeffizient eines Verfahrens ermittelt (vgl. GRAELLS 1999). 
Die Reliabilität der Spielbeobachtung bezeichnet den Grad der Genauigkeit, mit dem ein bestimmtes Spielerverhalten erkannt (gemessen) wird. Die vollkommene Reliabilität einer Spielbeobachtung im Hinblick auf einen Spieler ist dann gewährleistet, wenn die mittels der Spielbeobachtung registrierten Ergebnisse den Spieler in seinem Verhalten genau erfasst haben. So ist zum Beispiel die Beobachtung eines Fußballspielers reliabel, die alle Körpertäuschungsfehler oder Schussfehler aufzeichnet, die er auch tatsächlich gemacht hat.

Die frühen Forderungen der Sportspielforscher nach der Sicherung der Gütekriterien im Rahmen der Systematischen Spielbeobachtung (SCHMIDT/ HAGEDORN 1972) stoßen aber an ihre Grenzen. Sogar als „ein Dilemma der Sportspielforschung“ bezeichnet LAMES (1991) den Versuch, die erbrachte Leistung in einem Sportspiel mit messtheoretischen Instrumentarien zu erfassen, da diese mit ihren Bedingungen nicht mit dem Charakter der Sportspiele vereinbar sind. Das Aufeinandertreffen zweier Parteien in einem Spiel beinhaltet die Abhängigkeit vom gegnerischen Verhalten und verhindert somit eine Bedingungskonstanz, wie sie bei sportmotorischen Tests vorliegt. Diese wäre aber erforderlich, um einen komplexen Koeffizienten ermitteln zu können.

Die von taktischem Handeln geprägte Suche nach einem der Spielsituation gemäßen Verhalten mit einer entsprechenden Erfolgsaussicht verhindert eine Merkmalskonstanz (nach GRAELLS 1999). Diese Mängel lassen sich nicht beseitigen, da sie ein Charakteristikum der Sportspiele sind. Deshalb reicht es zur Sicherung der Qualität der Systematischen Sportspielbeobachtung aus, die Reliabilität im Sinne der sog. Instrumentellen Konsistenz zu kontrollieren (LAMES 1994). Die Beobachtungskonstanz i.S. einer Instrumentellen Konsistenz schließt die Wahrnehmungs- und Beurteilungskonstanz über mehrere Spiele, innerhalb eines Spiels und auch über die jeweiligen Einzelspieler mit ein. Während die Wahrnehmungskonstanz von der Differenzierungsfähigkeit und der Aufmerksamkeit abhängt (KONZAG 1981), wird die Beurteilungskonstanz in erster Linie von der Entscheidungsfähigkeit bestimmt. Die Gefahr einer fehlenden Beurteilungskonstanz wird umso geringer, je mehr die Beobachtung als Messung ausgelegt ist (SCHMIDT 1976). Fehler in der Beurteilung von Vorgängen, die mit der Systematischen Spielbeobachtung erfasst werden, können auch in der mangelnden Objektivität des Beobachters liegen. Die Notwendigkeit der instru- 
mentellen Präzision leitet sich aus der definitorischen Genauigkeit des ausgewählten Kategoriensystems ab. Um die Zuverlässigkeit der Beobachtung nicht zu gefährden, müssen die Beobachtungskategorien und -einheiten genau formuliert werden. (vgl. 4.2.1).

Beim Spielverhalten ergibt sich die Reliabilität aus der Bedingungs- und Merkmalskonstanz. Durch die vom Beobachter nicht beeinflussbaren Faktoren wie Schiedsrichter, Zuschauer und vor allem die verschiedenen gegnerischen Mannschaften, die beobachtet werden, verringert sich die Bedingungskonstanz. Diese Tatsache wird als die Reliabilität mindernd angesehen, da die Homogenität der Spielanforderungen nicht gegeben ist (CZWALINA 1984).

Durch die Mischung von quantitativen und qualitativen Messverfahren besteht in Grenzbereichen das Problem der Reliabilität. Hier geht die Reliabilität einerseits in die Objektivität über, weil die Messgenauigkeit eben auch ein Objektivitätsproblem darstellt (vgl. 5.1). Daneben treten allerdings die Probleme des möglichen Messfehlers. Am Beispiel der Unterscheidung zwischen kurzen Pässen/langen Pässen sei dies verdeutlicht. Im Endspiel der WM Frankreich gegen Brasilien kam es insgesamt zu 808 Pässen, hiervon wurden 309 Pässen von der französischen Mannschaft und 499 von der brasilianischen Mannschaft durchgeführt. Pässe lassen sich im Prinzip durch Definition von Torschüssen abgrenzen. Da die Fernsehbilder jedoch nicht digitalisiert wurden, besteht eine Abgrenzungsproblematik zwischen kurzen (bis $10 \mathrm{~m}$ ) und langen (ab 10 m) Pässen. Im WM Endspiel waren 12 Pässe (= 1,5 \%) der französischen und 15 (=1,9 \%) der brasilianischen Mannschaft in einem Grenzbereich zwischen ca. 9 und ca. $11 \mathrm{~m}$ und erforderten daher durch erhebliche Verlangsamung der Bildsequenzen einen erhöhten Analyseaufwand und somit einen möglichen Messfehler. Der mögliche Messfehler bei diesem Kriterium, das sich von allen am schlechtesten abgrenzen lässt, liegt somit bei ca. $2 \%$. Der mögliche Messfehler der anderen 20 Analyse-Einheiten ist kaum größer. Insgesamt kann man daher im Rahmen des Dilemmas der Systematischen Sportspielbeobachtung von einem geringen Messfehler und hoher bis sehr hoher Reliabilität sprechen. 
Auch beim Pressing hinten/Mitte/vorn kann es einen Messfehler geben. Die Abgrenzung ist im Anhang wiedergegeben. Beim selben Endspiel kam es zu 211 Situationen mit Pressing. Diese verteilten sich auf 118 für die französische und 93 für die brasilianische Mannschaft. Im Grenzbereich von hinten zu Mitte und im Grenzbereich von Mitte zu vorne lagen hiervon 3 bei der französischen und 2 bei der brasilianischen Mannschaft. Dies entspricht 1,4 \% bzw. 1,0\%. Auch hier kann man sehen, dass die Reliabilität nur gering beeinträchtigt ist.

Bei den anderen Kriterien ist die Wahrscheinlichkeit des Messfehlers noch geringer, sodass insgesamt - je nach Maßstab (vgl. KRÜGER 1985) von einer sehr guten Reliabilität auszugehen ist.

Dem Vorwurf der fehlenden Homogenität der Spielanforderungen (CZWALINA 1984) kann in diesem Fall teilweise widersprochen werden, da durch die analysierte Weltmeisterschaft für die untersuchende Mannschaften annährend gleiche Bedingungen bestanden haben. Durch die Verwendung von Relationen (z.B. kurzer Pass zu langem Pass) werden typische Elemente einer Spielweise reliabler deutlich als durch absolute Zahlen, die stärker von dem abhängen, was der Gegner zulässt.

\subsubsection{Validität}

Mit Validität der Spielerbeobachtung meint man den Genauigkeitsgrad, mit dem die Spielerbeobachtung dasjenige Spielverhalten, das sie zu beobachten vorgibt, auch tatsächlich beobachtet und registriert ${ }^{1}$. Wenn also durch Spielerbeobachtung erfolgreiches und erfolgloses Verhalten von Spielern festgehalten werden soll, muss sich die Spielbeobachtung auch auf spezifisches Verhalten beziehen (CZWALINA 1988). Die Inhaltsanalyse eines Tests ermöglicht es, von der Verhaltensstichprobe nicht nur auf sich selbst, sondern auch auf Verhaltenskonstanten zu schließen. Man muss jedoch vorsichtig sein, dass man die realen Situation nicht zu weit von den Standardbedingungen des Tests ansiedelt (KRÜGER 1985, 14).

Die Frage der Gültigkeit einer Untersuchung hängt aber in starkem Maße von de Reliabilität ab. Vor dem Hintergrund dieses Problems hat der Begriff der Validität in der

${ }^{1}$ „Die Validität eines Tests gibt der Genauigkeit an, mit dem dieser Test dasjenige Persönlichkeitsmerkmal oder diejenige Verhaltensweise, das (die) ermessen soll oder zu messen Vorgibt, tatsächlich misst“" (BALLREICH 1970, 49). 
Systematischen Spielbeobachtung eine andere Qualität. Es steht daher weniger die Gültigkeit einer Untersuchung, sondern die Aussagekraft der Untersuchungsergebnisse für die Sportspielforschung im Mittelpunkt. Demnach geht es also um Frage, wie gültig Daten einer Beobachtung für die daraus resultierenden Entscheidungen eines Wissenschaftlers oder Trainers sind (MEES 1977, 42).

\subsubsection{Relevanz und Ökonomie}

Die Relevanz und Ökonomie sind die bekanntesten Nebengütekriterien, deren Einhaltung bei der Durchführung eines Meßverfahrens gefordert wird.

Den Anspruch der Nützlichkeit (Relevanz) erfüllt eine Spielbeobachtung, wenn sie ein Spielverhalten beobachtet, für dessen Registrierung ein praktisches Bedürfnis besteht. Sie hat außerdem ein hohe Nützlichkeit, wenn sie in ihrer Funktion durch keine andere empirische- analytische Methode ersetzt werden kann, und umgekehrt eine geringe Nützlichkeit, wenn sie ein Spielverhalten beobachtet, das mit einer Reihe andere Methoden ebenso gut untersucht werden kann. Da das sportspielmotorische Leistungsverhalten von Spieler während des Spiels nach den bisherigen Erkenntnissen am besten durch Spielbeobachtung erfasst werden kann, ist deren Nützlichkeit gegeben (CZWALINA 1988).

Ökonomie und Praktikabilität kann Spielbeobachtung dann für sich beanspruchen, wenn sie wenig Personal- und Materialkosten verursacht, einfach gehandhabt sowie schnell ausgewertet werden kann. Dieses Nebengütekriterium ist für die Arbeit des Sportpraktikers nicht gering einzuschätzen. Gerade der Wunsch, sportwissenschaftliche Erkenntnisse der Spielbeobachtung auch in Beobachtungspraxis umzusetzen, gibt inm ein besonderes Gewicht. Dabei bleibt allerdings nicht aus, dass gegebenenfalls andere Gütekriterien zu seinen Gunsten vernachlässigt oder doch zumindest eingeschränkt werden müssen (CZWALINA 1988).

Mit der notwendigen Erfüllung von Gütekriterien sei allerdings noch auf weitere Probleme hingewiesen. So beschränkt man sich durch die Wahl der systematischen Spielbeobachtung auf Phänomene der sichtbaren Oberflächen des Spiels. Daraus ergeben sich weitreichende Konsequenzen. Lediglich das Verhalten der Spieler kann beobachtet werden. Innere, nicht sichtbare Prozesse sind der systematischen Spielbeobachtung nicht zugänglich. Ihr Zuständigkeitsbereich weist also auch deutlich 
Grenzen auf. Sie kann nicht unmittelbar die "Spielfähigkeit" eines Spielers erfassen, wenn darunter dessen Kreativität, Spontaneität oder kommunikative Kompetenz verstanden wird. Das Verhalten im Sportspiel kann lediglich als Indikator für die zugrunde liegenden inneren Prozesse aufgefasst werden (LAMES 1994, 34). Dem Zugang der systematischen Spielbeobachtung entziehen sich aber auch näher liegende Dinge als ein didaktischen motivierter Spielfähigkeitsbegriff: So bleiben versteckte Interaktionen zwischen den Spielern verborgen, wie die gelegentlich sehr wirkungsvollen Gesten oder bösen Worte.

Genauso verborgen bleibt das aktuelle und generelle Fähigkeitsniveau. Es umfasst die individuellen Leistungsvoraussetzungen und deren situative Ausprägung (z.B. Ermüdungszustand). Schließlich bleibt die Wahrnehmung der Spieler in Entscheidungssituationen, die von zentraler Bedeutung für taktisches Handeln ist, nur sehr unvollkommen zugänglich (LAMES 1994, 34).

\subsection{Untersuchungsplanung und -durchführung (Methode)}

In diesem Abschnitt wird die angewendete Untersuchungsmethode dargestellt. Dabei werden zunächst die einzelnen Beobachtungseinheiten erläutert, die vor Untersuchungsbeginn festzulegen sind. Dies erfolgt anhand von Kategorien, denen eine besondere Relevanz in Bezug auf das Erkenntnisleitende Interesse (nämlich Gründe für den Spielerfolg zu finden) zuzuschreiben ist. Sie sind als Bausteine i.S. eines Baukastensystems für eine komplexe Spielbeobachtung zu verstehen. Die Ergebnisse werden in dem resultierenden Auswertungsbogen zusammengefasst. Es folgt eine Erläuterung der Datenerhebung und der Datenauswertung (incl. der Weiterverarbeitung), zu denen auch die eingesetzte technische Ausstattung gehört.

\subsubsection{Beobachtungseinheiten}

Um die Beobachtungseinheiten schriftlich festhalten zu können, wird zunächst ein Auswertungsbogen entwickelt. Dieser sollte ermöglichen, sowohl quantitative als auch qualitative Aspekte zu erfassen. Dabei stellt sich die Frage, welche Elemente bzw. Fertigkeiten und welche übergreifenden Kategorien er enthalten soll. 
Hier die einzelnen Elemente/ Kategorien beschreiben:

\section{Technische Fähigkeiten}

Sämtliche nachfolgend behandelte technische Fähigkeiten beschreiben Fähigkeiten im Umgang mit dem Ball, setzen also unbedingt den Ballbesitz des Spielers der untersuchten Mannschaft voraus.

\section{Dribbling}

Das Dribbling ist eine individuelle Eigenschaft und beschreibt die Fähigkeit eines einzelnen Spielers, sich mit dem Ball am Fuß im Raum zu bewegen. Hierbei wurde nur als Dribbling gezählt, wenn der Spieler wenigstens 8 Schritte mit dem Ball ausführte. Als hochkarätig galt, wenn das Dribbling in der Nähe des gegnerischen Tores erfolgreich beendet wurde (oder es auch weiter vom Tor weg in einem Torschuss mündete). Als normal galt, wenn es im Mittelfeld oder der eigenen Hälfte erfolgte und die eigene Mannschaft in Ballbesitz blieb. Ein schlechtes Dribbling endet in einem Ballverlust.

\section{Körpertäuschung}

Die Körpertäuschung ist eine individuelle Eigenschaft (auf dem ganzen Spielfeld) und beschreibt die Fähigkeit eines einzelnen Spielers, einen Gegenspieler durch Bewegungen des eigenen Körpers über sein eigenes Vorhaben zu täuschen und diesen so zu fehlerhaften Handlungen zu zwingen. Bei einer exzellenten Körpertäuschung wird der Gegner in der Nähe des gegnerischen Tores überwunden, bzw. die Körpertäuschung führt weiter weg vom Tor zu einem Torschuss.

\section{Torschuss}

Der Torschuss ist eine individuelle Eigenschaft und beschreibt die Fähigkeit eines einzelnen Spielers, den Ball zielgenau und im Bemühen um den Torerfolg in Richtung gegnerisches Tor zu schießen. Bei einem exzellenten Torschuss wird ein Tor erzielt, bei einem normalen Torschuss wird dieser vom Torhüter abgewehrt bzw. das Tor wird nur knapp (nicht mehr als $1 \mathrm{~m}$ ) verfehlt; ein schwacher Torschuss verfehlt das Tor weiter als 1 Meter. 


\section{Kurze Pässe}

Kurze Pässe sind eine individuelle Eigenschaft und beschreiben die Fähigkeit eines einzelnen Spielers, den Ball zielgenau und über eine Maximaldistanz von zehn Metern zu einem Mitspieler zu schießen. Durch einen hochkarätigen kurzen Pass gerät das gegnerische Tor in Gefahr; der normale kurze Pass erfolgt im Mittelfeld, bzw. der eigenen Hälfte, und erreicht einen Spieler der eigenen Mannschaft; der schlechte kurze Pass erreicht einen Gegenspieler.

\section{Lange Pässe}

Lange Pässe sind eine individuelle Eigenschaft und beschreiben die Fähigkeit eines einzelnen Spielers, den Ball zielgenau und über eine Distanz von mehr als zehn Metern zu einem Mitspieler zu schießen. Durch einen hochkarätigen langen Pass gerät das gegnerische Tor in Gefahr; der normale lange Pass erfolgt im Mittelfeld, bzw. der eigenen Hälfte, und erreicht einen Spieler der eigenen Mannschaft; der schlechte lange Pass erreicht einen Gegenspieler.

\section{Ballkontrolle}

Die Ballkontrolle ist eine individuelle Eigenschaft und beschreibt die Fähigkeit eines einzelnen Spielers, den Ball so zu behandeln, dass dessen Verhalten für diesen jederzeit kontrollierbar ist. Aus einer Situation mit exzellenter Ballkontrolle entsteht ein Tor, bei der normalen Ballkontrolle bleibt die eigene Mannschaft in Ballbesitz, bei schlechter Ballkontrolle geht der Ball verloren.

\section{Offensiv - taktische Fähigkeiten}

Sämtliche nachfolgend behandelte taktische Fähigkeiten sind auf taktische Fähigkeiten in der Offensive beschränkt, setzen also unbedingt den Ballbesitz der untersuchten Mannschaft voraus.

\section{Freistoß}

Der (direkte) Freistoß als Standardsituation ist eine individuelle Eigenschaft und beschreibt die Fähigkeit eines einzelnen Spielers, einen Freistoß so auszuführen, dass Gefahr für die gegnerische Mannschaft entsteht. Ein exzellenter Freistoß mündet in einem Tor, ein normaler und ein schlechter Freistoß ist wie ein Torschuss (s. diesem) zu bewerten. 


\section{Eckball}

Der Eckball als Standardsituation ist eine mannschaftliche Eigenschaft und beschreibt die Fähigkeit der Mannschaft bzw. Teilen der Mannschaft einschließlich des Schützen, sich vor, während und nach Ausführung des Eckballs so zu verhalten, dass ein Torerfolg möglich ist. Ein exzellenter Eckball mündet in einem Torschuss, ein normaler Eckball wird zwar auch vor das Tor gebracht, aus inm resultiert aber keine torgefährliche Situation. Bei einem schlechten Eckball geht für die angreifende Mannschaft der Ball verloren.

\section{Unterstützen}

Das Unterstützen ist eine individuelle Eigenschaft und beschreibt die Fähigkeit eines einzelnen Spielers (auf dem ganzen Spielfeld), sich so in Richtung des ballführenden Mitspielers zu bewegen, dass diesem möglichst optimale Unterstützung zukommt. Hochkarätig ist die Unterstützung, wenn in Tornähe hieraus eine Torschuss-Situation entsteht, normale Unterstützung findet dann statt, wenn sich eine solche Situation nicht in Tornähe befindet und die unterstützte Mannschaft in Ballbesitz bleibt; wenn der Ball verloren geht, war die Unterstützung schlecht. Fehlende Unterstützung, obwohl es in der Situation erforderlich wäre, wird wie schlechte Unterstützung gewertet. Durch die Kameraführung ist nicht auszuschließen, dass fehlende (= schlechte) Unterstützung nicht in jedem Fall gesehen werden kann.

\section{Doppelpass}

Der Doppelpass ist eine mannschaftliche Eigenschaft und beschreibt die Fähigkeit von genau zwei Spielern der Mannschaft, sich den Ball über direktes Kurzpassspiel so zuzuspielen, dass dadurch ein taktischer Vorteil entsteht. Für die Bewertung vgl. Kurze Pässe.

\section{Raumaufteilung}

Die Raumaufteilung ist eine mannschaftliche Eigenschaft und beschreibt die Fähigkeit der Mannschaft bzw. Teilen der Mannschaft, sich so im Raum zu verteilen, dass dieser Raum möglichst optimal ausgenutzt wird. Für die Raumaufteilung wurden jeweils mindestens drei Spieler berücksichtigt. Exzellente Raumaufteilung liegt vor, wenn die Situation in der Nähe des Tors des Gegners stattfand, bzw. hieraus ein Torschuss resultierte. Bei der normalen Raumaufteilung haben die Spieler eine gute 
Position und den Ball nicht verloren. Bei der schlechten Raumaufteilung war die Position so eingenommen, dass der Ball verloren wurde (für die Abgrenzung vgl. Unterstützen). Durch die Kameraführung kann man nicht ausschließen, dass nur zwei Spieler im Bild gezeigt wurden, obwohl ein dritter Spieler sich entsprechend im Raum befand.

\section{Nachrücken}

Das Nachrücken ist eine individuelle Eigenschaft und beschreibt die Fähigkeit eines einzelnen Spielers, sich so in Richtung des gegnerischen Tores zu bewegen, dass dadurch die Angriffsbemühungen der eigenen Mannschaft möglichst optimal unterstützt werden. Das Nachrücken wird ggf. durch die Kamera erst relativ spät in Ballnähe erfasst. Durch exzellentes Nachrücken entsteht in der Nähe des Tores des Gegners eine torgefährliche Situation. Bei normalem nachrücken entsteht hieraus keine gefährliche Situation, aber die Mannschaft bleibt im Ballbesitz. Bei schlechtem Nachrücken geht der Ball verloren.

\section{"In die Breite ziehen“}

Das „In die Breite ziehen“ des Spiels ist eine mannschaftliche Eigenschaft und beschreibt die Fähigkeit der Mannschaft bzw. Teilen der Mannschaft, sich parallel zur Torauslinie so zu verteilen, dass möglichst die gesamte Breite des Spielfeldes (wenigstens $40 \mathrm{~m}$ ) ausgenutzt wird. Hochkarätiges In die Breite ziehen liegt dann vor, wenn hieraus eine torgefährliche Situation entsteht). Bei normalem In die Breite ziehen bleibt die angreifende Mannschaft in Ballbesitz, aber es entsteht keine gefährliche Situation. Bei schlechtem In die Breite ziehen geht der Ball verloren.

\section{Defensiv - taktische Fähigkeiten}

Sämtliche nachfolgend behandelte taktische Fähigkeiten sind auf taktische Fähigkeiten in der Defensive beschränkt, setzen also unbedingt voraus, dass die untersuchte Mannschaft nicht in Ballbesitz ist. 


\section{Pressing hinten}

Das „Pressing hinten“ ist eine mannschaftliche Eigenschaft und beschreibt die Fähigkeit der Mannschaft bzw. Teilen der Mannschaft, sich im eigenen Drittel des Spielfeldes so nah an den ballführenden Gegenspieler und seine möglichen Anspielpartner heranzugewegen, dass dieser unter Druck gerät und zu einem Fehler gezwungen wird. Exzellent ist das Pressing hinten, wenn dem Gegenspieler in Tornähe oder einer Tor gefährlichen Situation durch das Pressing der Ball abgenommen werden kann. Ein normales Pressing hinten liegt vor, wenn dem Gegner der Ball abgenommen wird, ohne dass durch den Ballbesitz des Gegners unmittelbar Gefahr ausgegangen wäre; bei einem schlechten Pressing hinten begeht der pressende Spieler entweder ein Foul oder das Pressing ist erfolglos und der Ballbesitz bleibt bei der angreifenden Mannschaft.

\section{Pressing Mitte}

Das „Pressing Mitte“ ist eine mannschaftliche Eigenschaft und beschreibt die Fähigkeit der Mannschaft bzw. Teilen der Mannschaft, sich im mittleren Drittel des Spielfeldes so nah an den ballführenden Gegenspieler und seine möglichen Anspielpartner heranzugewegen, dass dieser unter Druck gerät und zu einem Fehler gezwungen wird. Bei einem exzellenten Pressing in der Mitte wird der gegnerischen Mannschaft der Ball abgenommen und es entsteht durch einen schnellen Konter eine torgefährliche Situation; bei einem normalen Presse in der Mitte wird der gegnerischen Mannschaft zwar der Ball abgenommen, hieraus entsteht aber keine gefährliche Situation; beim schlechten Pressing in der Mitte bleibt die gegnerische Mannschaft unmittelbar oder nach einem begangen Foul in Ballbesitz.

\section{Pressing vorne}

Das „Pressing vorne“ ist eine mannschaftliche Eigenschaft und beschreibt die Fähigkeit der Mannschaft bzw. Teilen der Mannschaft, sich im gegnerischen Drittel des Spielfeldes so nah an den ballführenden Gegenspieler und seine möglichen Anspielpartner heranzugewegen, dass dieser unter Druck gerät und zu einem Fehler gezwungen wird. Ein exzellentes Pressing vorne liegt vor, wenn hierdurch die gegnerische Mannschaft in der Nähe des eigenen Tores den Ball verliert oder auch in größerer Torentfernung hierdurch eine torgefährliche Situation entsteht. Ein normales Pressing vorn liegt vor, wenn der Ball zwar abgenommen wurde, hieraus aber nicht 
unmittelbar Gefahr entsteht. Bei einem schlechten Pressing vorn wird entweder ein Foul begangen oder die Handlung war erfolglos.

\section{Raumaufteilung}

Die Raumaufteilung ist eine mannschaftliche Eigenschaft und beschreibt die Fähigkeit der Mannschaft bzw. Teilen der Mannschaft, sich so im Raum zu verteilen, dass der gegnerischen Mannschaft möglichst wenig Raum zur Verfügung steht (vgl. Raumaufteilung im Angriff).

\section{Zurückkommen}

Das Zurückkommen ist eine individuelle Eigenschaft und beschreibt die Fähigkeit eines einzelnen Spielers, sich direkt nach einem Ballverlust der eigenen Mannschaft so zu bewegen, dass die Defensivarbeit möglichst optimal unterstützt wird. Nicht jedes Zurückkommen wird von der Kamera erfasst, da diese die Spieler häufig erst wieder in Ballnähe zeigt. Beim Zurückkommen muss die jeweilige Spielposition berücksichtigt werden. Bewertet wurde, wenn die Spieler eine Position einnahmen, die sonst von anderen eingenommen wurde (= aus Angreifern wurden Mittelfeldspieler, aus Mittelfeldspielern Verteidiger etc.), d. h. es handelte sich um Bewegungen, die fast das halb Spielfeld umfassten. Ein hochkarätiges Zurückkommen lag dann vor, wenn durch das Zurückkommen eine torgefährliche Situation der gegnerischen Mannschaft unterbunden werden konnte. Ein normales Zurückkommen lag vor, wenn der Ball dem Gegner zwar abgenommen werden konnte, es sich aber nicht hat um eine torgefährliche Situation handelte. Bei einem schlechten Zurückkommen war der zurückkommende Spieler erfolglos und die gegnerische Mannschaft blieb unmittelbar oder mittelbar (nach Foulspiel) in Ballbesitz.

\section{Unterstützen}

Das Unterstützen ist eine individuelle Eigenschaft und beschreibt die Fähigkeit eines einzelnen Spielers, sich so in Richtung eines Mitspielers zu bewegen, dass diesem bei der Bekämpfung des ballführenden Gegenspielers möglichst optimale Unterstützung zukommt. (vgl. Unterstützen im Angriff). 


\section{Rückraum sichern}

Das Unterstützen ist eine individuelle Eigenschaft und beschreibt die Fähigkeit eines einzelnen Spielers, bei der Defensivarbeit auch das Geschehen auf dem Spielfeld zu kontrollieren, dass nicht in dessen Blickwinkel (meist hinter ihm) liegt. Als exzellent wird das Sichern des eigenen Rückraumes angesehen, wenn dies in der Nähe des Tores erfolgt und hierdurch eine gegnerische Torchance verhindert wird; ein normales Sichern des Rückraumes liegt vor, wenn hinter dem sichernden Spieler kein Angreifer in Position ist und der sichernde Spieler in Ballbesitz kommen kann; ein schlechtes Rückraum sichern liegt vor, wenn der Verteidiger nicht in Ballbesitz kommen kann, sondern der gegnerische Spieler in seinem Rückraum.

\section{Abseitsfalle}

Die Abseitsfalle ist eine mannschaftliche Eigenschaft und beschreibt die Fähigkeit der Mannschaft bzw. Teilen der Mannschaft, sich gleichzeitig so zu bewegen, dass im Moment der Ballabgabe des Gegners dadurch ein gegnerischer Spieler im Abseits steht. Bei Abseitsfallen wurde nur eine Zweierwertung eingeführt. Sie ist entweder erfolgreich (= exzellent) oder nicht erfolgreich (=schlecht).

Anhand dieser Elemente / Kategorien sollte sich die Leistung der Mannschaften erheben lassen. In schriftlich gebundener Form müssen zunächst die quantitativen Aspekte, nämlich die auftretende Anzahl der einzelnen Elemente, erhoben werden. In einem zweiten Schritt ist dann aber auch deren Qualität zu beurteilen. Dabei orientiere ich mich an den schon von TIEGEL (1973) und JASCHOK et al. (1976) vorgenommenen dreistufigen Einteilung. Anhand eines Spieles (Deutschland - USA) wurden die qualitativen Kategorien i.S. einer Pilotstudie durch einen weiteren Experten validiert. Eine zusammenfassende Betrachtung der Prüfergebnisse wies für die vorliegende Arbeit hinsichtlich der Gütekriterien Objektivität durchaus zufrieden stellende Werte auf (s.u.). 


\subsubsection{Datenerhebung}

Der unter den Gütekriterien genannten Aspekte einer systematischen Spielbeobachtung sind auch auf die Datenerhebung zu beziehen. Um eine möglichst objektive, reliable, valide und ökonomische Datenerhebung zu gewährleisten, wurden verschiedene Spiele (s.u.) mehrfach auf Videobändern abgespielt und beurteilt. Die Videoaufnahmen (von Live-Übertragungen der ARD, des ZDF und des DSF) gewährleisteten, dass der Untersucher nicht in die Situation involviert war, etwaige Merkmale übersehen und ein zumutbarer Aufwand vorlag. Die technische Ausstattung wurde vom Institut für Sportwissenschaften der Georg-August-Universität Göttingen zur Verfügung gestellt. Es handelt sich dabei um Videorekorder (Philips SVHS, s/l Play System, Zeitlupe; Grundig s/l Play System), Monitor und 16 Videokassetten (BASF180/240).

Bei der Fußballweltmeisterschaft in Frankreich wurden verschiedene Spiele ausgewählt, da nicht das ganze Turnier vollständig beobachtet wurde. Da ab dem Achtelfinale im K.O.-System gespielt wurde, war davon auszugehen, dass die Mannschaften auch ihr volles Potenzial ausschöpften und aus taktischen Gründen nicht "nur" auf unentschieden spielten. So wurden nur die Spiele ab Achtelfinale beobachtet und analysiert. Folgende Tabelle (7) gibt einen Überblick über den Turnierverlauf. 


\begin{tabular}{|c|c|c|c|c|}
\hline Datum & $\begin{array}{l}\text { Uhr- } \\
\text { zeit }\end{array}$ & Ort & Achtelfinale & $\begin{array}{l}\text { Ergebnis- } \\
\text { se }\end{array}$ \\
\hline 27.6.1998 & $21: 00$ & Paris & Brasilien : Chile & $4: 1(3: 0)$ \\
\hline 27.6 .1998 & $16: 30$ & Marseille & Italien : Norwegen & $1: 0(1: 0)$ \\
\hline 28.6 .1998 & $16: 30$ & Lens & Frankreich : Paraguay & $\begin{array}{l}1: 0(1: 0) \\
\text { G. Goal }\end{array}$ \\
\hline 28.6 .1998 & $21: 00$ & Saint-Denis & Nigeria : Dänemark & $1: 4(0: 2)$ \\
\hline 29.6 .1998 & $21: 00$ & Toulouse & Niederland : Jugoslawien & $2: 1(1: 0)$ \\
\hline 29.6 .1998 & $16: 30$ & Montpellier & Deutschland : Mexiko & $2: 1(0: 0)$ \\
\hline $30: 6: 1998$ & 16.30 & Bordeaux & Rumänien : Kroatien & $0: 1(0: 1)$ \\
\hline 30.6 .1998 & $21: 00$ & Saint Etienne & Argentinien : England & $\begin{array}{l}6: 5 \text { n. E. } \\
(2: 2,2: 2)\end{array}$ \\
\hline \multicolumn{5}{|c|}{ Viertelfinale } \\
\hline 03.07 .1998 & $21: 00$ & Nantes & Brasilien : Dänemark & $3: 2(2: 1)$ \\
\hline 04.07 .1998 & $16: 30$ & Saint-Denis & Italien : Frankreich & $\begin{array}{l}3: 4 \text { n. E. } \\
(0: 0,0: 0)\end{array}$ \\
\hline 04.07 .1998 & $16: 30$ & Marseille & Niederland : Argentinien & $2: 1(1: 1)$ \\
\hline 04.07 .1998 & $21: 00$ & Lens & Kroatien : Deutschland & $3: 0$ \\
\hline \multicolumn{5}{|c|}{ Halbfinale } \\
\hline 07.07.1998 & $21: 00$ & Marseille & Brasilien : Niederland & $\begin{array}{l}5: 3 \text { n. E. } \\
(1: 1,0: 0)\end{array}$ \\
\hline 08.07 .1998 & $21: 00$ & Saint-Denis & Frankreich : Kroatien & $2: 1(0: 0)$ \\
\hline \multicolumn{5}{|c|}{ Spiel um den 3.Platz } \\
\hline 11.07 .1998 & 21:00 & Saint-Denis & Niederland : Kroatien & $1: 2(1: 2)$ \\
\hline \multicolumn{5}{|c|}{ Finalspiel } \\
\hline 12.07 .1998 & $21: 00$ & Paris & Brasilien : Frankreich & $0: 3(0: 2)$ \\
\hline
\end{tabular}

Tab.3: Weltmeisterschaftsspiel ab Achtelfinale. 


\subsection{Datenauswertung}

Die Datenauswertung erfolgte anhand des bereits vorgestellten Auswertungsbogens. Die zuvor festgelegten Beobachtungseinheiten reichten nach Ansicht des Verfassers aus, um die Struktur der Leistungsfaktoren beurteilen zu können. Da die Beobachtung anhand von Videoaufzeichnungen erfolgte, konnte zudem jeder beliebige Aspekt der Spiele mehrfach analysiert werden.

Der Verfasser hat die Ergebnisse für jede Mannschaft mit Werte berechnet, um zwischen den Mannschaften vergleichen zu können.

Auf dieser Grundlage wurden dann die Leistungen anhand der quantitativen und qualitativen Aspekte bestimmt. Besonders bedeutsam ist dabei die Bildung eines Qualitötsquotienten für jedes einzelne Element. Daraufhin werden die Quotienten für Gewinner und Verlierer je nach Achtelfinale, Viertelfinale und Halbfinale bestimmt und im Anschluss verglichen. Daraus sollten sich Ableitungen vornehmen lassen, inwiefern sich Gewinner und Verlierer unterscheiden. Dazu wird in der folgenden Ergebnisdarstellung weitergehend diskutiert, warum dies so sein kann. 


\section{Untersuchungsergebnisse}

Die Nationenergebnisse der sechzehn Mannschaften, die bei der Weltmeisterschaft das Achtelfinale erreichten, wurden in diesem Abschnitt als Darstellung zu Technik und taktischen zusammengefasst. Anhand des Turniers beginnen wir mit den nach einem Spiel ausgeschiedenen Mannschaften (England, Nigeria, Paraguay, Chile, Rumänien, Jugoslawien, Norwegen und Mexiko), die zuvor erläuterten Beobachtungsgrößen sind ausführlich tabellarisch und grafisch dargestellt. Die Anwendung eines Diagramms bot sich vor allem für die Zuteilung der verschiedenen technischen und taktischen Größen, die Aufschlüsselung der unterschiedlichen Möglichkeiten zum Beschreiben einer Hinausstellung sowie für die Erläuterung der Torschüsse mit den jeweiligen Erfolgshäufigkeiten an. Vor der Darstellung der Kennziffern zu Technik und Taktik werden die Prüfergebnisse der Gütekriterien dargestellt. Damit wird die Unabhängigkeit des angewandten Beobachtungssystems vom jeweiligen Untersucher erfasst.

\subsection{Gütekriterien Prüferergebnisse}

Wie bereits unter 4.1.1 beschrieben, ist eine Überprüfung des Beobachtungsvorgangs hinsichtlich seiner Objektivität unerlässlich. Bei der vorliegenden Arbeit wurden mit Hilfe einer Übereinstimmungsmatrix die einzelnen Parameter „Erwirken der Hinausstellung" und "Technische-Taktische Fähigkeiten“ bezüglich ihrer Aussagefähigkeit für das Hauptgütekriterium Objektivität überprüft. Dabei wertet ein zweiter Beobachter willkürlich ausgewählte Spiele (Deutschland: USA) anhand des vorliegenden Videomaterials ein weiteres Mal unabhängig aus. Bei dem Beobachter handelt es sich um einen Experten für den Bereich Fußball und somit um einen für derartige Überprüfung geeignet Person. Christian Becker arbeitete als Dozent für Fußball am Institut für Sportwissenschaften der Georg- August- Universität Göttingen.

Durch einfaches Radizieren der Ergebnisse aus der Übereinstimmungsmatrix werden entsprechende Objektivitätskoeffizienten ermittelt (siehe Tabelle im Anhang 301). Dabei hat sich für eine Bewertung dieser festgestellten Resultate eine entsprechende Annahme durchgesetzt, die bereits von KIRKENDALL u. a. 1980; MATHEWS 1978; COLLINS/ HODGE 1978; MAYER/ BLESH 1962; NEUMEIR 1983 angewandt wurden. 
Demnach gilt eine Bewertung als „ausgezeichnet“, wenn der ermittelte Koeffizient zwischen 0,95 und 1,0 liegt. Als "sehr gut" gilt ein Wert, der sich zwischen 0,90 und 0,94 bewegt. „Annehmbar“ ist ein ermittelter Koeffizient dann noch, wenn er zwischen 0,80 und 0,98 liegt. Mit "gering“ wird ein Wert zwischen 0,70 und 0,79 bezeichnet. Fraglich erscheint ein Ergebnis, wenn sich der ermittelte Koeffizient zwischen 0,60 und 0,69 bewegt (KRÜGER 1985).

Mit $r=0,99$ handelt es sich somit um ausgezeichnete Objektivität.

Wie bereits unter 4.1.2 beschrieben, ist eine Überprüfung des Beobachtungsvorgangs hinsichtlich seiner Reliabilität unerlässlich. Bei der vorliegenden Arbeit wurden mit Hilfe einer Übereinstimmungsmatrix die Parameter "Erwirken der Hinausstellung" und "technisch-taktische Fähigkeiten“ bezüglich ihrer Aussagefähigkeit für das Hauptgütekriterium Reliabilität überprüft. Werden dieselben Test an derselben Stichprobe zweimal durchgeführt und korrelieren die Werte des ersten mit denen des zweiten Tests, so kann die Retest-Reliabilität bestimmt werden (s. Tabelle im Anhang).

Durch einfaches Radizieren der Ergebnisse aus der Übereinstimmungsmatrix werden entsprechende Reliabilitätskoeffizienten ermittelt (siehe Tabelle im Anhang 304). Dabei hat sich für eine Bewertung dieser festgestellten Resultate eine entsprechende Annahme durchgesetzt, die bereits von KIRKENDALL u. a. 1980; MATHEWS 1978; COLLINS/ HODGE 1978; MAYER/BLESH 1962 formuliert wurden.

Demnach gilt eine Bewertung als „ausgezeichnet“, wenn der ermittelte Koeffizient zwischen 0,95 und 1,0 liegt. Als "sehr gut" gilt ein Wert, der sich zwischen 0,90 und 0,94 bewegt. „Annehmbar" ist ein ermittelter Koeffizient dann noch, wenn er zwischen 0,80 und 0,98 liegt. Mit "gering“ wird ein Wert zwischen 0,70 und 0,79 bewertet. "fraglich“ erscheint ein Ergebnis, wenn sich der ermittelte Koeffizient zwischen 0,60 und 0,69 bewegt (KRÜGER 1985,22).

Mit $r=0,99$ handelt es sich somit im Rahmen des Messfehlers um eine ausgezeichnete Reliabilität. 


\subsection{Darstellung der Kennziffern von technischen Fähigkeiten}

Nachfolgend werden die Ergebnisse der Auswertungsbögen, die aus der Analyse des Videomaterials resultieren, für die jeweiligen Nationen dargestellt. Die Kennziffern zu den technischen und taktischen Fähigkeiten richten sich dabei in ihrer Reihenfolge nach den jeweiligen Nationsmannschaften, die im Achtelfinale verloren hatten. Insgesamt 21 verschiedene Untersuchungsparameter werden für jedes Nationenteam erläutert (sechs technische Fertigkeiten, sieben offensive-taktische, acht defensiv-taktische).

\subsection{Prozentuale Qualitätsanteile technischer Elemente}

Im folgenden Kapitel wird die Qualität der technischen Fähigkeiten der einzelnen Mannschaften beschrieben. Es wird aufgezeigt, mit welcher prozentualen Häufigkeit eine bestimmte Mannschaft bezüglich eines bestimmten technischen Elementes den Bewertungsklassen hochkarätig, das heißt "gut", mittel, d.h. "normal“ und schlecht, d.h. „schwach“ zugeordnet wurde. Aufgrund der unterschiedlichen Anzahl bestrittener Spiele werden die Mannschaften hierbei in Achtel-, Viertel- und Halbfinalisten aufgeteilt.

\subsubsection{Achtelfinalisten}

Als Achtelfinalisten werden diejenigen Mannschaften bezeichnet, die im Achtelfinale ausgeschieden sind und zu deren Untersuchung deshalb nur ein Spiel herangezogen werden konnte. Zu diesen Achtelfinalisten gehören England, Chile, Mexiko, Jugoslawien, Nigeria, Paraguay, Rumänien und Norwegen, die anhand ausgewählter Ergebnisse dargestellt werden sollen. Dabei wird der gleitende Übergang zwischen quantitativen und qualitativen Beobachtungsresultaten deutlich. Sämtliche nachfolgend behandelte technische Fähigkeiten beschreiben Fähigkeiten im Umgang mit dem Ball, setzen also unbedingt den Ballbesitz der untersuchten Mannschaft voraus.

\subsubsection{Dribblings}

Das Dribbling ist eine Einzeltechnik und beschreibt die Fähigkeit eines einzelnen Spielers, sich mit dem Ball am Fuß über den Platz zu bewegen. Es ist eine sehr wichtige Fußballtaktik, weil der Dribbler den Ball sehr schnell ins Drittel des Gegners bringen kann. 
Mit schnellem Dribbling kann die Mannschaft ihren Gegner überraschen, bevor sie ihre Verteidigung organisieren kann. Der Dribbler muss Schnelligkeit haben und gleichzeitig den Ball kontrollieren, insbesondere wenn er in das Drittel des Gegners laufen will, weil die Verteidiger hoch bemüht sind, ihm den Ball abzunehmen.

Hinsichtlich der Qualität des Dribblings wurde festgestellt: Von den registrierten 20 Dribblings der englischen Mannschaft wurden vier, d.h. $20 \%$, als „,hochkarätig“ bezeichnet, das ist der größte Wert aller Mannschaften. Absteigend folgen nun alle übrigen Mannschaften mit jeweils nur einem als „hochkarätig“ bewerteten Dribbling: Jugoslawien einem von 7 Dribblings (14\%), Nigeria mit einem von 11 (9\%), Mexiko hatte bei 12 Dribblings ein (8\%) als „hochkarätig“ bewertetes, Norwegen hatte von 14 Dribbling ebenso nur ein (7\%) „hochkarätiges“. Auch Chile erhielt bei 17 Dribblings nur 1 (6\%) als „hochkarätig“ bezeichnetes, Paraguay erzielte bei 22 Dribblings nur ein (5\%) und auch Rumänien erhielt trotz 24-maligem Dribbling nur einmal die Bewertung "hochkarätig“ und mit $4 \%$ damit den kleinsten Anteil an hochkarätigen Dribblings.

Das folgende Diagramm zeigt die unterschiedlichen Qualitätsanteile des technischen Elements „hochkarätiges Dribbling“ der Achtelfinalisten in Prozent.

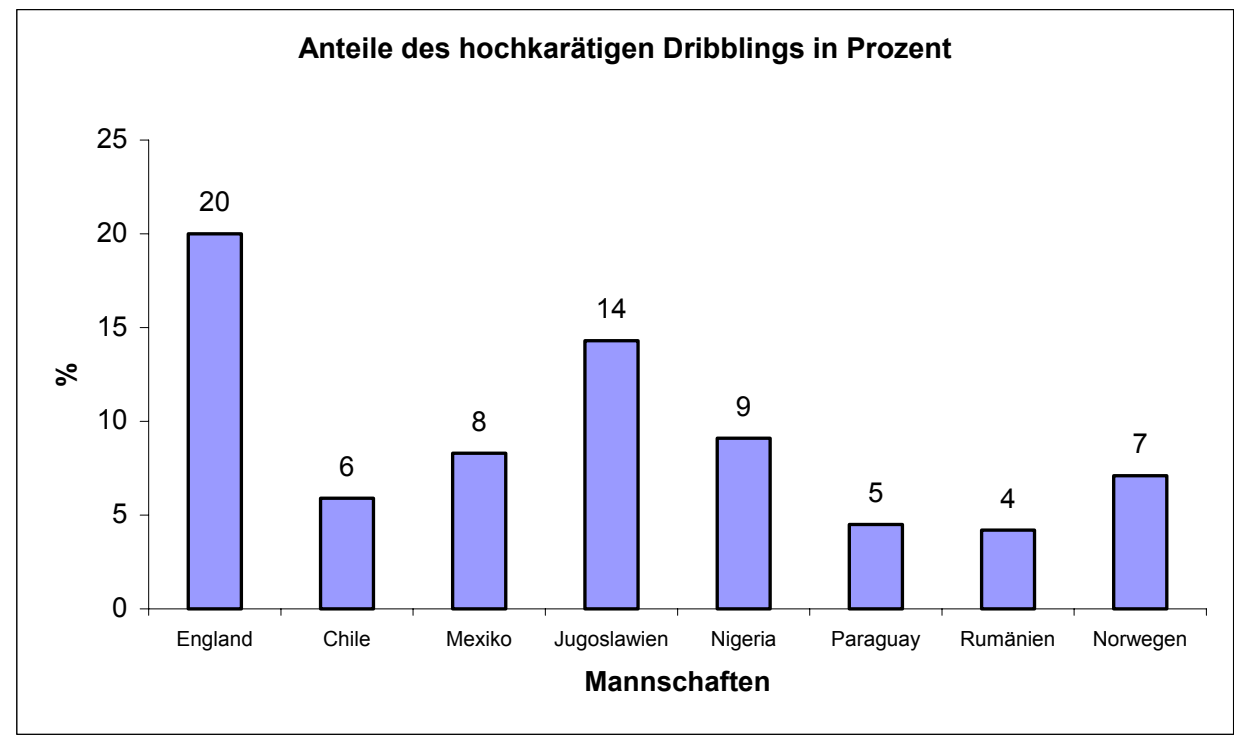

Diagramm 1: Anteile des hochkarätigen Dribblings in Prozent bei den Achtelfinalisten. 
Mit den registrierten 12 (86 \%) als "mittel“ bewerteten Dribblings hatte Norwegen den größten Anteil. Die weiteren Anteile „mittlerer“ Dribblings vor Nigeria mit 9 (82 \%), Paraguay 16 (73\%), Chile 11 (65\%), England 20 (60\%), Rumänien 14 (58\%), Jugoslawien 4 (57 \%) und Mexiko mit 5 (42\%).

Das folgende Diagramm zeigt die unterschiedlichen Qualitätsanteile des technischen Elements „normales Dribbling“ der Achtelfinalisten in Prozent.

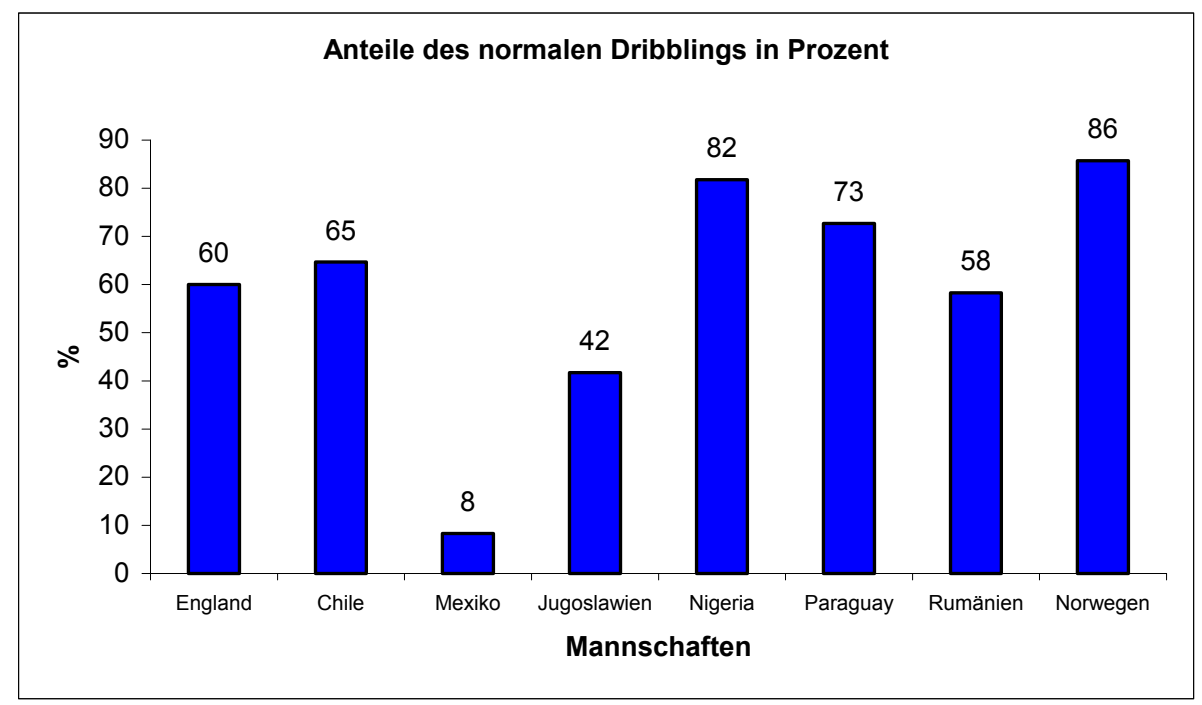

Diagramm 2: Anteile des normalen Dribblings in Prozent bei den Achtelfinalisten.

Ein "schwaches“ Dribbling wurde mit einem Anteil von 6 (50\%) am häufigsten bei Mexiko festgestellt. Es folgen Rumänien 9 (38\%), Chile 5 (29\%), Jugoslawien 2 (29\%), Paraguay 5 (23\%), England 4 (20\%), Nigeria 1 (9\%) und schließlich Norwegen mit nur einem (7\%).

Das untenstehende Diagramm zeigt die unterschiedlichen Qualitätsanteile des technischen Elements „schwaches Dribbling“ der Achtelfinalisten in Prozent. 


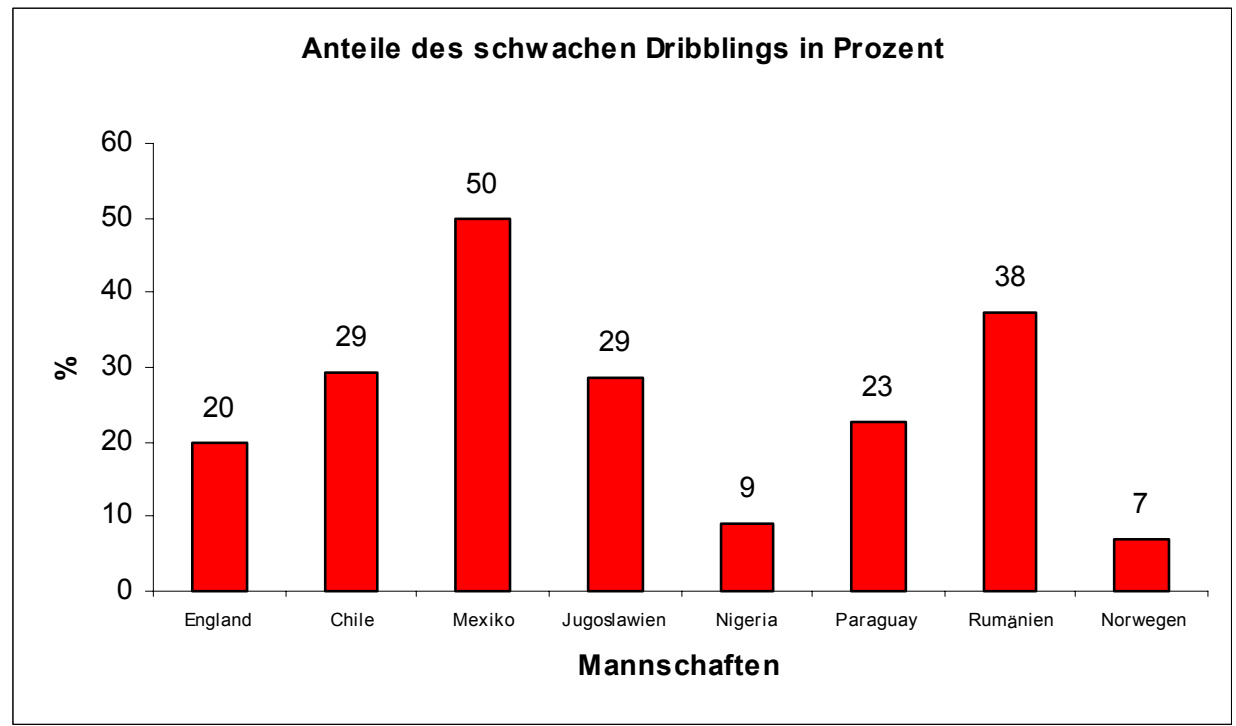

Diagramm 3: Anteile des schwachen Dribblings in Prozent bei den Achtelfinalisten. Die Diagramme 1, 2 und 3 erklären die Werte der Mannschaften:

Diagramm 1 erklärt, dass hochkarätiges Dribbling in einem Anteil zwischen $4 \%$ und $20 \%$ aller Dribblings auftrat. Die englische Mannschaft hatte die besten und die rumänische die schlechtesten Werte. Diagramm 2 zeigt, dass gutes Dribbling zwischen $42 \%$ und $86 \%$ vorkam. Die norwegische Mannschaft hatte hier die besten Werte und die mexikanische die schlechtesten. Diagramm 3 schließlich stellt dar, dass schwaches Dribbling einen Anteil zwischen $7 \%$ und $50 \%$ hatte. Demzufolge hatte die mexikanische Mannschaft die schwächsten und die nigerianische die besten Werte.

Aus den Diagrammen lässt sich schlussfolgern, dass die normalen Dribblings bei allen Achtelfinalisten einen größeren Anteil hatten als die hochkarätigen und die schlechten Dribblings. Dabei hatten alle außer der englische Mannschaft mehr schlechte als hochkarätige Dribblings. Die Spieler können den Ball im Lauf offenbar relativ schlecht kontrollieren.

Nun könnte man versuchen, die drei Gütemaßstäbe zusammenzufassen. Dann müssten aber diese qualitativ erhobenen Daten in quantitative umwandelt werden (z.B. mit der Bewertung 2 für hochkarätig, 1 für normal und 0 für schlecht). Aber die Abstände zwischen den Qualitäten blieben die gleichen, nämlich 1. Ich habe daher darauf verzichtet, durch die Umwandlung in Ziffern eine scheinbare Objektivität vorzuspiegeln. Wichtig ist hier festzuhalten, dass auch die englische Mannschaft trotz ihrem sehr guten Dribbling zu den Verlierern gehörte. 


\subsubsection{Körpertäuschung}

Die Körpertäuschung ist eine Einzeltaktik und beschreibt die Fähigkeit eines Spielers, einen Gegenspieler durch Bewegungen des eigenen Körpers über sein eigenes Vorhaben zu täuschen und diesen so zu spieltaktisch fehlerhaften Handlungen zu verführen. Die Körpertäuschung ist ein sehr wichtiges Fußballelement, weil der Täuscher auf das Tor zuläuft, wenn er im letzten Drittel den Gegner täuschen konnte.

Hinsichtlich der Qualität der Körpertäuschung wurde festgestellt, dass bei Nigeria von 22-maliger Körpertäuschung $9(41 \%)$ als hochkarätig bezeichnet wurden und damit den größten Anteil hatte. Absteigend folgen England mit 6 von 23 (26\%), Norwegen mit einer von 8 (13\%), Paraguay mit zwei von 18 (11\%), Mexiko mit zwei von 23 (9\%), Jugoslawien mit einer von $12(8 \%)$, Chile und Rumänien hatten mit jeweils einer von $22(5 \%)$ die kleinsten Anteile als hochkarätig bezeichneter Körpertäuschungen.

Das folgende Diagramm zeigt die unterschiedlichen Qualitätsanteile des technischen Elements „hochkarätige Körpertäuschung“ der Achtelfinalisten in Prozent.

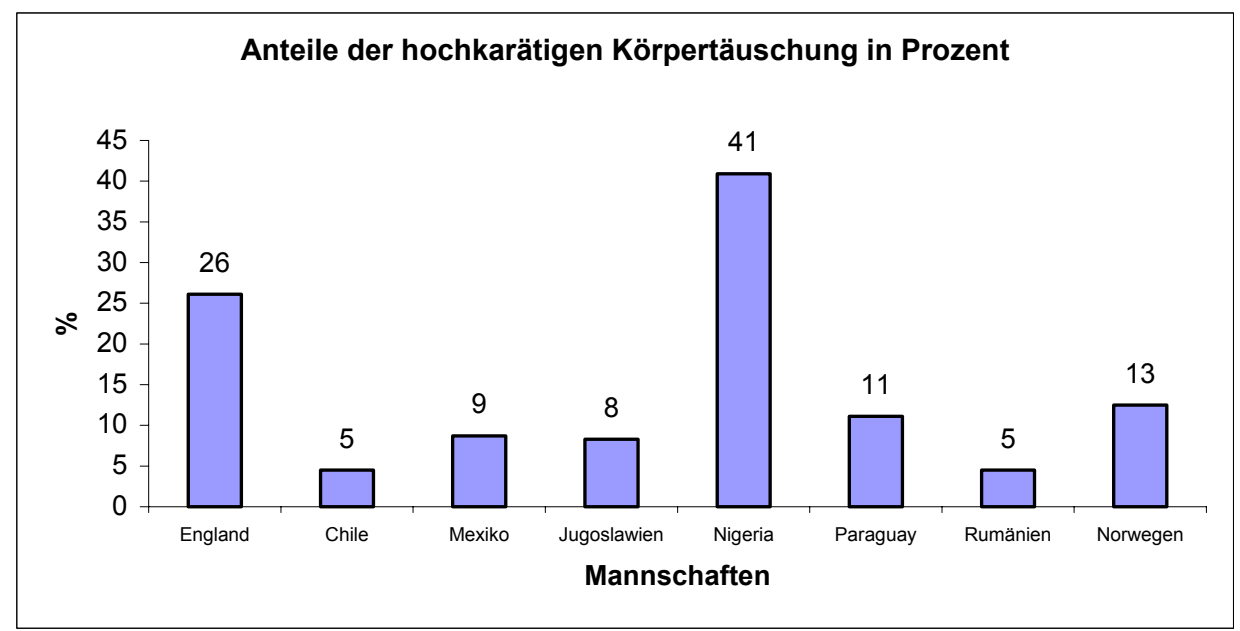

Diagramm 4: Anteile der hochkarätigen Körpertäuschung in Prozent bei den Achtelfinalisten.

16 (73\%) der Körpertäuschungen von Chile wurden mit "normal“ bewertet und bildeten den größten Anteil. Die weiteren Anteile „normaler“ Körpertäuschungen lauten 16 (70 \%) von Mexiko, 14 (64 \%) von Rumänien, 13 (57 \%) von England, 8 (44 \%) von Paraguay, 5 (42 \%) hatte Jugoslawien, 2 (25\%) hatte Norwegen und 4 (18\%) Nigeria. 
Das folgende Diagramm zeigt die unterschiedlichen Qualitätsanteile des technischen Elements „normale Körpertäuschung“ der Achtelfinalisten in Prozent.

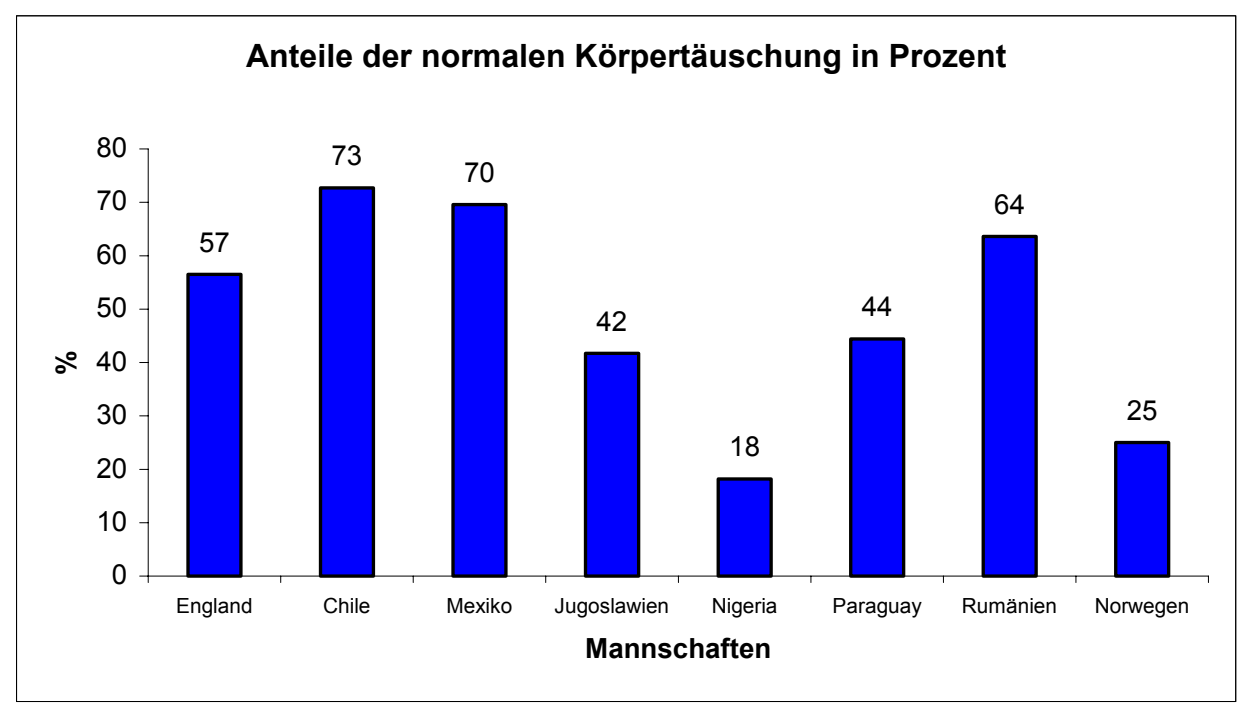

Diagramm 5: Anteile der normalen Körpertäuschung in Prozent bei der Achtelfinalisten.

Eine „schwache“ Körpertäuschung wurde mit einem Anteil von $63 \%$ aller Körpertäuschungen am häufigsten bei Norwegen festgestellt. Es folgen Jugoslawien 6 (50\%), Paraguay 8 (44\%), Nigeria 9 (40\%), Rumänien 7 (32\%), Chile 5 (23\%), Mexiko 5 (22\%) und schließlich England 4 (17\%).

Das folgende Diagramm zeigt die unterschiedlichen Qualitätsanteile des technischen Elements „schwache Körpertäuschung“ der Achtelfinalisten in Prozent.

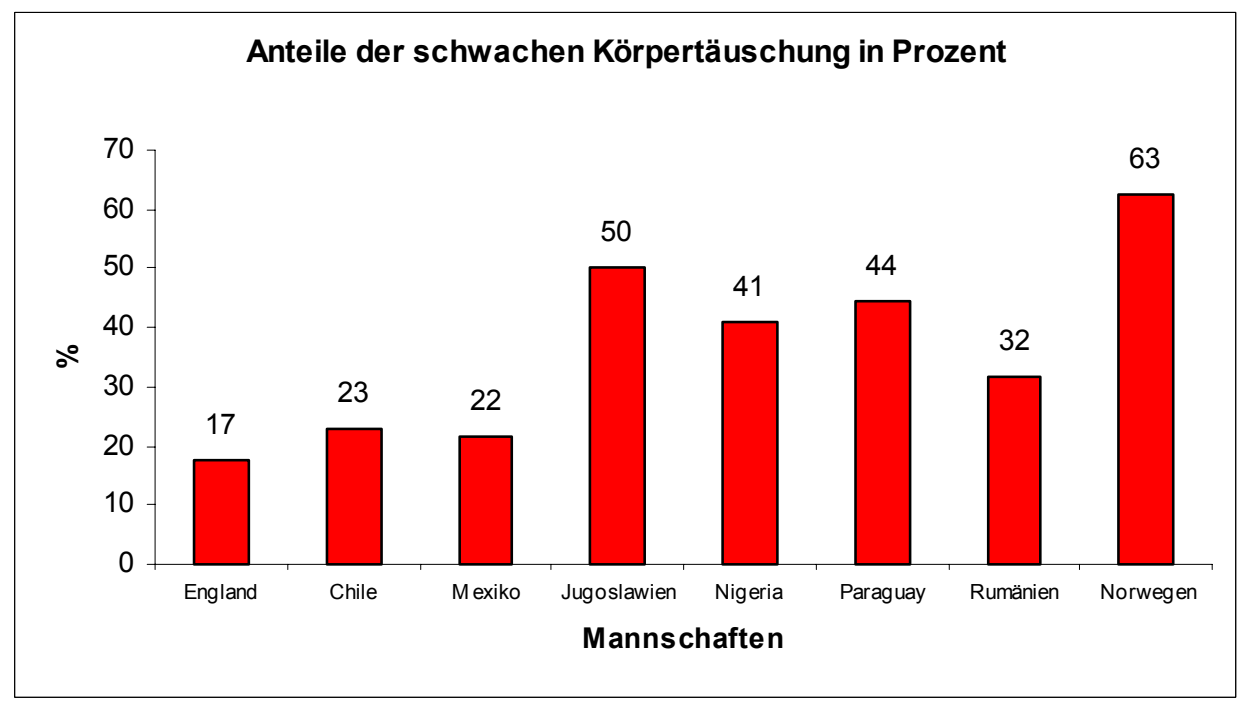

Diagramm 6: Anteile der schwachen Körpertäuschung in Prozent bei der Achtelfinalisten. 
Die Diagramme 4, 5 und 6 erklären die Werte der Mannschaften:

Diagramm 4 zeigt, dass hochkarätige Körpertäuschung zu einem Anteil zwischen 5 $\%$ und $41 \%$ aller Körpertäuschungen vorkam. Die nigerianische Mannschaft hatte die besten Werte und die chilenische und rumänische die schlechtesten. Diagramm 5 erklärt, dass gute Körpertäuschung zwischen $18 \%$ und $73 \%$ Die norwegische Mannschaft hatte hier die besten Werte und die mexikanische hatte die schlechtesten. Diagramm 6 erklärt, dass schwache Körpertäuschung zwischen $17 \%$ und $63 \%$ ausmacht. Die norwegische Mannschaft hatte die schwächsten Werte und die englische die besten Werte.

Aus den Diagrammen lässt sich die Schlussfolgerung ziehen, dass die normalen Körpertäuschungen bei allen Achtelfinalisten öfter als die hochkarätigen sind und hochkarätigen Körpertäuschungen seltener als schwache, außer bei der englischen Mannschaft. Erfolgreichende Körpertäuschungen hatten die Achtelfinalisten meistens, wenn der Ballbesitzer weit vom letzten Drittel des gegnerischen Spielfeld entfernt war und entweder das Pressing vom Gegner im letzten Drittel sehr stark war oder er in Mittelfeld ein leichtes Pressing ausübte.

\subsubsection{Torschuss}

Der Torschuss ist eine Einzeltechnik und beschreibt die Fähigkeit eines Spielers, den Ball zielgenau und im Bemühen um einen Torerfolg in Richtung gegnerisches Tor zu schießen. Denn nach den internationalen Fußballregeln gewinnt die Mannschaft, die in einem Spiel die meisten Tore erzielt!

Hinsichtlich der Qualität der Torschuss wurde festgestellt: Von den registrierten 24 Torschüssen wurden bei der englischen Mannschaft 6 (25\%) als „hochkarätig“ bewertet und bildeten den größten Wert. Absteigend folgen nun mit jeweils einem Torschuß Jugoslawien mit einem von 6 (17\%), Chile einem von 12 (8\%), Mexiko mit einem von 14 (7\%), Nigeria hatte einen von $26(4 \%)$. Keinen hochkarätigen Torschuß hatten Paraguay bei 9 , Rumänien 13 und Norwegen 10 Torschüssen mit jeweils $0(0,0 \%)$.

Das folgende Diagramm zeigt die unterschiedlichen Qualitätsanteile des technischen Elements „hochkarätiger Torschuss“ der Achtelfinalisten in Prozent. 


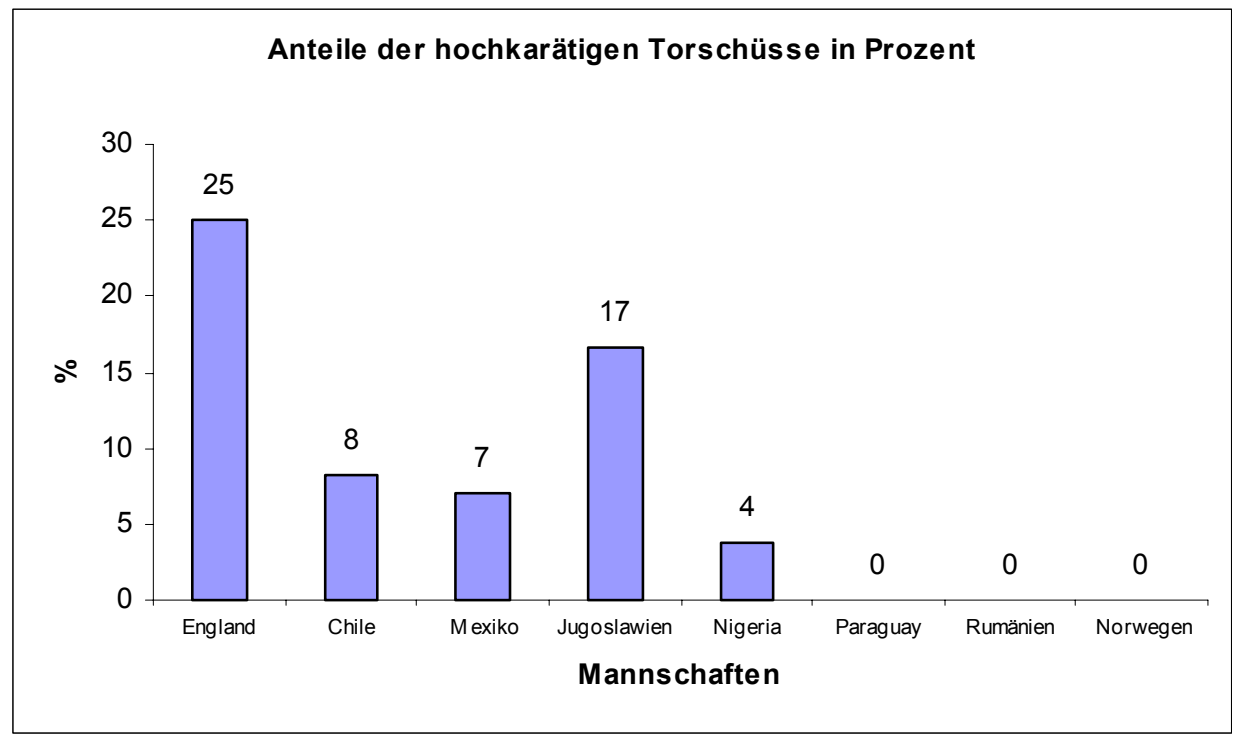

Diagramm 7: Anteile der hochkarätigen Torschüsse in Prozent bei den Achtelfinalisten.

$10(71 \%)$ der Torschüsse von Mexiko wurden mit „mittel“ bewertet und bildeten den größten Anteil. Die weiteren Anteile „mittlere“ Torschüsse lauten 4 (40\%) für Norwegen, 4 (33 \%) für Chile, 2 (33\%) für Jugoslawien, 3 (23 \%) für Rumänien, 4 (17\%) für England, 4 (15\%) für Nigeria und 0 (0,0\%) für Paraguay.

Das folgende Diagramm zeigt die unterschiedlichen Qualitätsanteile des technischen Elements „mittlerer Torschuss“ der Achtelfinalisten in Prozent.

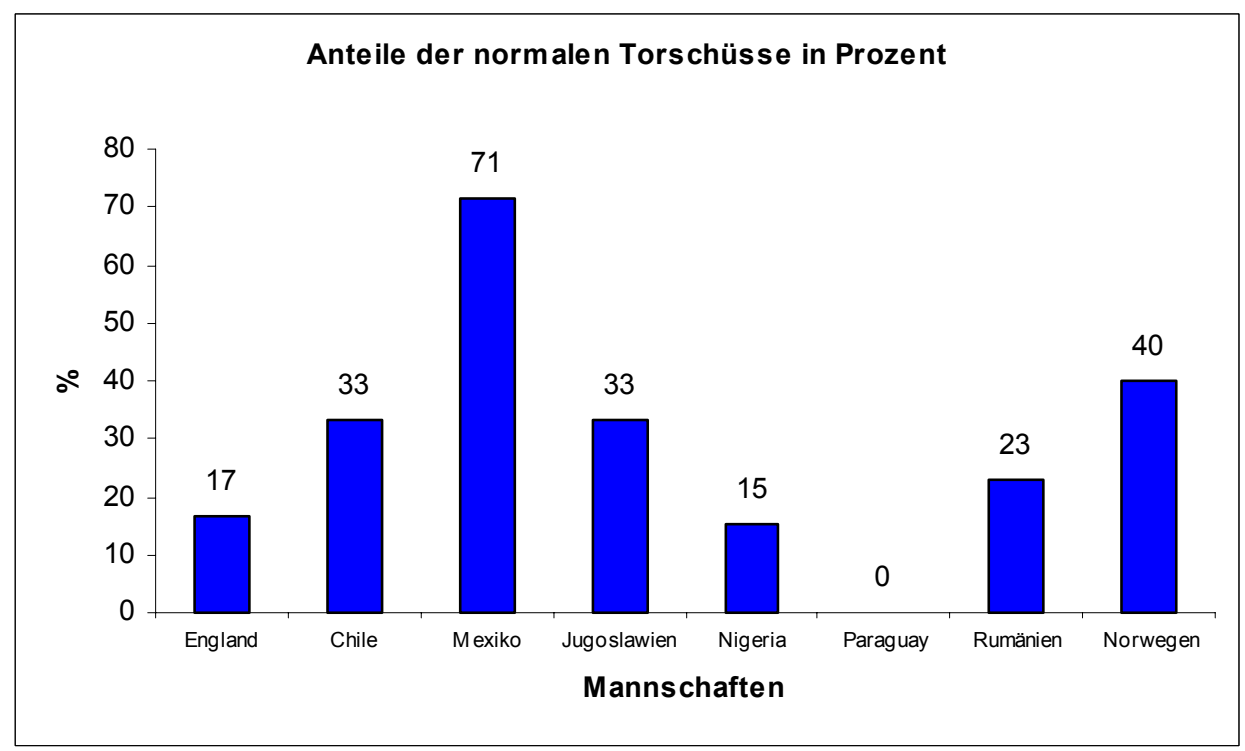

Diagramm 8: Anteile der normalen Torschüsse in Prozent bei der Achtelfinalisten. 
Ein "schwacher“ Torschuss wurde mit einem Anteil von 9 (100\%) am häufigsten bei Paraguay festgestellt. Es folgen Nigeria 21 (81\%), Rumänien 10 (77\%), Norwegen 6 (60\%), England 14 (58\%), Chile 7 (58\%60 \%), En474.25n Nigerc6 (60\%), En480.25n Niger 
der Ballbesitzer einen Alibipass auf einen schlechter postierten Mitspieler spielt, anstatt selbst mutig mit einem Solo in den Strafraum einzudringen, oder dass der mögliche Torschütze nicht auf eine für den Torabschluss günstige Position läuft und deshalb nicht gut angespielt werden kann. Nahe vor dem Tor zugespielte Bälle können wahrscheinlich nicht direkt verwandelt werden. Es darf hierbei nicht vergessen werden, dass alle Mannschaften aus dem Turnier nach diesem Spielen ausschieden, wozu die schwachen Torschüsse beigefragen haben.

\subsubsection{Kurze und lange Pässe}

Kurze Pässe sind eine Einzeltechnik und bezeichnen die Fähigkeit eines einzelnen Spielers, den Ball zielgenau über eine maximale Distanz von zehn Metern zu einem Mitspieler zu schießen. Lange Pässe gehen dagegen über eine Distanz von mehr als zehn Metern zu einem Mitspieler. Pässe haben unter den Spieltechniken die höchste Anzahl und sind dabei auch eine der leichtesten auszuführenden Techniken im Fußball, um den Ball zu behalten. Der Spieler muss darauf achten, wohin und wann er den Ball günstigenfalls abgeben kann und wie kräftig der Pass zu seinem Mitspieler auszuführen ist.

Hinsichtlich der Qualität der kurzen Pässe wurde festgestellt: Norwegen bei 344 mal, wurden mit 6 (2 \%) als „,hochkarätig“ bezeichnet. Absteigend folgen Jugoslawien mit 203 und $3(2 \%)$, Nigeria mit 406 und $6(2 \%)$. England mit 223 und $3(1 \%)$, Mexiko mit 310 und $3(1 \%)$, Paraguay mit 344 und $2(1 \%)$, Rumänien mit 480 und $3(1 \%)$, Chile von 515, wurden $3(1 \%)$, hatten mit jeweils $1 \%$ den kleinsten Anteil „hochkarätiger" kurzer Pässe.

Das folgende Diagramm zeigt die unterschiedlichen Qualitätsanteile des technischen Elements "hochkarätige kurze Pässe“ der Achtelfinalisten in Prozent. 


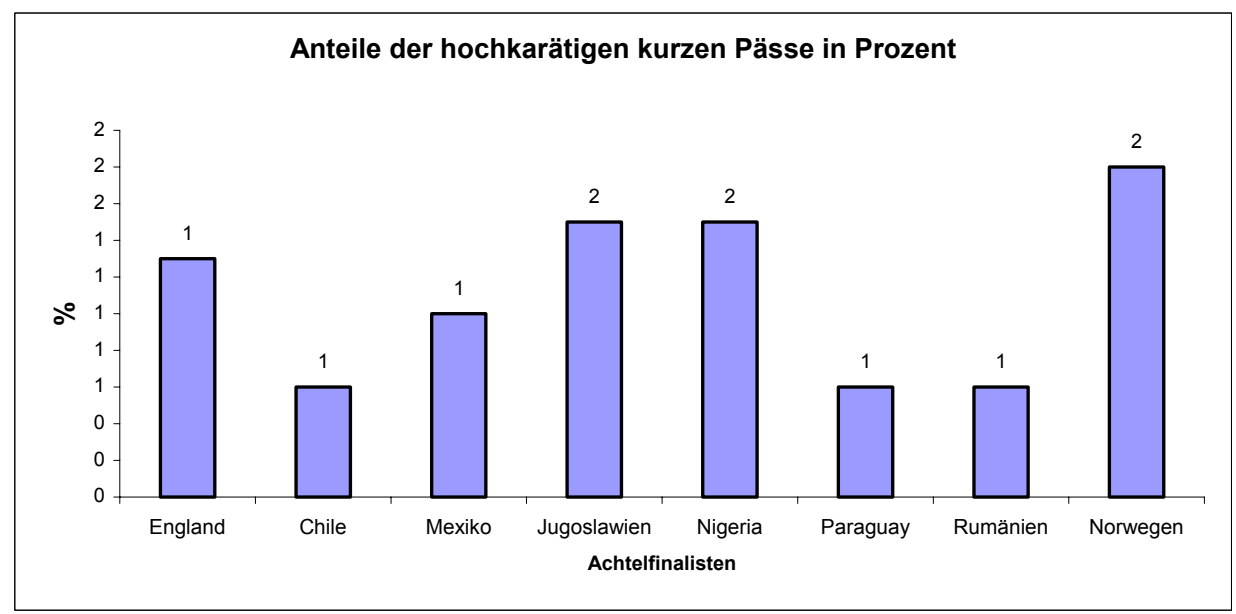

Diagramm 10: Anteile der hochkarätigen kurzen Pässe in Prozent bei den Achtelfinalisten.

371 (91 \%) der kurzen Pässe von Nigeria wurden mit „mittel“ bewertet und bildeten den größten Anteil. Die weiteren Anteile „mittlerer“ kurzer Pässe lauteten 457 (89 \%) für Chile, 178 (88 \%) für Jugoslawien, 421 (88\%) für Rumänien, 299 (87 \%) für Paraguay, 289 (87 \%) für Norwegen, 265 (86 \%) für Mexiko und 188 (84 \%) für England. Das folgende Diagramm zeigt die unterschiedlichen Qualitätsanteile des technischen Elements „mittlere kurze Pässe“ der Achtelfinalisten in Prozent.

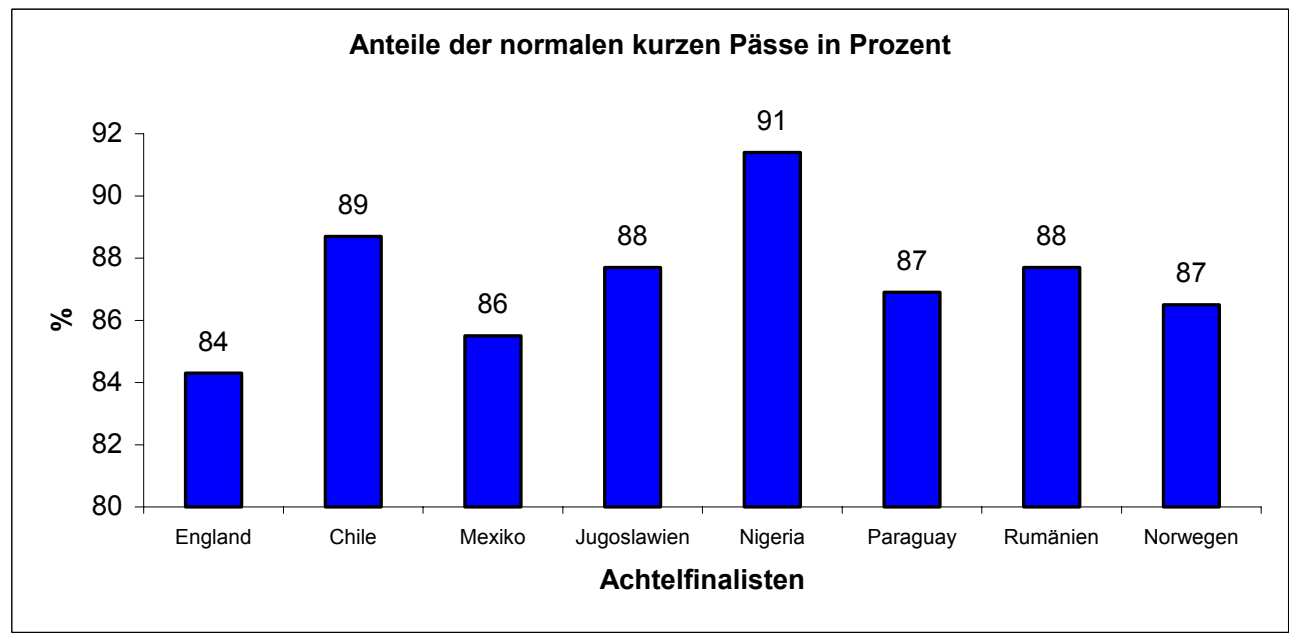

Diagramm 11: Anteile der normalen kurzen Pässe in Prozent der Achtelfinalisten.

Ein „schwacher" kurzer Pass wurde mit einem Anteil von 32 (14 \%) am häufigsten bei England festgestellt. Es folgen Mexiko 42 (14 \%), Paraguay 43 (13\%), Norwegen 39 (12\%) und Rumänien 56 (12\%), Jugoslawien 22 (11\%), Chile 55 (11\%) und schließlich Nigeria 29 (7\%).

Das folgende Diagramm zeigt die unterschiedlichen Qualitätsanteile des technischen Elements „schwache kurze Pässe“ der Achtelfinalisten in Prozent. 


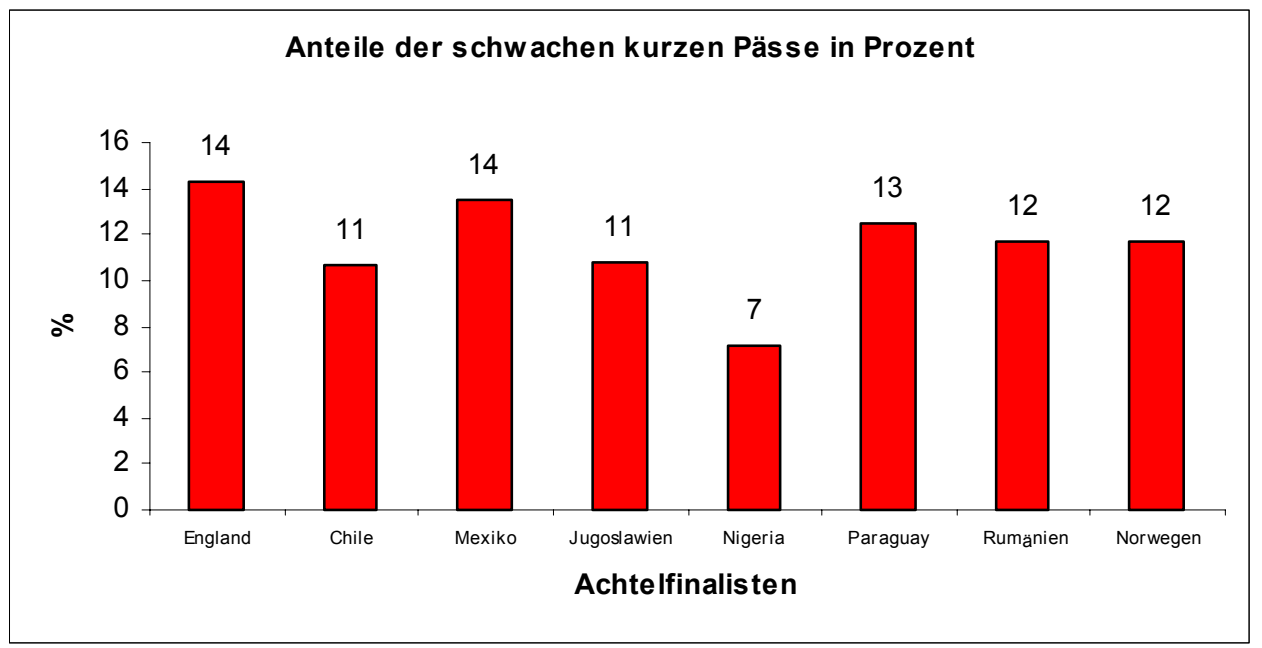

Diagramm 12: Anteile der schwachen kurzen Pässe in Prozent bei den Achtelfinalisten.

Lange Pässe hatte Nigeria 68-mal, davon wurden 4 (6 \%) als hochkarätige bewertet und bildeten den größten Wert. Absteigend folgen Rumänien von 72 wurden 3 (4\%), Norwegen von 83 wurden 3 (4 \%), Paraguay von 139, wurden 4 (3\%), Chile von 57, wurde 1 (2\%), England von 134 wurden 2 (2\%), Jugoslawien von 85 wurde 1 (1\%), und bei Mexiko von 106 ebenfalls 1 (1\%) als „hochkarätige“ lange Pässe identifiziert. Das folgende Diagramm zeigt die Anteile der „hochkarätigen langen Pässe“ in Prozent für die Achtelfinalisten.

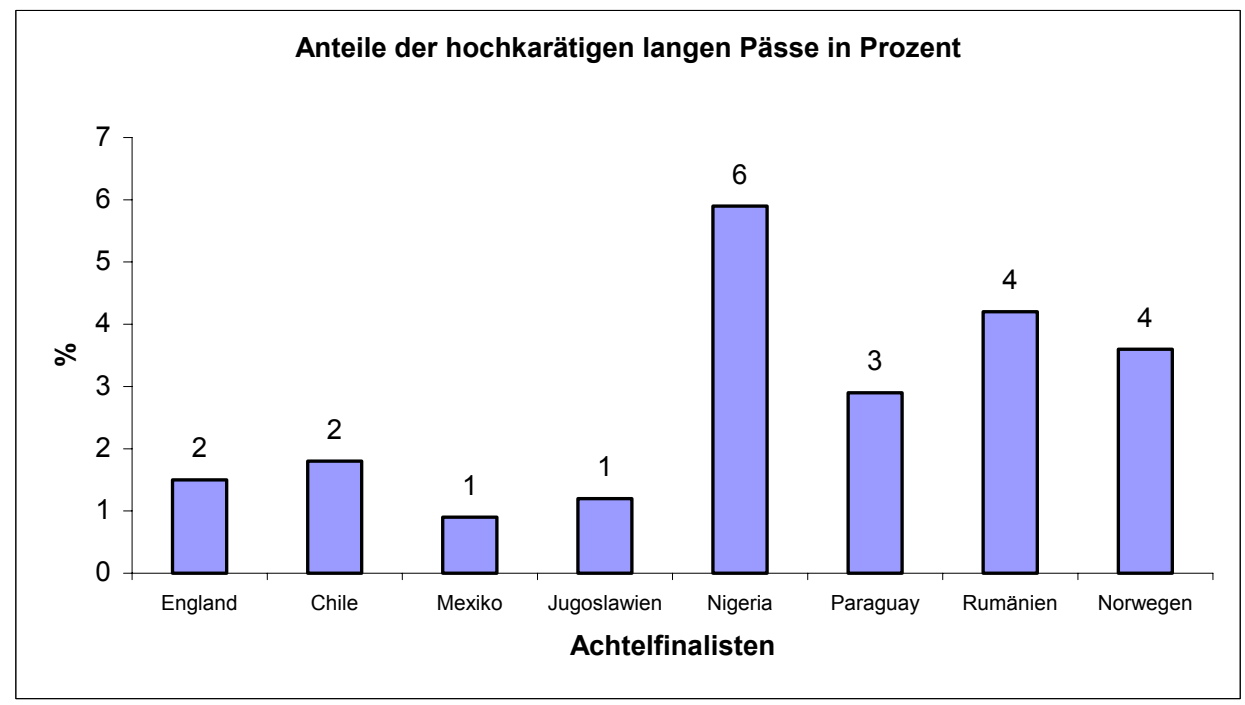

Diagramm 13: Anteile der hochkarätigen langen Pässe in Prozent der Achtelfinalisten. 
$28(41 \%)$ der langen Pässe von Nigeria wurden mit „mittel“ bewertet und bildeten den größten Anteil. Die weiteren Anteile „mittlerer“ langer Pässe lauteten 20 (35\%) für Chile, 22 (31 \%) für Rumänien, 25 (30 \%) für Norwegen, 29 (27\%) für Mexiko, 35 (26 \%) für England, 20 (24\%) für Jugoslawien und 28 (20\%) für Paraguay.

Das folgende Diagramm zeigt die unterschiedlichen Qualitätsanteile des technischen Elements „mittlere lange Pässe“ der Achtelfinalisten in Prozent.

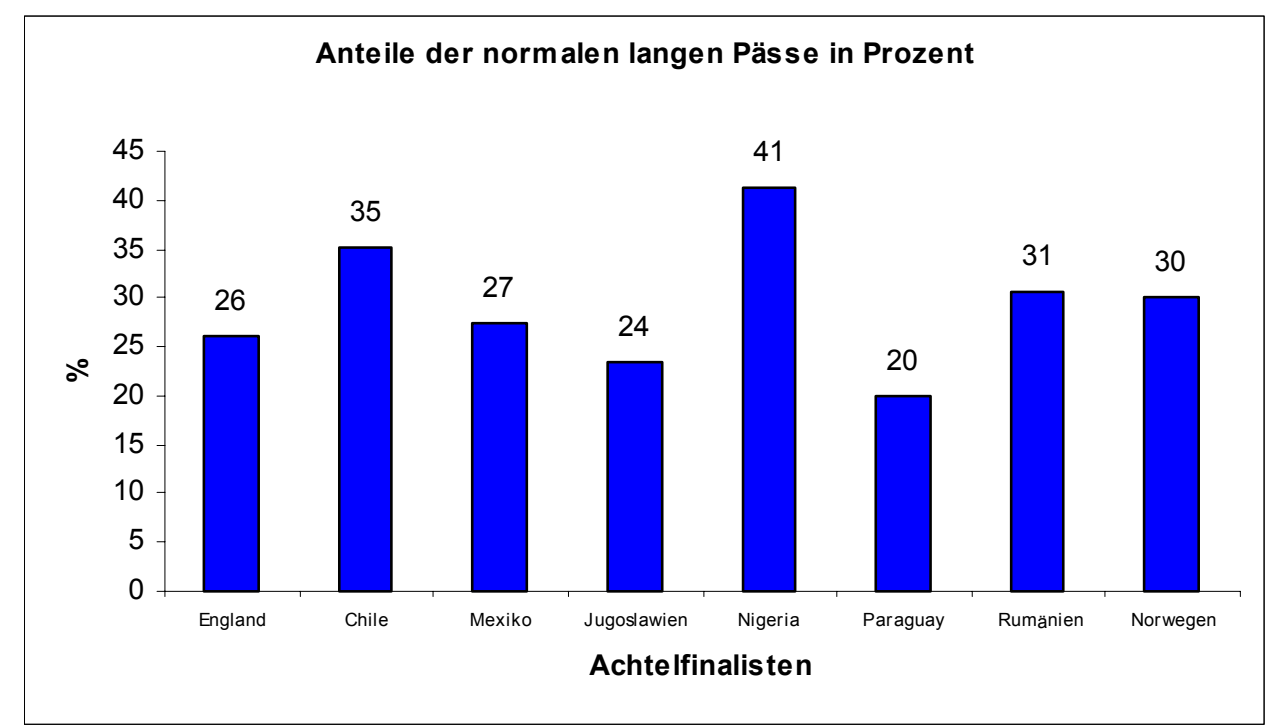

Diagramm 14: Anteile der normalen langen Pässe in Prozent der Achtelfinalisten.

Ein „schwacher“ langer Pass wurde mit einem Anteil von 107 (77 \%) am häufigsten bei Paraguay festgestellt. Es folgen Jugoslawien 64 (75\%), England 97 (72 \%), Mexiko 76 (72\%), Norwegen 55 (66\%), Rumänien 47 (65\%), Chile 36 (63\%) und schließlich Nigeria 36 (53\%).

Das folgende Diagramm zeigt die unterschiedlichen Qualitätsanteile des technischen Elements „schwache lange Pässe“ der Achtelfinalisten in Prozent. 


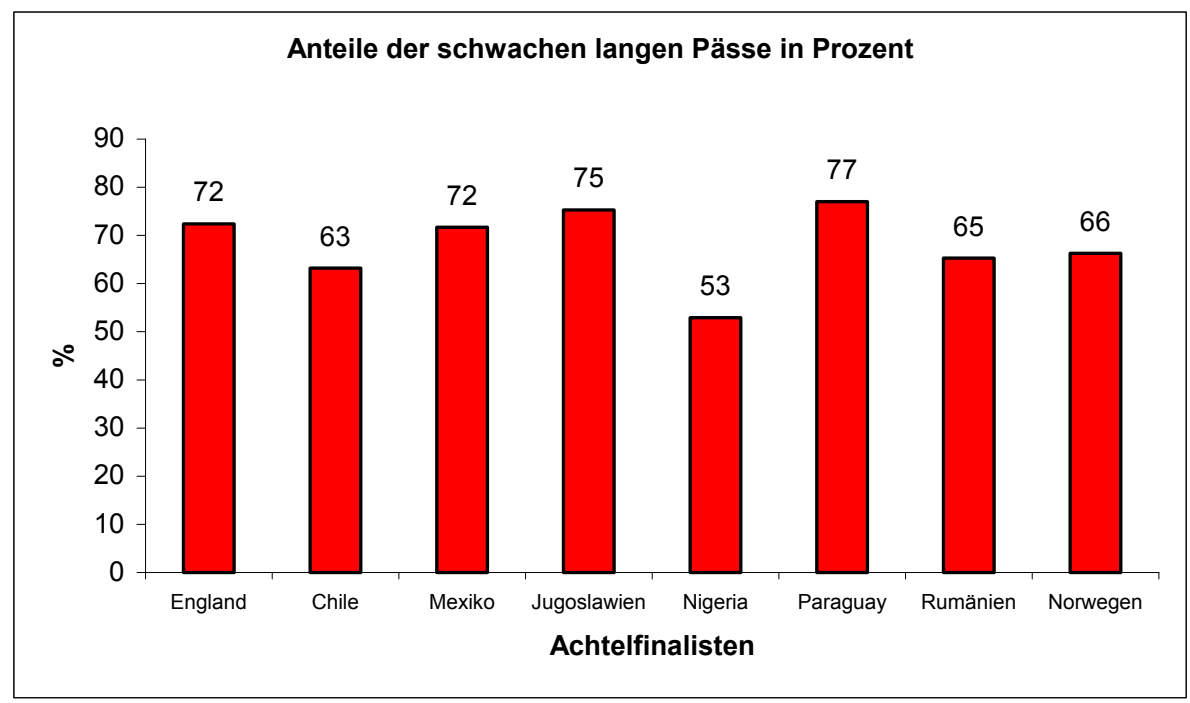

Diagramm 15: Anteile der schwachen langen Pässe in Prozent der Achtelfinalisten.

Die Diagramme 10, 11,12,13, 14 und 15 erklären die Werte der Mannschaften:

Diagramm 10 erklärt, dass die hochkarätigen kurzen Pässe zwischen $1 \%$ und $2 \%$ lagen. Die englische Mannschaft hatte die besten Werte und die rumänische, paraguayische und norwegische die schlechtesten. Diagramm 11 erklärt, dass die normalen kurzen Pässe zwischen 84 \% und 91 \% lagen. Die mexikanische Mannschaft hatte hier die besten Werte und die paraguayische hatte die schlechtesten. Diagramm 12 zeigt, dass die schwachen kurzen Pässe zwischen $7 \%$ und $14 \%$ lag. Die paraguayische Mannschaft hatte die schwächsten Werte und die mexikanische hatte die besten Werte.

Es lässt sich die Schlussfolgerung aus den Diagrammen ziehen, dass die normalen und schwachen kurzen Pässe bei allem Achtelfinalisten häufiger sind als die hochkarätigen. Wahrscheinlich hatten die Mannschaften eine unfangreicher oder langsame Vorbereitung in Mittelfeld, wodurch sie der anderen Mannschaft eine gute Chance gaben, um ihre Verteidigung zu organisieren.

Diagramm 13 erklärt, dass die langen Pässe zwischen $1 \%$ und $6 \%$ ausmachten. Die nigerianische Mannschaft hatte die besten Werte, die jugoslawische und mexikanische die schlechtesten. Diagramm 14 erklärt, dass gute lange Pässe zwischen $20 \%$ und $41 \%$ lagen. Die nigerianische Mannschaft hatte hier die besten Werte und die paraguayische hatten die schlechtesten. Diagramm 15 erklärt, dass schwache lange Pässe zwischen $53 \%$ und $77 \%$ lagen. Die paraguayische Mannschaft hatte die schwächsten Werte und die nigerianische hatte die besten Werte. 
Es lässt sich die Schlussfolgerung aus den Diagrammen ziehen, dass die schwachen langen Pässe bei allem Achtelfinalisten häufiger sind als die hochkarätigen und die normalen. Bei langen Pässen muss auf jeden Fall der Spieler mit großer Genauigkeit spielen müssen, weil der Ball sehr leicht abzunehmen ist und es dauert länger als bei kurzen Pässen bis der Ball zum anderen Mitspieler kommt. Die Pässe wurden unkonzentriert, technisch unsauber, schlecht getimt (zu scharf / zu schwach) und oft mit Effet gespielt. Dadurch hat der annehmende Spieler Probleme mit der Ballkontrolle oder mit der Schuss- Direktannahme.

\subsubsection{Ballkontrolle}

Die Ballkontrolle ist eine Einzeltechnik, die die Fähigkeit eines Spielers beschreibt, den Ball so zu behandeln, dass dessen Verhalten für diesen jederzeit kontrollierbar ist. Mit der Verbesserung des Fußballspiels ist die Verteidigung effektiver geworden, da die gegnerischen Mannschaften sich immer bemühen, Tore zu verhindern. Körpertäuschung ist deswegen für den Angreifer von großer Bedeutung geworden, da er seinen Ball schnell unter Kontrolle bringen muss, besonders in Tornähe.

Hinsichtlich der Qualität der Ballkontrolle wurde festgestellt: Die Ballkontrolle hatte Nigeria 61-mal hiervon wurden 4 (7\%) als „hochkarätig“ bewertet und bildete den größten Wert. Absteigend folgen Mexiko mit 42, hiervon wurden 2 (5\%), Jugoslawien 56, hiervon 2 (4\%), Rumänien von 29 wurde 1 (3\%), Norwegen von 34 wurde 1 (3\%), Chile von 39 wurde 1 (3\%) als hochkarätig bewertet.

Den kleinsten Anteil an „hochkarätiger“ Ballkontrolle hatten England von 45 nur 1 (2 \%). Paraguay von 61 ebenfalls nur 1 (2\%).

Das folgende Diagramm zeigt die unterschiedlichen Qualitätsanteile des technischen Elements „hochkarätige Ballkontrolle“ der Achtelfinalisten in Prozent. 


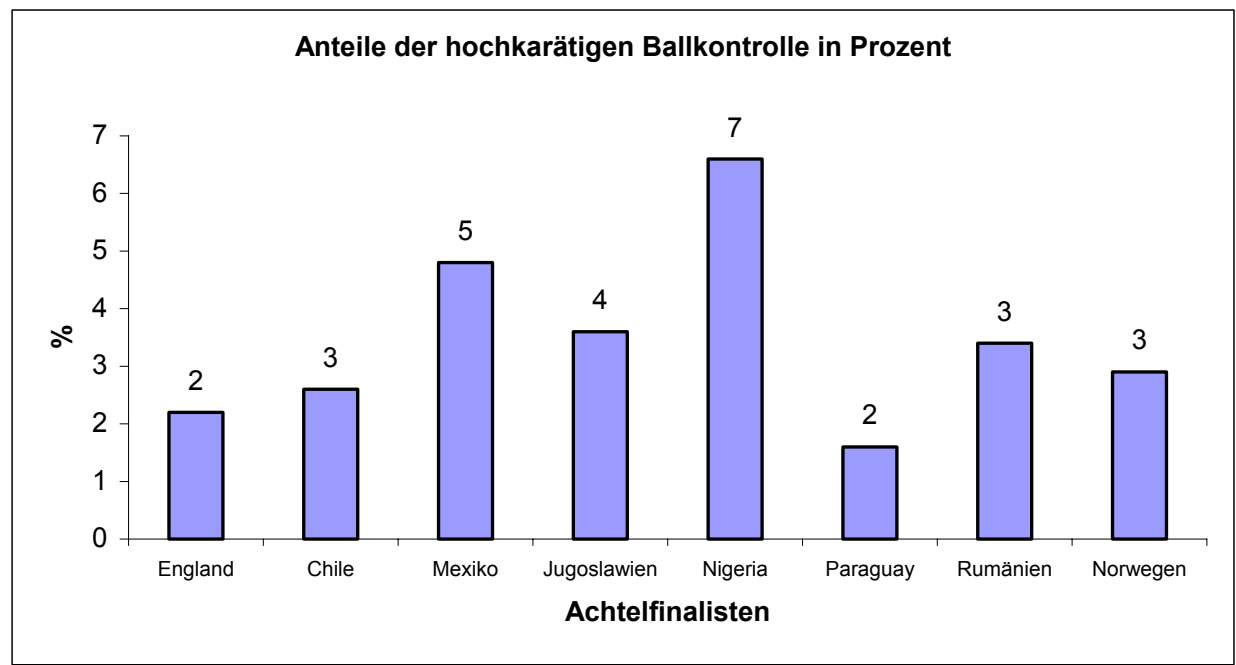

Diagramm 16: Anteile der hochkarätigen Ballkontrolle in Prozent der Achtelfinalisten.

26 (62 \%) der Ballkontrollen von Mexiko wurden mit „mittel“ bewertet und bildeten den größten Anteil. Die weiteren Anteile „mittlerer“ Ballkontrollen lauten 29 (52 \%) für Jugoslawien, 25 (41\%) für Nigeria, 17 (38 \%) für England, 19 (31\%) für Paraguay, 11 (28 \%) für Chile, 7 (24 \%) für Rumänien und 8 (24 \%) für Norwegen.

Das folgende Diagramm zeigt die unterschiedlichen Qualitätsanteile des technischen Elements „mittlere Ballkontrolle“ der Achtelfinalisten in Prozent.

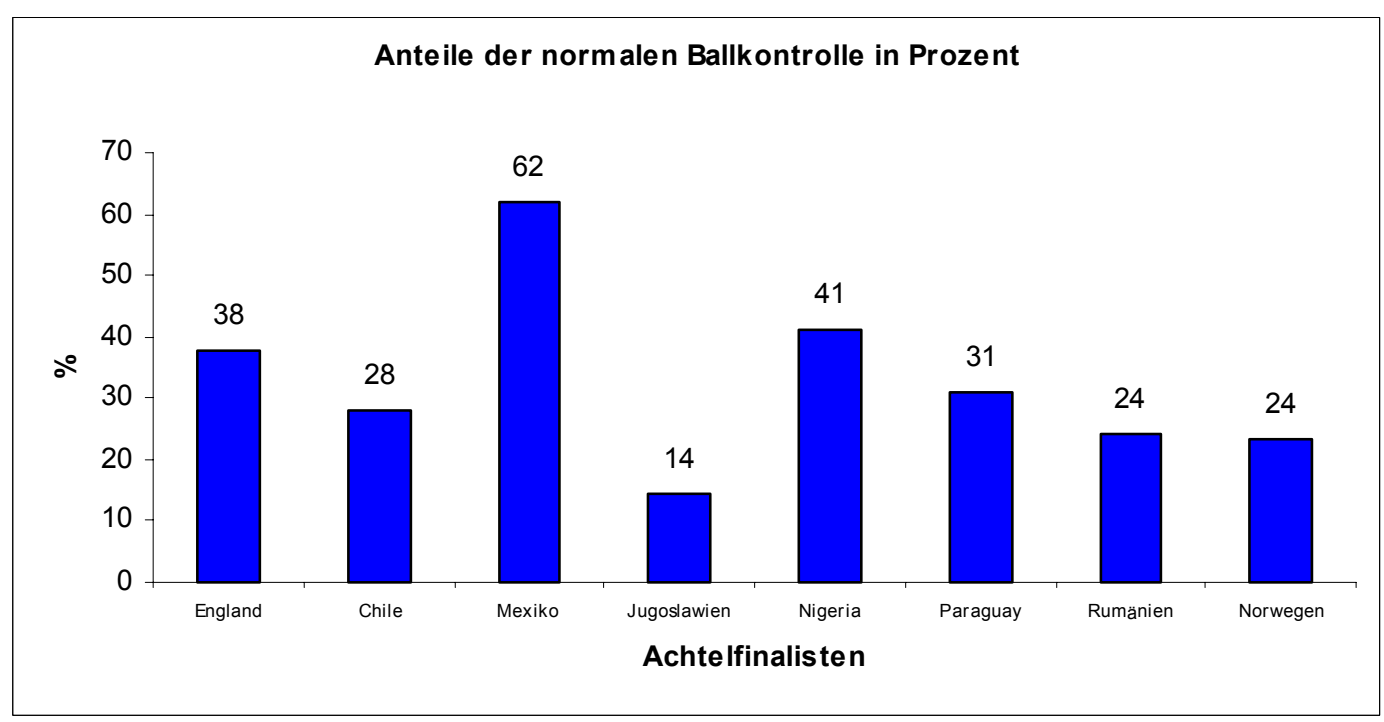

Diagramm 17: Anteile der normalen Ballkontrolle in Prozent bei den Achtelfinalisten.

Eine „schwache“ Ballkontrolle wurde mit einer Anzahl von 25 (74 \%) am häufigsten bei Norwegen festgestellt. Es folgen Rumänien 21 (72 \%), Chile 27 (69 \%), Paraguay 
$41(67 \%)$, England 27 (60\%), Nigeria 32 (53\%), Jugoslawien 25 (45\%) und schließlich Mexiko 14 (33\%).

Das folgende Diagramm zeigt die unterschiedlichen Qualitätsanteile des technischen Elements „schwache Ballkontrolle“ der Achtelfinalisten in Prozent.

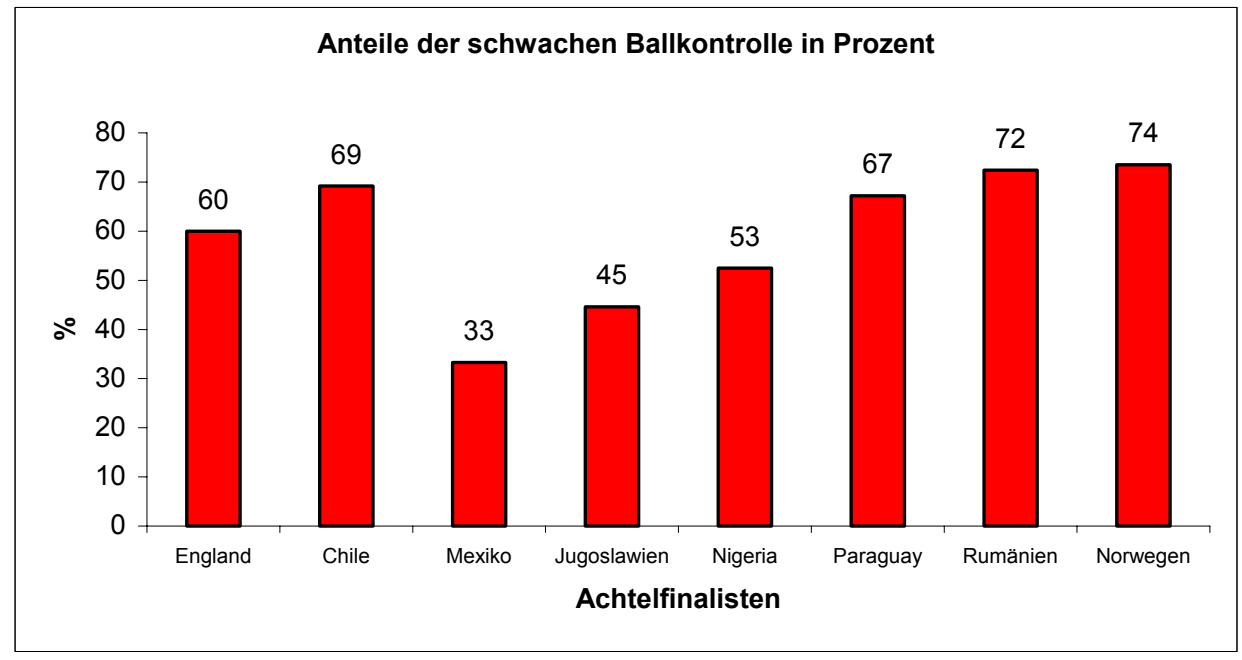

Diagramm 18: Anteile der schwachen Ballkontrolle in Prozent bei den Achtelfinalisten.

Die Diagramme 16, 17 und 18 erklären die Werte der Mannschaften:

Diagramm 16 erklärt, dass die hochkarätige Ballkontrolle einen Anteil zwischen $2 \%$ und $7 \%$ hat. Die nigerianische Mannschaft hatte die besten Werte, die englische und paraguayische die schlechtesten. Diagramm 17 erklärt, dass der Anteil der guten Ballkontrolle zwischen $14 \%$ und $62 \%$ beträgt. Die mexikanische Mannschaft hat hier die besten Werte und die jugoslawische die schlechtesten. Diagramm 18 erklärt, dass der Anteil der schwachen Ballkontrolle zwischen $33 \%$ und $74 \%$ beträgt. Die norwegische Mannschaft hatte die niedrigsten Werte und die mexikanische die besten.

Aus den Diagrammen lässt sich die Schlussfolgerung ziehen, dass die schwache Ballkontrolle bei allen Achtelfinalisten einen größeren Anteil hat als die hochkarätige und die hochkarätige Ballkontrolle auch seltener ist als die normale. Das ist dadurch zu erklären, dass die Angreifer ihren Ball oftmals verlieren, wenn das Pressing im Drittel des Gegners stark ist und bei dem schnellen Spielverlauf die Spieler ihren Ball schnell unter ihre Kontrolle bringen müssen. 


\subsubsection{Viertelfinalisten}

Als Viertelfinalisten werden diejenigen Mannschaften bezeichnet, die im Viertelfinale ausgeschieden sind und zu deren Analyse deshalb zwei Spiele herangezogen werden konnten, deren Ergebnisse addiert wurden. Zu diesen Viertelfinalisten gehören Deutschland, Italien, Argentinien und Dänemark.

\subsubsection{Dribblings}

Hinsichtlich der Qualität des Dribblings hatte Italien 28-mal (aus zwei Spielen), hiervon wurde $4(14 \%)$ als hochkarätig bezeichnet und bildeten den größten Wert. Absteigend folgen Dänemark von 29 wurde 1 (3\%), Deutschland von 36, wurde 1 (3 $\%)$ als hochkarätig bezeichnet. Argentinien hatte bei 51 Dribblings mit 1 (2\%) den kleinsten Anteil des hochkarätigen Dribblings.

Das folgende Diagramm zeigt die unterschiedlichen Qualitätsanteile des technischen Elements „hochkarätiges Dribbling“ der Viertelfinalisten in Prozent.

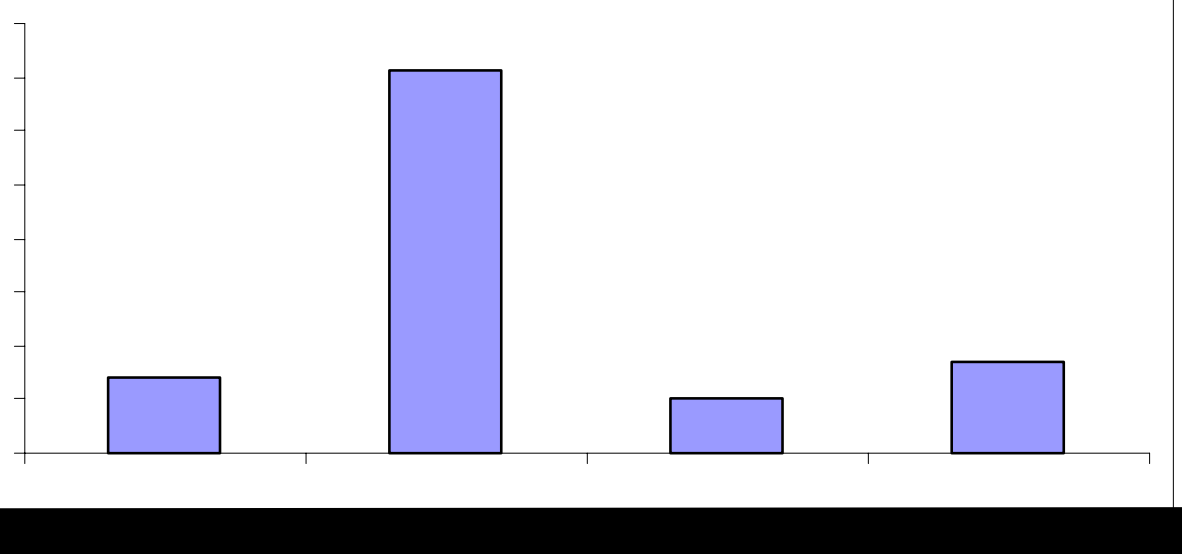




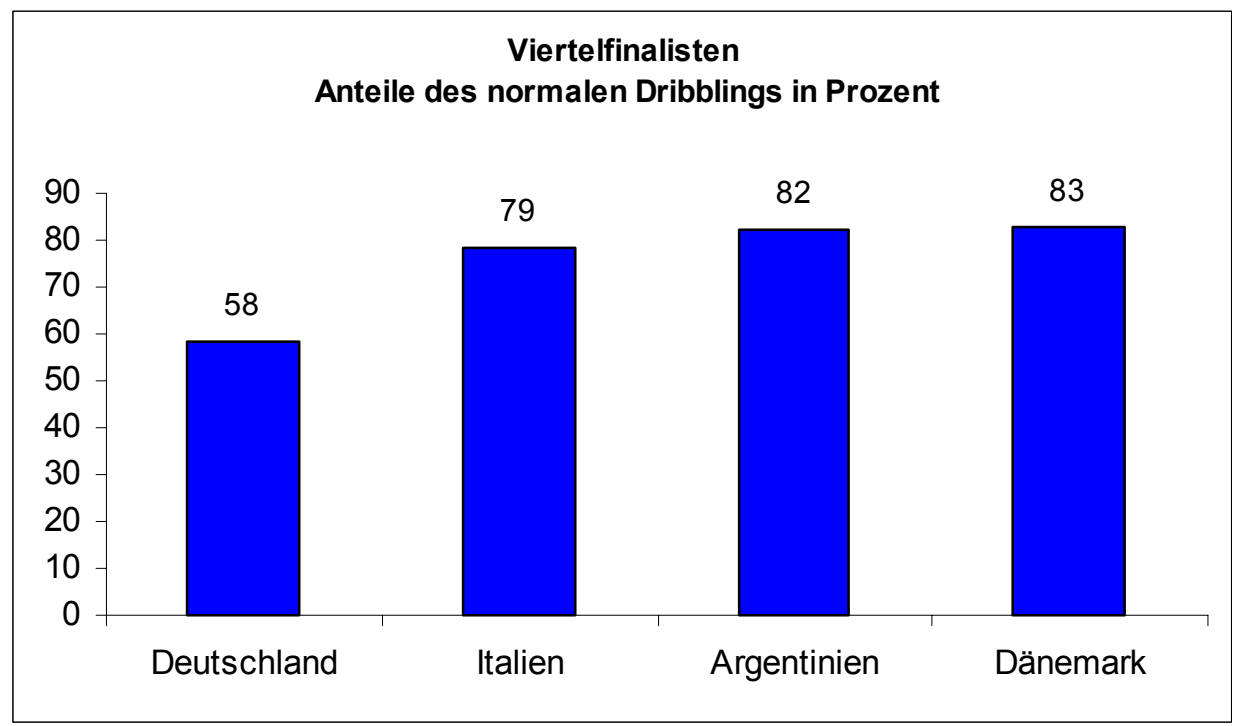

Diagramm 20: Anteile des normalen Dribblings in Prozent der Viertelfinalisten.

Ein „schwaches“ Dribbling wurde mit einem Anteil von 14 (39\%) am häufigsten bei Deutschland festgestellt. Es folgen Argentinien 8 (16\%), Dänemark 4 (14\%) und schließlich Italien 2 (7\%).

Das folgende Diagramm zeigt die unterschiedlichen Qualitätsanteile des technischen Elements „schwaches Dribbling“ der Viertelfinalisten in Prozent.

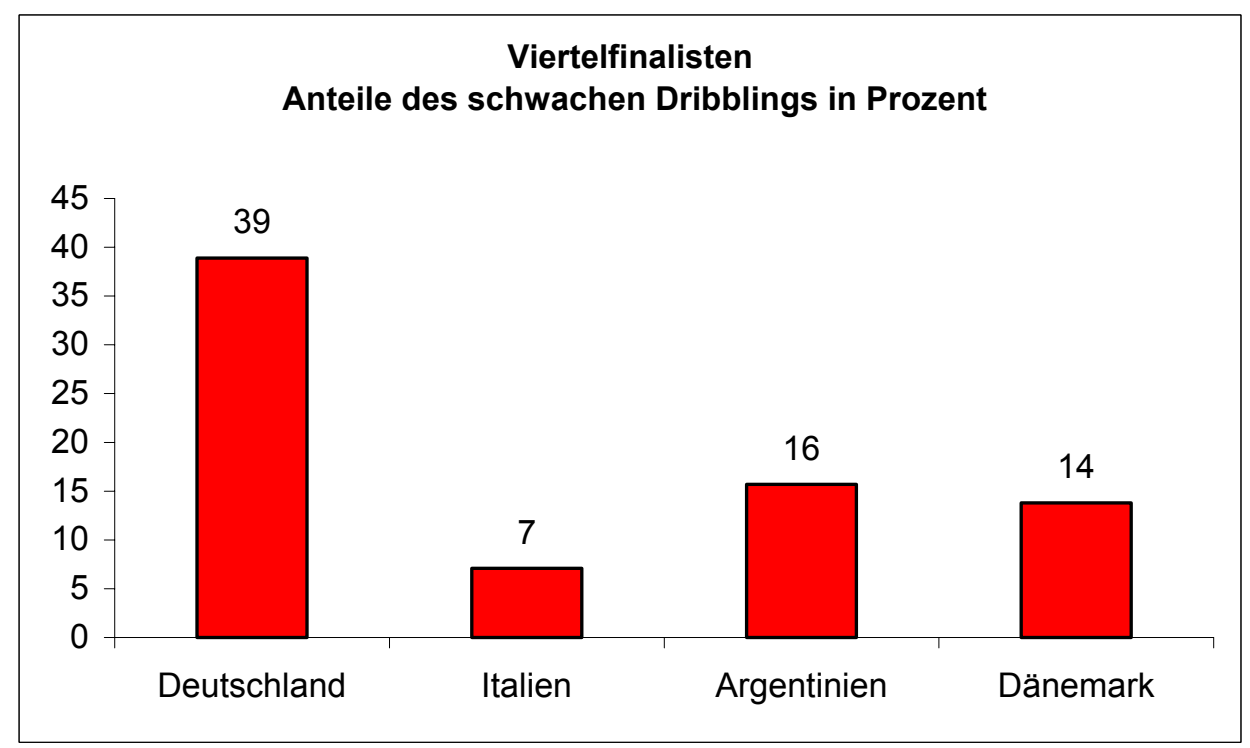

Diagramm 21: Anteile des schwachen Dribblings in Prozent bei den Viertelfinalisten. 
Die Diagramme 19, 20 und 21 erklären die Werte der Mannschaften:

Diagramm 20 erklärt, dass hochkarätiges Dribbling zwischen $2 \%$ und $14 \%$ vorlag. Die italienische Mannschaft hatte die besten Werte und die argentinische hatte die schlechtesten.

Diagramm 22 erklärt, dass normales Dribbling zwischen $58 \%$ und $83 \%$ vorkam. Die dänische Mannschaft hatte hier die besten Werte und die deutsche hatte die schlechtesten. Diagramm 23 erklärt, dass schwaches Dribbling zwischen $7 \%$ und 39 $\%$ lag. Die deutsche Mannschaft hatte die schwächsten und die italienische die besten Werte.

Es lässt sich die Schlussfolgerung aus den Diagrammen ziehen, dass das normale Dribbling bei allem Viertelfinalisten nicht nur häufiger als das hochkarätige ist, sondern auch die schwachen Dribblings der besten Mannschaften häufiger sind als ihre hochkarätigen.

\subsubsection{Körpertäuschung}

Von 45 Körpertäuschungen waren bei Argentinien 5 (11\%) hochkarätig und bildeten den größten Wert. Absteigend folgen Dänemark mit 43 hiervon 4 (9\%), Deutschland mit 29 hiervon 2 (7\%). Italien hatte bei 29 mit $2(5 \%)$ die kleinsten Anteile an hochkarätigen Körpertäuschungen.

Das folgende Diagramm zeigt die unterschiedlichen Qualitätsanteile des technischen Elements "hochkarätige Körpertäuschung“ der Viertelfinalisten in Prozent.

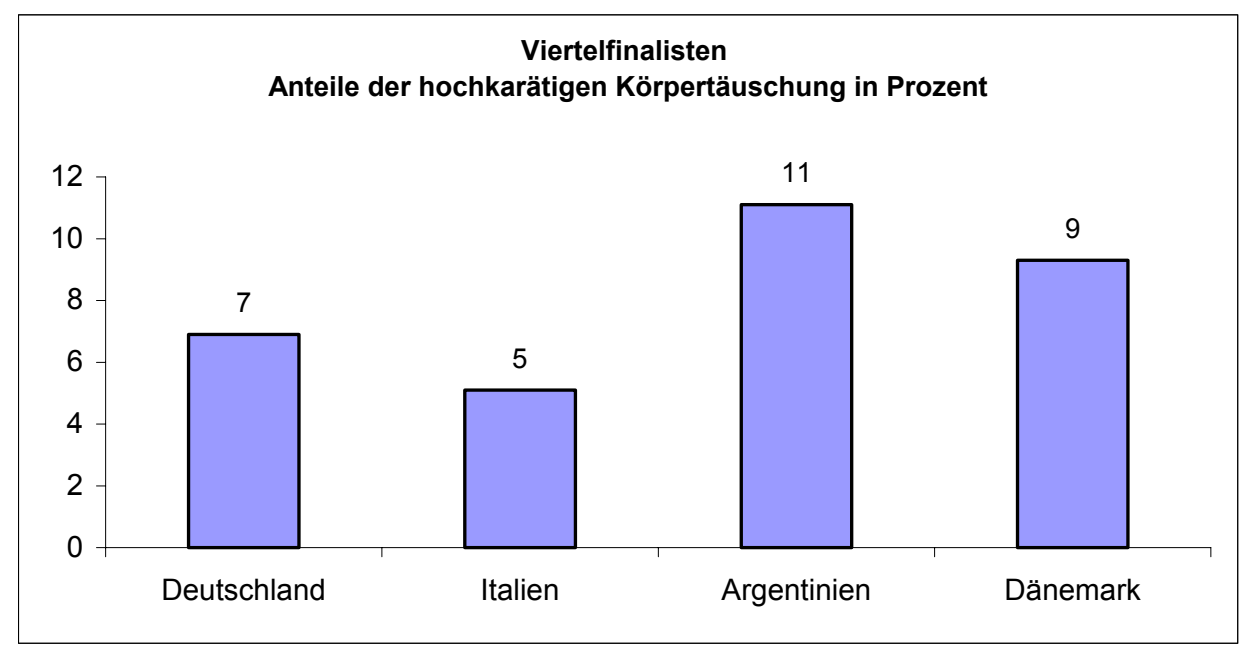

Diagramm 22: Anteile der hochkarätigen Körpertäuschung in Prozent bei den Viertelfinalisten. 
20 (69 \%) der Körpertäuschungen von Deutschland wurden mit „mittel“ bewertet und bildeten den größten Anteil. Die weiteren Anteile „normaler“ Körpertäuschungen lauteten 28 (62 \%) für Argentinien, 23 (59 \%) für Italien und 21 (49 \%) für Dänemark. Das folgende Diagramm zeigt die unterschiedlichen Qualitätsanteile des technischen Elements „mittlere Körpertäuschung“ der Viertelfinalisten in Prozent.

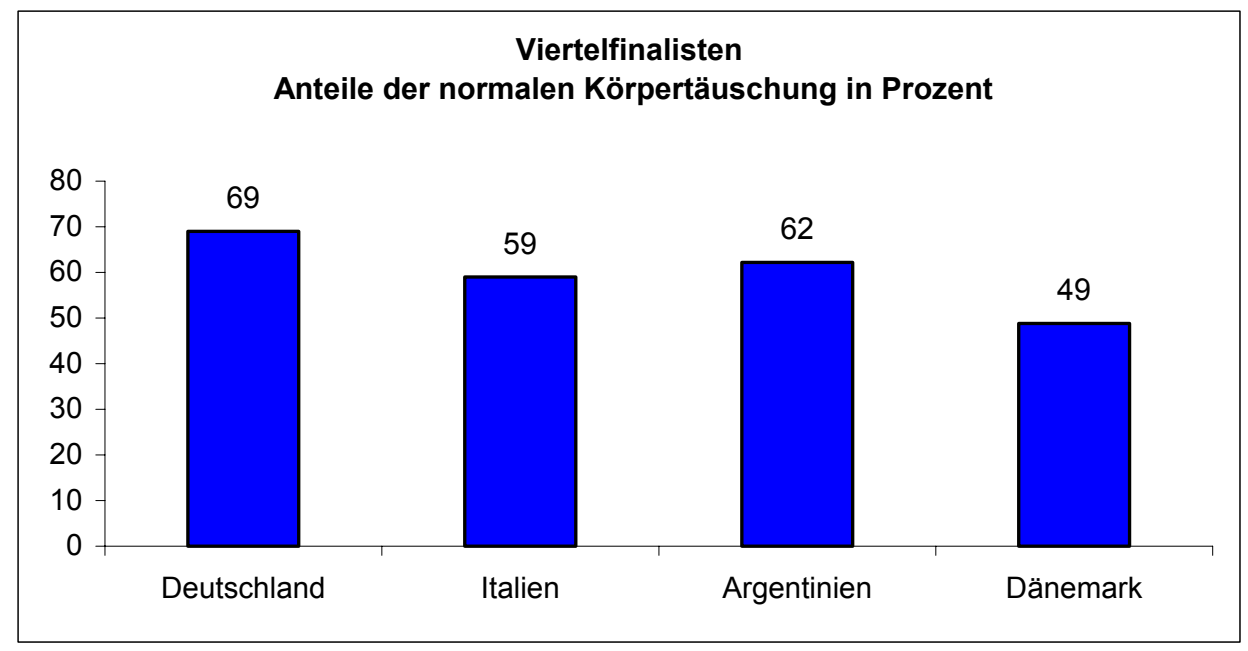

Diagramm 23: Anteile der normalen Körpertäuschung in Prozent der Viertelfinalisten.

Eine „schwache“ Körpertäuschung wurde mit einem Anteil von 18 (42 \%) am häufigsten bei Dänemark festgestellt. Es folgen Italien 14 (36 \%), Argentinien $12(27 \%)$ und schließlich Deutschland 7 (24\%).

Das folgende Diagramm zeigt die unterschiedlichen Qualitätsanteile des technischen Elements „schwache Körpertäuschung“ der Viertelfinalisten in Prozent.

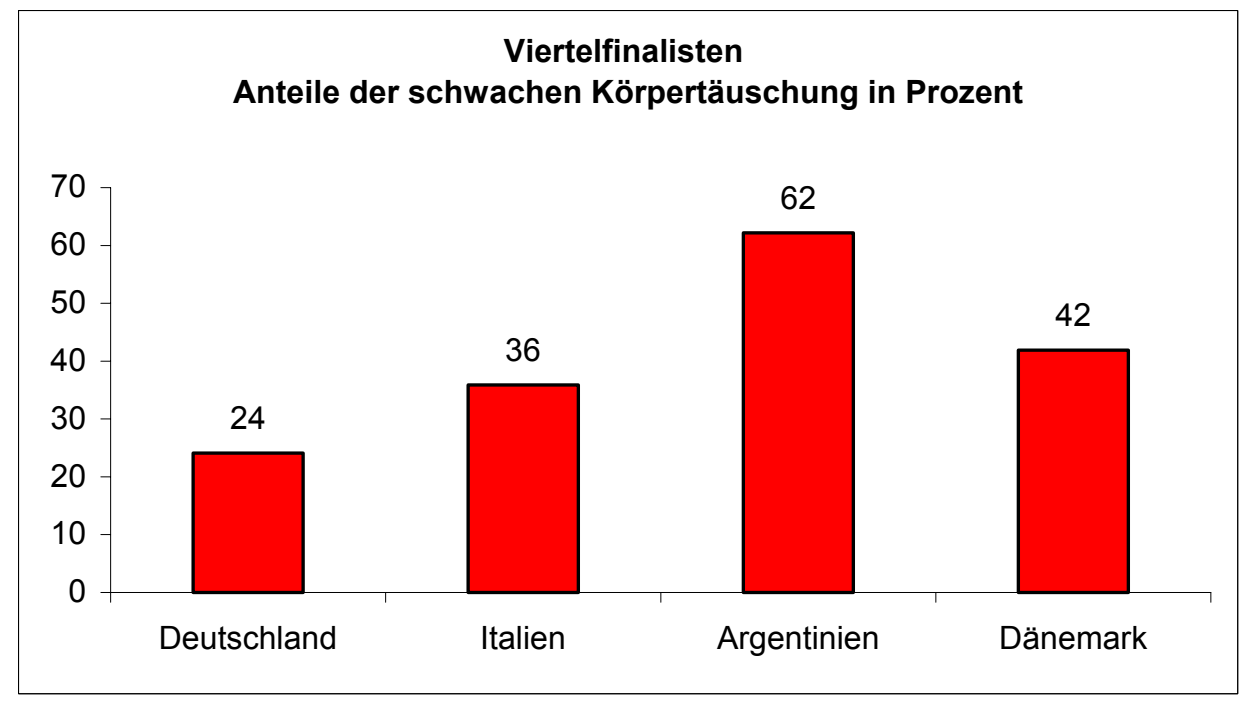

Diagramm 24: Anteile der schwachen Körpertäuschung in Prozent bei den Viertelfinalisten. 
Diagramme 22, 23 und 24 erklären die Werte der Mannschaften:

Diagramm 22 erklärt, dass hochkarätigen Körpertäuschung zwischen $5 \%$ und $11 \%$ lag. Die argentinische Mannschaft hatte die besten Werte und die italienische hatte die schlechtesten. Diagramm 23 erklärt, dass gute Körpertäuschung zwischen $49 \%$ und $69 \%$ lag. Die deutsche Mannschaft hatte hier die besten Werte und die dänische hatte die schlechtesten. Diagramm 23 erklärt, dass schwache Körpertäuschungen zwischen $24 \%$ und $62 \%$ vorkamen. Die argentinische Mannschaft hatte die schwächsten Werte und die deutsche hatte die besten Werte.

Aus den Diagrammen lässt sich die Schlussfolgerung ziehen, dass die schwache Körpertäuschung bei allen Viertelfinalisten einen größeren Anteil hat als die hochkarätige und die hochkarätige Körpertäuschung auch seltener ist als die normale. Das ist dadurch zu erklären, dass die Angreifer ihren Ball oftmals verlieren, wenn das Pressing im Drittel des Gegners stark ist und bei dem schnellen Spielverlauf die Spieler ihren Ball schnell unter ihre Kontrolle bringen müssen.

\subsubsection{Torschuss}

Hinsichtlich der Qualität des Torschusses wurde festgestellt: Dänemark hatte 25 hiervon 6 (24 \%) "hochkarätigen“ und dies bildete den größten Wert. Absteigend folgen Italien von 29 nur 4 (14\%), Argentinien von 37 nur 5 (14\%). Deutschland hatte 31 , wovon nur $2(7 \%)$ hochkarätig waren, was den kleinsten Anteil an „,hochkarätigen“ Torschüssen darstellt.

Das folgende Diagramm zeigt die unterschiedlichen Qualitätsanteile des technischen Elements „hochkarätiger Torschuss“ der Viertelfinalisten in Prozent. 


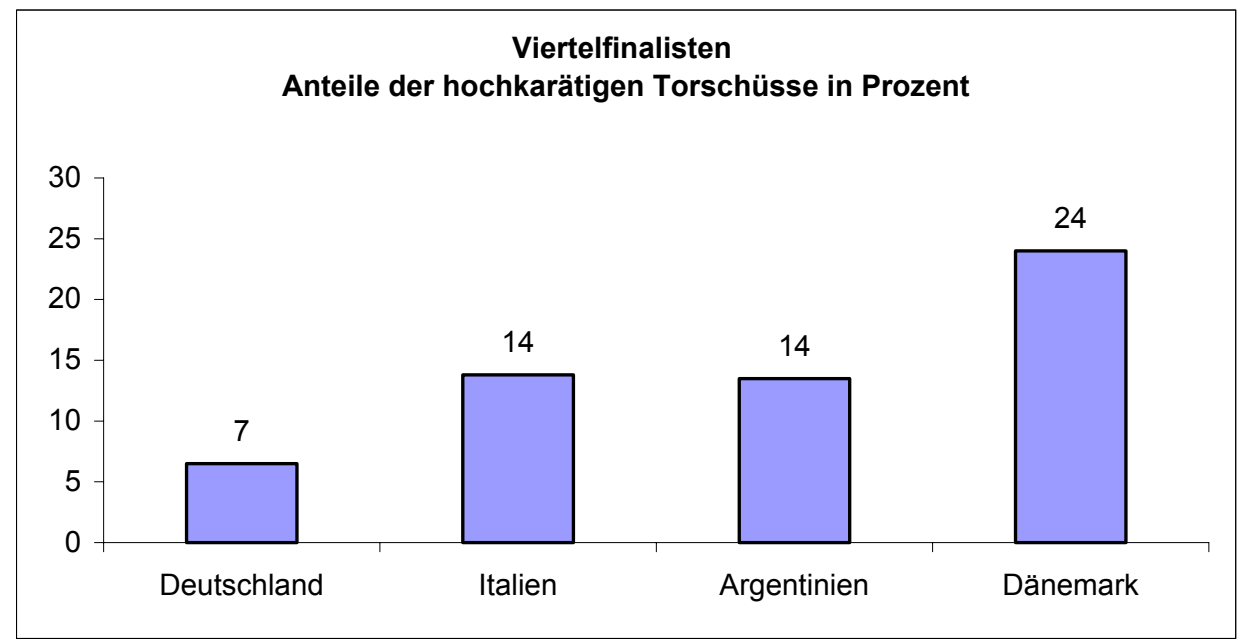

Diagramm 25: Anteile der hochkarätigen Torschüsse in Prozent bei den Viertelfinalisten.

9 (36 \%) der Torschüsse von Dänemark wurden mit "mittel“ bewertet und bildeten den größten Anteil. Die weiteren Anteile „mittlerer“ Torschüsse lauteten 9 (29 \%) für Deutschland, 7 (24\%) für Italien und 8 (22\%) für Argentinien. Das folgende Diagramm zeigt die unterschiedlichen Qualitätsanteile des technischen Elements „mittlerer Torschuss" der Viertelfinalisten in Prozent.

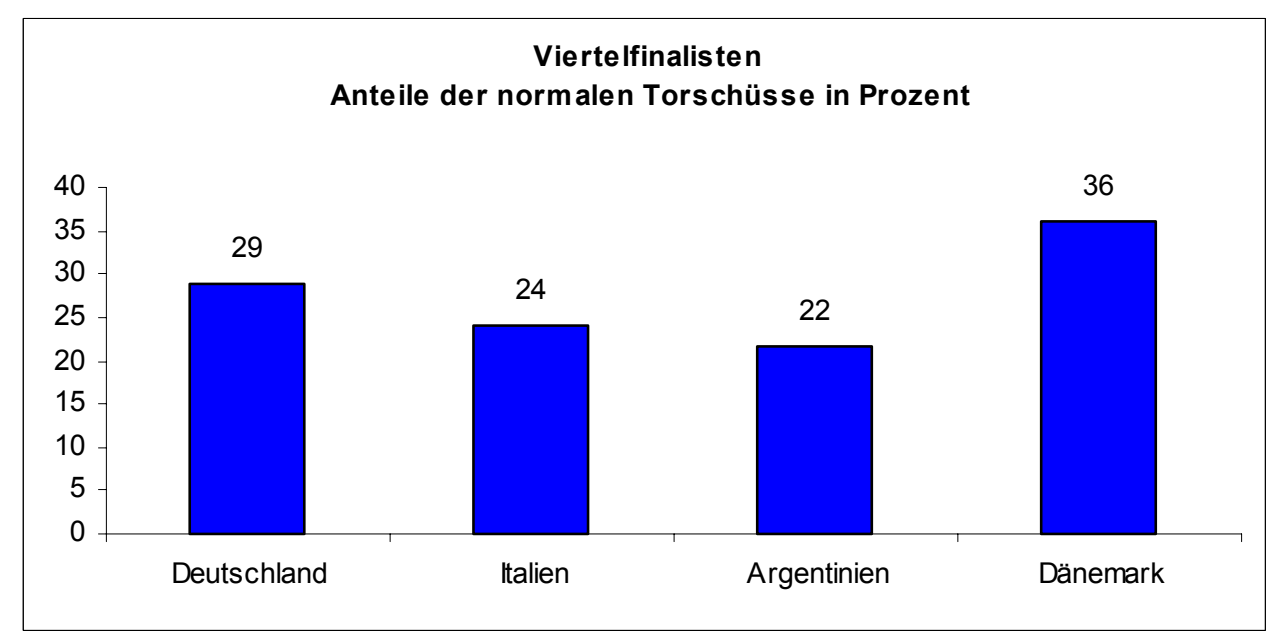

Diagramm 26: Anteile der normalen Torschüsse in Prozent der Viertelfinalisten.

Ein „schwacher" Torschuss wurde mit einem Anteil von 24 (65 \%) am häufigsten bei Argentinien festgestellt. Es folgen Deutschland 20 (65\%), Italien 18 (62\%) und schließlich Dänemark 10 (40\%). Das folgende Diagramm zeigt die unterschiedlichen Qualitätsanteile des technischen Elements „schwacher Torschuss“ der Viertelfinalisten in Prozent. 


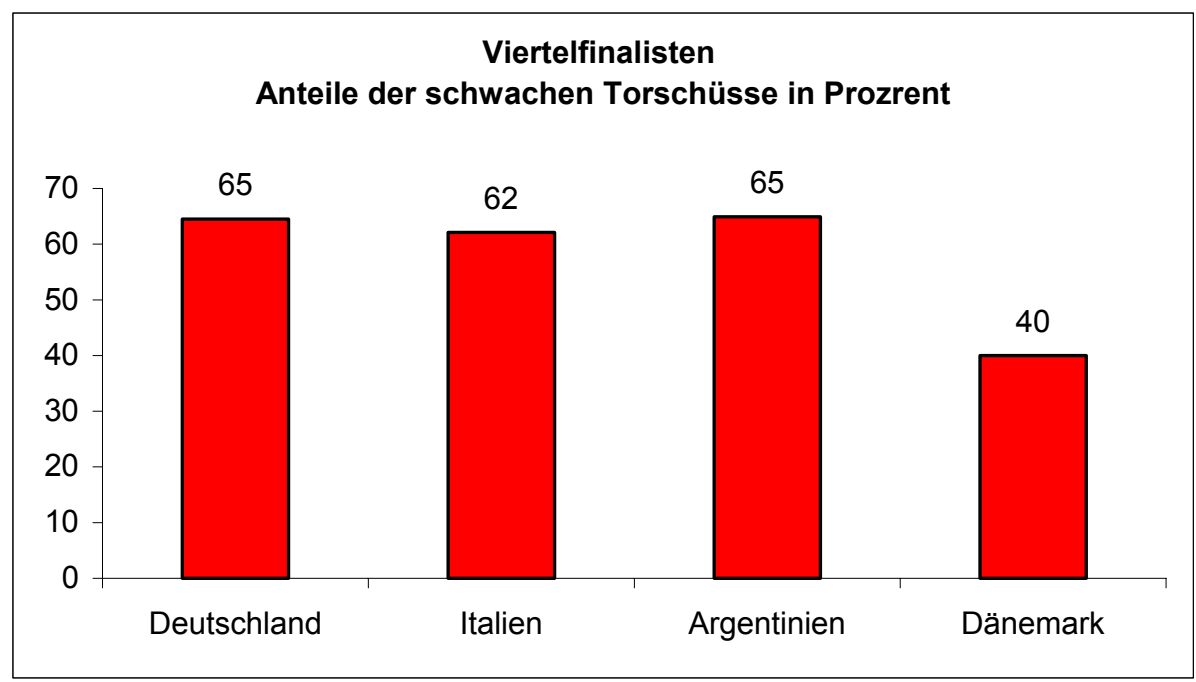

Diagramm 27: Anteile der schwachen Torschüsse in Prozent bei den Viertelfinalisten.

Diagramme 25, 26 und 27 erklären die Werte der Mannschaften:

Diagramm 25 erklärt, dass hochkarätigen Torschüsse zwischen $7 \%$ und $24 \%$ lagen. Die dänische Mannschaft hatte die besten Werte und die deutsche hatte die schlechtesten.

Diagramm 26 erklärt, dass gute Torschüsse zwischen $29 \%$ und $36 \%$ lagen. Die dänische Mannschaft hatte hier die besten Werte und die argentinische hatte die schlechtesten. Diagramm 27 erklärt, dass schwache Torschüsse zwischen $40 \%$ und $65 \%$ lagen. Die deutsche Mannschaft hatte die schwächsten Werte und die dänische hatte die besten Werte.

Daraus lässt sich Schlussfolgerung ziehen, dass alle Viertelfinalisten mehr schwache als hochkarätige Torschüsse erzielten und die Anzahl der schlechten-wie zu erwarten-größer als die der guten und hochkarätigen Torschüsse ist. Die Gründe dafür sind darin zu sehnen, dass der Ballbesitzer möglicherweise-oft von weit außerhalb des Strafraums - auf das Tor schießt, ohne eine echte Torchance zu haben. Oder der Ballbesitzer einen Alibipass auf einen schlechter postierten Mitspieler spielt, anstatt selbst mutig mit einem Solo in den Strafraum einzudringen, oder dass der mögliche Torschütze nicht auf eine für den Torabschluss günstige Position läuft und deshalb nicht gut angespielt werden kann. Nahe vor dem Tor zugespielte Bälle können wahrscheinlich nicht direkt verwandelt werden. Es darf hierbei nicht vergessen werden, dass alle Mannschaften aus dem Turnier nach diesem Spielen ausschieden, wozu die schwachen Torschüsse beigefragen haben. 


\subsubsection{Kurze / lange Pässe}

Hinsichtlich der Qualität der kurzen Pässen wurde festgestellt: Dänemark hatte 749, hiervon wurden 19 (3\%) als hochkarätig bezeichnet und bildeten den größten Wert. Absteigend folgen Italien von 791 wurden 13 (2\%), Deutschland hatte 621 es wurden 7 (1\%) und Argentinien von 920 wurden 7 (1\%) als "hochkarätige“ kurze Pässe bewertet.

Das folgende Diagramm zeigt die unterschiedlichen Qualitätsanteile des technischen Elements „hochkarätige kurze Pässe“ der Viertelfinalisten in Prozent.

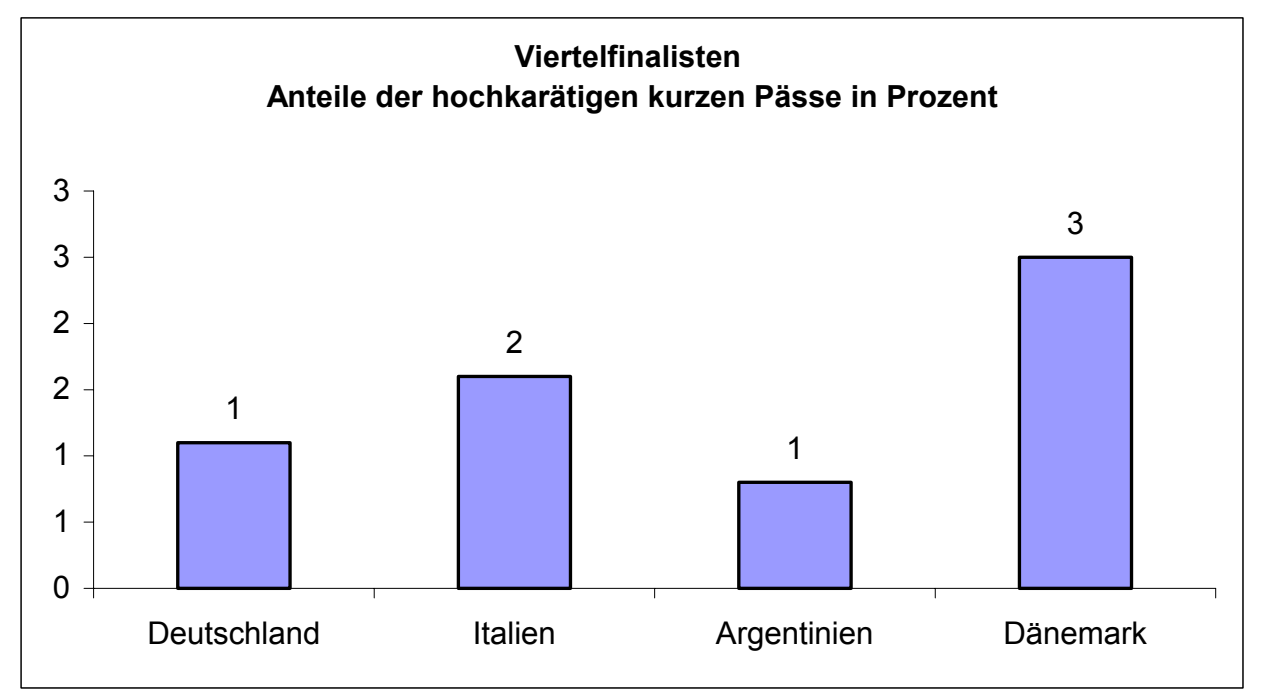

Diagramm 28: Anteile der hochkarätigen kurzen Pässe in Prozent bei den Viertelfinalisten.

663 (89 \%) der kurzen Pässe von Dänemark wurden mit "mittel“ bewertet und bildeten den größten Anteil. Die weiteren Anteile "mittlerer" kurze Pässe lauteten 800 (87\%) für Argentinien, 685 (87 \%) für Italien und 532 (86 \%) für Deutschland.

Das folgende Diagramm zeigt die unterschiedlichen Qualitätsanteile des technischen Elements "mittlere kurze Pässe“ der Viertelfinalisten in Prozent. 


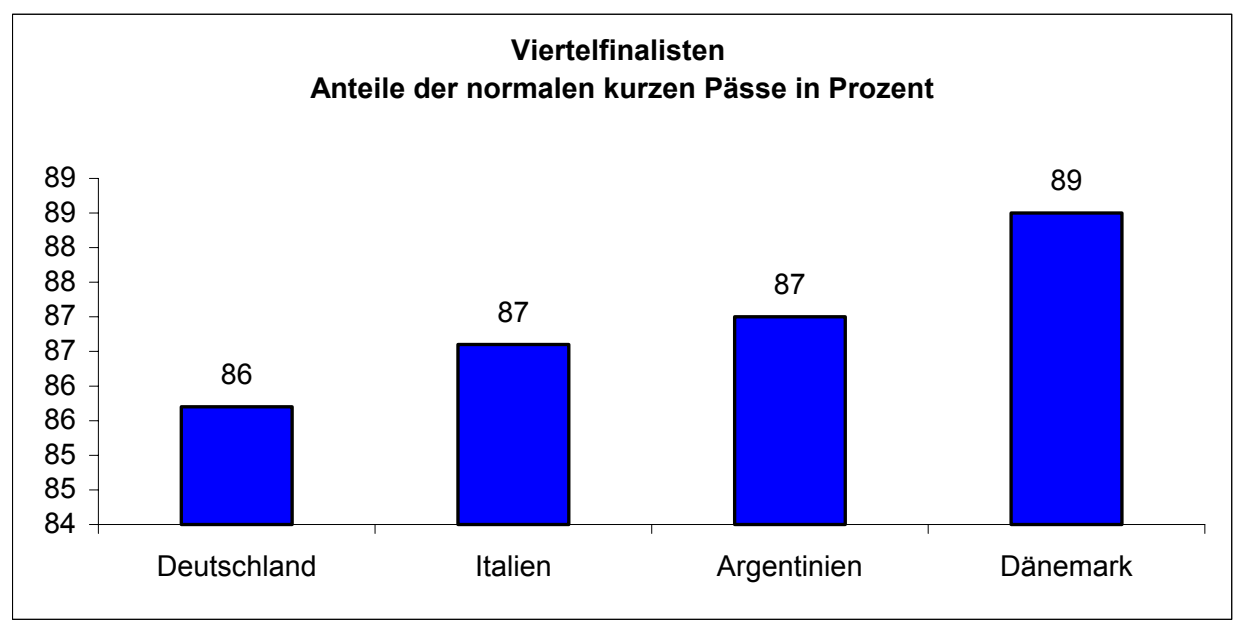

Diagramm 29: Anteile der normalen kurzen Pässe in Prozent bei den Viertelfinalisten.

Ein "schwacher" kurzer Pass wurde mit einem Anteil von 82 (13\%) am häufigsten bei Deutschland festgestellt. Es folgen Argentinien 113 (12\%), Italien 93 (12\%) und schließlich Dänemark 67 (9\%).

Das folgende Diagramm zeigt die unterschiedlichen Qualitätsanteile des technischen Elements „schwache kurze Pässe“ der Viertelfinalisten in Prozent.

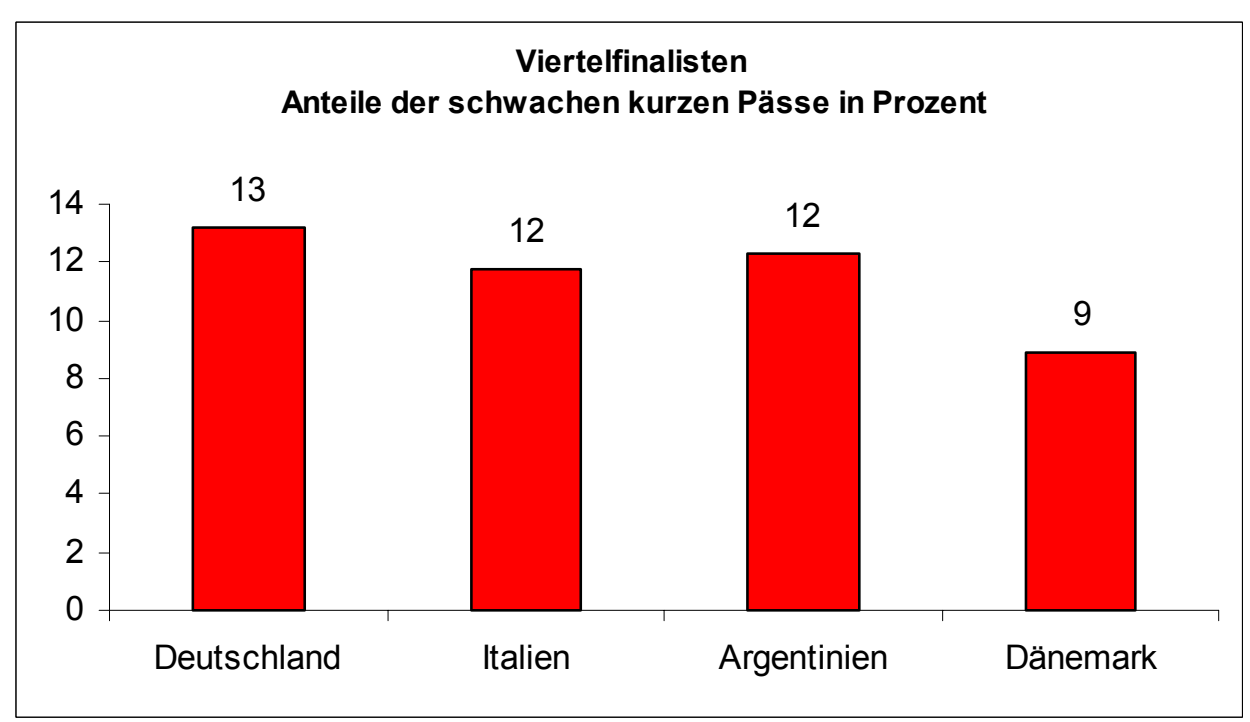

Diagramm 30: Anteile der schwachen kurzen Pässe in Prozent bei den Viertelfinalisten. 
An hochkarätigen langen Pässe hatte Argentinien 102, hiervon wurden 6 (6\%) als hochkarätig bewertet und bildeten den größten Wert. Absteigend folgen Italien von 190 wurden 7 (4\%), Deutschland von 198 wurden 6 (3\%) entsprechend positiv bewertet. Dänemark hatte 140 mit $1(1 \%)$ die kleinsten Anteile an "hochkarätigen“ langen Pässe.

Das folgende Diagramm zeigt die unterschiedlichen Qualitätsanteile des technischen Elements „hochkarätige lange Pässe" der Viertelfinalisten in Prozent.

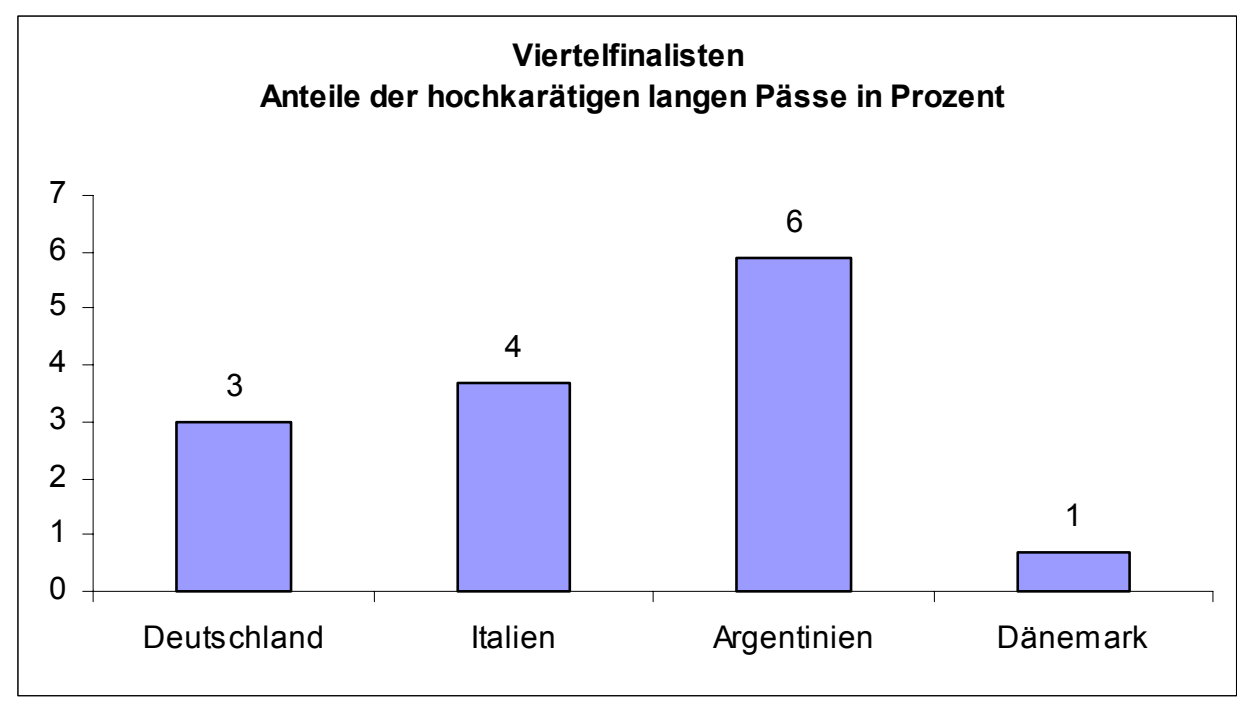

Diagramm 31: Anteile der hochkarätigen langen Pässe in Prozent der Viertelfinalisten.

64 (32 \%) der langen Pässe von Deutschland wurden mit „mittel“ bewertet und bildeten den größten Anteil. Die weiteren Anteile „normaler“ langer Pässe lauteten 41 (29 \%) für Dänemark, 50 (26 \%) für Italien und 19 (19\%) für Argentinien.

Das folgende Diagramm zeigt die unterschiedlichen Qualitätsanteile des technischen Elements „normale lange Pässe“ der Viertelfinalisten in Prozent. 


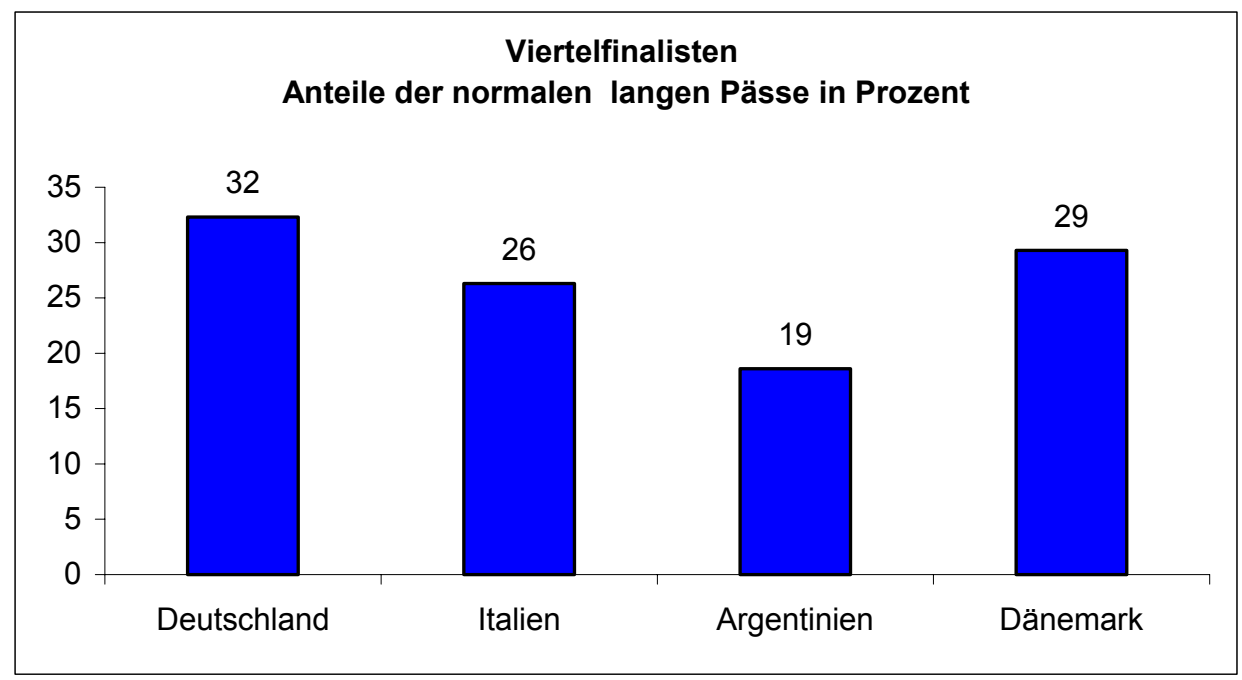

Diagramm 32: Anteile der normalen langen Pässe in Prozent bei den Viertelfinalisten.

Ein „schwacher“ langer Pass wurde mit einem Anteil von 77 (76 \%) am häufigsten bei Argentinien festgestellt. Es folgen Italien 133 (70\%), Dänemark 98 (70\%) und schließlich Deutschland 128 (65\%).

Das folgende Diagramm zeigt die unterschiedlichen Qualitätsanteile des technischen Elements „schwache lange Pässe“ der Viertelfinalisten in Prozent.

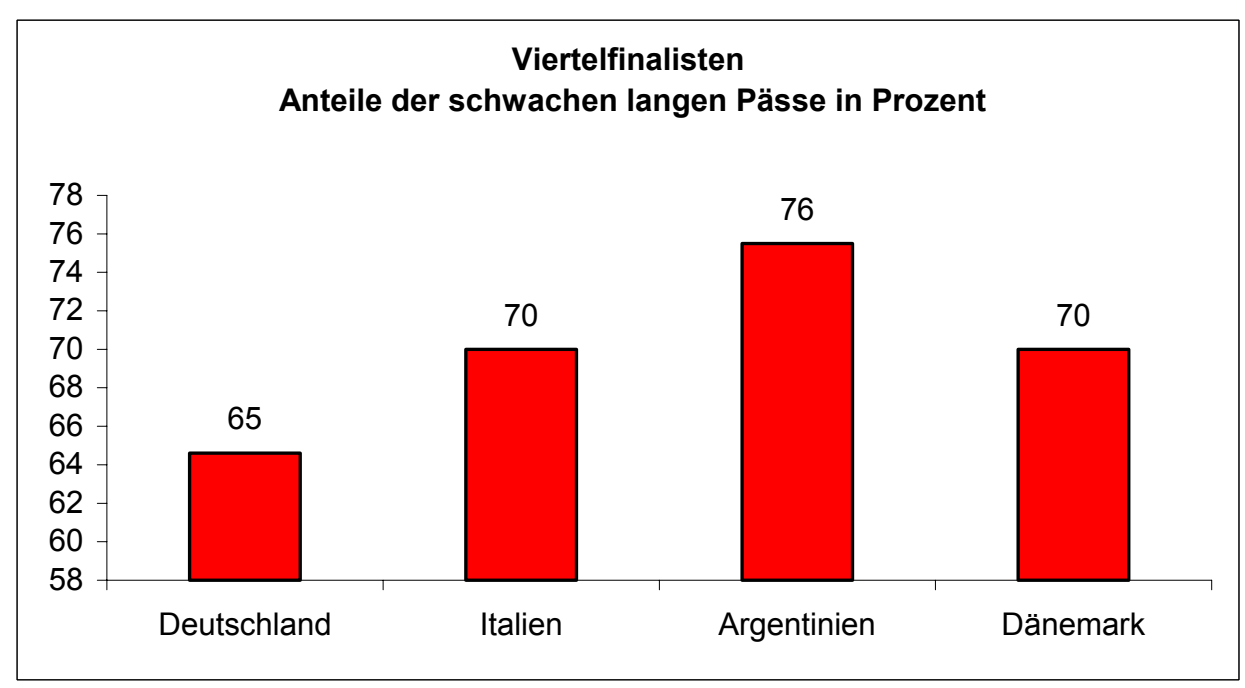

Diagramm 33: Anteile der schwachen langen Pässe in Prozent den Viertelfinalisten.

Diagramme 28, 29, 30, 31, 32 und 33 erklären die Werte der Mannschaften:

Diagramm 28 erklärt, dass die hochkarätigen kurzen Pässe zwischen $1 \%$ und $3 \%$ lagen. Die dänische Mannschaft hatte die besten Werte und die deutsche hatte die schlechtesten. Diagramm 29 erklärt, dass normale kurze Pässe zwischen $86 \%$ und $89 \%$ lagen. Die dänische Mannschaft hatte hier die besten Werte und die deutsche Mannschaft hatte die schlechtesten. Diagramm 30 erklärt, dass schwache kurze 
Pässe zwischen $9 \%$ und $13 \%$ lagen. Die deutsche Mannschaft hatte die schwächsten Werte und die dänische hatte die besten Werte.

Es lässt sich die Schlussfolgerung aus den Diagrammen ziehen, dass die normalen und schwachen kurzen Pässe bei allem Viertelfinalisten häufiger sind als die hochkarätigen. Wahrscheinlich hatten die Mannschaften eine unfangreicher oder langsame Vorbereitung in Mittelfeld, wodurch sie der anderen Mannschaft eine gute Chance gaben, um ihre Verteidigung zu organisieren.

Diagramm 31 erklärt, dass die langen hochkarätigen Pässe zwischen $1 \%$ und $3 \%$ lagen. Die deutsche Mannschaft hatte die besten Werte, die dänische hatte die schlechtesten. Diagramm 32 erklärt, dass gute lange Pässe zwischen $29 \%$ und 32 $\%$ lagen. Die deutsche Mannschaft hatte hier die besten Werte und die argentinische hatten die schlechtesten. Diagramm 33 erklärt, dass schwache lange Pässe zwischen $65 \%$ und $76 \%$ lagen. Die argentinische Mannschaft hatte die schwächsten Werte und die deutsche Mannschaft hatte die besten Werte.

Es lässt sich die Schlussfolgerung aus den Diagrammen ziehen, dass die schwachen langen Pässe bei allem Viertelfinalisten häufiger sind als die hochkarätigen und die normalen. Bei langen Pässen muss auf jeden Fall der Spieler mit großer Genauigkeit spielen müssen, weil der Ball sehr leicht abzunehmen ist und es dauert länger als bei kurzen Pässen bis der Ball zum anderen Mitspieler kommt. Die Pässe wurden unkonzentriert, technisch unsauber, schlecht getimt (zu scharf / zu schwach) und oft mit Effet gespielt. Dadurch hat der annehmende Spieler Probleme mit der Ballkontrolle oder mit der Schuss- Direktannahme.

\subsubsection{Ballkontrolle}

Ballkontrolle wurde bei Italien 95-mal beobachtet, hiervon 2-mal (2 \%) als hochkarätige bewertet und bildeten den größten Wert. Absteigend folgen Argentinien von 71 wurde 1 (1\%), Dänemark von 72, wurde 1 (1\%) und Deutschland von 78 wurde 1 (1 $\%)$ als „hochkarätige" Ballkontrolle bewertet.

Das folgende Diagramm zeigt die unterschiedlichen Qualitätsanteile des technischen Elements "hochkarätige Ballkontrolle" der Viertelfinalisten in Prozent. 


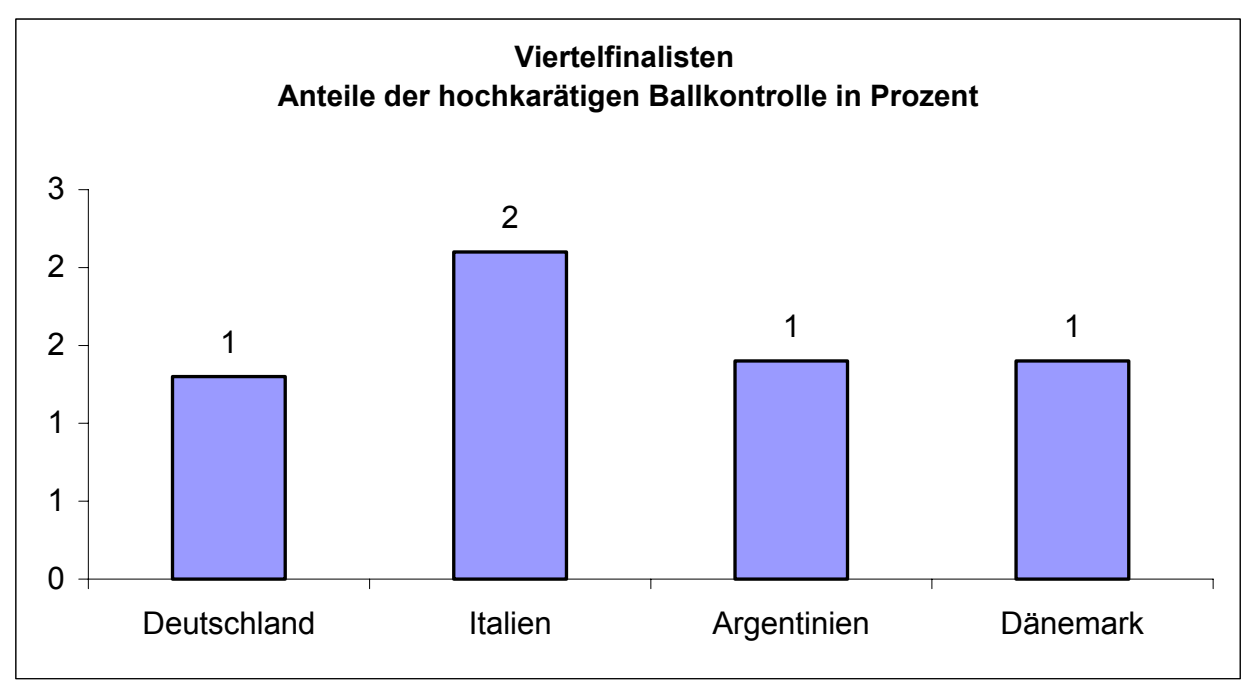

Diagramm 34: Anteile der hochkarätigen Ballkontrolle in Prozent bei den Viertelfinalisten.

33 (42 \%) der Ballkontrollen von Deutschland wurden mit „normal“ bewertet und bildeten den größten Anteil. Die weiteren Anteile mittlerer „normaler“ Ballkontrollen lauteten 39 (41\%) für Italien, 17 (24\%) für Argentinien und 17 (24\%) für Dänemark. Das folgende Diagramm zeigt die unterschiedlichen Qualitätsanteile des technischen Elements „mittlere Ballkontrolle“ der Viertelfinalisten in Prozent.

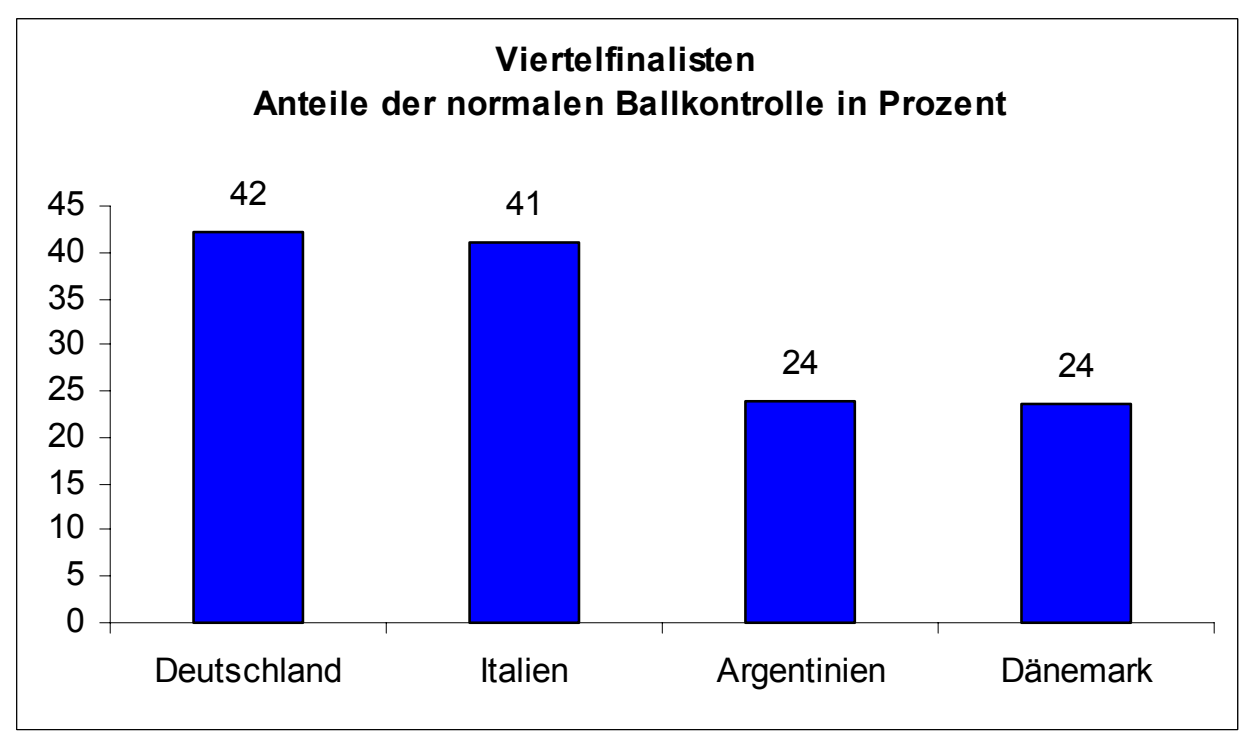

Diagramm 35: Anteile der normalen Ballkontrolle in Prozent bei den Viertelfinalisten.

Eine „schwache“ Ballkontrolle wurde mit einem Anteil von 54 (75\%) am häufigsten bei Dänemark und Argentinien 53 (74 \%) festgestellt. Es folgen Italien 54 (57\%) und schließlich Deutschland 44 (56\%). 
Das folgende Diagramm zeigt die unterschiedlichen Qualitätsanteile des technischen Elements „schwache Ballkontrolle“ der Viertelfinalisten in Prozent.

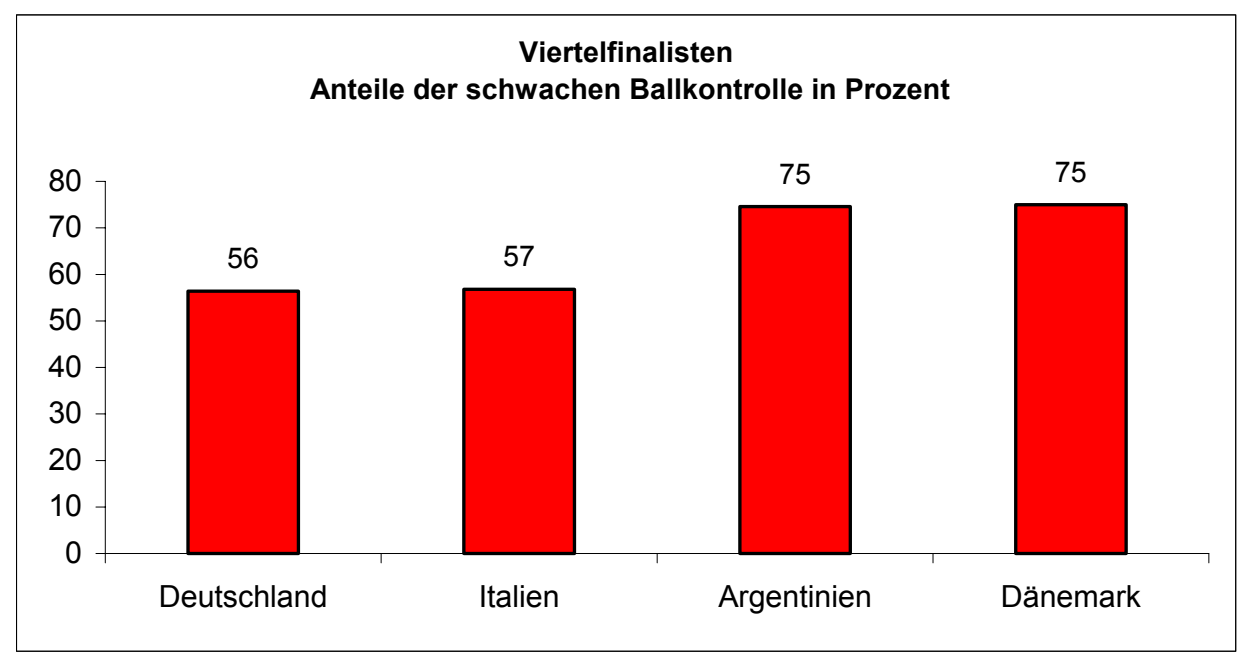

Diagramm 36: Anteile der schwachen Ballkontrolle in Prozent bei den Viertelfinalisten.

Die Diagramme 34, 35 und 36 erklären die Werte der Mannschaften:

Diagramm 34 erklärt, dass hochkarätige Ballkontrolle nur zwischen $1 \%$ und $2 \%$ vorkam. Die argentinische Mannschaft hatte die besten Werte und die italienische hatte die schlechtesten. Diagramm 35 erklärt, dass gute Ballkontrolle zwischen $24 \%$ und $42 \%$ erzielt wurde. Die deutsche Mannschaft hatte hier die besten Werte und die dänische und argentinische hatten die schlechtesten. Diagramm 36 erklärt, dass schwache Ballkontrolle zwischen $56 \%$ und $75 \%$ vorkam und die dänische und argentinische Mannschaft die schwächsten Werte und die deutsche die besten Werte hatte.

Aus den Diagrammen lässt sich die Schlussfolgerung ziehen, dass die schwache Ballkontrolle bei allen Viertelfinalisten einen größeren Anteil hat als die hochkarätige und die hochkarätige Ballkontrolle auch seltener ist als die normale. Obwohl die Viertelfinalisten ein Spiel mehr als die Achtelfinalisten hatten, hatten sie auch die gleichen Voraussetzungen. Die schlechten Werte sind dadurch zu erklären, dass die Angreifer ihren Ball oftmals verlieren, wenn das Pressing im Drittel des Gegners stark ist und bei dem schnellen Spielverlauf die Spieler ihren Ball schnell unter ihre Kontrolle bringen müssen. 


\subsubsection{Halbfinalisten}

Als Halbfinalisten werden diejenigen Mannschaften bezeichnet, die das Halbfinale erreicht haben und zu deren Analyse aufgrund der automatischen Qualifikation für ein weiteres Spiel (Endspiel bzw. Spiel um Platz Drei) vier Spiele herangezogen werden konnten, wobei die Zahlen addiert wurden. Zu diesen Halbfinalisten gehören Frankreich, Brasilien, Kroatien und die Niederlande.

\subsubsection{Dribbling}

Hinsichtlich der Qualität des Dribblings wurde festgestellt: Von den registrierten 96mal Dribbling von Frankreich wurden 10 (10 \%) als "hochkarätig“ bezeichnet, der größte Wert. Absteigend folgen Kroatien mit 47 hiervon $3(6 \%)$ und Brasilien mit 80 hiervon 5 (6 \%) hochkarätiges Dribbling. Die Niederlande hatte mit 1 von 36 (3\%) den kleinsten Anteil „hochkarätigen“ Dribblings.

Das folgende Diagramm zeigt die unterschiedlichen Qualitätsanteile des technischen Elements „hochkarätiges Dribbling“ der Halbfinalisten in Prozent.

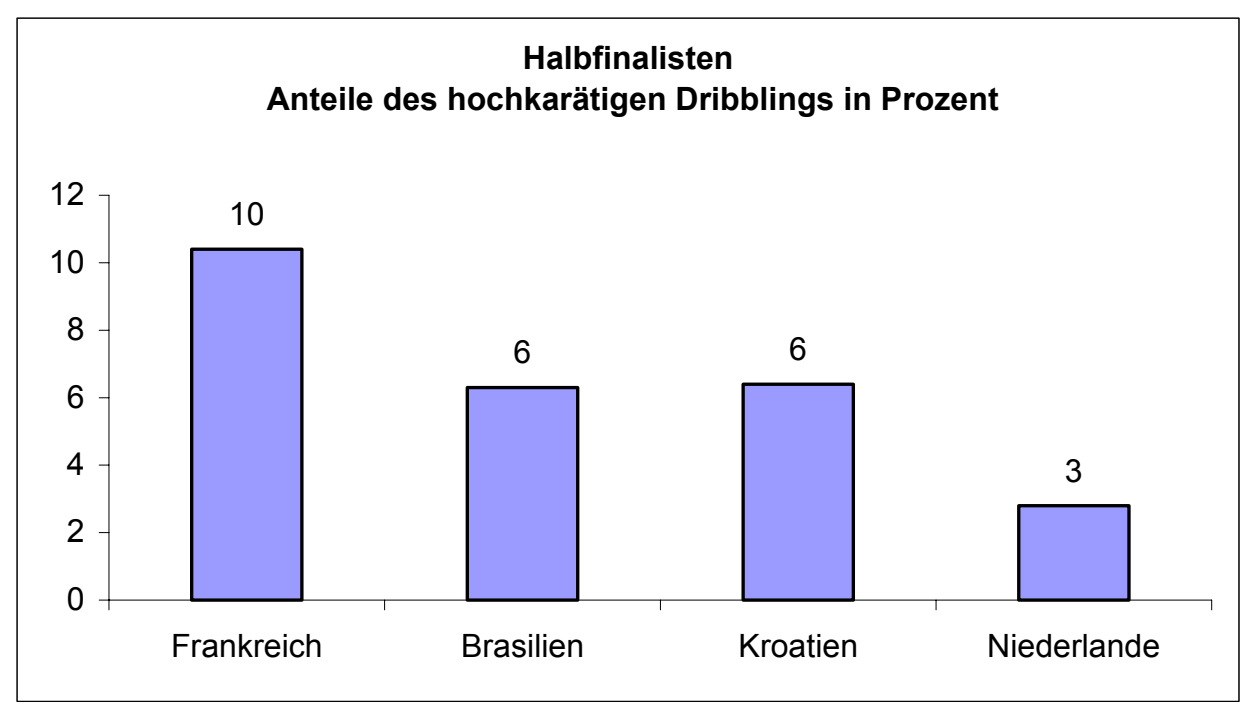

Diagramm 37: Anteile des hochkarätigen Dribblings in Prozent bei den Halbfinalisten.

$82(85 \%)$ der Dribblings von Frankreich wurden mit "normal" bewertet und bildeten den größten Anteil. Die weiteren Anteile ,normaler“ Dribblings lauteten 30 (83 \%) für die Niederlande, 35 (75\%) für Kroatien und 58 (73\%) für Brasilien.

Das folgende Diagramm zeigt die unterschiedlichen Qualitätsanteile des technischen Elements „normales Dribbling“ der Halbfinalisten in Prozent. 


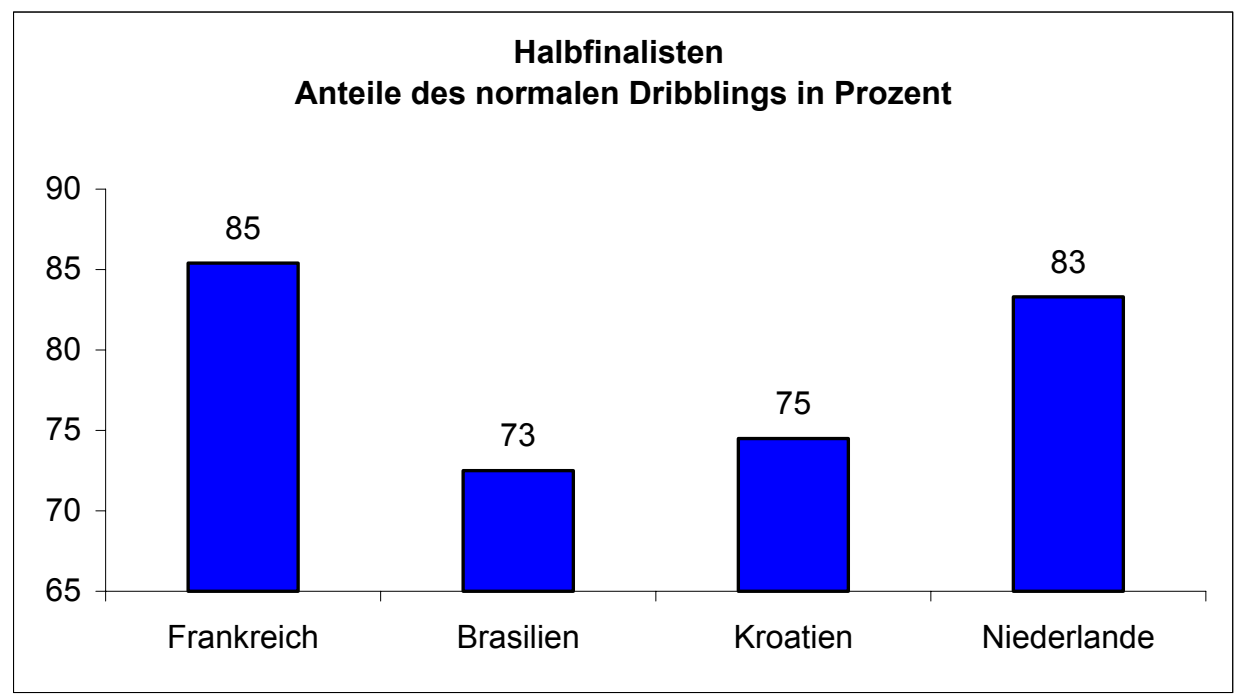

Diagramm 38: Anteile des normalen Dribblings in Prozent bei den Halbfinalisten.

Ein „schwaches“ Dribbling wurde mit einem Anteil von 17- mal (21\%) am häufigsten bei Brasilien festgestellt. Es folgen Kroatien 9 (19\%), die Niederlande 5 (14\%) und schließlich Frankreich 4 (4 \%).

Das folgende Diagramm zeigt die unterschiedlichen Qualitätsanteile des technischen Elements „schwaches Dribbling“ der Halbfinalisten in Prozent.

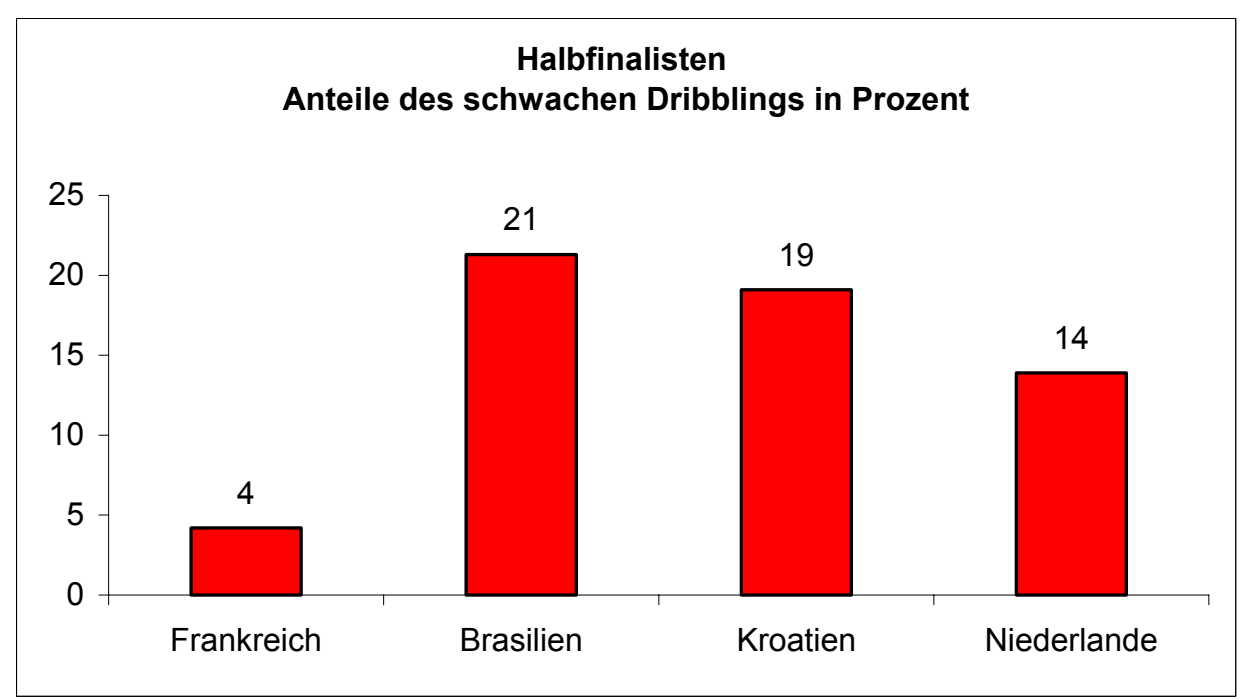

Diagramm 39: Anteile des schwachen Dribblings in Prozent bei den Halbfinalisten.

Die Diagramme 37, 38 und 39 erklären die Werte der Mannschaften:

Diagramm 37 erklärt, dass hochkarätiges Dribbling zwischen $3 \%$ und $10 \%$ vorkam. Die französische Mannschaft hatte die besten Werte und die niederländische hatte die schlechtesten. 
Diagramm 38 erklärt, dass gutes Dribbling zwischen $73 \%$ und $85 \%$ erzielt wurde. Die französische Mannschaft hatte hier die besten Werte und die brasilianische hatte die schlechtesten. Diagramm 39 erklärt, dass schwaches Dribbling zwischen $4 \%$ und $21 \%$ vorkam. Die französische Mannschaft hatte die besten Werte und die brasilianische Mannschaft hatte die schlechten Werte.

Aus den Diagrammen lässt sich schlussfolgern, dass die normalen Dribblings bei allen Halbfinalisten einen größeren Anteil hatten als die hochkarätigen und die schlechten Dribblings. Dabei hatten alle außer der französische Mannschaft mehr schlechte als hochkarätige Dribblings. Die Spieler können den Ball im Lauf selbst auf diesem Weltniveau offenbar relativ schlecht kontrollieren.

\subsubsection{Körpertäuschung}

Hinsichtlich der Qualität der Körpertäuschung wurde festgestellt: Von den registrierten 83-mal der Niederlande wurden 14 (17\%) als hochkarätig bewertet. Absteigend folgt Kroatien von 60 wurden 10 (17\%), Frankreich von 103 wurden 13 (13\%), Brasilien von 119 wurden 5 (4\%) als kleinsten Anteil von hochkarätigen Körpertäuschungen bewertet.

Das folgende Diagramm zeigt die unterschiedlichen Qualitätsanteile des technischen Elements „hochkarätige Körpertäuschung“ der Halbfinalisten in Prozent.

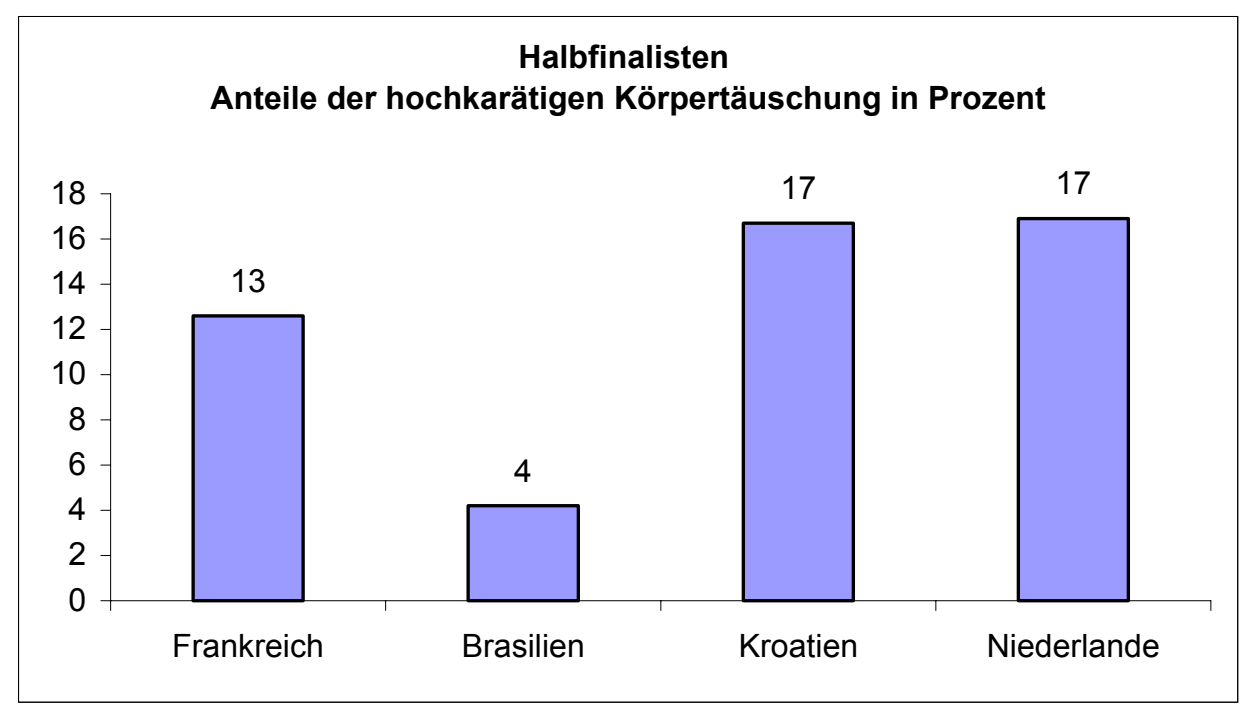

Diagramm 40: Anteile der hochkarätigen Körpertäuschung in Prozent bei den Halbfinalisten. 
54 (65 \%) der Körpertäuschungen der Niederlande wurden mit „normal“ bewertet und bildeten den größten Anteil. Die weiteren Anteile „normaler“ Körpertäuschungen lauteten 60 (58 \%) für Frankreich, 33 (55\%) für Kroatien und 56 (47\%) für Brasilien. Das folgende Diagramm zeigt die unterschiedlichen Qualitätsanteile des technischen Elements „normale Körpertäuschung“ der Halbfinalisten in Prozent.

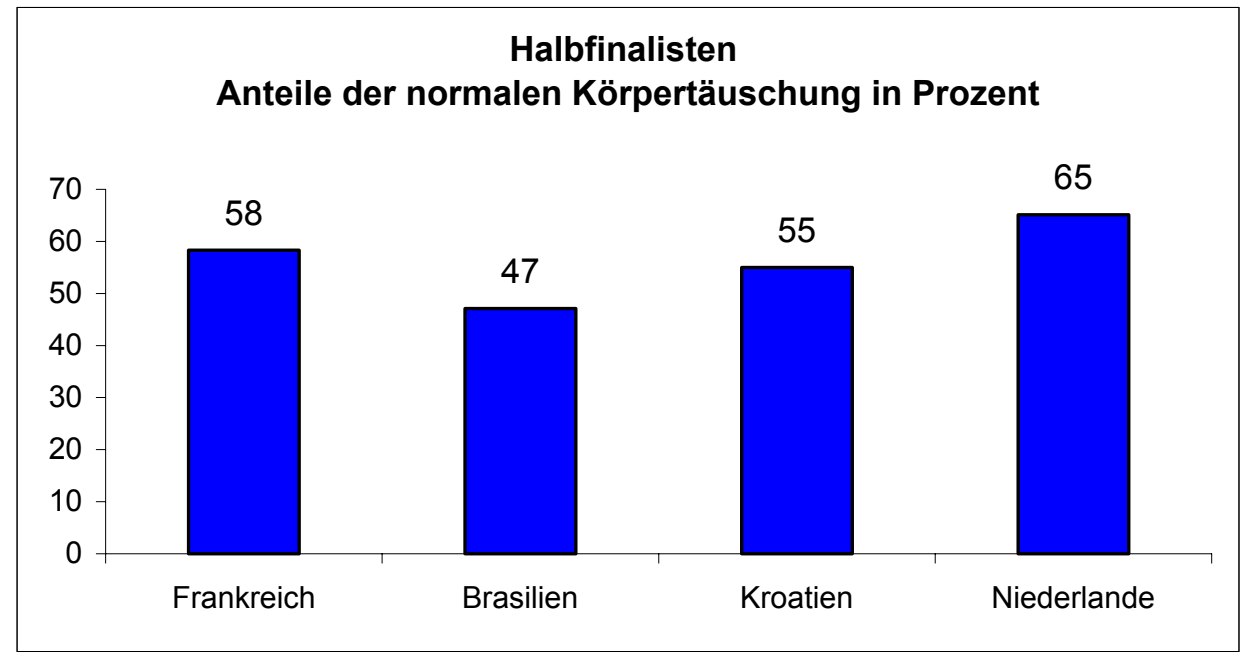

Diagramm 41: Anteile der normalen Körpertäuschung in Prozent bei den Halbfinalisten.

Eine „schwache“ Körpertäuschung wurde mit einem Anteil von 58 (49 \%) am häufigsten bei Brasilien festgestellt. Es folgen Frankreich 30 (29\%), Kroatien 17 (28\%) und schließlich die Niederlande 15 (18\%).

Das folgende Diagramm zeigt die unterschiedlichen Qualitätsanteile des technischen Elements „schwache Körpertäuschung“ der Halbfinalisten in Prozent.

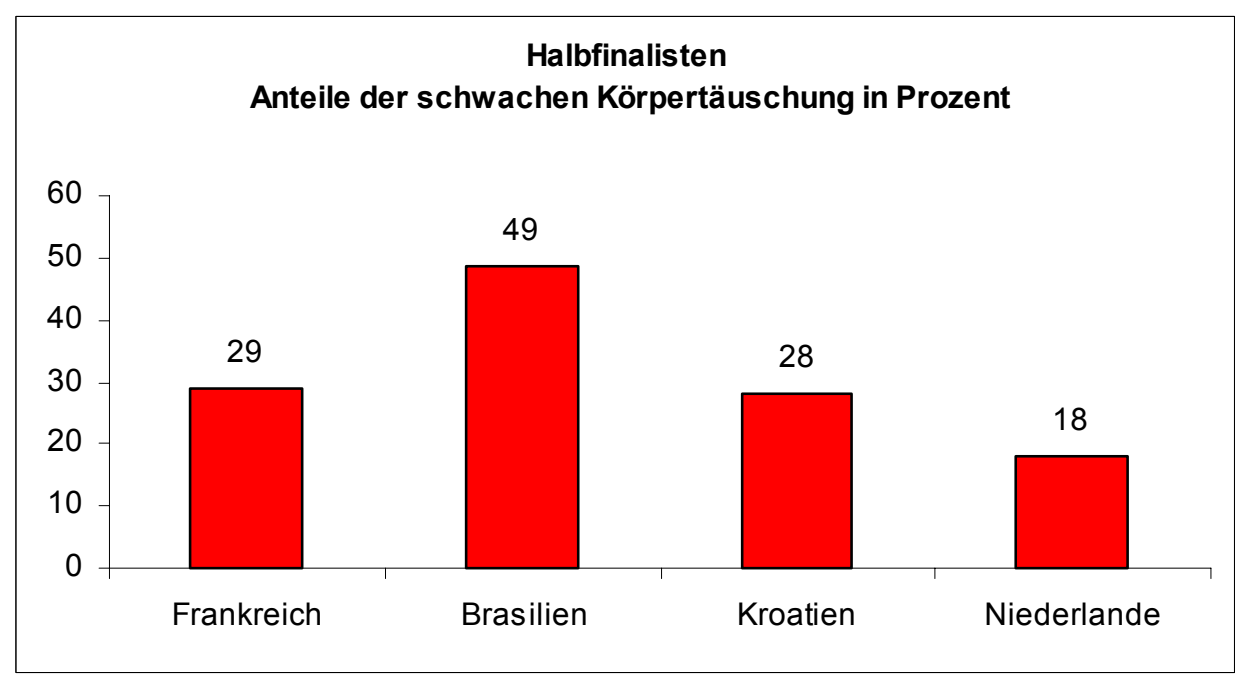

Diagramm 42: Anteile der schwachen Körpertäuschung in Prozent bei den Halbfinalisten. 
Die Diagramme 40, 41 und 42 erklären die Werte der Mannschaften:

Diagramm 40 erklärt, dass hochkarätige Körpertäuschungen zwischen $4 \%$ und $17 \%$ lagen. Die niederländische und die kroatische Mannschaft hatten die besten Werte und die brasilianische hatte die schlechtesten.

Diagramm 41 erklärt, dass normale Körpertäuschung zwischen $47 \%$ und $65 \%$ lagen. Die niederländische Mannschaft hatte hier die besten Werte und die brasilianische hatte die schlechteste.

Diagramm 42 erklärt, dass die schwache Körpertäuschung zwischen $18 \%$ und $49 \%$ lagen. Die brasilianische Mannschaft hatte die schwächsten Werte und die niederländische hatte die besten Werte.

Es lässt sich die Schlussfolgerung aus den Diagrammen ziehen, dass die normale Körpertäuschung bei allem Halbfinalisten am häufigsten war und dass es weniger hochkarätige als schlechte Körpertäuschung gab. Das ist dadurch zu erklären, dass die Angreifer ihren Ball oftmals verlieren, wenn das Pressing im Drittel des Gegners stark ist und bei dem schnellen Spielverlauf die Spieler ihren Ball schnell unter ihre Kontrolle bringen müssen.

\subsubsection{Torschuss}

Hinsichtlich der Qualität des Torschusses wurde festgestellt: Bei den registrierten Torschüssen hatte Brasilien 62 hiervon $13(21 \%)$ hochkarätige und dies bildete den größten Wert. Absteigend folgen die Niederlande mit 60 Torschüssen hiervon 8 hochkarätige (13\%) und Kroatien hatte bei 47 Torschüssen 5 (11\%) hochkarätige. Frankreich hatte 102, von denen $10(10 \%)$ als hochkarätige bezeichnet wurden und dies bildete den kleinsten Anteil "sehr guter" Torschüsse.

Das folgende Diagramm zeigt die unterschiedlichen Qualitätsanteile des technischen Elements "hochkarätiger Torschuss“ der Halbfinalisten in Prozent. 


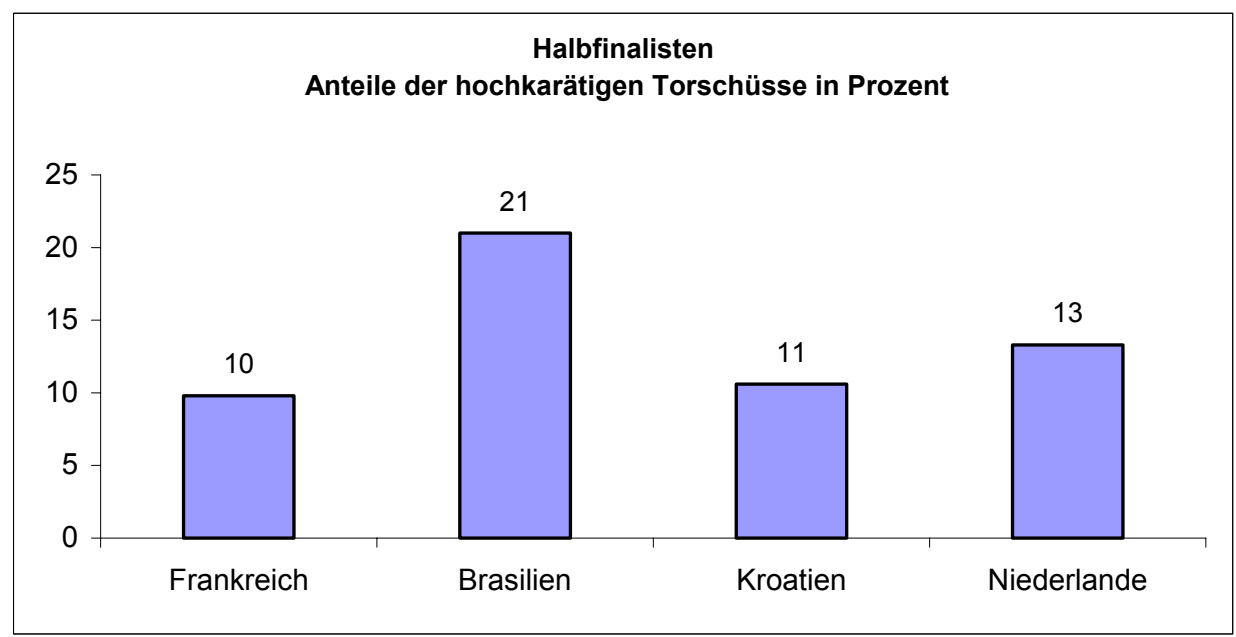

Diagramm 43: Anteile der hochkarätigen Torschüsse in Prozent bei den Halbfinalisten.

25 (42 \%) der Torschüsse der Niederlande wurden mit „normal“ bewertet und bildeten den größten Anteil. Die weiteren Anteile „normaler“ Torschüsse lauteten 20 (32 \%) für Brasilien, 14 (30 \%) für Kroatien und 25 (25 \%) für Frankreich.

Das folgende Diagramm zeigt die unterschiedlichen Qualitätsanteile des technischen Elements „mittlerer Torschuss“ der Halbfinalisten in Prozent.

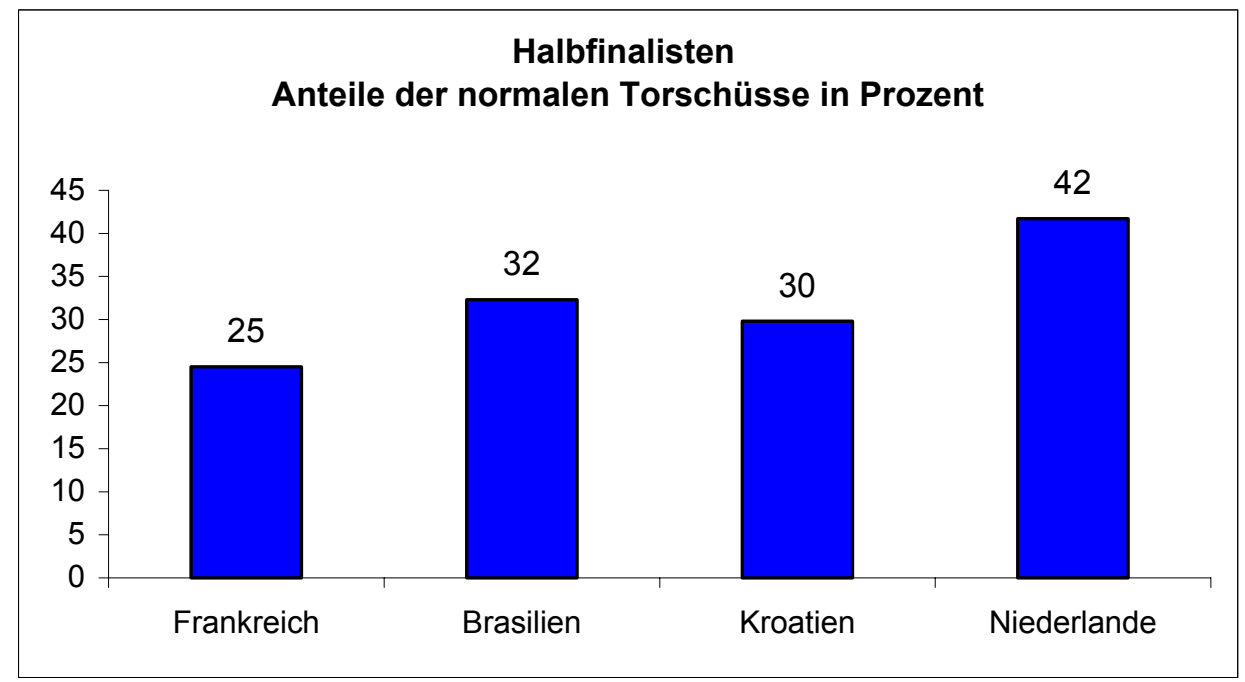

Diagramm 44: Anteile der normalen Torschüsse in Prozent der Halbfinalisten.

Ein „schwacher“ Torschuss wurde mit einem Anteil von 67 (66\%) am häufigsten bei Frankreich festgestellt. Es folgen Kroatien 28 (60\%), Brasilien 29 (47\%) und schließlich die Niederlande 27 (45\%).

Das folgende Diagramm zeigt die unterschiedlichen Qualitätsanteile des technischen Elements „schwacher Torschuss“ der Halbfinalisten in Prozent. 


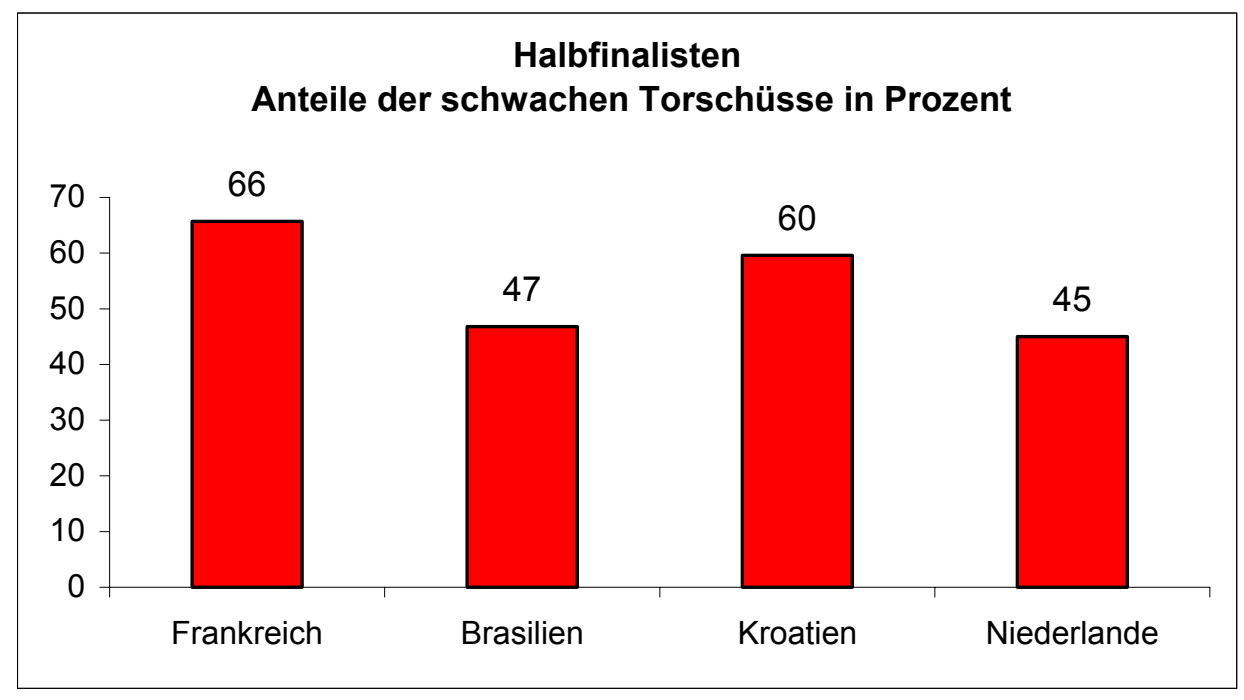

Diagramm 45: Anteile der schwachen Torschüsse in Prozent bei den Halbfinalisten. Die Diagramme 43, 44 und 45 erklären die Werte der Mannschaften:

Diagramm 43 erklärt, dass hochkarätige Torschüsse zwischen $10 \%$ und $21 \%$ vorkamen. Die brasilianische Mannschaft hatte die besten Werte und die französische hatte die schlechtesten.

Diagramm 44 erklärt, dass gute Torschüsse zwischen $25 \%$ und $42 \%$ vorkamen. Die niederländische Mannschaft hatte hier die besten Werte und die französische hatte die schlechtesten. Diagramm 45 erklärt, dass schwache Torschüsse zwischen $45 \%$ und $66 \%$ erzielt wurden. Die französische Mannschaft hatte die schwächsten Werte und die niederländische hatte die besten Werte.

Daraus lässt sich die Schlussfolgerung ziehen, dass alle Viertelfinalisten mehr schwache als hochkarätige Torschüsse erzielten und die Anzahl der schlechten wie zu erwarten größer als die der guten und hochkarätigen Torschüsse ist. Die Gründe dafür sind darin zu sehnen, dass der Ballbesitzer möglicherweise oft von weit außerhalb des Strafraums auf das Tor schießt, ohne eine echte Torchance zu haben. Es kann auch vorkommen, dass der Ballbesitzer einen Alibipass auf einen schlechter postierten Mitspieler spielt, anstatt selbst mutig mit einem Solo in den Strafraum einzudringen, oder dass der mögliche Torschütze nicht auf eine für den Torabschluss günstige Position läuft und deshalb nicht gut angespielt werden kann. Nahe vor dem Tor zugespielte Bälle können wahrscheinlich nicht direkt verwandelt werden. Es darf hierbei nicht vergessen werden, dass alle Mannschaften aus dem Turnier nach diesem Spielen ausschieden, wozu die schwachen Torschüsse beigetragen haben. 


\subsubsection{Kurze / lange Pässe}

Hinsichtlich der Qualität der kurzen Pässe wurde festgestellt: Kroatien hatte 679, hiervon wurden $(2 \%)$ als hochkarätige bezeichnet und dies bildete den größten Wert. Absteigend folgt Frankreich, es erzielte 1606-mal kurze Pässe, hiervon wurden 26 (2 \%) als hochkarätige bezeichnet. Brasilien erzielte 1679-mal mit $20(1 \%)$ und die Niederlande erzielte 1668 ebenfalls mit 20 (1\%) „hochkarätigen“ kurzen Pässen. Das folgende Diagramm zeigt die unterschiedlichen Qualitätsanteile des technischen Elements "hochkarätige kurze Pässe“ der Halbfinalisten in Prozent.

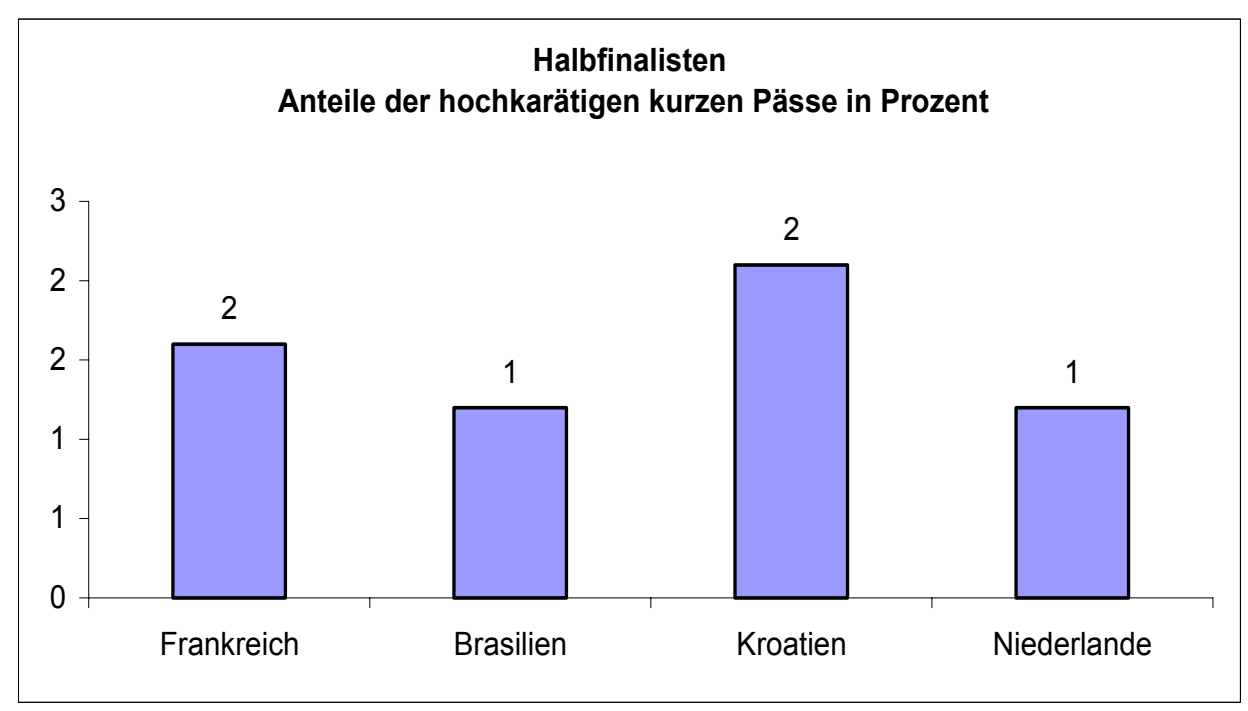

Diagramm 46: Anteile der hochkarätigen kurzen Pässe in Prozent bei den Halbfinalisten.

1503 (90 \%) der kurzen Pässe der Niederlande wurden mit "normal“ bewertet und bildeten den größten Anteil. Die weiteren Anteile "normaler" kurzer Pässe lauteten 1510 (90 \%) für Brasilien, 1372 (85 \%) für Frankreich und mit 679 (84 \%) hatte Kroatien den kleinsten Anteil mittlerer kurze Pässe.

Das folgende Diagramm zeigt die unterschiedlichen Qualitätsanteile des technischen Elements „mittlere kurze Pässe“ der Halbfinalisten in Prozent. 


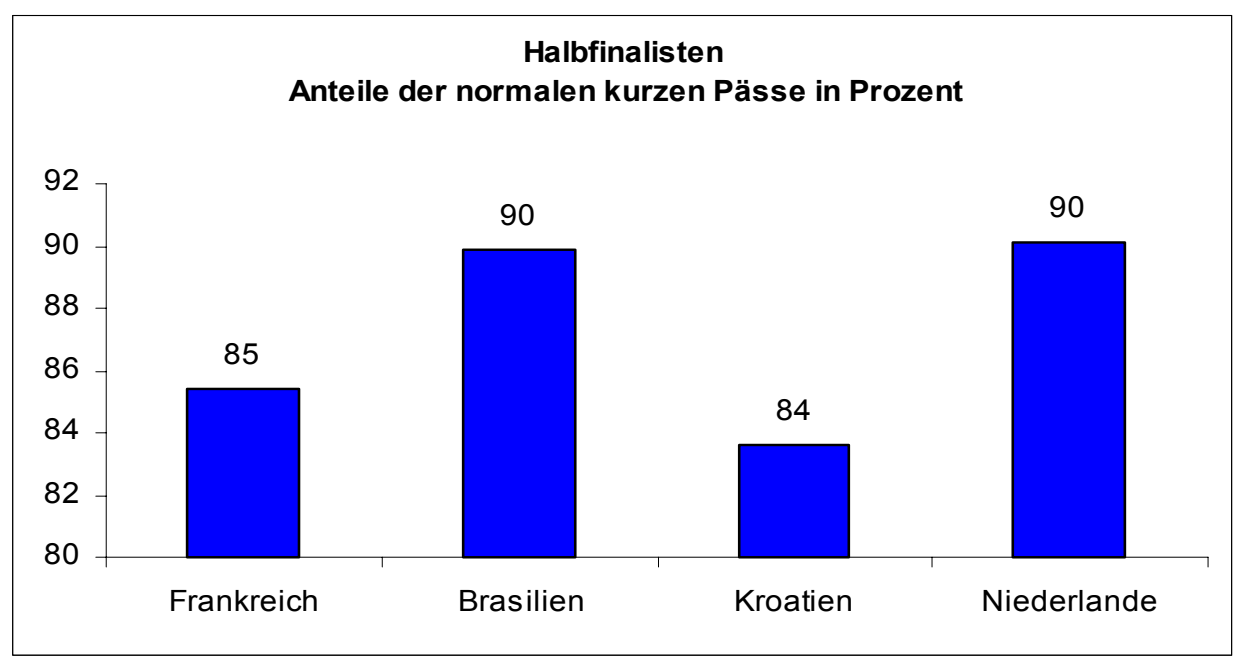

Diagramm 47: Anteile der normalen kurzen Pässe in Prozent bei den Halbfinalisten.

Ein „schwacher“ kurzer Pass wurde mit einem Anteil von 116 (14 \%) am häufigsten bei Kroatien festgestellt. Es folgen Frankreich 208 (13\%), Brasilien 149 (9\%) und schließlich die Niederlande 145 (9\%). Das folgende Diagramm zeigt die unterschiedlichen Qualitätsanteile des technischen Elements „schwache kurze Pässe“ der Halbfinalisten in Prozent.

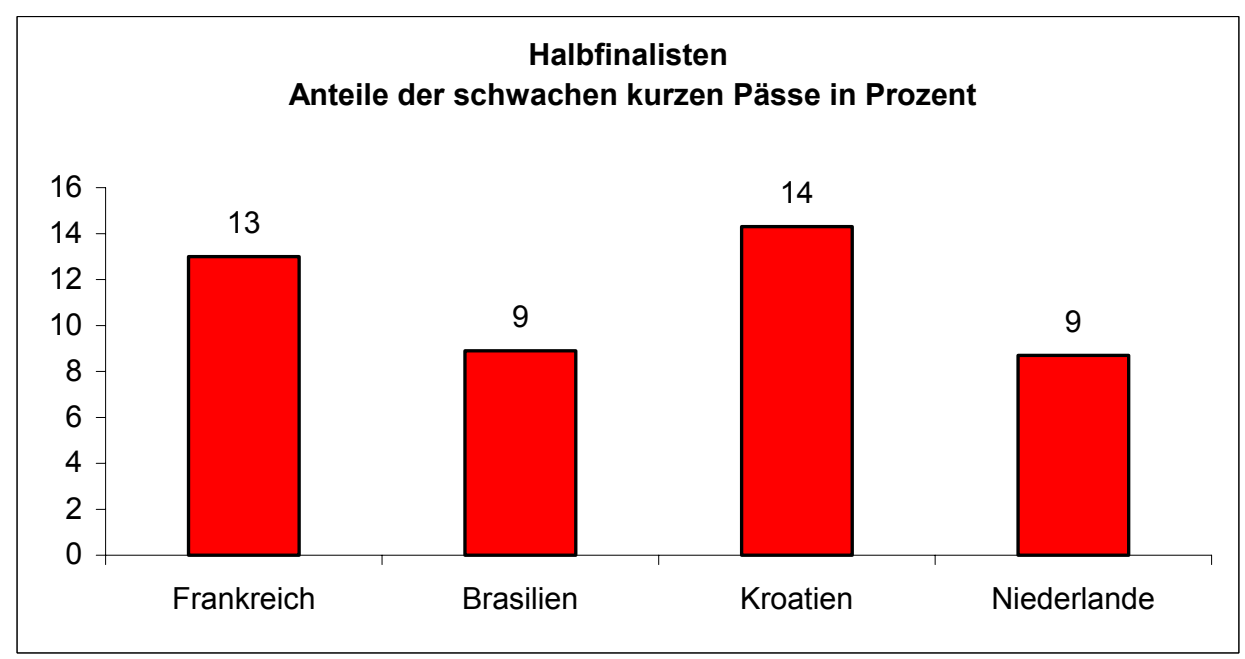

Diagramm 48: Anteile der schwachen kurzen Pässe in Prozent bei den Halbfinalisten.

Lange Pässe hatten Niederlande 232-mal, hiervon 16 (7\%) hochkarätige. Dies bildete den größten Wert. Absteigend folgen Kroatien mit 250 und 9 (4 \%), Frankreich hatte 343 und 11 (3\%), Brasilien 285 und 7 (3\%) „hochkarätige“ lange Pässe. Das folgende Diagramm zeigt die unterschiedlichen Qualitätsanteile des technischen Elements „hochkarätige lange Pässe“ der Halbfinalisten in Prozent. 


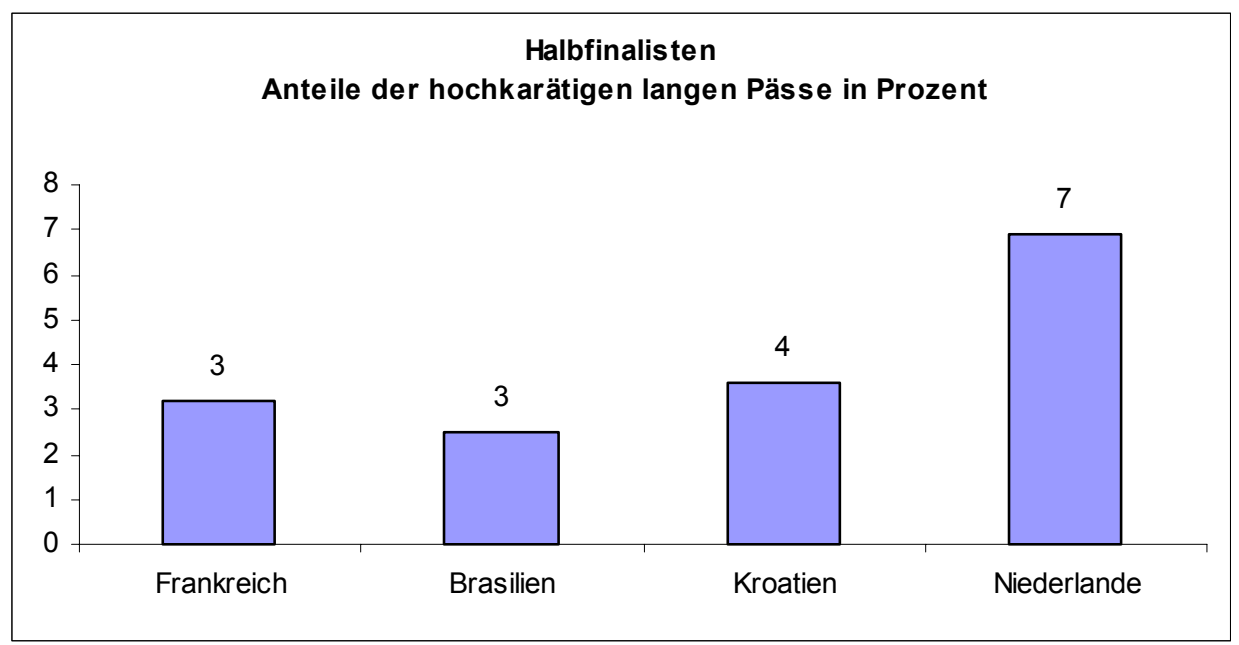

Diagramm 49: Anteile der hochkarätigen langen Pässe in Prozent bei den Halbfinalisten.

119 (35 \%) der langen Pässe von Frankreich wurden mit "normal“ bewertet und bildeten den größten Anteil. Die weiteren Anteile „normaler“ langer Pässe lauteten 73 (32\%) für die Niederlande, 84 (30\%) für Brasilien und 70 (28 \%) für Kroatien.

Das folgende Diagramm zeigt die unterschiedlichen Qualitätsanteile des technischen Elements "normalen langen Pässe“ der Halbfinalisten in Prozent.

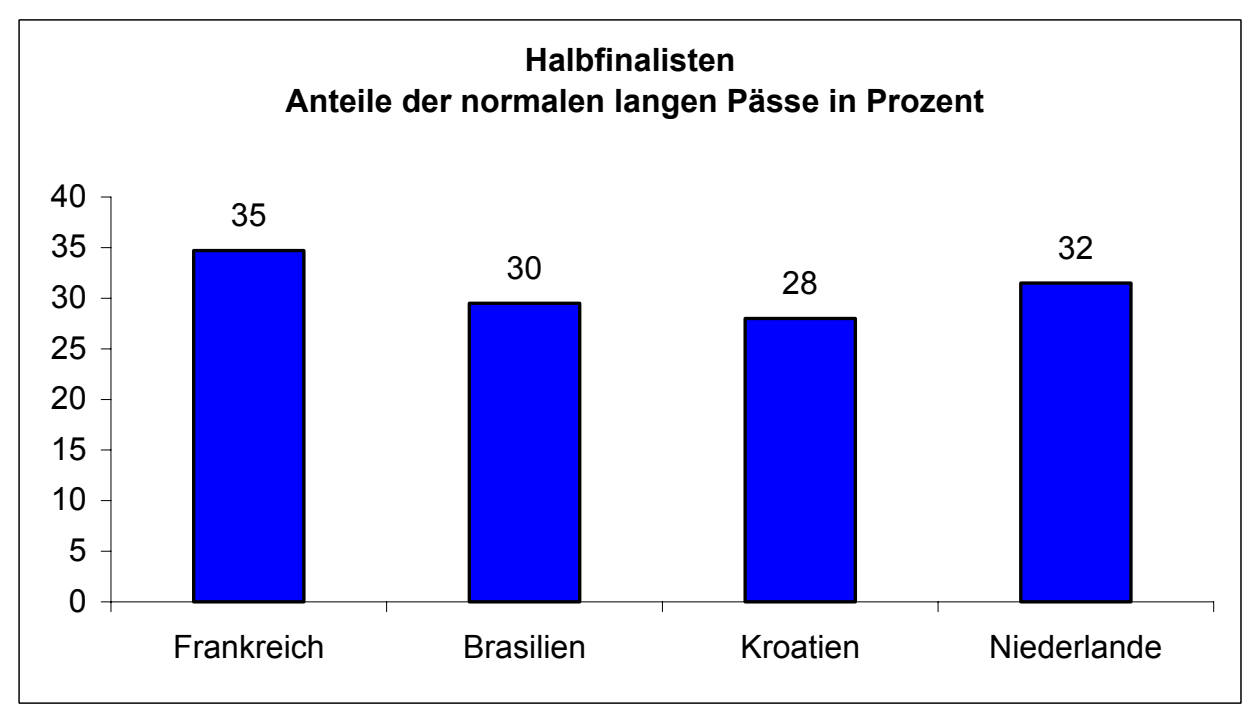

Diagramm 50: Anteile der normalen langen Pässe in Prozent bei den Halbfinalisten.

Ein "schwacher" langer Pass wurde mit einem Anteil von 171 (68 \%) am häufigsten bei Kroatien festgestellt. Es folgen Brasilien 194 (68 \%), Frankreich 213 (62\%) und schließlich die Niederlande 143 (62\%). 
Das folgende Diagramm zeigt die unterschiedlichen Qualitätsanteile des technischen Elements „schwache lange Pässe“ der Halbfinalisten in Prozent.

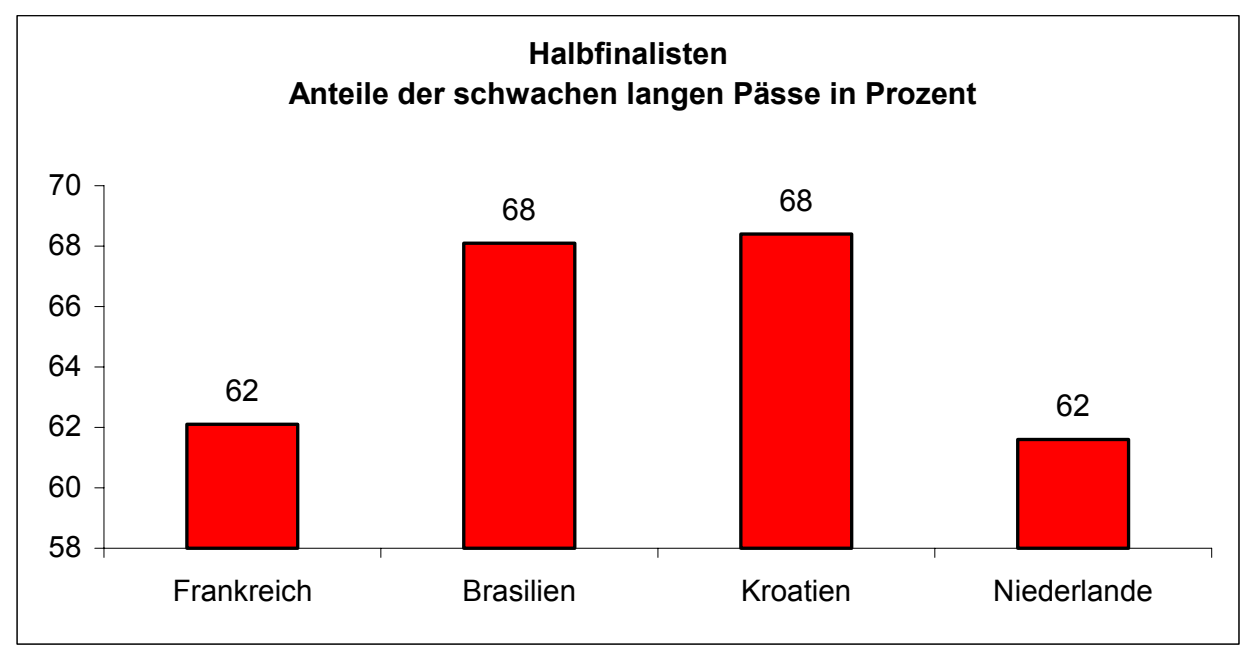

Diagramm 51: Anteile der schwachen langen Pässe in Prozent bei den Halbfinalisten.

Die Diagramme 46, 47, 48, 49, 50 und 51 erklären die Werte der Mannschaften:

Diagramm 46 erklärt, dass die hochkarätige kurze Pässe zwischen $1 \%$ und $2 \%$. Die kroatische Mannschaft hatte die besten Werte und die brasilianische und niederländische hatten die schlechtesten. Diagramm 47 erklärt, dass gute kurze Pässe zwischen $84 \%$ und $90 \%$ vorkamen. Die niederländische und brasilianische Mannschaft hatten hier die besten Werte und die kroatische Mannschaft hatte die schlechtesten. Diagramm 48 erklärt, dass schwachen kurzen Pässe zwischen $9 \%$ und $14 \%$ vorkamen. Die kroatische Mannschaft hatte die schwächsten Werte und die niederländische und brasilianische hatten die besten Werte.

Es lässt sich die Schlussfolgerung aus den Diagrammen ziehen, dass die normalen und schwachen kurzen Pässe bei allen Halbfinalisten häufiger sind als die hochkarätigen. Wahrscheinlich hatten die Mannschaften eine unfangreiche oder langsame Vorbereitung in Mittelfeld, wodurch sie der anderen Mannschaft eine gute Chance gaben, um ihre Verteidigung zu organisieren.

Diagramm 49 erklärt, dass die hochkarätigen langen Pässe zwischen $3 \%$ und $7 \%$ vorkamen. Die niederländische Mannschaft hatte die besten Werte, die brasilianische hatte die schlechtesten. Diagramm 50 erklärt, dass gute lange Pässen zwischen 28 $\%$ und $35 \%$ vorkamen. Die französische Mannschaft hatte hier die besten Werte 
und die kroatische hatte die schlechtesten. Diagramm 51 erklärt, dass die schwachen langen Pässe zwischen $62 \%$ und 68 \% vorkamen. Die französische und niederländische Mannschaft hatten die besten Werte und die kroatische und brasilianische Mannschaft hatten die schlechtesten Werte.

Es lässt sich die Schlussfolgerung aus den Diagrammen ziehen, dass die schwachen langen Pässe bei allen Halbfinalisten häufiger sind als die hochkarätigen und die normalen. Bei langen Pässen muss auf jeden Fall der Spieler mit großer Genauigkeit spielen, weil der Ball sehr leicht abzunehmen ist und es dauert länger als bei kurzen Pässen bis der Ball zum anderen Mitspieler kommt. Die Pässe wurden unkonzentriert, technisch unsauber, schlecht getimt (zu scharf / zu schwach) und oft mit Effet gespielt. Dadurch hat der annehmende Spieler Probleme mit der Ballkontrolle oder mit der Schuss- Direktannahme.

\subsubsection{Ballkontrolle}

Hinsichtlich der Qualität der Ballkontrolle wurde festgestellt: von 117 fallen an Ballkontrolle hatte Kroatien, hiervon wurden 7 (6\%) als hochkarätige bewertet und bildeten den größten Wert. Absteigend folgen Brasilien mit 181und 4 (2\%), die Niederlande mit 151und 3 (2\%), Frankreich mit 240 und 3 (1\%) hochkarätige Ballkontrolle. Das folgende Diagramm zeigt die unterschiedlichen Qualitätsanteile des technischen Elements "hochkarätige Ballkontrolle" der Halbfinalisten in Prozent.

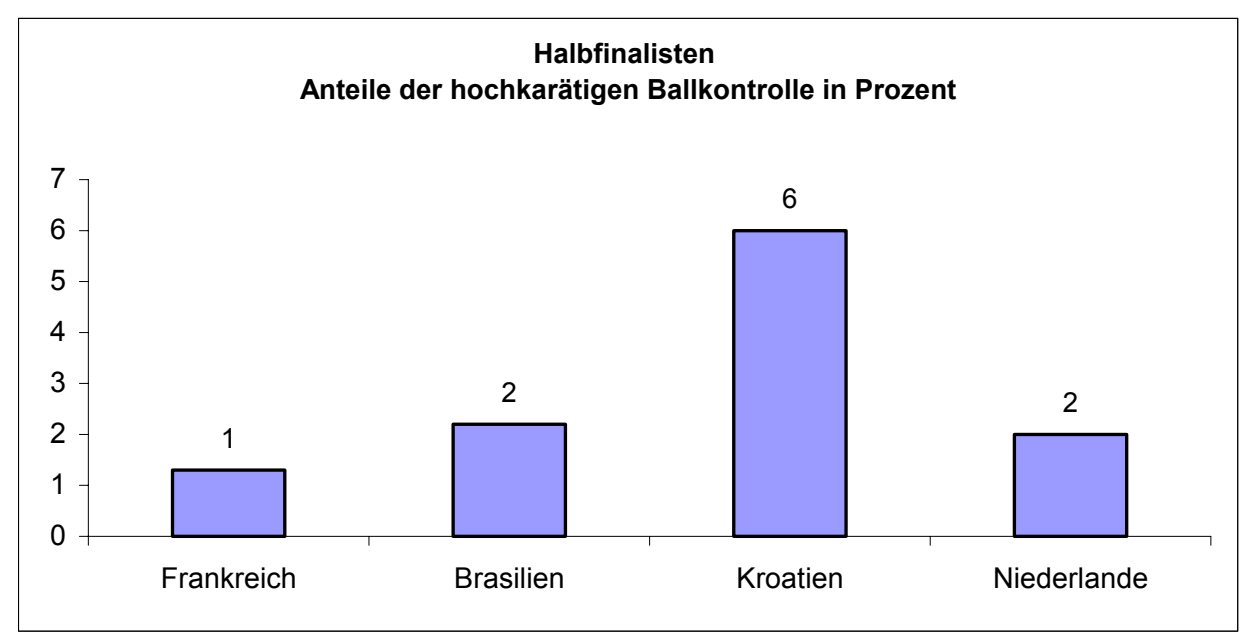

Diagramm 52: Anteile der hochkarätigen Ballkontrolle in Prozent bei den Halbfinalisten. 
67-mal (44 \%) der Ballkontrollen der Niederlande wurden mit „normal“ bewertet und bildeten den größten Anteil. Die weiteren Anteile „normaler“ Ballkontrollen lauteten 106-mal (44 \%) für Frankreich, 73-mal (40 \%) für Brasilien und 44-mal (38 \%) für Kroatien.

Das folgende Diagramm zeigt die unterschiedlichen Qualitätsanteile des technischen Elements „mittlere Ballkontrolle“ der Halbfinalisten in Prozent.

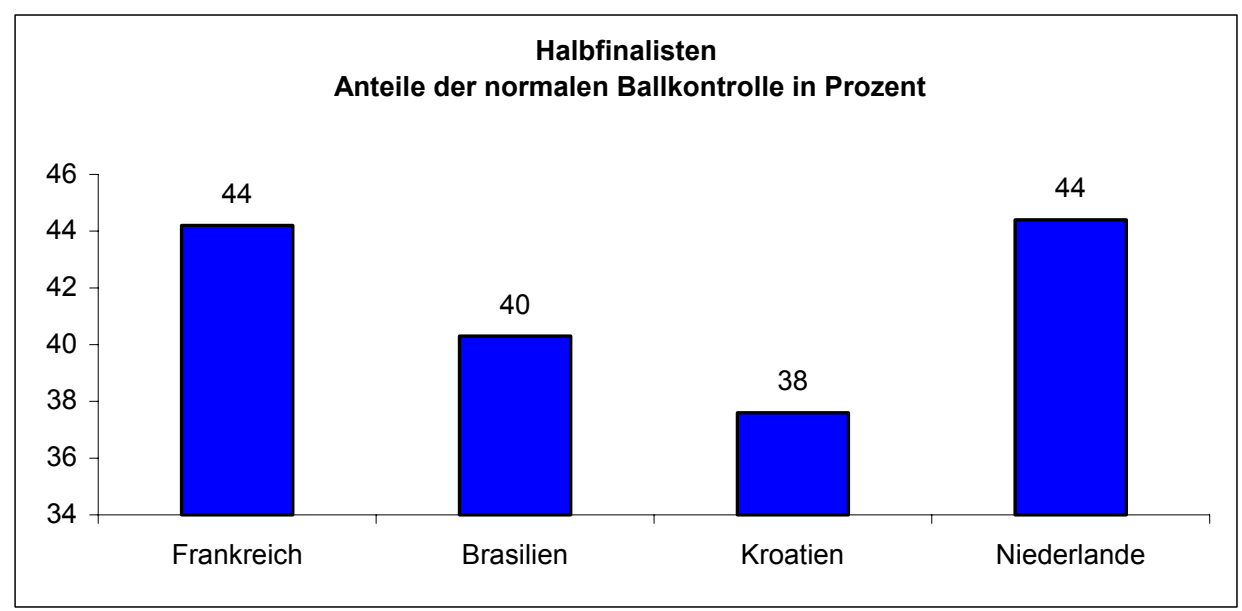

Diagramm 53: Anteile der normalen Ballkontrolle in Prozent bei den Halbfinalisten.

Eine „schwache“ Ballkontrolle wurde mit einem Anteil von 104 (58 \%) am häufigsten bei Brasilien festgestellt. Es folgen Kroatien 66 (56 \%), Frankreich 131 (55\%) und schließlich die Niederlande 81 (54\%).

Das folgende Diagramm zeigt die unterschiedlichen Qualitätsanteile des technischen Elements „schwache Ballkontrolle“ der Halbfinalisten in Prozent.

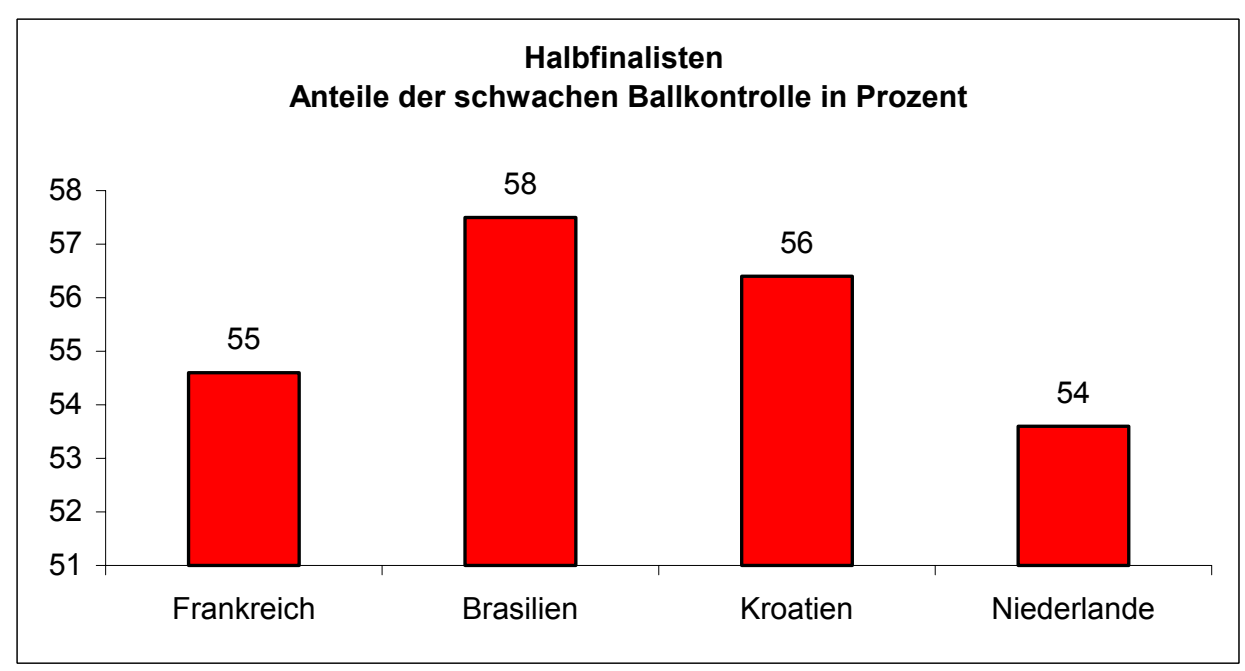

Diagramm 54: Anteile der schwachen Ballkontrolle in Prozent bei den Halbfinalisten. 
Die Diagramme 52, 53 und 54 erklären die Werte der Mannschaften:

Diagramm 52 erklärt, dass hochkarätigen Ballkontrolle nur zwischen $1 \%$ und $6 \%$ lag. Die kroatische Mannschaft hatte die besten Werte und die französische hatte die schlechtesten.

Diagramm 53 erklärt, dass gute Ballkontrolle zwischen $38 \%$ und $44 \%$ lag. Die französische und niederländische Mannschaft hatten hier die besten Werte und die kroatische hatte die schlechtesten. Diagramm 54 erklärt, dass schwache Ballkontrolle zwischen $54 \%$ und $58 \%$ lag. Die brasilianische Mannschaft hatte die schwächsten Werte und die niederländische hatte die besten Werte.

Es lässt sich die Schlussfolgerung aus den Diagrammen ziehen, dass die hochkarätigen Ballkontrolle bei allen Halbfinalisten am seltensten vorkommen und auch die schlechten hoch häufiger sind als die „normalen“. Das ist dadurch zu erklären, dass die Angreifer ihren Ball oftmals verlieren, wenn das Pressing im Drittel des Gegners stark ist und bei dem schnellen Spielverlauf die Spieler ihren Ball schnell unter ihre Kontrolle bringen müssen.

\subsection{Prozentuale Qualitätsanteile offensiv- taktischer Elemente}

Im folgenden Kapitel wird die Qualität der offensiv-taktischen Fähigkeiten der einzelnen Mannschaften beschrieben. Es wird aufgezeigt, mit welcher prozentualen Häufigkeit eine bestimmte Mannschaft bezüglich eines bestimmten offensiv-taktischen Elementes den Bewertungsklassen „gut“, „normal“ und „schwach“ zugeordnet wurde. Aufgrund der unterschiedlichen Anzahl von bestrittenen Spielen werden die Mannschaften hierbei in Achtel-, Viertel- und Halbfinalisten aufgeteilt.

\subsubsection{Achtelfinalisten}

Als Achtelfinalisten werden diejenigen Mannschaften bezeichnet, die im Achtelfinale ausgeschieden sind und zu deren Analyse deshalb nur ein Spiel herangezogen werden konnte. Zu diesen Achtelfinalisten gehören England, Chile, Mexiko, Jugoslawien, Nigeria, Paraguay, Rumänien und Norwegen. 


\subsubsection{Freistöße}

Der (indirekte) Freistoß als Standardsituation ist eine mannschaftliche Eigenschaft und beschreibt die Fähigkeit der Mannschaft Gefahr für den Gegner auszuüben bzw. der (direkte) Freistoß als Standardsituation ist eine individuelle Eigenschaft und beschreibt die Fähigkeit eines einzelnen Spielers, einen Freistoß so auszuführen, dass Gefahr für die gegnerische Mannschaft entsteht. Teile der Mannschaft einschließlich des Schützen, müssen sich vor, während und nach Ausführung des Freistoßes so verhalten, dass ein Torerfolg möglich ist, zumindest aber eine für die Mannschaft günstige Situation entsteht. Eine der besten Gelegenheiten, um in einem Wettkampf ein Tor zu erzielen, bietet sich beim Freistoß, besonders wenn er nahe am Strafraum erfolgt.

Hinsichtlich der Qualität des Freistoßes wurde festgestellt: Jugoslawien hatte 4 Freistösse, hiervon wurde $1(25 \%)$ als hochkarätig bezeichnet und bildete den größten Wert. Absteigend folgt England mit 14 und 1 (7\%). Chile, Mexiko, Nigeria, Paraguay, Rumänien und Norwegen hatten 7, 10, 7, 3, 6, 8-Freistößen von denen jeweils 0,0 \% als den kleinsten Anteil als "hochkarätig" bewertet wurden.

Das folgende Diagramm zeigt die Anteile der hochkarätigen Freistöße in Prozent für die Achtelfinalisten.

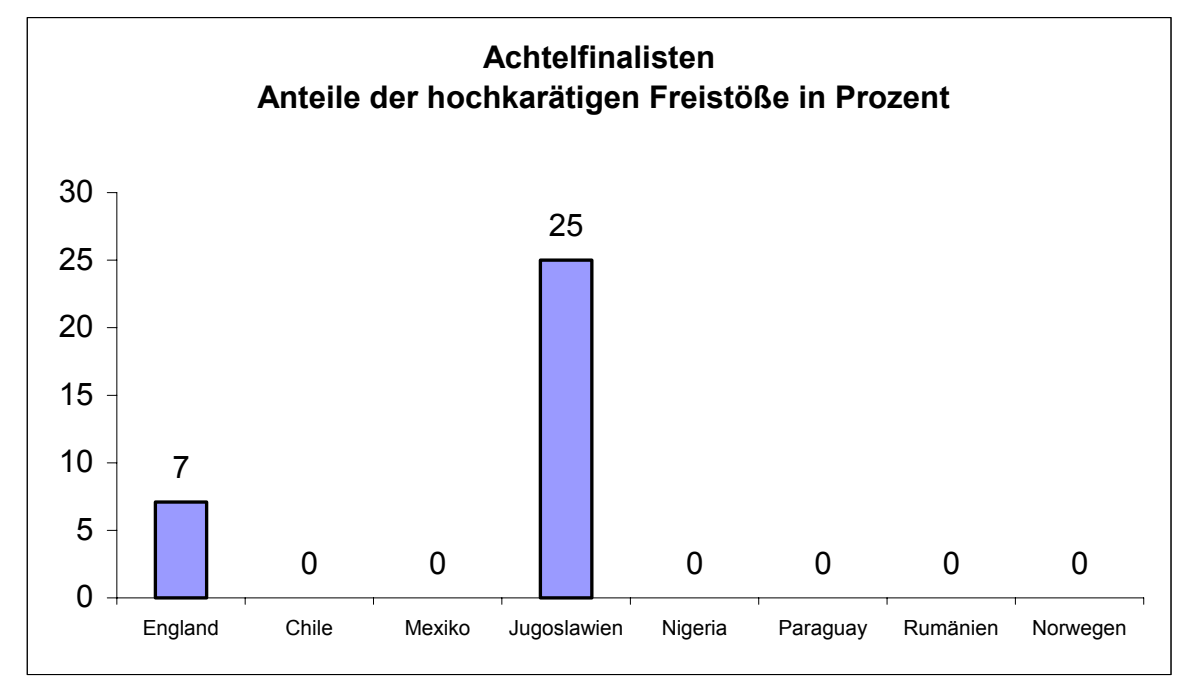

Diagramm 55: Anteile der hochkarätigen Freistöße in Prozent bei den Achtelfinalisten. 
5 (83 \%) der Freistöße von Rumänien wurden mit „normal“ bewertet und bildeten den größten Anteil. Die weiteren Anteile „normaler“ Freistöße lauteten 7-mal (70 \%) für Mexiko, 4 (57 \%) für Chile, 2 (50 \%) für Jugoslawien, 3 (43\%) für Nigeria, 3 (38 \%) für Norwegen, 1 (33 \%) für Paraguay und 3 (21 \%) für England.

Das folgende Diagramm zeigt die Anteile der mittleren Freistösse in Prozent für die Achtelfinalisten.

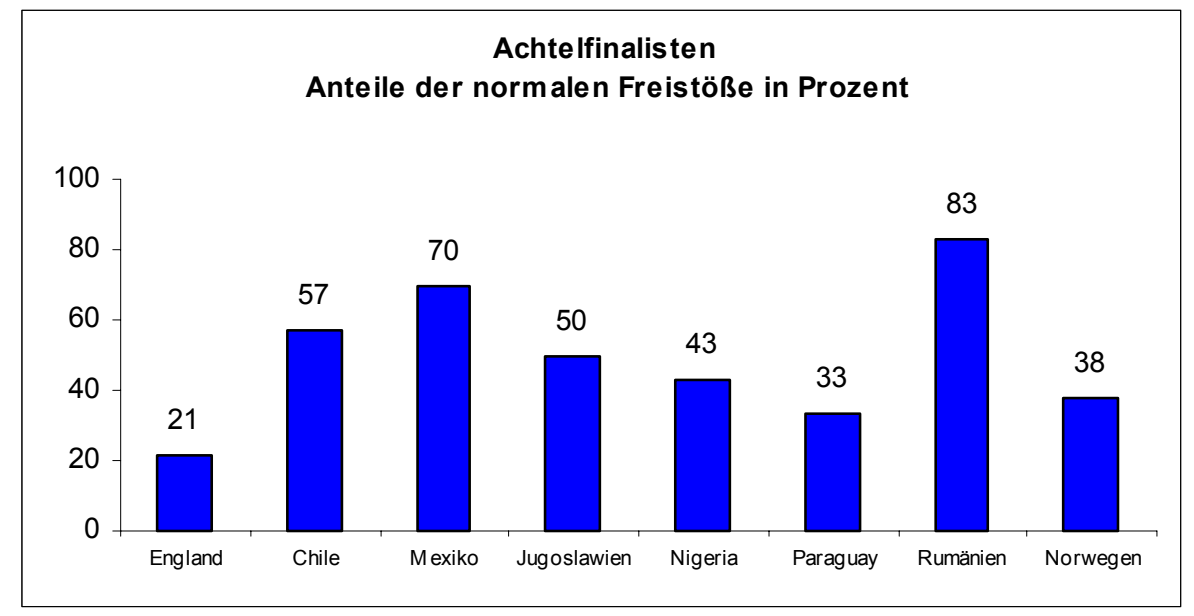

Diagramm 56: Anteile der normalen Freistöße in Prozent bei den Achtelfinalisten.

Ein „schwacher“ Freistoß wurde mit einem Anteil von 10-mal (71 \%) am häufigsten bei England festgestellt. Es folgen Paraguay 2 (67\%), Norwegen 5 (63\%), Nigeria 4 (57\%), Chile 3 (43\%), Mexiko 3 (30\%), Jugoslawien 1 (25\%) und schließlich Rumänien $1(17 \%)$.

Das folgende Diagramm zeigt die Anteile der schwachen Freistöße in Prozent für die Achtelfinalisten.

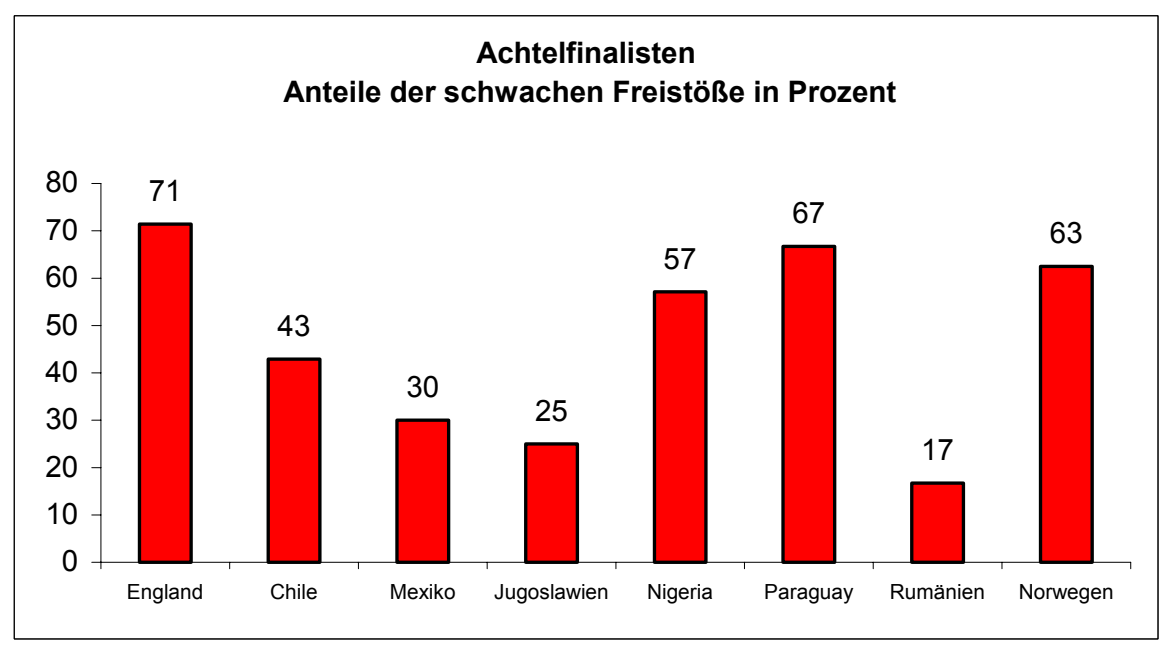

Diagramm 57: Anteile der schwachen Freistöße in Prozent bei den Achtelfinalisten. 
Die Diagramme 55, 56 und 57 erklären die Werte der Mannschaften:

Diagramm 55 erklärt, dass die hochkarätigen Freistöße zwischen $0 \%$ und 25 \% lagen. Die jugoslawische und die englische Mannschaft hatten die besten Werte, und alle anderen hatten die schlechtesten. Diagramm 56 erklärt, dass normale Freistöße zwischen $21 \%$ und $83 \%$ vorkamen. Die rumänische Mannschaft hatte hier die besten Werte und die englische hatte die schlechtesten. Diagramm 57 erklärt, dass schwache Freistöße zwischen $17 \%$ und $71 \%$ vorkamen. Die englische Mannschaft hatte die schwächsten Werte und die rumänische hatte die besten Werte.

Es lässt sich Schlussfolgerung aus den Diagrammen ziehen: Mit Ausnahme von Jugoslawien und England ist die Ausführung der Freistöße im Achtelfinale als recht schwach anzusehen, d.h., dass die meisten der Freistöße entweder als "normal“ oder "schwach“ zu bewerten sind. Das ist auf die mangelhafte Aufstellung des Angriffs zurückzuführen, besonders wenn der Freistoß von einem Platz weit vom Tor gespielt wird oder ungenau bei direktem Schuss geschossen wird. Die Streuung der Werte zwischen den besten und den schlechtesten Mannschaften war bei diesem Wert sehr groß.

\subsubsection{Eckball}

Der Eckball als Standardsituation ist eine mannschaftliche Leistung und beschreibt die Fähigkeit der Mannschaft bzw. Teilen der Mannschaft einschließlich des Schützen, sich vor, während und nach Ausführung des Eckballs so zu verhalten, dass ein Torerfolg möglich ist. Eckbälle gelten als gute Möglichkeit, um den Spielverlauf und das -ergebnis zu beeinflussen, wenn sie gut ausgeführt werden. Sie bieten der ausführenden Mannschaft die Gelegenheit, die gegnerische Verteidigung zu überwinden.

Von 7 Ecken für England wurden 1 (14 \%) mit hochkarätig bezeichnet. Absteigend folgt Norwegen mit 8 und 1 (13\%) als hochkarätig bezeichneten Eckball. Chile, Mexiko, Jugoslawien, Nigeria, Paraguay und Rumänien hatten 5, 5, 2, 6, 4, 2 Ecken, von denen jeweils $0,0 \%$ als "hochkarätiger" Eckbälle bezeichnet wurde.

Das folgende Diagramm zeigt die Anteile den hochkarätigen Eckbällen in Prozent für die Achtelfinalisten. 


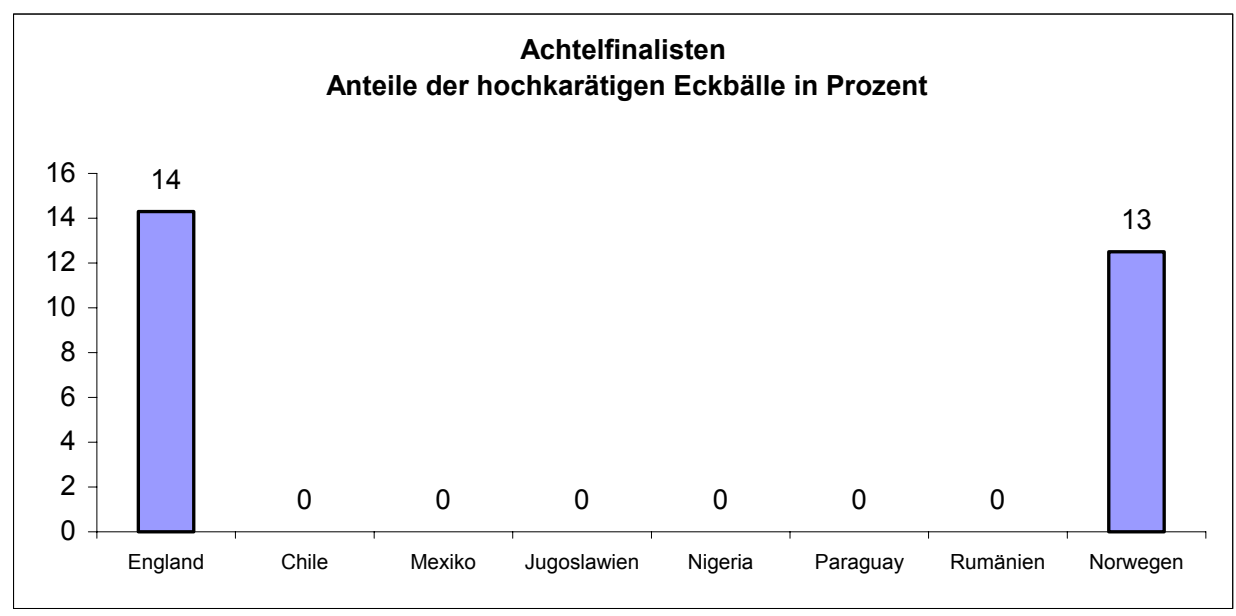

Diagramm 58: Anteile der hochkarätigen Eckbälle in Prozent bei den Achtelfinalisten.

Jugoslawien, Nigeria und Rumänien hatten 1, 3, 1 normale Eckbälle, was jeweils (50 \%) entsprach und den größten Anteil bildete. Die weiteren Anteile „normaler“ Eckbälle lauteten 2 (40 \%) für Mexiko, 2 (29 \%) hatte England, 2 (25\%) für Norwegen, 1 (20\%) hatte Chile, und 0,0\% für Paraguay.

Das folgende Diagramm zeigt die Anteile der mittleren Eckbälle in Prozent für Achtelfinalisten.

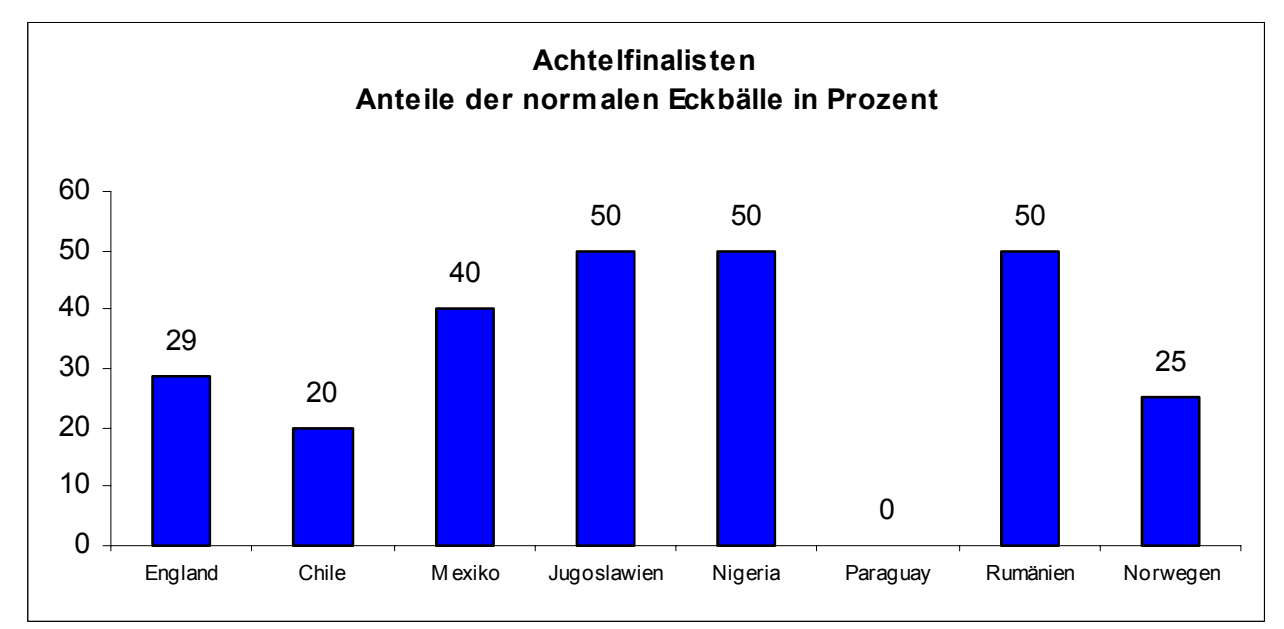

Diagramm 59: Anteile der normalen Eckbälle in Prozent bei den Achtelfinalisten.

Ein „schwacher“ Eckball wurde mit einem Anteil von 4-mal (100\%) am häufigsten bei Paraguay festgestellt. Es folgen Chile 4 (80 \%), Norwegen 5 (63\%), Mexiko 3 (60 $\%$ ), England 4 (57\%), Jugoslawien 1 (50\%), Nigeria 3 (50\%) und schließlich Rumänien $1(50 \%)$. 
Das folgende Diagramm zeigt die Anteile der schwachen Eckbälle in Prozent für die Achtelfinalisten.

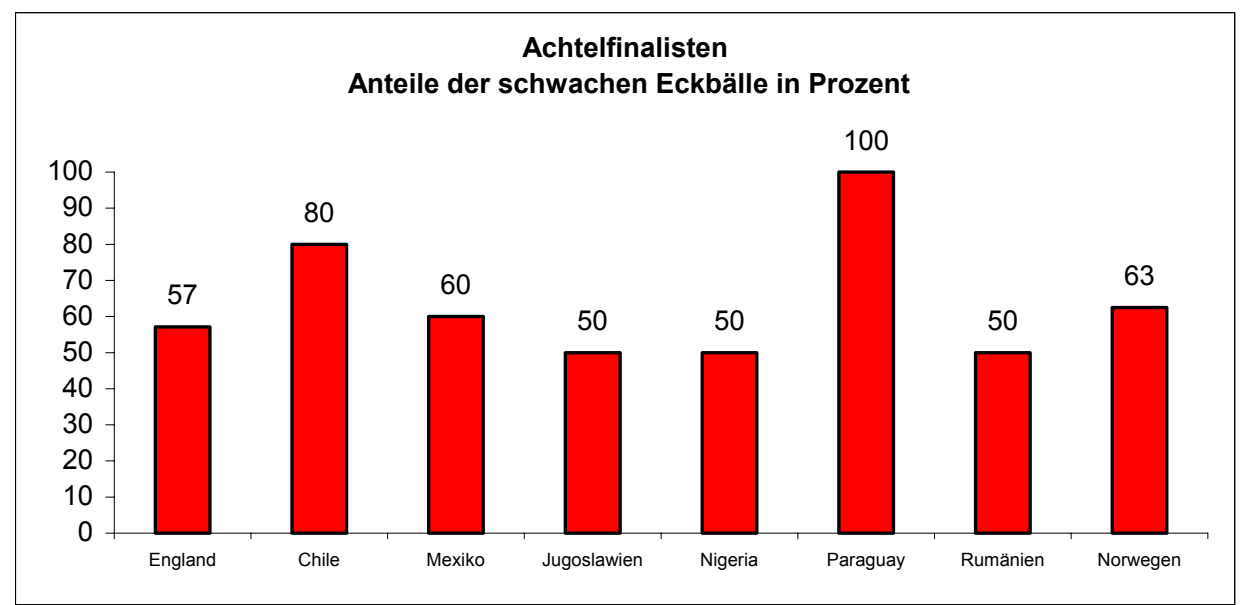

Diagramm 60: Anteile der schwachen Eckbälle in Prozent bei den Achtelfinalisten.

Aus den Werten der Diagramme 58, 59 und 60 ist die geringe Anzahl der hochkarätigen im Vergleich zu den guten und schwachen Eckbällen ersichtlich. Dabei ist die Anzahl der schwachen Eckbälle nicht nur größer als die der hochkarätigen und normalen. Wir führen das auf die schlechte Organisation des Angriffs bei der Ausführung des Eckballs oder schlechte Taktik zurück. Mit einer guten Taktik kann die ausführende Mannschaft die gegnerische Mannschaft täuschen und die gegnerische Abwehr überwinden.

\subsubsection{Unterstützen}

Das Unterstützen ist eine mannschaftliche Eigenschaft und beschreibt die Fähigkeit der Mannschaft bzw. Teilen der Mannschaft, sich so in Richtung des Ballführenden Mitspielers zu bewegen, dass diesem möglichst optimale Unterstützung zukommt. Das Unterstützen ist ein wichtiger Spielzug beim Wettkampf, da der Spieler, der unterstützt, vom Gegner nicht beschattet wird, da auch der Ballbesitzende Spieler nicht alleine durch die gegnerische Abwehr laufen kann.

Hinsichtlich der Qualität des Unterstützen wurde festgestellt: Paraguay erzielte mit 3mal 1 (33 \%) den größten als hochkarätig bezeichneten Wert. Absteigend folgen Mexiko 5-mal mit 1 (20\%), England 6-mal, mit 1 (17\%) als hochkarätiger bezeichnet, 
Nigeria 8-mal, mit 1 (13\%), Chile 9-mal, mit 1 (11\%), Jugoslawien 19-mal, mit 1 (5 \%) und Norwegen 21-mal, mit 1 (5\%). Rumänien hatte 55-mal mit 1 (2\%) den kleinsten Anteil "guten“ Unterstützens.

Dem folgenden Diagramm sind die Anteile des hochkarätigen Unterstützens in Prozent für die Achtelfinalisten zu entnehmen.

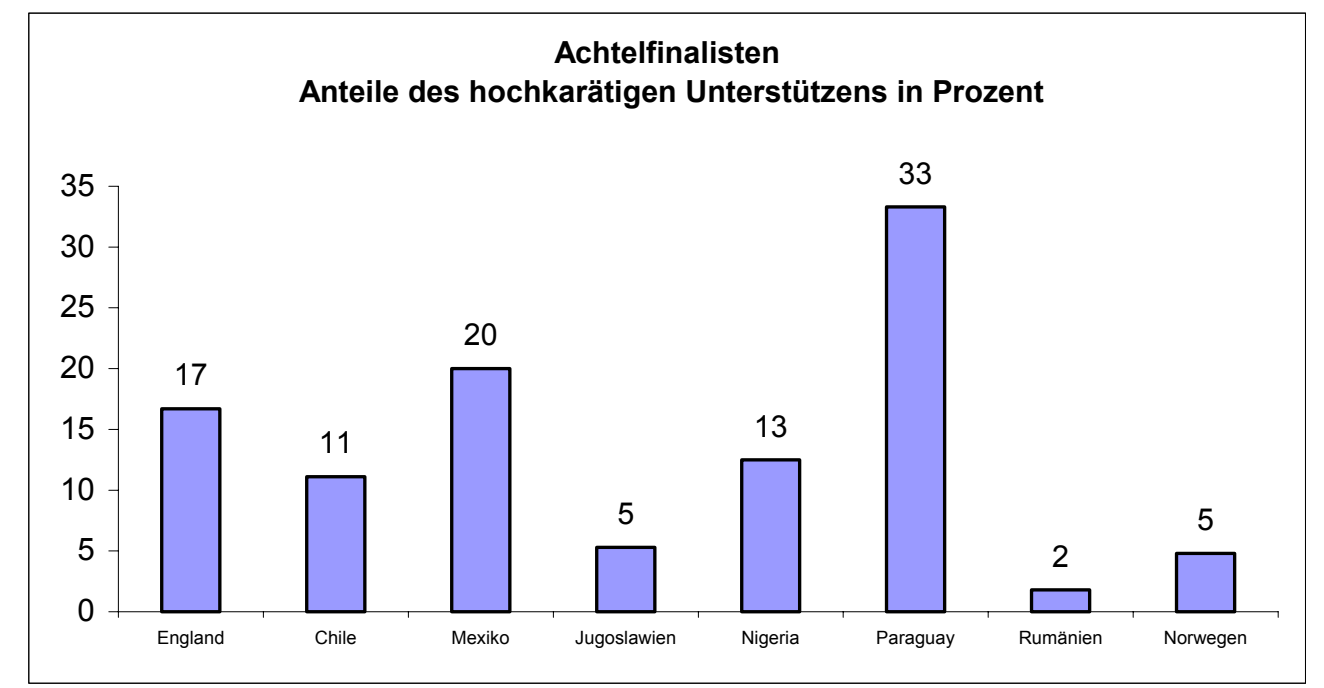

Diagramm 61: Anteile des hochkarätigen Unterstützens in Prozent bei den Achtelfinalisten.

53-mal (96 \%) des Unterstützens von Rumänien wurde mit "normal“ bewertet und bildete den größten Anteil. Die weiteren Anteile „normalen“ Unterstützens erzielte Jugoslawien 17 (90\%), 18 (86 \%) für Norwegen, 7 (78 \%) für Chile, 6 (75 \%) für Nigeria, 4 (67 \%) für England, 3 (60 \%) für Mexiko und 1 (33 \%) für Paraguay. Das folgende Diagramm zeigt die Anteile des normalen Unterstützens in Prozent für die Achtelfinalisten. 


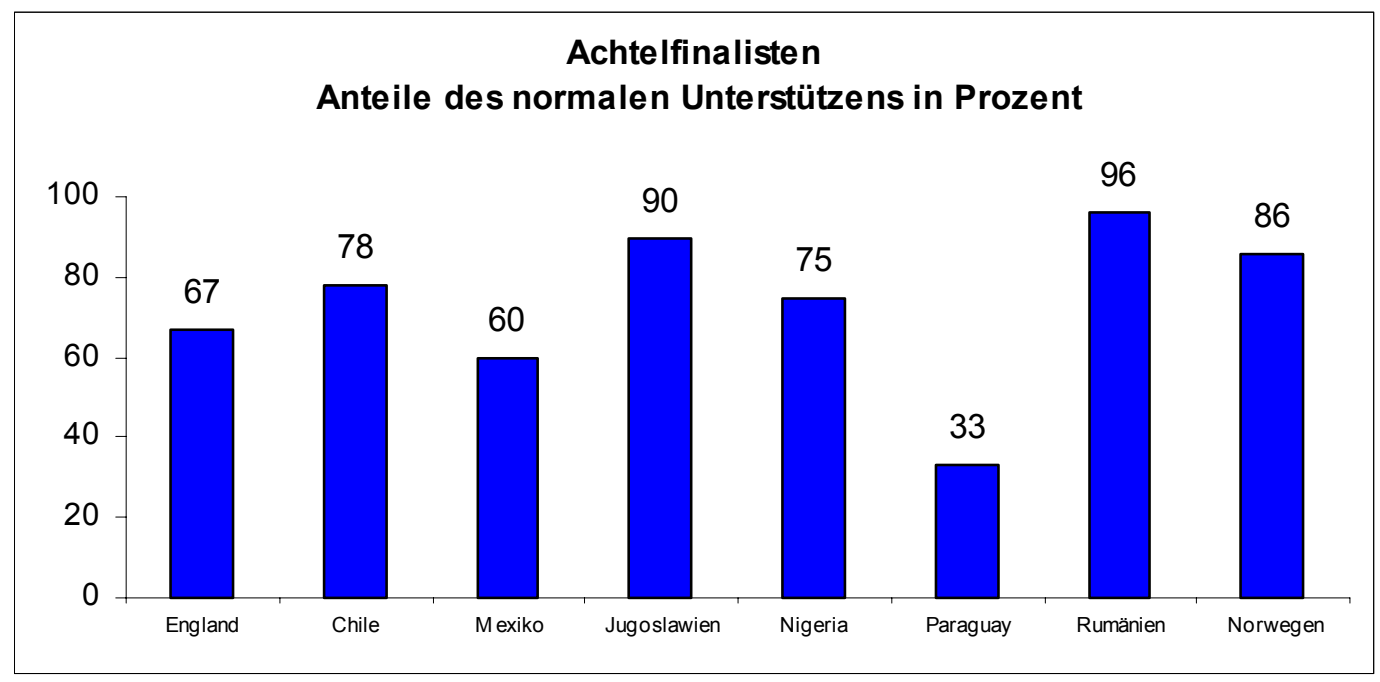

Diagramm 62: Anteile des normalen Unterstützens in Prozent bei den Achtelfinalisten.

Ein „schwaches“ Unterstützen wurde mit einem Anteil von 1 (33 \%) am häufigsten bei Paraguay festgestellt. Es folgen Mexiko 1 (20\%), England 1 (17 \%), Nigeria 1 (13 $\%)$, Chile 1 (11\%), Norwegen $2(10 \%)$, Jugoslawien 1 (5\%) und schließlich Rumänien $1(2 \%)$.

Das folgende Diagramm zeigt die Anteile des schwachen Unterstützens in Prozent für die Achtelfinalisten.

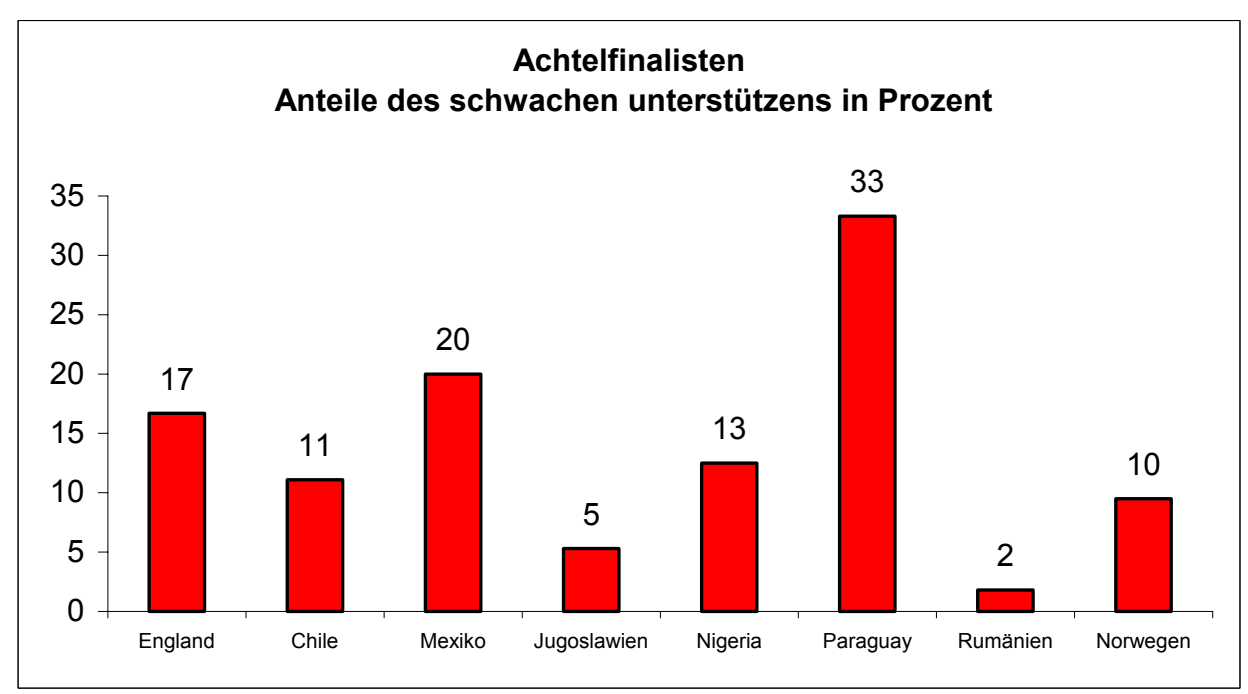

Diagramm 63: Anteile des schwachen Unterstützens in Prozent bei den Achtelfinalisten. 
Aus der Darstellung wird deutlich, dass normales Unterstützen häufiger ist als hochkarätiges oder schwaches, da die unterstützenden Spieler einen Platz einnehmen, von dem aus sie ihren Mitspielern so helfen können, dass sie gut mit dem Ball vorankommen können. Manchmal unterstützen die Mitspieler nicht zur rechten Zeit oder der richtige Position.

\subsubsection{Doppelpass}

Der Doppelpass ist eine mannschaftliche Eigenschaft und beschreibt die Fähigkeit von genau zwei Spielern der Mannschaft, sich den Ball über direktes Kurzpassspiel so zuzuspielen, dass dadurch ein taktischer Vorteil entsteht. Der Doppelpass ist eine Weiterentwicklung der Standardkombinationen. Er wurde als Gegenmittel zur engen Manndeckung entwickelt. Mit seiner Hilfe können sich die Stürmer auch gegen Abwehrspieler, die eng decken und aggressiv bereits bei der Ballkontrolle stören, durchsetzen.

„Guten“ Doppelpass hatte Nigeria 1 von 3-mal, wovon mit (33 \%) der größte Wert als „hochkarätig“ bezeichnet wurde. Absteigend folgt Chile mit 10 und 1 (10\%); England, Mexiko, Jugoslawien, Paraguay, Rumänien und Norwegen hatten 4,1, 3, 3, 2, 2, wovon jeweils $0,0 \%$ der kleinste Anteil als "hochkarätiger" Doppelpass bezeichnet wurde.

Das folgende Diagramm zeigt die Anteile der hochkarätigen Doppelpässe in Prozent für die Achtelfinalisten.

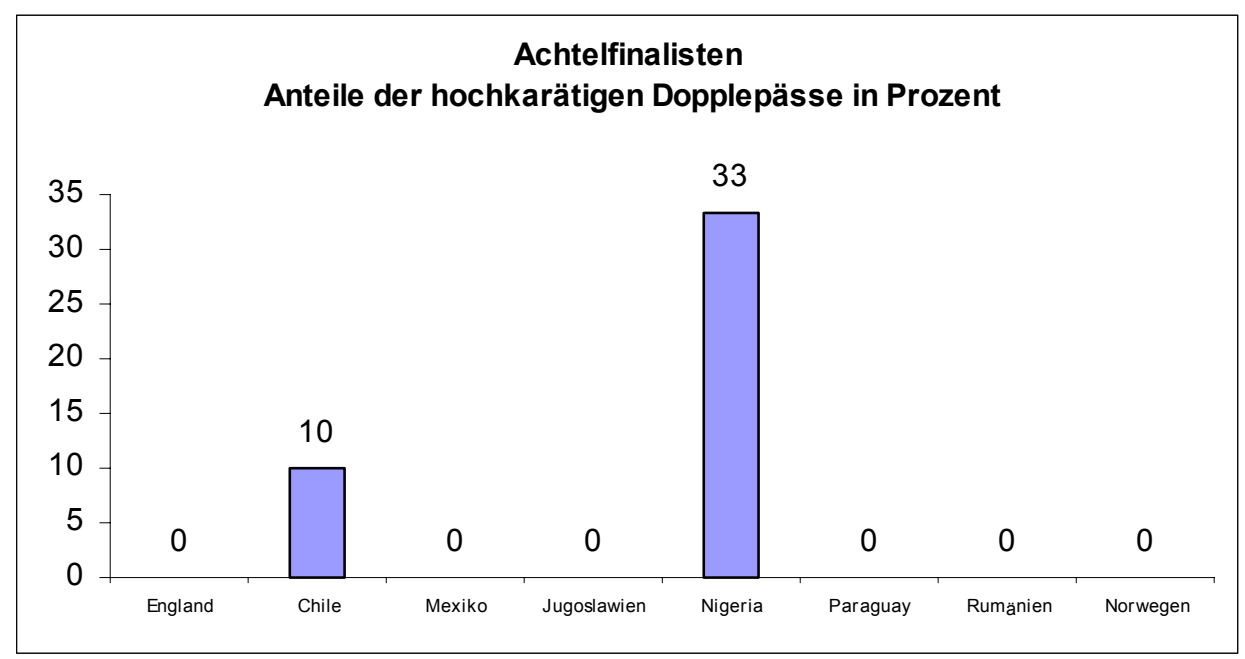

Diagramm 64: Anteile der hochkarätigen Doppelpässe in Prozent bei den Achtelfinalisten. 
8-mal ( $80 \%$ ) der Doppelpässe von Chile wurden mit "normal“ bewertet und bildeten den größten Anteil. Die weiteren Anteile „normaler“ Doppelpässe lauteten 3 (75\%) für England, 2-mal (67 \%) für Jugoslawien und Paraguay, 1 (50 \%) für Rumänien und Norwegen, 1 (33 \%) für Nigeria und 0,0 \% für Mexiko.

Das folgende Diagramm zeigt die Anteile der normalen Doppelpässe in Prozent für die Achtelfinalisten.

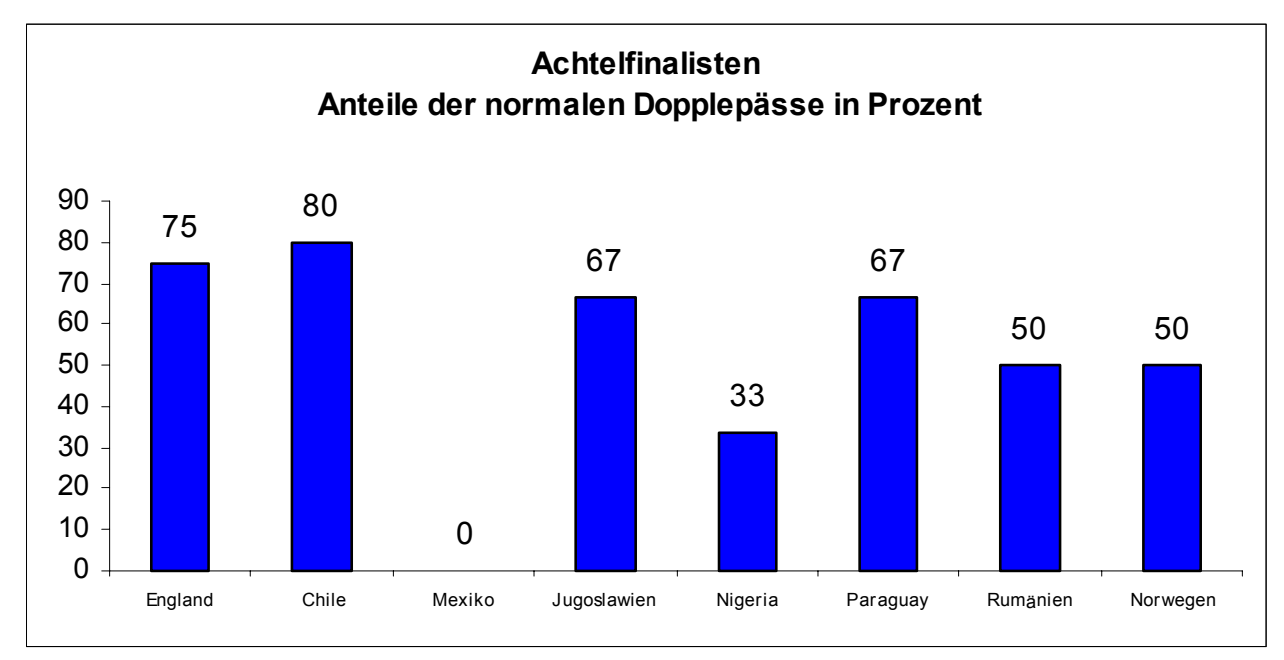

Diagramm 65: Anteile der normalen Doppelpässe in Prozent bei den Achtelfinalisten. Ein „schwacher“ Doppelpass wurde mit einem Anteil von 1-mal (100 \%) am häufigsten bei Mexiko festgestellt. Es folgen Rumänien und Norwegen 1 (50\%), Jugoslawien, Nigeria und Paraguay hatten 1 (33\%), England hatte 1 (25\%) und schließlich Chile 1 (10 \%). Das folgende Diagramm zeigt die Anteile des schwachen Doppelpasses in Prozent für die Achtelfinalisten.

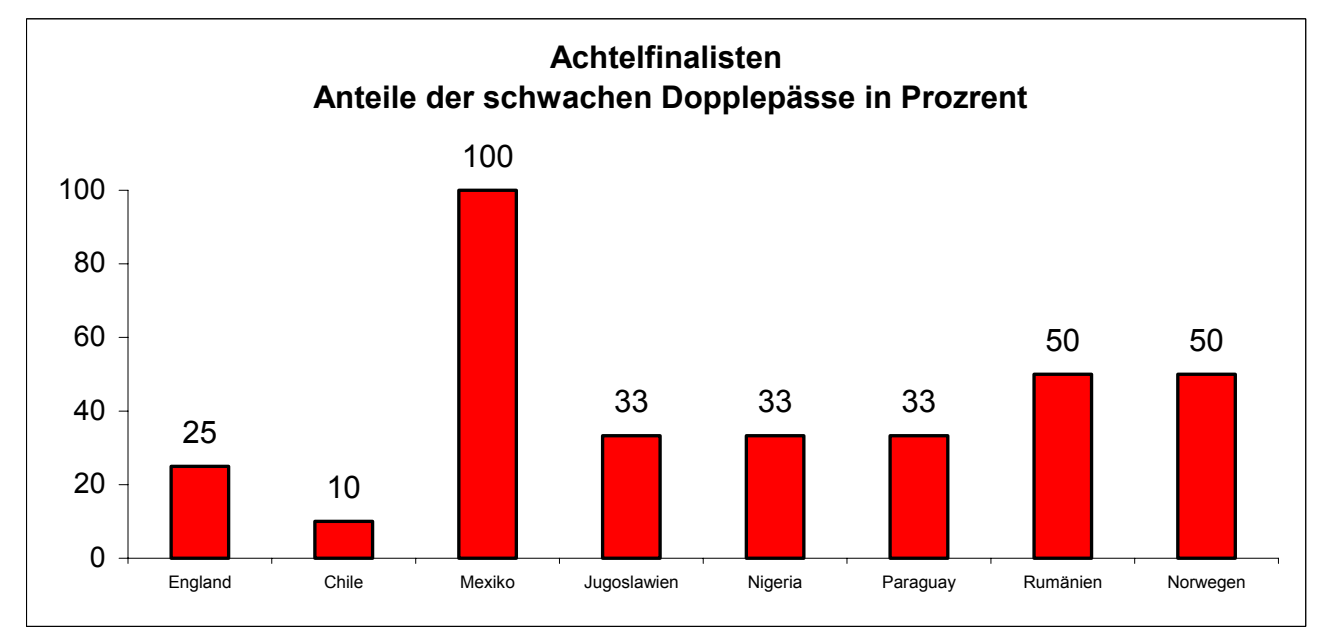

Diagramm 66: Anteile der schwachen Doppelpässe in Prozent bei den Achtelfinalisten. 
Die Diagramme 64, 65 und 66 erklären die Werte der Mannschaften:

Diagramm 64 erklärt, dass die hochkarätigen Doppelpässe einen Anteil zwischen 2 $\%$ und $7 \%$ hat. Die nigerianische Mannschaft hatte die besten Werte, die englische und paraguayische die schlechtesten. Diagramm 65 erklärt, dass der Anteil der guten Doppelpässe zwischen $14 \%$ und $62 \%$ beträgt. Die mexikanische Mannschaft hat hier die besten Werte und die jugoslawische die schlechtesten. Diagramm 66 erklärt, dass der Anteil der schwachen Doppelpässe zwischen $33 \%$ und $74 \%$ beträgt. Die norwegische Mannschaft hatte die niedrigsten Werte und die mexikanische die besten.

Aus den Diagrammen lässt sich die Schlussfolgerung ziehen, dass die normalen Doppelpässe bei allen Achtelfinalisten einen größeren Anteil hatten als die hochkarätigen und die hochkarätigen Doppelpässe auch seltener sind als die schwachen. Das ist dadurch zu erklären, dass die Angreifer ihren Ball oftmals verlieren, wenn das Pressing im Drittel des Gegners stark ist.

\subsubsection{Raumaufteilung}

Die Raumaufteilung ist eine mannschaftliche Eigenschaft und beschreibt die Fähigkeit der Mannschaft bzw. Teilen der Mannschaft, sich so im Raum zu verteilen, dass dieser Raum möglichst optimal ausgenutzt wird. Die Raumaufteilung ist die Verteilung der Spieler auf dem Spielfeld. Sie soll es dem Spieler in Ballbesitz ermöglichen, den Ball leicht an einen anderen Spieler der Mannschaft abzugeben. Wenn es die Raumaufteilung bis zu einem Drittel auf das gegnerische Spielfeld geschafft hat, wird es sehr gefährlich, denn, wenn ein Spieler ohne Ball eine gute Position im Schussbereich des gegnerischen Tores erreicht hat, so ist ein Tor leicht möglich.

Hinsichtlich der Qualität der Raumaufteilung wurde festgestellt: Nigeria hatte von 66mal mit $17(26 \%)$ den größten Wert, der als hochkarätig bezeichnet wurde. Absteigend folgen England mit 45 und 9 (20\%), Norwegen mit 52 und $8(15 \%)$, Chile mit 56 und $6(11 \%)$, Mexiko mit 43 und $3(7 \%)$, Rumänien mit 53 und $3(6 \%)$ und Paraguay mit 71 und 4 (6\%). Jugoslawien hatte bei 50 mit 2 (4\%) den kleinsten Anteil „hochkarätiger" Raumaufteilung. 
Das folgende Diagramm zeigt die Anteile der hochkarätigen Raumaufteilung in Prozent für die Achtelfinalisten.

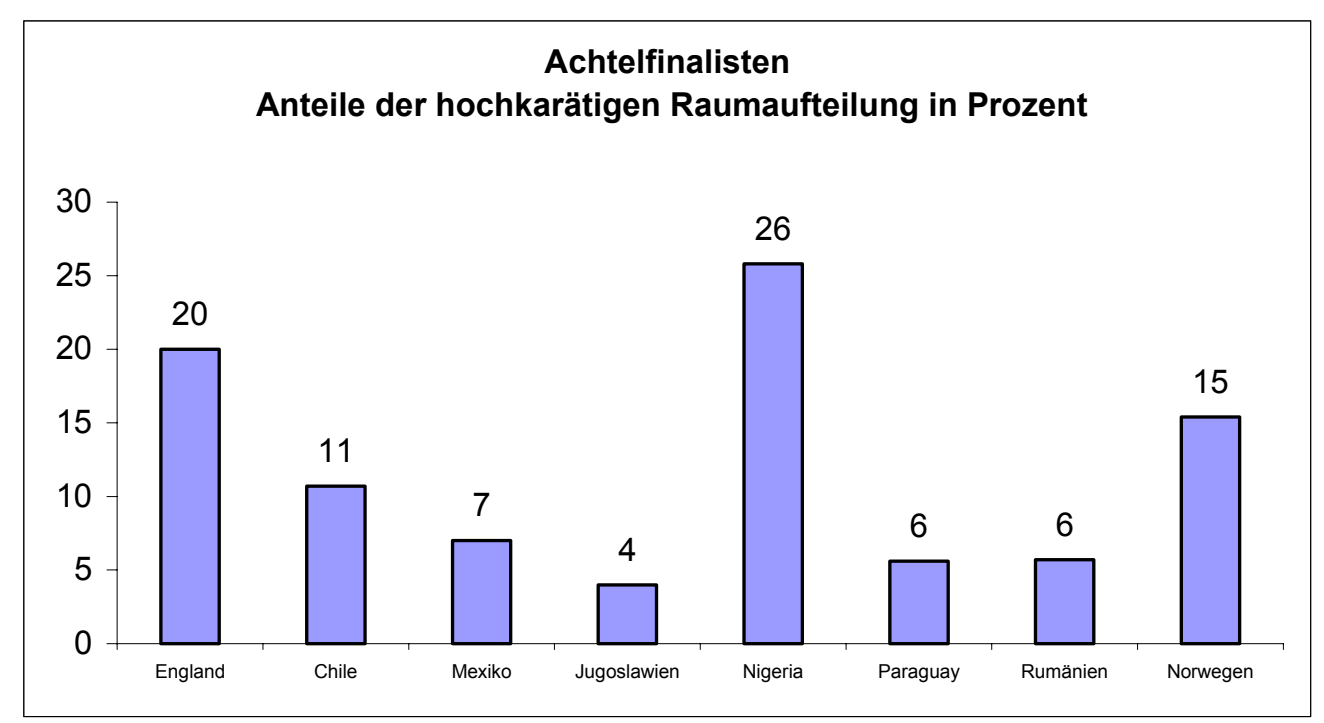

Diagramm 67: Anteile der hochkarätigen Raumaufteilung in Prozent bei den Achtelfinalisten.

34-mal (79 \%) der Raumaufteilungen von Mexiko wurden mit „normal“ bewertet und bildeten den größten Anteil. Die weiteren Anteile „normaler“ Raumaufteilungen lauteten 43 (77 \%) für Chile, 31 (62 \%) für Jugoslawien, 28 (54 \%) für Norwegen, 31 (47 \%) für Nigeria, 18 (40 \%) für England, 21 (40 \%) für Rumänien und 26 (37\%) für Paraguay. Das folgende Diagramm zeigt die Anteile der normalen Raumaufteilung in Prozent für die Achtelfinalisten.

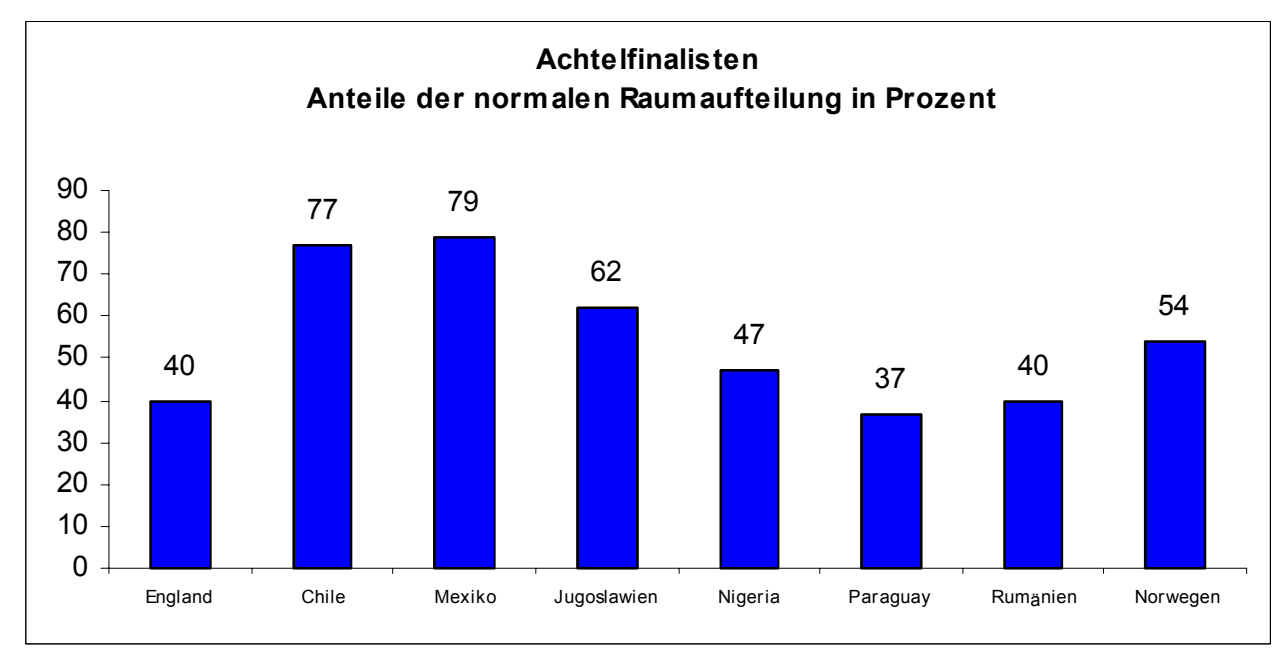

Diagramm 68: Anteile der normalen Raumaufteilung in Prozent bei den Achtelfinalisten. 
Eine „schwache“ Raumaufteilung wurde mit einem Anteil von 41-mal (58 \%) am häufigsten bei Paraguay festgestellt. Es folgen Rumänien mit 29 (55\%), England mit 18 (40\%), Jugoslawien mit 17 (34\%), Norwegen mit 16 (31\%), Nigeria mit $18(27 \%)$, Mexiko mit 6 (14\%) und schließlich Chile mit 7 (13\%). Das folgende Diagramm zeigt die Anteile der schwachen Raumaufteilung in Prozent für die Achtelfinalisten.

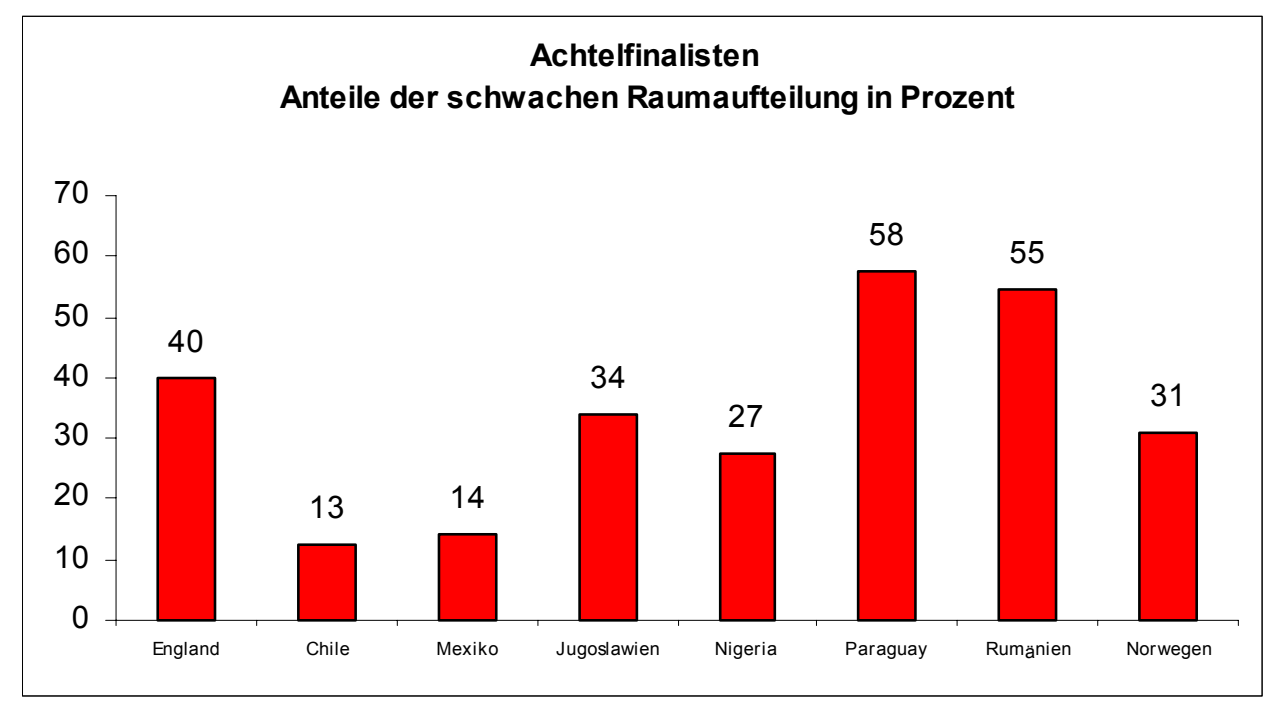

Diagramm 69: Anteile der schwachen Raumaufteilung in Prozent bei den Achtelfinalisten.

Aus den Diagrammen lässt sich die Schlussfolgerung ziehen, dass die normalen Raumaufteilungen bei allen Achtelfinalisten einen größeren Anteil hatten als die hochkarätigen und die hochkarätigen Raumaufteilungen auch seltener sind als die schwachen. Wir führen das auf die schlechte Organisation des Angriffs zurück, wenn der Mannschaft den Ball abgenommen wurde, besonders im Drittel des Gegners, das erklärt, dass die Verteilung der Spieler auf dem Spielfeld schlecht war und die Spieler ohne Ball keine gute Position im Schussbereich des gegnerischen Tores erreicht hatten.

\subsubsection{Nachrücken}

Das Nachrücken ist eine mannschaftliche Eigenschaft und beschreibt die Fähigkeit der Mannschaft bzw. Teilen der Mannschaft, sich so in Richtung des gegnerischen Tores zu bewegen, dass dadurch die Angriffsbemühungen möglichst optimal unterstützt werden. Das Nachrücken ist der Versuch, von der Mitte aus bis ins gegnerische Tor zu kommen. Dazu muss ein Spieler seine Fähigkeit zum Laufen mit dem 
Ball und zum Täuschen einsetzen. Die Fähigkeit zum Nachrücken ist dann wichtig, wenn die Verteidiger großen Druck auf die Angreifer ausüben.

Nachrücken kam bei England 6-mal vor, hiervon wurde 2 (33 \%) als "hochkarätig“ bewertet, was den größten Wert darstellt. Absteigend folgen Norwegen bei 4-mal mit $1(25 \%)$ als hochkarätig, Jugoslawien, Nigeria und Rumänien hatten je 5-mal, mit 1 (20\%), Chile hatte 6-mal mit 1 (17\%). Mexiko und Paraguay hatten 8-mal mit jeweils $1(13 \%)$ den kleinsten Anteil des „hochkarätigen“ Nachrückens.

Das folgende Diagramm zeigt die Anteile des hochkarätigen Nachrückens in Prozent für die Achtelfinalisten.

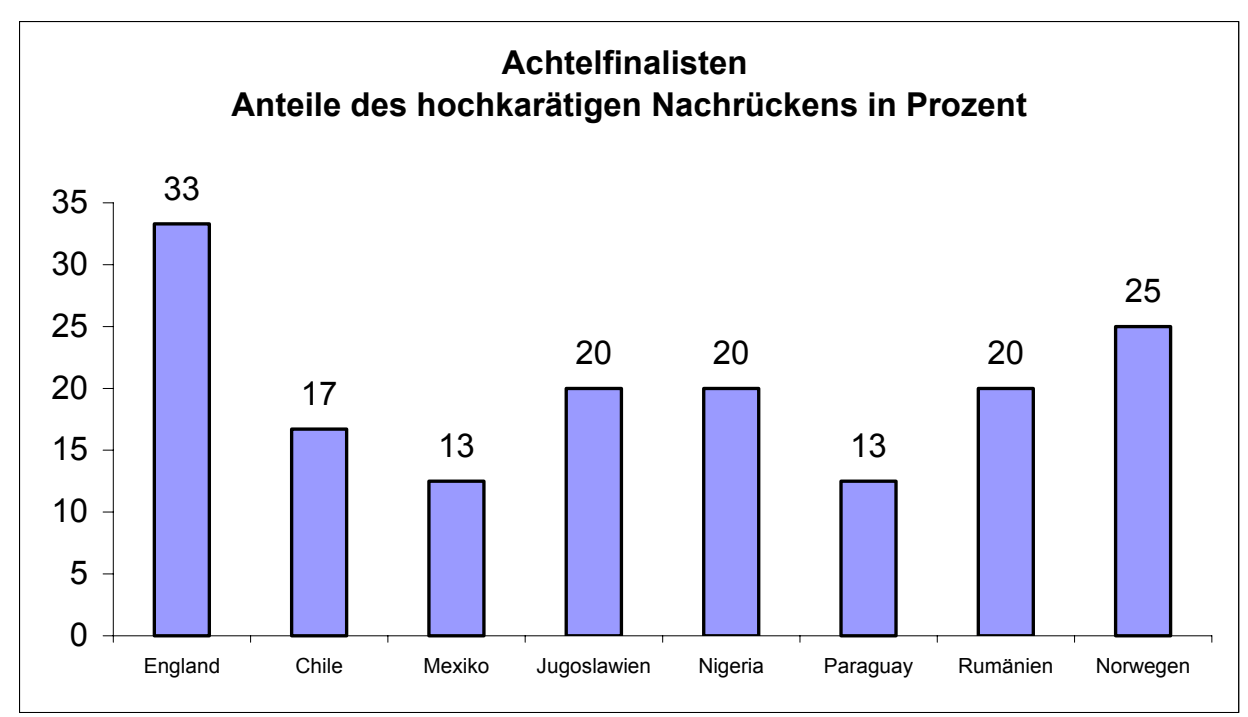

Diagramm 70: Anteile des hochkarätigen Nachrückens in Prozent bei den Achtelfinalisten.

3-mal (50 \%) des Nachrückens von England wurden mit „normal“ bewertet und bildeten den größten Anteil. Die weiteren Anteile „normalen“ Nachrückens lauteten 2 (40 $\%$ ) für Nigeria, 2 (25 \%) für Mexiko, 1 (25\%) für Norwegen, 1 (20\%) für Jugoslawien und Rumänien, 1 (17\%) für Chile und 1 (13\%) für Paraguay.

Das folgende Diagramm zeigt die Anteile des normalen Nachrückens in Prozent für die Achtelfinalisten. 


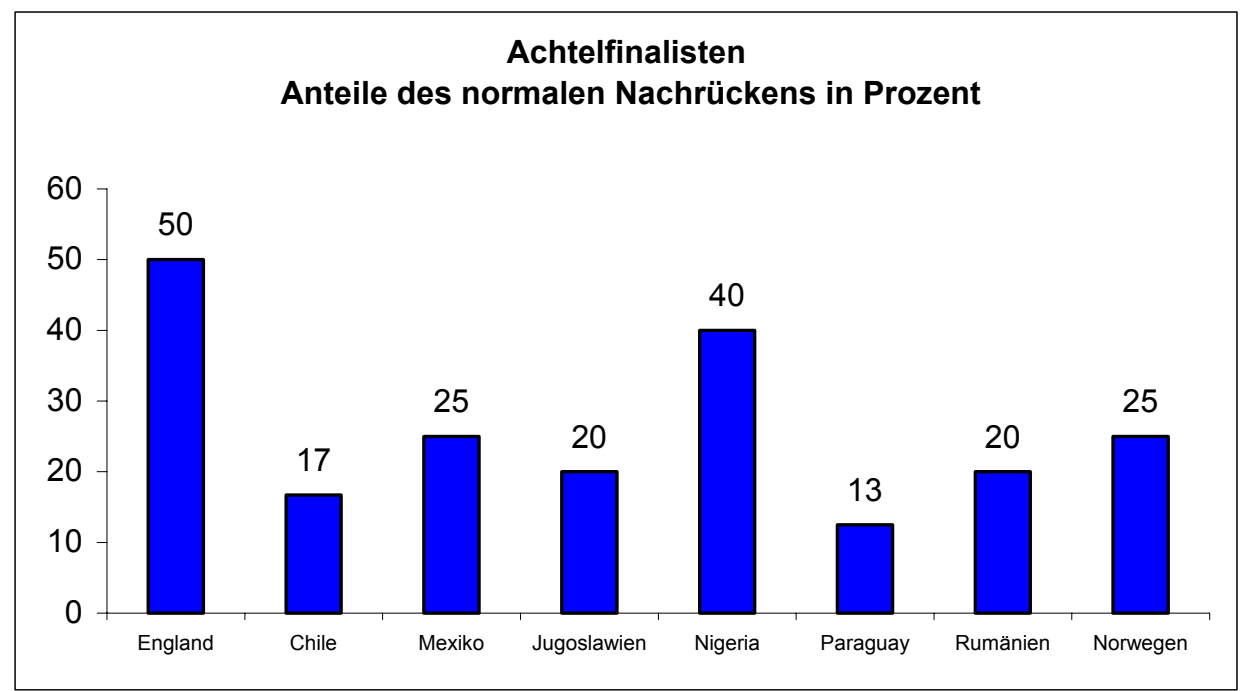

Diagramm 71: Anteile des normalen Nachrückens in Prozent bei den Achtelfinalisten.

Ein „schwaches“ Nachrücken wurde mit einem Anteil von 6-mal (75 \%) am häufigsten bei Paraguay festgestellt. Es folgen Chile 4 (67\%), Mexiko 5 (63\%), Jugoslawien und Rumänien 3 (60\%), Norwegen 2 (50\%), Nigeria 2 (40\%) und schließlich England 1 (17\%).

Das folgende Diagramm zeigt die Anteile des schwachen Nachrückens in Prozent für die Achtelfinalisten.

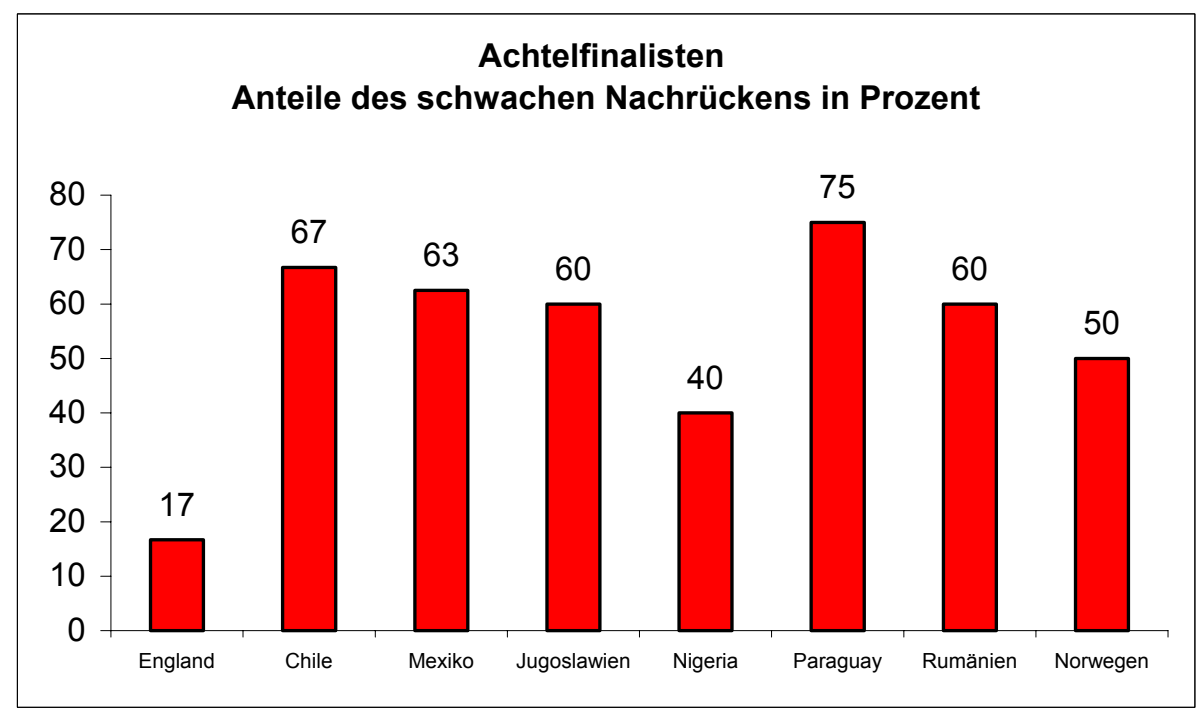

Diagramm 72: Anteile des schwachen Nachrückens in Prozent bei den Achtelfinalisten. 
Aus den Diagrammen Nr. 70, 71 und 72 ist ersichtlich, dass das normale Nachrücken verbreiteter ist als das hochkarätige, und letzteres gleich häufig wie das gescheiterte. Das kann daran liegen, dass die Spieler nicht mit dem Ball laufen und täuschen können und die Ballbesitzer nicht genug Hilfe von den Mitspielern ohne Ball bekommen.

\subsubsection{In die Breite ziehen}

Das „In die Breite ziehen" des Spiels ist eine mannschaftliche Eigenschaft und beschreibt die Fähigkeit der Mannschaft bzw. Teilen der Mannschaft, sich parallel zur Torauslinie so zu bewegen, dass möglichst die gesamte Breite des Spielfeldes ausgenutzt wird. Das in die Breite ziehen ist eine Angriffstaktik, bei der die Stürmer der angreifenden Mannschaft die Verteidiger auf die andere Seite des Spielfelds ziehen und dann die Spielrichtung wechseln. Die Taktik beruht auf einer Folge von kurzen oder langen Pässen, die den Ball von einer Längsseite zur anderen führen.

Hinsichtlich der Qualität des „In die Breite ziehen“ wurde festgestellt: England wandet diese Technik 6-mal an, hiervon 2-mal (33 \%) hochkarätig. Absteigend folgen Nigeria 9-mal mit 1 (11\%), Mexiko und Jugoslawien 10-mal mit 1 (10\%), Norwegen 11-mal mit 1 (9 \%) und Chile 13-mal mit 1 (8 \%). Paraguay und Rumänien hatten 14mal mit jeweils 1 (7\%) den kleinsten Anteil des „hochkarätigen“ „In die Breite ziehen".

Das folgende Diagramm zeigt die Anteile des hochkarätigen „In die Breite ziehen“ in Prozent für die Achtelfinalisten.

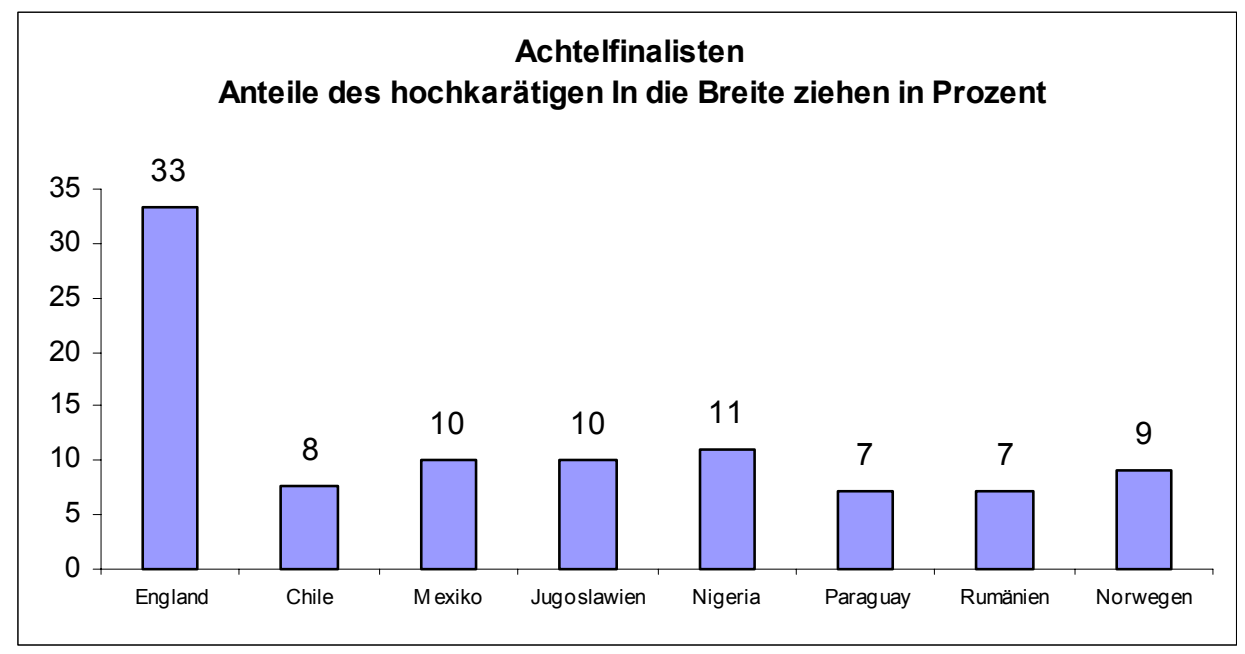

Diagramm 73: Anteile des hochkarätigen „In die Breite ziehen“ in Prozent bei den Achtelfinalisten. 
10-mal (77 \%) des „In die Breite ziehen“ von Chile wurden mit „normal“ bewertet und bildete den größten Anteil. Die weiteren Anteile „normalen“ „In die Breite ziehen“ lauteten 8 (77 \%) für Norwegen, 10 (71 \%) für Paraguay und Rumänien, $6(67 \%)$ für Nigeria, 6 (60 \%) für Mexiko und Jugoslawien und 3 (50 \%) für England.

Dem folgenden Diagramm sind die Anteile des normalen In die Breite ziehen in Prozent für die Achtelfinalisten zu entnehmen.

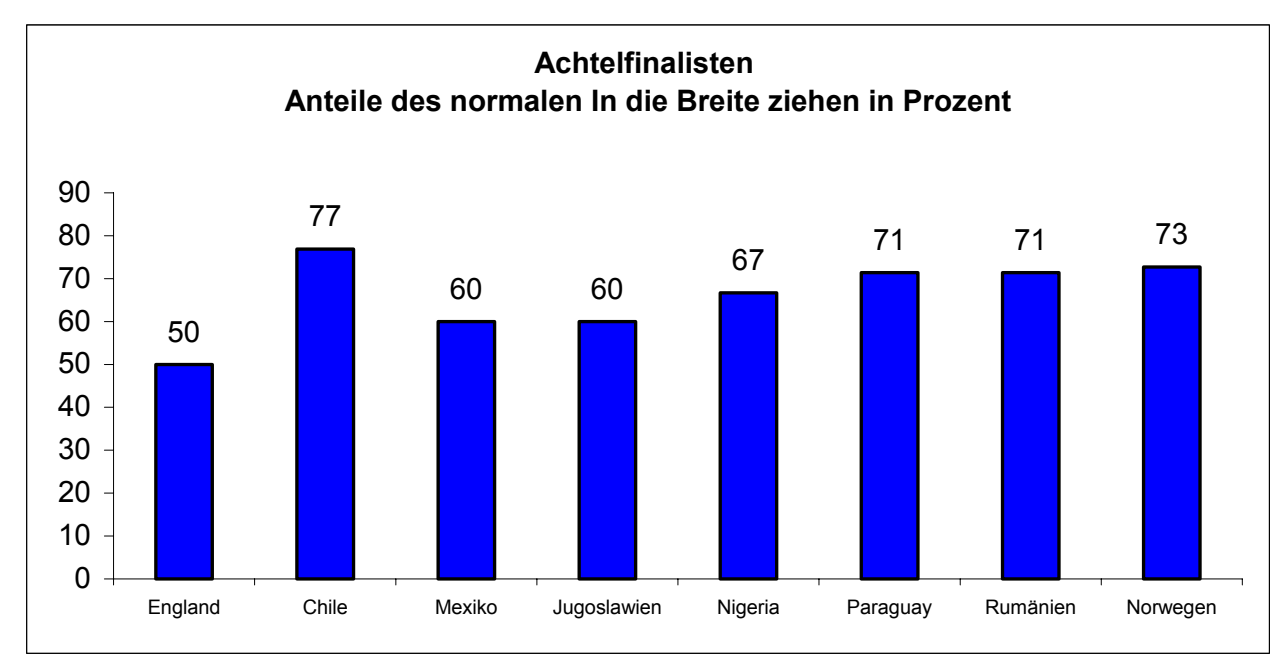

Diagramm 74: Anteile des normalen „In die Breite ziehen“ in Prozent bei den Achtelfinalisten.

Ein "schwaches“ „In die Breite ziehen“ wurde mit einem Anteil von 3-mal (30\%) am häufigsten bei Mexiko und Jugoslawien festgestellt. Es folgen Nigeria 2 (22\%), Paraguay und Rumänien 3 (21\%), Norwegen 2 (18\%), England 1 (17\%) und schließlich Chile 1 (15\%).

Dem folgenden Diagramm sind die Anteile des schwachen In die Breite ziehen in Prozent für die Achtelfinalisten zu entnehmen. 


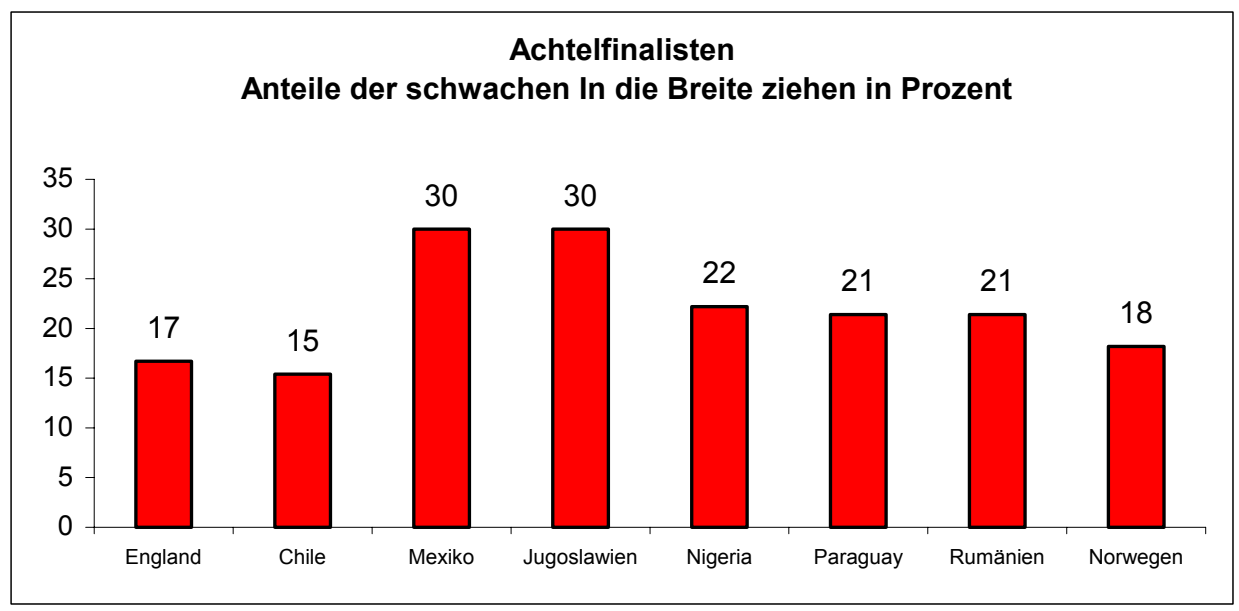

Diagramm 75: Anteile des schwachen „In die Breite ziehen“ in Prozent bei den Achtelfinalisten.

Aus den Diagrammen 73, 74 und 75 lässt sich schließen, dass das normale „In die Breite ziehen" häufiger ist als das hochkarätige und das misslungene. Die Gründe dafür liegen vielleicht darin, dass die Spieler das „In die Breite ziehen“ zu weit vom gegnerischen Tor entfernt unternehmen, wodurch der Druck auf die Angreifer geringer ist.

\subsubsection{Viertelfinalisten}

Als Viertelfinalisten werden diejenigen Mannschaften bezeichnet, die im Viertelfinale ausgeschieden sind und zu deren Analyse deshalb zwei Spiele herangezogen werden konnten. Zu diesen Viertelfinalisten gehören Deutschland, Italien, Argentinien und Dänemark. Es handelt sich jeweils um die addierten Werte der beiden Spiele.

\subsubsection{Freistöße}

Hinsichtlich der Qualität des „Freistoßes“ wurde festgestellt: Dänemark erzielte 15mal Freistöße, hiervon 2-mal (13\%) hochkarätige; dies bildete den größten Wert. Absteigend folgen Argentinien von 12-mal, wurde 1 (8\%) als hochkarätige bezeichnet und Deutschland erzielte von 191 (5\%). Italien hatte 11, hiervon mit 0,0\% den kleinsten Anteil „hochkarätiger“ Freistöße.

Dem folgenden Diagramm sind die Anteile der hochkarätige Freistößes in Prozent für die Viertelfinalisten zu entnehmen. 


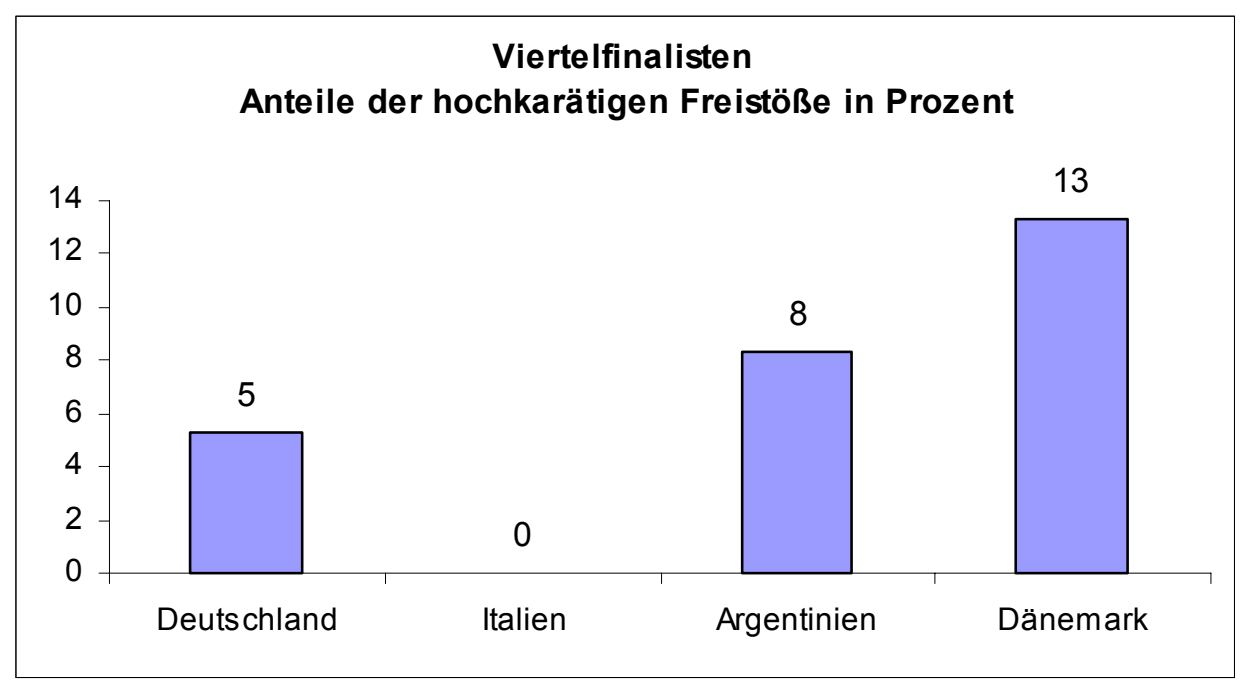

Diagramm 76: Anteile der hochkarätigen Freistöße in Prozent bei den Viertelfinalisten.

7-mal (64 \%) der Freistöße von Italien wurden mit "normal“ bewertet und bildeten den größten Anteil. Die weiteren Anteile "normaler" Freistöße erzielte Dänemark 9 (60 $\%), 7$ (58 \%) für Argentinien und 9 (47 \%) für Deutschland.

Dem folgenden Diagramm sind die Anteile der normalen Freistöße in Prozent für die Viertelfinalisten zu entnehmen.

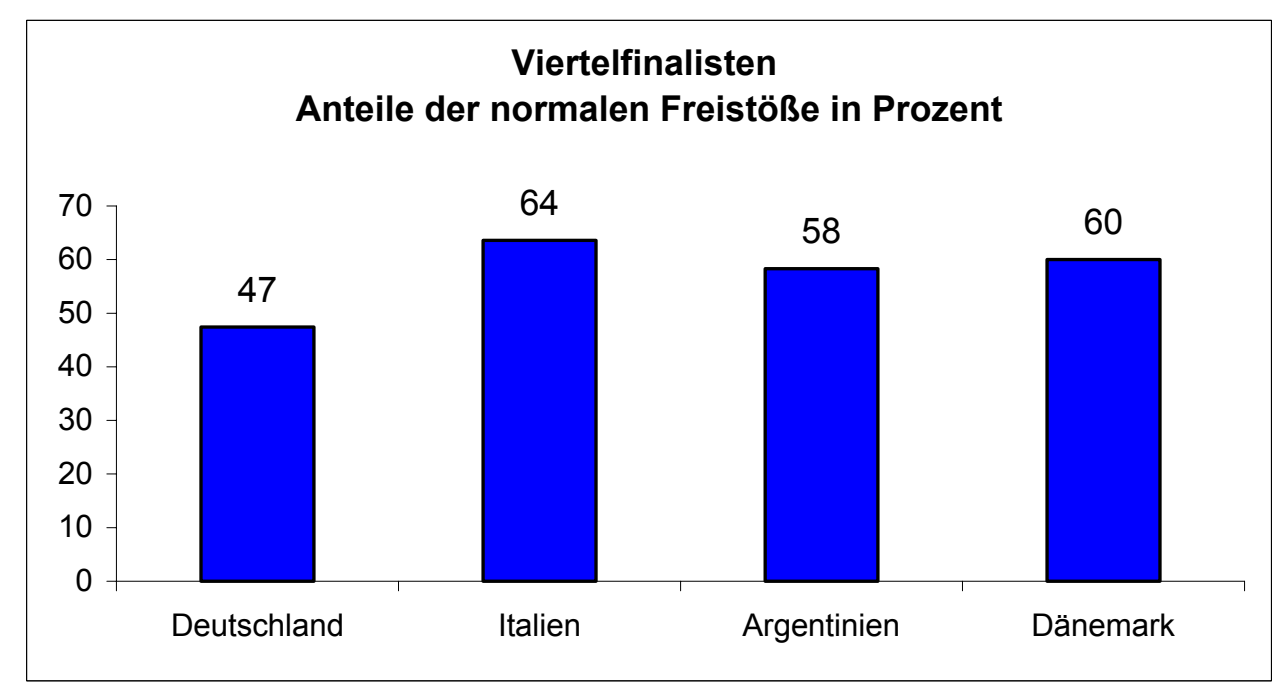

Diagramm 77: Anteile der normalen Freistöße in Prozent der Viertelfinalisten.

Ein „schwacher" Freistoß wurde mit einem Anteil von 9-mal (47\%) am häufigsten bei Deutschland festgestellt. Es folgen Italien mit 4 (36\%), Argentinien mit 4 (33\%) und schließlich Dänemark mit 4 (27\%). Dem folgenden Diagramm sind die Anteile der schwachen Freistöße in Prozent für die Viertelfinalisten zu entnehmen. 


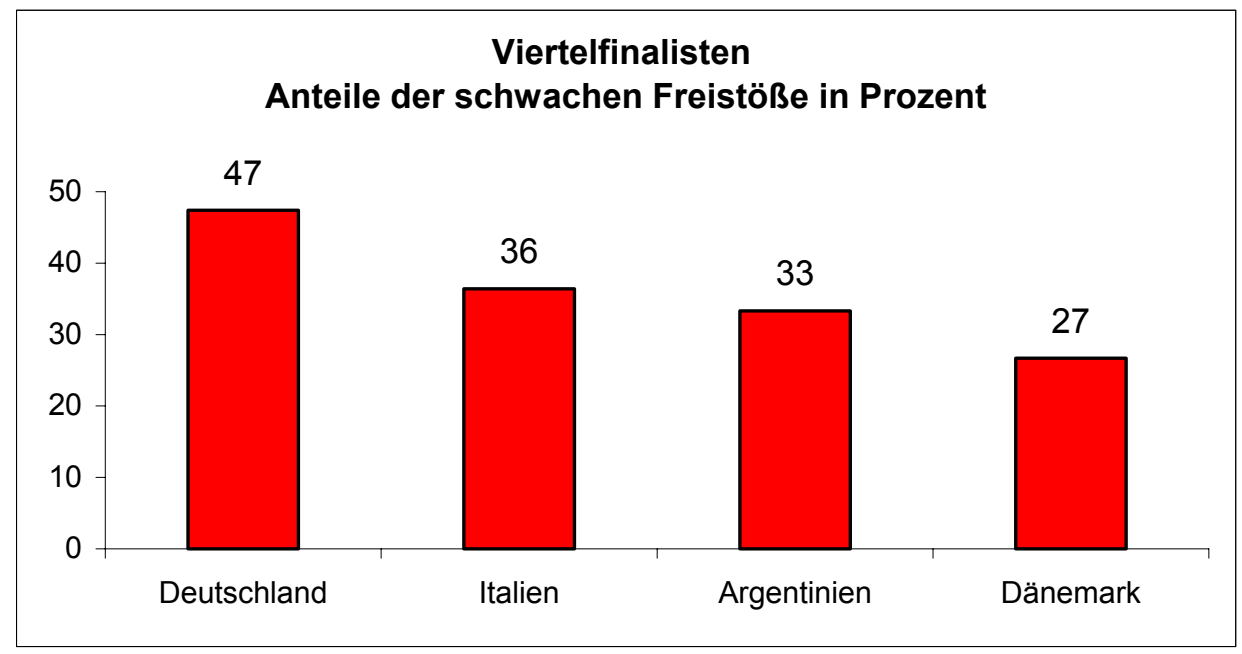

Diagramm 78: Anteile der schwachen Freistöße in Prozent bei den Viertelfinalisten.

Diagramm 76 erklärt, dass die hochkarätigen Freistöße zwischen 0 \% und $13 \%$ lagen. Die dänische Mannschaft hatte die besten Werte und die italienische hatte die schlechtesten. Diagramm 77 erklärt, dass normale Freistöße zwischen $47 \%$ und 64 \% lagen. Die dänische Mannschaft hatte hier die besten Werte und die deutsche hatte die schlechtesten. Diagramm 78 erklärt, dass schwache Freistöße zwischen 27 \% und $47 \%$ Die deutsche Mannschaft hatte die schwächsten Werte und die dänische hatte die besten Werte.

Es scheint, als könnten die Nationalmannschaften die Freistöße noch häufiger üben, um den Anteil der hochkarätigen Freistöße zu erhöhen und den der schwachen zu verringern.

\subsubsection{Eckball}

Italien hatte 4-mal Eckball, hiervon wurde mit 1 (25\%) als hochkarätig bezeichnet und bildete den größten Wert. Absteigend folgen Argentinien von 11-mal wurden 2 (18\%) als hochkarätig bezeichnet und Deutschland hatte 14-mal mit 1 (7\%). Dänemark hatte 11-mal Eckball, hiervon wurde mit 0,0 \% der kleinste Anteil als „hochkarätiger" Eckball bewertet.

Dem folgenden Diagramm sind die Anteile der hochkarätige Eckbälle in Prozent für die Viertelfinalisten zu entnehmen. 


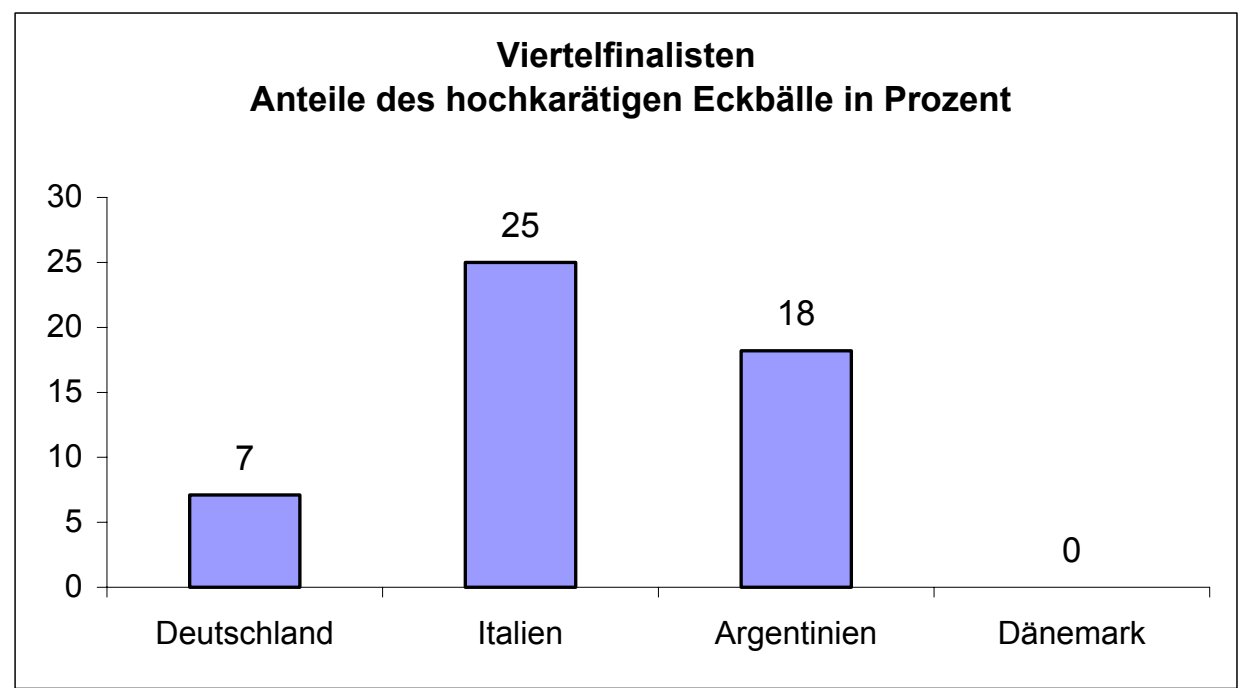

Diagramm 79: Anteile der hochkarätigen Eckbälle in Prozent bei den Viertelfinalisten.

8-mal (73 \%) der Eckbälle von Dänemark wurden mit „normal“ bewertet und bildeten den größten Anteil. Die weiteren Anteile „normaler“ Eckbälle lauteten 2 (50 \%) für Italien, 5 (36 \%) für Deutschland und 2 (18\%) für Argentinien.

Dem folgenden Diagramm sind die Anteile der mittleren Eckbälle in Prozent für die Viertelfinalisten zu entnehmen.

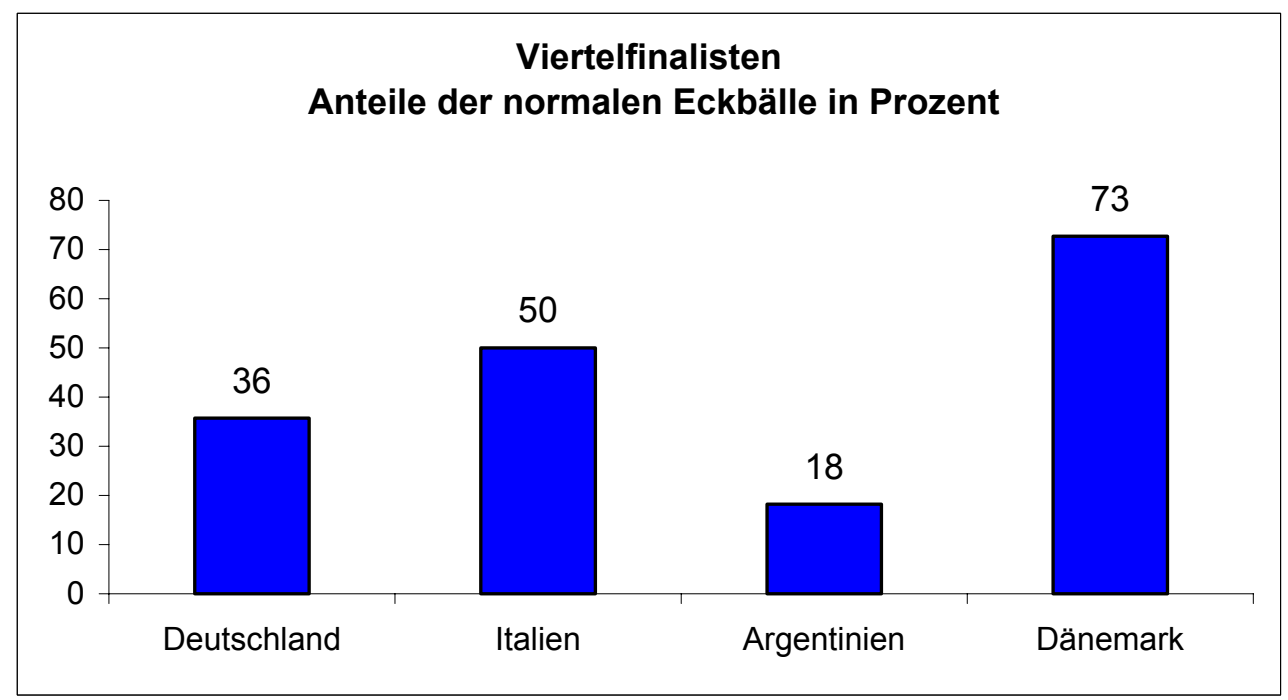

Diagramm 80: Anteile der normalen Eckbälle in Prozent bei den Viertelfinalisten.

Ein "schwacher" Eckball wurde mit einem Anteil von 7-mal (64 \%) am häufigsten bei Argentinien festgestellt. Es folgen Deutschland mit 8 (57\%), Dänemark mit $3(27 \%)$ und schließlich Italien mit 1 (25\%). 
Dem folgenden Diagramm sind die Anteile der schwachen Eckbälle in Prozent für die Viertelfinalisten zu entnehmen.

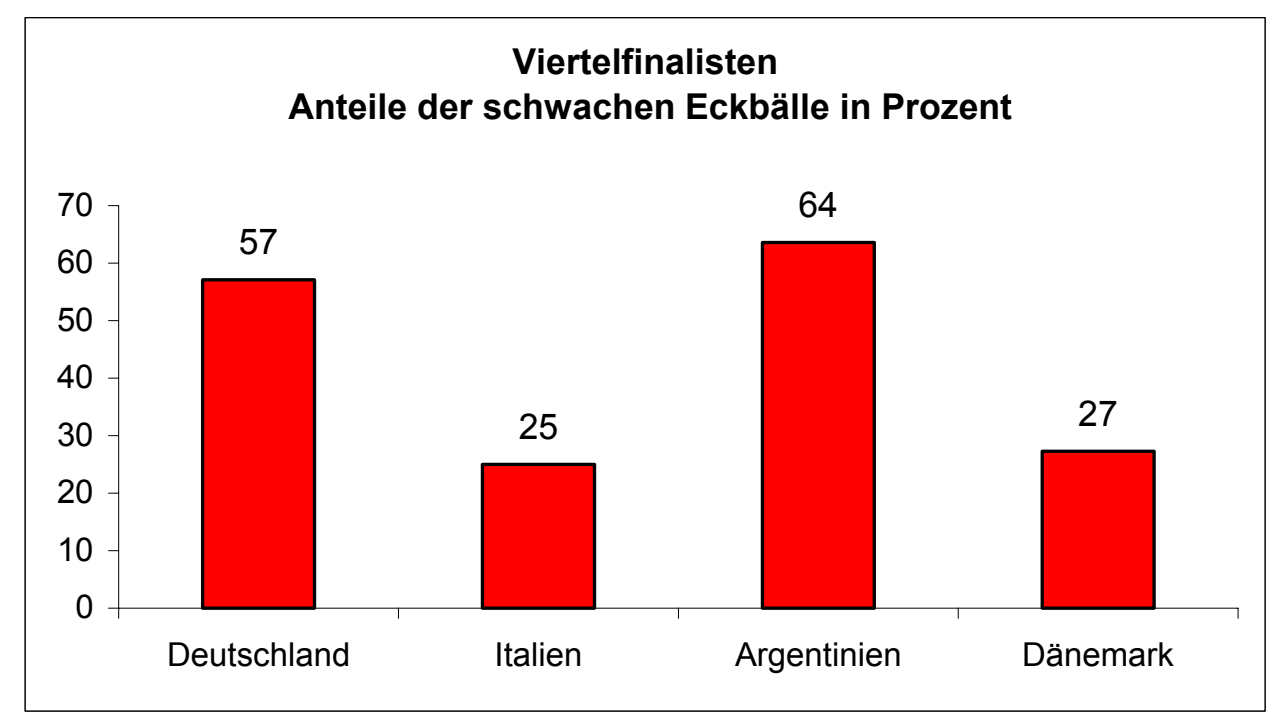

Diagramm 81: Anteile der schwachen Eckbälle in Prozent bei den Viertelfinalisten.

Aus den Werten der Diagramme 79, 80 und 81 ist die geringe Anzahl der hochkarätigen im Vergleich zu den guten und schwachen Eckbällen ersichtlich. Dabei ist die Anzahl der normalen Eckbälle nicht nur größer als die der hochkarätigen und schwachen, sondern auch die hochkarätigen sind seltener als die schlechten.

Wir führen das auf die schlechte Organisation des Angriffs bei der Ausführung des Eckballs oder schlechte Taktik zurück. Mit einer guten Taktik kann die ausführende Mannschaft die gegnerische Mannschaft täuschen und die gegnerische Abwehr überwinden. Auch dies weist darauf hin, dass Nationalmannschaften auch mit Formationen den letzten Acht in einem Weltturnier Standardsituationen häufiger üben müssen.

\subsubsection{Unterstützen}

Hinsichtlich der Qualität des „Unterstützens“ wurde festgestellt: Argentinien „unterstützte“ 32-mal, hiervon wurde mit 2-mal (6 \%) der größten Wert als hochkarätig bezeichnet. Absteigend folgen Dänemark mit 26-mal „Unterstützungen“, hiervon wurde 1 (4\%) als hochkarätig bezeichnet und Italien mit 40, hiervon wurde 1 (3\%) als hochkarätig bezeichnet. Deutschland erzielte 45-mal und hatte mit 1 (2\%) den kleinsten Anteil „guten“ Unterstützens. 
Dem folgenden Diagramm sind die Anteile des hochkarätigen Unterstützens in Prozent für die Viertelfinalisten zu entnehmen.

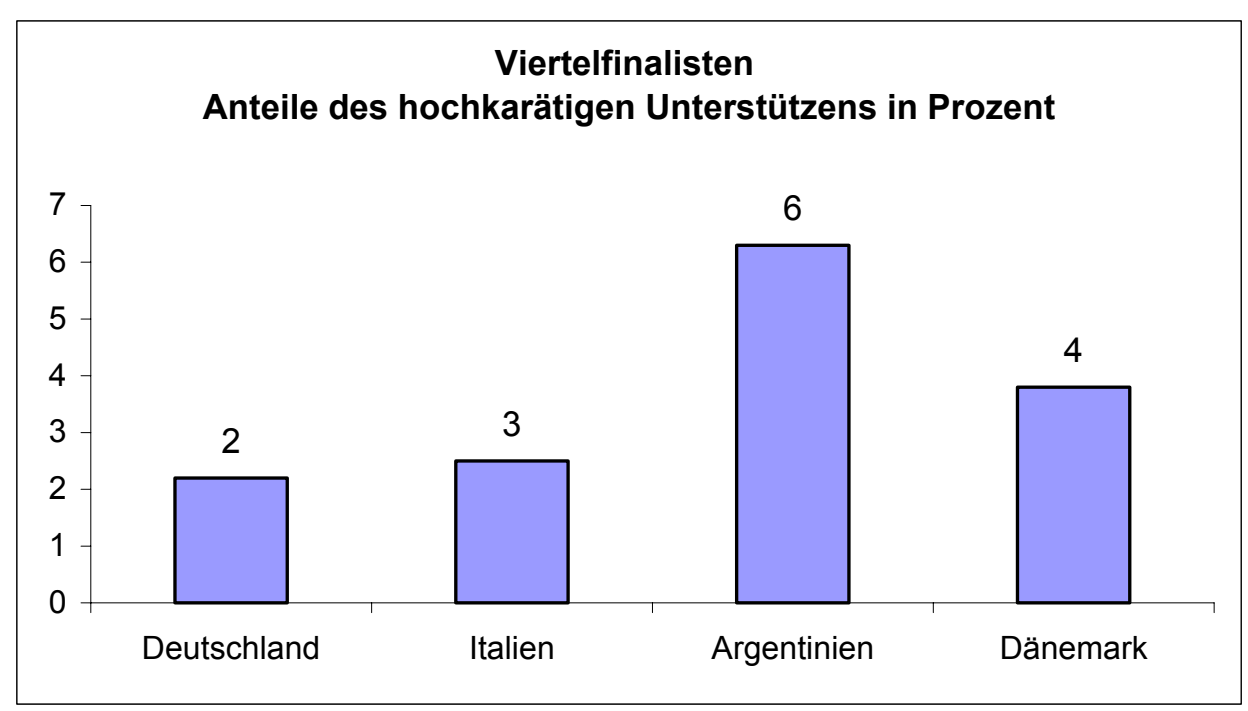

Diagramm 82: Anteile des hochkarätigen Unterstützens in Prozent bei den Viertelfinalisten.

43 (96 \%) des Unterstützens von Deutschland wurde mit "normal“ bewertet und bildete den größten Anteil. Die weiteren Anteile „normalen“ Unterstützens lauteten 38 (95\%) für Italien, 24 (92 \%) für Dänemark und 29 (91 \%) für Argentinien. Dem folgenden Diagramm sind die Anteile des normalen Unterstützens in Prozent für die Viertelfinalisten zu entnehmen.

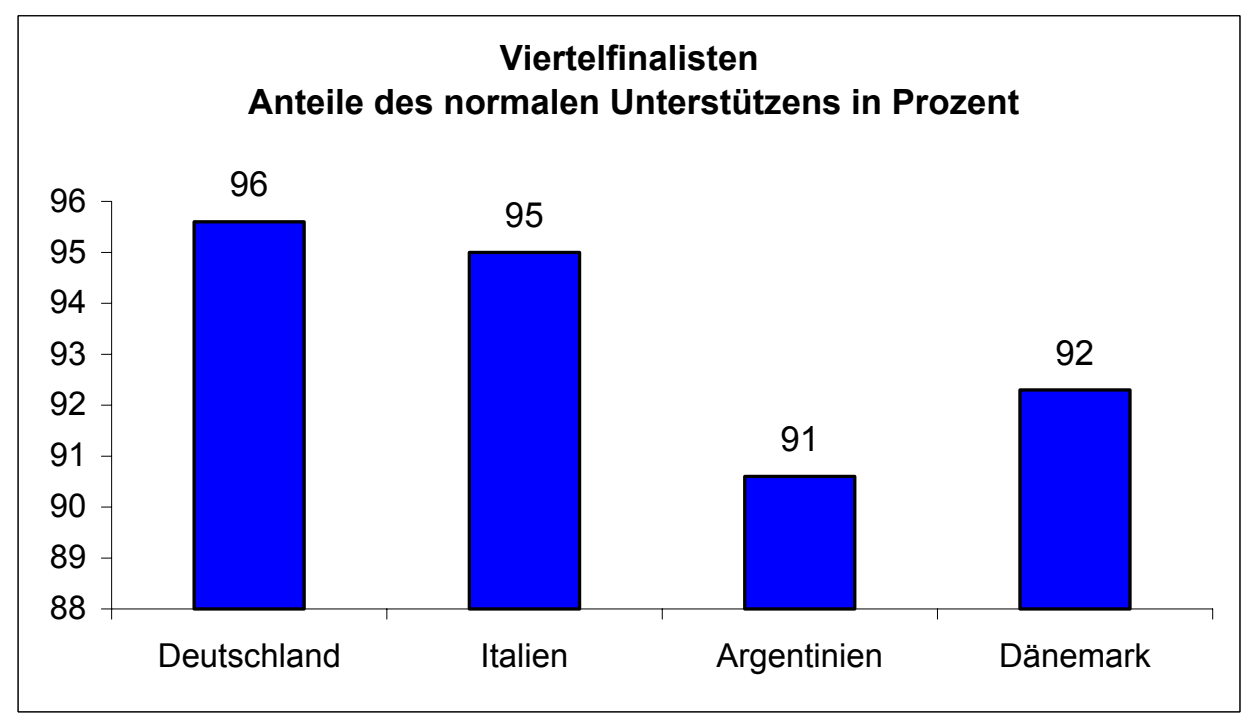

Diagramm 83: Anteile des normalen Unterstützens in Prozent der Viertelfinalisten. 
Ein „schwaches“ Unterstützen wurde mit einem Anteil von 1 (4 \%) am häufigsten bei Dänemark festgestellt. Es folgen Argentinien mit 1 (3\%), Italien mit 1 (3\%) und Deutschland mit 1 (2\%).

Dem folgenden Diagramm sind die Anteile des schwachen Unterstützens in Prozent für die Viertelfinalisten zu entnehmen.

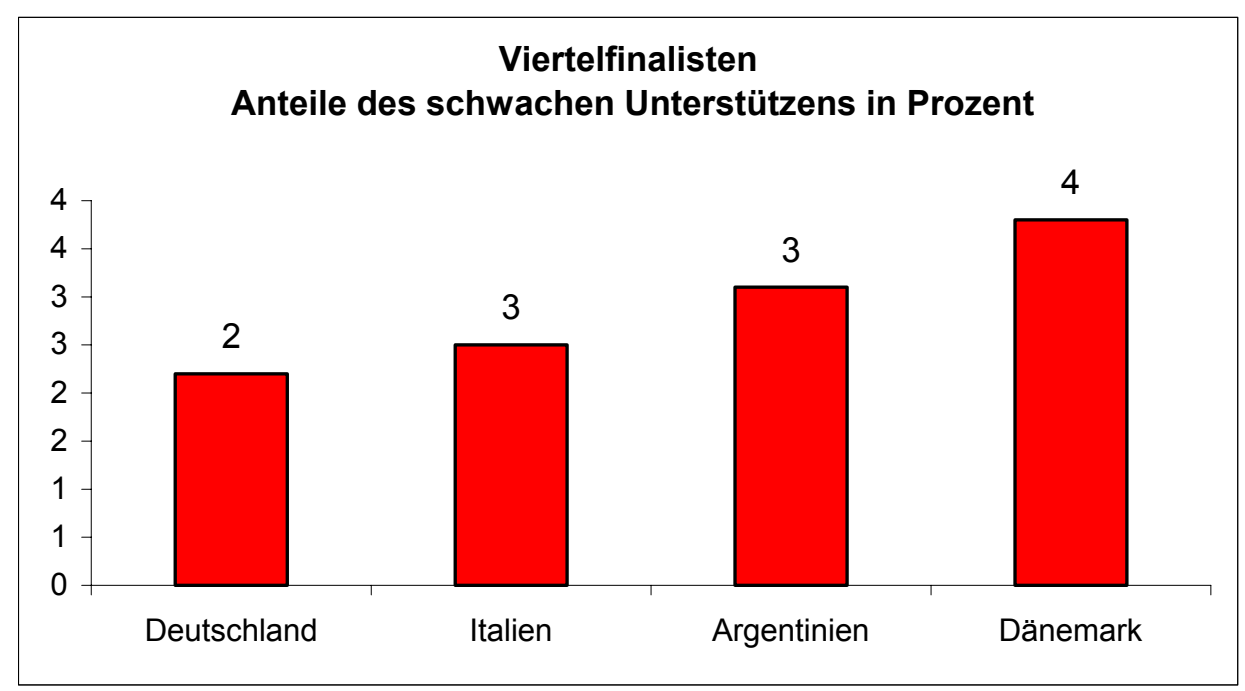

Diagramm 84: Anteile des schwachen Unterstützens in Prozent bei den Viertelfinalisten.

Aus der Darstellung von der Diagrammen 82, 83 und 84 wird deutlich, dass das normalen Unterstützen häufiger ist als das hochkarätige und schwache, da die unterstützenden Spieler einen Platz einnehmen, von dem aus sie ihre Mitspieler so helfen können, dass sie gut mit dem Ball vorankommen können. Manchmal unterstützen die Mitspieler nicht zur rechten Zeit oder auf der richtigen Position.

\subsubsection{Doppelpass}

Doppelpässe hatte Italien 7 Doppelpässe wovon mit 1 (14 \%) der größte Wert als ", hochkarätig“ bezeichnet wurde. Deutschland, Argentinien und Dänemark erzielten 5, 4, 6-mal Doppelpässe, wovon jeweils 0,0\% der kleinste Anteil als „hochkarätiger“ Doppelpass bezeichnet wurde.

Das folgende Diagramm zeigt die Anteile der hochkarätigen Doppelpässe in Prozent für die Viertelfinalisten. 


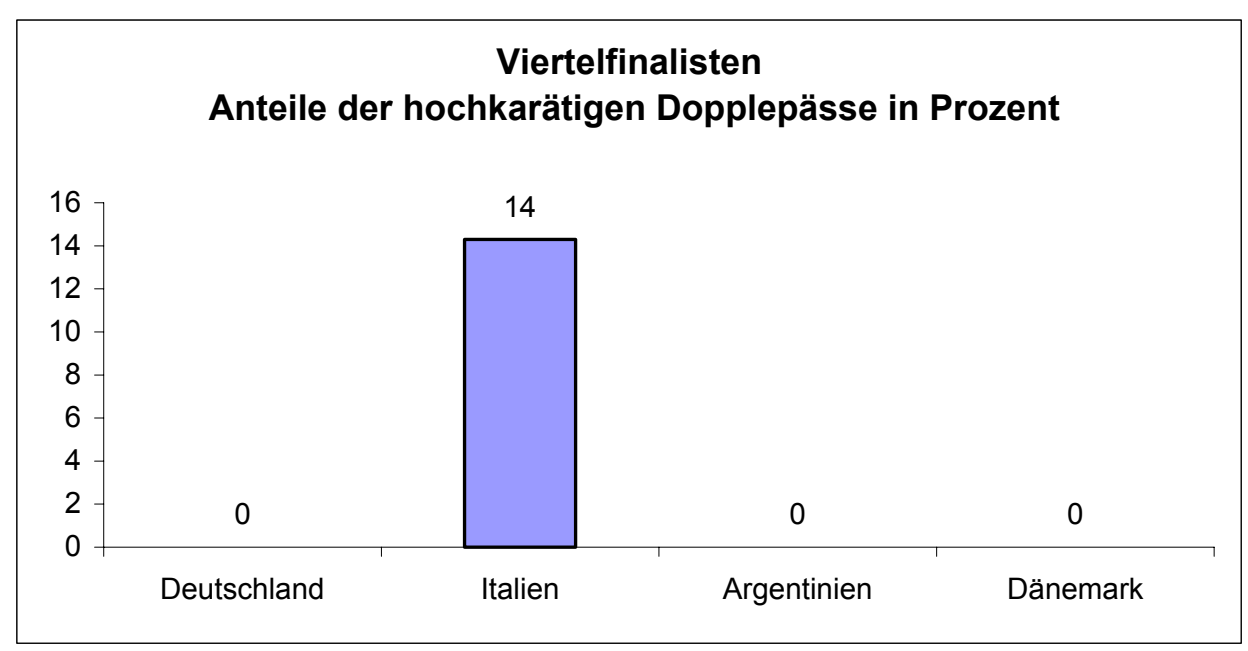

Diagramm 85: Anteile der hochkarätigen Doppelpässe in Prozent bei den Viertelfinalisten.

3-mal (60 \%) der Doppelpässe von Deutschland wurden mit "normal“ bewertet und bildeten den größten Anteil. Die weiteren Anteile „normaler" Doppelpässe lauteten 2 (50 \%) für Argentinien, 3 (43 \%) für Italien und 2 (33 \%) für Dänemark.

Dem folgenden Diagramm sind die Anteile der normalen Doppelpässe in Prozent für die Viertelfinalisten zu entnehmen.

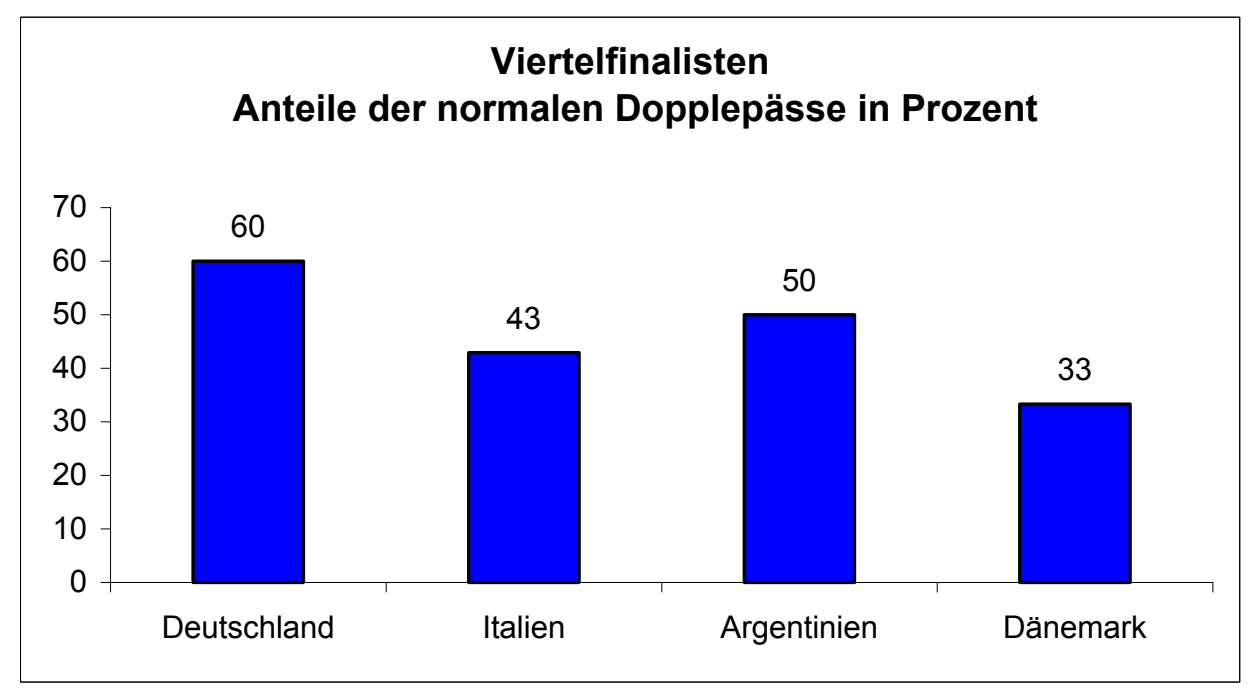

Diagramm 86: Anteile der normalen Doppelpässe in Prozent bei den Viertelfinalisten.

Ein "schwacher" Doppelpass wurde mit einem Anteil von 4-mal (67\%) am häufigsten bei Dänemark festgestellt. Es folgen Argentinien 2 (50\%), Italien $3(43 \%)$ und schließlich Deutschland 2 (40\%).

Das folgende Diagramm zeigt die Anteile der schwachen Doppelpässe in Prozent für die Viertelfinalisten. 


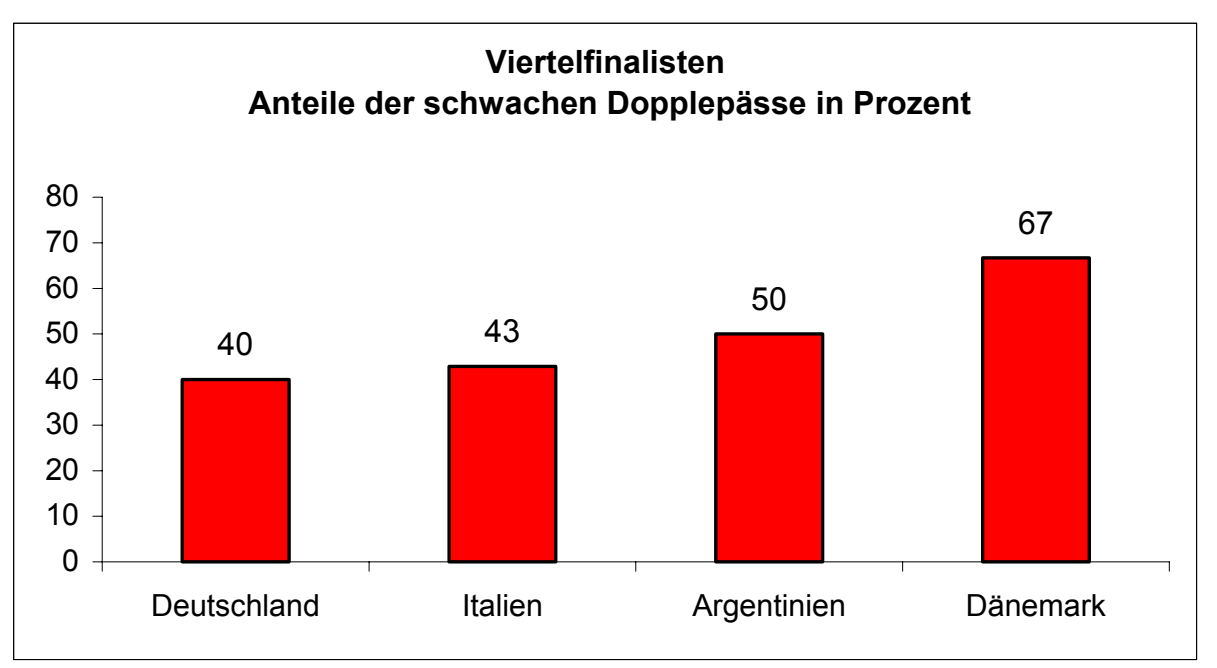

Diagramm 87: Anteile der schwachen Doppelpässe in Prozent bei den Viertelfinalisten.

Aus den Diagrammen lässt sich die Schlussfolgerung ziehen, dass die schwachen Doppelpässe bei allen Viertelfinalisten einen größeren Anteil hatten als die hochkarätigen und die hochkarätigen Doppelpässe auch seltener sind als die normalen. Das ist dadurch zu erklären, dass die Angreifer ihren Ball oftmals verlieren, wenn das Pressing im Drittel des Gegners stark ist, und die Verteidiger die Zweikämpfe leicht gewinnen.

\subsubsection{Raumaufteilung}

„Gute“ Raumaufteilung hatte Italien 105-mal, hiervon wurde mit 13 (12\%) als hochkarätig bezeichnet und bildete den größten Wert. Absteigend folgen Deutschland mit 94, hiervon wurde 10 (11\%) als hochkarätig bezeichnet und Argentinien hatte 130, hiervon wurden $13(10 \%)$ als hochkarätig bezeichnet. Dänemark hatte bei 155 mit 15 (10\%) den kleinsten Anteil „guter“ Raumaufteilung.

Das folgende Diagramm zeigt die Anteile der hochkarätigen Raumaufteilung in Prozent für die Viertelfinalisten. 


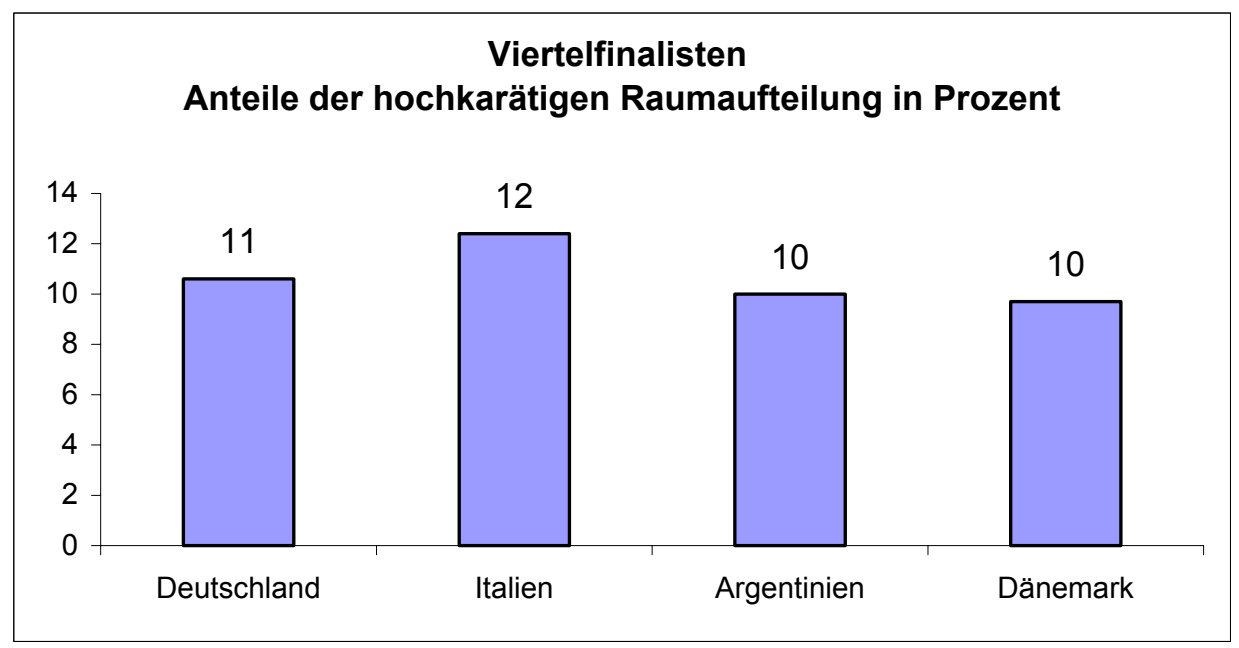

Diagramm 88: Anteile der hochkarätigen Raumaufteilung in Prozent bei den Viertelfinalisten.

112 (72 \%) der Raumaufteilungen von Dänemark wurden mit "normal“ bewertet und bildeten den größten Anteil. Die weiteren Anteile „normaler" Raumaufteilungen lauteten 52 (55\%) für Deutschland, 57 (54\%) für Italien und 58 (45\%) für Argentinien. Dem folgenden Diagramm sind die Anteile der normalen Raumaufteilung in Prozent für die Viertelfinalisten zu entnehmen.

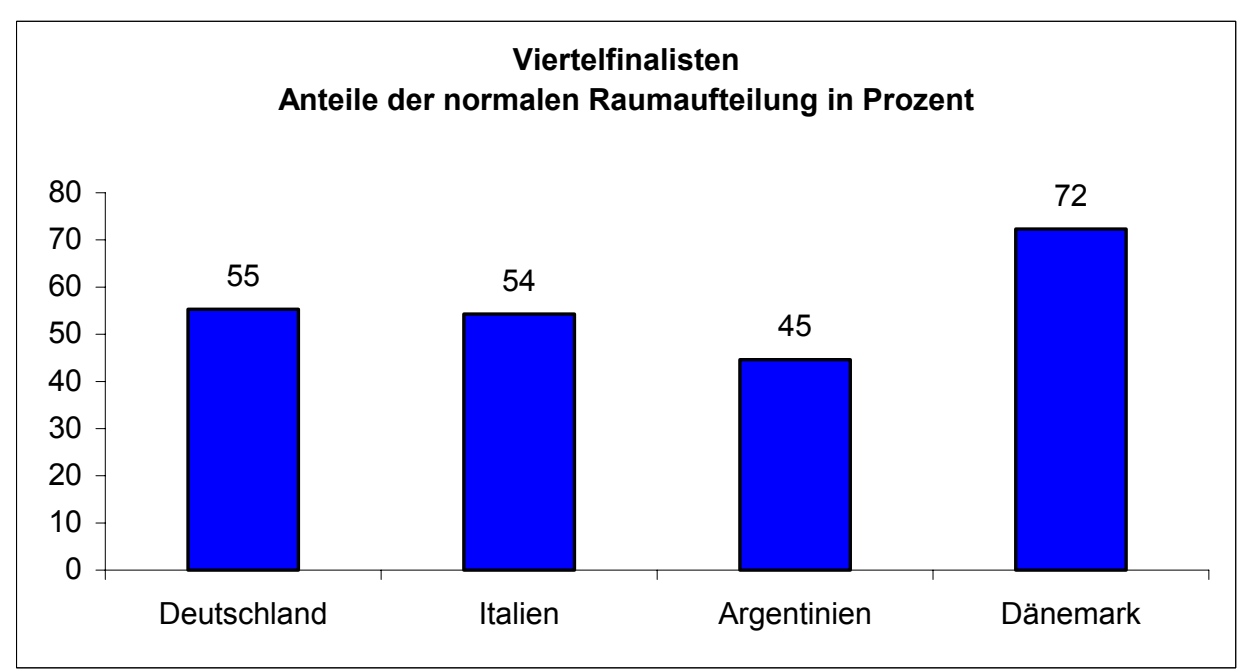

Diagramm 89: Anteile der normalen Raumaufteilung in Prozent bei den Viertelfinalisten. 
Eine „schwache" Raumaufteilung wurde mit einem Anteil von 59 (45 \%) am häufigsten bei Argentinien festgestellt. Es folgen Deutschland 32 (34\%), Italien 35 (33\%) und schließlich Dänemark 28 (18\%).

Dem folgenden Diagramm sind die Anteile der schwachen Raumaufteilung in Prozent für die Viertelfinalisten zu entnehmen.

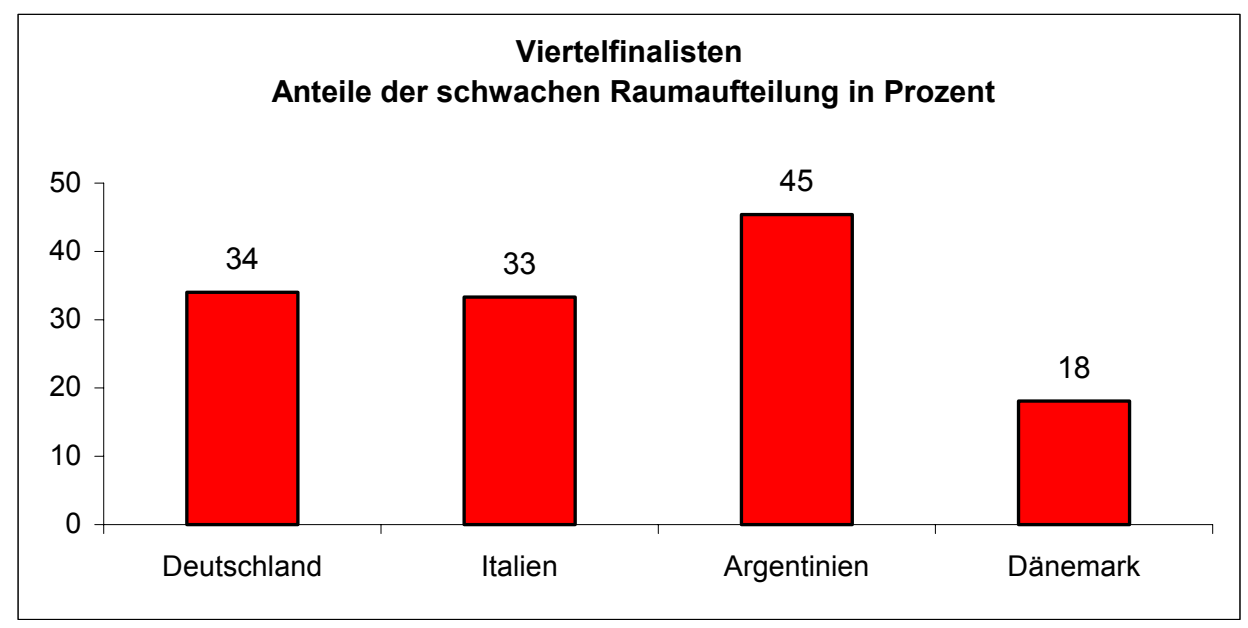

Diagramm 90: Anteile der schwachen Raumaufteilung in Prozent bei den Viertelfinalisten.

Aus den Diagrammen lässt sich die Schlussfolgerung ziehen, dass die normalen Raumaufteilungen bei allen Viertelfinalisten einen größeren Anteil hatten als die hochkarätigen und die hochkarätigen Raumaufteilungen auch seltener sind als die schwachen. Wir führen das auf die schlechte Organisation des Angriffs zurück, wenn der Mannschaft der Ball abgenommen wurde, besonders im Drittel des Gegners; das erklärt, dass die Verteilung der Spieler auf dem Spielfeld schlecht war und die Spieler ohne Ball keine gute Position im Schussbereich des gegnerischen Tores erreicht hatten.

\subsubsection{Nachrücken}

Hinsichtlich der Qualität des „Nachrückens“ wurde festgestellt: Italien hatte dies 6mal, wovon 3 (50 \%) als hochkarätig bezeichnet wurde und den größten Wert bildete. Absteigend folgen Dänemark mit 4 und 1 (25\%), Deutschland mit 8 und 1 (13\%). Argentinien mit 10 und 1 (10\%) hatte den kleinsten Anteil „hochkarätigen“ Nachrückens. Das folgende Diagramm zeigt die Anteile des hochkarätigen Nachrückens in Prozent für die Viertelfinalisten. 


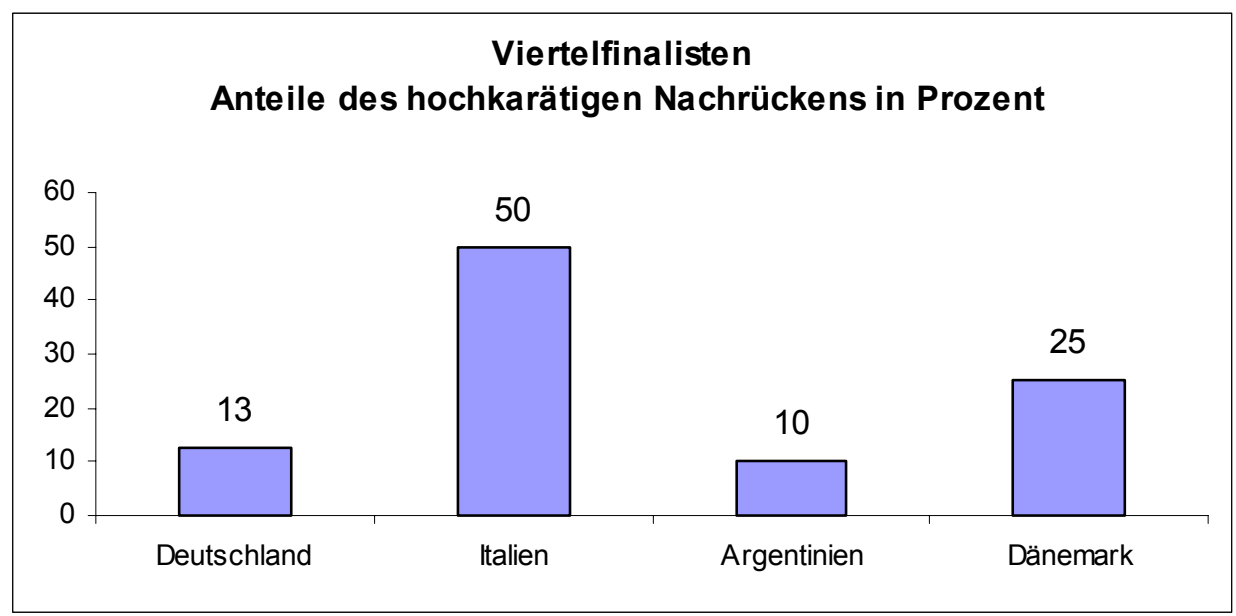

Diagramm 91: Anteile des hochkarätigen Nachrückens in Prozent bei den Viertelfinalisten.

2-mal (50 \%) des Nachrückens von Dänemark wurden mit „normal“ bewertet und bildeten den größten Anteil. Die weiteren Anteile „normalen“ Nachrückens lauteten 4 (40 \%) für Argentinien, 2 (33 \%) für Italien und 2 (25 \%) für Deutschland.

Das folgende Diagramm zeigt die Anteile des normalen Nachrückens in Prozent für die Viertelfinalisten.

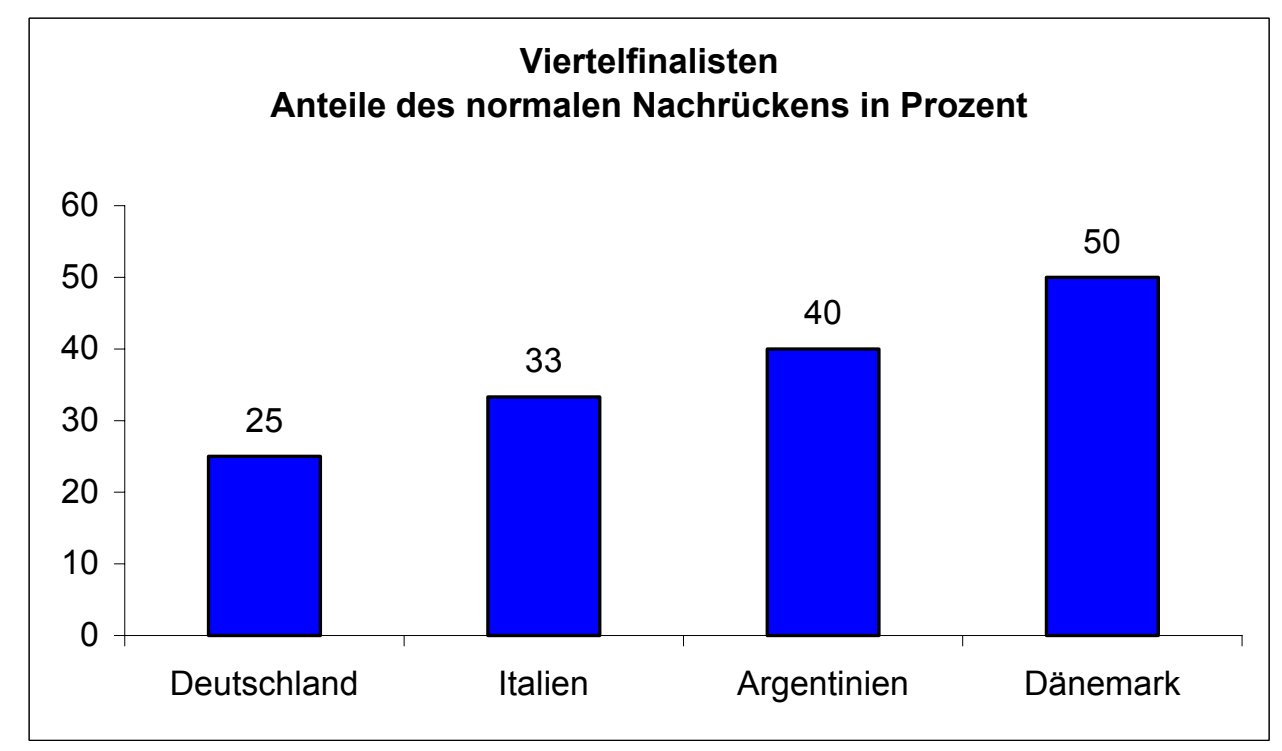

Diagramm 92: Anteile des normalen Nachrückens in Prozent bei den Viertelfinalisten.

Ein „schwaches“ Nachrücken wurde mit einem Anteil von 5 (63\%) am häufigsten bei Deutschland festgestellt. Es folgen Argentinien mit 5 (50\%), Dänemark mit 1 (25\%) und schließlich Italien mit 1 (17\%).

Das folgende Diagramm zeigt die Anteile des schwachen Nachrückens in Prozent für die Viertelfinalisten. 


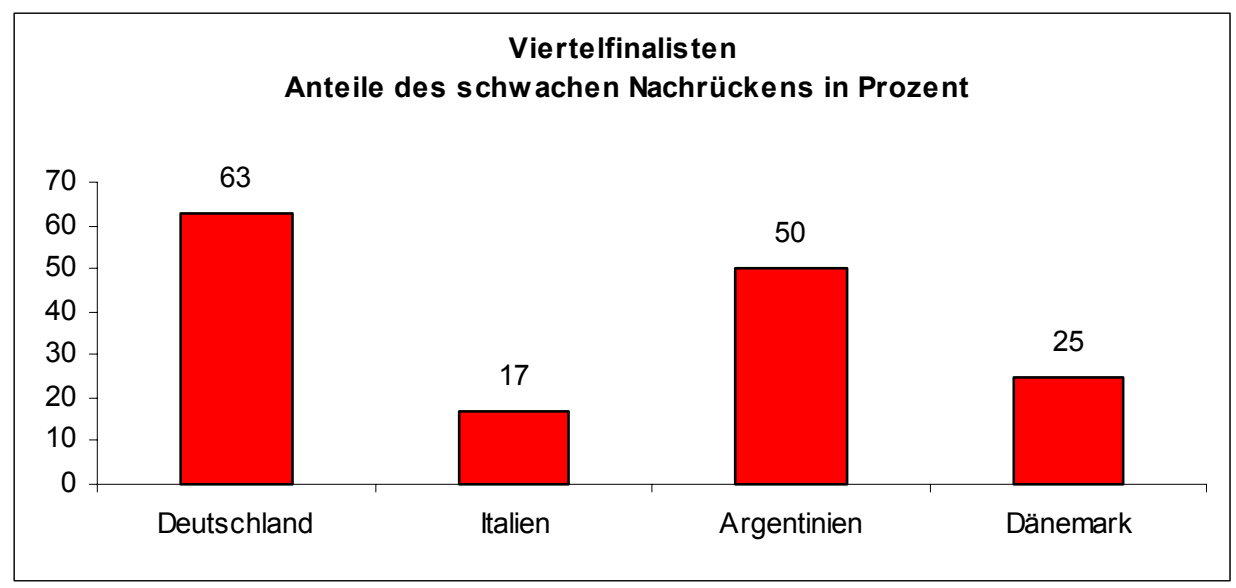

Diagramm 93: Anteile des schwachen Nachrückens in Prozent bei den Viertelfinalisten.

Aus den Diagrammen Nr. 91, 92 und 93 ist ersichtlich, dass das schwache Nachrücken bei allen Mannschaften außer Italien verbreiteter ist als das hochkarätige, und dass normale Nachrücken häufiger sind als die hochkarätigen. Das kann daran liegen, dass die Spieler nicht mit dem Ball laufen und täuschen können, und der Ballbesitzer nicht genug Hilfe von den Mitspielern bekommt.

\subsubsection{In die Breite ziehen}

„In die Breite ziehen“ wandte Argentinien als Technik 17-mal an, hiervon 2-mal (12 $\%)$ hochkarätig. Absteigend folgen Italien mit 13 und 1 (8 \%) und Deutschland 30-mal mit 1 (3\%). Dänemark 39-mal mit 1 (3\%) hatte die kleinsten Anteile "hochkarätigen“ „In die Breite ziehens".

Das folgende Diagramm zeigt die Anteile des hochkarätigen "In die Breite ziehens“ in Prozent für die Viertelfinalisten.

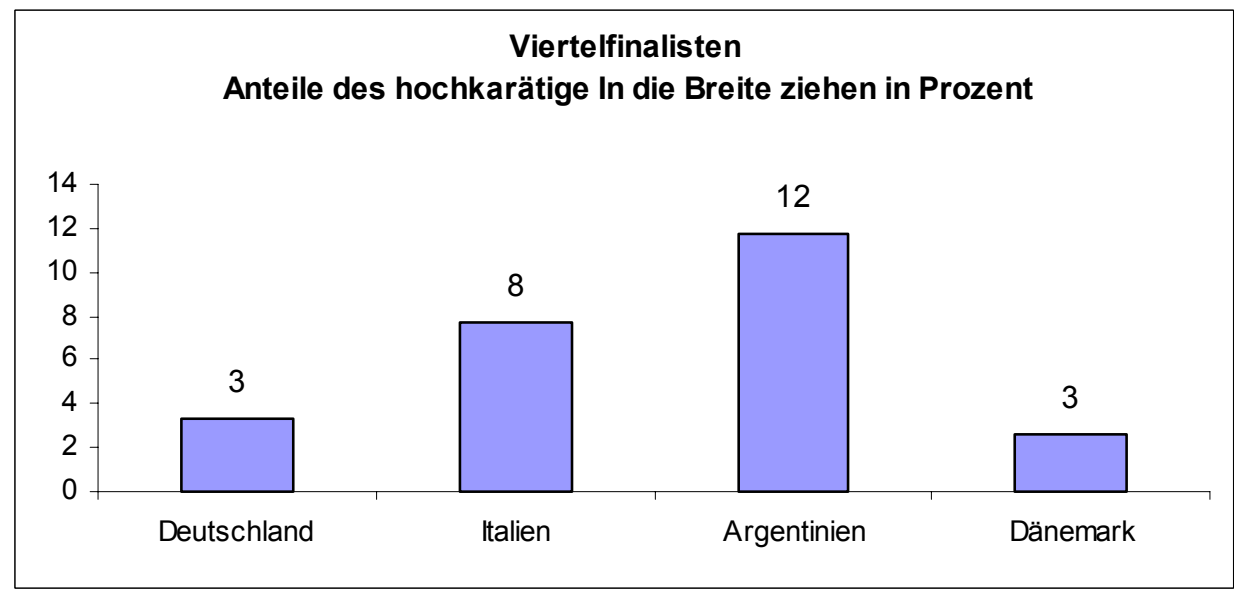

Diagramm 94: Anteile des hochkarätigen „In die Breite ziehen“ in Prozent bei den Viertelfinalisten. 
34-mal (87 \%) des „In die Breite ziehen“ von Dänemark wurden mit „normal“ bewertet und bildeten den größten Anteil. Die weiteren Anteile „normalen“ „In die Breite ziehen“ lauteten 11 (85\%) für Italien, 22 (73\%) für Deutschland und 7 (41\%) für Argentinien.

Dem folgenden Diagramm sind die Anteile des normalen „In die Breite ziehen“ in Prozent für die Viertelfinalisten zu entnehmen.

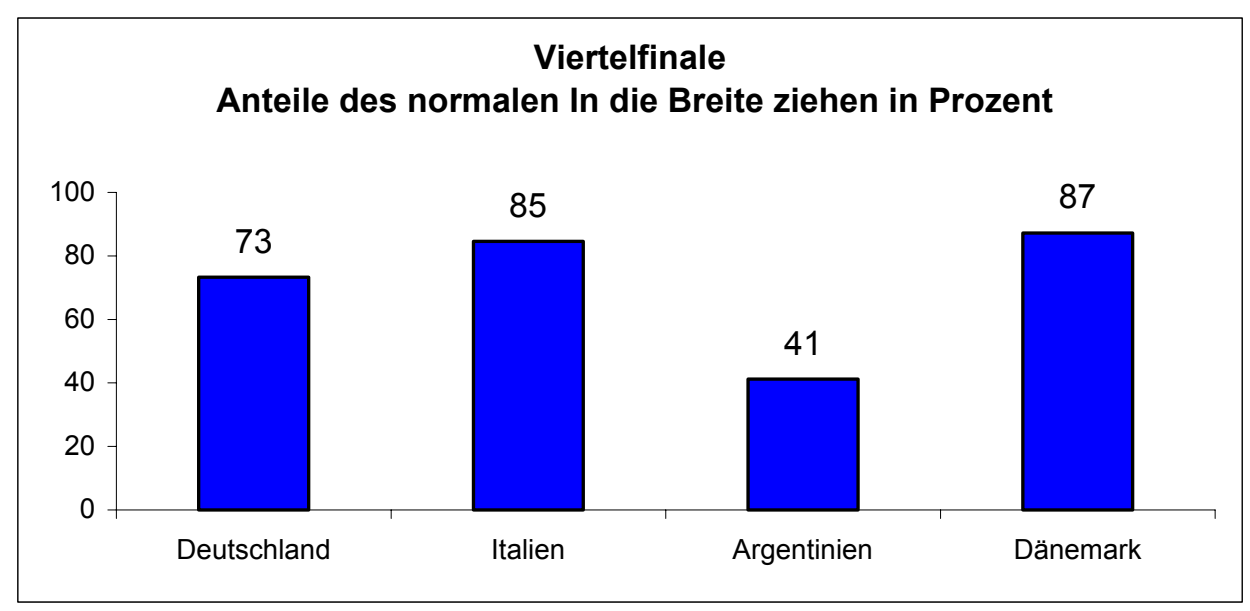

Diagramm 95: Anteile des normalen „In die Breite ziehen“ in Prozent bei den Viertelfinalisten.

Ein „schwaches“ „In die Breite ziehen“ wurde mit einem Anteil von 8-mal (47\%) am häufigsten bei Argentinien festgestellt. Es folgen Deutschland mit 7 (23\%), Dänemark mit 4 (10\%) und schließlich Italien mit 1 (8\%).

Das folgende Diagramm zeigt die Anteile des schwachen „In die Breite ziehen“ in Prozent für die Viertelfinalisten.

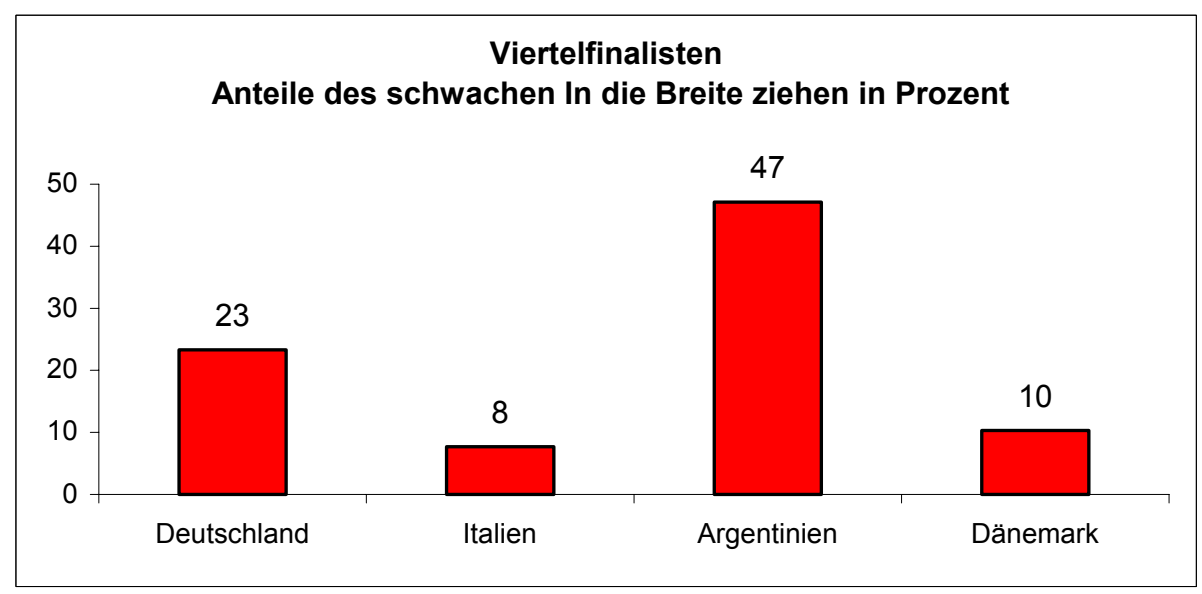

Diagramm 96: Anteile des schwachen „In die Breite ziehen“ in Prozent bei den Viertelfinalisten. 
Aus den Diagrammen 94, 95 und 96 lässt sich schließen, dass das normale In die Breite ziehen häufiger ist als das hochkarätige und das misslungene. Die Gründe dafür liegen vielleicht darin, dass die Spieler das „In die Breite ziehen“ zu weit vom gegnerischen Tor entfernt unternehmen, wodurch der Druck auf die Angreifer geringer ist.

\subsubsection{Halbfinalisten}

Als Halbfinalisten werden diejenigen Mannschaften bezeichnet, die das Halbfinale erreicht haben und zu deren Analyse aufgrund der automatischen Qualifikation für ein weiteres Spiel (Endspiel bzw. Spiel um Platz Drei) vier Spiele herangezogen werden konnten. Zu diesen Halbfinalisten gehören Frankreich, Brasilien, Kroatien und die Niederlande.

\subsubsection{Freistöße}

Freistoß hatte Brasilien 26-mal, hiervon 3-mal (12 \%) hochkarätig. Dies bildete den größten Wert. Absteigend folgen Kroatien mit 20 und 2 (10\%), Frankreich mit 38 und 2 (5 \%). Die Niederlande hatte mit 23 und 1 (4\%) die kleinste Anteile „hochkarätiger“ Freistöße.

Dem folgenden Diagramm sind die Anteile der hochkarätigen Freistöße in Prozent für die Halbfinalisten zu entnehmen.

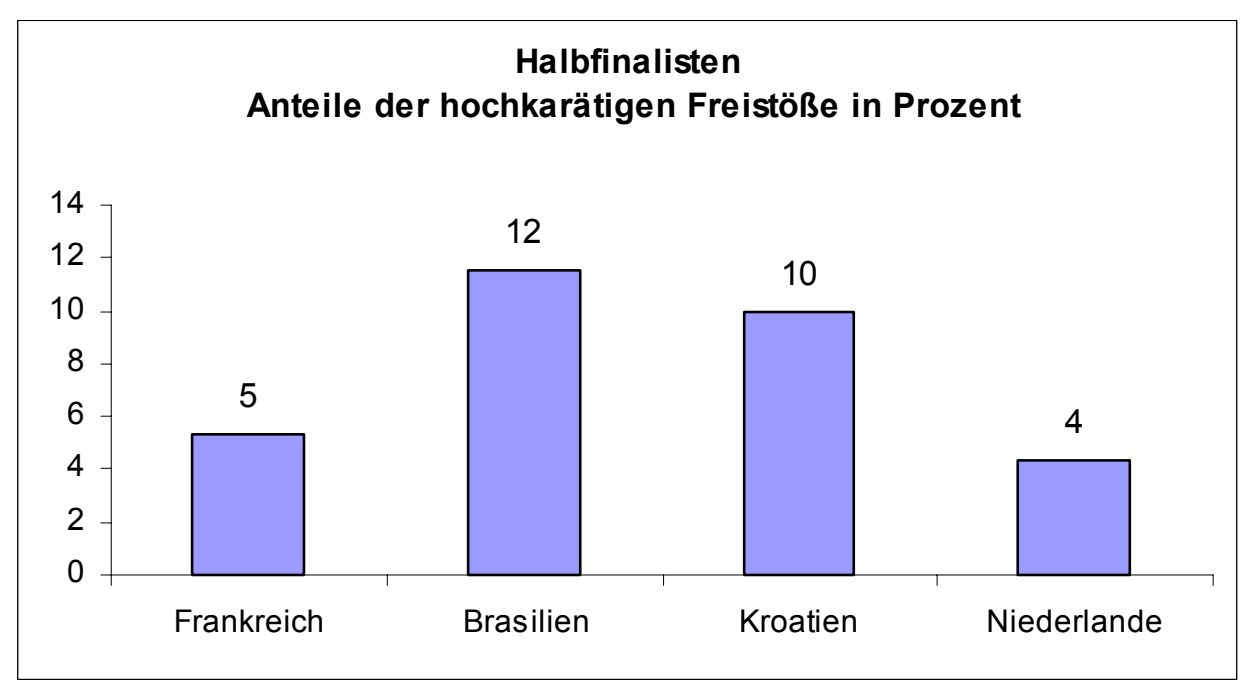

Diagramm 97: Anteile der hochkarätigen Freistöße in Prozent bei den Halbfinalisten. 
3-mal (54 \%) der Freistöße von Brasilien wurden mit "normal“ bewertet und bildete der größte Anteil. Die weiteren Anteile "normaler" Freistöße lauteten 20 (53 \%) für Frankreich, 12 (52 \%) für die Niederlande und 6 (30 \%) für Kroatien.

Dem folgenden Diagramm sind die Anteile der normalen Freistöße in Prozent für die Halbfinalisten zu entnehmen.

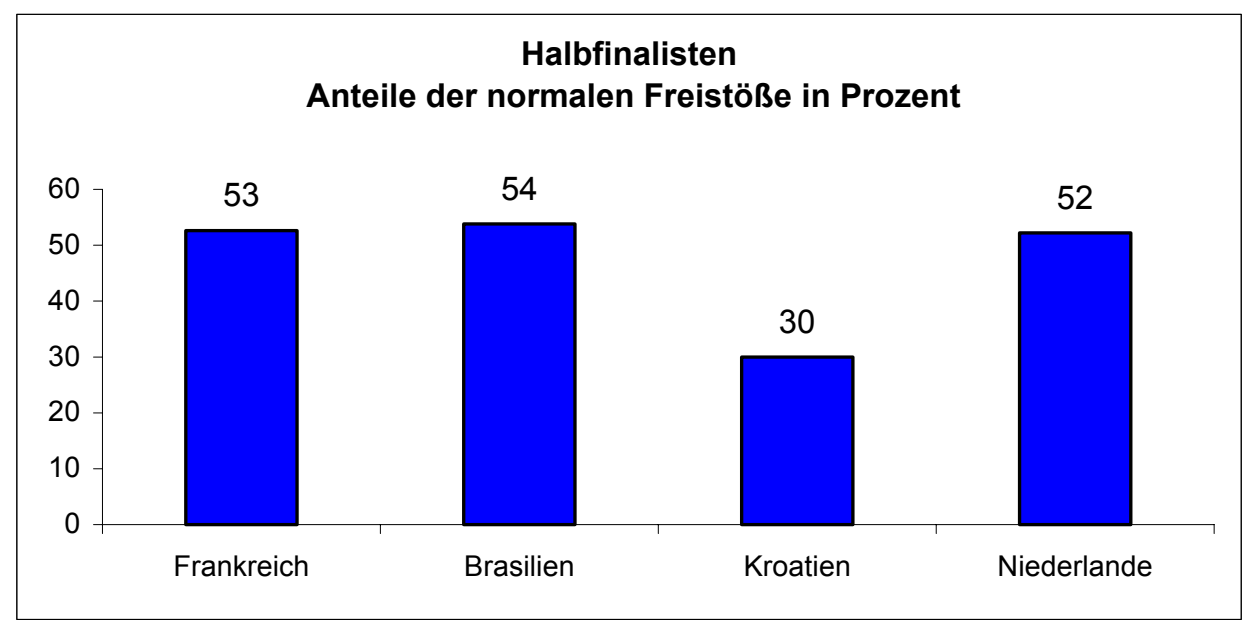

Diagramm 98: Anteile der normalen Freistöße in Prozent bei den Halbfinalisten.

Ein "schwacher" Freistoß wurde mit einem Anteil von 12 (60 \%) am häufigsten bei Kroatien festgestellt. Es folgen die Niederlande mit 10 (44\%), Frankreich mit 16 (42 $\%)$ und schließlich Brasilien mit 9 (35\%).

Dem folgenden Diagramm sind die Anteile der schwachen Freistöße in Prozent für die Halbfinalisten zu entnehmen.

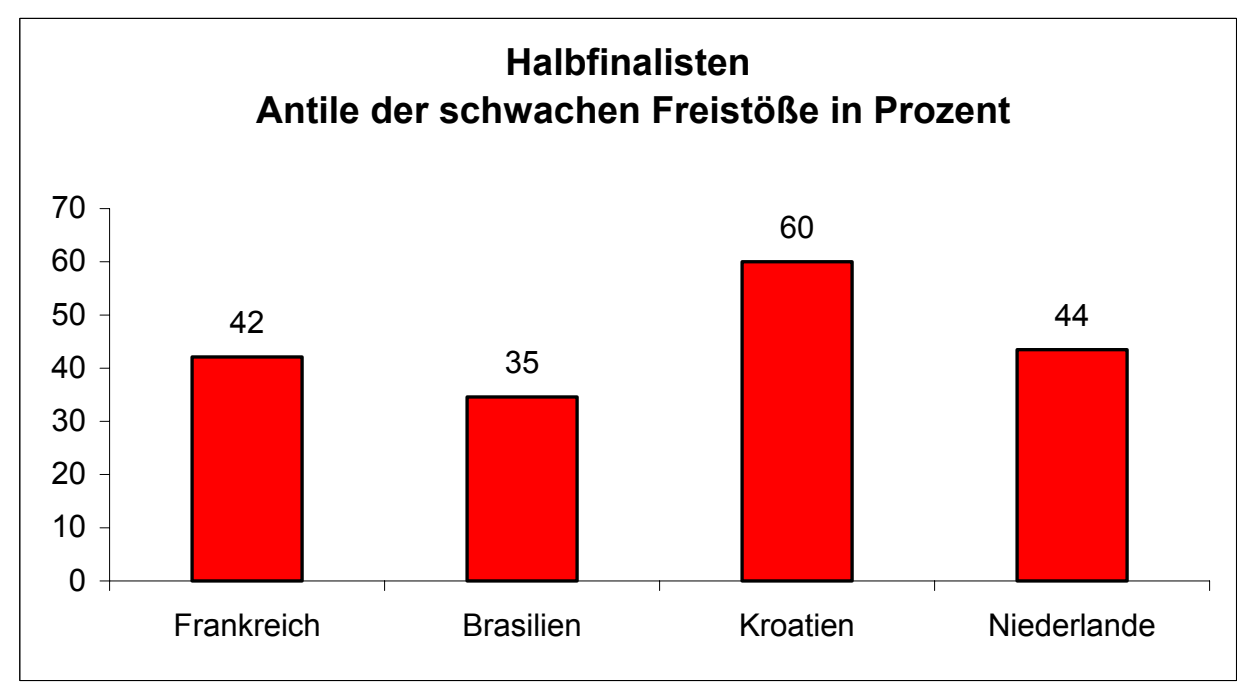

Diagramm 99: Anteile der schwachen Freistöße in Prozent bei den Halbfinalisten. 
Diagramm 97 erklärt, dass die hochkarätigen Freistöße zwischen 4 \% und 12 \% lagen. Die brasilianische Mannschaft hatte die besten Werte, und die niederländische hatte die schlechtesten. Diagramm 98 erklärt, dass normale Freistöße zwischen 30 $\%$ und $54 \%$ lagen. Die brasilianische Mannschaft hatte hier die besten Werte und die kroatische hatte die schlechtesten. Diagramm 99 erklärt, dass schwache Freistöße zwischen $35 \%$ und 60 \% lagen. Die kroatische Mannschaft hatte die schwächsten Werte und die brasilianische hatte die besten Werte.

Es lässt sich Schlussfolgerung aus den Diagrammen ziehen: Mit Ausnahme von Brasilien ist die Ausführung der Freistöße bei den Halbfinalisten als recht schwach anzusehen, d.h., dass die meisten der Freistöße entweder als „normal“ oder „schwach“ zu bewerten sind. Das ist auf die mangelhafte Aufstellung des Angriffs zurückzuführen, besonders wenn der Freistoß von einem Platz weit vom Tor gespielt wird oder ungenau bei direktem Schuss geschossen wird. Die Streuung der Werte zwischen den besten und den schlechtesten Mannschaften war bei diesem Wert sehr groß.

\subsubsection{Eckball}

Hinsichtlich der Qualität der „Eckbälle“ wurde festgestellt: Die Niederlande hatten 22-mal, hiervon 4-mal (18 \%) den größten Wert als hochkarätig. Absteigend folgen Frankreich mit 38 und 5 (13\%). Brasilien und Kroatien hatten 20, 19 mit jeweils 0,0\% den kleinsten Anteil „hochkarätigen“ Eckbälle.

Das folgende Diagramm zeigt die Anteile der hochkarätigen Eckbälle in Prozent für die Halbfinalisten.

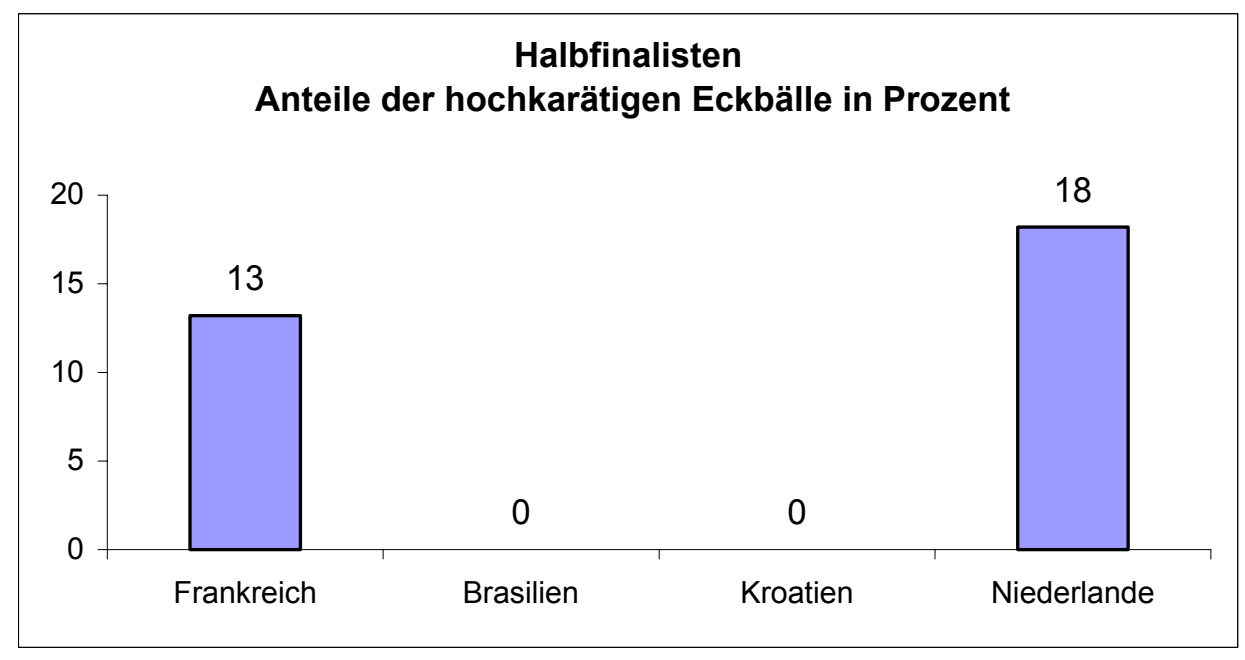

Diagramm 100: Anteile der hochkarätigen Eckbälle in Prozent bei den Halbfinalisten. 
12-mal (63 \%) der Eckbälle von Kroatien wurden mit „normal“ bewertet und bildeten den größten Anteil. Die weiteren Anteile „normaler“ Eckbälle lauteten 10 (50 \%) für Brasilien, die Niederlande hatte 11 (50\%) und 18 (47\%) Frankreich.

Das folgende Diagramm zeigt die Anteile der normalen Eckbälle in Prozent für die Halbfinalisten.

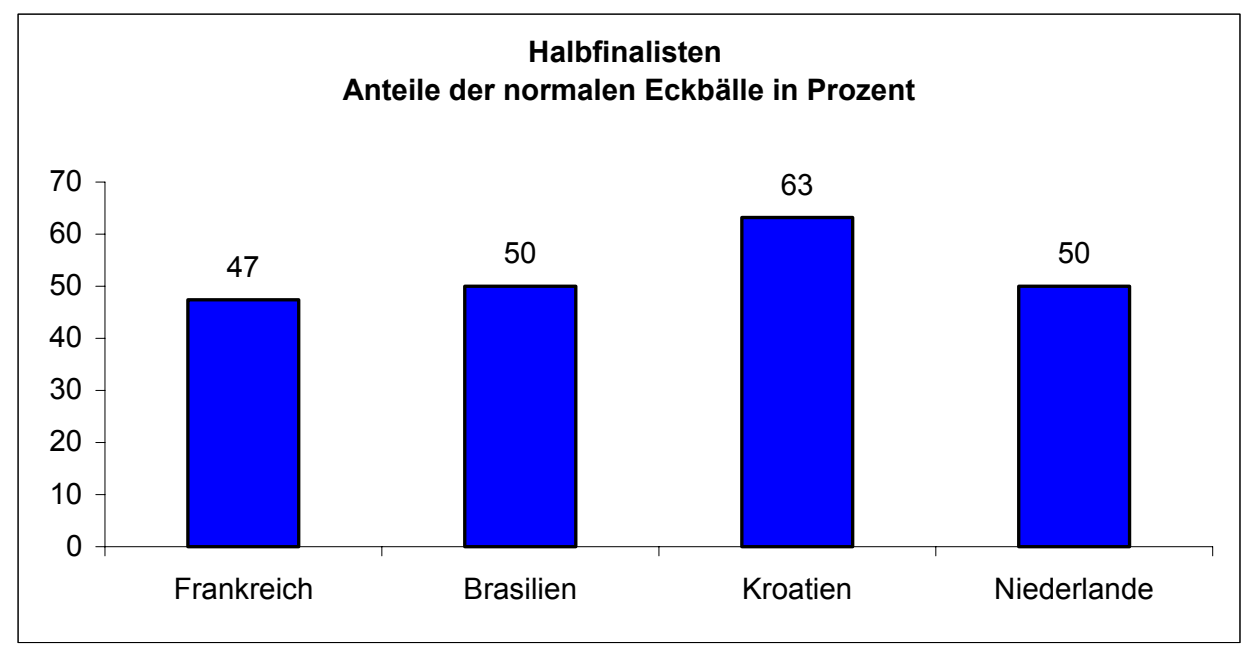

Diagramm 101: Anteile der normalen Eckbälle in Prozent bei den Halbfinalisten.

Ein „schwacher“ Eckball wurde mit einem Anteil von 10 (50 \%) am häufigsten bei Brasilien festgestellt. Es folgen Frankreich mit 15 (40\%), Kroatien mit 7 (37\%) und schließlich die Niederlande mit 7 (31\%).

Das folgende Diagramm zeigt die Anteile der schwachen Eckbälle in Prozent für die Halbfinalisten.

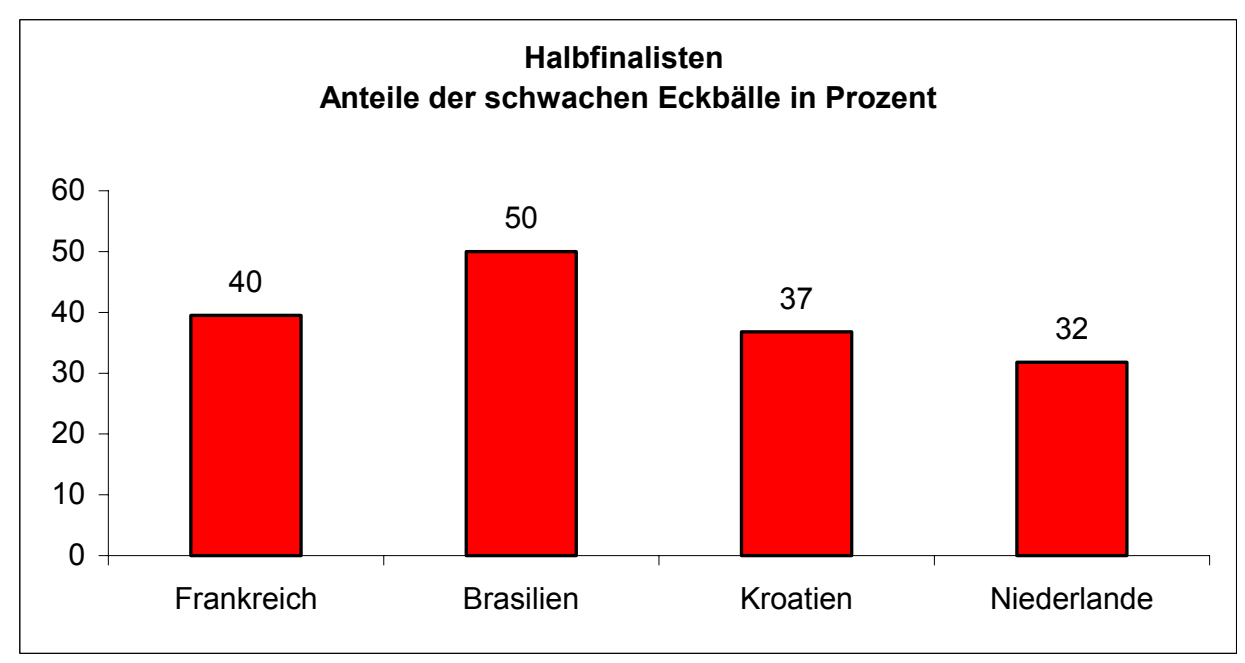

Diagramm 102: Anteile der schwachen Eckbälle in Prozent bei den Halbfinalisten. 
Aus den Werten der Diagramme 100, 101 und 102 ist die geringe Anzahl der hochkarätigen im Vergleich zu den guten und schwachen Eckbällen ersichtlich. Dabei ist die Anzahl der guten Eckbälle größer als die der hochkarätigen und schwachen.

Wir führen das auf die schlechte Organisation des Angriffs bei der Ausführung des Eckballs oder schlechte Taktik zurück. Mit einer guten Taktik kann die ausführende Mannschaft die gegnerische Mannschaft täuschen und die gegnerische Aberwehr überwinden.

\subsubsection{Unterstützen}

Hinsichtlich der Qualität des „Unterstützens“ wurde festgestellt: Brasilien „unterstützte“ 87-mal, hiervon wurde mit 5 (6\%) der größten Wert festgestellt. Absteigend folgen Frankreich mit 99 und 3 (3\%), die Niederlande mit 123 und 2 (2\%). Kroatien hatte 97, hiervon wurde mit 1 (1\%) den kleinsten Anteil „hochkarätigen“ Unterstützens ermittelt.

Das folgende Diagramm zeigt die Anteile des hochkarätigen Unterstützens in Prozent für die Halbfinalisten.

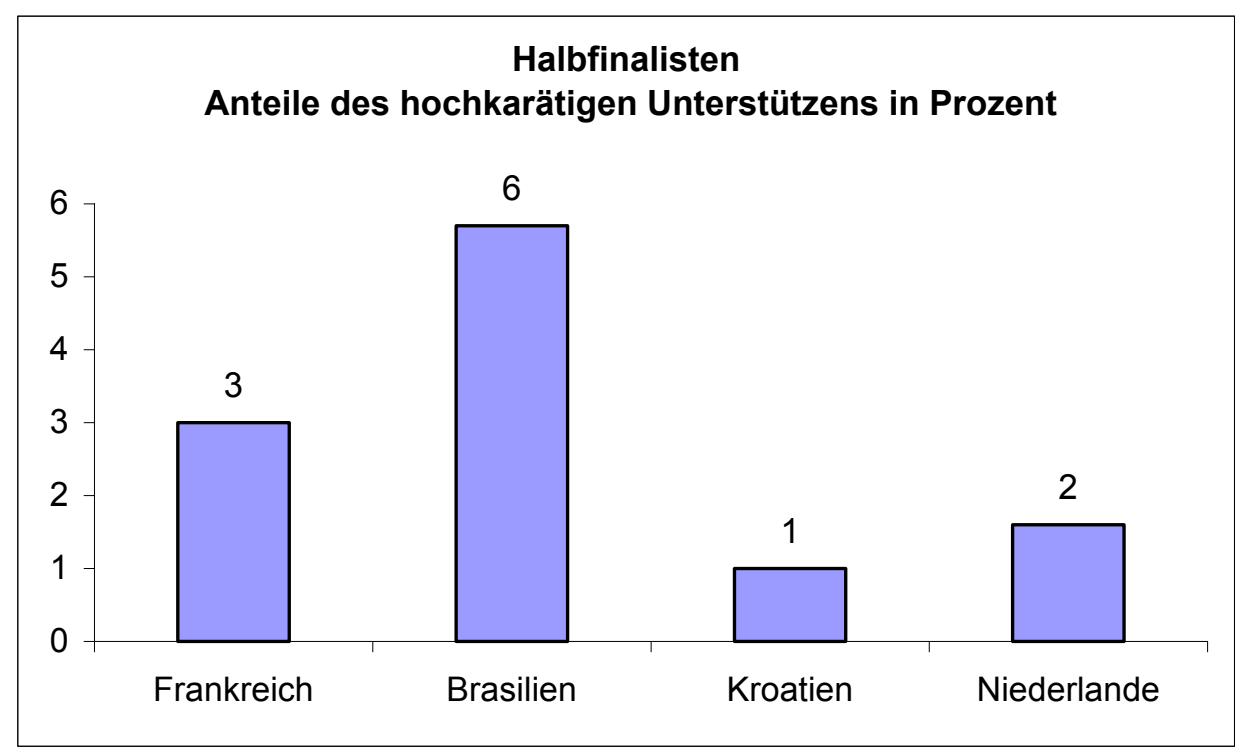

Diagramm 103: Anteile des hochkarätigen Unterstützens in Prozent bei den Halbfinalisten.

$120(98 \%)$ des Unterstützens von den Niederlanden wurde mit „normal“ bewertet und bildete den größten Anteil. Die weiteren Anteile „normalen“ Unterstützens lauteten 93 (96 \%) für Kroatien, 94 (95\%) für Frankreich und 75 (86 \%) für Brasilien. 
Das folgende Diagramm zeigt die Anteile des normalen Unterstützens in Prozent für die Halbfinalisten.

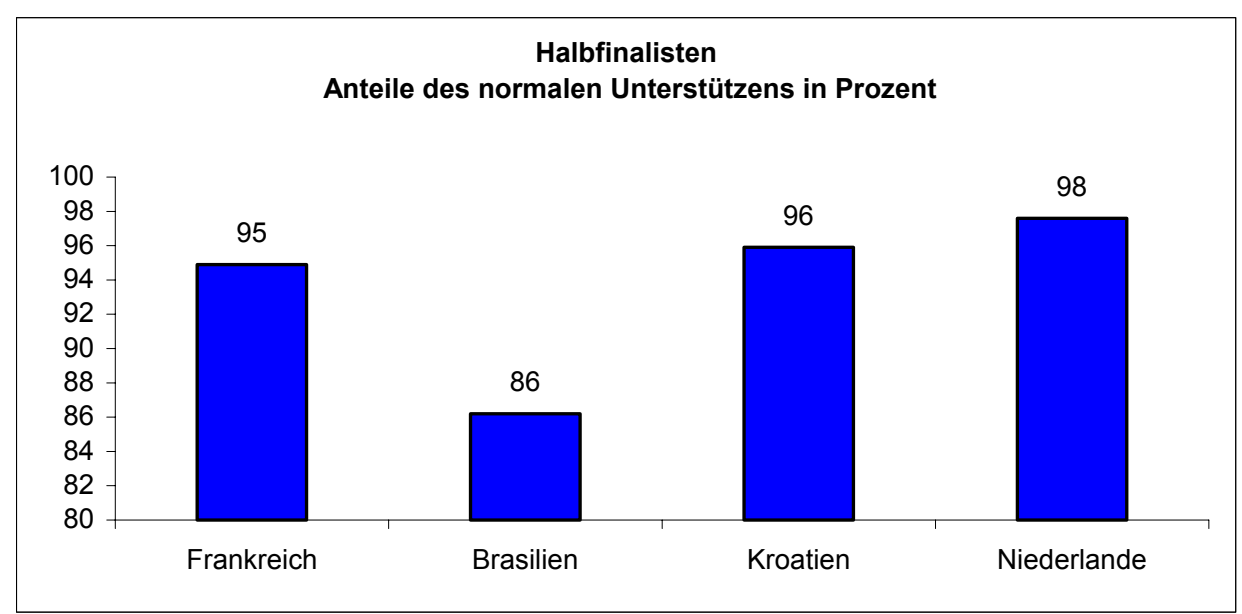

Diagramm 104: Anteile des normalen Unterstützens in Prozent bei den Halbfinalisten.

Ein „schwaches“ Unterstützen wurde mit einem Anteil von 7 (8\%) am häufigsten bei Brasilien festgestellt. Es folgen Kroatien mit 3 (3\%), Frankreich mit 2 (2\%) und schließlich die Niederlande mit 1 (1\%). Das folgende Diagramm zeigt die Anteile des schwachen Unterstützens in Prozent für die Halbfinalisten.

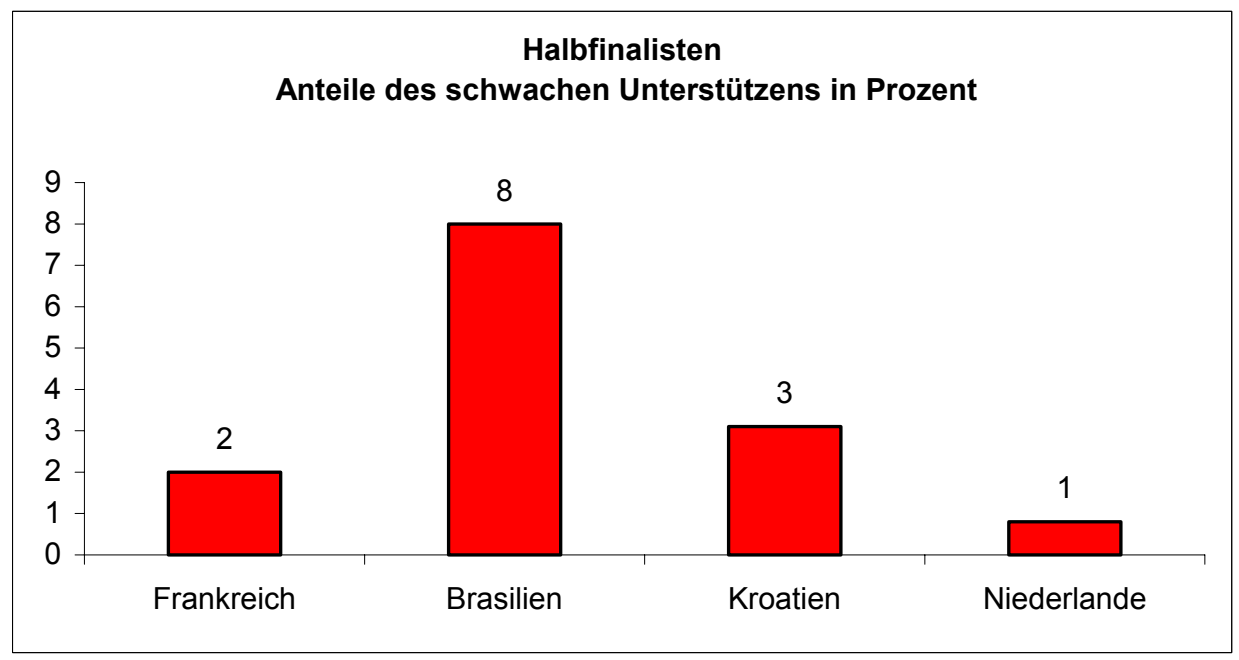

Diagramm 105: Anteile des schwachen Unterstützens in Prozent bei den Halbfinalisten. 
Aus der Darstellung von den Diagrammen 103,104 und 105 wird deutlich, dass das normalen Unterstützen häufiger ist als das hochkarätige und schwache, da die unterstützenden Spieler einen Platz einnehmen, von dem aus sie ihre Mitspieler so helfen können, dass sie gut mit dem Ball vorankommen können. Manchmal unterstützen die Mitspieler nicht zur rechten Zeit oder richtigen Position.

\subsubsection{Doppelpass}

Doppelpass hatten die Niederlande 12-mal, wovon mit 2 (17\%) der größte Wert als „hochkarätig“ bezeichnet wurde. Absteigend folgen Brasilien mit 19, und 3 (16\%), Frankreich mit 26 und 3 (12\%). Kroatien mit 2, wovon mit 0,0\% der kleinsten Anteil „hochkarätiger“ Doppelpässe.

Dem folgenden Diagramm sind die Anteile der hochkarätigen Doppelpässe in Prozent für die Halbfinalisten zu entnehmen.

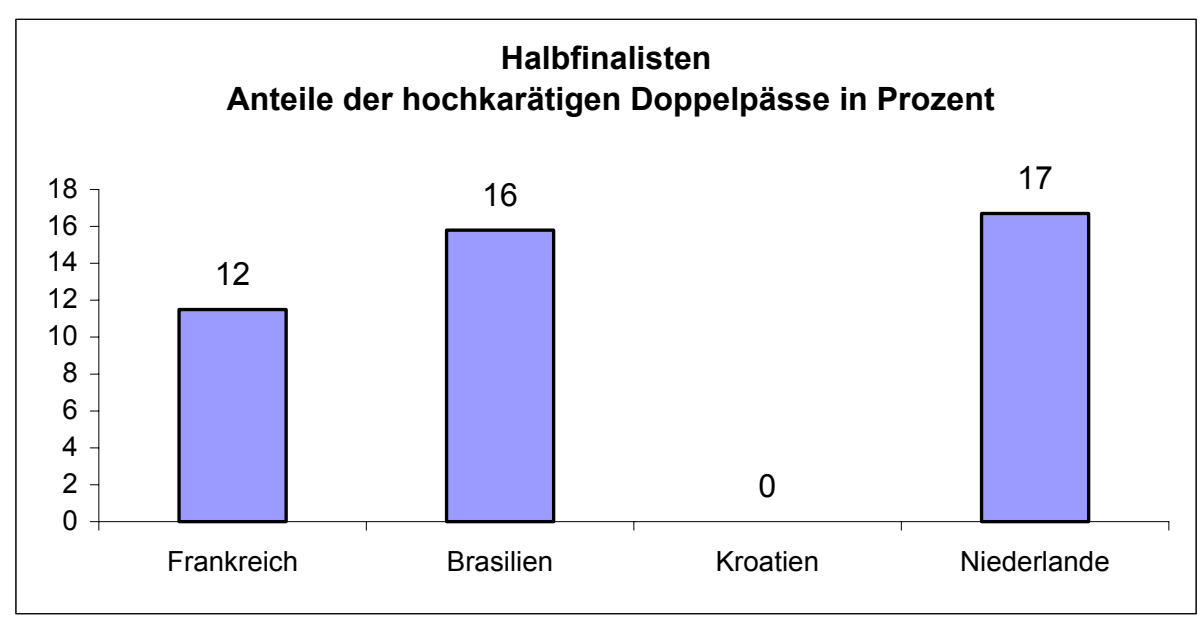

Diagramm 106: Anteile der hochkarätigen Doppelpässe in Prozent bei den Halbfinalisten.

$8(67 \%)$ der Doppelpässe von den Niederlanden wurden mit „normal“ bewertet und bildeten den größten Anteil. Die weiteren Anteile „normaler“ Doppelpässe lauteten 16 (62 \%) für Frankreich, 11 (58 \%) für Brasilien und 1 (50 \%) für Kroatien.

Das folgende Diagramm zeigt die Anteile der normalen Doppelpässe in Prozent für die Halbfinalisten. 


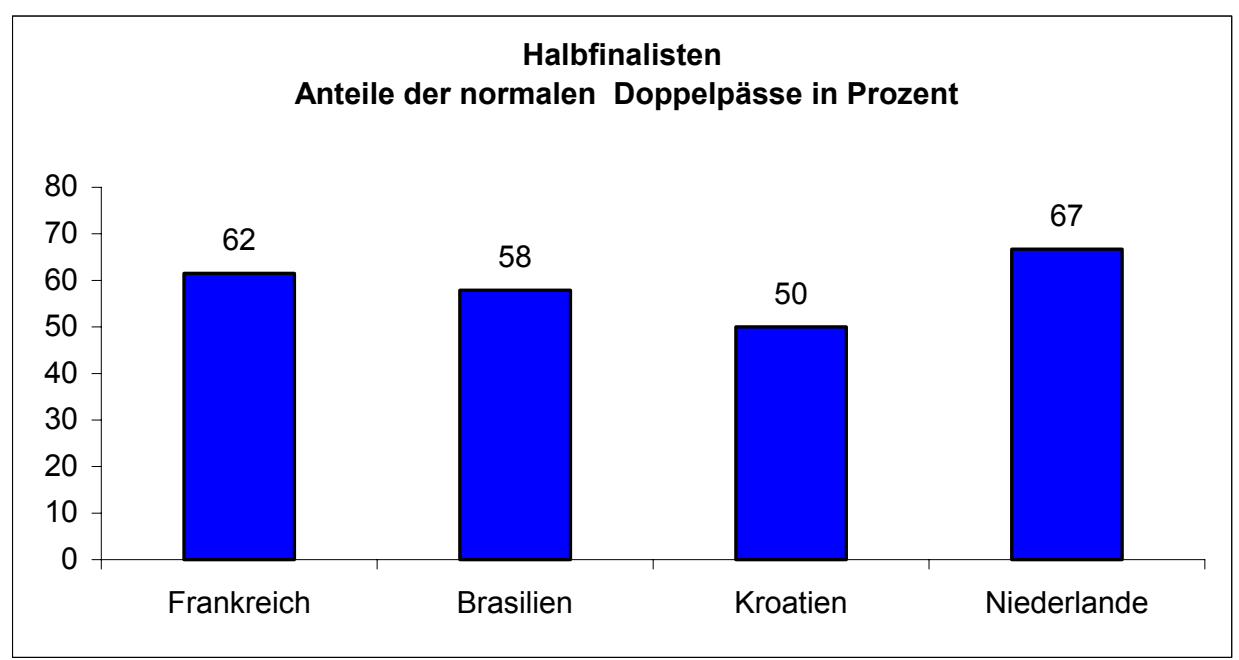

Diagramm 107: Anteile der normalen Doppelpässe in Prozent bei den Halbfinalisten.

Ein „schwacher" Doppelpass wurde mit einem Anteil von 1 (50 \%) am häufigsten bei Kroatien festgestellt. Es folgen Frankreich mit 7 (27\%), Brasilien mit $5(26 \%)$ und schließlich die Niederlande mit 2 (17\%).

Dem folgenden Diagramm sind die Anteile der schwachen Doppelpässe in Prozent für die Halbfinalisten zu entnehmen.

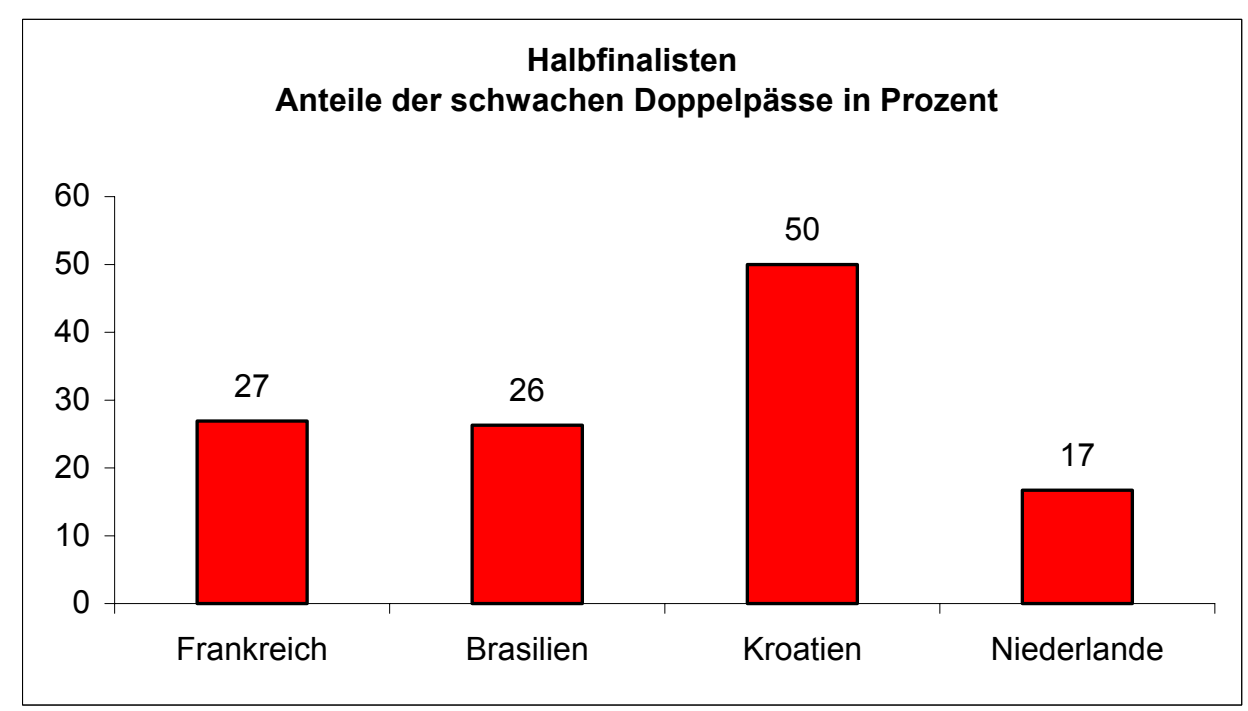

Diagramm 108: Anteile der schwachen Doppelpässe in Prozent bei den Halbfinalisten.

Aus den Diagrammen lässt sich die Schlussfolgerung ziehen, dass die normalen Doppelpäße bei allen Halbfinalisten einen größeren Anteil hatten als die hochkarätigen und die hochkarätigen Doppelpäße auch seltener sind als die schwachen. Das 
ist dadurch zu erklärt, dass die Angreifer ihren Ball oftmals verlieren, wenn das Pressing im Drittel des Gegners stark ist, und die Verteidigern leicht gewonnen die Zweikämpfe.

\subsubsection{Raumaufteilung}

Hinsichtlich der Qualität der „Raumaufteilung“ wurde festgestellt: Die Niederlande hatten bei 184-mal mit 28 (15\%) den größten Wert. Absteigend folgen Frankreich hatte 243 mit 36 (15\%), Kroatien 137 mit 19 (14\%). Brasilien hatte bei 227 mit 29 (13\%) den kleinsten Anteil "guter" Raumaufteilung.

Das folgende Diagramm zeigt die Anteile der hochkarätigen Raumaufteilung in Prozent für die Halbfinalisten.

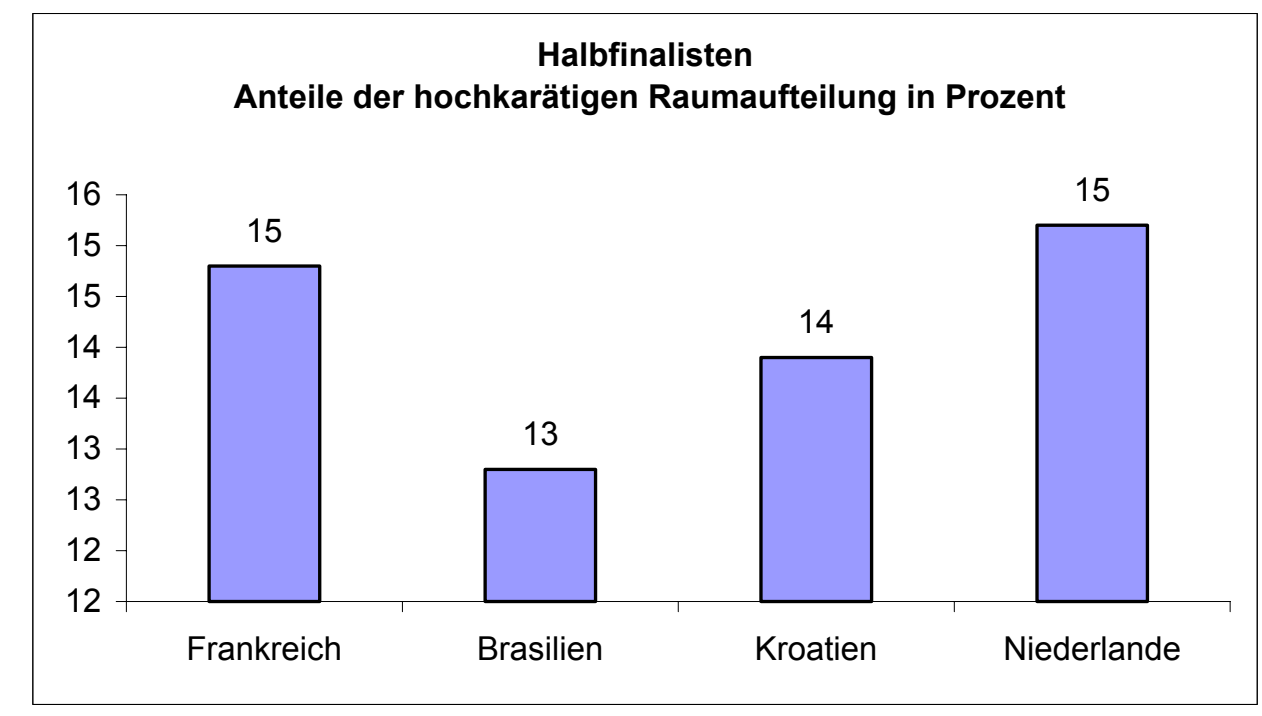

Diagramm 109: Anteile der hochkarätigen Raumaufteilung in Prozent bei den Halbfinalisten.

121 (66 \%) der Raumaufteilungen von den Niederlanden wurden mit „normal“ bewertet und bildeten den größten Anteil. Die weiteren Anteile "normaler" Raumaufteilungen lauteten 133 (59\%) für Brasilien, 130 (54 \%) für Frankreich und 55 (40\%) für Kroatien.

Das folgende Diagramm zeigt die Anteile der normalen Raumaufteilung in Prozent für die Halbfinalisten. 


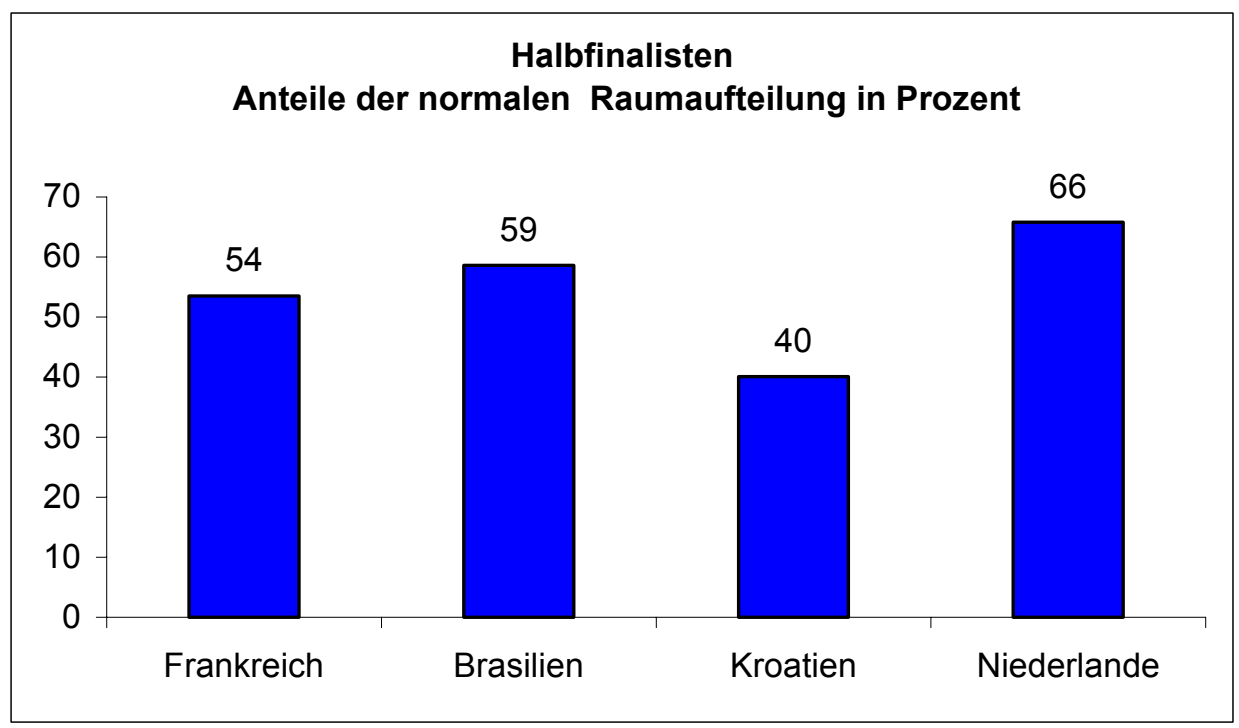

Diagramm 110 : Anteile der normalen Raumaufteilung in Prozent bei den Halbfinalisten.

Eine „schwache“ Raumaufteilung wurde mit einem Anteil von 63 (46 \%) am häufigsten bei Kroatien festgestellt. Es folgen Frankreich mit 77 (32 \%), Brasilien mit 65 (29 $\%)$ und schließlich die Niederlande mit 35 (19\%).

Dem folgenden Diagramm sind die Anteile der schwachen Raumaufteilung in Prozent für die Halbfinalisten zu entnehmen.

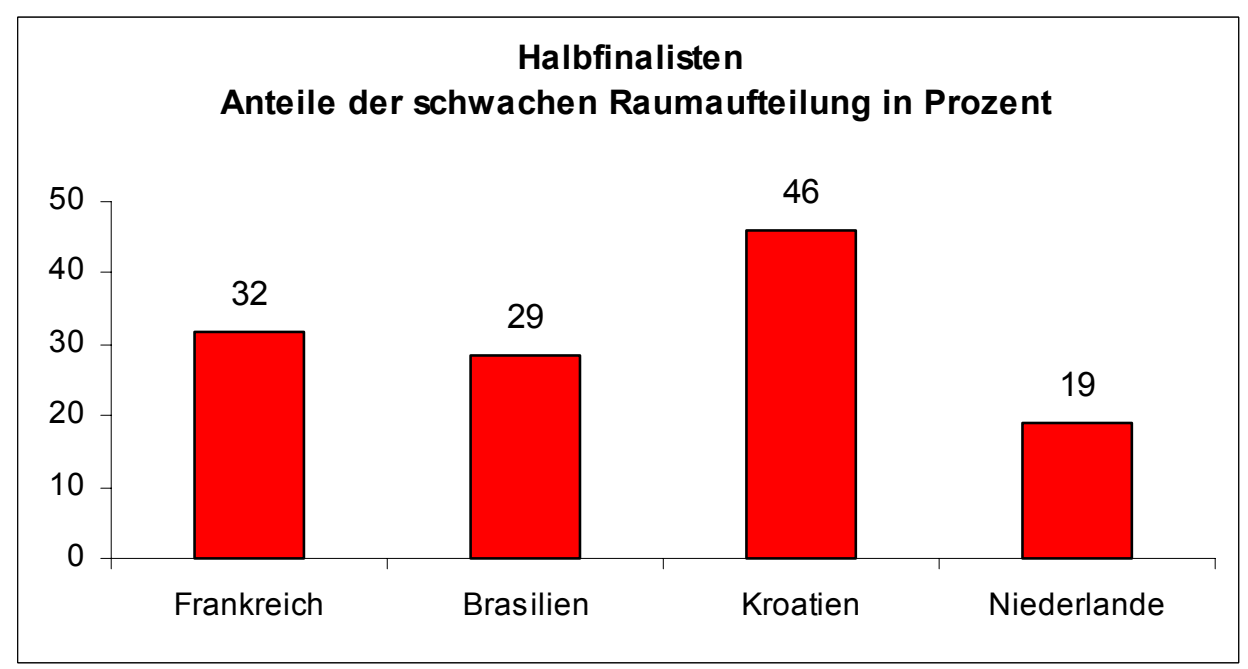

Diagramm 111 : Anteile der schwachen Raumaufteilung in Prozent bei den Halbfinalisten. 
Aus den Diagrammen lässt sich die Schlussfolgerung ziehen, dass die normalen Raumaufteilungen bei allen Halbfinalisten einen größeren Anteil hatten als die hochkarätigen und die hochkarätigen Raumaufteilungen auch seltener sind als die schwachen. Wir führen das auf die schlechte Organisation des Angriffs wenn die Mannschaft den Ball abgenommen wurde, besonders Im Drittel des Gegners, das erklärt, dass die Verteilung der Spieler auf dem Spielfeld schlecht war. Die Spieler ohne Ball kein gute Position im Schussbereich des gegnerischen Tores erreicht hatten.

\subsubsection{Nachrücken}

„Hochkarätiges“ Nachrücken kam bei Brasilien 21-mal vor, hiervon wurden 3 (14 \%) als "hochkarätig“ bewertet. Absteigend folgen die Niederlande mit 12-mal und 1 (8 $\%$ ), Kroatien mit 13 und 1 (8\%), Frankreich mit 21 und 1 (5\%) den kleinsten Anteil „hochkarätigen“ Nachrückens.

Dem folgenden Diagramm sind die Anteile des hochkarätigen Nachrückens in Prozent für die Halbfinalisten zu entnehmen.

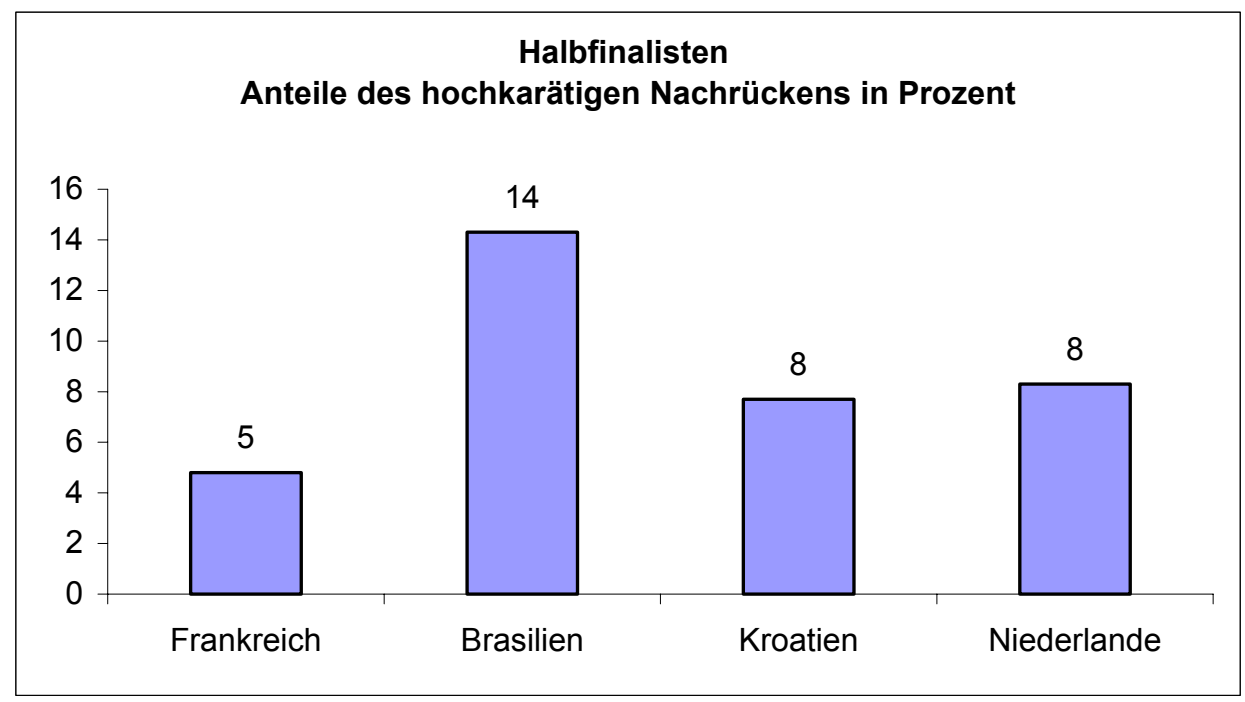

Diagramm 112 : Anteile des hochkarätigen Nachrückens in Prozent bei den Halbfinalisten.

8 (62 \%) des Nachrückens von Kroatien wurden mit „normal“ bewertet und bildeten den größten Anteil. Die weiteren Anteile „normalen“ Nachrückens lauteten 9 (43\%) für Frankreich, 6 (29 \%) für Brasilien und 2 (17\%) für die Niederlande. 
Das folgende Diagramm zeigt die Anteile des normalen Nachrückens in Prozent für die Halbfinalisten.

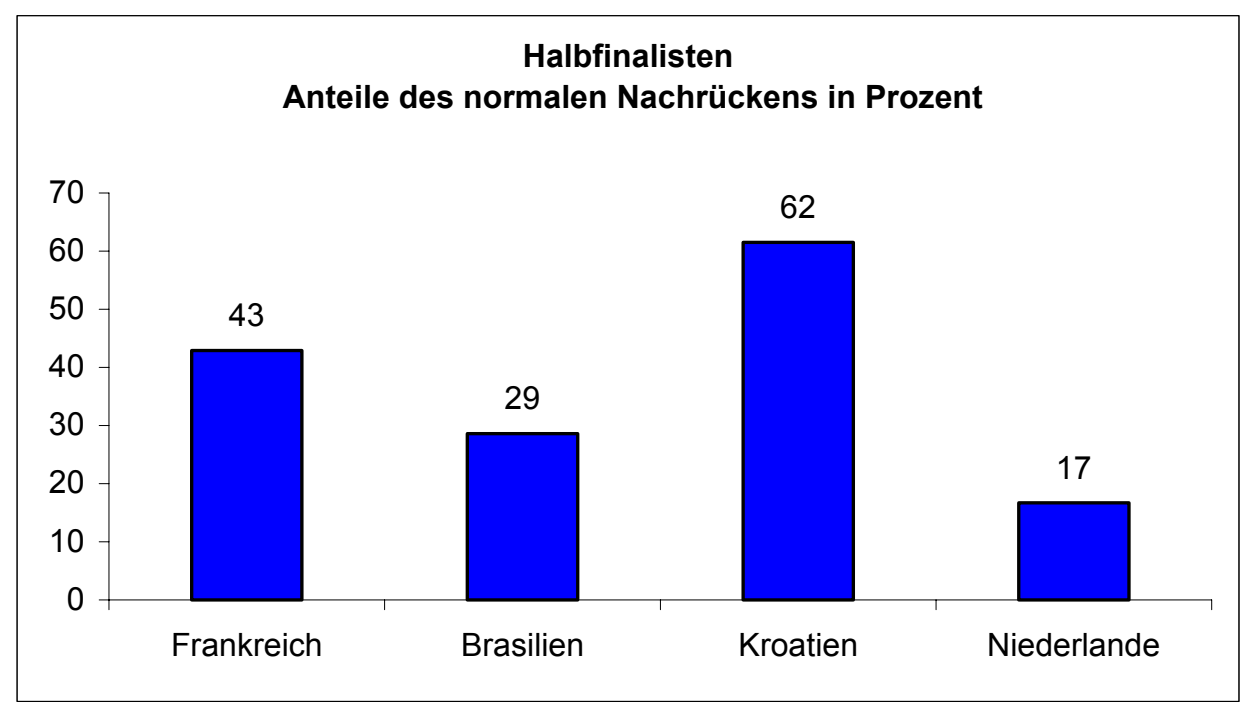

Diagramm 113 : Anteile des normalen Nachrückens in Prozent bei den Halbfinalisten.

Ein „schwaches“ Nachrücken wurde mit einem Anteil von 9 (75\% am häufigsten bei den Niederlanden festgestellt. Es folgen Brasilien mit 12 (57\%), Frankreich mit 11 (52\%) und schließlich Kroatien mit 4 (31\%).

Das folgende Diagramm zeigt die Anteile des schwachen Nachrückens in Prozent für die Halbfinalisten.

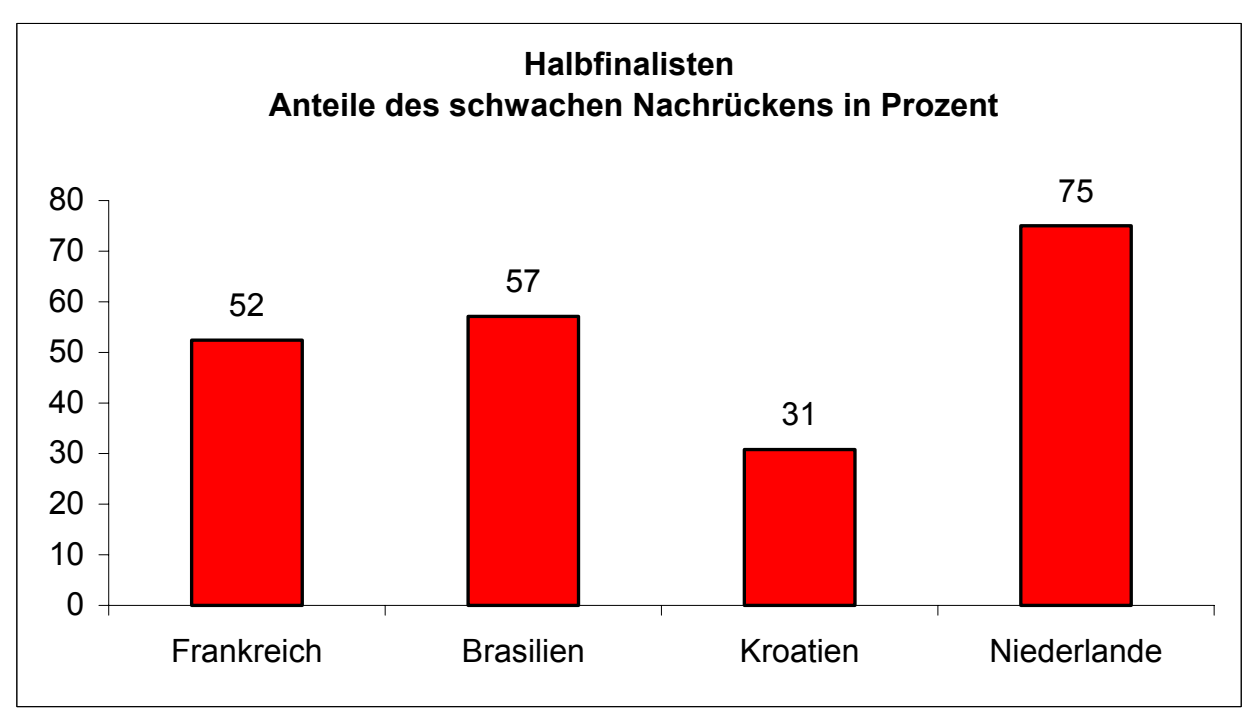

Diagramm 114 : Anteile des schwachen Nachrückens in Prozent bei den Halbfinalisten. 
Aus den Diagrammen Nr. 112, 113 und 114 ist ersichtlich, dass das schwache Nachrücken bei allen Mannschaften außer Kroatien verbreiteter ist als das hochkarätige und das normalen. Das kann daran liegen, dass die Spieler nicht mit dem Ball laufen und täuschen können, und nicht genug Hilfe den Ballbesitzer von den Mitspielern bekommen hatten.

\subsubsection{In die Breite ziehen}

Hinsichtlich der Qualität des „In die Breite ziehen“ wurde festgestellt, dass diese Technik von Frankreich 56-mal angewandt wurde, hiervon wurden 4 (7\%) als „hochkarätig" bewertet. Absteigend folgen Kroatien mit 33 und 1 (3\%), die Niederlande mit 45 und 1 (2\%). Brasilien hatte mit 73-mal und 1 (1\%) den kleinsten Anteil des „hochkarätigen“ „In die Breite ziehen“.

Dem folgenden Diagramm sind die Anteile des hochkarätigen „In die Breite ziehen“ in Prozent für die Halbfinalisten zu entnehmen.

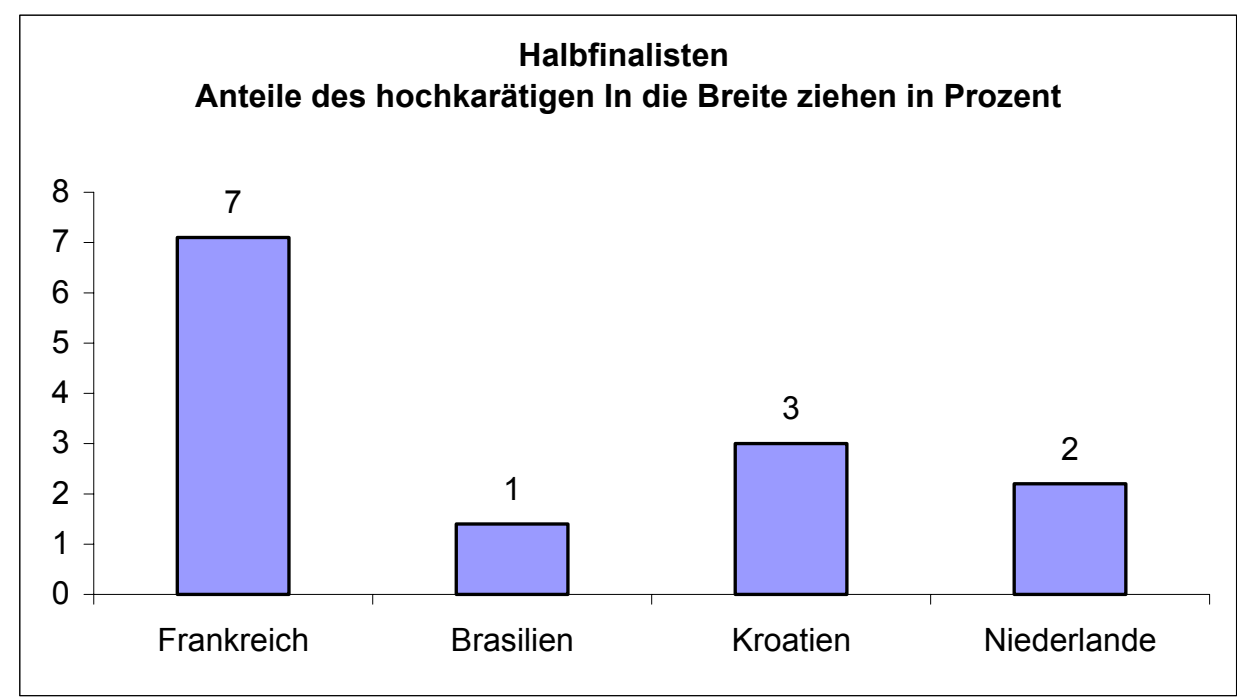

Diagramm 115: Anteile des hochkarätigen „In die Breite ziehen“ in Prozent bei den Halbfinalisten.

39 (87 \%) des „In die Breite ziehen“ von den Niederlanden wurden mit „normal“ bewertet und bildeten den größten Anteil. Die weiteren Anteile „normalen“ „In die Breite ziehen" lauteten 61 (84 \%) für Brasilien, 44 (79 \%) für Frankreich und 23 (70 \%) für Kroatien.

Dem folgenden Diagramm sind die Anteile des normalen „In die Breite ziehen“ in Prozent für die Halbfinalisten zu entnehmen. 


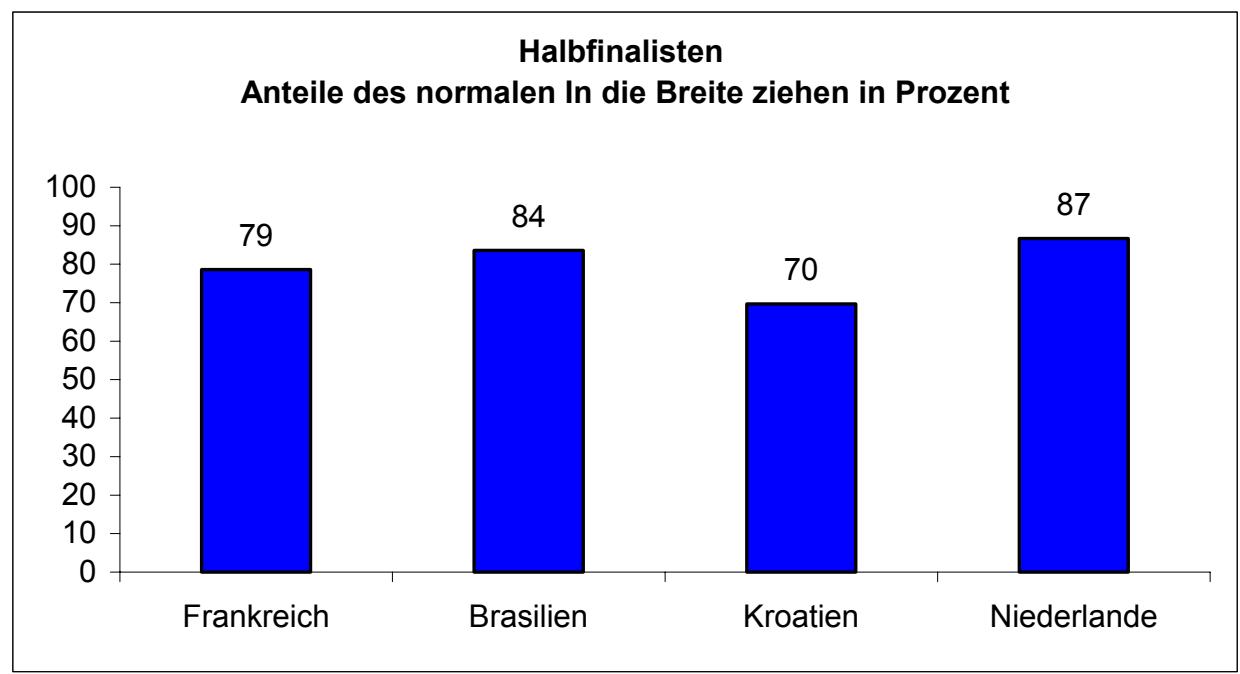

Diagramm 116: Anteile des normalen „In die Breite ziehen“ in Prozent bei den Halbfinalisten.

Ein „schwaches“ „in die Breite ziehen“ wurde mit einem Anteil von 9 (27\%) am häufigsten bei Kroatien festgestellt. Es folgen Brasilien mit 11 (15\%), Frankreich mit 8 (14\%) und schließlich die Niederlande mit 5 (11\%).

Dem folgenden Diagramm sind die Anteile des schwachen „In die Breite ziehen“ in Prozent für die Halbfinalisten zu entnehmen.

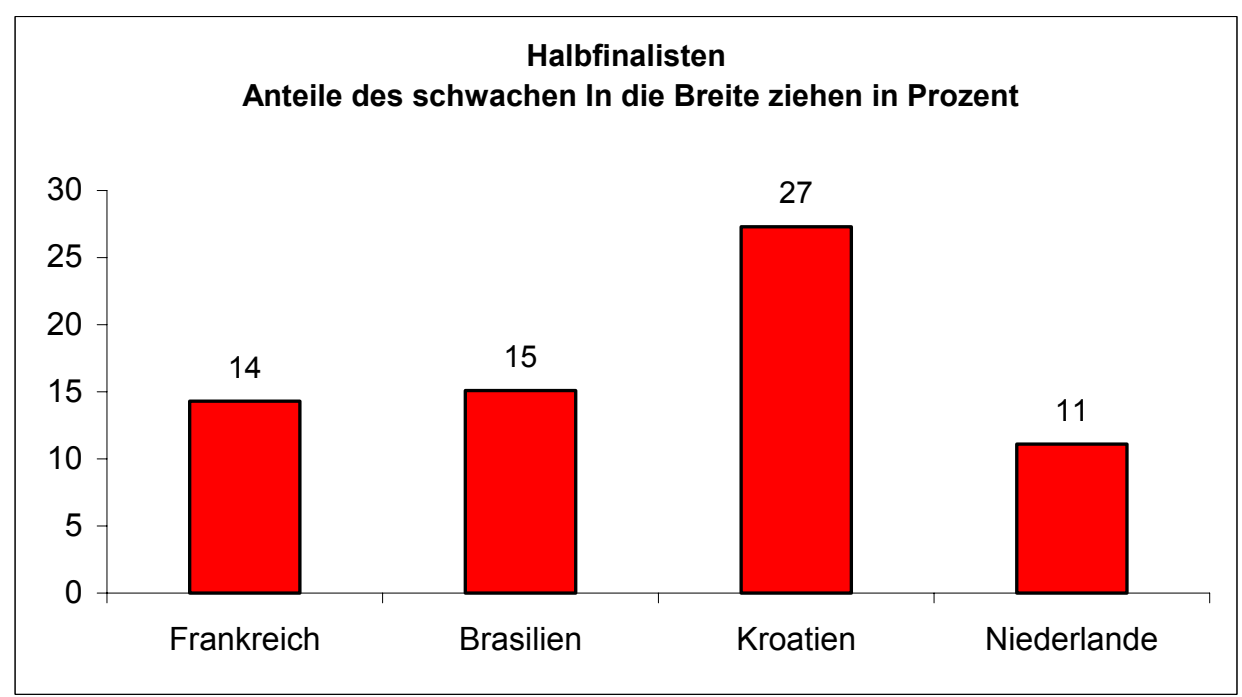

Diagramm 117: Anteile des schwachen "In die Breite ziehen“ in Prozent bei den Halbfinalisten.

Aus den Diagrammen 115, 116 und 117 lässt sich schließen, dass das normale In die Breite ziehen besser ist als das hochkarätige und das misslungene. Die Gründe 
dafür liegen vielleicht darin, dass die Spieler das In die breite ziehen zu weit vom gegnerischen Tor entfernt unternehmen, wodurch der Druck auf die Angreifer geringer ist.

\subsection{Prozentuale Qualitätsanteile defensiv-taktischer Elemente}

Im folgenden Kapitel wird die Qualität der defensiv-taktischen Fähigkeiten der einzelnen Mannschaften beschrieben. Es wird aufgezeigt, mit welcher prozentualen Häufigkeit eine bestimmte Mannschaft bezüglich eines bestimmten offensiv-taktischen Elementes den Bewertungsklassen „gut“, „normal“ und „schwach“ zugeordnet wurde. Aufgrund der unterschiedlichen Anzahl von bestrittenen Spielen werden die Mannschaften hierbei in Achtel-, Viertel- und Halbfinalisten aufgeteilt.

\subsubsection{Achtelfinalisten}

Als Achtelfinalisten werden diejenigen Mannschaften bezeichnet, die im Achtelfinale ausgeschieden sind und zu deren Analyse deshalb nur ein Spiel herangezogen werden konnte. Zu diesen Achtelfinalisten gehören England, Chile, Mexiko, Jugoslawien, Nigeria, Paraguay, Rumänien und Norwegen.

\subsubsection{Pressing hinten}

Das „Pressing hinten“ ist eine mannschaftliche Eigenschaft und beschreibt die Fähigkeit der Mannschaft bzw. Teilen der Mannschaft, sich im eigenen Drittel des Spielfeldes so nah an den ballführenden Gegenspieler und seine möglichen Anspielpartner heranzubewegen, dass dieser unter Druck gerät und zu einem Fehler gezwungen wird. Eine wichtige Taktik ist das Pressing hinten, da der Verteidigungsraum der hinterste Bereich der Mannschaft auf dem Spielfeld ist. Wenn ein Angreifen einen Verteidiger täuscht, so steht er schnell vor dem Tor.

Hinsichtlich der Qualität der „Pressing hinten“ wurde festgestellt: England hatte mit 27 von 102 Mal (26 \%) den größten Wert an hochkarätigen Pressing hinten. Absteigend folgen Nigeria mit 13 von 56 (23\%), Norwegen mit 7 von 38 (18\%), Paraguay hatte 24 von 131 (18\%), Rumänien mit 8 von 45 (18\%), Jugoslawien mit 11 von 74 (15\%) Mexiko mit 6 von $41(15 \%)$. Chile mit 3 von 54 (6\%) hatte die kleinste Anteil „hochkarätig Pressing hinten“. 
Dem folgenden Diagramm sind die Anteile des hochkarätigen Pressing hinten in Prozent für die Achtelfinalisten zu entnehmen.

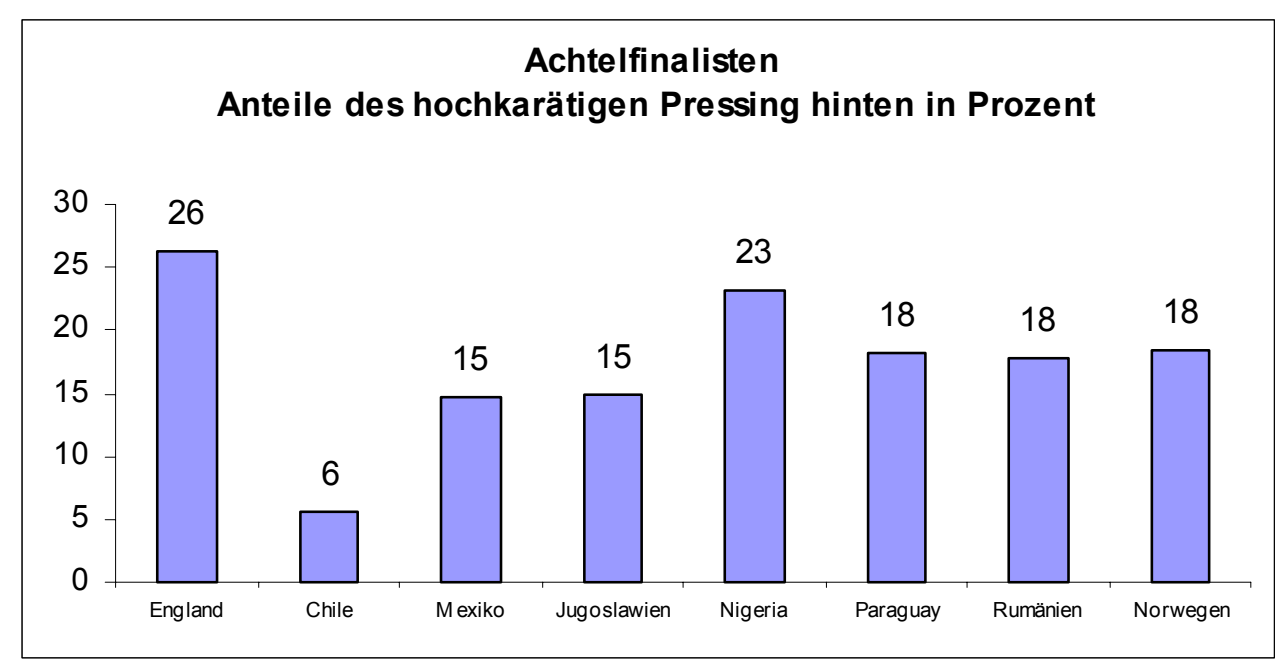

Diagramm 118: Anteile des hochkarätigen „Pressing hinten“ in Prozent bei den Achtelfinalisten.

38 (70 \%) der „Pressing hinten“ von Chile wurden mit „normal“ bewertet und bildeten den größten Anteil. Die weiteren Anteile „normalen Pressing hinten“ lauteten 92 (70 \%) für Paraguay, 28 (68 \%) für Mexiko, 25 (66 \%) für Norwegen, 61 (59 \%) für England, 33 (59 \%) für Nigeria, 26 (58 \%) für Rumänien und 42 (57 \%) für Jugoslawien. Das folgende Diagramm zeigt die Anteile des „normalen Pressing hinten“ in Prozent für die Achtelfinalisten.

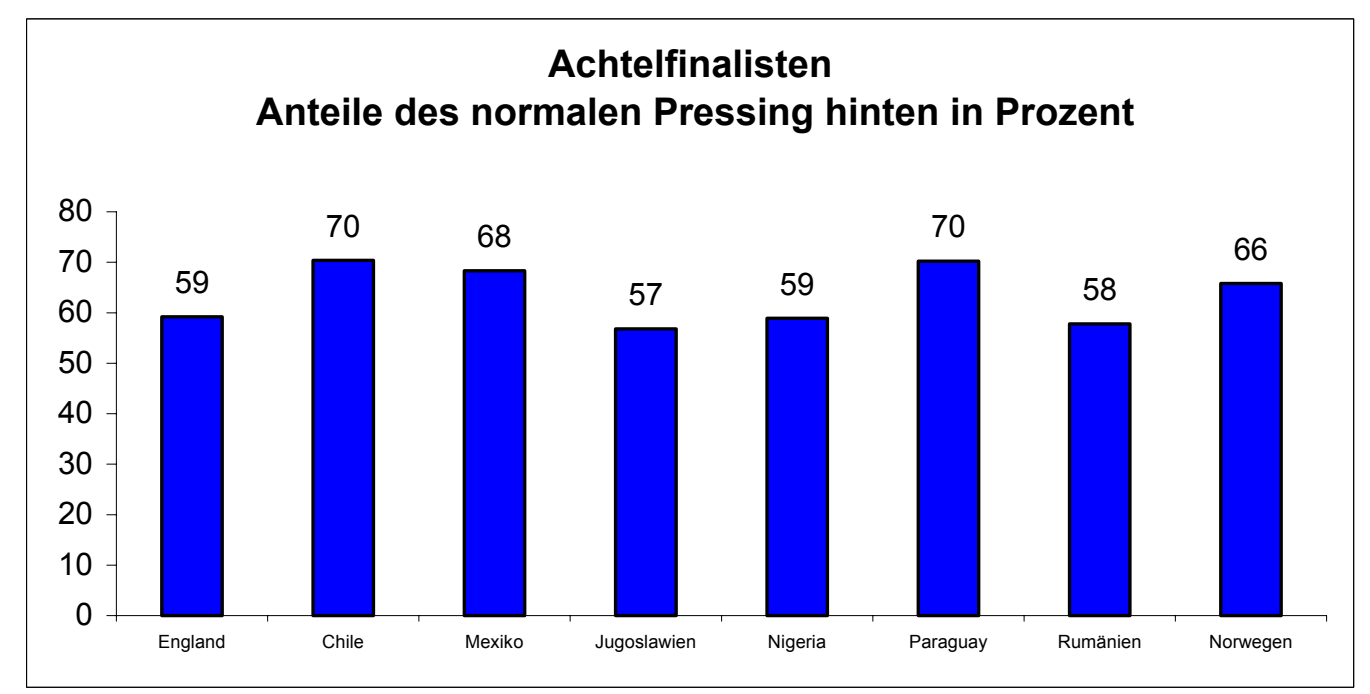

Diagramm 119: Anteile des „normalen Pressing hinten“ in Prozent bei den Achtelfinalisten 
Ein „schwaches Pressing hinten“ wurde mit einem Anteil von 21 (28 \%) am häufigsten bei Jugoslawien festgestellt. Es folgen Rumänien $11(24 \%)$, Chile $13(24 \%)$, Nigeria 10 (18\%), Mexiko 7 (17\%), Norwegen 6 (16\%), England 15 (15\%) und schließlich Paraguay 15 (12\%).

Das folgende Diagramm zeigt die Anteile des „schwachen Pressing hinten“ in Prozent für die Achtelfinalisten.

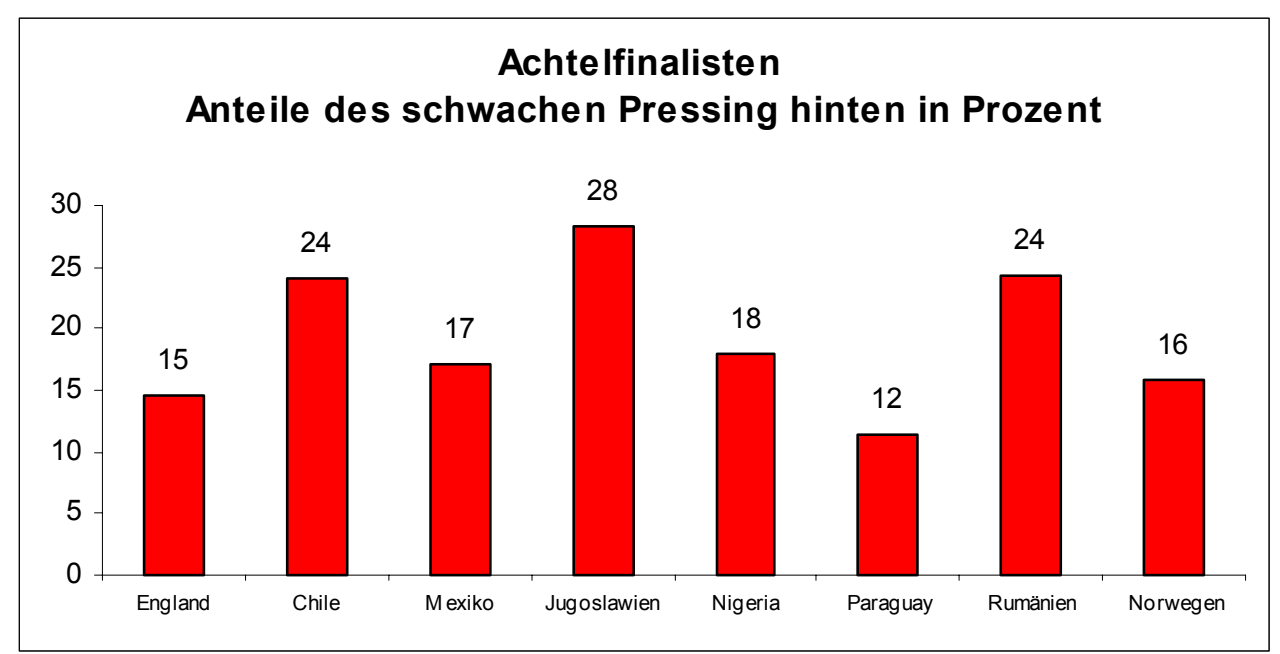

Diagramm 120: Anteile des „schwachen Pressing hinten“ in Prozent bei den Achtelfinalisten.

Aus den Diagrammen 118, 119 und 120 wird deutlich, dass der Anteil des hochkarätigen Pressing hinten geringer ist als das gescheiterte und das gute Pressing hinten weit öfter geschieht als die beiden anderen Kategorien. Der Grund liegt wahrscheinlich darin, dass die meisten Mannschaften im Achtelfinale sich überwiegend auf das Verteidigungsspiel stützen. Das führt dazu, dass die Mannschaft einige Fehler in der Nähe von 12 Metern vom Tor begeht, was eine große Gefahr für das Tor darstellt.

\subsubsection{Pressing Mitte}

Das „Pressing Mitte" ist eine Mannschaftstaktik und beschreibt die Fähigkeit der Mannschaft bzw. Teilen der Mannschaft, sich im mittleren Drittel des Spielfeldes so nah an den ballführenden Gegenspieler und seine möglichen Anspielpartner heranzubewegen, dass dieser unter Druck gerät und zu einem Fehler gezwungen wird. Das Pressing Mitte ist der zweite Bereich, in dem die Mannschaft ihr Tor sichern kann, ohne sich einer Gefahr auszusetzen. Wenn das Pressing Mitte schnell ausgeführt wird, kann die Mannschaft ihren Gegnern gefährlich werden. 
„Hochkarätigen Pressing Mitte" erzielten sämtliche Achtelfinalisten 0 \%.

Das folgende Diagramm zeigt die Anteile des "hochkarätigen Pressing“ hinten in Prozent für die Achtelfinalisten.

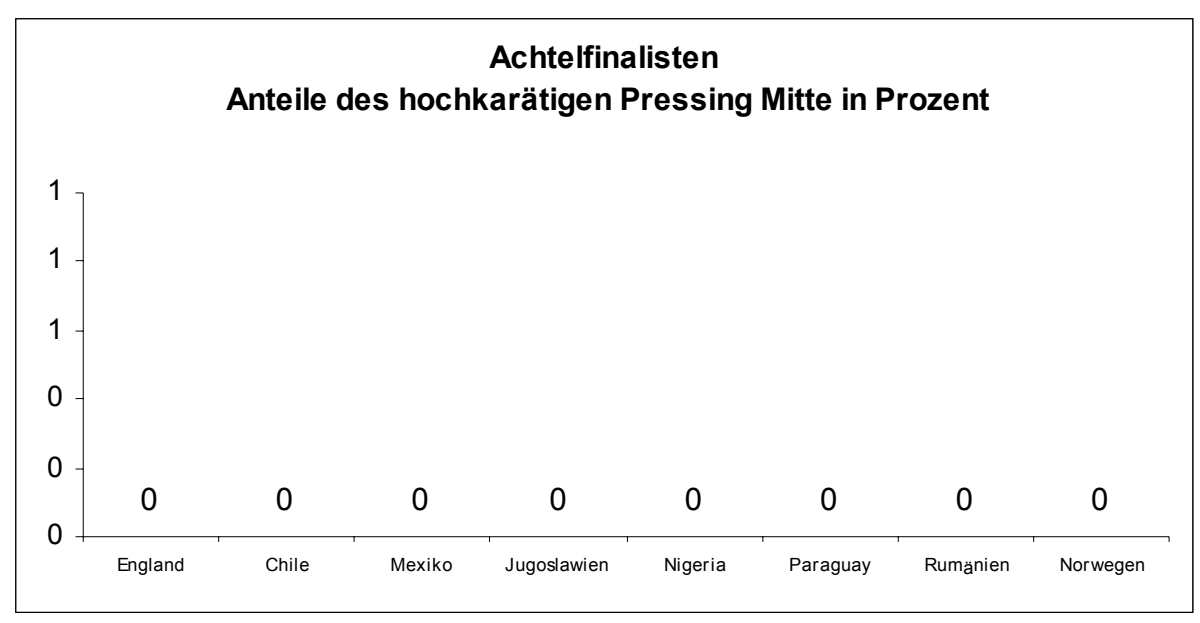

Diagramm 121: Anteile des "hochkarätige Pressing Mitte“ in Prozent bei den Achtelfinalisten.

31 von 33 (94\%) des „Pressing Mitte" von Chile wurden mit "normal“ bewertet und bildete die größte Anteil. Die weiteren Anteile „normalen Pressing Mitte“ lauteten 23 von 25 (92 \%) von Jugoslawien, 20 von 22 (91 \%) von Mexiko, 18 von 20 (90 \%) für England, 33 von 37 (89 \%) für Rumänien, 38 von 47 (81\%) für Nigeria, 28 von 35 (80 \%) für Norwegen und 39 von 50 (78 \%) für Paraguay.

Das folgende Diagramm zeigt die Anteile des "normalen Pressing Mitte“ in Prozent für die Achtelfinalisten.

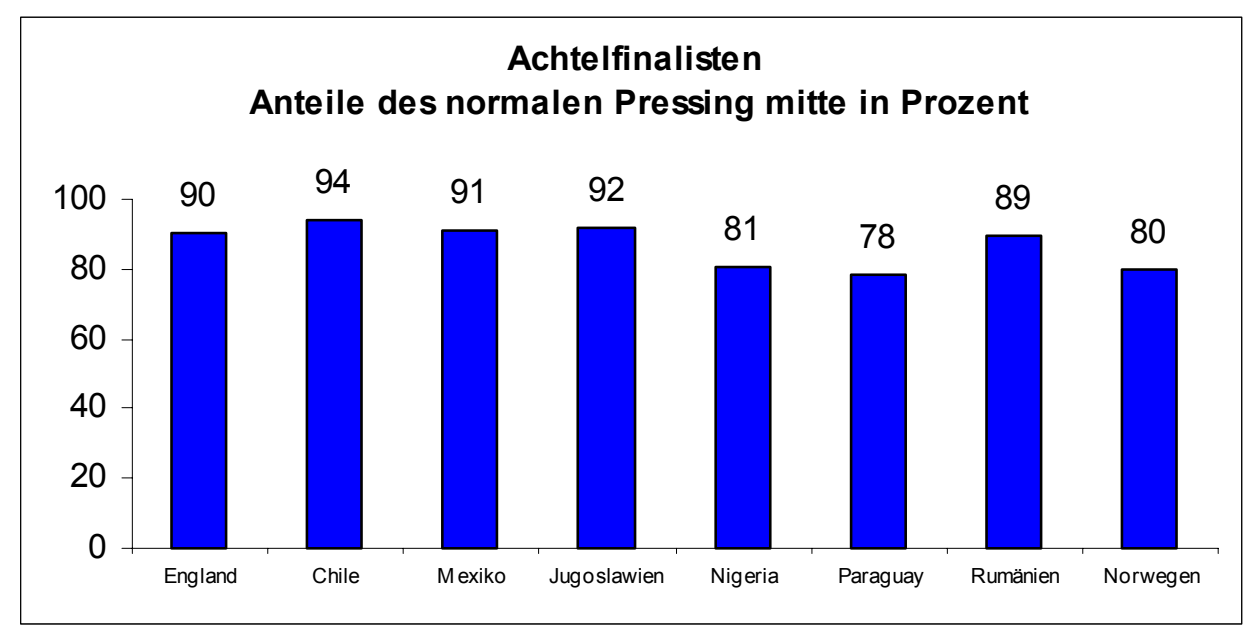

Diagramm 122: Anteile des "normalen Pressing Mitte“ in Prozent bei den Achtelfinalisten. 
Ein „schwaches Pressing Mitte“ wurde mit einem Anteil von 11 (22 \%) am häufigsten bei Paraguay festgestellt. Es folgen Norwegen mit 7 (20\%), Nigeria mit 9 (19\%), Rumänien mit 4 (11\%), England mit 2 (10\%), Mexiko mit 2 (9\%), Jugoslawien mit 2 (8\%) und schließlich Chile mit 2 (6\%).

Das folgende Diagramm zeigt die Anteile des schwachen Pressing Mitte in Prozent für die Achtelfinalisten.

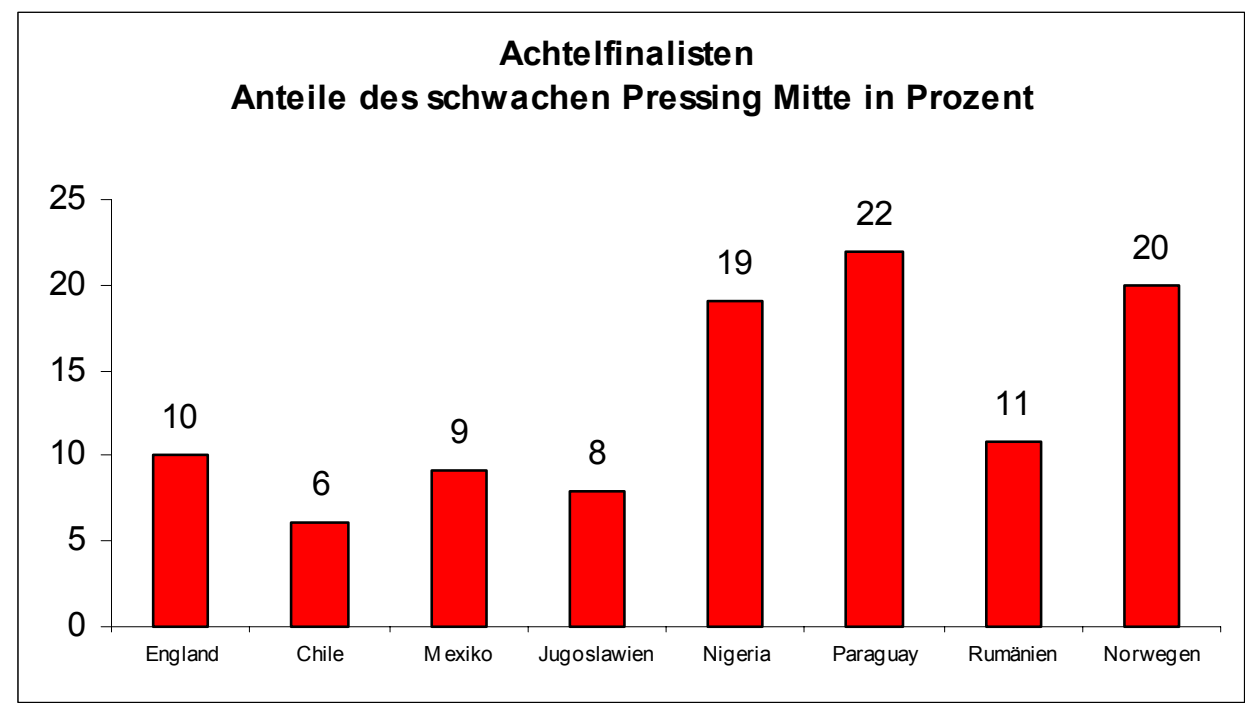

Diagramm 123: Anteile des „schwachen Pressing Mitte“ in Prozent bei den Achtelfinalisten.

Aus den Diagrammen 121, 122 und 123 geht hervor, dass das „hochkarätige Pressing Mitte" sehr schwach in seiner Wirkung ist, da keine der beiden Mannschaften den Ball aus der Mitte abnehmen und ein Tor erzielen kann. Zu begründen ist das mit der Langsamkeit der Spieler beim gekonterten Angriff.

\subsubsection{Pressing vorne}

Das „Pressing vorne“ ist eine Mannschaftstaktik und beschreibt die Fähigkeit der Mannschaft bzw. Teilen der Mannschaft, sich im gegnerischen Drittel des Spielfeldes so nah an den ballführenden Gegenspieler und seine möglichen Anspielpartner heranzubewegen, dass dieser unter Druck gerät und zu einem Fehler gezwungen wird. Hatte Nigeria und Rumänien je 1 von 6 „Pressing vorne“ als hochkarätig mit jeweils (17 \%) und bildeten den größten Wert. England, Chile, Mexiko, Jugoslawien, Paraguay und Norwegen hatten mit jeweils $0 \%$ den kleinsten Anteil „,hochkarätigen Pressing vorne“. 
Dem folgenden Diagramm sind die Anteile hochkarätigen Pressing vorne in Prozent für die Achtelfinalisten zu entnehmen.

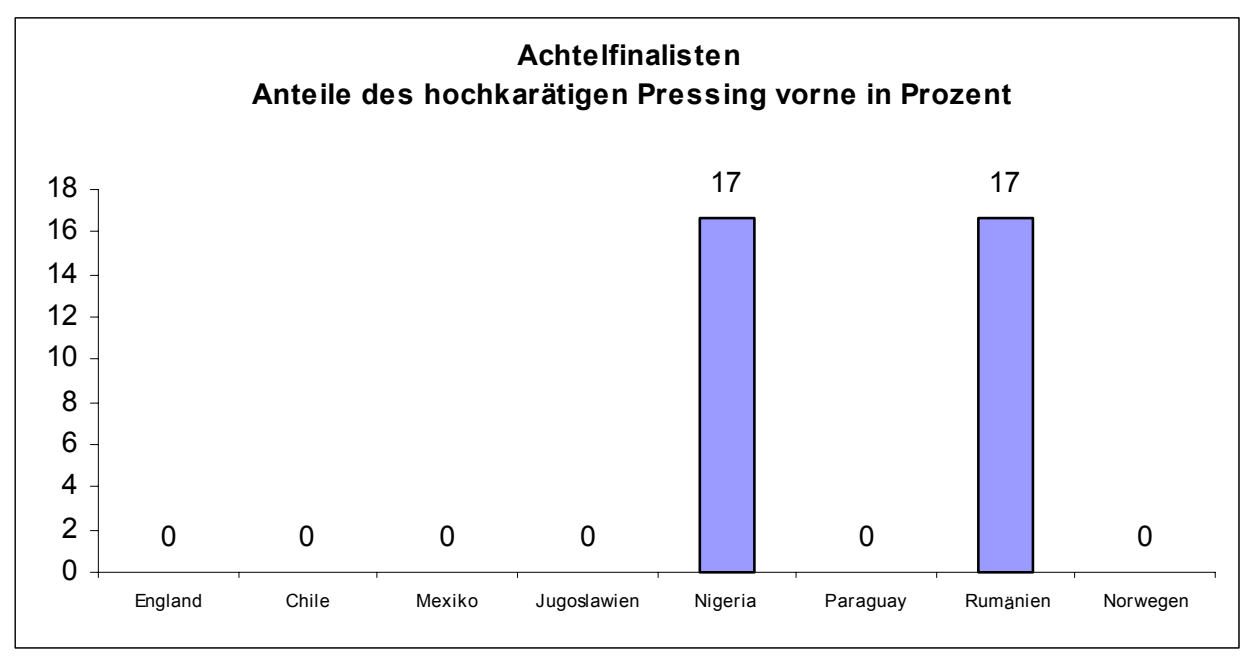

Diagramm 124: Anteile des „hochkarätigen Pressing vorne“ in Prozent bei den Achtelfinalisten.

6 von 7 (86 \%) des „Pressing vorne“ von England wurden mit „normal“ bewertet und bildete den größten Anteil. Die weiteren Anteile „normalen Pressing vorne“ lauteten 5 von 6 (83 \%) bei Chile, bei Paraguay und Norwegen je 4 von 5 (80 \%) für Jugoslawien beträgt es 3 von 6 (50 \%), für Nigeria und Rumänien und 1 von 2 (50\%) für Mexiko.

Dem folgenden Diagramm zeigt die Anteile des normalen „Pressing vorne“ in Prozent für die Achtelfinalisten zu entnehmen.

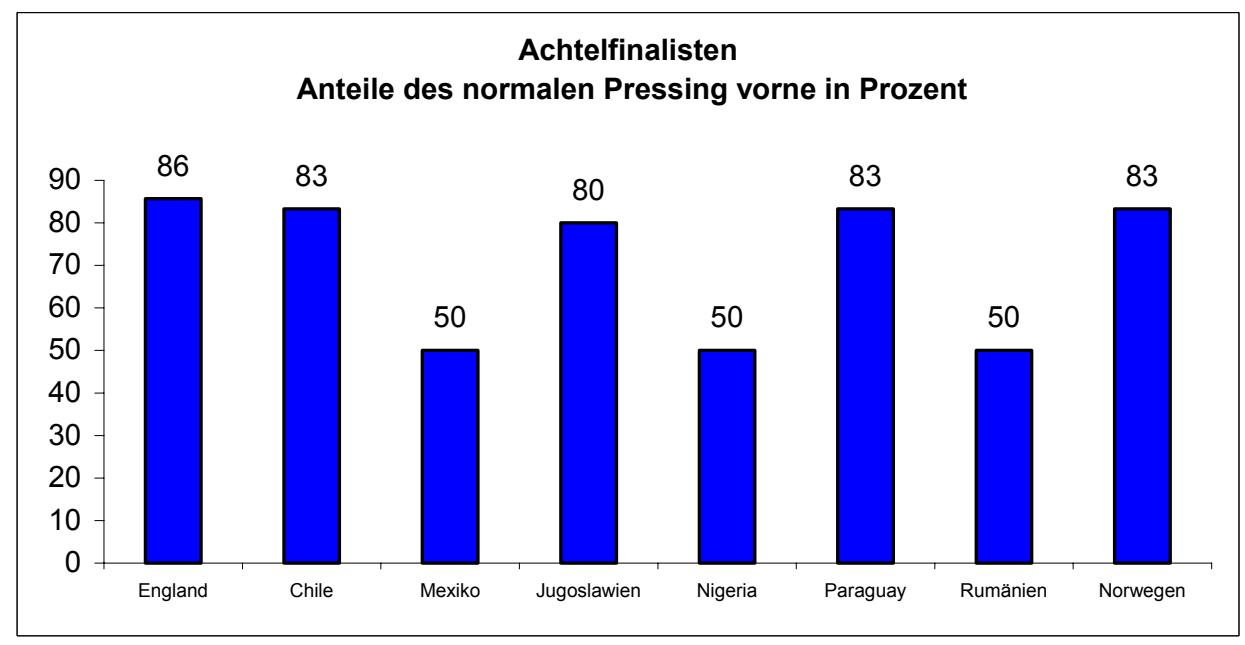

Diagramm 125: Anteile des „normalen Pressing vorne“ in Prozent bei den Achtelfinalisten. 
Ein „schwaches Pressing vorne“ wurde mit einem Anteil von 1 (50 \%) am häufigsten bei Mexiko festgestellt. Es folgen Nigeria und Rumänien 2 (33\%), Jugoslawien 1 (20 $\%)$, England 1 (14\%) und schließlich Chile, Paraguay und Norwegen 1 (17\%).

Das folgende Diagramm zeigt die Anteile des schwachen Pressing vorne in Prozent für die Achtelfinalisten.

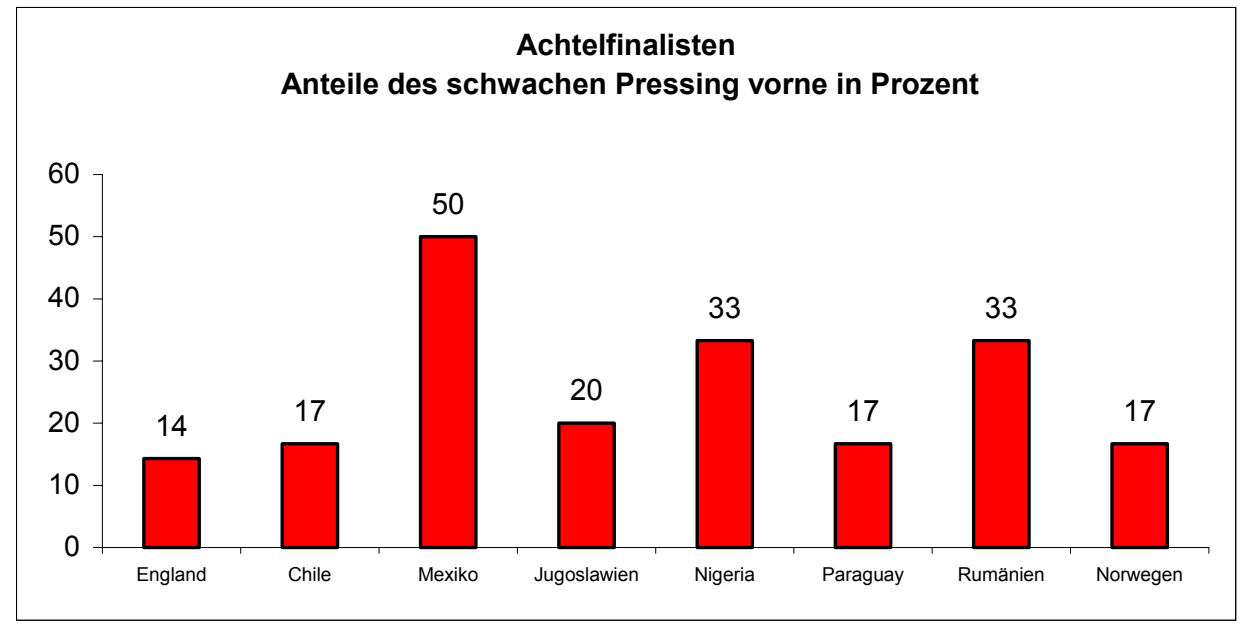

Diagramm 126: Anteile des „schwachen Pressing vorne“ in Prozent bei den Achtelfinalisten.

Aus den Diagrammen 124, 125 und 126 wird deutlich, dass die Anteile des hochkarätigen Pressing vorne geringer sind als das gescheiterte, und das normale Pressing hinten weit öfter geschieht als die beiden anderen Kategorien. Der Grund liegt wahrscheinlich darin, dass die meisten Mannschaften im Achtelfinale sich überwiegend auf das Verteidigungsspiel stützten. Das führte dazu, dass in dem Drittel des Gegners nach Ballübernahme versucht wurde, mit wenigen Angreifern den Ball wieder abzunehmen. Keine Mannschaft außer Nigeria und Rumänien konnte den Ball vorne nach dem Abnehmen wieder in ihren Besitz bringen und ein Tor zu schießen versuchen.

\subsubsection{Raumaufteilung}

Die Raumaufteilung ist eine Mannschaftstaktik und beschreibt die Fähigkeit der Mannschaft bzw. Teilen der Mannschaft, sich so im Raum zu verteilen, dass der gegnerischen Mannschaft möglichst wenig Raum zur Verfügung steht. Die Raumaufteilung ist die Verteilung der Spieler auf dem Spielfeld, wenn der Ball von der Mannschaft abgenommen wird. Sie soll es dem Angreifer ermöglichen, den Ball leicht abzunehmen. 
Hinsichtlich der Qualität der Raumaufteilung wurde festgestellt: England hatte mit 25 von 98 (26\%) den größten Wert. Absteigend folgen Nigeria mit 7 von 59 (12\%), Rumänien mit 6 von 70 (9\%), Jugoslawien mit 5 von 65 (8\%), Mexiko mit 4 von 61 (7\%), Norwegen mit 5 von 76 (7\%) und Paraguay mit 4 von 88 (5\%). Chile hatte mit 1 von 74 (1\%) den kleinsten Anteil "hochkarätiger" Raumaufteilung.

Das folgende Diagramm zeigt die Anteile der hochkarätigen Raumaufteilung in Prozent für die Achtelfinalisten.

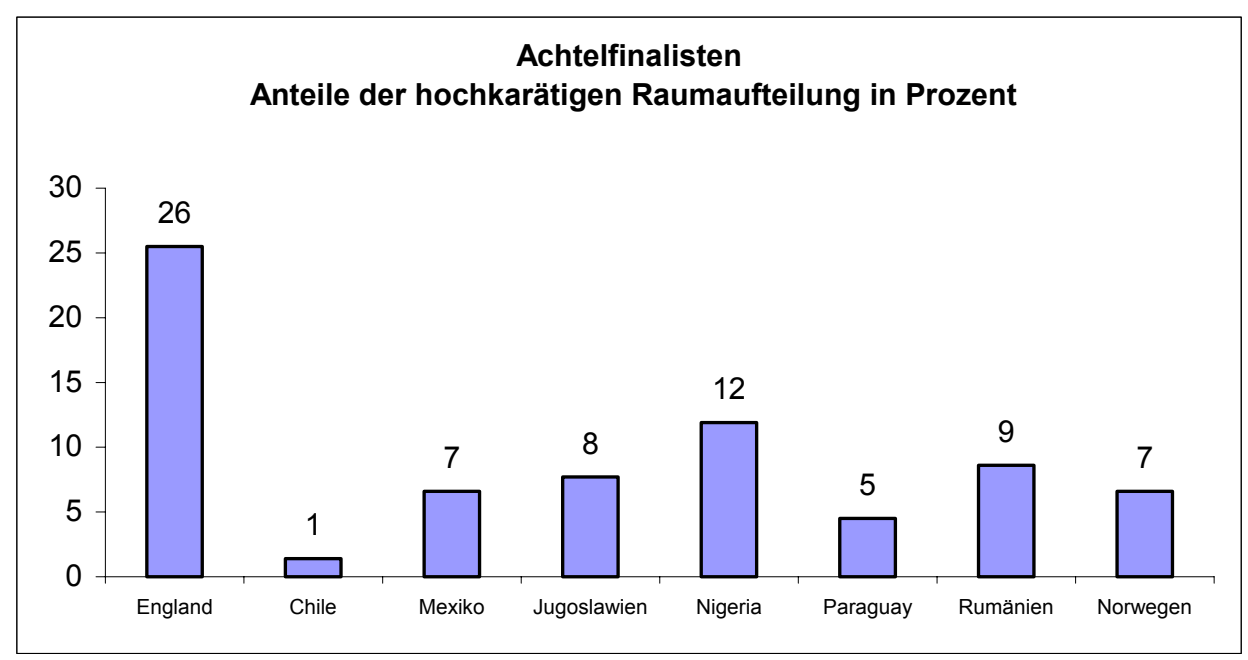

Diagramm 127: Anteile der „hochkarätigen“ Raumaufteilung in Prozent bei den Achtelfinalisten.

66 (89 \%) der Raumaufteilungen von Chile wurden mit "normal“ bewertet und bildeten den größten Anteil. Die weiteren Anteile „normaler" Raumaufteilungen lauteten 60 (86 \%) für Rumänien, 50 (82 \%) für Mexiko, 71 (81 \%) für Paraguay, 47 (80 \%) für Nigeria, 60 (79 \%) für Norwegen, 47 (72\%) für Jugoslawien und 67 (68 \%) für England.

Dem folgenden Diagramm sind die Anteile der "normalen Raumaufteilung“ in Prozent für die Achtelfinalisten zu entnehmen. 


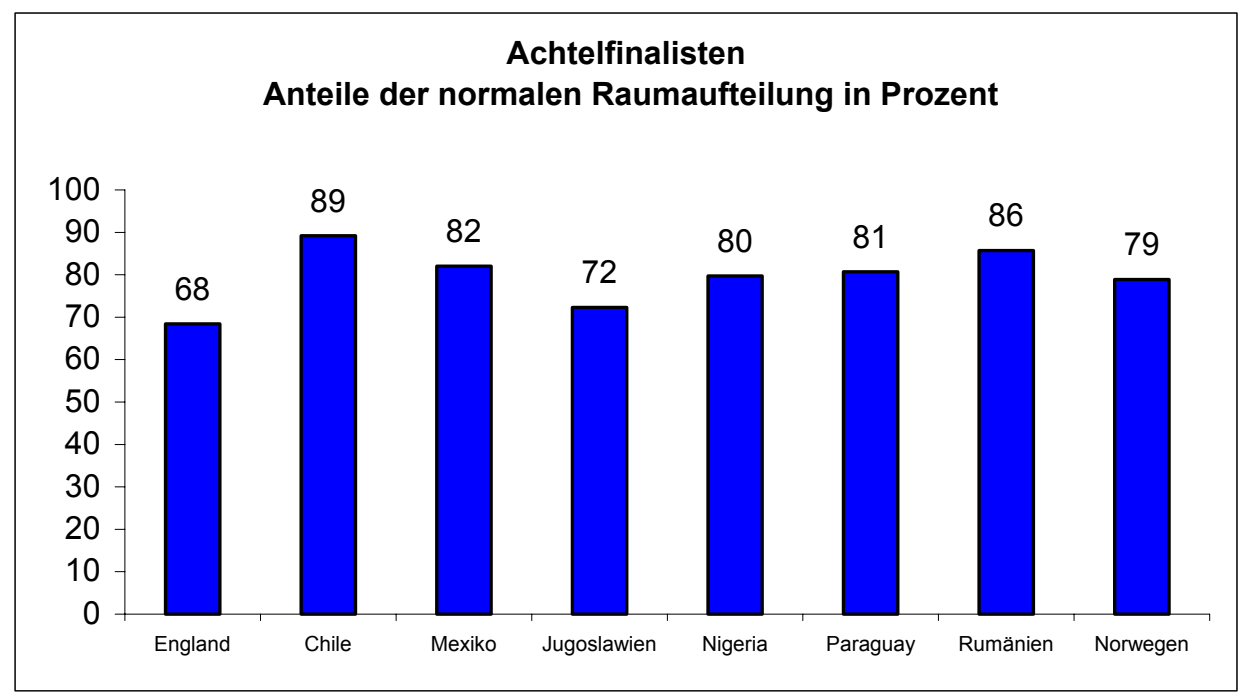

Diagramm 128: Anteile der „normalen“ Raumaufteilung in Prozent bei den Achtelfinalisten.

Eine „schwache“ Raumaufteilung wurde mit einem Anteil von 13 (20\%) am häufigsten bei Jugoslawien festgestellt. Es folgen Paraguay mit 13 (15\%), Norwegen mit 11 (15\%), Mexiko mit 7 (12\%), Chile mit 7 (10\%), Nigeria mit 5 (9\%), England mit 6 (6 $\%)$ und schließlich Rumänien mit 4 (6\%).

Das folgende Diagramm zeigt die Anteile der schwachen Raumaufteilung in Prozent für die Achtelfinalisten.

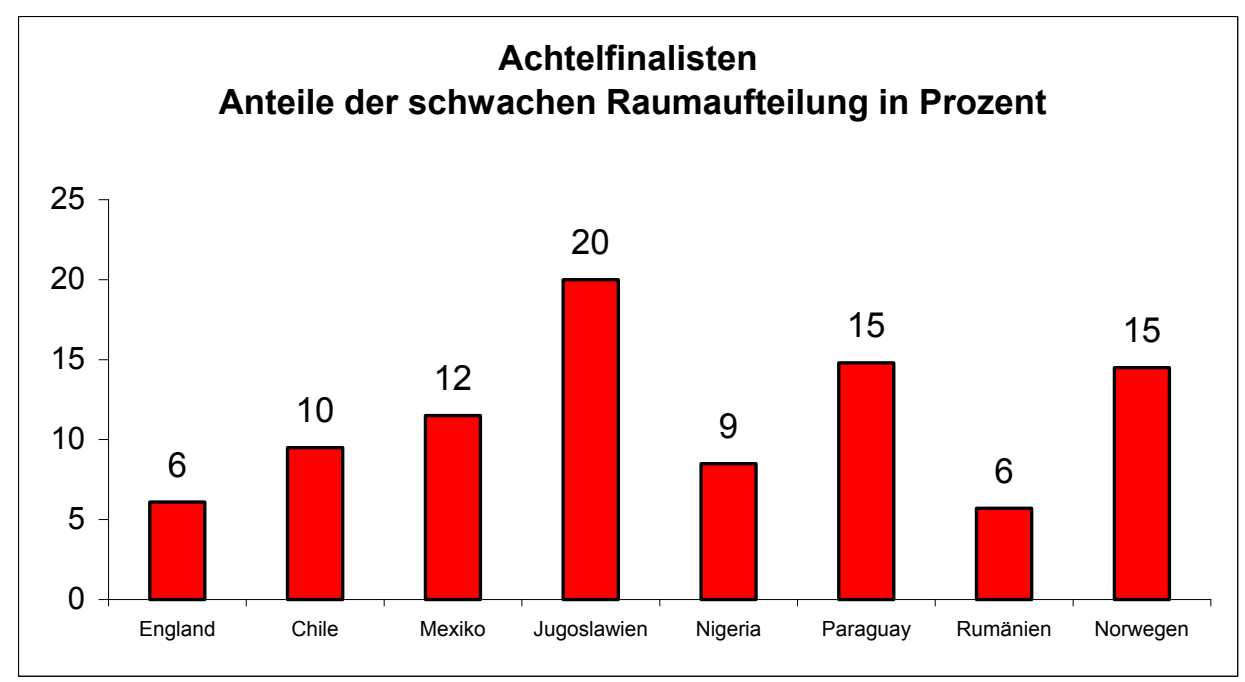

Diagramm 129: Anteile der „schwachen“ Raumaufteilung in Prozent bei den Achtelfinalisten. 
Aus den Diagrammen 127, 128 und 129 geht hervor, dass die normale Raumaufteilung die am meisten angewandte ist, da die beiden spielenden Mannschaften die Spieler besser im mittleren Bereich des Spielfelds verteilen als schwache oder hochkarätige Raufaufteilung. Der Grund liegt wahrscheinlich darin, dass die meisten Mannschaften im Achtelfinale sich überwiegend auf das Verteidigungsspiel stützten.

\subsubsection{Zurückkommen}

Das Zurückkommen ist eine Einzeltaktik und beschreibt die Fähigkeit eines einzelnen Spielers, sich direkt nach einem Ballverlust der eigenen Mannschaft so zu bewegen, dass die Defensivarbeit möglichst optimal unterstützt wird.

Zurückkommen hatte England 28-mal, wovon mit 9 (32 \%) als hochkarätig bezeichnet wurde. Absteigend folgen Rumänien mit 2 von 27 (7\%), Mexiko und Nigeria mit 2 von 35 (6 \%), Norwegen mit 1 von 19 (5\%), Jugoslawien mit 2 von 39 (5\%) und Paraguay mit 2 von 52 (4\%). Chile hatte mit 1 von 33 (3\%) den kleinsten Anteil „hochkarätigen“ Zurückkommens.

Das folgende Diagramm zeigt die Anteile des hochkarätigen Zurückkommens in Prozent für die Achtelfinalisten.

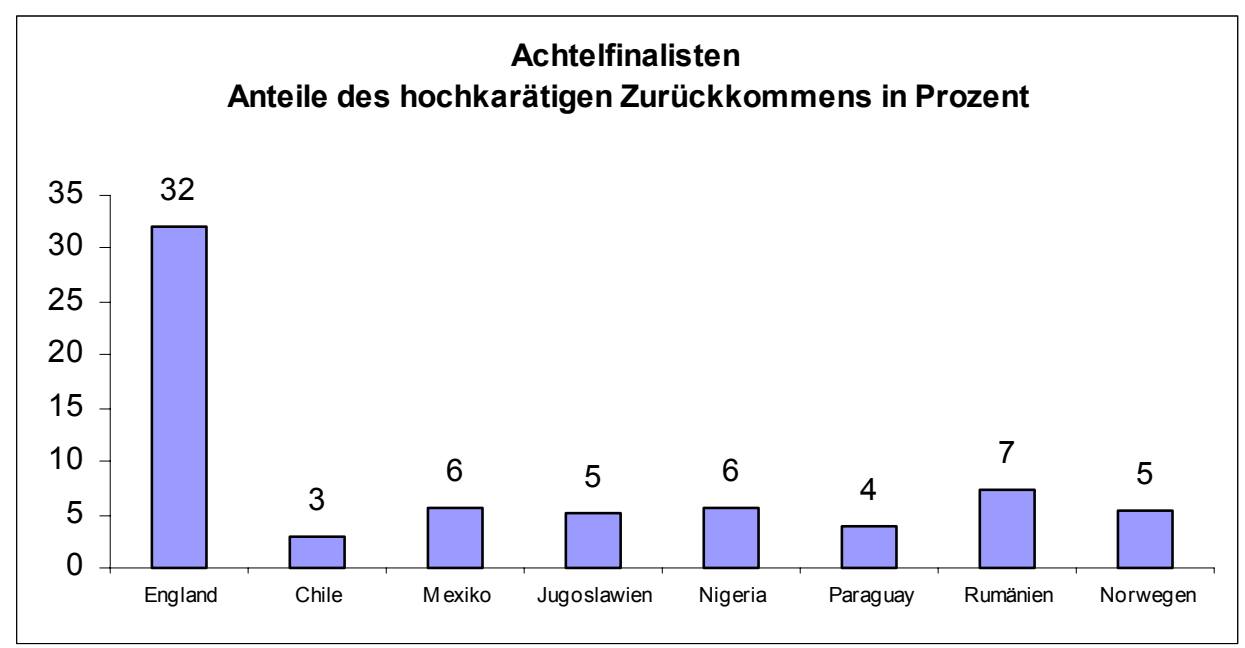

Diagramm 130: Anteile des „hochkarätigen“ Zurückkommens in Prozent bei den Achtelfinalisten.

49 (94 \%) des „Zurückkommens“ von Paraguay wurden mit „normal“ bewertet und bildete den größten Anteil. Die weiteren Anteile „normalen“ Zurückkommens lauten 31 (94 \%) für Chile, 36 (92 \%) für Jugoslawien, 32 (91 \%) für Mexiko und Nigeria, 17 (90 \%) für Norwegen, 24 (89 \%) für Rumänien und 18 (64 \%) für England. 
Dem folgenden Diagramm sind die Anteile des "normalen Zurückkommens“ in Prozent für die Achtelfinalisten zu entnehmen.

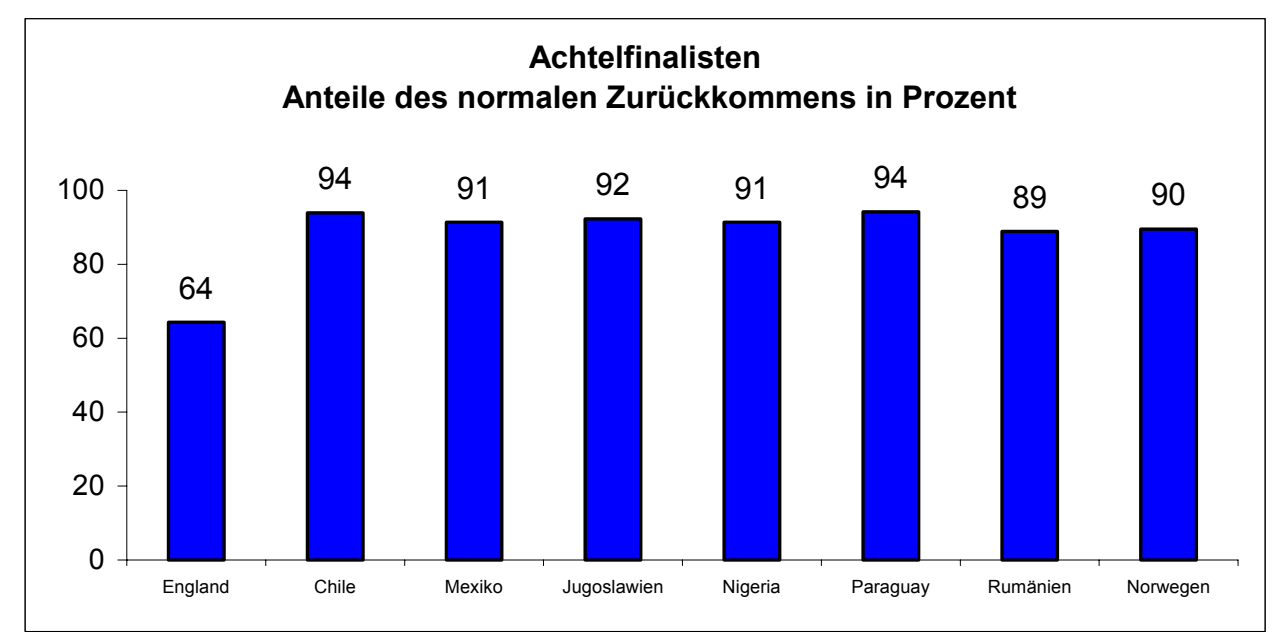

Diagramm 131: Anteile des „normalen“ Zurückkommens in Prozent bei den Achtelfinalisten.

Ein „schwaches" Zurückkommen wurde mit einem Anteil von 1 (5\%) am häufigsten bei Norwegen festgestellt. Es folgen Rumänien 1 (4\%), England 1 (4\%), Chile 1 (3 \%), Mexiko und Nigeria 1 (3\%), Jugoslawien 1 (3 \%) und schließlich Paraguay 1 (2 $\%)$.

Das folgende Diagramm zeigt die Anteile des schwachen Zurückkommens in Prozent für die Achtelfinalisten.

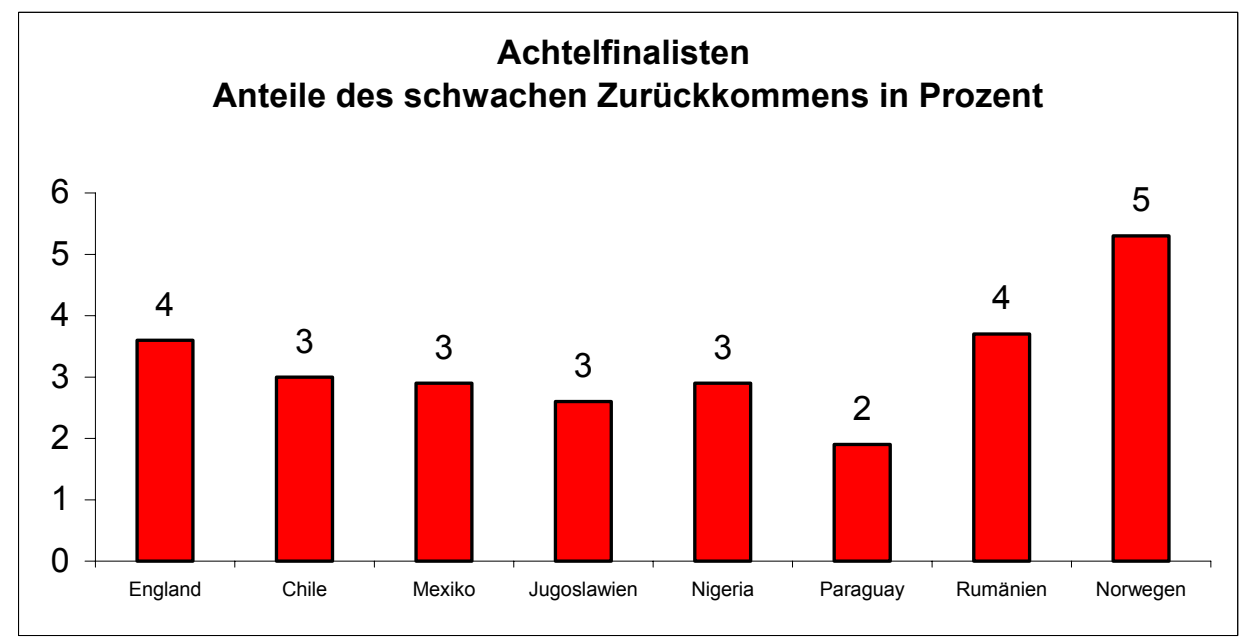

Diagramm 132: Anteile des „schwachen“ Zurückkommens in Prozent bei den Achtelfinalisten. 
Aus der Darstellung der Diagramme 130, 131 und 132 wird deutlich, dass das normalen Zurückkommen öfter vorkam als das hochkarätige und schwache, da die Spieler einen Platz einnehmen, von dem aus sie ihre Mitspieler helfen können.

\subsubsection{Unterstützen}

Das Unterstützen ist eine individuelle Eigenschaft und beschreibt die Fähigkeit eines einzelnen Spielers, sich so in Richtung eines Mitspielers zu bewegen, dass diesem bei der Bekämpfung des ballführenden Gegenspielers möglichst optimale Unterstützung zukommt.

„Gute" Unterstützen hatte Nigeria mit 2-mal, wovon 3 (67\%) als hochkarätig bezeichnet wurde. Absteigend folgen Mexiko mit 1 von 2 (50 \%), England und Jugoslawien mit 1 von 4 (25\%). Chile, Paraguay, Rumänien und Norwegen hatten mit jeweils $0 \%$ den kleinsten Anteil „hochkarätigen“ Unterstützens.

Dem folgenden Diagramm sind die Anteile des hochkarätigen Unterstützens in Prozent für die Achtelfinalisten zu entnehmen.

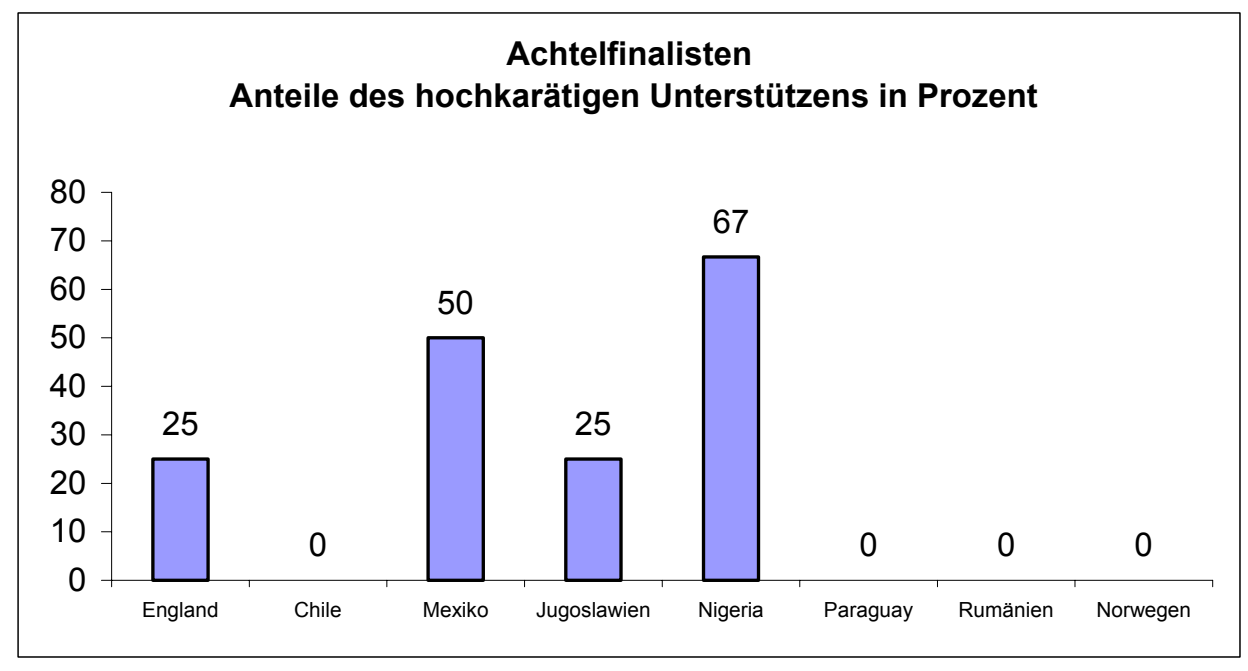

Diagramm 133: Anteile des „hochkarätigen“ Unterstützens in Prozent bei den Achtelfinalisten.

3 von 4 (75 \%) des Unterstützens von Paraguay wurden mit "normal“ bewertet und bildete den größten Anteil. Die weiteren Anteile „normalen“ Unterstützens lauten 2 (50 \%) für England, Chile, Jugoslawien, Rumänien und Norwegen und 0\% für Mexiko und Nigeria. 
Das folgende Diagramm zeigt die Anteile des normalen Unterstützens in Prozent für die Achtelfinalisten.

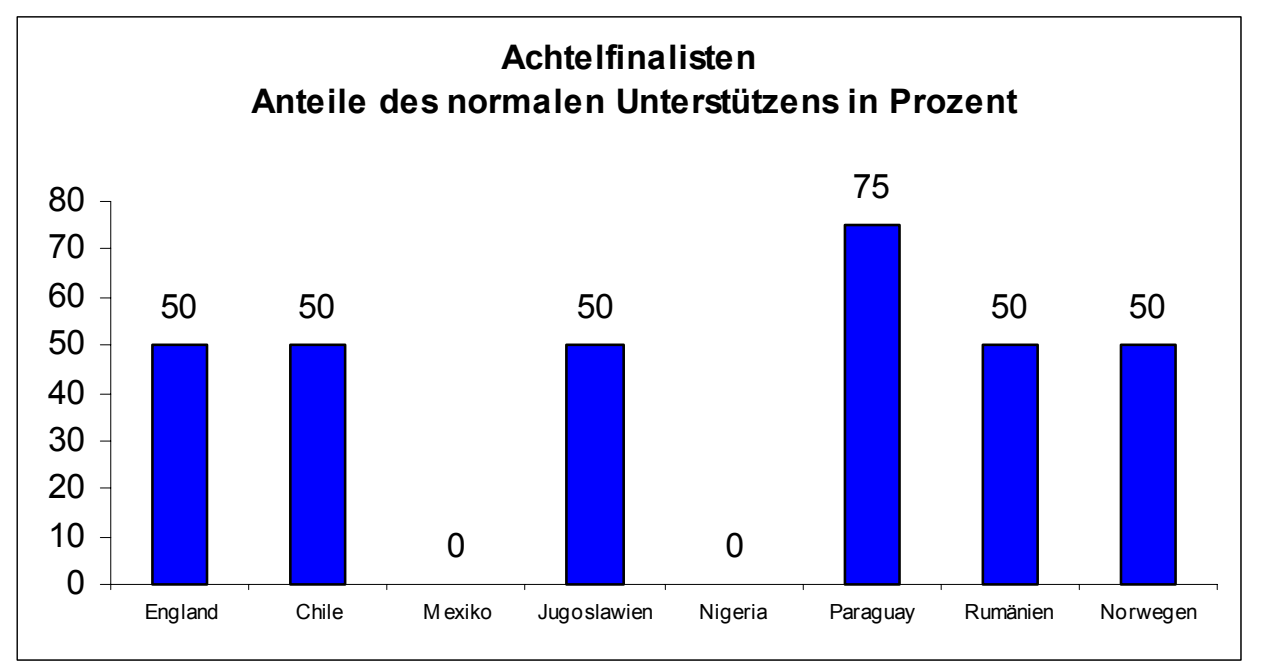

Diagramm 134: Anteile des „normalen“ Unterstützens in Prozent bei den Achtelfinalisten.

Ein "schwaches“ Unterstützen wurde mit einem Anteil von 1 (50 \%) am häufigsten bei Chile, Mexiko, Rumänien und Norwegen festgestellt. Es folgen Nigeria 1 (33\%) und schließlich England, Jugoslawien und Paraguay mit je 1 (25\%).

Das folgende Diagramm zeigt die Anteile des schwachen Unterstützens in Prozent für die Achtelfinalisten.

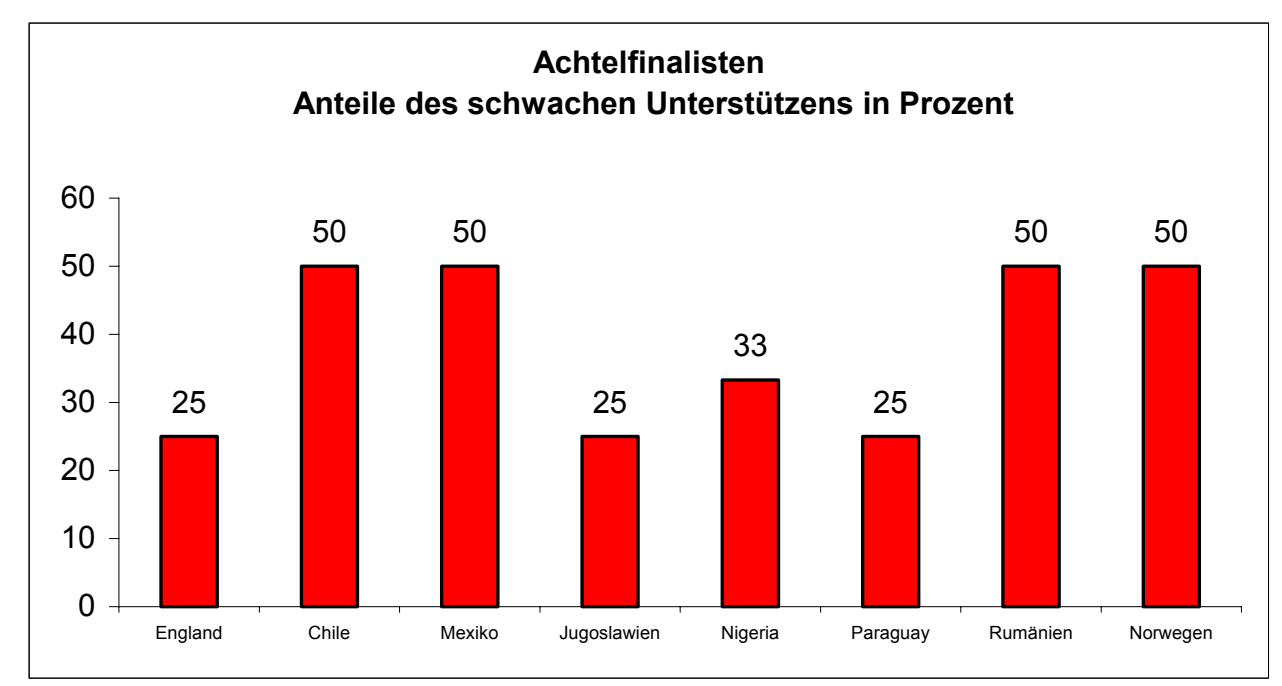

Diagramm 135: Anteile des „schwachen“ Unterstützens in Prozent bei den Achtelfinalisten. 
Aus der Darstellung der Diagramme 133, 134 und 135 wird deutlich, dass das normale Unterstützen öfters zu finden ist als das hochkarätige und schwache. Manchmal unterstützen die Mitspieler nicht zur rechten Zeit oder in der richtigen Position.

\subsubsection{Rückraum sichern}

Das „Rückraum sichern“ ist eine Einzeltaktik und beschreibt die Fähigkeit eines einzelnen Spielers, bei der Defensivarbeit auch das Geschehen auf dem Spielfeld zu kontrollieren, das nicht in dessen Blickwinkel liegt.

Rückraum sichern hatten England und Chile mit jeweils 5-mal, wovon 1 (20\%) als hochkarätig bezeichnet wurde. Absteigend folgen Nigeria und Norwegen mit 1 von 7 (14 \%), Paraguay mit 1 von 9 (11\%). Mexiko, Jugoslawien und Rumänien hatten mit jeweils 1 von 10 (10\%) den kleinsten Anteil "guten“ Rückraumsicherns.

Das folgende Diagramm zeigt die Anteile des "hochkarätigen Rückraumsichern“ in Prozent für die Achtelfinalisten.

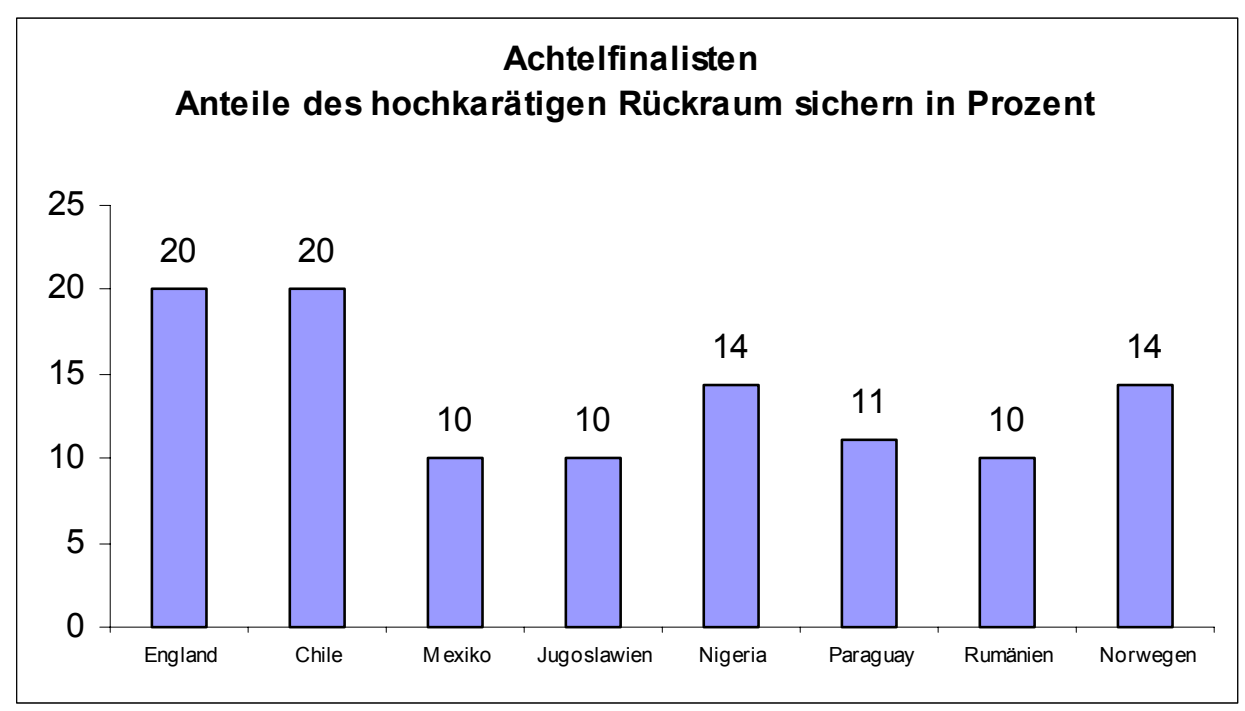

Diagramm 136: Anteile des "hochkarätigen Rückraum sichern“ in Prozent bei den Achtelfinalisten.

$8(80 \%)$ des „Rückraum sichern“ von Mexiko und Jugoslawien wurden mit „normal“ bewertet und bildete den größten Anteil. Die weiteren Anteile „normalen“ „Rückraum sichern“ lauteten 7 (78 \%) für Paraguay, 5 (71 \%) für Norwegen, 3 (60 \%) für England, Chile und Rumänien. 3 (43\%) für Nigeria. 
Dem folgenden Diagramm sind die Anteile des normalen Rückraumsichern in Prozent für die Achtelfinalisten zu entnehmen.

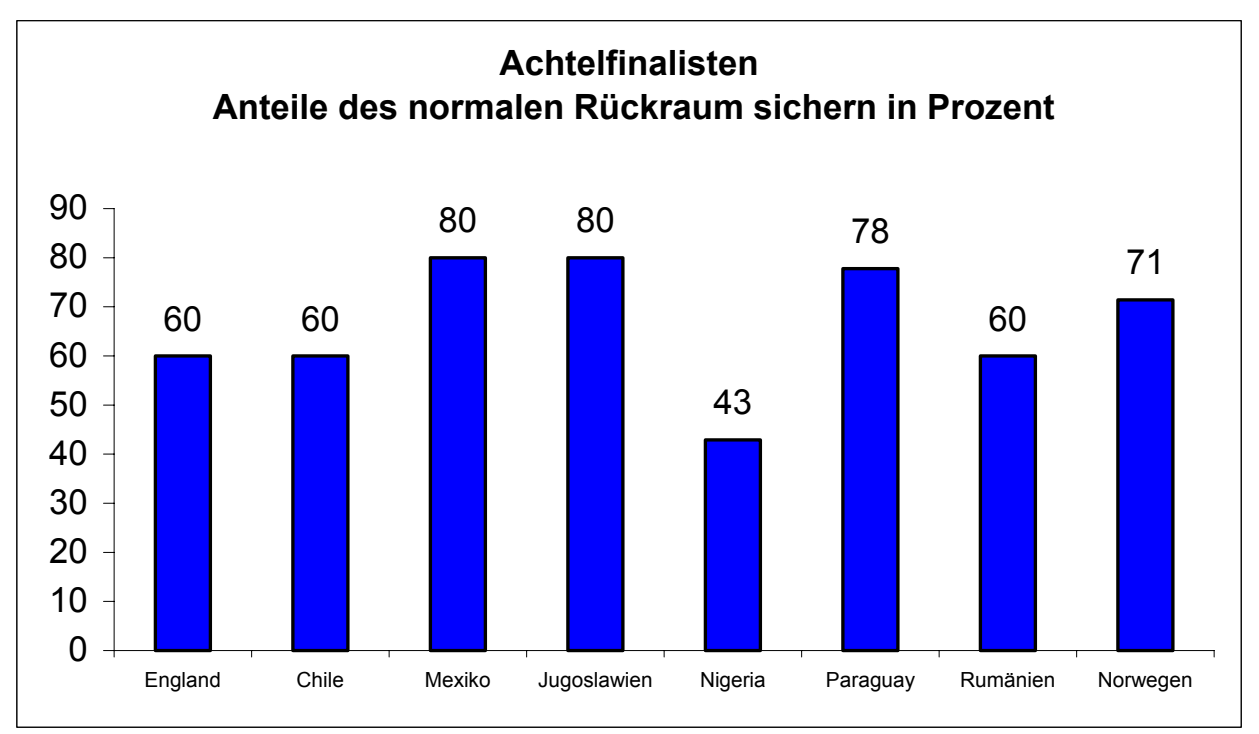

Diagramm 137: Anteile des „normalen“ Rückraum sichern in Prozent bei den Achtelfinalisten.

Ein „schwaches“ Rückraum sichern wurde mit einem Anteil von 3 (43 \%) am häufigsten bei Nigeria festgestellt. Es folgen Rumänien 3 (30 \%), England und Chile 1 (20 $\%)$, Norwegen 1 (14 \%), Paraguay 1 (11\%) und schließlich Mexiko und Jugoslawien je $1(10 \%)$.

Das folgende Diagramm zeigt die Anteile des schwachen Rückraumsicherns in Prozent für die Achtelfinalisten.

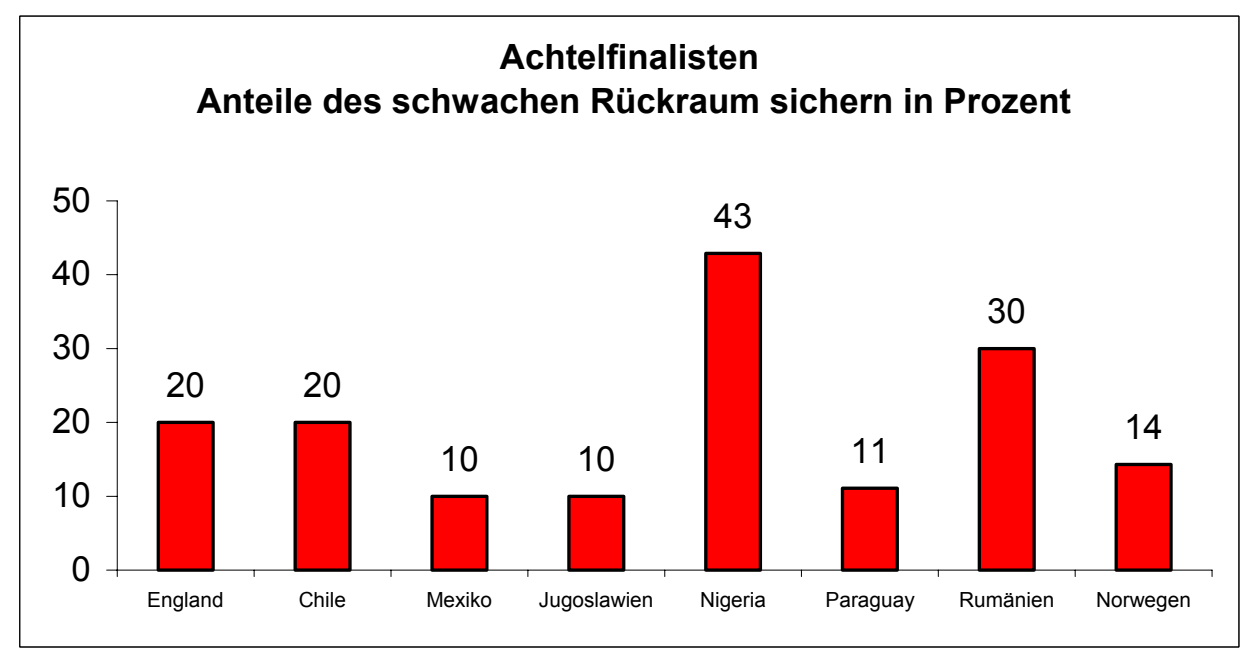

Diagramm 138: Anteile des „schwachen“ Rückraum sichern in Prozent bei den Achtelfinalisten. 
Aus den Diagrammen 136, 137 und 138 wird deutlich, dass das normalen und hochkarätigen Rückraum sichern mehr angewandt wird als das schwache, da die Spieler hinten den Torraum sichern und bei der Defensivarbeit helfen können.

\subsubsection{Abseitsfallen}

Die Abseitsfalle ist eine Mannschaftstaktik und beschreibt die Fähigkeit der Mannschaft bzw. Teilen der Mannschaft, sich gleichzeitig so zu bewegen, dass im Moment der Ballabgabe des Gegners ein gegnerischer Spieler im Abseits steht.

Abseitsfallen wandte England 7-mal an, wovon 6-mal (86 \%) als hochkarätig bewertet wurde. Absteigend folgen Rumänien und Norwegen mit 5 von 6 (83\%), Jugoslawien und Nigeria 3 von 4 (75\%). Chile, Mexiko und Paraguay hatten mit jeweils $0 \%$ der kleinste Anteil „hochkarätiger“ Abseitsfälle.

Dem folgenden Diagramm sind die Anteile der hochkarätigen Abseitsfallen in Prozent für die Achtelfinalisten zu entnehmen.

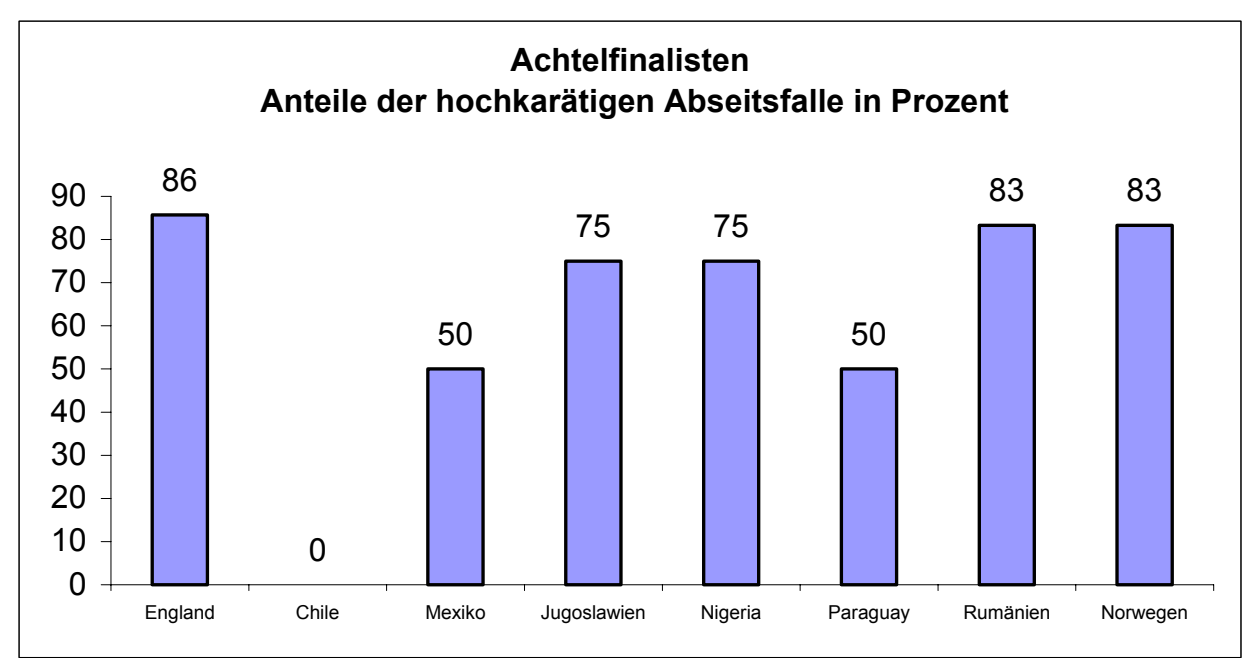

Diagramm 139: Anteile der hochkarätigen Abseitsfallen in Prozent für bei den Achtelfinalisten.

Eine „schwache“ Abseitsfalle gab es bei allen Mannschaften nur je einmal. Mit einem Anteil von 1 bei keinen anderen ist dadurch bei Chile ein relativer Anteil von $100 \%$ festzustellen. Es folgen Mexiko und Paraguay 1 (50 \%), Jugoslawien und Nigeria 1 (25\%), Rumänien und Norwegen 1 (17\%) und schließlich England 1 (14 \%). 
Das folgende Diagramm zeigt die Anteile der schwachen Abseitsfalle in Prozent für die Achtelfinalisten.

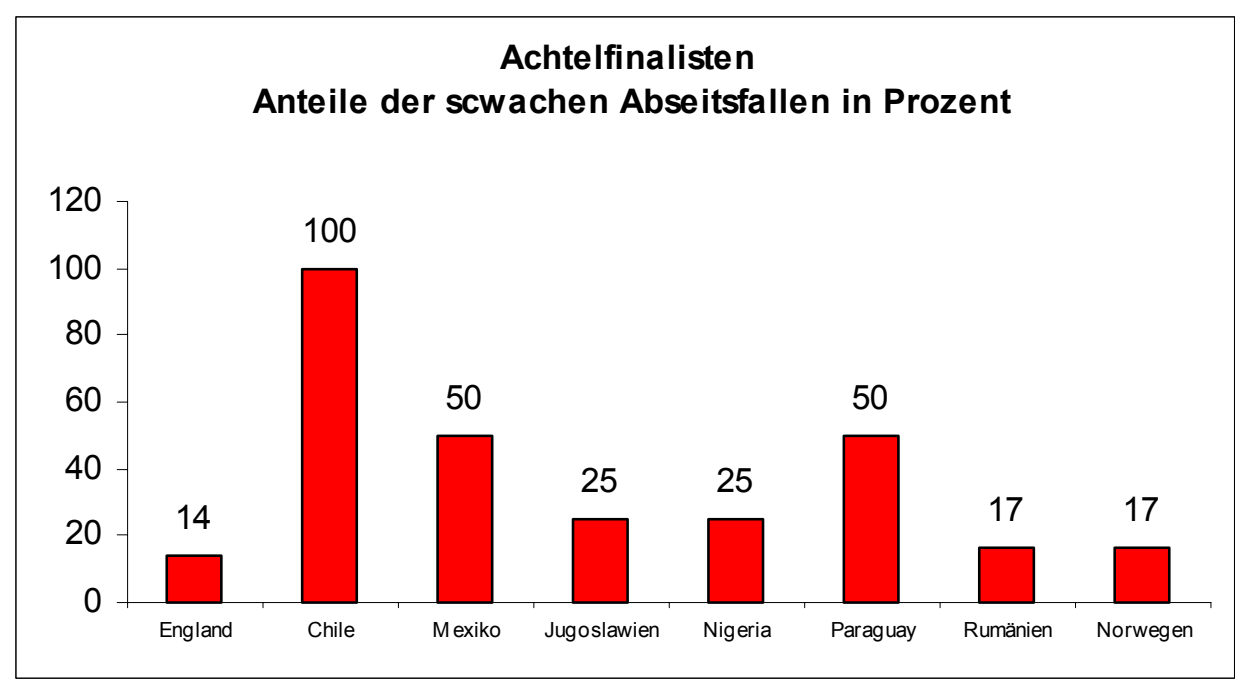

Diagramm 140: Anteile der „schwachen“ Abseitsfallen in Prozent bei den Achtelfinalisten.

Beim Vergleich der Diagramme 138, 139 und 140 finden wir, dass mehr hochkarätigen Abseitsfallen gestellt wurden als schwache, jedoch mit großen Unterschieden zwischen den Mannschaften. Bei Abseitsfalle darf die Mannschaften keine Fehler machen, weil sonst die Verteidigung fehlt, das mögliche Resultat ist also alles oder nichts, wodurch hier keine „normalen“ Abseitsfallen angeführt sind.

\subsubsection{Viertelfinalisten}

Als Viertelfinalisten werden diejenigen Mannschaften bezeichnet, die im Viertelfinale ausgeschieden sind und zu deren Analyse deshalb zwei Spiele herangezogen werden konnten. Zu diesen Viertelfinalisten gehören Deutschland, Italien, Argentinien und Dänemark.

\subsubsection{Pressing hinten}

„Hochkarätigen“ Pressing hinten hatte Italien mit 35 von 169 (21\%) den größten Wert. Absteigend folgen Deutschland 20 von 111 (18 \%), Dänemark 19 von 111 (17 \%). Argentinien mit 12 von 136 (9\%) den kleinste Anteil „hochkarätig“ „Pressing hinten". 
Das folgende Diagramm zeigt die Anteile des hochkarätigen „Pressing hinten“ in Prozent für die Viertelfinalisten.

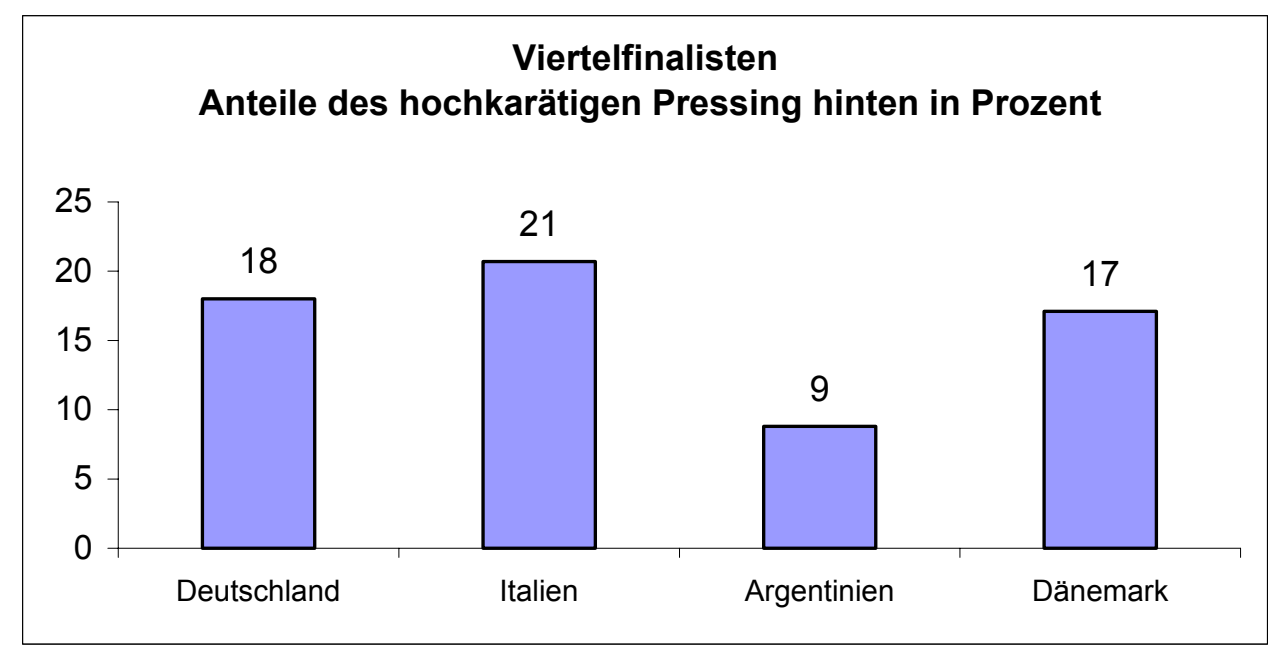

Diagramm 141: Anteile des hochkarätigen „Pressing hinten“ in Prozent bei den Viertelfinalisten.

99 (73 \%) des „Pressing hinten“ von Argentinien wurden mit „normal“ bewertet und bildeten den größten Anteil. Die weiteren Anteile „normalen“ „Pressing hinten“ lauteten 104 (62 \%) für Italien, 66 (60 \%) für Dänemark und 65 (59 \%) für Deutschland. Dem folgenden Diagramm sind die Anteile des normalen Pressing hinten in Prozent für die Viertelfinalisten zu entnehmen.

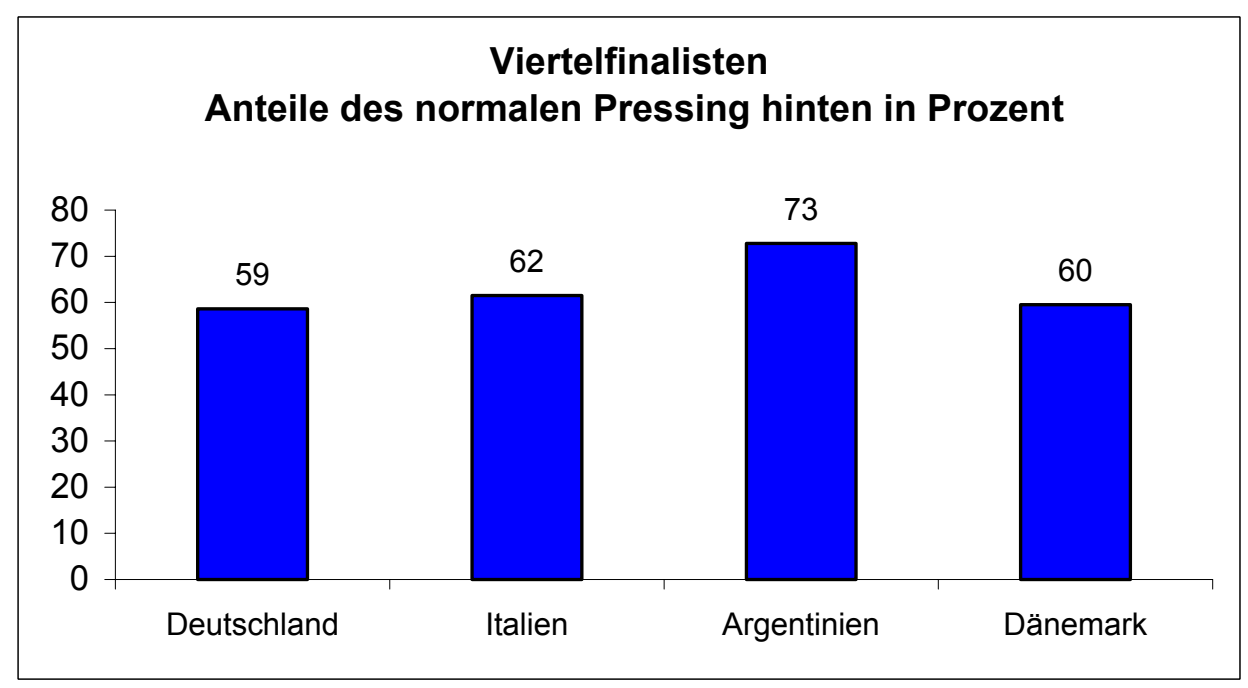

Diagramm 142: Anteile des normalen „Pressing hinten“ in Prozent bei den Viertelfinalisten. 
Ein „schwaches“ „Pressing hinten“ wurde mit einem Anteil von jeweils 26 (23\%) am häufigsten bei Dänemark und Deutschland festgestellt. Es folgen Argentinien mit 25 (18\%) und schließlich Italien mit 30 (18\%).

Das folgende Diagramm zeigt die Anteile des schwachen Pressing hinten in Prozent für die Viertelfinalisten.

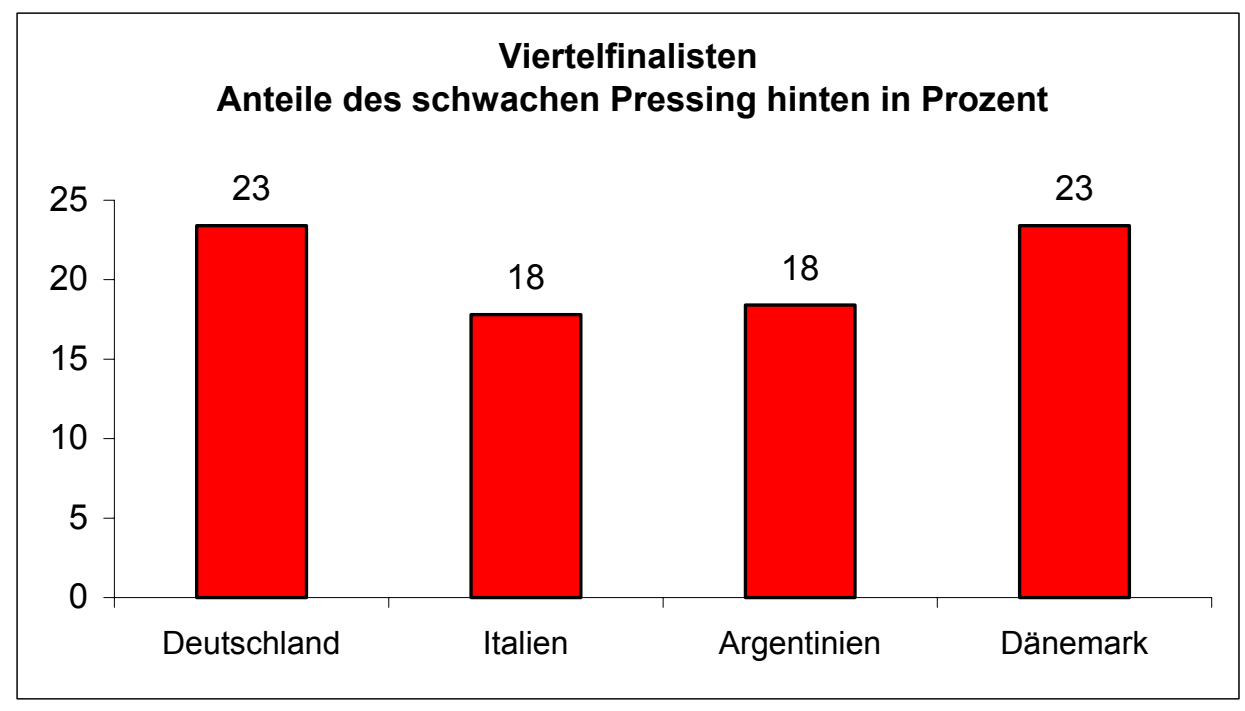

Diagramm 143: Anteile des schwachen „Pressing hinten“ in Prozent bei den Viertelfinalisten.

Diagramm 141 zeigt, dass hochkarätiges Pressing hinten zu einem Anteil zwischen 9 $\%$ und $21 \%$ vorkam. Die italienische Mannschaft hatte hier die besten Werte und die argentinische die schlechtesten. Diagramm 142 besagt, dass der Anteil des normalen Pressings hinten zwischen $59 \%$ und $73 \%$ betrug. Die argentinische Mannschaft hatte hier die besten Werte und die deutsche die schlechtesten. Aus Diagramm 143 geht hervor, dass schwaches Dribbling zwischen $18 \%$ und $23 \%$ aller Dribblings ausmachte. Die italienische und argentinische Mannschaft hatten die besten Werte und die deutsche und dänische Mannschaft die schlechtesten Werte.

Es lässt sich aus den Diagrammen folgern, dass das normalen Pressing hinten bei allen Viertelfinalisten nicht nur öfter als das hochkarätige, sondern auch als das schwache Pressing hinten ausgeführt wurde. Das führt dazu, dass die Mannschaft einige Fehler in der Nähe von 12 Metern vom Tor begeht, was eine große Gefahr für das Tor darstellt. 


\subsubsection{Pressing Mitte}

Bei „Hochkarätigem Pressing Mitte“ erzielten sämtliche Viertelfinalisten einen Wert von $0 \%$.

Das folgende Diagramm zeigt die Anteile des hochkarätigen Pressing Mitte in Prozent der Viertelfinalisten.

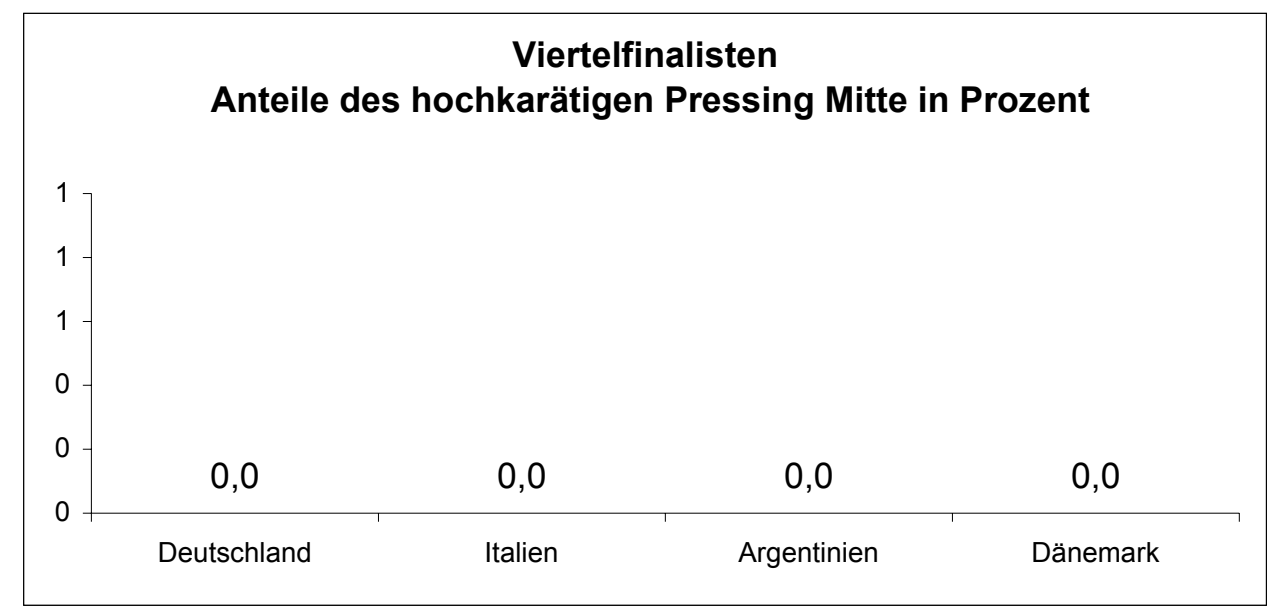

Diagramm 144: Anteile des „hochkarätigen Pressing Mitte“ in Prozent bei den Viertelfinalisten.

44 von 52 (85\%) des „Pressing Mitte“ von Italien wurde mit „normal“ bewertet und bildeten damit den größten Anteil. Die weiteren Anteile „normalen“ Pressing Mitte lauten 61 von 77 (79\%) für Argentinien, 46 von 66 (70 \%) für Deutschland und 43 von 67 (64 \%) für Dänemark.

Das folgende Diagramm zeigt die Anteile des normalen Pressing Mitte in Prozent der Viertelfinalisten.

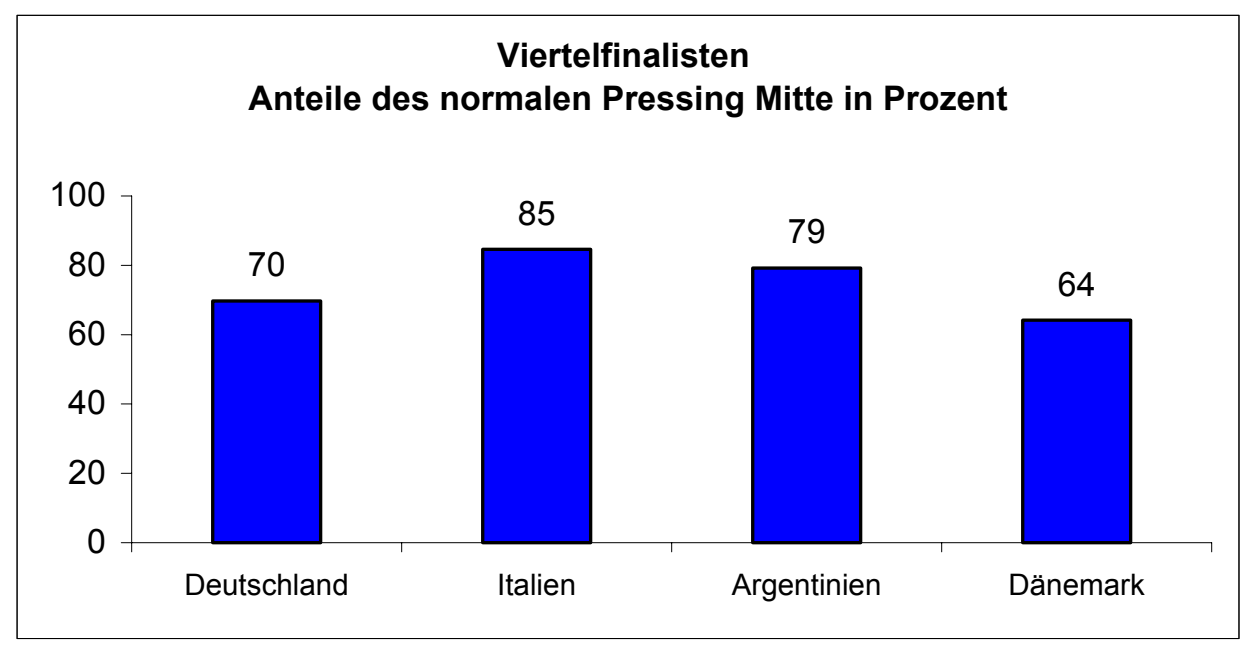

Diagramm 145: Anteile des „normalen Pressing Mitte“ in Prozent bei den Viertelfinalisten. 
Ein „schwaches Pressing Mitte“ wurde mit einem Anteil von 24 (36 \%) am häufigsten bei Dänemark festgestellt. Es folgen Deutschland 20 (30\%), Argentinien 16 (21\%) und schließlich Italien 8 (15\%).

Das folgende Diagramm zeigt anteile die schwache Pressing mitte in Prozent der Viertelfinalisten.

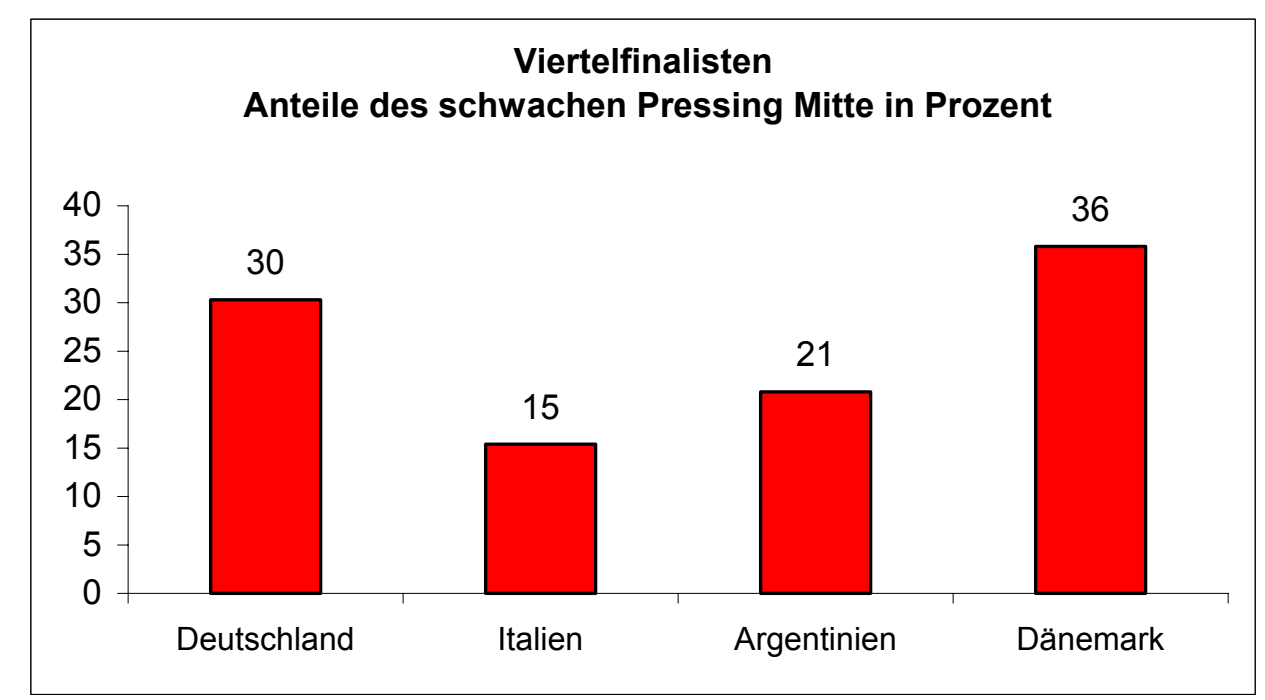

Diagramm 146: Anteile des „schwachen Pressing Mitte“ in Prozent bei den Viertelfinalisten.

Diagramm 144 gibt die Werte für hochkarätiges Pressing Mitte mit $0 \%$ für alle. Aus Diagramm 145 gehen die Werte für normales Pressing Mitte zwischen $64 \%$ und 85 $\%$ hervor. Die italienische Mannschaft hatte hier die besten Werte und die dänische hatte die schlechtesten. Diagramm 146 veranschaulicht, dass schwaches Pressing Mitte zu Anteilen zwischen $15 \%$ und $36 \%$ vorkam, wobei die italienische Mannschaft die besten Werte hatte und die dänische Mannschaft die schlechtesten.

Aus den Diagrammen geht hervor, dass das hochkarätige Pressing Mitte sehr schwach in seiner Wirkung ist, da keine der beiden Mannschaften den Ball aus der Mitte abnehmen und ein Tor erzielen konnte. Zu begründen ist das mit der Langsamkeit der Spieler beim gekonterten Angriff. 


\subsubsection{Pressing vorne}

Bei „Hochkarätigem“ „Pressing vorne“ hatte Argentinien mit 2 von 21 (10\%) den größten Wert. Absteigend folgen Dänemark mit 1 von 12 (8\%), Deutschland mit 0 von $21(0 \%)$, Italien mit 0 von $11(0 \%)$ mit dem kleinsten Anteil „hochkarätigen Pressing vorne“.

Das folgende Diagramm verdeutlicht die Anteile des hochkarätigen Pressing vorne in Prozent der Viertelfinalisten.

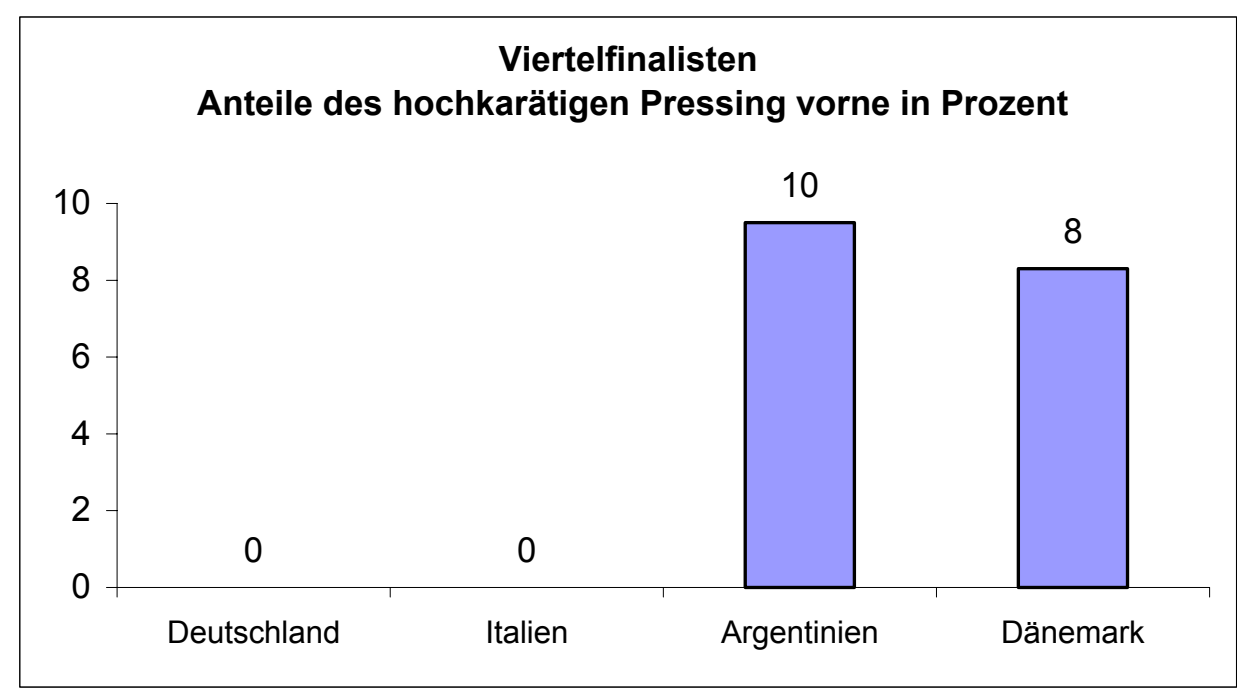

Diagramm 147: Anteile des „hochkarätigen Pressing vorne“ in Prozent bei den Viertelfinalisten.

16 (76 \%) des „Pressing vorne“ von Argentinien wurden mit „normal“ bewertet und bildeten den größten Anteil. Die weiteren Anteile „normalen“ „Pressing vorne“ lauteten 8 (73 \%) für Italien und 14, 8 (je $67 \%$ ) für Deutschland und Dänemark.

Das folgende Diagramm zeigt die Anteile des normalen Pressing vorne in Prozent der Viertelfinalisten. 


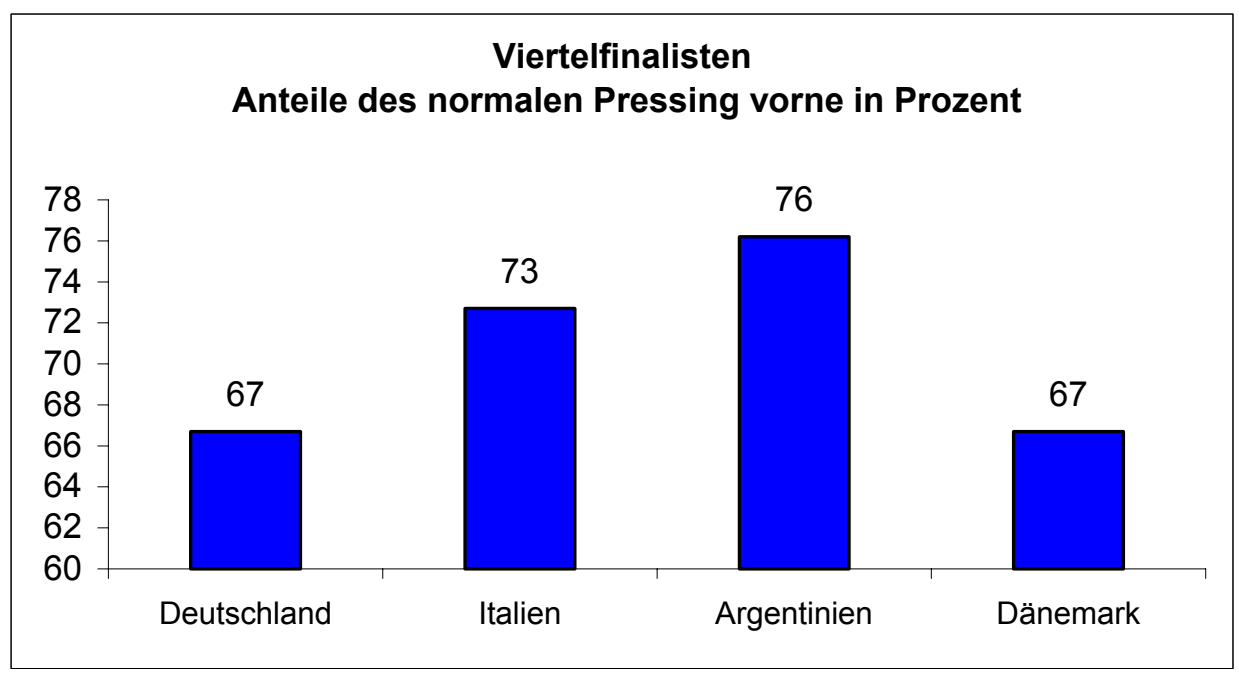

Diagramm 148: Anteile des normalen „Pressing vorne“ in Prozent bei den Viertelfinalisten.

Ein „schwaches“ „Pressing vorne“ wurde mit einem Anteil von 7 (33\%) am häufigsten bei Deutschland festgestellt. Es folgen Italien 3 (27\%), Dänemark 3 (25\%) und schließlich Argentinien 3 (14\%).

Das folgende Diagramm sind die Anteile des schwachen Pressing vorne in Prozent der Viertelfinalisten.

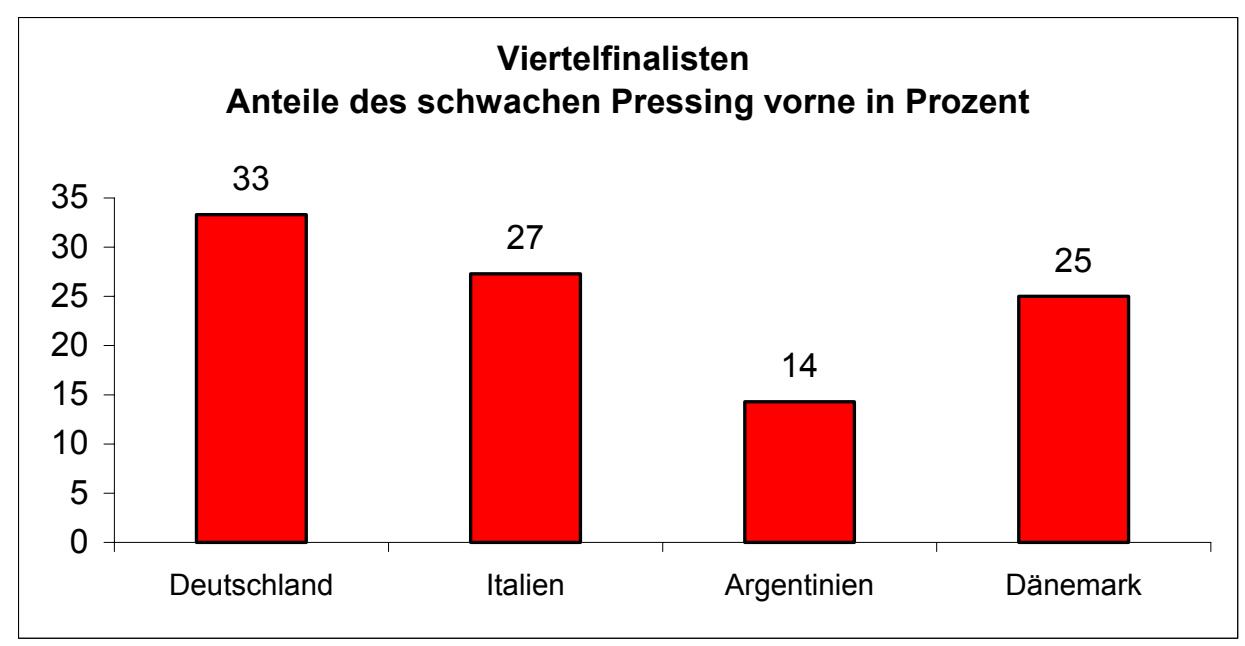

Diagramm 149: Anteile des schwachen „Pressing vorne“ in Prozent bei den Viertelfinalisten.

Diagramm 147 veranschaulicht, dass hochkarätiges Pressing vorne zwischen $0 \%$ und $10 \%$ vorkam, wobei die beiden Mannschaften von Argentinien und Dänemark die besten Werte hatten und die italienische und deutsche Mannschaften die 
schlechtesten. Diagramm 148 weist aus, dass normales Pressing vorne zwischen 67 $\%$ und $76 \%$ zur Ausführung kam. Die italienische und argentinische Mannschaft hatten hier die besten Werte, die deutsche und dänische hatten die schlechtesten.

Diagramm 149 gibt an, dass schwaches Pressing vorne zu $14 \%$ bis $33 \%$ gespielt wurde, die argentinische Mannschaft dabei die besten Werte hatte und die deutsche Mannschaft die schlechtesten.

Aus den Diagrammen wird deutlich, dass der Anteil des hochkarätigen Pressing vorne geringer ist als das des gescheiterten und das normale Pressing vorne weit öfter geschieht als die beiden anderen Kategorien. Der Grund liegt wahrscheinlich darin, dass die meisten Mannschaften im Achtelfinale sich überwiegend auf das Verteidigungsspiel stützten. Keine Mannschaften außer Nigeria und Rumänien konnten den Ball vorne abnehmen und ein Tor erzielen. Das liegt daran, dass mit wenigen Angreifern in dem Drittel des Gegners versucht wurde, den Ball wieder zunehmen.

\subsubsection{Raumaufteilung}

Das folgende Diagramm bildet die Anteile der hochkarätigen Raumaufteilung in Prozent der Viertelfinalisten ab. Bei der "hochkarätigen“ Raumaufteilung hatte Italien mit 32 von 152 (21\%) den größten Wert. Absteigend folgen Dänemark mit 19 von 129 (15\%) und Deutschland mit 10 von 120 (8\%). Argentinien hatte mit 12 von 146 (8 $\%)$ den kleinsten Anteil "hochkarätiger" Raumaufteilung. Dem folgenden Diagramm sind die Anteile des hochkarätigen „Raumaufteilung“ in Prozent der Viertelfinalisten zu entnehmen.

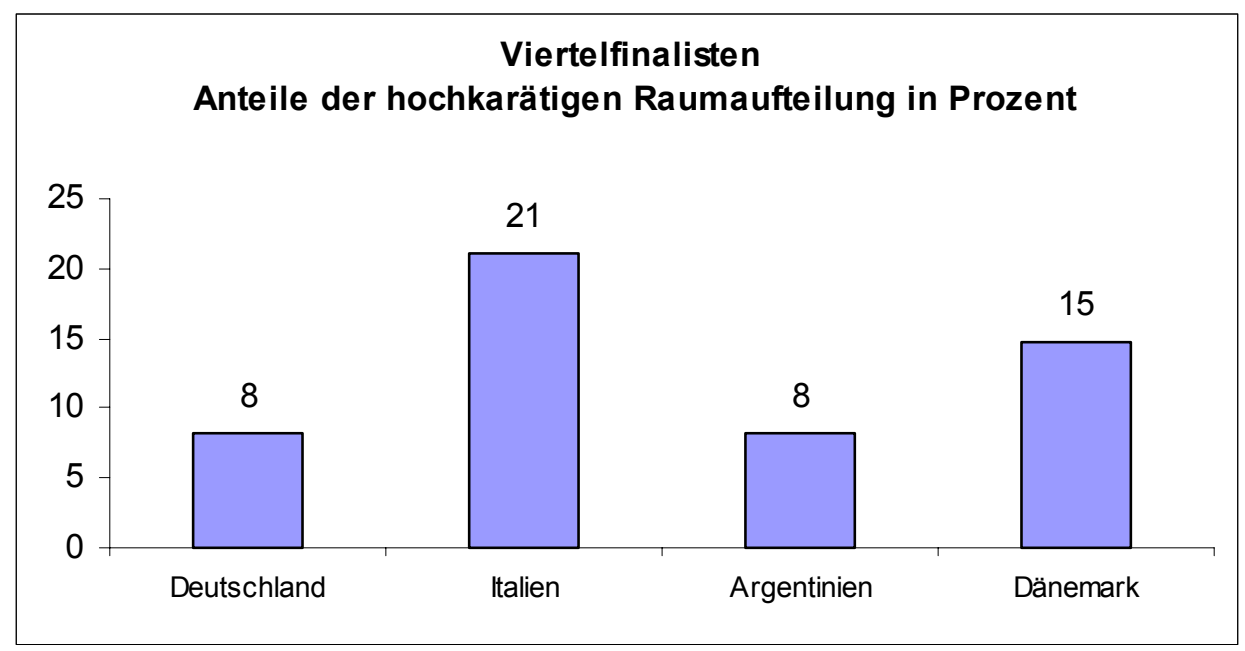

Diagramm 150: Anteile der "hochkarätigen“ Raumaufteilung in Prozent bei den Viertelfinalisten. 
$100(83 \%)$ der Raumaufteilungen von Deutschland wurden mit "normal“ bewertet und bildeten den größten Anteil. Die weiteren Anteile „normaler" Raumaufteilungen lauteten 117 (80\%) für Argentinien, 90 (70\%) für Dänemark und 105 (69 \%) für Italien.

Das folgende Diagramm veranschaulicht die Anteile der mittleren Raumaufteilung in Prozent bei den Viertelfinalisten.

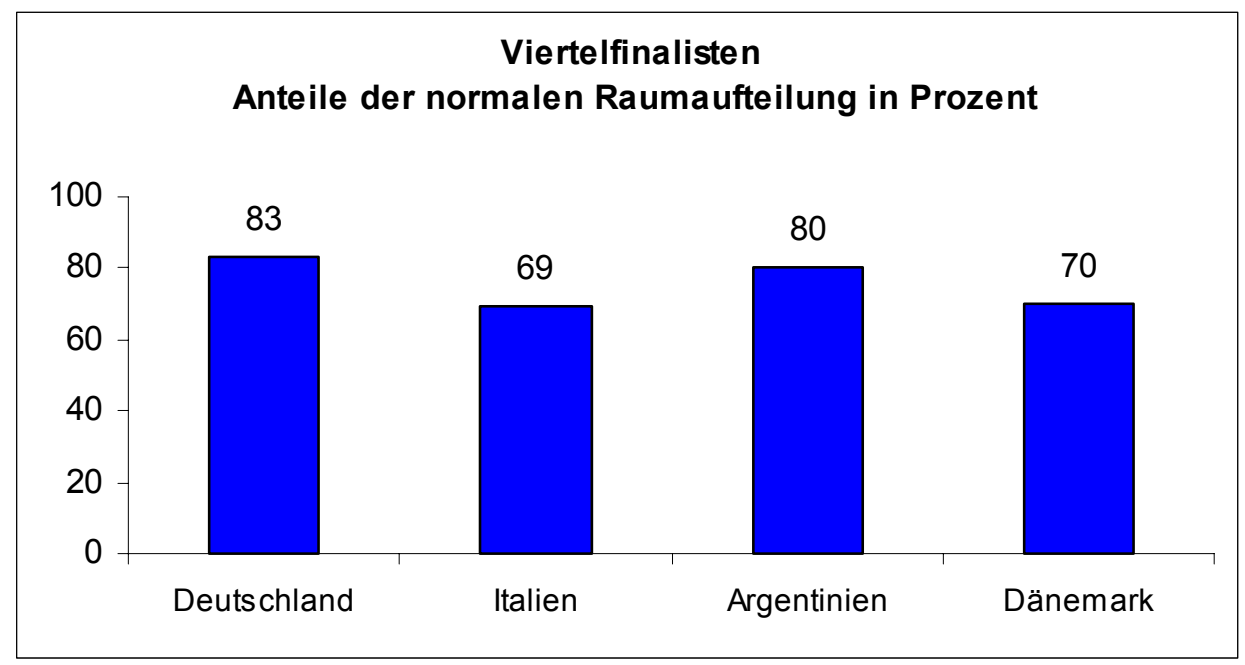

Diagramm 151: Anteile der „normalen“ Raumaufteilung in Prozent bei den Viertelfinalisten.

Eine „schwache“ Raumaufteilung wurde mit einem Anteil von 20 (16 \%) am häufigsten bei Dänemark festgestellt. Es folgen Argentinien 17 (12\%), Italien $15(10 \%)$ und schließlich Deutschland 10 (8\%). Das folgende Diagramm zeigt anteile die schwache Raumaufteilung in Prozent der Viertelfinalisten.

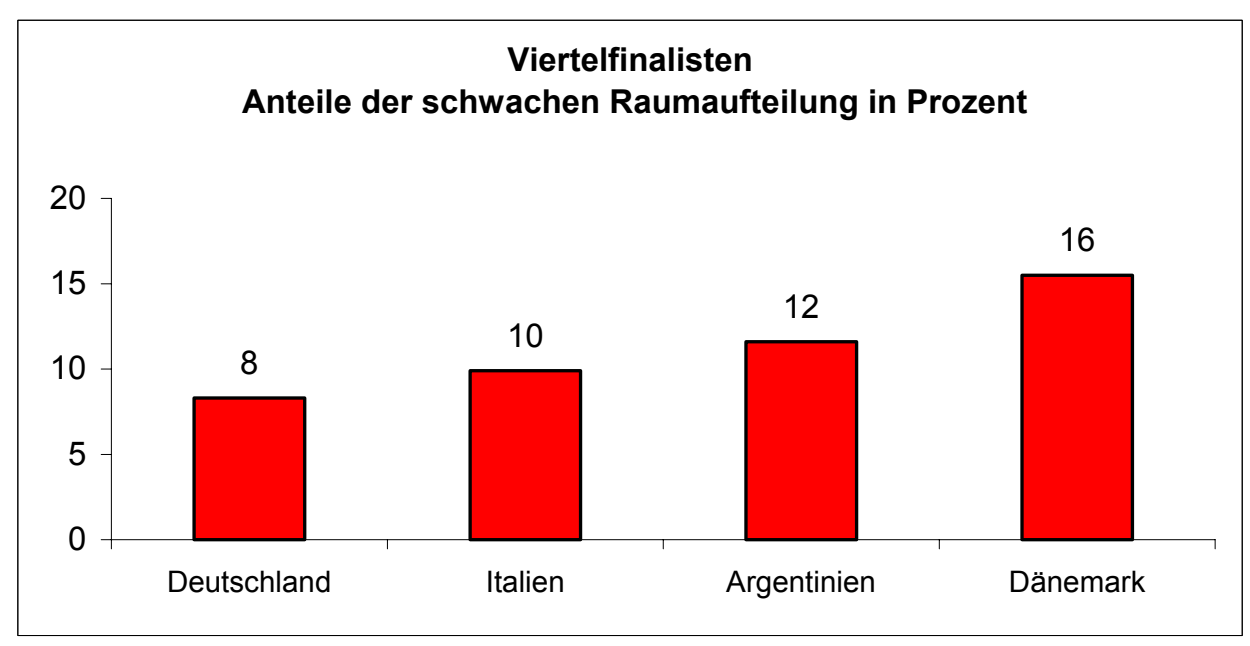

Diagramm 152: Anteile der schwachen "Raumaufteilung“ in Prozent bei den Viertelfinalisten. 
Diagramm 150 erklärt, dass schwaches Raumaufteilung zwischen 8 \% und $21 \%$ die italienische und dänische Mannschaften hatten die besten Werte und argentinische und deutsche Mannschaften hatten die schlechten Werte. Diagramm 151 erklärt, dass normalen Raumaufteilung zwischen $70 \%$ und $83 \%$ Die deutsche hatte hier die besten Werte und italienische hatte die schlechtesten. Diagramm 152 erklärt, dass schwaches Raumaufteilung zwischen $8 \%$ und $16 \%$ die deutsche hatte die besten Werte und die dänische Mannschaft hatte die schlechten Werte.

Aus den Diagrammen geht hervor, dass die normalen Raumaufteilung sehr häufig vorkommt, da die beiden Mannschaften die Spieler in die Mitte des Spielfelds am häufigsten verlieren. Der Grund liegt wahrscheinlich darin, dass die meisten Mannschaften im Achtelfinale sich überwiegend auf das Verteidigungsspiel stützen.

\subsubsection{Zurückkommen}

Bei „Gutem“ „Zurückkommen“ hatte Italien mit 14 von 106 (13 \%) den größten Wert. Absteigend folgen Argentinien mit 7 von 74 (10\%) und Dänemark mit 5 von 57 (9 \%). Deutschland hatte mit 1 von 52 (2 \%) den kleinsten Anteil „hochkarätigen“ „Zurückkommen“.

Das folgende Diagramm zeigt die Anteile des hochkarätigen Zurückkommens in Prozent der Viertelfinalisten.

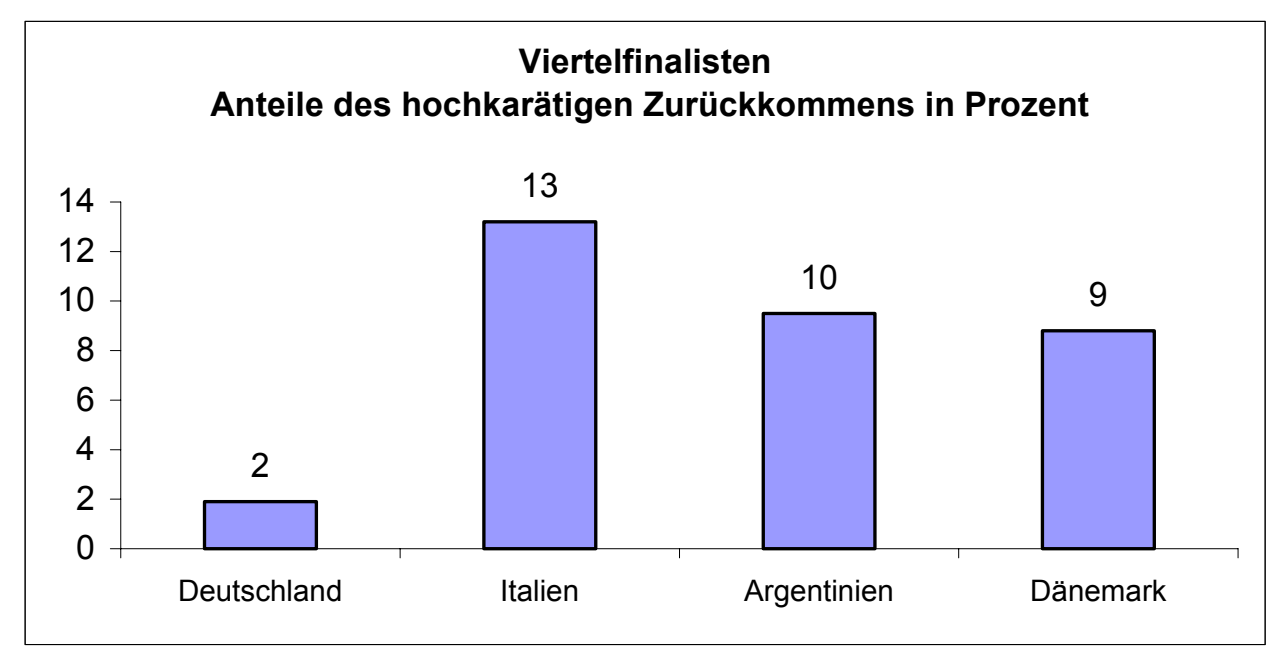

Diagramm 153: Anteile des hochkarätigen „Zurückkommens“ in Prozent bei den Viertelfinalisten. 
50 (96 \%) des „Zurückkommens“ von Deutschland wurden mit „normal“ bewertet und bildeten den größten Anteil. Die weiteren Anteile „normalen“ „Zurückkommens“ lauteten 51 (90 \%) für Dänemark, 66 (89 \%) für Argentinien und 91 (86 \%) für Italien.

Das folgende Diagramm zeigt die Anteile des normalen Zurückkommens in Prozent für die Viertelfinalisten.

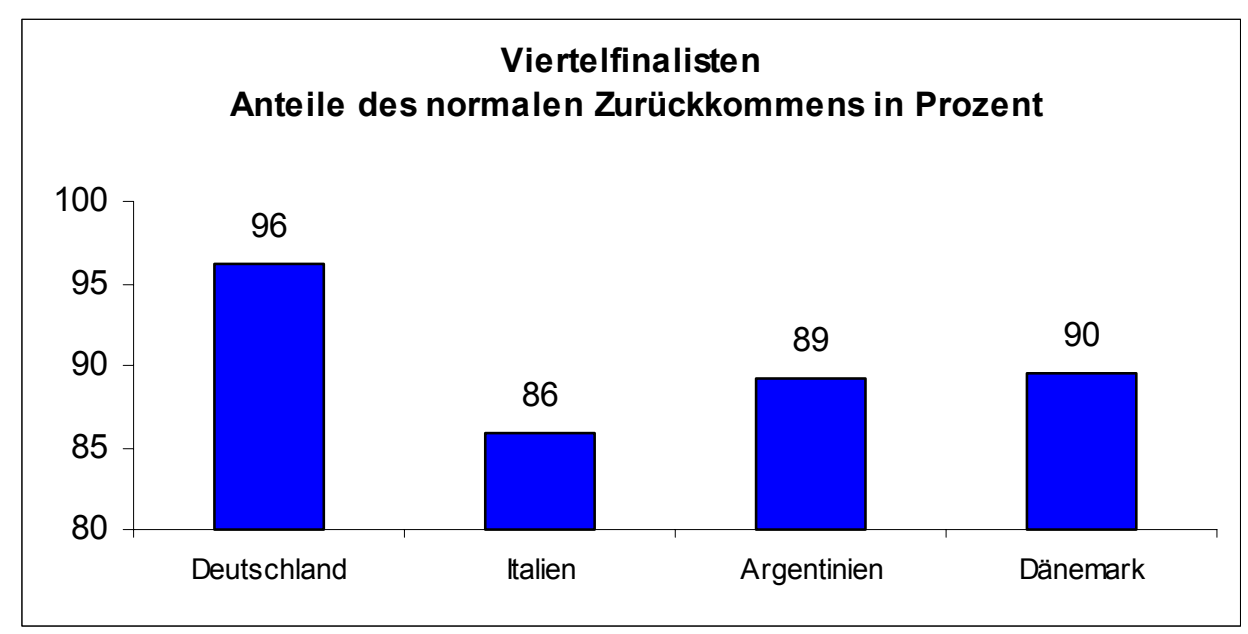

Diagramm 154: Anteile des normalen „Raumaufteilung“ in Prozent bei den Viertelfinalisten.

Ein „schwaches“ „Zurückkommen“ wurde mit einem Anteil von 1 (2 \%) am häufigsten bei Deutschland festgestellt. Es folgen Dänemark mit 1 (2 \%), Argentinien mit 1 (1\%) und schließlich Italien mit 1 (1\%).

Das folgende Diagramm zeigt die Anteile des schwachen Zurückkommens in Prozent der Viertelfinalisten.

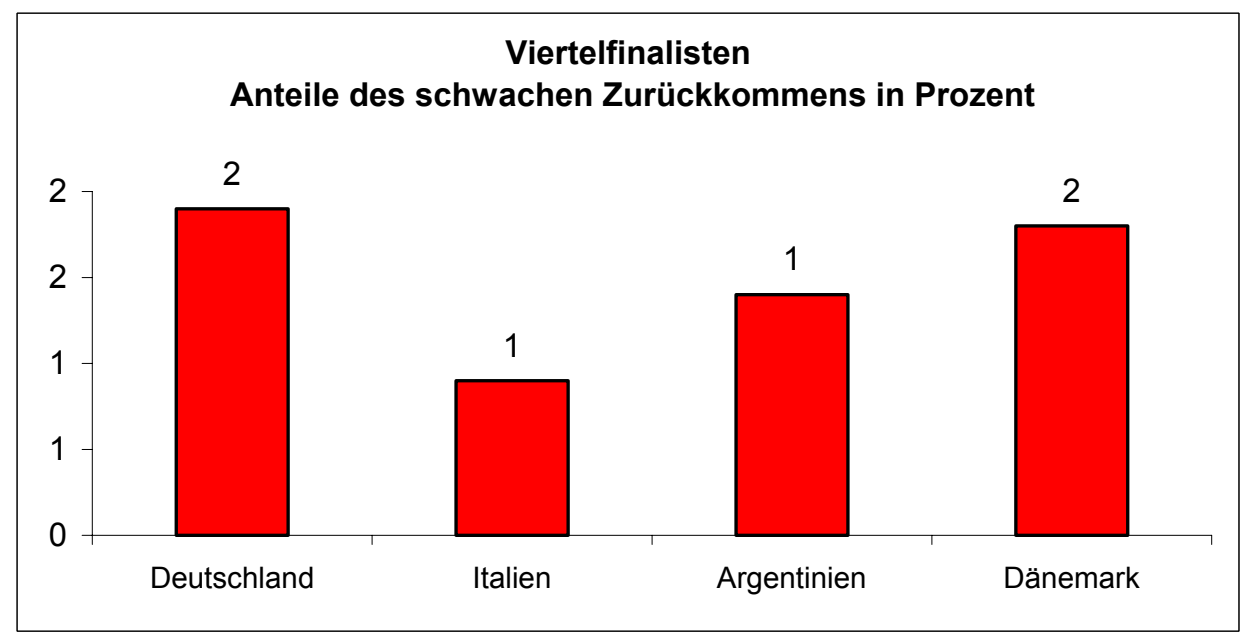

Diagramm 155: Anteile des schwachen „Zurückkommens“ in Prozent bei den Viertelfinalisten. 
Diagramm 153 zeigt, dass hochkarätiges Zurückkommen zwischen $2 \%$ und $13 \%$ vorkam. Die italienische Mannschaft hatte die besten Werte und die deutsche Mannschaft hatte die schlechten Werte. Diagramm 154 erklärt, dass normales Zurückkommen zwischen $86 \%$ und $96 \%$ vorkam. Die deutsche hatte hier die besten Werte und italienische hatte die schlechtesten. Diagramm 155 erklärt, dass schwaches Zurückkommen zwischen $1 \%$ und $2 \%$ vorkam. Die italienische und die argentinische Mannschaft hatten die besten Werte die deutsche und dänische Mannschaft hatten die schlechtesten.

Aus der Darstellung von der Diagrammen wird deutlich, dass das normalen Zurückkommen mehr besser ist als das hochkarätige und schwache, da die Spieler einen Platz einnehmen, von dem aus sie ihre Mitspieler so helfen können.

\subsubsection{Unterstützen}

Bei „Gutem“ Unterstützen erzielten Italien und Dänemark mit jeweils 1 von 8 (13 \%) den größten Wert. Deutschland und Argentinien hatten mit jeweils $0,0 \%$ den kleinsten Anteil "guten“ Unterstützens.

Das folgende Diagramm zeigt die Anteile des hochkarätigen Unterstützens in Prozent der Viertelfinalisten.

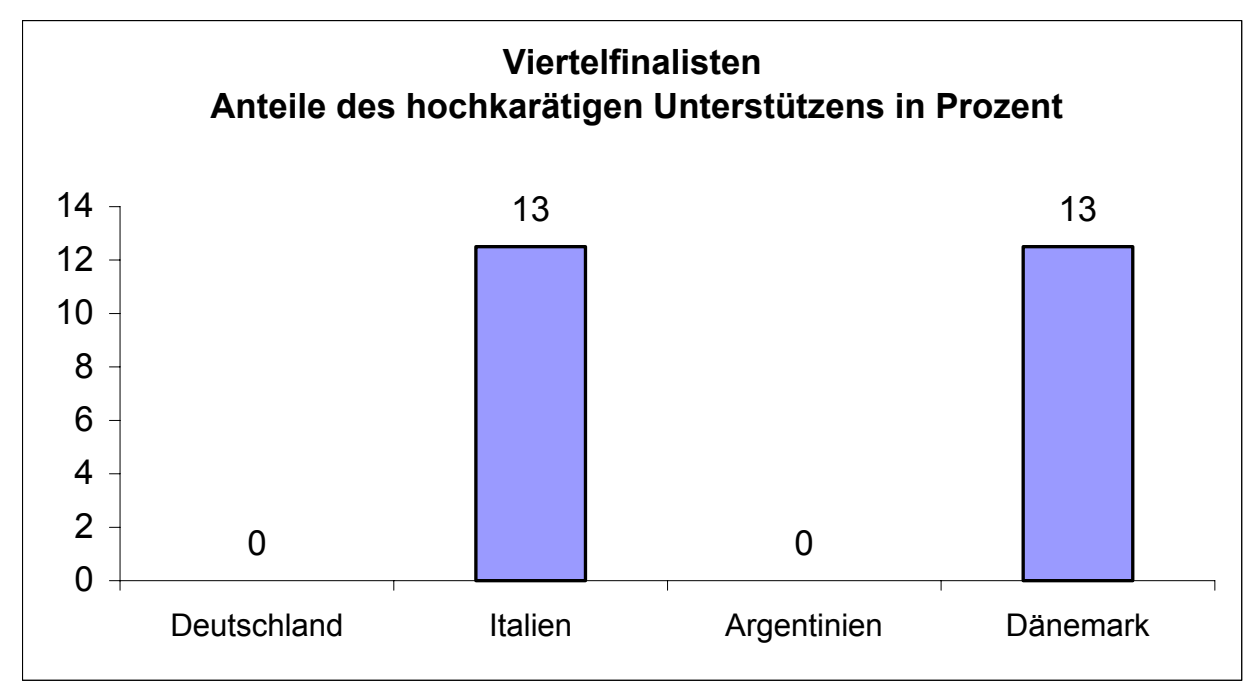

Diagramm 156: Anteile des hochkarätigen „Unterstützens“ in Prozent bei den Viertelfinalisten. 
$4(80 \%)$ des Unterstützens von Argentinien wurden mit „normal“ bewertet und bildete den größten Anteil. Die weiteren Anteile „normalen“ Unterstützens lauteten 6 (75 \%) für Italien, Dänemark und 2 (67\%) für Deutschland.

Das folgende Diagramm sind die Anteile des normalen Unterstützens in Prozent der Viertelfinalisten.

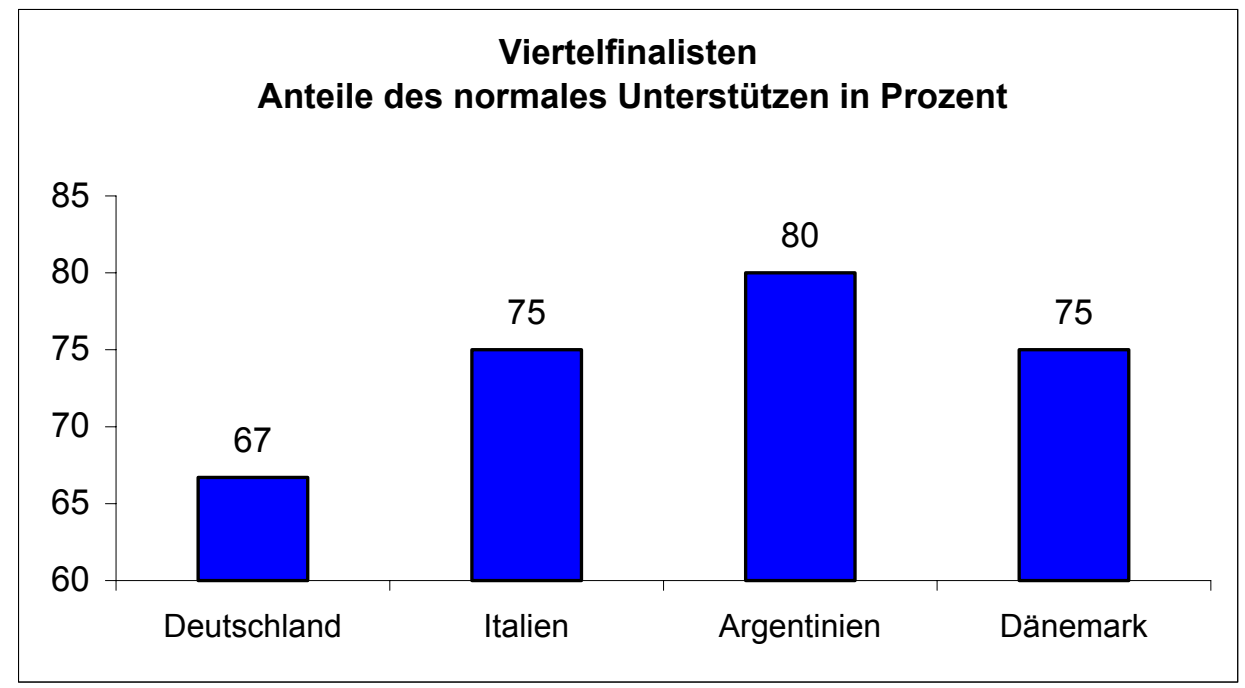

Diagramm 157: Anteile des normalen "Unterstützens“ in Prozent bei den Viertelfinalisten.

Ein „schwaches“ Unterstützen wurde mit einem Anteil von 1 (33\%) am häufigsten bei Deutschland festgestellt. Es folgen Argentinien mit 1 (20\%) und schließlich Italien und Dänemark mit 1 (13\%). Das folgende Diagramm zeigt die Anteile des schwachen Unterstützens in Prozent der Viertelfinalisten.

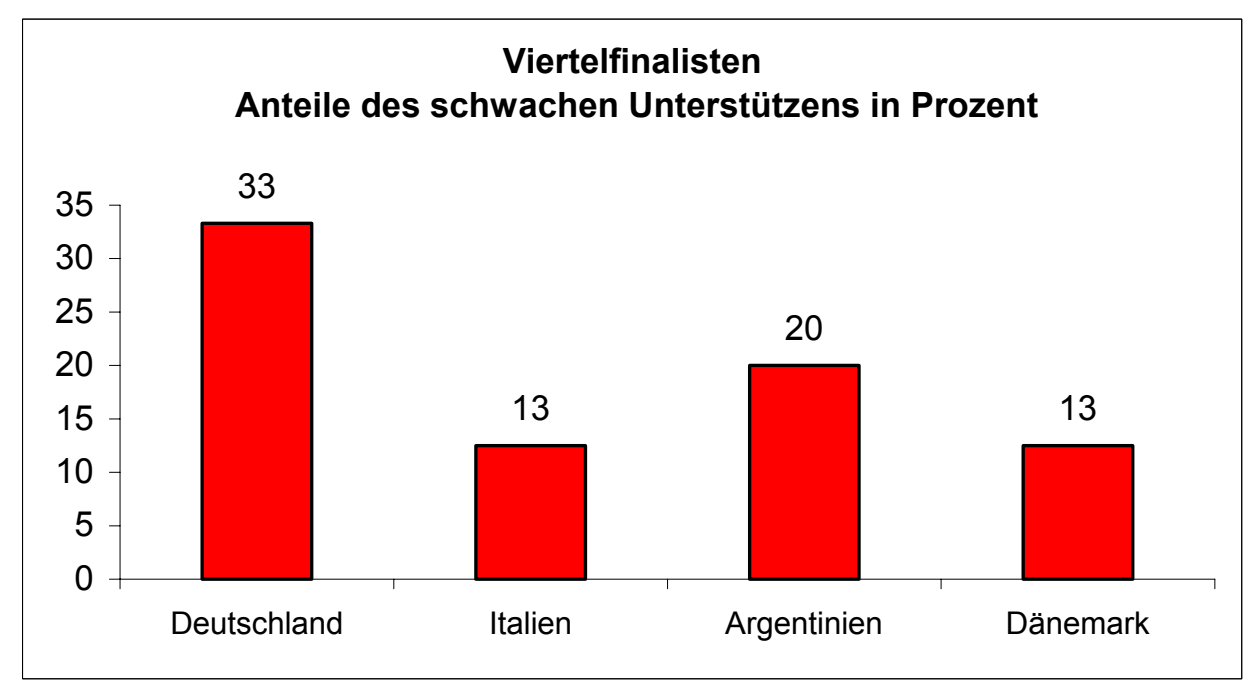

Diagramm 158: Anteile des schwachen „Unterstützens“ in Prozent bei den Viertelfinalisten. 
Diagramm 156 zeigt, dass bei hochkarätigem Unterstützen die Werte zwischen 0,0 $\%$ und $13 \%$ lagen und die italienische und die dänische Mannschaft die besten Werte und argentinische und deutsche Mannschaft die schlechten Werte hatten. Diagramm 157 zeigt, dass normales Unterstützen zwischen 67\% und $80 \%$ lag. Die argentinische Mannschaft hatte hier die besten Werte und deutsche hatte die schlechtesten. Diagramm 158 erklärt, dass schwaches Unterstützen zwischen $13 \%$ und 33 \% vorkam; dänische Mannschaft hatte die besten Werte und die deutsche Mannschaft hatte die schlechten Werte.

Aus der Darstellung der Diagramme wird deutlich, dass das normalen Unterstützen häufiger ist als das hochkarätige und schwache, da die unterstützenden Spieler einen Platz einnehmen, von dem aus sie ihre Mitspieler so helfen können, dass sie gut mit dem Ball voran kommen können. Manchmal unterstützen die Mitspieler nicht zur rechten Zeit oder richtigen Position.

\subsubsection{Rückraum Sichern}

Beim „Hochkarätigen“ „Rückraum Sichern“ erzielte Deutschland mit 1 von 8 (13\%) den größten Wert. Absteigend folgen Dänemark mit 1 von 16 (6\%) und Argentinien mit 1 von 17 (6\%). Italien hatte mit 1 von 19 (5\%) den kleinsten Anteil "guten“ „Rückraum Sicherns“.

Das folgende Diagramm zeigt die Anteile des hochkarätigen Rückraum Sicherns in Prozent der Viertelfinalisten.

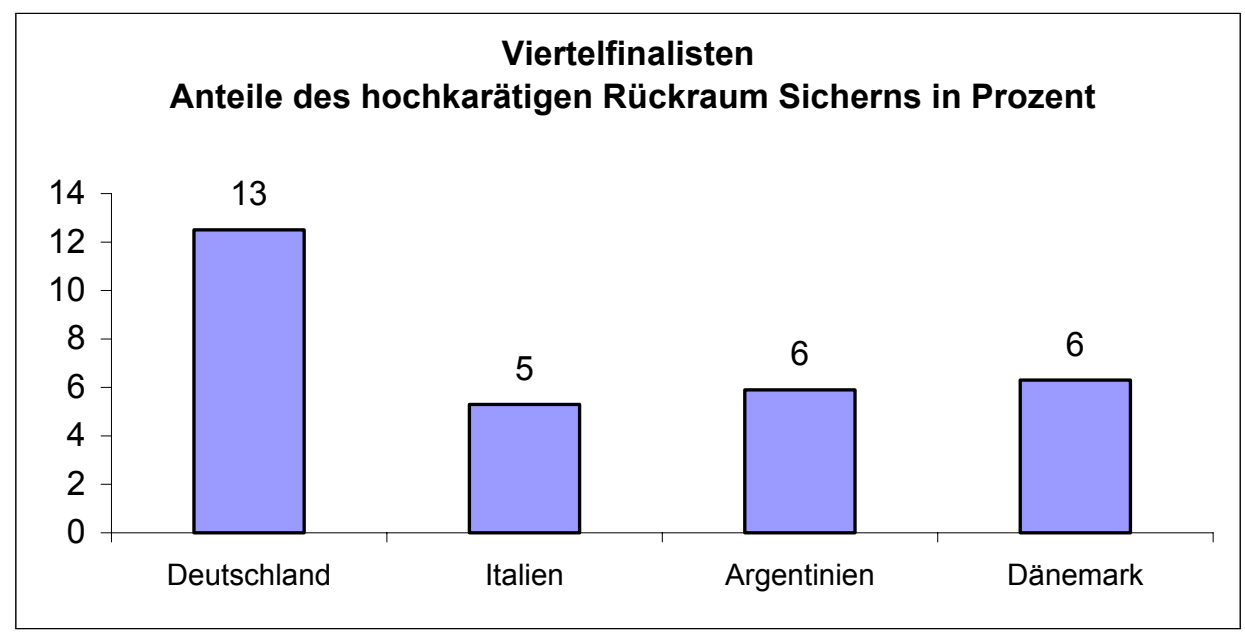

Diagramm 159: Anteile des hochkarätigen „Rückraum Sicherns“ in Prozent bei den Viertelfinalisten. 
$6(75 \%)$ des „Rückraum Sichern“ von Deutschland wurden mit „normal“ bewertet und bildeten den größten Anteil. Die weiteren Anteile „normalen“ „Rückraum Sichern“ lauteten 14 (74 \%) für Italien, 12 (71\%) für Argentinien und 11 (69 \%) für Dänemark. Dem folgenden Diagramm sind die Anteile des normalen Rückraum Sicherns in Prozent der Viertelfinalisten zu entnehmen.

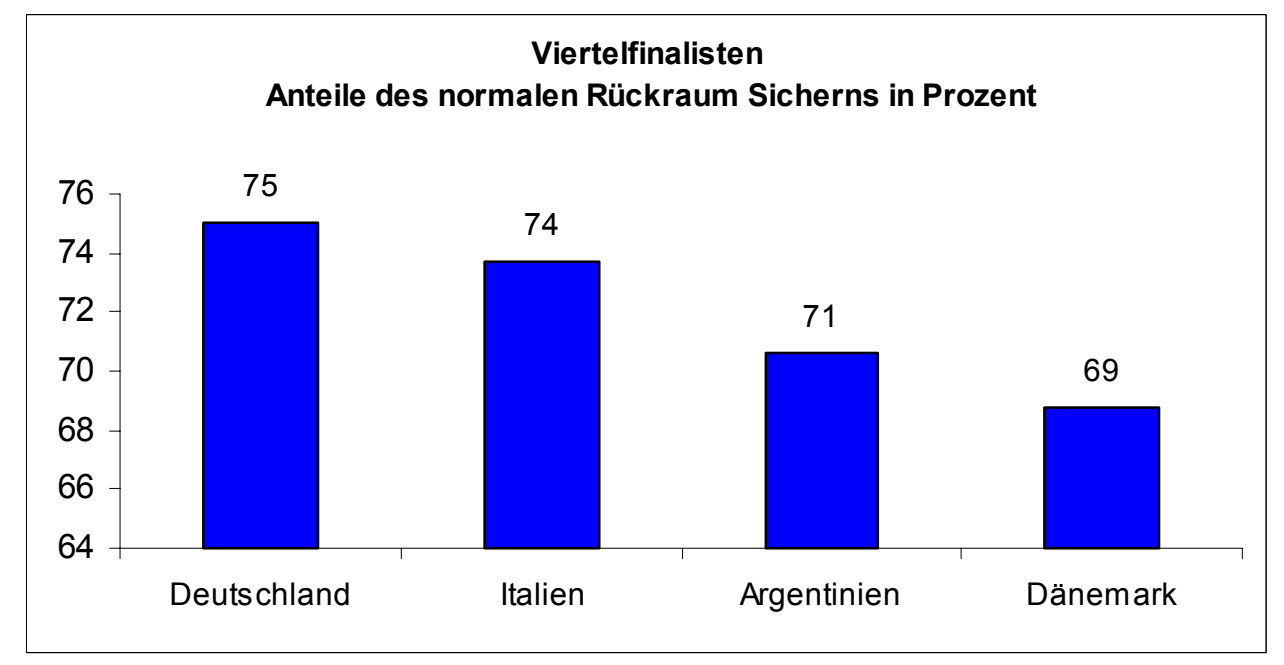

Diagramm 160: Anteile des normalen „Rückraum Sicherns“ in Prozent bei den Viertelfinalisten.

Ein „schwaches“ „Rückraum Sichern“ wurde mit einem Anteil von 4 (25\%) am häufigsten bei Dänemark festgestellt. Es folgen Argentinien 4 (24\%), Italien 4 (21\%), und schließlich Deutschland 1 (13\%).

Das folgende Diagramm zeigt die Anteile des schwachen Rückraum Sicherns in Prozent der Viertelfinalisten.

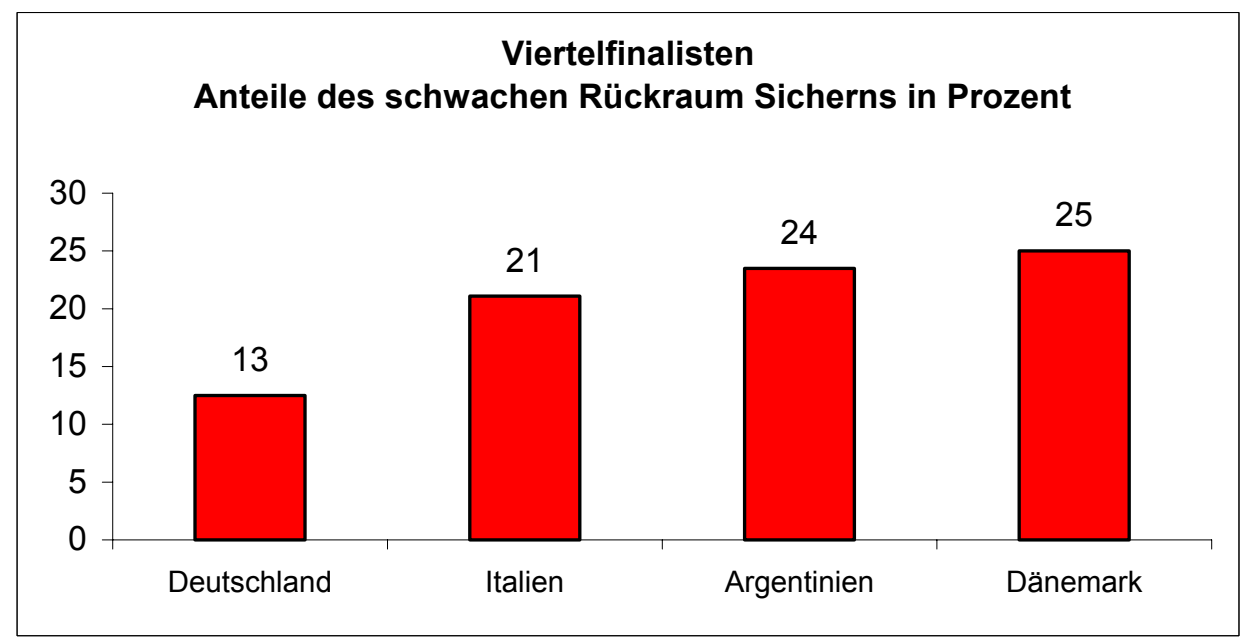

Diagramm 161: Anteile des schwachen „Rückraum Sicherns“ in Prozent bei den Viertelfinalisten. 
Diagramm 159 zeigt, dass beim hochkarätigen Rückraum Sichern die Werte zwischen $6 \%$ und $13 \%$ lagen und die deutsche Mannschaft die besten und die dänische Mannschaft die schlechten Werte hatte. Diagramm 160 zeigt, dass normales Rückraum Sichern zwischen $69 \%$ und $75 \%$ vorkam. Die deutsche Mannschaft hatte hier die besten Werte und dänische hatte die schlechtesten. Diagramm 161 zeigt, dass schwaches Rückraum Sichern zwischen $13 \%$ und $25 \%$ vorkam; die deutsche Mannschaft hatte die besten Werte und die dänische Mannschaft hatte die schlechten Werte.

Aus der Darstellung von den Diagrammen wird deutlich, dass das normale und hochkarätige Rückraum Sichern häufiger ist als das schwache, da die Spieler bei der Defensivarbeit ihre Gegenspieler gut bewachen.

\subsubsection{Abseitsfalle}

Bei "guten“ Abseitsfallen erzielte Dänemark mit 10 von 11 (91\%) den größten Wert. Absteigend folgen Deutschland und Italien mit jeweils 5 von 6 (83\%). Argentinien hatte mit 2 von 3 (67\%) den kleinsten Anteil "guter" Abseitsfallen.

Dem folgenden Diagramm sind die Anteile der hochkarätigen Abseitsfallen in Prozent der Viertelfinalisten zu entnehmen.

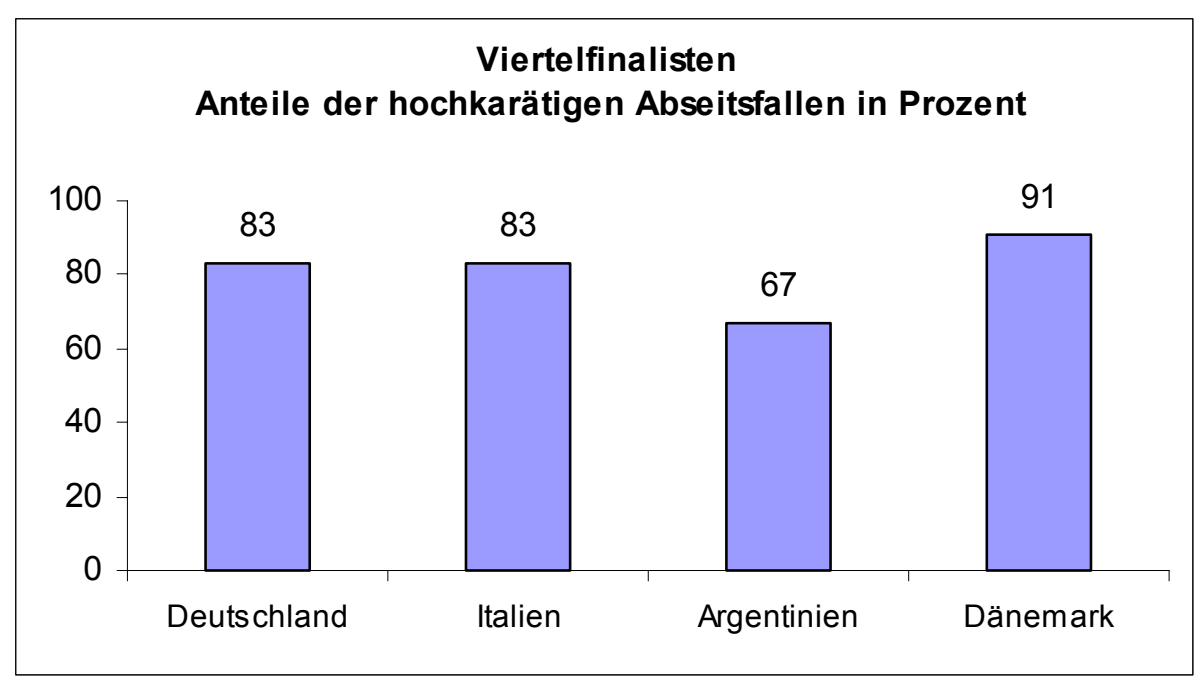

Diagramm 162: Anteile der hochkarätigen „Abseitsfallen“ in Prozent bei den Viertelfinalisten. 
Eine „schwache“ Abseitsfalle wurde mit einem Anteil von 1 (33\%) am häufigsten bei Argentinien festgestellt. Es folgen Deutschland und Italien mit 1 (17\%) und schließlich Dänemark mit 1 (9\%).

Das folgende Diagramm zeigt die Anteile der schwachen Abseitsfalle in Prozent der Viertelfinalisten.

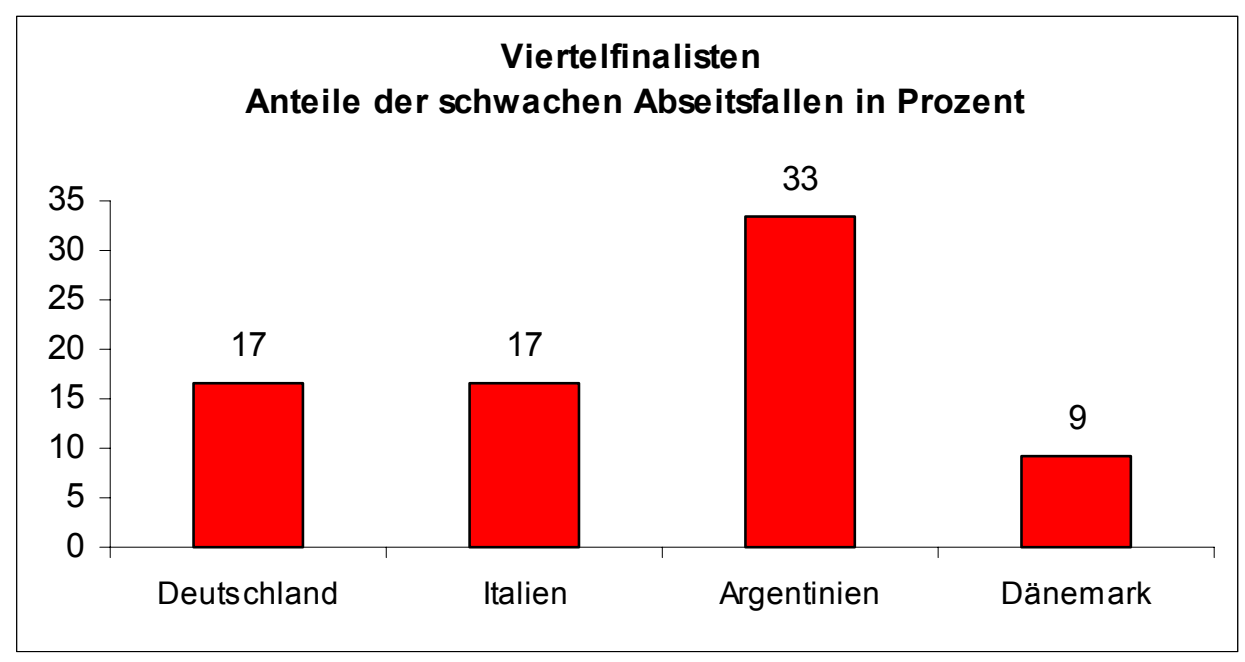

Diagramm 163: Anteile der schwachen „Abseitsfallen“ in Prozent bei den Viertelfinalisten.

Diagramm 162 erklärt, dass hochkarätigen Abseitsfalle zwischen $67 \%$ und $91 \%$ die dänische Mannschaft hatte die besten Werte und die argentinische Mannschaft hatte die schlechten Werte. Diagramm 163 erklärt, dass schwaches Abseitsfalle zwischen $9 \%$ und $33 \%$ die dänische hatte die besten Werte und die argentinische Mannschaft hatte die schlechten Werte.

Bei Vergleich die Diagrammen finden wir, dass die hochkarätige Abseitsfalle mehr besser als die schwache aber beim Abseitsfalle darf die Mannschaften kein Fehler haben, weil von der Abseitsfalle gleiche Möglichkeit Resultat alles oder nicht bekommen und wenn es Fehler gibt, dann wäre die Mannschaft in Gefahr sein, deswegen darf kein Fehler haben. 


\subsubsection{Halbfinalisten}

Als Halbfinalisten werden diejenigen Mannschaften bezeichnet, die das Halbfinale erreicht haben und zu deren Analyse aufgrund der automatischen Qualifikation für weiteres Spiel (Endspiel bzw. Spiel um Platz Drei) vier Spiele herangezogen werden konnten. Zu diesen Halbfinalisten gehören Frankreich, Brasilien, Kroatien und die Niederlande.

\subsubsection{Pressing hinten}

Bei „hochkarätigem“ „Pressing hinten“ hatte Kroatien mit 34 von 209 (16 \%) den größten Wert. Absteigend folgen die Niederlande mit 22 von 157 (14 \%) und Frankreich mit 29 von 225 (13\%). Brasilien mit 15 von 210 (7\%) hatte den kleinsten Anteil „guten“" „Pressing hinten“.

Das folgende Diagramm zeigt die Anteile des hochkarätigen Pressing hinten in Prozent der Halbfinalisten.

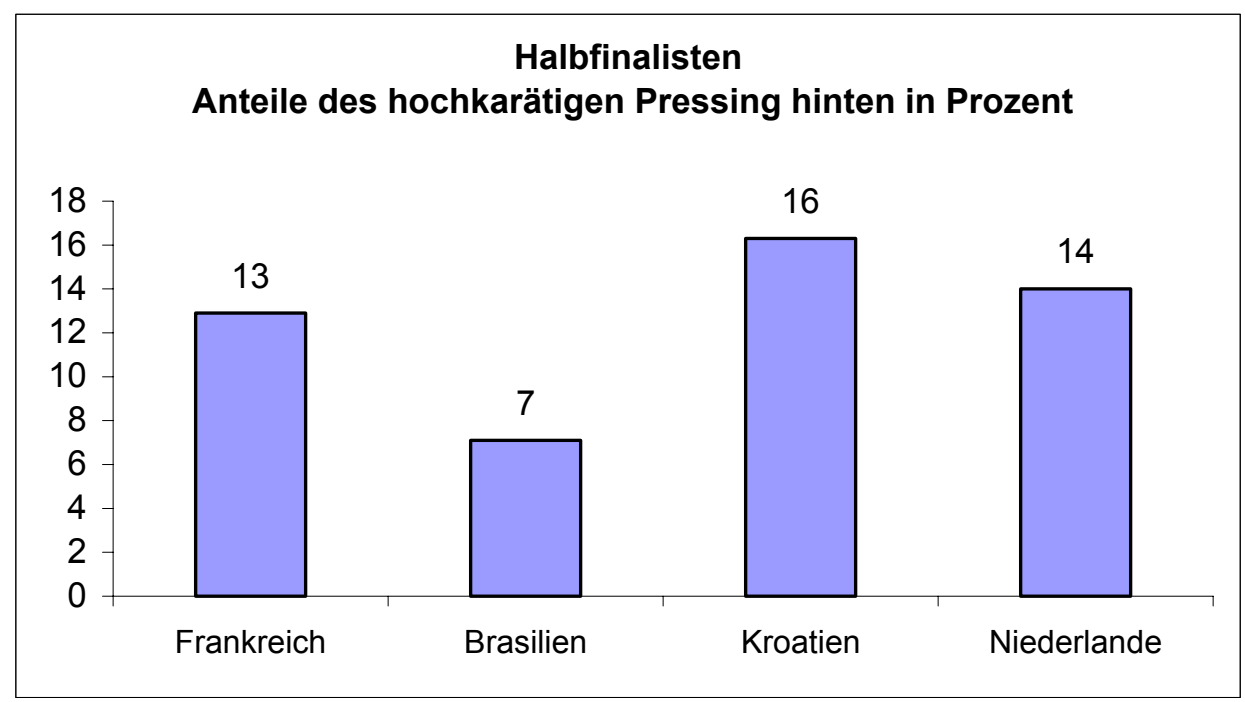

Diagramm 164: Anteile des hochkarätigen „Pressing hinten“ in Prozent bei den Halbfinalisten.

165 (79 \%) des „Pressing hinten“ von Brasilien wurden mit „normal“ bewertet und bildeten den größten Anteil. Die weiteren Anteile „normalen“ „Pressing hinten“ lauteten 163 (72 \%) für Frankreich, 147 (70 \%) für Kroatien und 109 (69 \%) für die Niederlande.

Dem folgenden Diagramm sind die Anteile des normalen Pressing hinten in Prozent der Halbfinalisten zu entnehmen. 


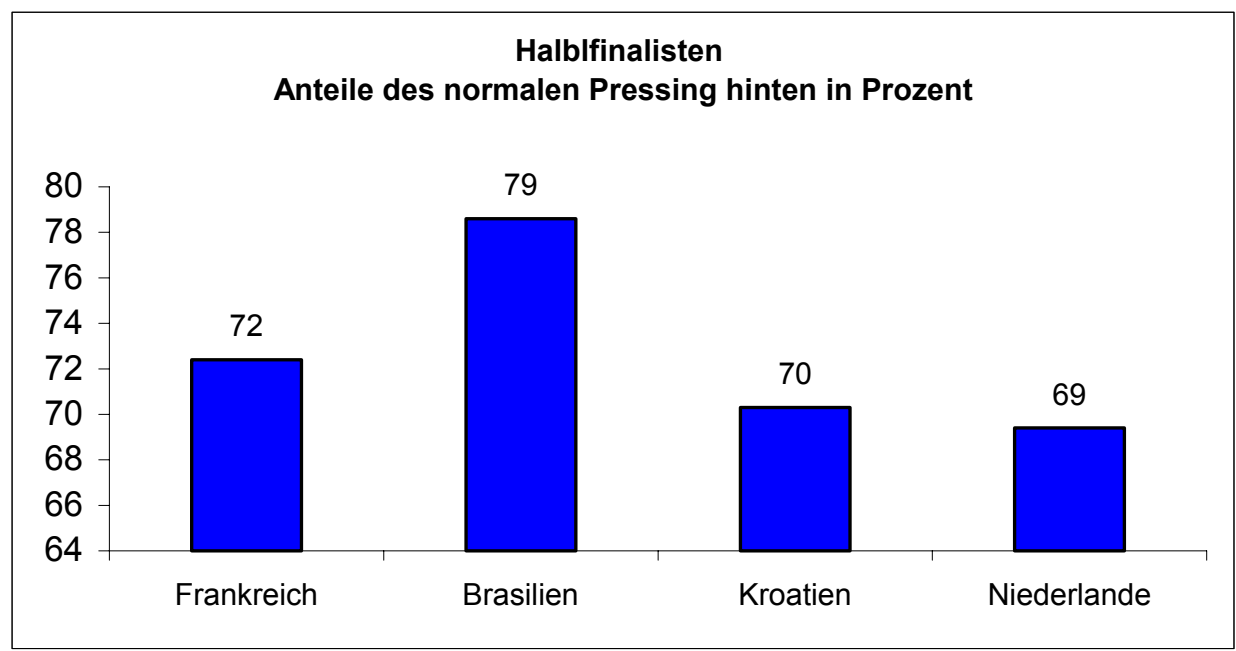

Diagramm 165: Anteile des normalen „Pressing hinten“ in Prozent bei den Halbfinalisten.

Ein „schwaches“ „Pressing hinten“ wurde mit einem Anteil von 26 (17\%) am häufigsten bei den Niederlanden festgestellt. Es folgen Frankreich 33 (15\%), Brasilien 30 (14\%), und schließlich Kroatien 28 (13\%).

Das folgende Diagramm zeigt die Anteile des schwachen Pressing hinten in Prozent der Halbfinalisten.

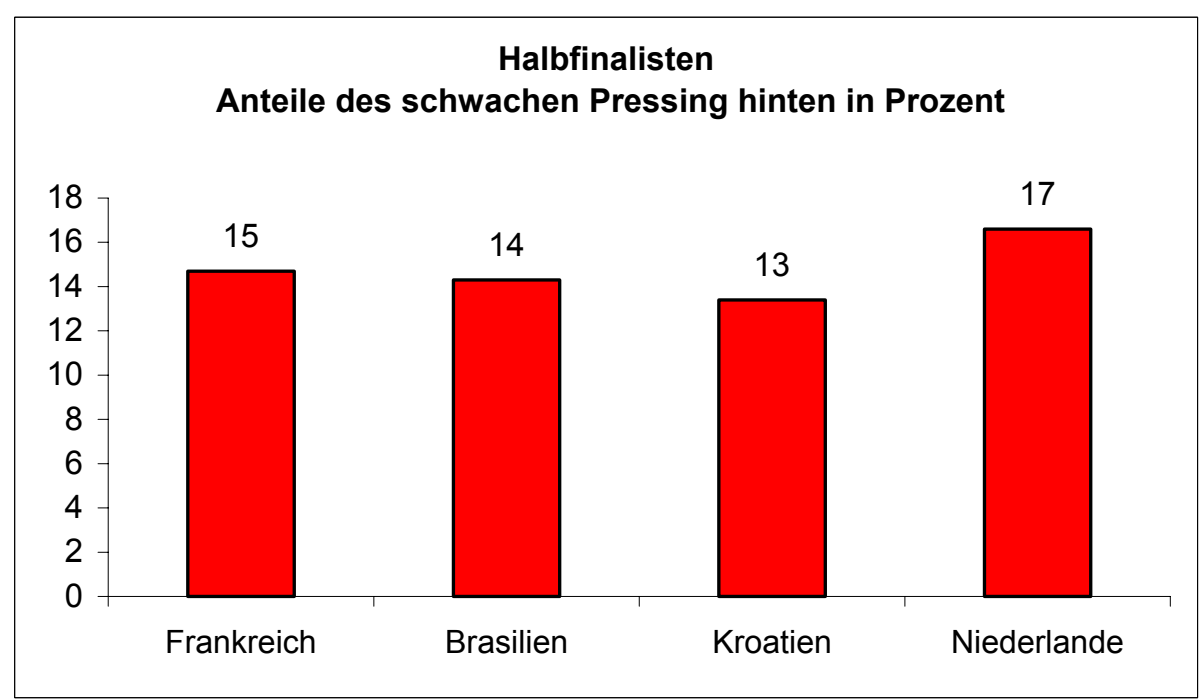

Diagramm 166: Anteile des schwachen „Pressing hinten“ in Prozent bei den Halbfinalisten.

Diagramm 164 erklärt, dass hochkarätiges Pressing hinten zwischen $7 \%$ und $16 \%$ Die kroatische hatte hier die besten Werte und die brasilianische hatte die schlechtesten. Diagramm 165 erklärt, dass normales Pressing hinten zwischen $69 \%$ und 79 
$\%$ vorkam. Die brasilianische hatte hier die besten Werte und die niederländische hatte die schlechtesten. Diagramm 166 erklärt, dass schwaches Pressing hinten zwischen $13 \%$ und $17 \%$ vorkam. Die kroatische Mannschaft hatte die besten Werte und die niederländische Mannschaft hatte die schlechten Werte.

Es lässt sich Schlussfolgerung aus den Diagrammen ziehen, dass das normale Pressing hinten bei allem Halbfinalisten nicht nur häufiger als die hochkarätige, sondern auch als die schwachen Pressing hinten vorkam.

\subsubsection{Pressing Mitte}

Beim „guten“ „Pressing Mitte“ erzielten sämtliche Halbfinalisten 0,0 \%.

Das folgende Diagramm zeigt die Anteile des hochkarätigen Pressing Mitte in Prozent der Halbfinalisten.

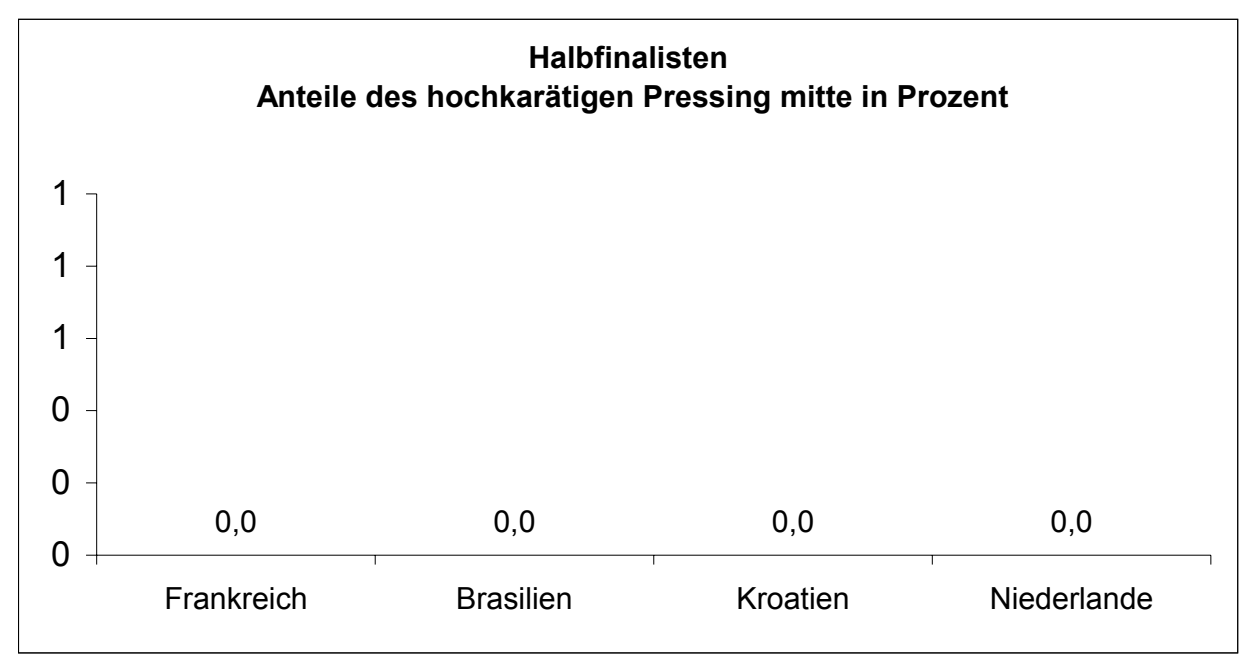

Diagramm 167: Anteile des hochkarätigen „Pressing Mitte“ in Prozent bei den Halbfinalisten.

185 von 209 (89 \%) des „Pressing Mitte“ wurden bei Frankreich mit „normal“ bewertet und dies bildete den größten Anteil. Die weiteren Anteile „normalen“ „Pressing Mitte" lauteten 136 von 165 (82 \%) für Brasilien, 64 von 87 (74 \%) für Kroatien und 102 von 144 (71\%) für die Niederlande.

Das folgende Diagramm zeigt die Anteile des normalen Pressing Mitte in Prozent der Halbfinalisten. 


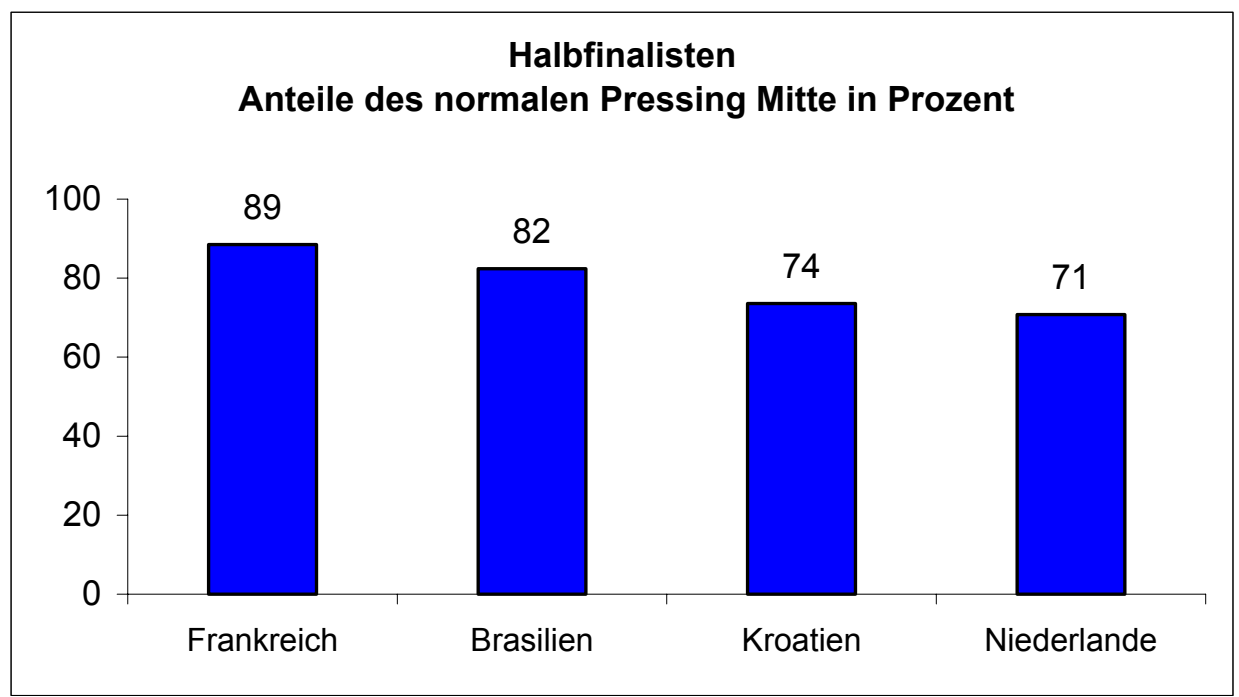

Diagramm 168: Anteile des normalen „Pressing Mitte“ in Prozent bei den Halbfinalisten.

Ein „schwaches“ „Pressing Mitte“ wurde mit einem Anteil von 42 (29 \%) am häufigsten bei den Niederlanden festgestellt. Es folgen Kroatien 23 (26\%), Brasilien 29 (18 $\%)$ und schließlich Frankreich 24 (12\%).

Dem folgenden Diagramm sind die Anteile des schwachen Pressing mitte in Prozent der Halbfinalisten zu entnehmen.

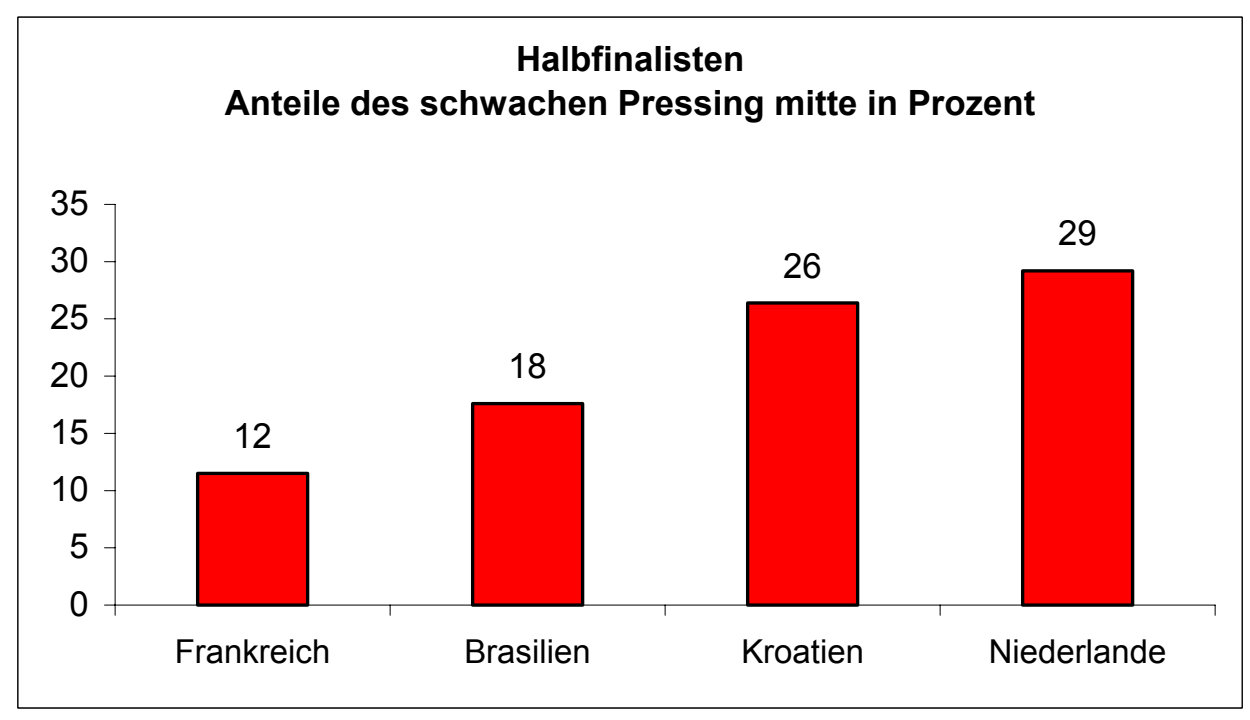

Diagramm 169: Anteile des schwachen „Pressing mitte“ in Prozent bei den Halbfinalisten. 
Diagramm 167 zeigt, dass hochkarätiges Pressing Mitte 0,0 \% für alle Mannschaften sind. Diagramm 168 zeigt, dass normales Pressing Mitte zwischen $71 \%$ und $89 \%$ vorkommt. Die französische Mannschaft hatte hier die besten Werte und die niederländische hatte die schlechtesten. Diagramm 169 zeigt, dass schwaches Pressing Mitte zwischen $12 \%$ und $29 \%$ vorkam. Die französische Mannschaft hatte die besten Werte und die niederländische Mannschaft hatte die schlechten Werte.

Aus den Diagrammen geht hervor, dass das hochkarätige Pressing Mitte sehr schwach in seiner Wirkung ist, da keine der beiden Mannschaften den Ball aus der Mitte abnehmen und ein Tor erzielen konnte. Zu Begründen ist das mit der Langsamkeit der Spieler beim gekonterten Angriff.

\subsubsection{Pressing vorne}

Beim "guten“ „Pressing vorne“ hatte Kroatien mit 4 von 24 (17\%) den größten Wert. Absteigend folgen Frankreich mit 7 von 48 (15\%), Brasilien mit 3 von 28 (11\%). Die Niederlande mit 1 von 42 (2\%) hatten den kleinsten Anteil „hochkarätigen“ „Pressing vorne".

Dem folgenden Diagramm sind die Anteile des hochkarätigen Pressing vorne in Prozent der Halbfinalisten zu entnehmen.

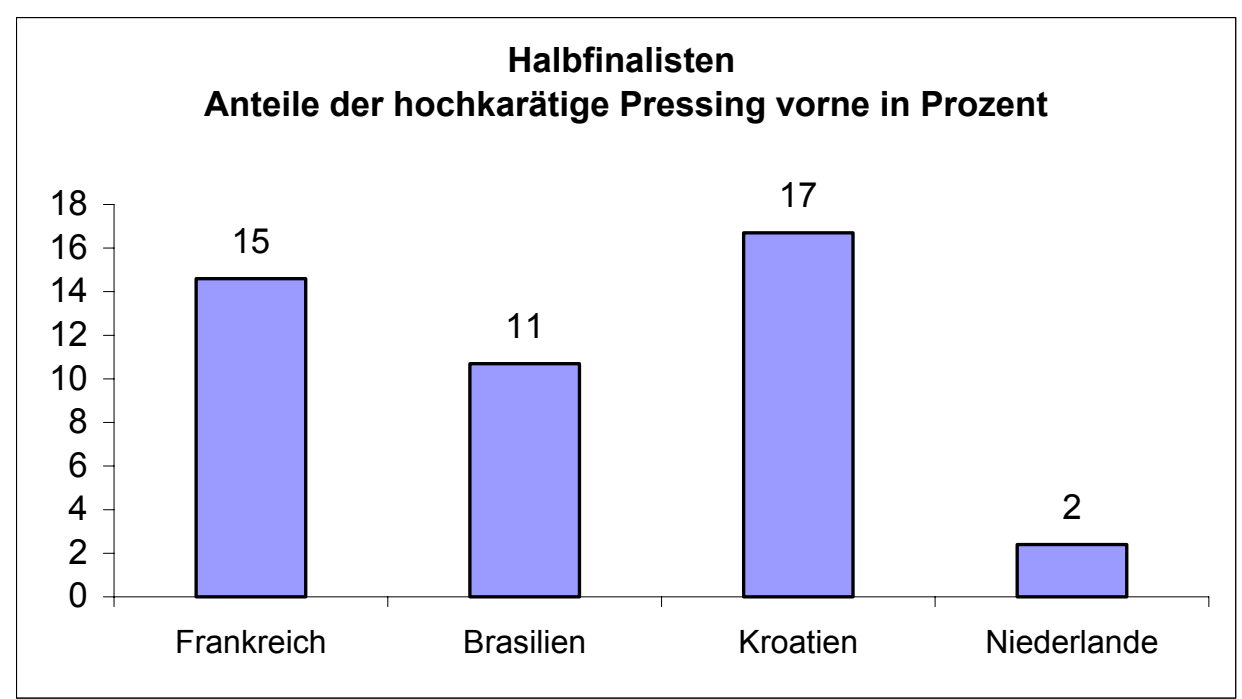

Diagramm 170: Anteile des hochkarätiges „Pressing vorne“ in Prozent bei den Halbfinalisten. 
$34(81 \%)$ des „Pressing vorne“ von den Niederlanden wurden mit „normal“ bewertet und bildeten den größten Anteil. Die weiteren Anteile „normalen“ „Pressing vorne“ lauteten 34 (71 \%) für Frankreich, 16 (57\%) für Brasilien und 12 (50 \%) für Kroatien. Dem folgenden Diagramm sind die Anteile des normalen Pressing vorne in Prozent der Halbfinalisten zu entnehmen.

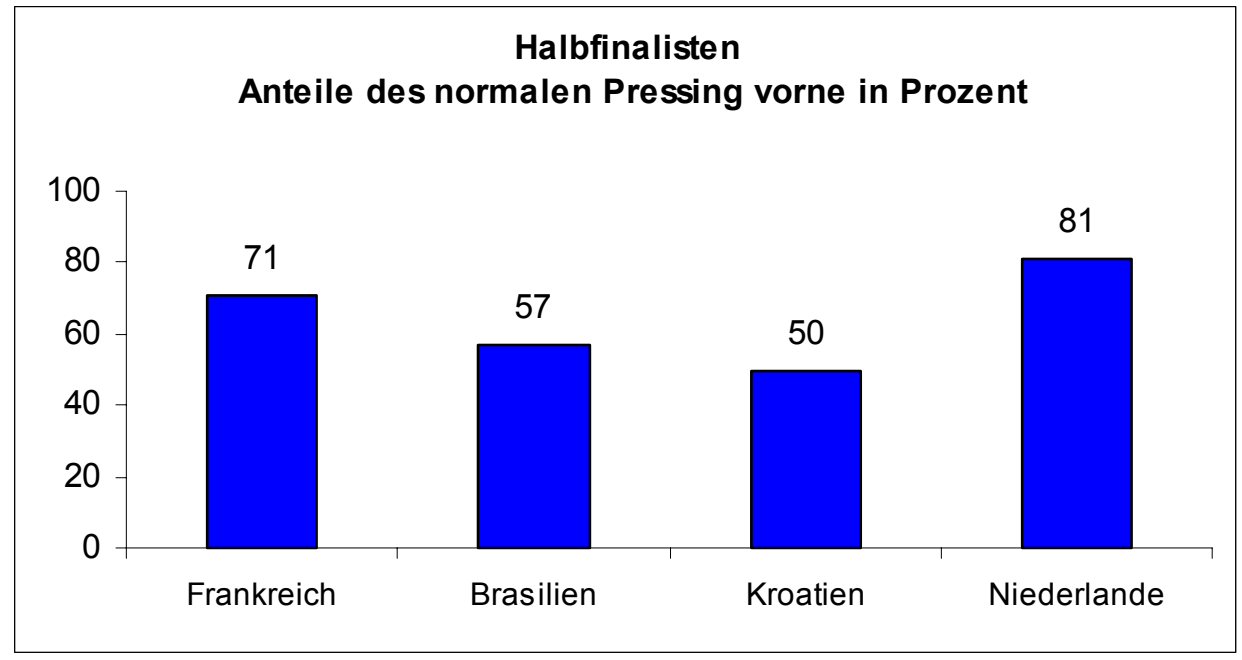

Diagramm 171: Anteile des normalen „Pressing vorne“ in Prozent bei den Halbfinalisten.

Ein „schwaches“ „Pressing vorne“ wurde mit einem Anteil von 8 (33 \%) am häufigsten bei Kroatien festgestellt. Es folgen Brasilien 9 (32\%), die Niederlande 7 (17\%) und schließlich Frankreich 7 (15\%).

Das folgende Diagramm zeigt die Anteile der schwachen Pressing vorne in Prozent der Halbfinalisten.

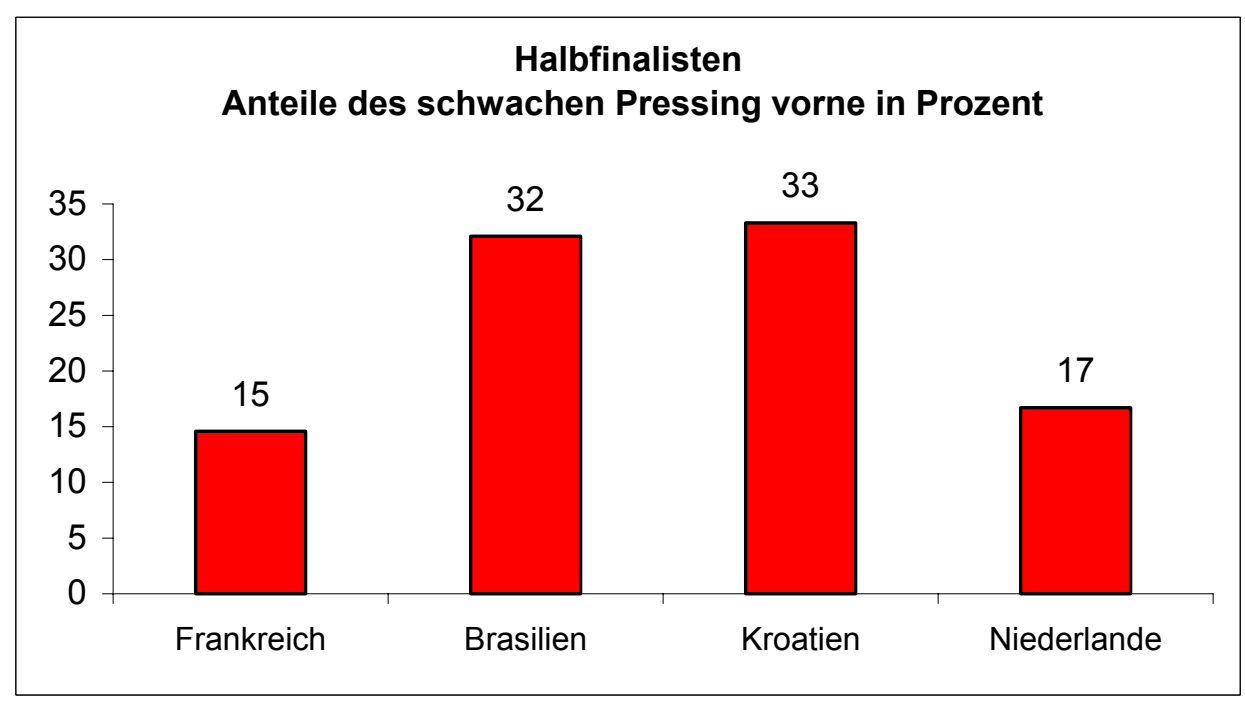

Diagramm 172: Anteile des schwachen „Pressing vorne“ in Prozent bei den Halbfinalisten. 
Diagramm 169 zeigt, dass schwaches Pressing vorne zwischen $2 \%$ und $17 \%$ vorkommt. Die kroatische Mannschaft hatte die besten Werte und niederländische Mannschaft hatte die schlechten Werte. Diagramm 170 zeigt, dass normales Pressing vorne zwischen $50 \%$ und $81 \%$ vorkommt. Die niederländische Mannschaft hatte hier die besten Werte und die kroatische hatte die schlechtesten. Diagramm 172 zeigt, dass schwaches Pressing vorne zwischen $15 \%$ und $33 \%$ erzielt wurde; Die französische Mannschaft hatte die besten Werte und die kroatische Mannschaft hatte die schlechten Werte.

Aus den Diagrammen wird deutlich, dass der Anteil des hochkarätigen Pressing vorne geringer ist als das gescheiterte und dass das normale Pressing vorne weit öfter geschieht als die beiden anderen Kategorien. Der Grund liegt wahrscheinlich darin, dass die meisten Mannschaften im Halbfinale sich überwiegend auf das Verteidigungsspiel stützen, und weniger versuchen den Ball vorne abzunehmen und ein Tor zu erzielen. Das führt dazu, dass weniger Angreifer in dem Drittel des Gegners versuchen, den Ball wieder in Besitz zu bekommen.

\subsubsection{Raumaufteilung}

Hinsichtlich der Qualität der Raumaufteilung wurde fest gestellt: Frankreich hatte mit 36 von 305 (12\%) den größten Wert. Absteigend folgen Kroatien mit 24 von 213 (11 $\%)$ und die Niederlande mit 16 von 186 (9\%). Brasilien hatte mit 19 von 279 (7\%) den kleinsten Anteil "guter" Raumaufteilung.

Das folgende Diagramm zeigt die Anteile der hochkarätigen „Raumaufteilung“ in Prozent der Halbfinalisten. 


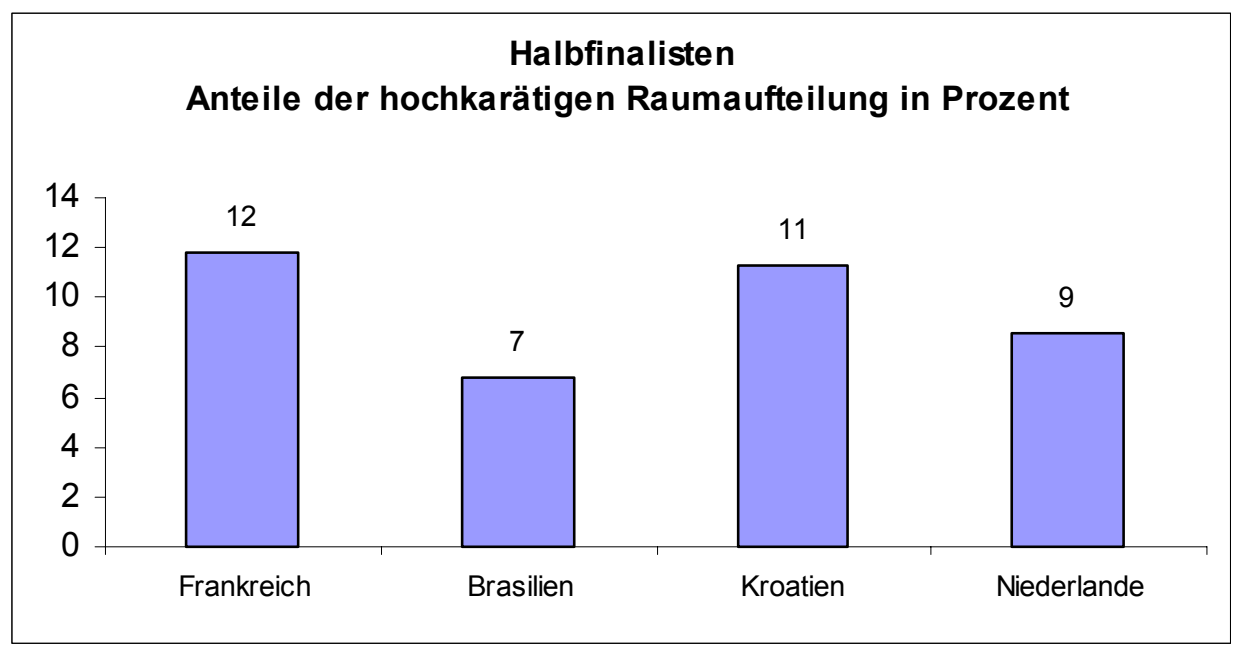

Diagramm 173: Anteile der hochkarätigen „Raumaufteilung“ in Prozent bei den Halbfinalisten.

229 (82 \%) der Raumaufteilungen von Brasilien wurden mit "normal“ bewertet und bildeten den größten Anteil. Die weiteren Anteile „normaler" Raumaufteilungen lauteten 174 (82 \%) für Kroatien, 149 (80 \%) für die Niederlande und 244 (80\%) für Frankreich.

Dem folgenden Diagramm sind die Anteile der normalen Raumaufteilung in Prozent der Halbfinalisten zu entnehmen.

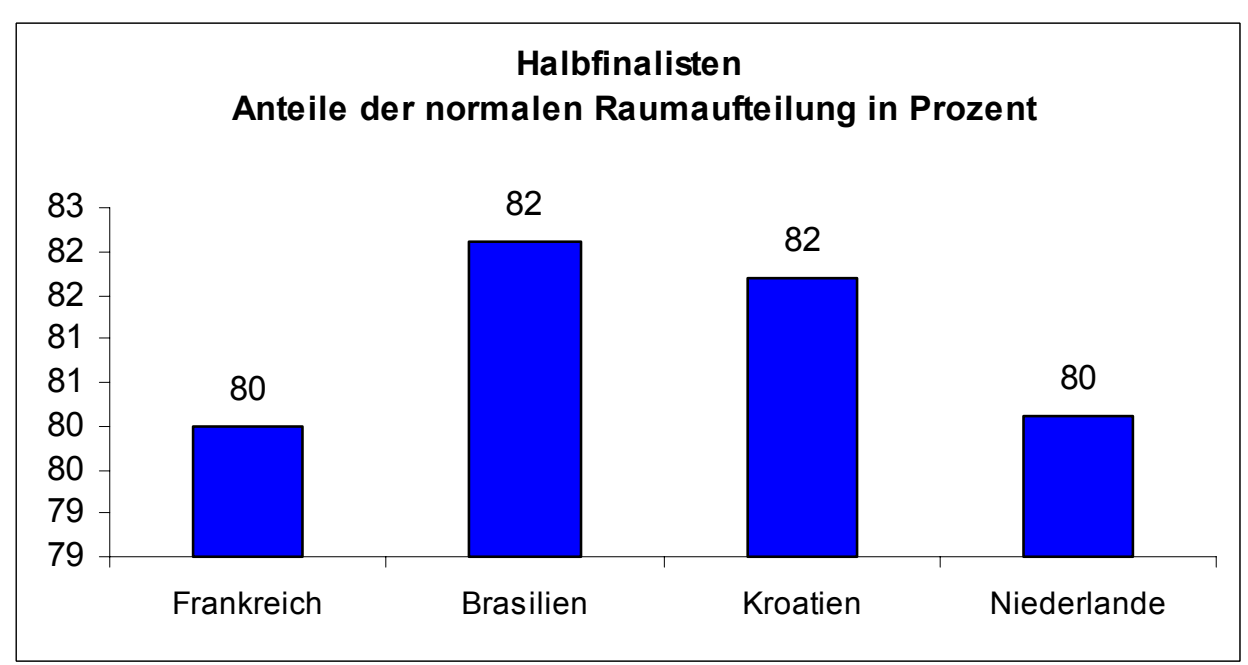

Diagramm 174: Anteile der normalen „Raumaufteilung“ in Prozent bei den Halbfinalisten. 
Eine „schwache“ Raumaufteilung wurde mit einem Anteil von 21 (11\%) am häufigsten bei den Niederlanden festgestellt. Es folgen Brasilien 31 (11\%), Frankreich 25 (8 \%) und schließlich Kroatien 15 (7\%).

Das folgende Diagramm zeigt die Anteile der schwachen Raumaufteilung in Prozent der Halbfinalisten.

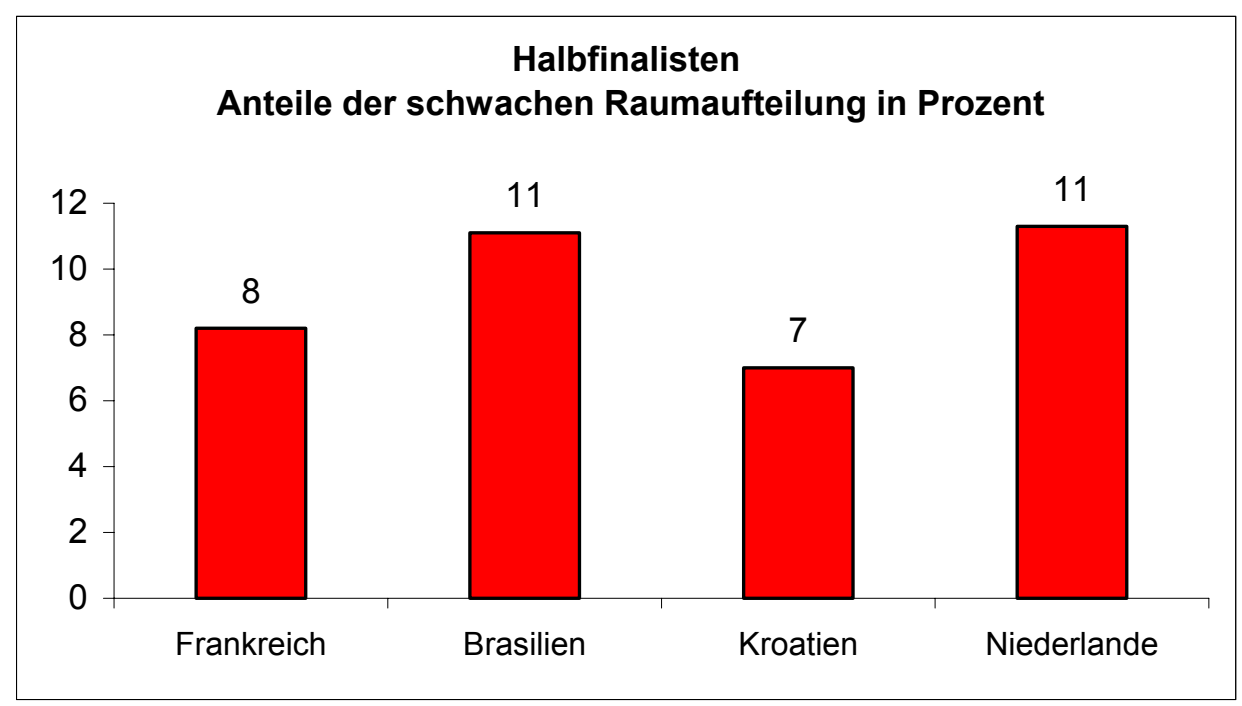

Diagramm 175: Anteile der schwachen „Raumaufteilung“ in Prozent bei den Halbfinalisten.

Diagramm 173 zeigt, dass schwache Raumaufteilung zwischen $7 \%$ und $12 \%$ vorkam; Die französische Mannschaft hatte die besten Werte und die brasilianische Mannschaft hatte die schlechten Werte. Diagramm 174 zeigt, dass normalen Raumaufteilung zwischen $80 \%$ und $82 \%$ vorkam. Die brasilianische und die kroatische Mannschaft hatten hier die besten Werte und die französische und die niederländische hatten die schlechtesten. Diagramm 175 zeigt, dass schwache Raumaufteilung zwischen $7 \%$ und $11 \%$ vorkam. Die kroatische hatte die besten Werte und die brasilianische und die niederländische Mannschaft hatten die schlechten Werte.

Aus den Diagrammen geht hervor, dass die normale Raumaufteilung sehr hoch in seiner Wirkung ist, da die beiden Mannschaften ihre Spieler besser in der Mitte des Spielfelds verteilen. Der Grund liegt wahrscheinlich darin, dass die meisten Mannschaften im Achtelfinale sich überwiegend auf das Verteidigungsspiel stützen. 


\subsubsection{Zurückkommen}

"Guten“ „Zurückkommen" hatte die Niederlande mit 4 von 118 (3 \%) den größten Wert. Absteigend folgen Frankreich mit 5 von 171 und Kroatien mit 3 von 103 mit jeweils (3\%). Brasilien hatte mit 2 von 134 (2\%) den kleinsten Anteil "hochkarätig“ „Zurückkommen“.

Dem folgenden Diagramm sind die Anteile des hochkarätigen Zurückkommens in Prozent der Halbfinalisten zu entnehmen.

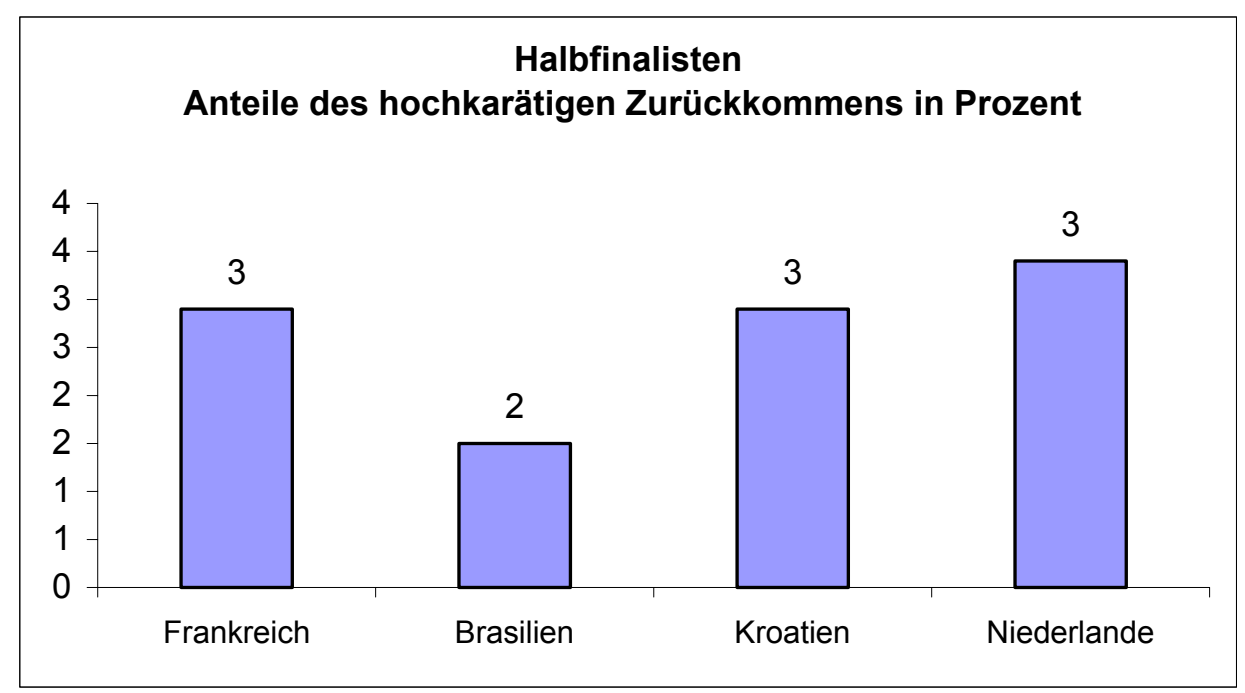

Diagramm 176: Anteile des hochkarätigen „Zurückkommens“ in Prozent bei den Halbfinalisten.

131 (98 \%) des „Zurückkommens“ von Brasilien wurden mit „normal“ bewertet und bildeten den größten Anteil. Die weiteren Anteile „normalen“ „Zurückkommens“ lauteten 165 (97 \%) für Frankreich, 99 (96 \%) für Kroatien und 113 (96 \%) für die Niederlande.

Das folgende Diagramm zeigt die Anteile des normalen Zurückkommens in Prozent der Halbfinalisten. 


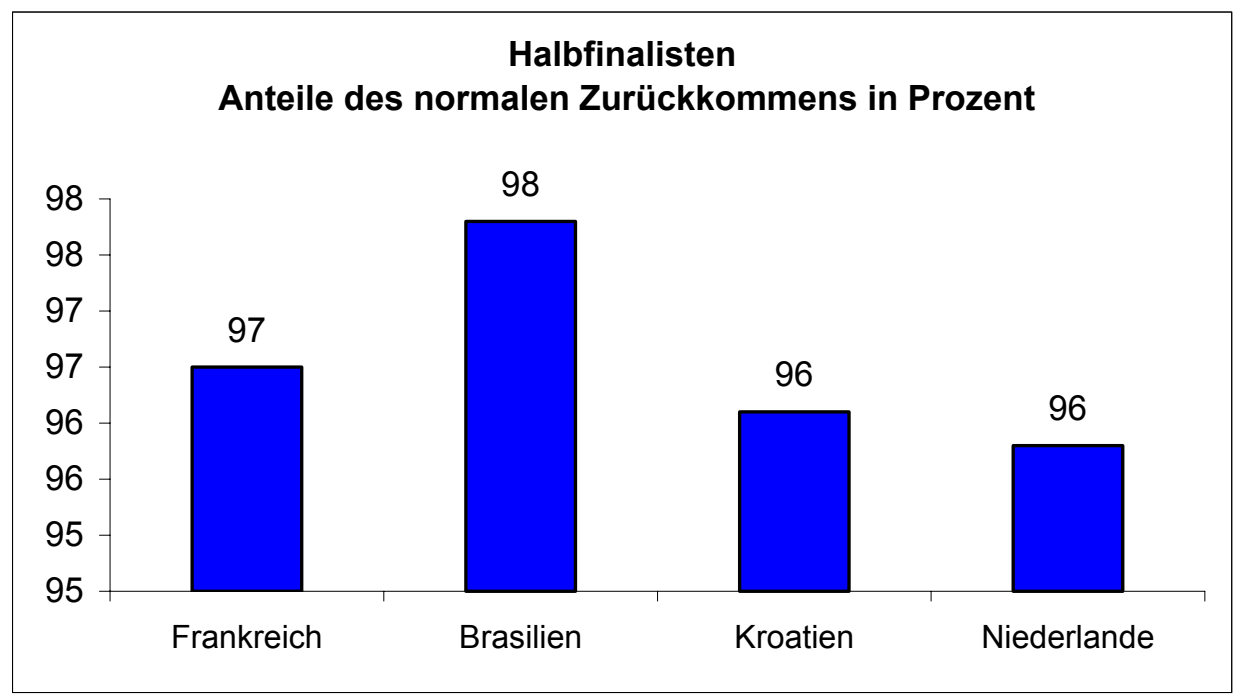

Diagramm 177: Anteile des normalen „Zurückkommens“ in Prozent bei den Halbfinalisten.

Ein „schwaches“ „Zurückkommen“ wurde mit einem Anteil von 1 (1\%) am häufigsten bei Kroatien festgestellt. Es folgen die Niederlande mit 1 (1\%), Brasilien mit 1 (1\%) und schließlich Frankreich mit 1 (1\%).

Das folgende Diagramm zeigt die Anteile des schwachen Zurückkommens in Prozent der Halbfinalisten.

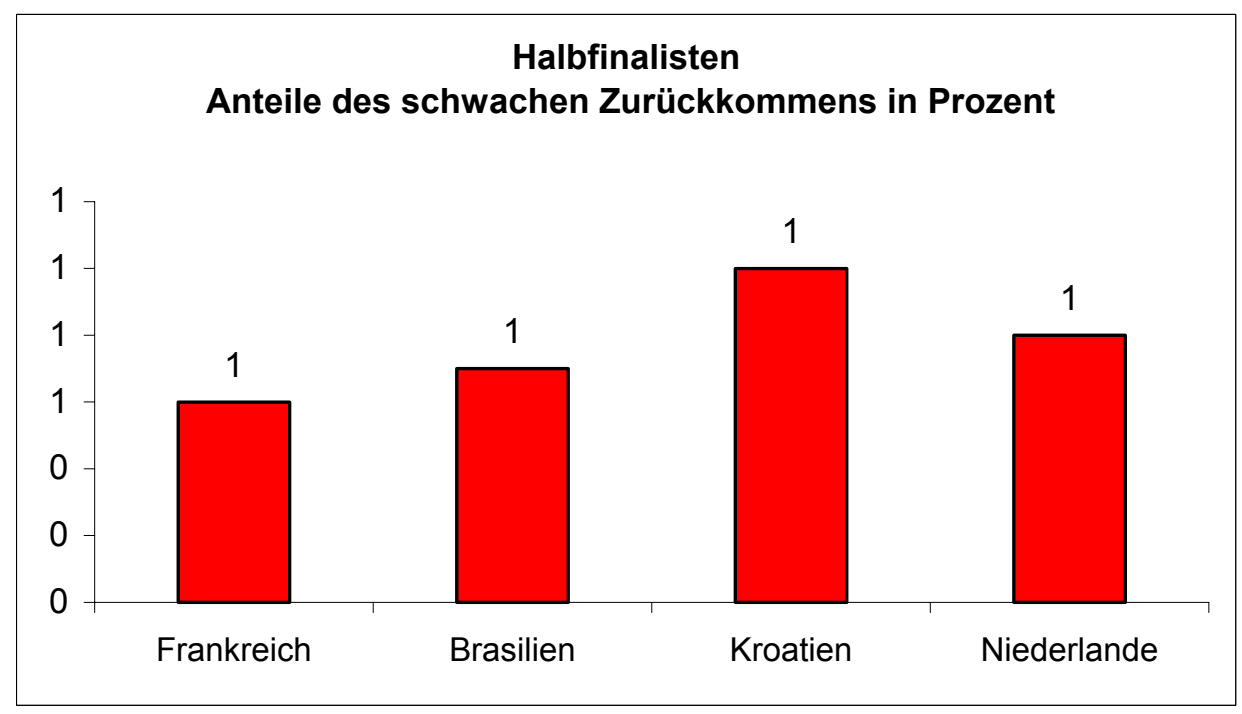

Diagramm 178: Anteile des schwachen „Zurückkommens“ in Prozent bei den Halbfinalisten. 
Diagramm 176 zeigt, dass hochkarätigen Zurückkommen zwischen $2 \%$ und $3 \%$ die französische, kroatische und niederländische Mannschaften hatten die gleichen besten Werte und die brasilianische Mannschaft hatte die schlechten Werte.

Diagramm 177 zeigt, dass normales Zurückkommen zwischen $96 \%$ und $98 \%$ vorkam. Die brasilianische hatte hier die besten Werte und niederländische und kroatische Mannschaften hatten die schlechtesten. Diagramm 178 zeigt, dass schwaches Zurückkommen für alle Mannschaften 0,0 ist.

Aus der Darstellung von der Diagrammen wird deutlich, dass das normalen Zurückkommen mehr besser ist als das hochkarätige und schwache, da die Spieler einen Platz einnehmen, von dem aus sie ihre Mitspieler so helfen können.

\subsubsection{Unterstützen}

Bei „Hochkarätigem“ Unterstützen hatten die Niederlande mit 1 von 5 (20\%) den größten Wert. Absteigend folgen Kroatien mit 2 von 11 (18\%) und Frankreich mit 5 von 30 (17\%). Brasilien hatte mit 1 von 13 (8\%) den kleinsten Anteil "guten“ Unterstützens.

Dem folgenden Diagramm sind die Anteile des hochkarätigen Unterstützens in Prozent der Halbfinalisten zu entnehmen.

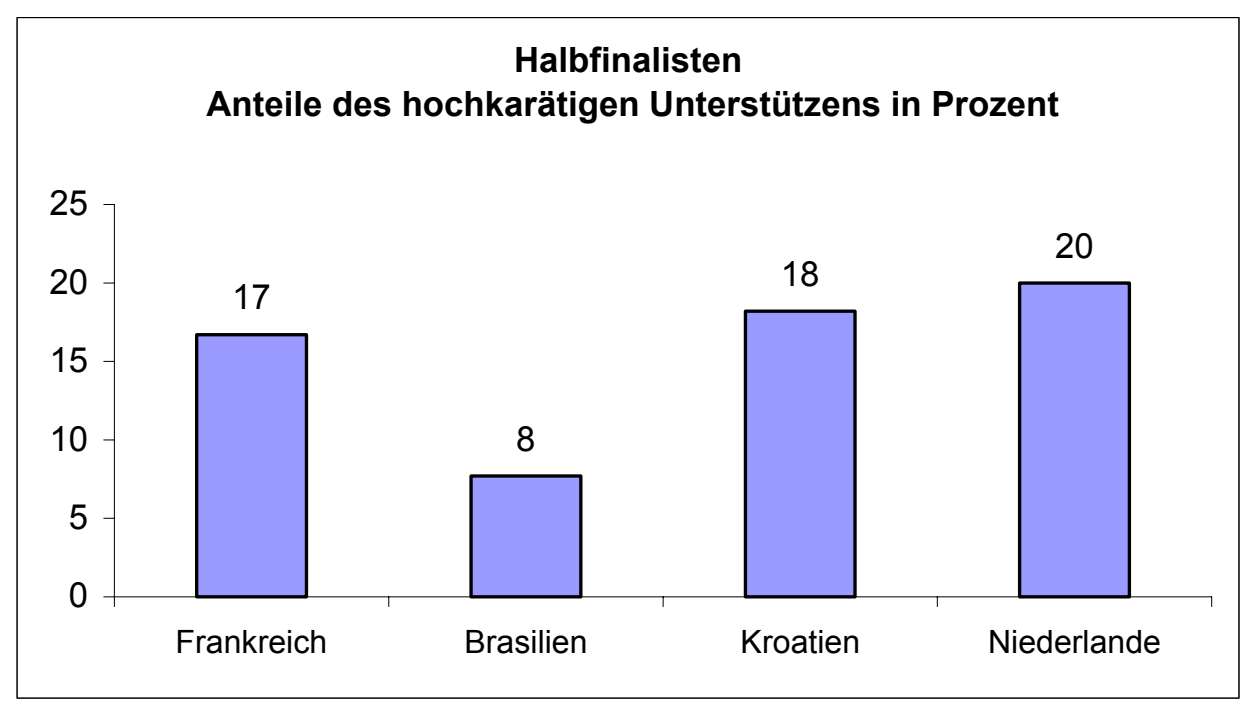

Diagramm 179: Anteile des hochkarätigen „Unterstützens“ in Prozent bei den Halbfinalisten. 
11 (85 \%) des Unterstützens von Brasilien wurde mit "normal“ bewertet und bildete den größten Anteil. Die weiteren Anteile „normalen“ Unterstützens lauteten 24 (80 \%) für Frankreich, 8 (73\%) für Kroatien und 3 (60 \%) für die Niederlande.

Das folgende Diagramm zeigt die Anteile des normalen Unterstützens in Prozent der Halbfinalisten.

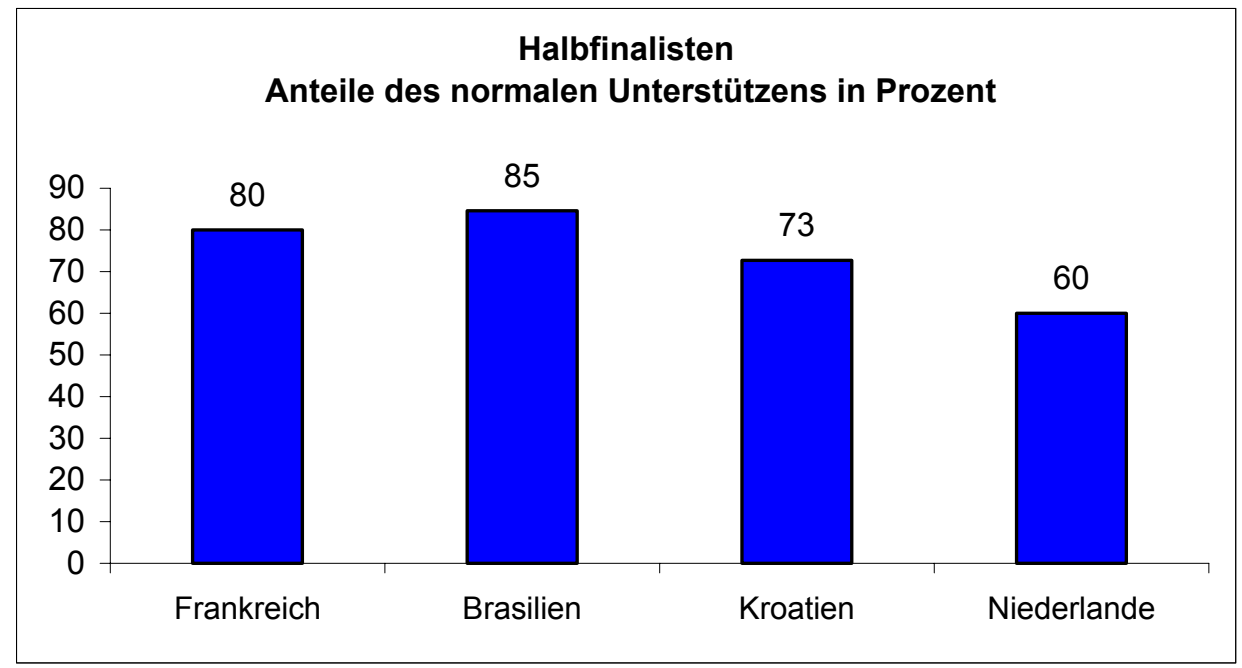

Diagramm 180: Anteile des normalen „Unterstützens“ in Prozent bei den Halbfinalisten.

Ein „schwaches“ Unterstützen wurde mit einem Anteil von 1 (20\%) am häufigsten bei den Niederlanden festgestellt. Es folgen Kroatien 1 (9\%), Brasilien $1(8 \%)$ und schließlich Frankreich 1 (3\%).

Das folgende Diagramm zeigt die Anteile des schwachen Unterstützens in Prozent der Halbfinalisten.

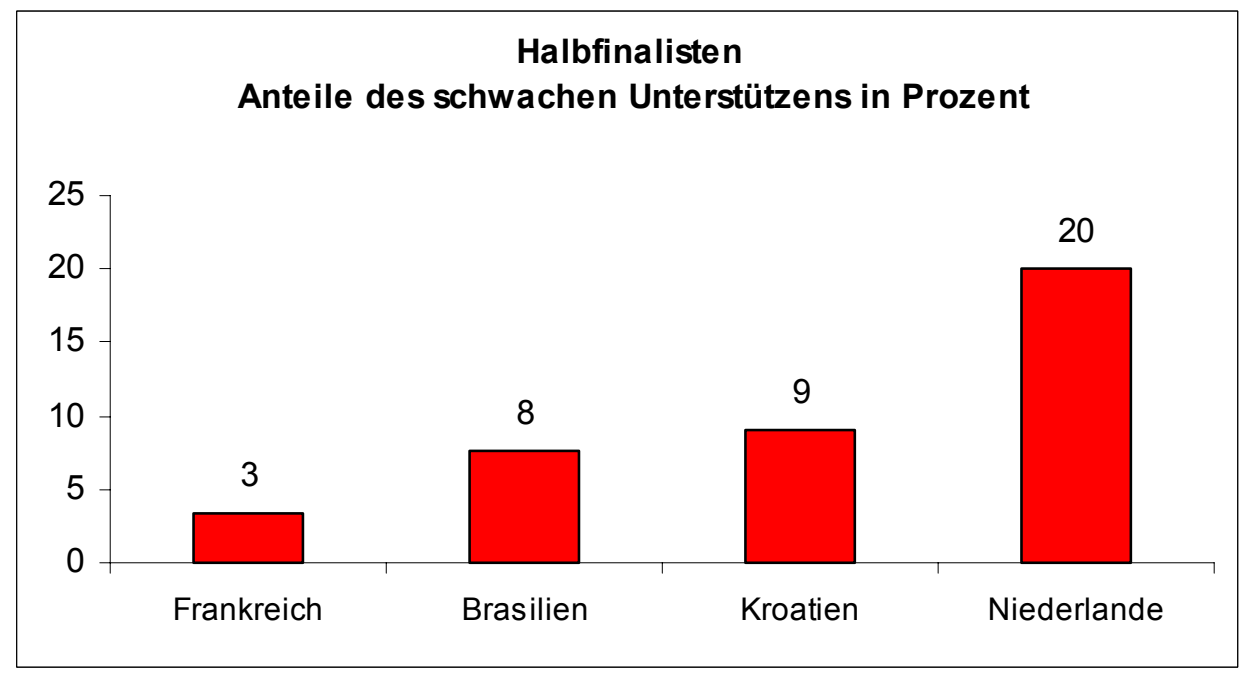

Diagramm 181: Anteile des schwachen „Unterstützens“ in Prozent bei den Halbfinalisten. 
Aus der Darstellung der Diagramme 179, 180 und 181 wird deutlich, dass das normalen Unterstützen häufiger ist als das hochkarätige und das schwache, da die unterstützenden Spieler einen Platz einnehmen, von dem aus sie ihren Mitspielern so helfen können, dass sie gut mit dem Ball vorankommen können. Manchmal unterstützen die Mitspieler nicht zur rechten Zeit oder richtigen Position.

\subsubsection{Rückraum sichern}

„Guten“ „Rückraum sichern“ hatte Brasilien mit 1 von 17 (6\%) den größten Wert. Absteigend folgen Kroatien mit 1 von 22 (5\%) und die Niederlande mit 1 von 23 (4\%). Frankreich hatte mit 1 von 35 (3\%) den kleinsten Anteil „guten“ „Rückraum sichern“. Das folgende Diagramm zeigt die Anteile des guten Rückraum sichern in Prozent der Halbfinalisten.

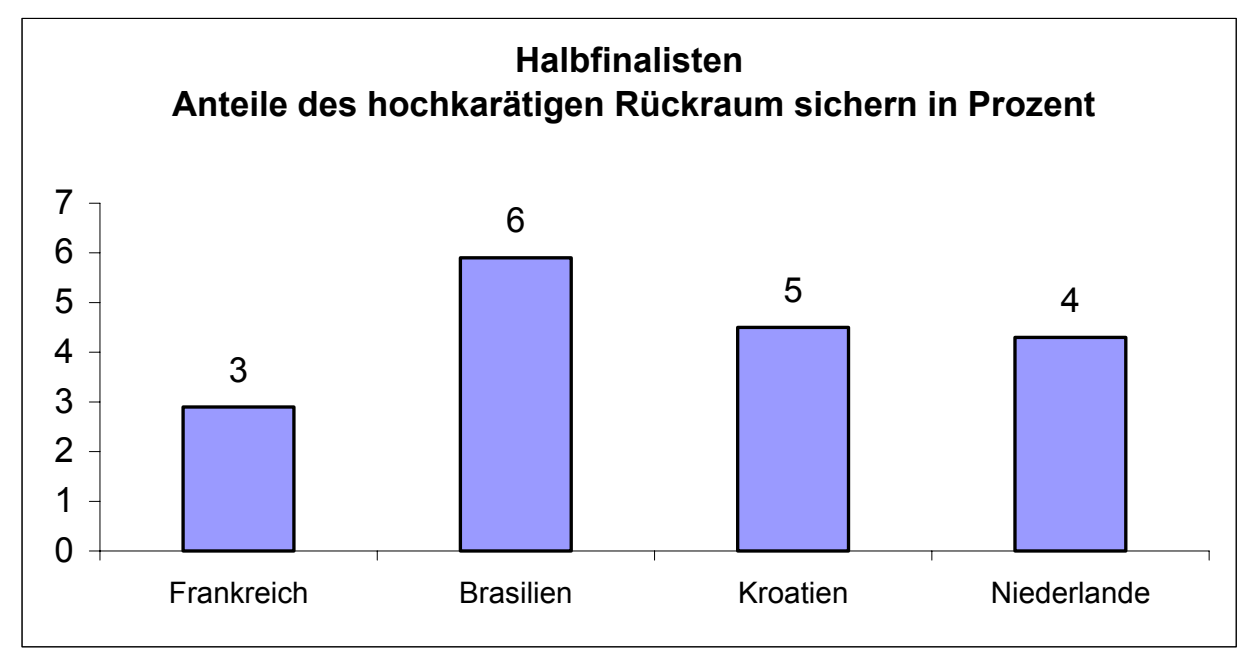

Diagramm 182: Anteile des hochkarätigen „Rückraum sichern“ in Prozent bei den Halbfinalisten.

20 (87 \%) des „Rückraum sichern“ von den Niederlanden wurden mit „normal“ bewertet und bildeten den größten Anteil. Die weiteren Anteile „normalen“ „Rückraum sichern“ lauteten 19 (86 \%) für Kroatien, 25 (71\%) für Frankreich und 10 (59\%) für Brasilien.

Das folgende Diagramm zeigt die Anteile des normalen Rückraum sichern in Prozent der Halbfinalisten. 


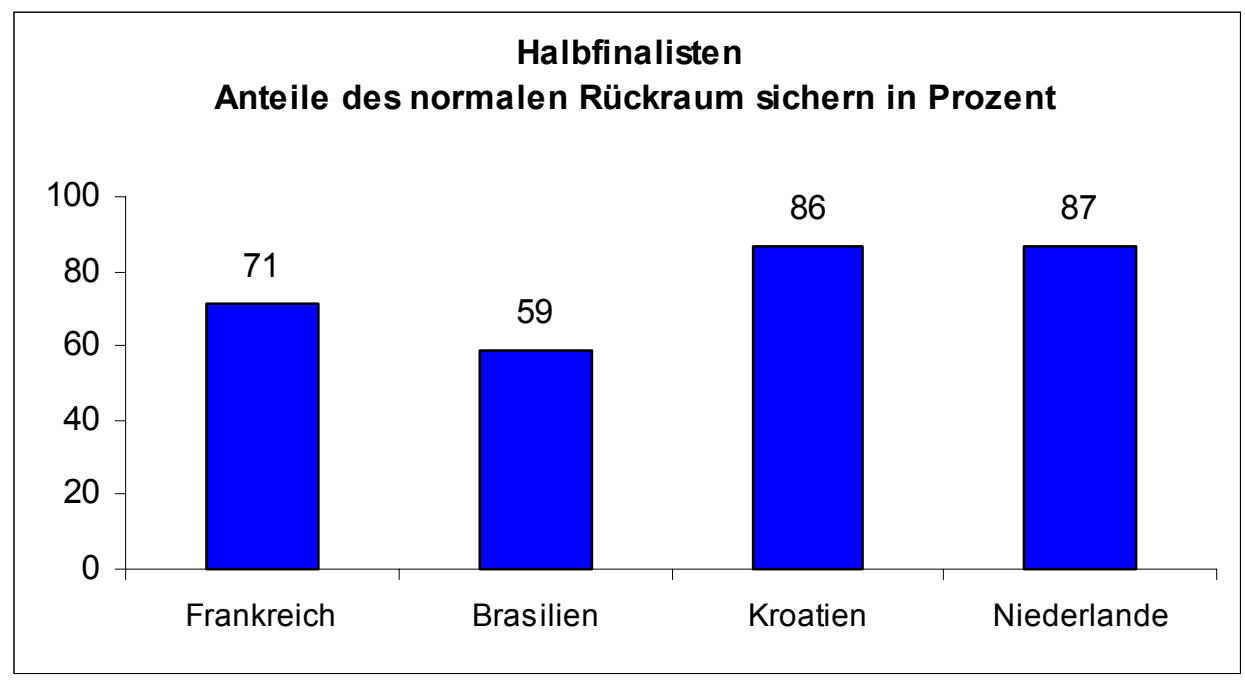

Diagramm 183: Anteile des normalen „Rückraum sichern“ in Prozent bei den Halbfinalisten.

Ein „schwaches“ „Rückraum sichern“ wurde mit einem Anteil von 6 (35 \%) am häufigsten bei Brasilien festgestellt. Es folgen Frankreich 9 (26\%), Kroatien 2 (9\%) und schließlich die Niederlande 2 (9\%).

Das folgende Diagramm zeigt die Anteile des schwachen Rückraum sichern in Prozent der Halbfinalisten.

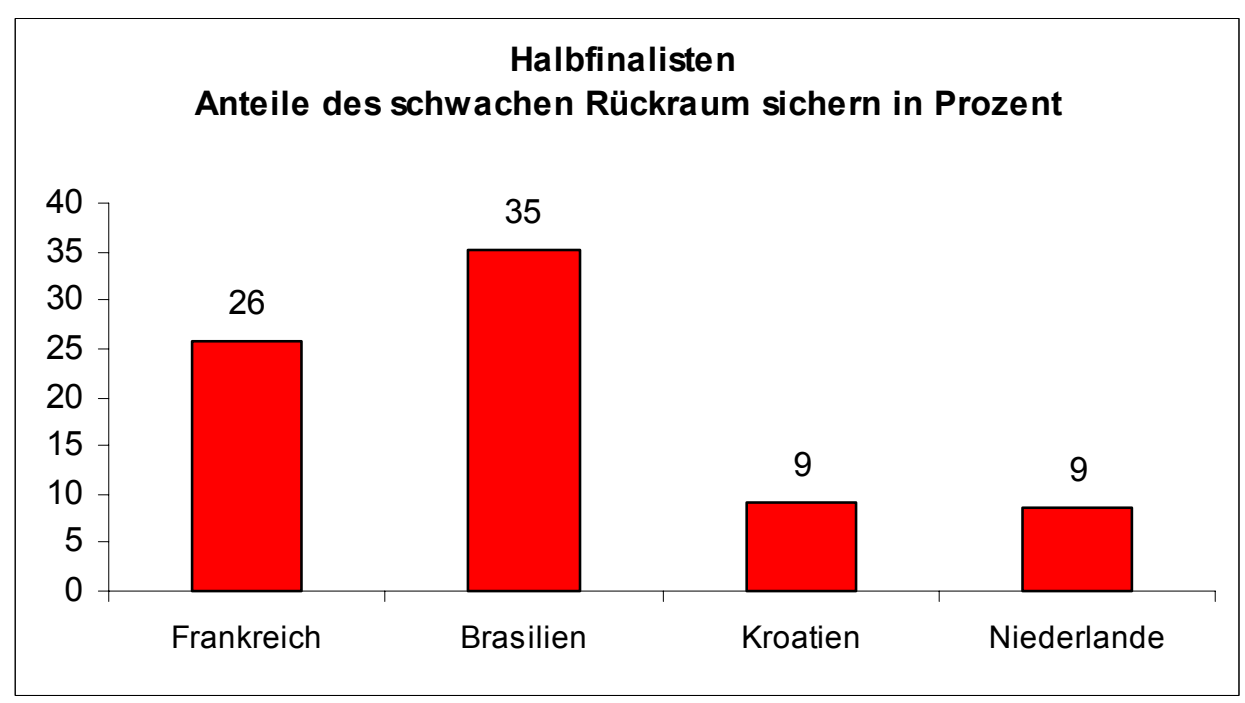

Diagramm 184: Anteile des „schwachen Rückraum sichern“ in Prozent bei den Halbfinalisten. 
Diagramm 182 zeigt, dass hochkarätiges Rückraum sichern zwischen $3 \%$ und $6 \%$ lag; die brasilianische Mannschaft hatte die besten Werte und die französische hatte die schlechten Werte. Diagramm 183 erklärt, dass normales Rückraum sichern zwischen $59 \%$ und $87 \%$ lag. Die niederländische hatte hier die besten Werte und die brasilianische hatte die schlechtesten Werte. Diagramm 184 erklärt, dass schwaches Rückraum sichern zwischen $9 \%$ und $35 \%$ lag. Die niederländische und die kroatische Mannschaft hatten die besten Werte und die brasilianische Mannschaft hatte die schlechten Werte.

Aus der Darstellung der Diagramme wird deutlich, dass das normalen und hochkarätigen Rückraum sichern häufiger ist als das schwache, da die Spieler bei der Defensivarbeit ihren Rückraum gut bewachen.

\subsubsection{Abseitsfalle}

Bei „Guten“ Abseitsfallen hatte Brasilien mit 13 von 14 (93 \%) den größten Wert. Absteigend folgen die Niederlande mit 12 von 13 (92 \%) und Frankreich mit 18 von 20 (90\%). Kroatien hatte mit 6 von 7 (86 \%) den kleinsten Anteil „guter“ Abseitsfallen. Das folgende Diagramm zeigt die Anteile der hochkarätigen Abseitsfallen in Prozent der Halbfinalisten.

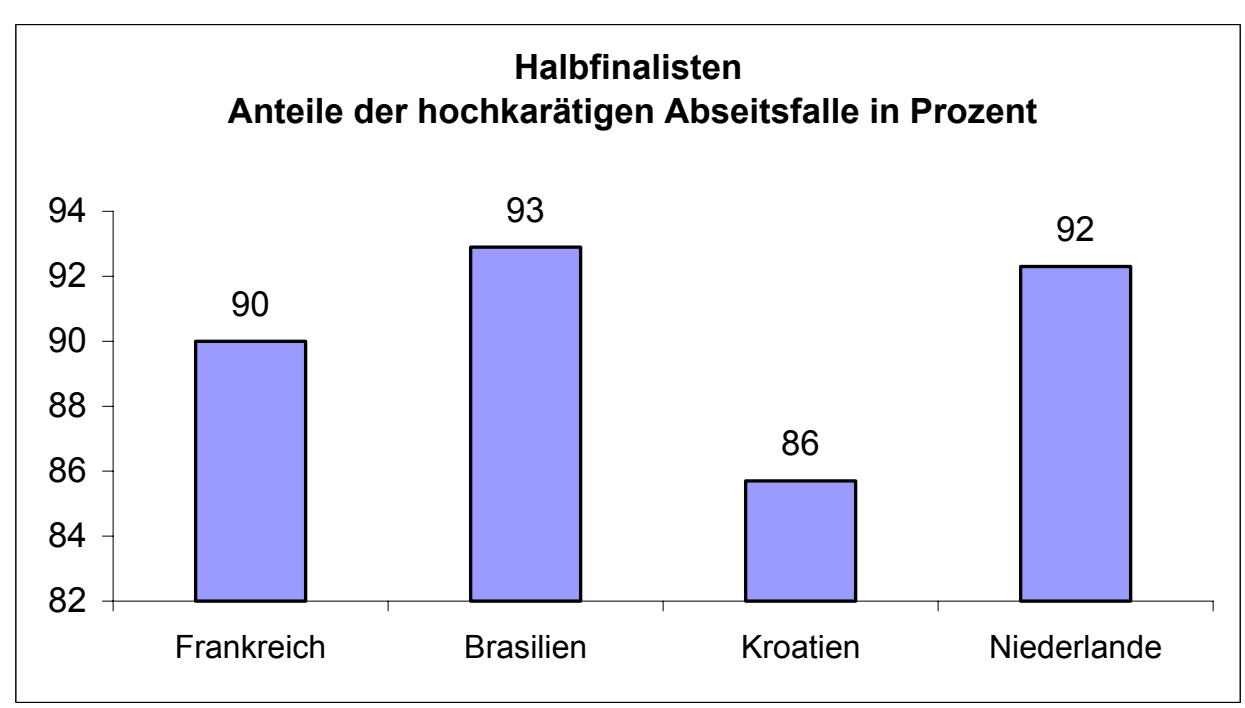

Diagramm 185: Anteile der „hochkarätigen Abseitsfalle“ in Prozent bei den Halbfinalisten. 
Eine „schwache“ Abseitsfalle wurde mit einem Anteil von 1 (14 \%) am häufigsten bei Kroatien festgestellt. Es folgen Frankreich mit 2 (10\%), die Niederlande mit 1 (8 \%) und schließlich Brasilien mit 1 (7\%).

Dem folgenden Diagramm sind die Anteile der schwachen Abseitsfallen in Prozent der Halbfinalisten zu entnehmen.

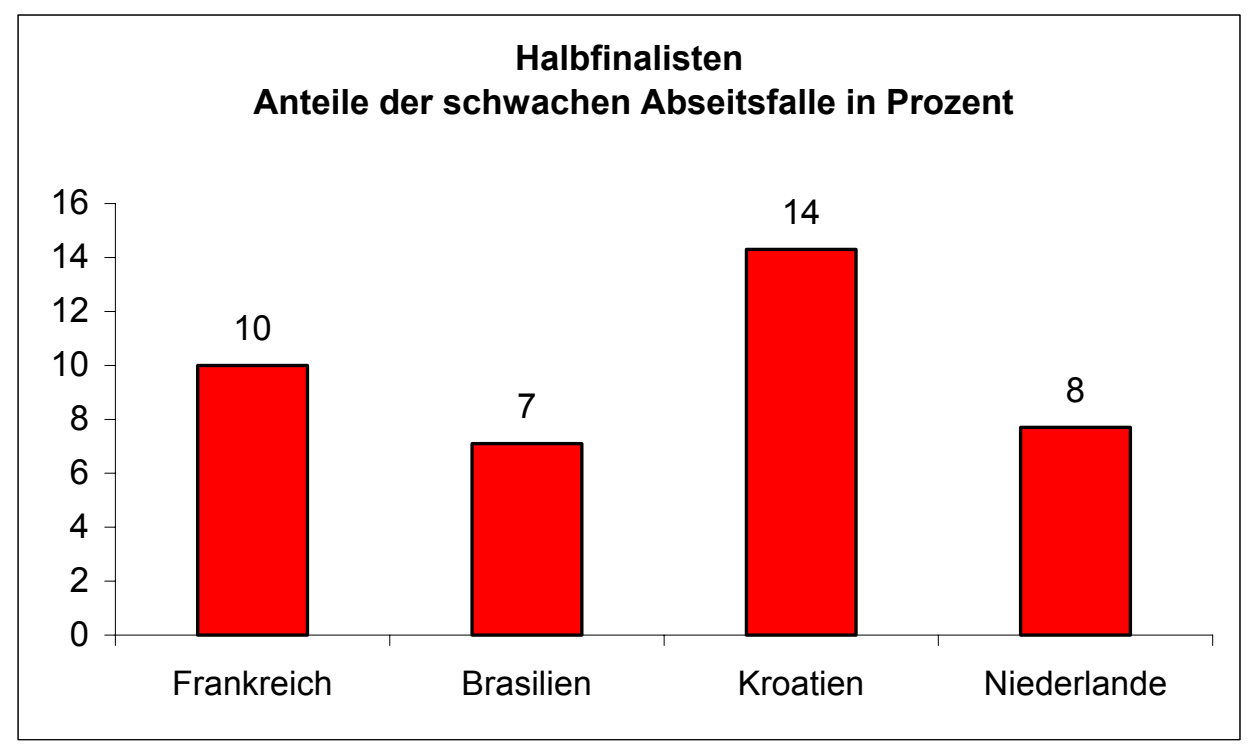

Diagramm 186: Anteile der „schwachen Abseitsfalle“ in Prozent bei den Halbfinalisten.

Diagramm 185 erklärt, dass bei der hochkarätigen Abseitsfalle die Werte zwischen $86 \%$ und $93 \%$ lagen und die brasilianische Mannschaft die besten Werte hatte, während der kroatischen Mannschaft die schlechten Werte hatte. Diagramm 186 erklärt, dass eine schwache Abseitsfalle nur zwischen $7 \%$ und $14 \%$ vorkam und die brasilianische Mannschaft die besten Werte und die kroatische Mannschaft die schlechten Werte hatte.

Bei Vergleich der Diagramme finden wir, dass die hochkarätigen Abseitsfallen deutlich häufiger als die schwachen Abseitsfallen vorkamen. Bei der Abseitsfalle dürfen die Mannschaften keinen Fehler machen, weil von der missglückten Abseitsfalle gleiche die Möglichkeit besteht, ein Tor zu bekommen. Deshalb sind Fehler hier unbedingt zu vermeiden, was diesen Mannschaften auch weitgehend gelungen ist.

Bei Vergleich der Halb- und Viertelfinalisten finden wir, dass die hochkarätigen Abseitsfallen bei den Halbfinalisten häufiger als die Viertelfinalisten sind und die schwachen Abseitsfallen bei den Viertelfinalisten häufiger als die Halbfinalisten sind. 


\subsection{Qualitätsquotienten technischer Elemente}

\subsubsection{Achtelfinalisten}

Auf der Suche nach einer einzigen Kennzahl, die die Qualität einer bestimmten Mannschaft hinsichtlich eines einzelnen technischen, offensiv-taktischen bzw. defensiv-taktischen Elements beschreibt, wurde die ursprüngliche Idee der Zuweisung von Zahlen an die einzelnen Kategorien (nämlich -1 für „schwach“, 0 für „normal“ und +1 für „gut") und die dadurch erzwungene metrische Meßbarkeit der Daten aufgrund mangelnder Schärfe bald verworfen.

An deren Stelle trat eine Einführung des sogenannten Qualitätsquotienten, einer Kennzahl, die das Verhältnis der Anzahl der "guten“ zu der der „schwachen“ ohne Berücksichtigung der „normalen“ Aktionen einer bestimmten Mannschaft bezüglich eines bestimmten Elements beschreibt. Der Qualitätsquotient ist aufgrund der getroffenen Definition eine Zahl, die zwischen Null und plus Unendlich liegt. Eine Zahl größer als Eins deutet auf eine größere Anzahl guter als schwacher, eine Zahl kleiner Eins auf eine größere Anzahl schwacher als guter Aktionen hin, wobei eine Mannschaft bezüglich eines Elements grundsätzlich umso höher zu bewerten ist, je größer der entsprechende Qualitätsquotient ist.

Die Einführung des Qualitätsquotienten geht zwar im Vergleich zu der ursprünglichen Datendarstellung in Form einer prozentualen Verteilung der einzelnen Kategorien mit einem gewissen Informationsverlust einher, dieser wird jedoch nach Ansicht des Verfassers durch die über die Reduzierung der Qualitätsbeschreibung auf eine einzige Zahl (statt vorher drei) gewonnene Übersichtlichkeit mehr als kompensiert. In den folgenden Kapiteln werden deshalb die prozentualen Verteilungen durch den Qualitätsquotienten ersetzt.

Der Qualitätsquotient einer bestimmten Mannschaft bezüglich eines einzelnen Elements ergibt sich, indem man (ungeachtet der „mittleren“ oder „normalen“ Aktionen) das Verhältnis der Anzahl „hochkarätiger" und „schwacher" Aktionen dieser Mannschaft bezüglich dieses Elements berechnet. Der Qualitätsquotient ist demnach eine Zahl, die zwischen „Null“ und „Unendlich“ liegt, wobei eine Zahl nahe „Null“ auf eine geringe, eine Zahl nahe „Unendlich“ auf eine (sehr) hohe Qualität hindeutet.

Qualitätsquotient $=$ Anzahl guter in \% / Anzahl schwacher in \% . 
Für die Kennzahlen entscheidend ist aber das Verhältnis zwischen „hochkarätigen“ und "schwachen“ Aktionen.

Die folgenden Diagramme zeigen die unterschiedlichen Qualitätsquotienten technischer Elemente der Achtelfinalisten.

Hinsichtlich der Qualität des Dribblings wurde festgestellt: Die englische, nigerianische und norwegische Mannschaft hatten die besten Qualitätsquotienten für das Dribblings nämlich 1,00, die Werte der anderen Mannschaften betrugen 0,20 für Chile, 0,16 für Mexiko, 0,50 für Jugoslawien, 0,20 für Paraguay und 0,11 für Rumänien. Das folgende Diagramm zeigt die Qualitätsquotienten des „Dribblings“ der Achtelfinalisten.

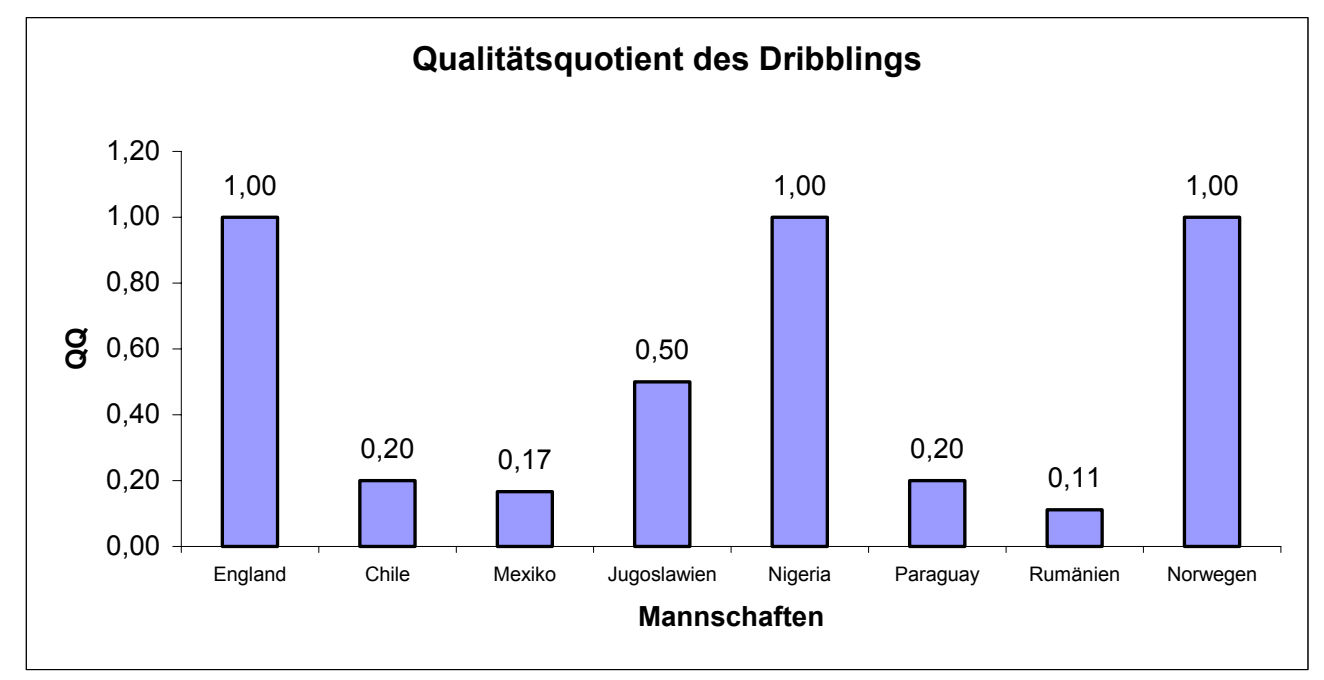

Diagramm 187: Qualitätsquotient des Dribblings bei den Achtelfinalisten.

Beim Vergleich der Werte der Qualitätsquotienten für die Dribblings wird ersichtlich, dass das Ergebnis immer weniger eins beträgt, außer bei England, Nigeria und Norwegen bei denen es gleich eins ist. Das gibt einen Hinweis darauf, dass die Dribblingfähigkeit, bei Pressing vom Gegner schwach ist und dass die Spieler dann den Ball verlieren, d.h. nach dieser Bewertung nicht gut dribbeln.

Bei der Körpertäuschung hat die englische Mannschaft den besten Qualitätsquotienten von 1,50, die anderen Mannschaften haben Werte von 0,20 für Chile, 0,40 für Mexiko, 0,16 für Jugoslawien, 1,00 für Nigeria, 0,25 für Paraguay, 0,14 für Rumänien und 0,20 für Norwegen. 
Untenstehendes Diagramm bildet die Qualitätsquotienten für das Leistungsmerkmal „Körpertäuschung“ der Achtelfinalisten ab.

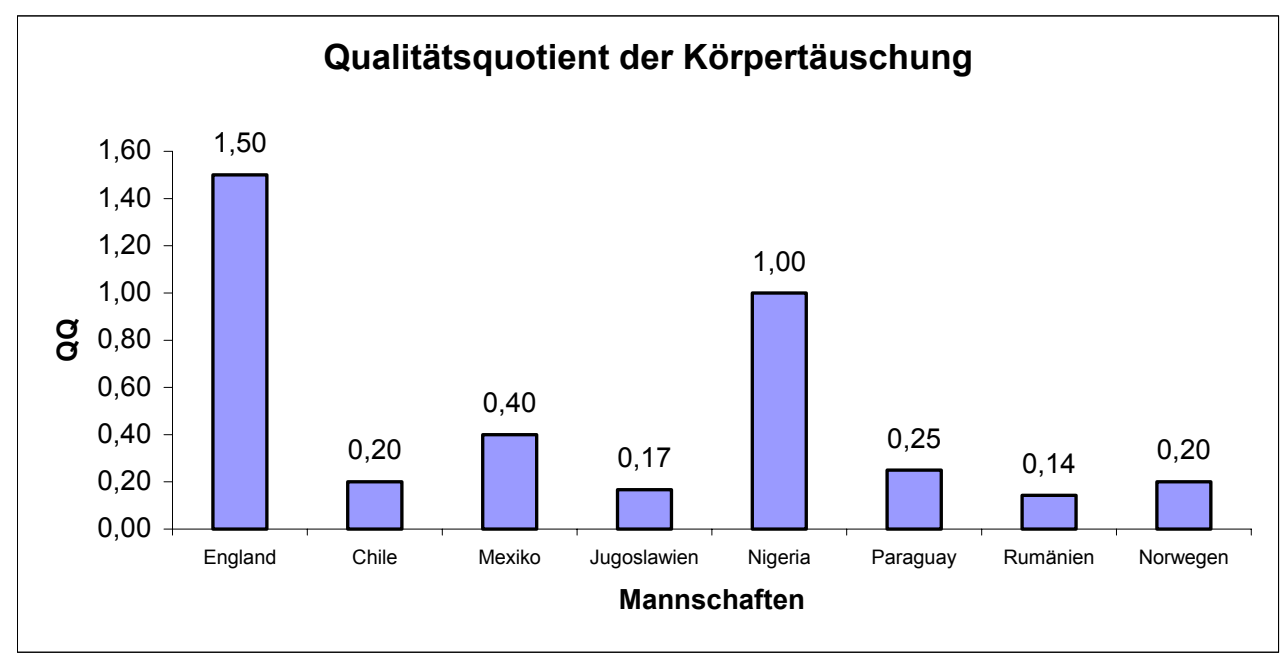

Diagramm 188: Qualitätsquotient der Körpertäuschung bei der Achtelfinalisten.

Beim Vergleich der guten mit schwachen Werte des Qualitätsquotienten für die Körpertäuschung wird ersichtlich, dass das Ergebnis immer weniger als eins beträgt, außer bei Nigeria und England wobei letzteres sogar einen Quotienten weit über eins hat und damit in dieser Bewertung sehr gut abschneidet. Die Körpertäuschungsfähigkeiten der meisten Mannschaften sind schwach, wenn sie Pressing vom Gegner in dessen Drittel erhalten. Sie können dann nicht mehr gut täuschen, d.h. sie verlieren den Ball.

Im Achtelfinale zeigt die englische Mannschaft mit 0,42 den besten Qualitätsquotient für den Torschuss, wohingegen die Quotienten bei den anderen Achtelfinalisten 0,14 für Chile, 0,33 für Mexiko, 0,33 für Jugoslawien, 0,04 für Nigeria und 0,00 für Paraguay, Rumänien und Norwegen betrugen.

Das folgende Diagramm zeigt die Qualitätsquotienten des „Torschusses“ der Achtelfinalisten. 


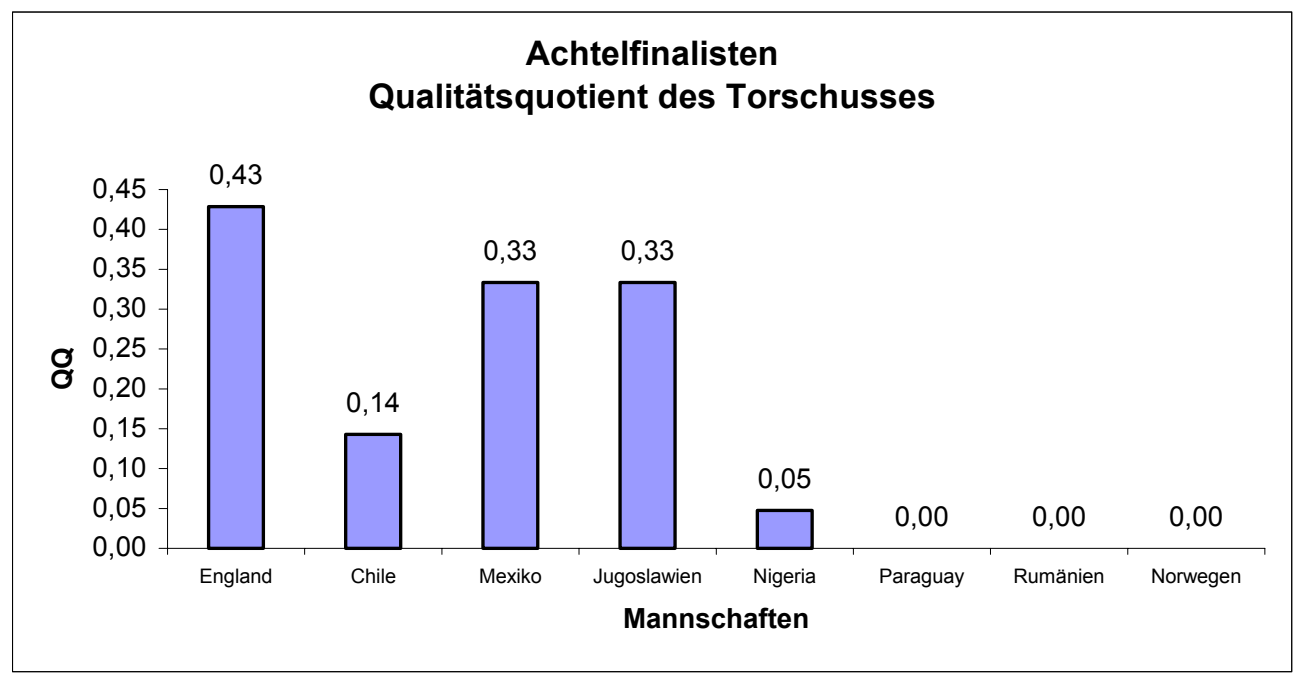

Diagramm 189: Qualitätsquotient des Torschusses bei den Achtelfinalisten.

Beim Vergleich der hochkarätigen und schwachen Werte für den Torschuss aus den Diagramm 189 ist zu ersehen, dass das Ergebnis durchweg deutlich weniger als eins, nämlich höchstens 0,43 beträgt. Damit sind die Torschussfähigkeiten als schwach zu beurteilen. Als Ursache kann man sich vorstellen, dass die Spieler, wenn sie Pressing vom Gegner erhalten, das Tor nicht genau anzielen und treffen können.

Bei den kurzen Pässen zeigt die nigerianische Mannschaft den besten Qualitätsquotienten mit 0,20. Bei den anderen Achtelfinalisten betrug er 0,09 für England, 0,05 für Chile, 0,07 für Mexiko, 0,13 für Jugoslawien, 0,04 für Paraguay, 0,05 für Rumänien und 0,15 für Norwegen.

Das folgende Diagramm zeigt die Qualitätsquotienten der „kurzen Pässe“ der Achtelfinalisten.

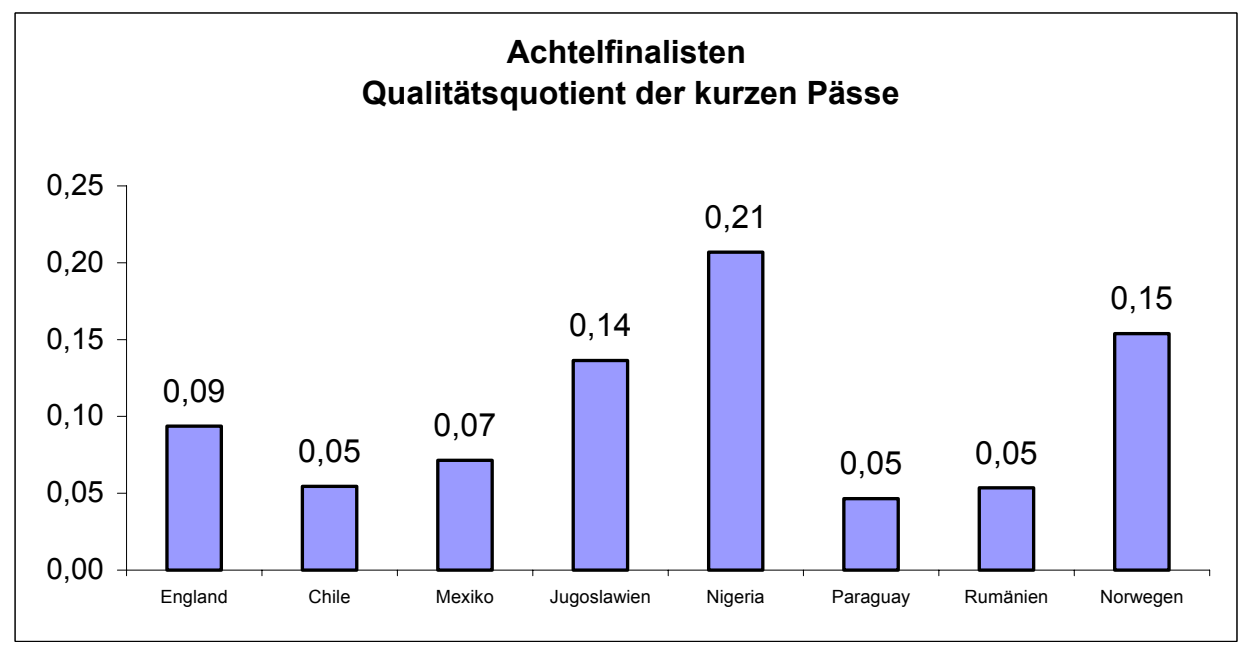

Diagramm 190: Qualitätsquotient der kurzen Pässe bei den Achtelfinalisten 
Beim Qualitätsquotienten für die kurzen Pässen in dem Diagramm Nr. 190 wird ersichtlich, dass die Ergebnisse weniger als eins betragen. Daraus lässt sich schließen, dass die Fähigkeiten zu kurzen Pässen schwach sind und dass die Spieler, wenn sie Pressing vom Gegner erhalten, den Ball nicht genau oder mit zu wenig Kraft zum Mitspieler abgeben und infolge dessen den Ball verlieren.

Die nigerianische Mannschaft zeigt auch die besten Qualitätsquotienten bei langen Pässen mit 0,11, während die anderen Achtelfinalisten hatten 0,02 für England, 0,02 für Chile, 0,01 für Mexiko, 0,01 für Jugoslawien, 0,03 für Paraguay, 0,06 für Rumänien und 0,05 für Norwegen hatten.

Das folgende Diagramm zeigt die Qualitätsquotienten der „langen Pässe“ der Achtelfinalisten.

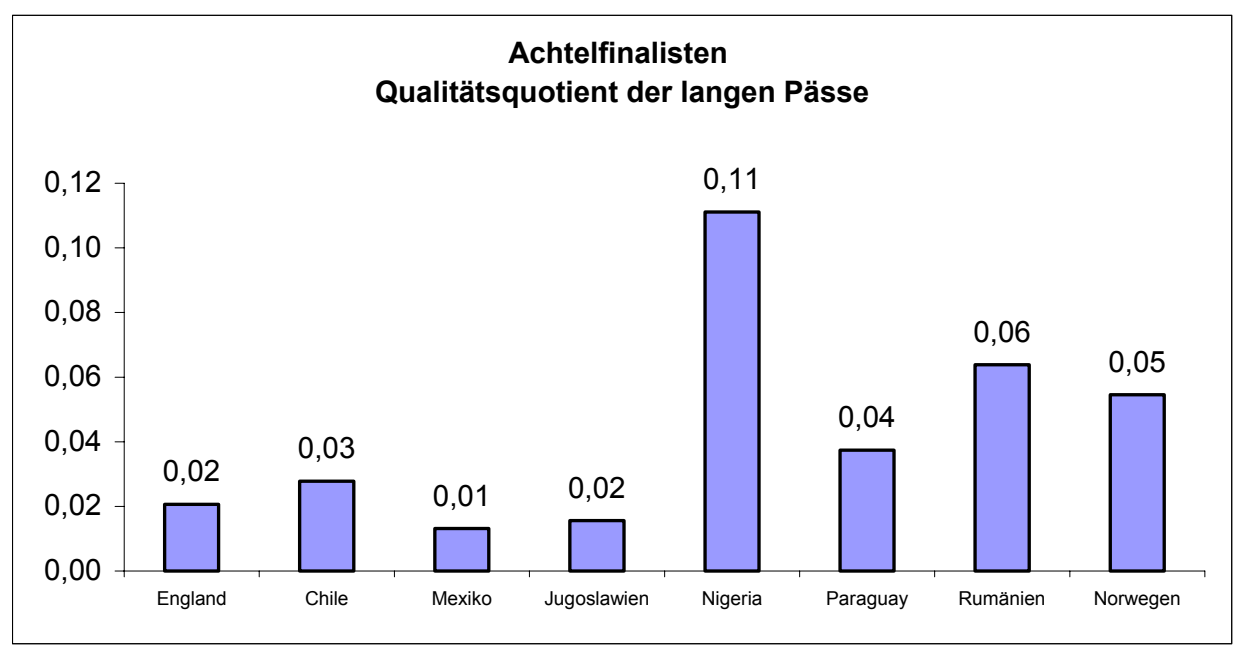

Diagramm 191: Qualitätsquotient der langen Pässe bei den Achtelfinalisten.

Beim Vergleich der hochkarätigen und schwachen Werte für die langen Pässen aus den Diagrammen 191 wird ersichtlich, dass das Ergebnis immer viel kleiner als eins beträgt. Das lässt erkennen, dass lange Pässe in den Wettkämpfen schwer zu spielen sind, die Spieler hatten entweder wegen Defensivaktionen der Gegner keine Gelegenheit, den Ball in die richtige Position zu schießen oder der annehmende Spieler wurde gehindert und konnte nicht zur rechten Zeit zum Mitspieler abgeben, worauf der Ball verloren wurde. Die durchweg, auch bei guten Mannschaften, sehr niedrigen Werte sind also nicht unbedingt ein Anzeichen für die fehlende Fähigkeit, Pässe zu spielen, sondern auch als Ausdruck der starken Verteidigung in diesem Wettkampf zu sehen. 
Das folgende Diagramm zeigt die Qualitätsquotienten der „Ballkontrolle“ der Achtelfinalisten.

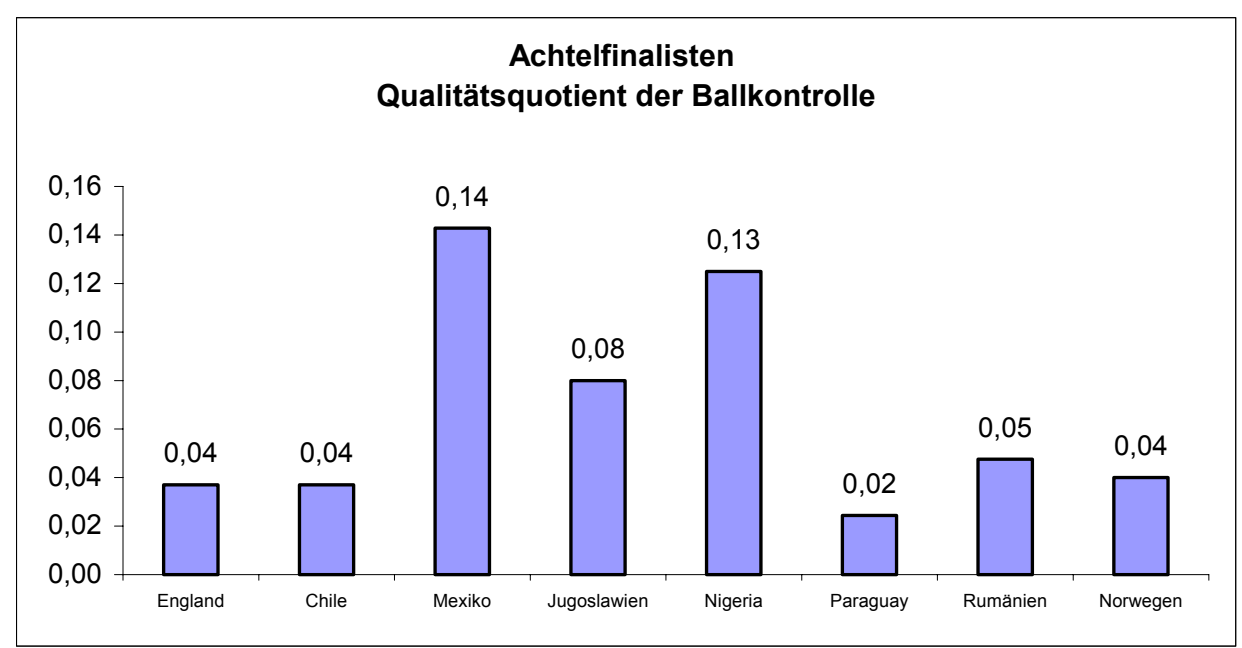

Diagramm 192 : Qualitätsquotient der Ballkontrolle bei den Achtelfinalisten.

Die mexikanische Mannschaft erhielt mit 0,14 den besten Qualitätsquotient für die Ballkontrolle, die Werte der andere Mannschaften betragen 0,03 für England, 0,03 für Chile, 0,08 für Jugoslawien, 0,12 für Nigeria, 0,02 für Paraguay, 0,04 für Rumänien und 0,04 für Norwegen.

Auch aus diesem Diagramm lässt sich ein durchweg niedriger Qualitätsquotient bei allen Achtelfinalisten erkennen. Die Mannschaften hatten weit mehr schwache als hochkarätige Ballkontrollen. Die Ballkontrollenfähigkeiten der meisten Spieler sind schwach, wenn sie Pressing vom Gegner erhalten, sie können den Ball nicht mehr kontrollieren, der Gegner vermag ihnen also den Ball abzunehmen.

\subsubsection{Viertelfinalisten}

Die Ergebnisse sind wie folgt: Die italienische Mannschaft zeigt den besseren Qualitätsquotient bei den Dribblings (2,00), während der Wert für die anderen Mannschaften $(0,07)$ für Deutschland, $(0,13)$ für Argentinien und $(0,25)$ für Dänemark beträgt.

Das folgende Diagramm zeigt die Qualitätsquotienten für Dribbling der Viertelfinalisten, die im Viertelfinale ausgeschieden sind. 


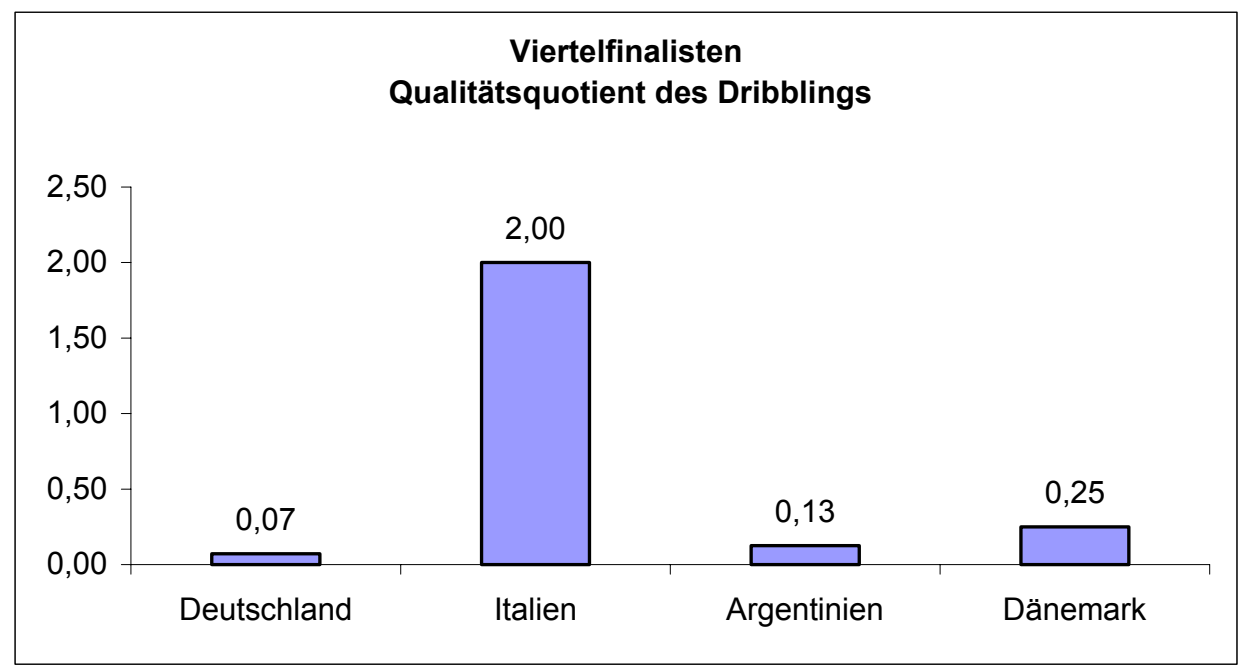

Diagramm 193 : Qualitätsquotient des Dribblings bei der Viertelfinalisten.

Bei den Dribblings zeigen sich sehr große Unterschiede zwischen den Mannschaften. Die Qualitätsquotienten der meisten Viertelfinalisten sind sehr niedrig, nur Italien hat einen sehr hohen Wert. Das erklärt, dass die Mannschaften außer Italien hatten mehr schwache Dribblings als hochkarätigen hatten.

Die argentinische Mannschaft zeigt sich als die beste beim Qualitätsquotient Körpertäuschung $(0,42)$, während die anderen Mannschaften $(0,29)$ für Deutschland, $(0,14)$ für Italien und $(0,22)$ für Dänemark hatten.

Das folgende Diagramm zeigt den unterschiedlichen Qualitätsquotienten Körpertäuschung der Viertelfinalisten.

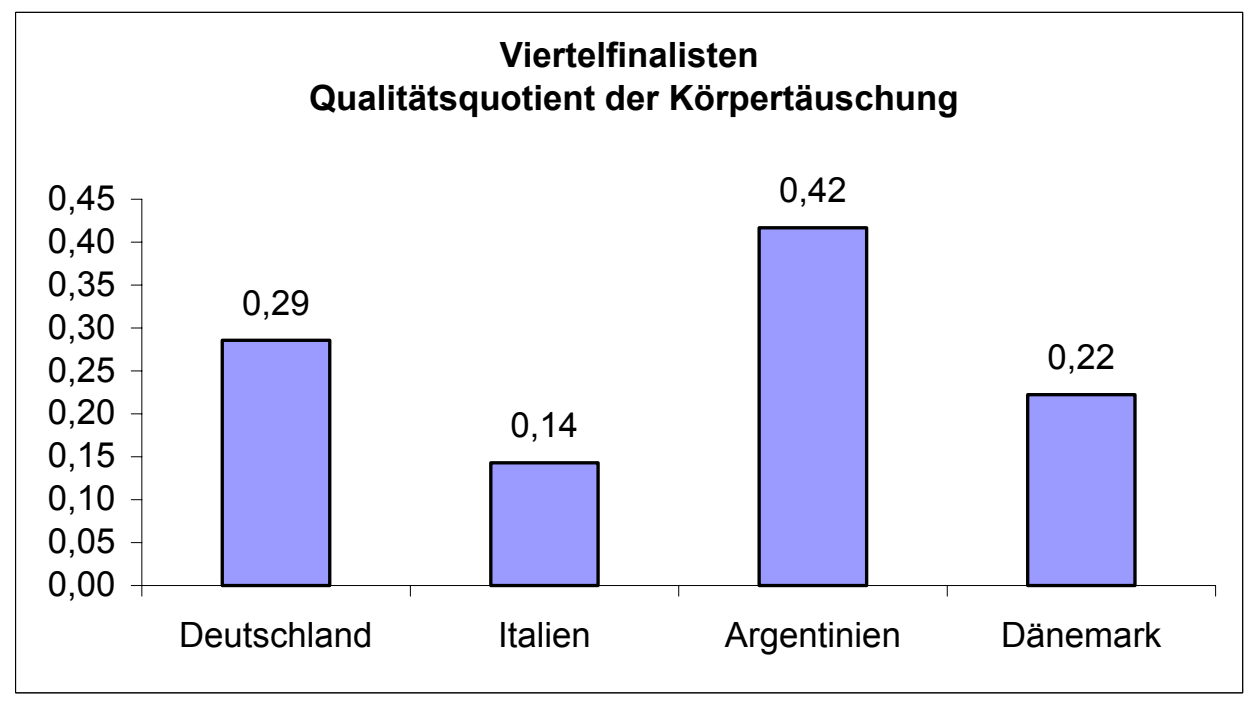

Diagramm 194 : Qualitätsquotient der Körpertäuschung bei den Viertelfinalisten. 
Die besten Qualitätsquotienten für Torschuss zeigte sich bei der dänischen Mannschaft $(0,60)$, während die anderen Mannschaften $(0,10)$ für Deutschland, $(0,22)$ für Italien und $(0,20)$ für Argentinien hatten.

Das folgende Diagramm zeigt den unterschiedlichen Qualitätsquotienten Torschuss der Viertelfinalisten.

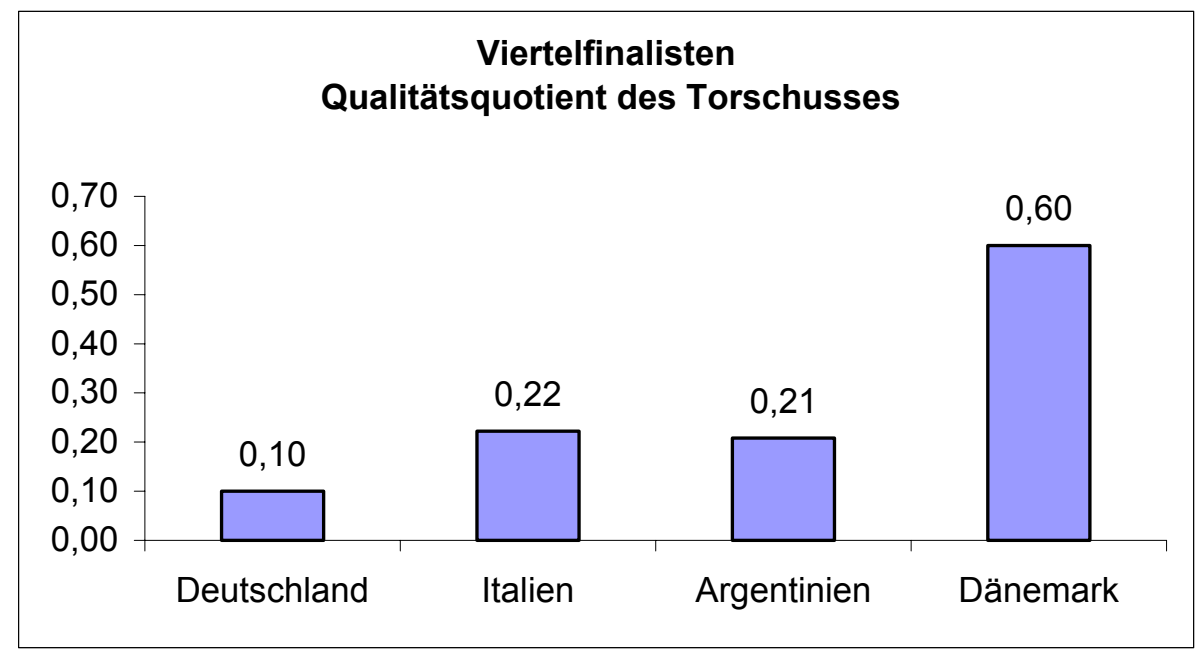

Diagramm 195 : Qualitätsquotient des Torschusses bei den Viertelfinalisten.

Die dänische Mannschaft zeigt auch den besten Qualitätsquotient bei kurzen Pässen $(0,28)$, während die anderen Mannschaften $(0,05)$ für Deutschland, $(0,13)$ für Italien und $(0,06)$ für Argentinien hatten.

Das folgende Diagramm zeigt den unterschiedlichen Qualitätsquotienten für kurze Pässe der Viertelfinalisten.

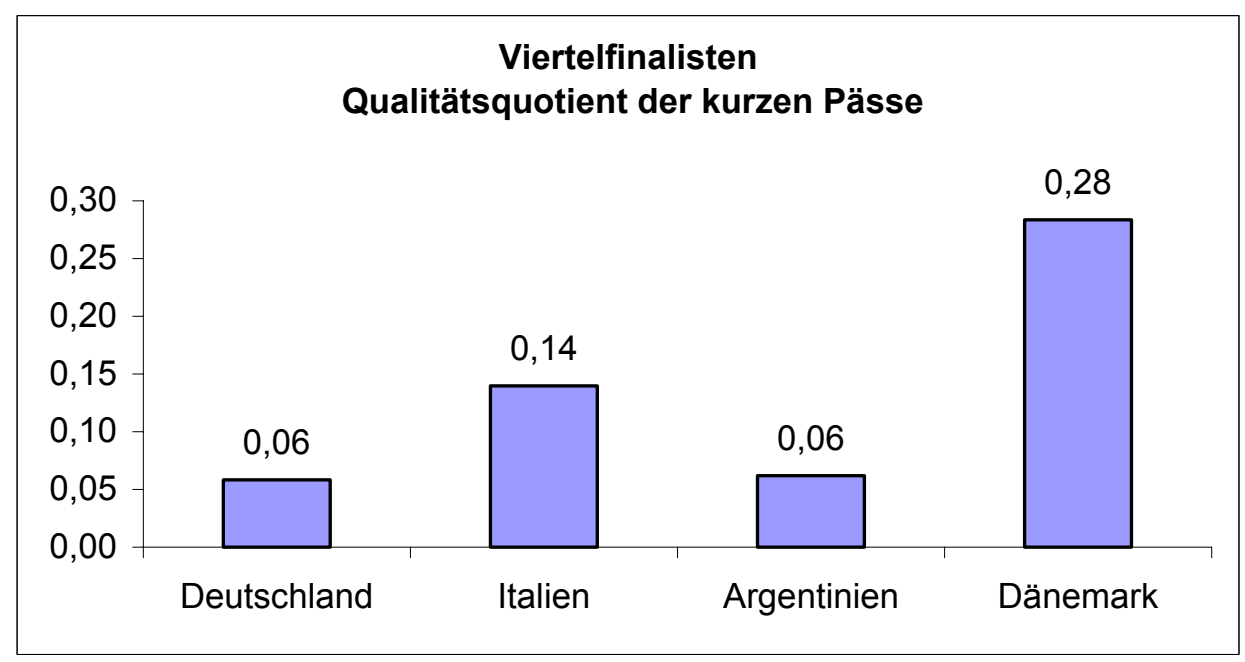

Diagramm 196: Qualitätsquotient der kurzen Pässe bei den Viertelfinalisten. 
Die argentinische Mannschaft zeigt den besten Qualitätsquotient für lange Pässe $(0,07)$, während die anderen Mannschaften $(0,04)$ für Deutschland, $(0,05)$ für Italien und $(0,01)$ für Dänemark hatten.

Das folgende Diagramm zeigt den unterschiedlichen Qualitätsquotienten für lange Pässe der Viertelfinalisten.

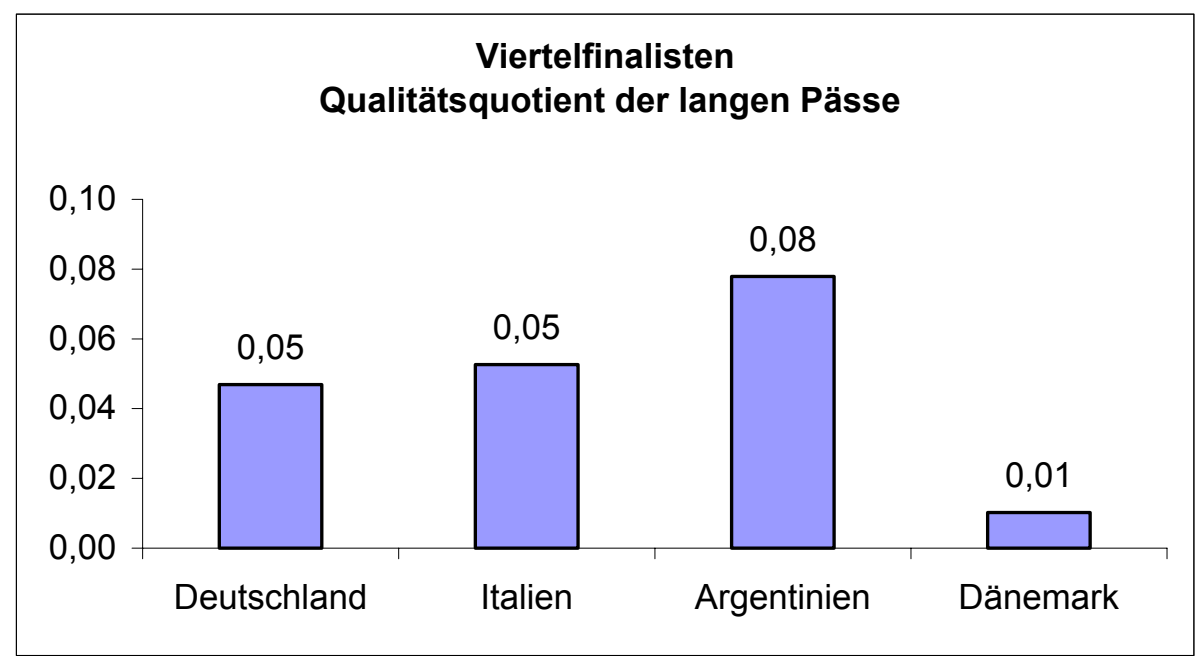

Diagramm 197 : Qualitätsquotient der langen Pässe bei den Viertelfinalisten.

Die italienische Mannschaft zeigt den besten Qualitätsquotient für Ballkontrolle $(0,03)$, während die anderen Mannschaften $(0,02)$ für Deutschland, $(0,01)$ für Argentinien und $(0,01)$ für Dänemark hatten.

Das folgende Diagramm zeigt die unterschiedlichen Qualitätsquotienten für Ballkontrolle der Viertelfinalisten.

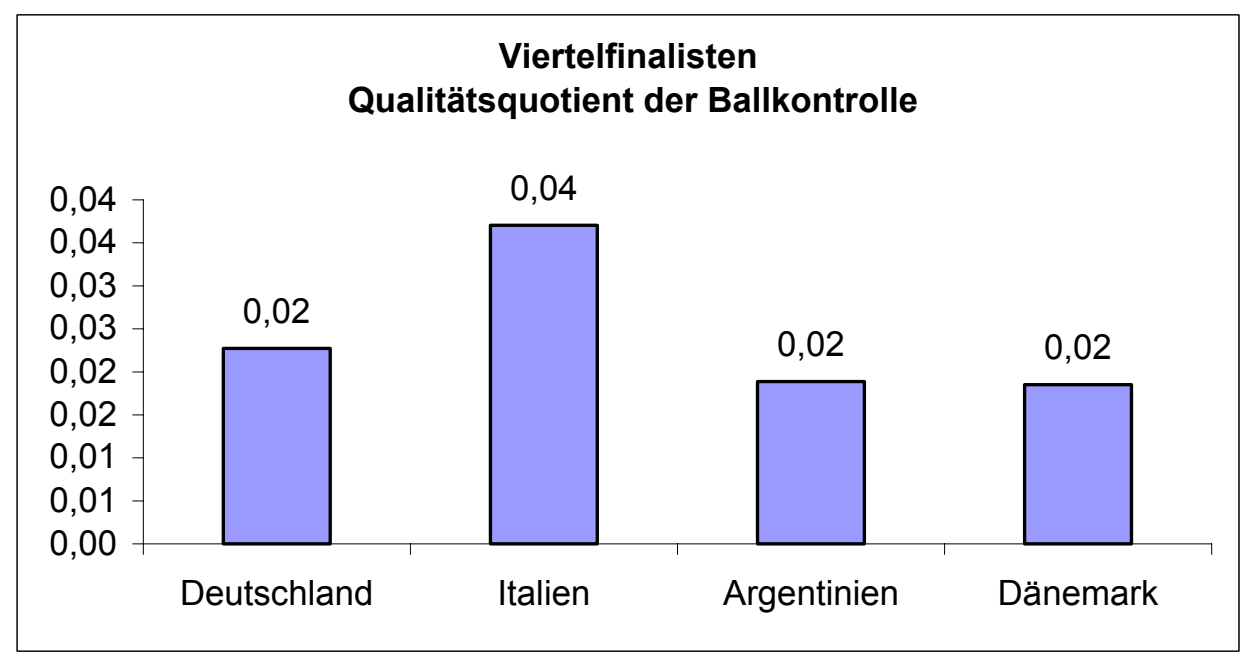

Diagramm 198 : Qualitätsquotient der Ballkontrolle bei den Viertelfinalisten. 


\subsubsection{Halbfinalisten}

Das folgende Diagramm zeigt die Qualitätsquotienten des Dribblings für die Halbfinalisten. Wie zu sehen ist, hat die französische Mannschaft die besten Qualitätsquotienten für Dribblings $(2,50)$, während die anderen Mannschaften $(0,29)$ für Brasilien, $(0,33)$ für Kroatien und $(0,20)$ für Niederland hatten.

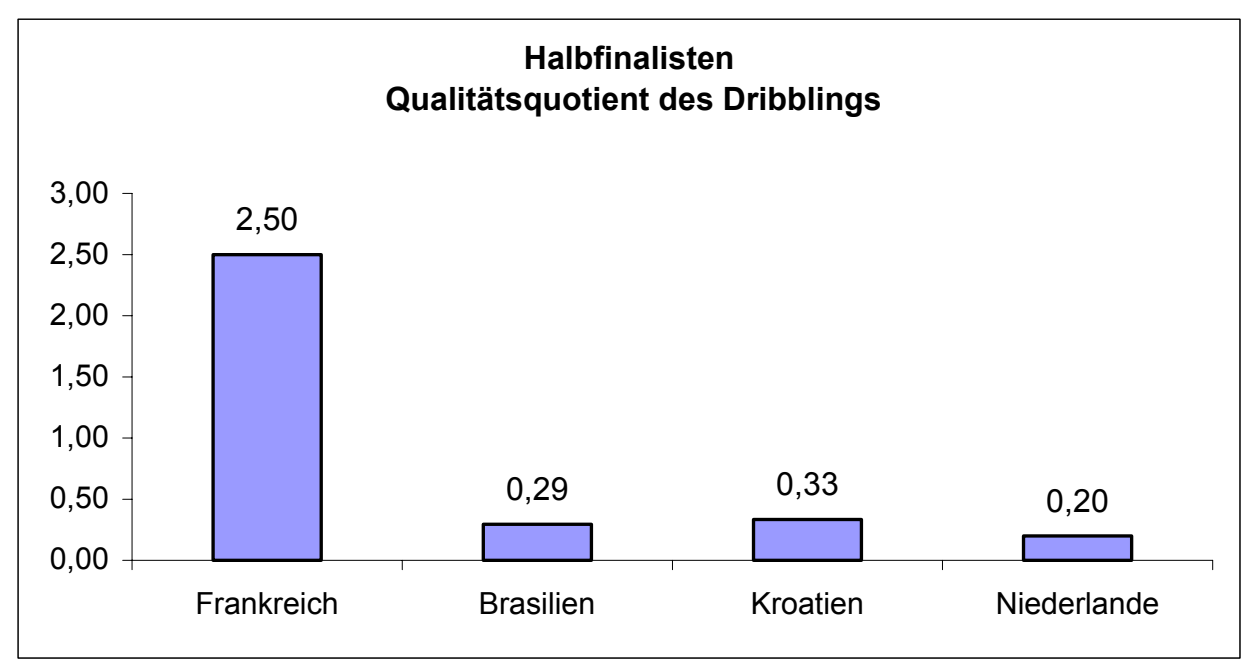

Diagramm 199 : Qualitätsquotient des Dribblings bei den Halbfinalisten.

Das folgende Diagramm zeigt die Qualitätsquotienten der Körpertäuschung für die Halbfinalisten.

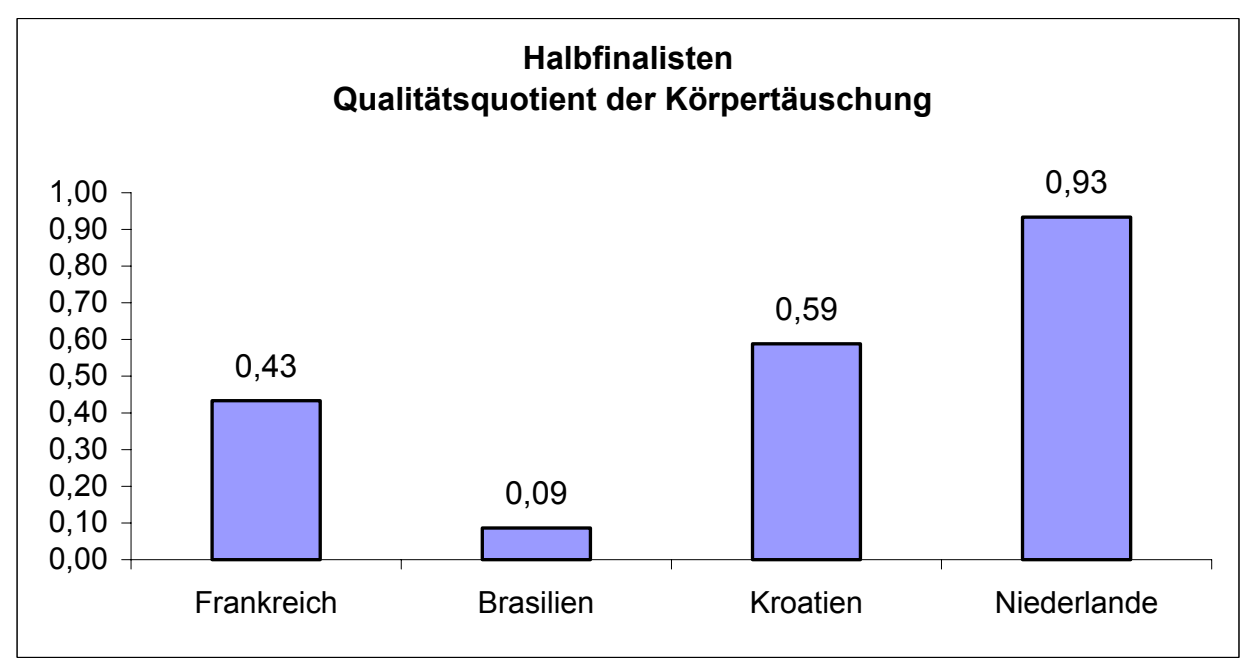

Diagramm 200 : Qualitätsquotient der Körpertäuschung den Halbfinalisten. 
Die Niederländer zeigten den besten Qualitätsquotient für Körpertäuschung $(0,93)$, neben Kroatien $(0,59)$, beide Mannschaften schieden jedoch nach dieser Runde aus. Es stellt sich also so dar, dass die Mannschaften mit den geringsten Werten in die Finalrunde kamen, dabei Brasilien mit einem vergleichsweise sehr niedrigen Wert.

Das folgende Diagramm zeigt die Qualitätsquotienten des Torschusses für die Halbfinalisten.

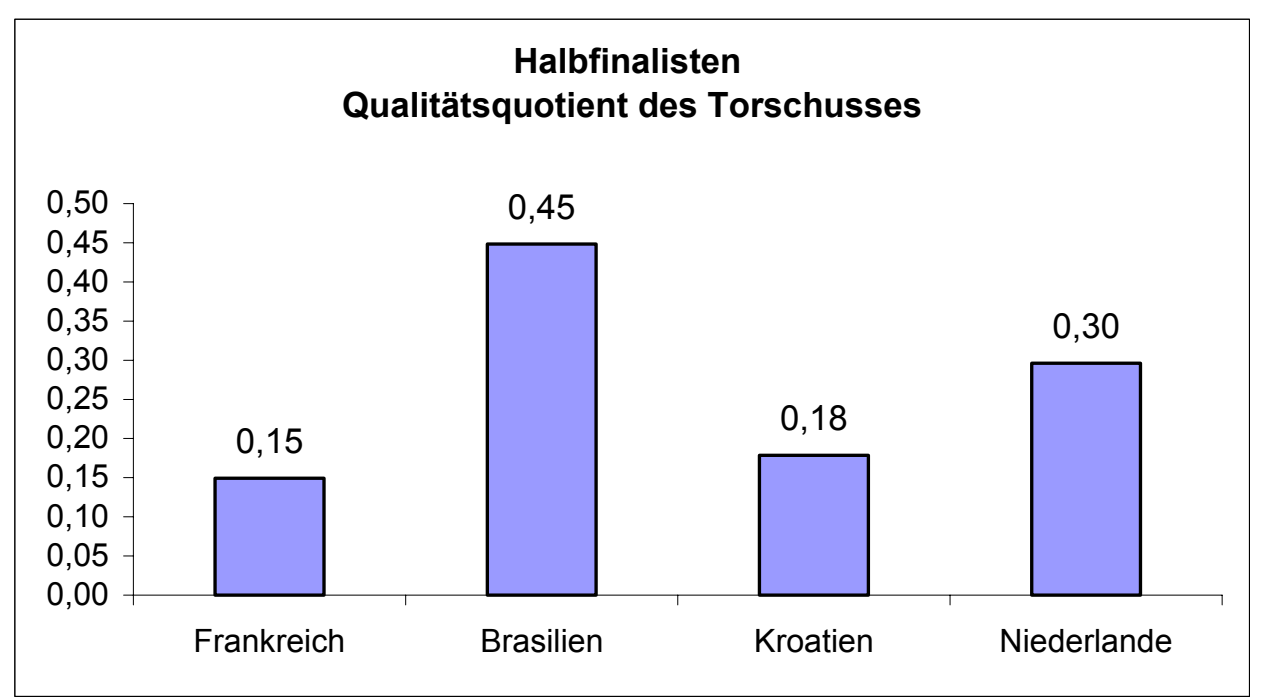

Diagramm 201 : Qualitätsquotient des Torschusses bei den Halbfinalisten.

Den besten Qualitätsquotienten Torschuss erhielt die brasilianische Mannschaft $(0,45)$, mit deutlichem Abstand folgen die Niederlande, Kroatien und Frankreich. Der Weltmeister hat hier den niedrigsten.

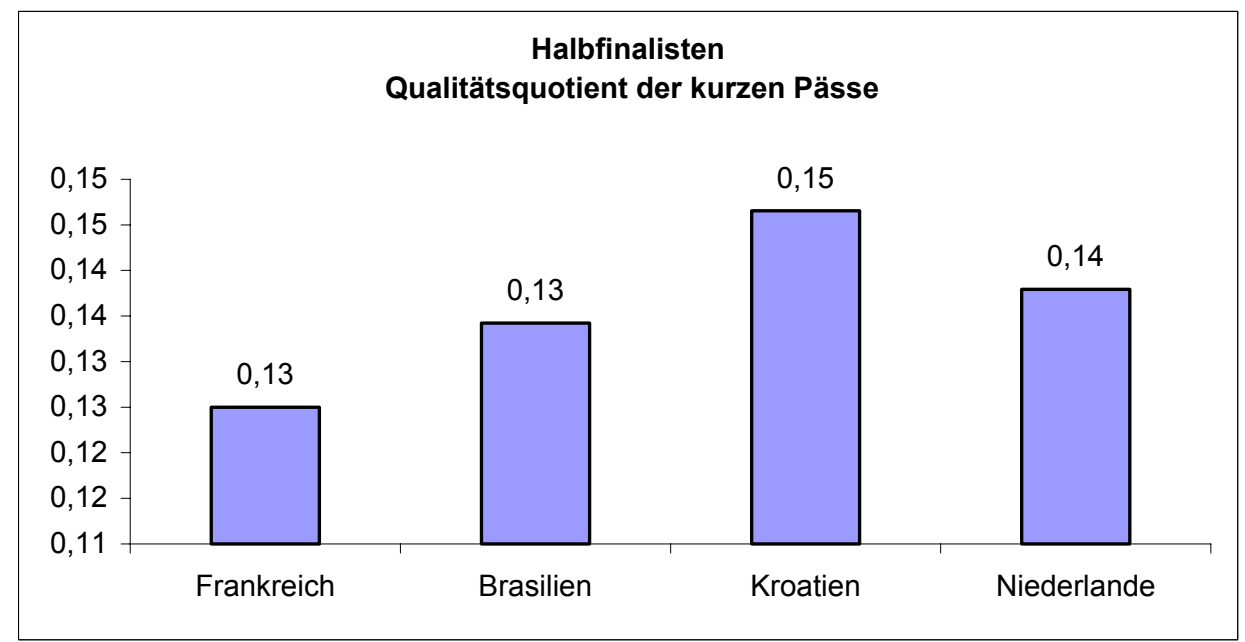

Diagramm 202 : Qualitätsquotient der kurzen Pässe bei den Halbfinalisten. 
Alle Halbfinalisten zeigen sehr schwache Werte, mit unwesentlichen Unterschieden. Das Ergebnis scheint auch wieder stark durch die Abwehr beeinflusst zu sein und gibt nicht nur die Mannschaftsstärke wieder.

Das folgende Diagramm zeigt die Qualitätsquotienten der langen Pässe für Halbfinalisten.

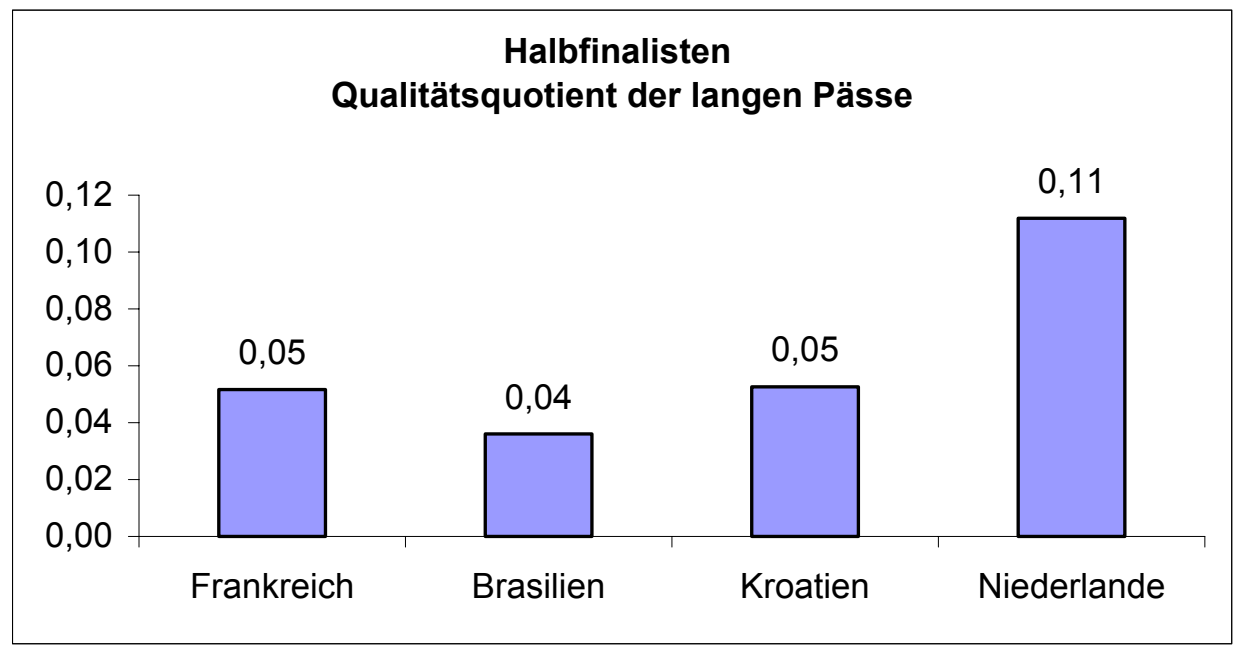

Diagramm 203 : Qualitätsquotient der langen Pässe bei den Halbfinalisten.

Die Niederländer zeigen den besten Qualitätsquotienten bei den langen Pässen, doch ist auch dieser Wert sehr gering, erwartungsgemäß etwas geringer als bei den kurzen Pässen, bei denen die Störungsmöglichkeiten naturgemäß vielfältiger sind. Unter der hohen Anzahl von Pässen, hier langen, war also nur ein recht geringer Anteil hochkarätig zu nennen und hatte den erwünschten Erfolg. Es ist also fraglich, ob diese Versuche, den Ball abzugeben, insgesamt das erstrebte Ergebnis brachten oder im Gegenteil eher der gegnerische Mannschaft Chancen bot, während die anderen Mannschaften $(0,02)$ für Frankreich, $(0,03)$ für Brasilien und $(0,03)$ für die Niederlande hatten.

Das folgende Diagramm zeigt die Qualitätsquotienten der Ballkontrolle für Halbfinalisten. 


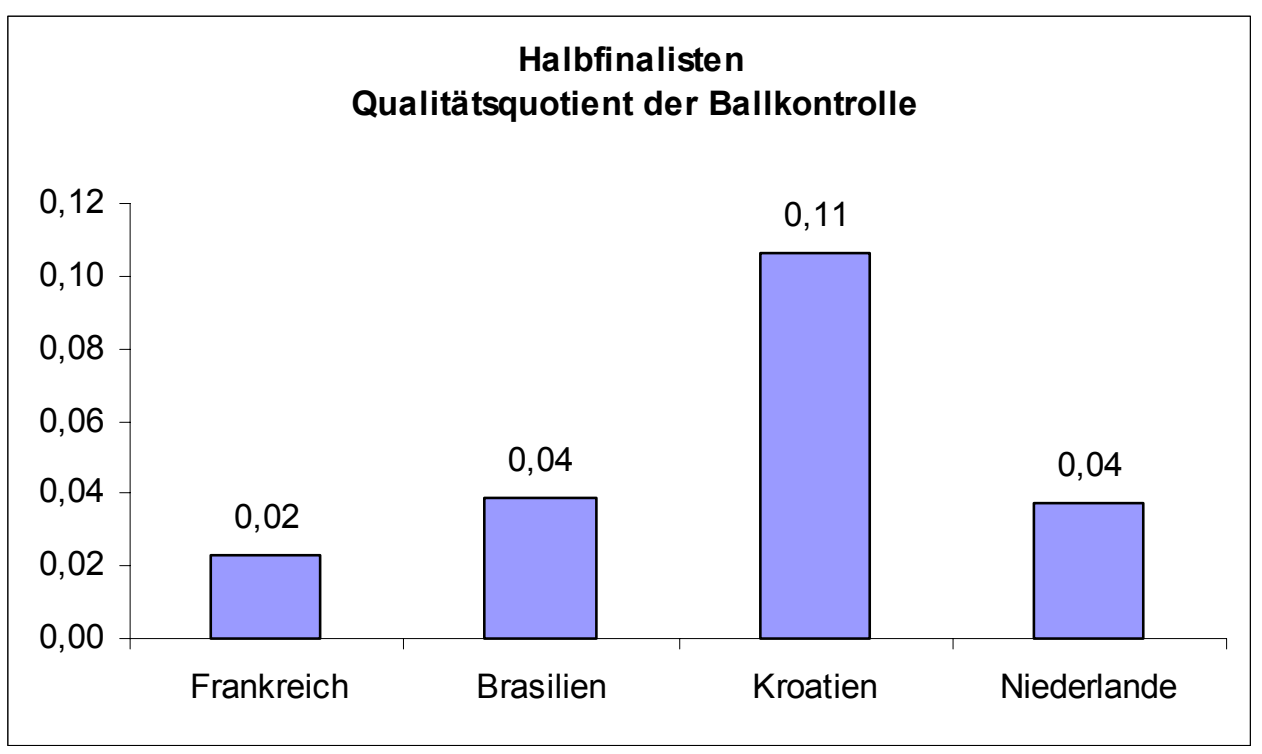

Diagramm 204 : Qualitätsquotient der Ballkontrolle bei den Halbfinalisten.

Für die kroatische Mannschaft ergab sich der beste Qualitätsquotient Ballkontrolle mit 0,11 und damit gut doppelt so hoch wie die übrigen. Auch hier sind die Werte jedoch durchweg gering und nicht mit dem Erfolg im Wettkampf direkt verbunden.

Aus den vorangehenden Diagrammen über die Qualitätsquotienten der technischen Fähigkeiten ist zu ersehen, dass die Leistungen der meisten Mannschaften recht schwach waren, sogar die der siegreichen. Das deutet auf ein geringes Talent oder mangelnde Beherrschung der Technik. Spieltaktisch gesehen liegt es jedoch zu einem guten Teil daran, dass das Spiel stark auf Abwehrtechniken beruhte, die gegnerische Mannschaft also ständig versuchte, den Ball zu erlangen.

\subsection{Qualitätsquotienten offensiv taktischer Elemente}

\subsubsection{Achtelfinalisten}

Das folgende Diagramm zeigt den Qualitätsquotienten des offensiv-taktischen Elementes „Freistoß“ der Achtelfinalisten. 


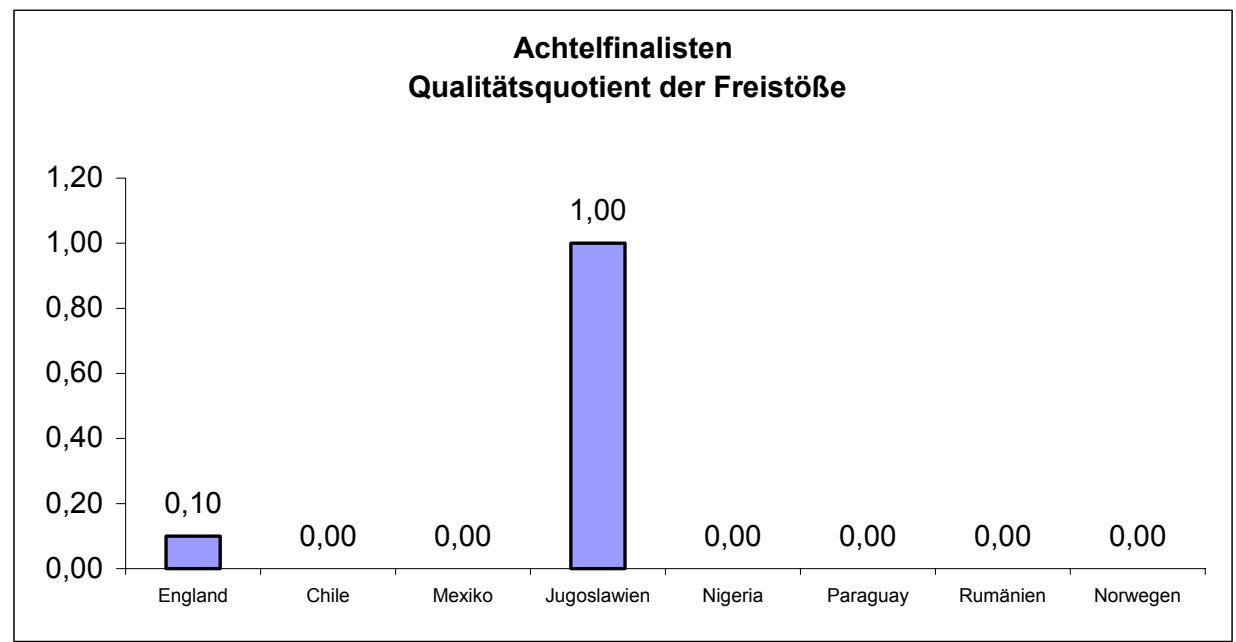

Diagramm 205 : Qualitätsquotient der Freistöße bei den Achtelfinalisten.

Die jugoslawische Mannschaft zeigt sich hier eindeutig als die beste mit einem Qualitätsquotienten im Freistoss von 1,00, also mit einem im angelegten Maßstab zumindest mit gut zu bewertenden Ergebnis, während die andere Mannschaften keinen hochkarätigen Freistoß hatten, d.h. das Tor verfehlten. England erhielt wegen vieler schlechter Freistöße nur ein 0,10. Es mangelt wahrscheinlich an der Einübung der Freistöße.

Das folgende Diagramm zeigt die Qualitätsquotienten Eckball für die Achtelfinalisten.

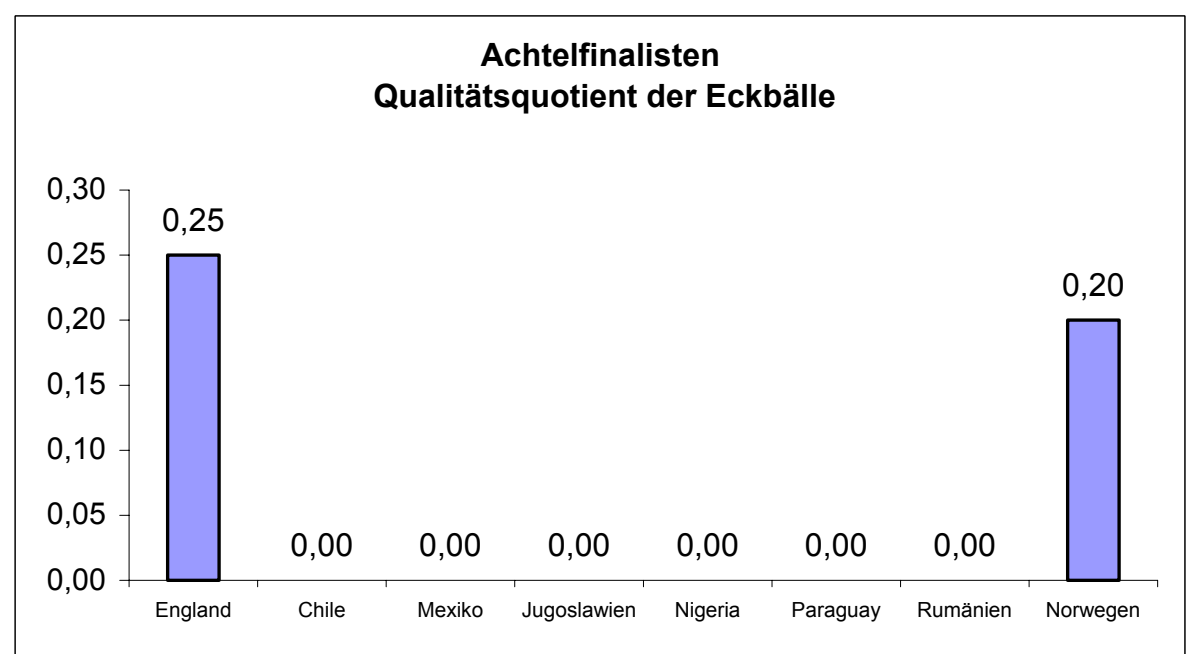

Diagramm 206 : Qualitätsquotient der Eckbälle bei den Achtelfinalisten. 
Bei den Eckbällen ergibt der Qualitätsquotient für die englische Mannschaft 0,25 und damit das beste Ergebnis vor Norwegen, während die anderen Mannschaften keine hochkarätigen Eckbälle spielten.

Das folgende Diagramm zeigt die Qualitätsquotienten Unterstützen für Achtelfinalisten.

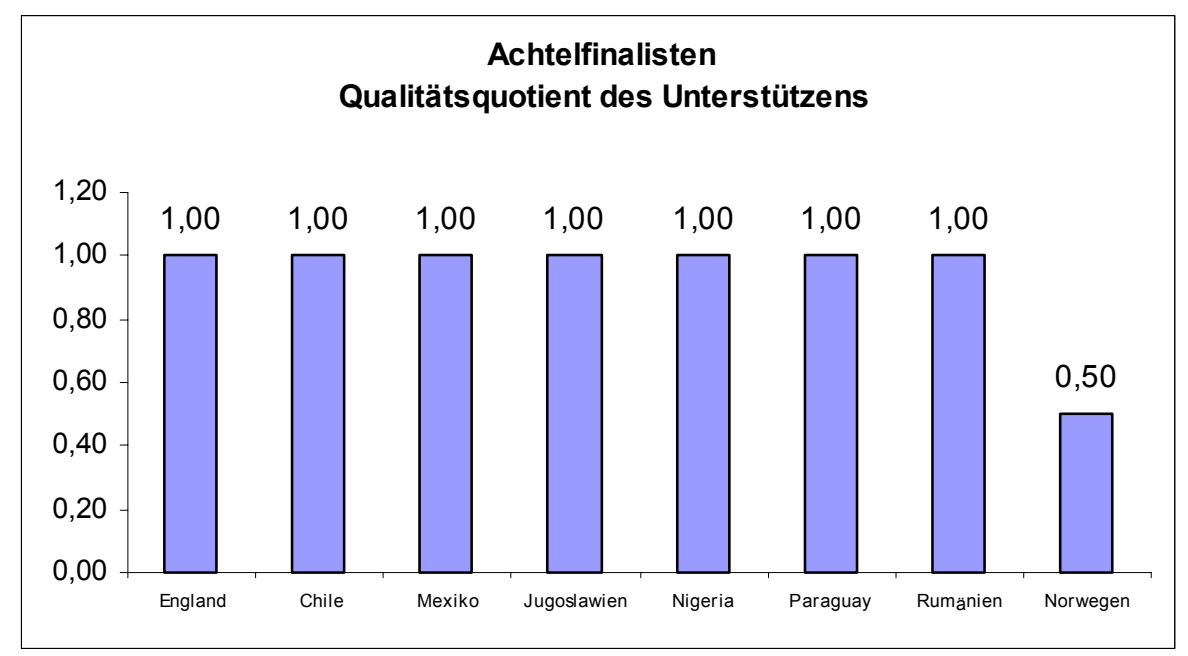

Diagramm 207: Qualitätsquotient des Unterstützens bei den Achtelfinalisten.

Alle Mannschaften außer Norwegen haben im Unterstützen hier den gleichen, hohen Qualitätsquotienten von 1,0, während die norwegische Mannschaft nur ein 0,50 bekommt. Die Leistung im Unterstützen beim Angriff kann also fast durchweg als gut bezeichnet werden. Das liegt aber auch an der Art der Bewertung, da nicht alle möglichen Spieler, sondern nur der am geeignetsten erscheinende als Unterstützer bewertet wurde. Deswegen ist die Bewertung hier anders zu sehen als beim Dribbling oder bei den Pässen, wo jede Aktion bewertet und der Qualitätsquotient dadurch niedriger wurde (vgl. Kap 5.6).

Das folgende Diagramm zeigt die Qualitätsquotienten der Doppelpässe für die Achtelfinalisten. 


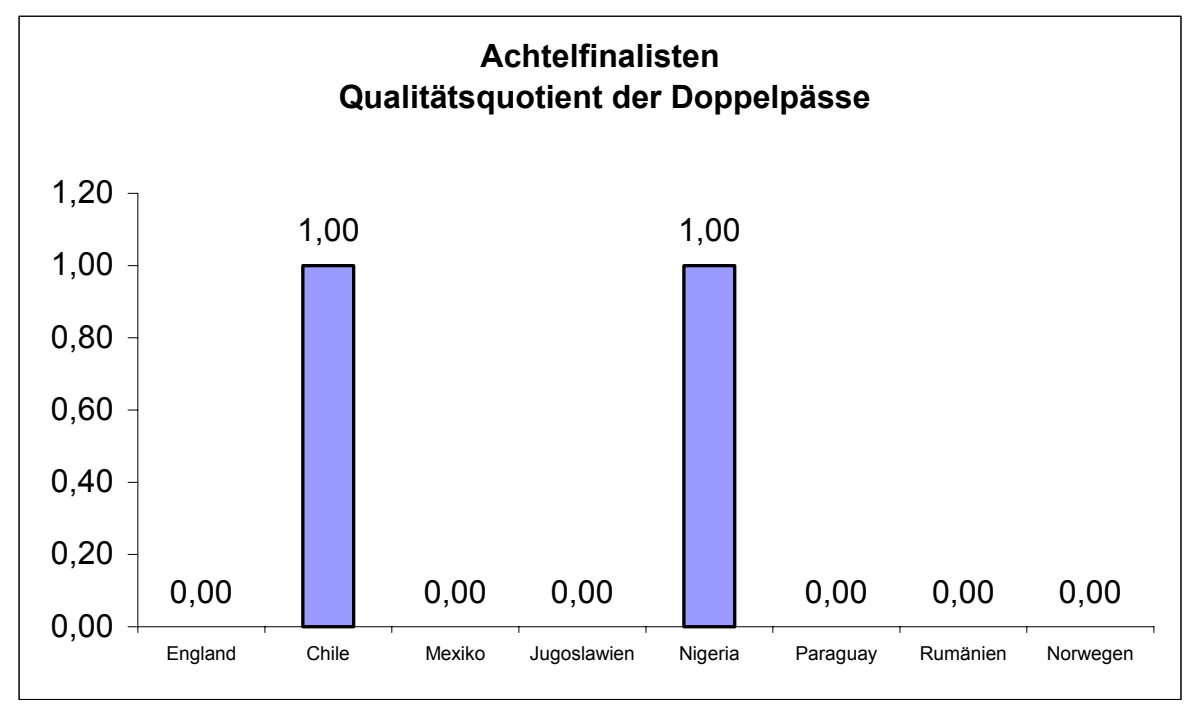

Diagramm 208 : Qualitätsquotient der Doppelpässe bei den Achtelfinalisten.

Die nigerianische und chilenische Mannschaft zeigen die besseren Qualitätsquotienten im Doppelpass mit 1,00; die anderen Mannschaften hatten keine hochkarätigen Doppelpässe zustande gebracht.

Das folgende Diagramm zeigt die Qualitätsquotienten für Raumaufteilung für die Achtelfinalisten.

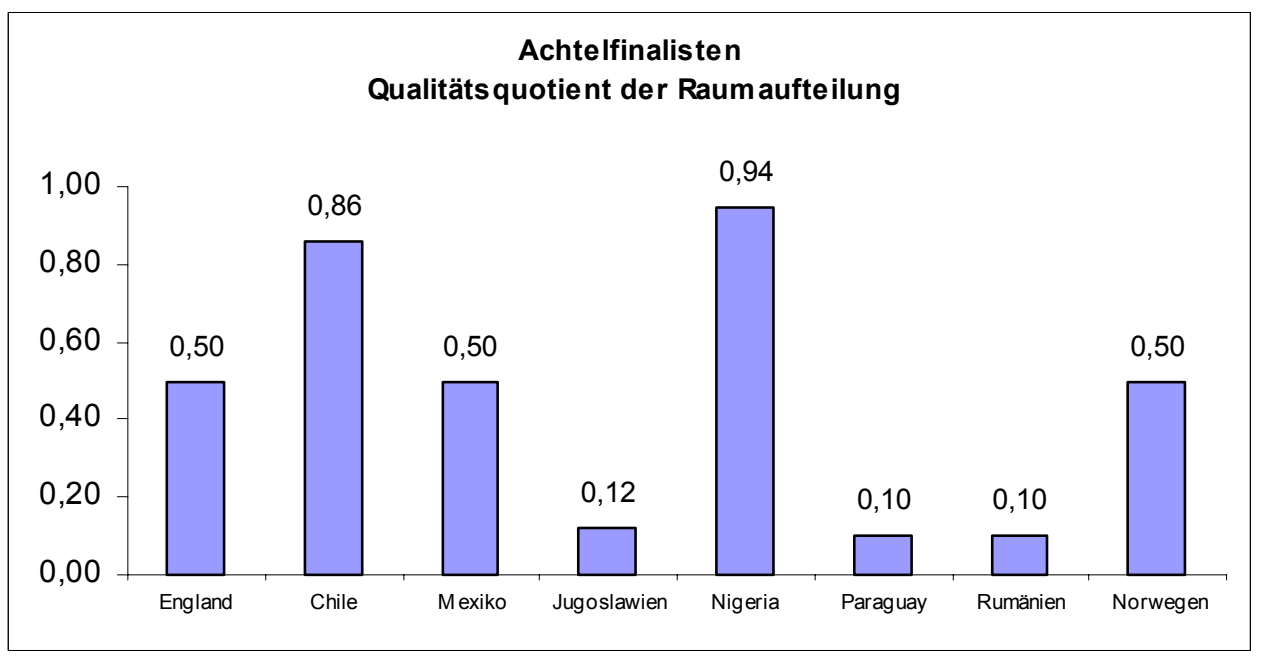

Diagramm 209 : Qualitätsquotient der Raumaufteilung bei den Achtelfinalisten.

Die nigerianische Mannschaft erreicht mit 0,94 den besten Qualitätsquotient der Raumaufteilung, Chile folgt mit 0,86. Die Werte der anderen Mannschaften mit 0,50 für England und ebenfalls für Mexiko sind schon als ungenügend anzusehen, erst recht die noch niedrigeren.

Das folgende Diagramm zeigt die Qualitätsquotienten Nachrücken für Achtelfinalisten. 


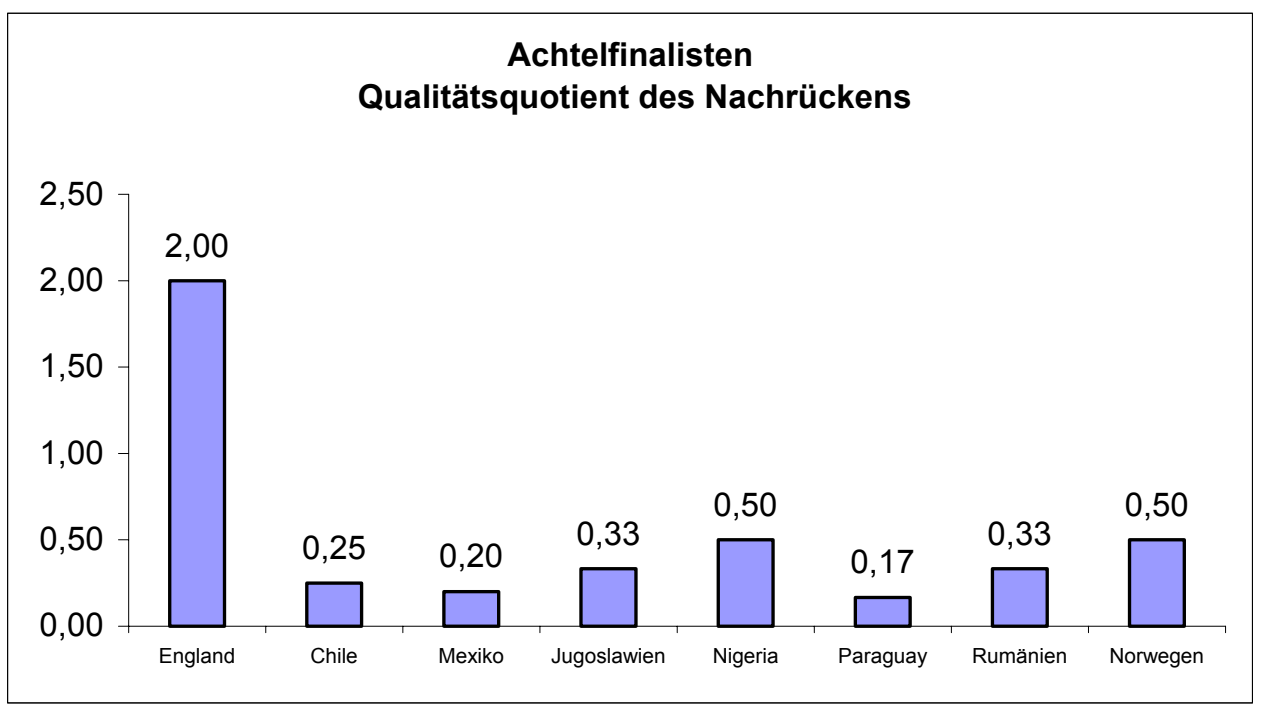

Diagramm 210 : Qualitätsquotient des Nachrückens bei den Achtelfinalisten.

Die englische Mannschaft zeigt den besten Qualitätsquotient im Nachrücken mit 2,00 und liegt damit weit vor 0,50 für Nigeria und für Norwegen. Diese Mannschaften sollte das Nachrücken also noch verbessern.

Das folgende Diagramm zeigt die Qualitätsquotienten „In die Breite ziehen“ für die Achtelfinalisten.

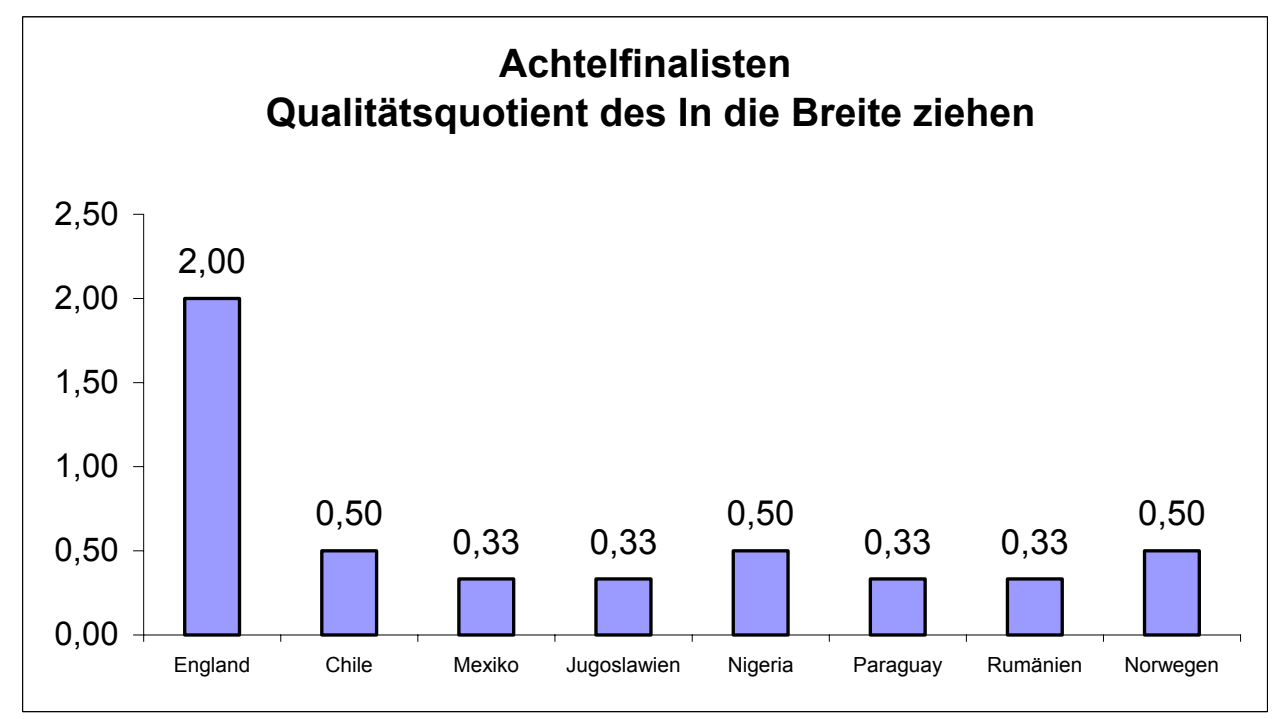

Diagramm 211: Qualitätsquotient des In die Breite ziehen bei den Achtelfinalisten.

Für die englische Mannschaft ergibt sich auch der beste Qualitätsquotient beim Ball in die Breite ziehen mit wiederum 2,00. Auch hier liegen die anderen Mannschaften abgeschlagen und verbesserungsbedürftig im niedrigen Bereich. Die englische Mannschaft ist offensichtlich überaus lauffreudig. 


\subsubsection{Viertelfinalisten}

Das folgende Diagramm zeigt die Qualitätsquotienten des offensiv-taktischen Spielzugs „Freistoß“ der Viertelfinalisten.

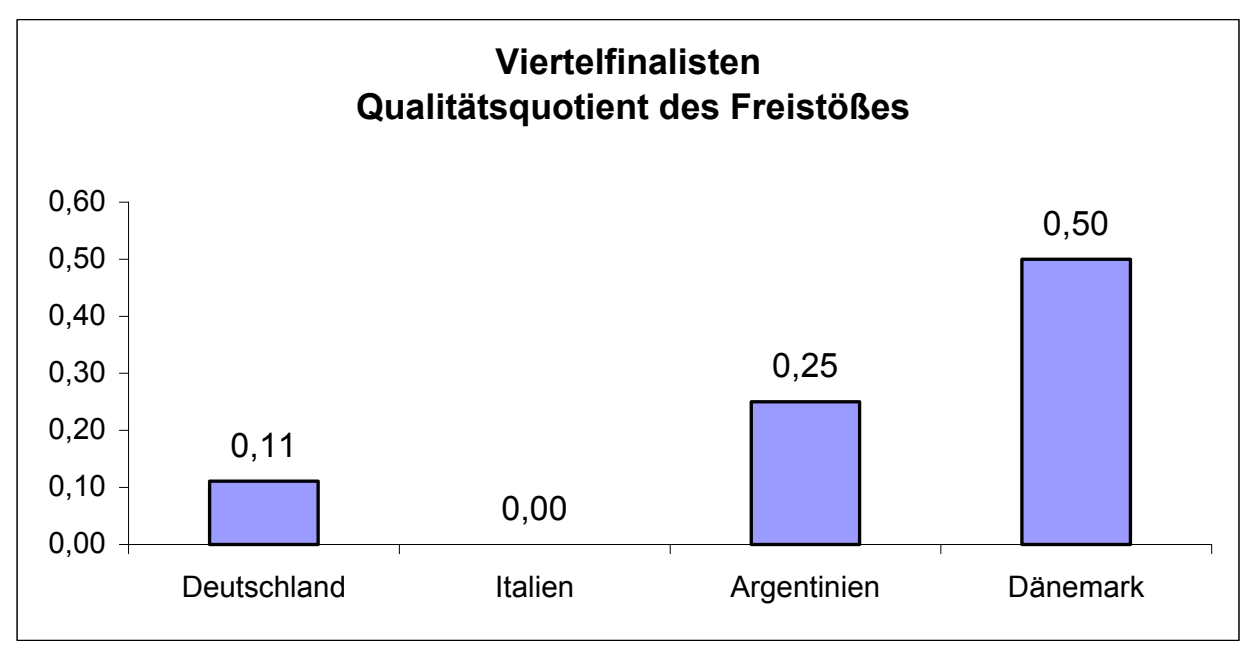

Diagramm 212 : Qualitätsquotient des Freistoßes bei den Viertelfinalisten.

Die dänische Mannschaft hat mit einem Qualitätsquotient im Freistoß von 0,50 noch einen einigermaßen akzeptablen Wert erlangt, während die anderen Mannschaften schlecht abschnitten.

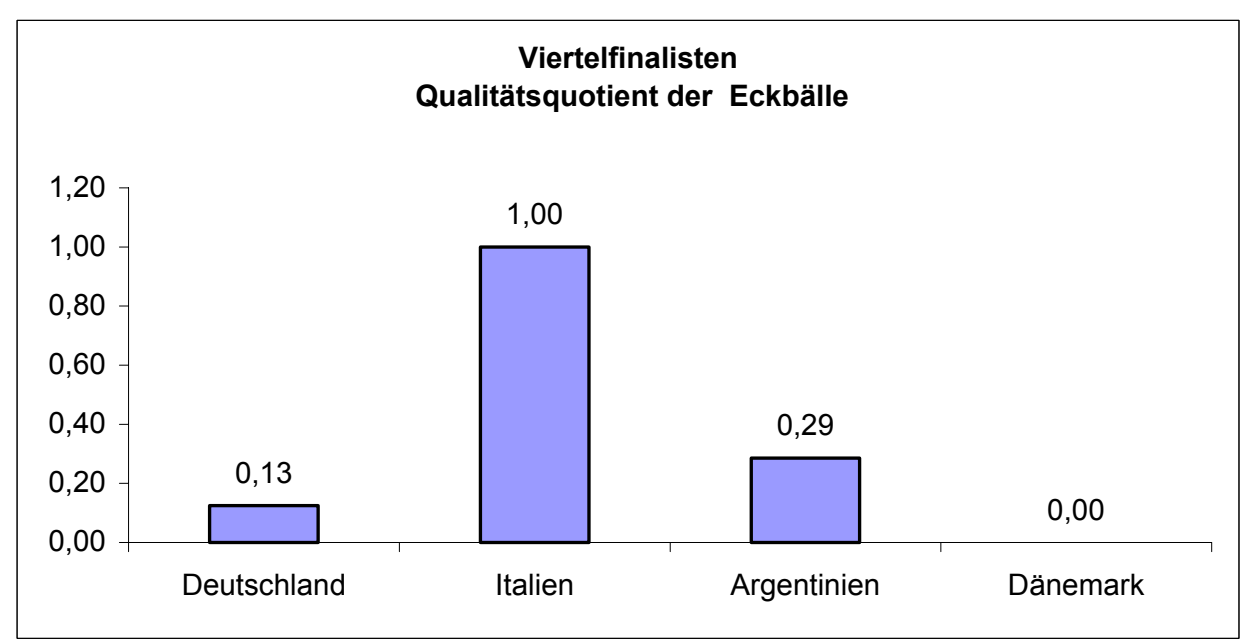

Diagramm 213 : Qualitätsquotient der Eckbälle bei den Viertelfinalisten.

Bei den Eckbällen ist die italienische Mannschaft der Favorit mit dem Wert 1,00. Es stellt sich ein stark differenzierendes Ergebnis im Vergleich der Eckbälle mit den Freistößen dar, da die besten in der jeweils anderen Disziplin nur eine Null erhielten. 
Offenbar spielte der Zufall auch eine große Rolle bei einer nur niedrigen Anzahl von bewertbaren Spielzügen.

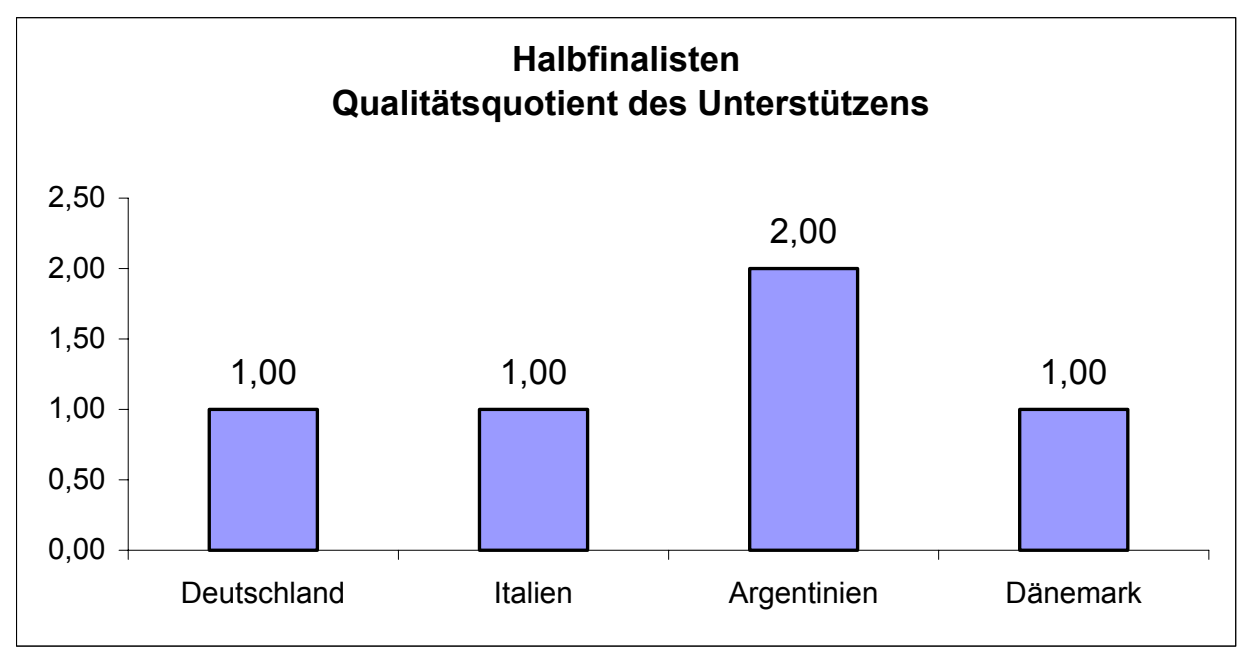

Diagramm 214 : Qualitätsquotient des Unterstützens bei den Viertelfinalisten.

Den besten Qualitätsquotient im Unterstützen erreichte die argentinische Mannschaft $(2,00)$, alle anderen Mannschaften hatten mit 1,00 immer noch einen guten Wert.

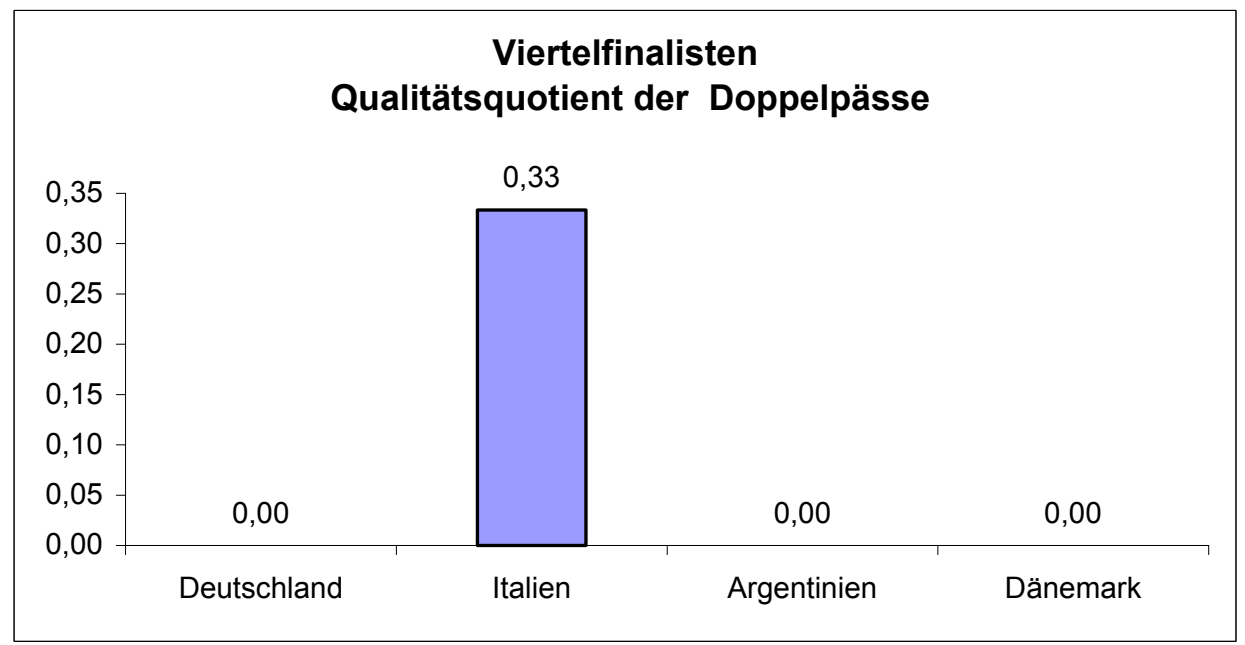

Diagramm 215 : Qualitätsquotient der Doppelpässe bei den Viertelfinalisten.

Die italienische Mannschaft zeigt sich als die besten Doppelpassspieler mit einem Ergebnis von 0,33, während alle anderen Mannschaften eine Null hatten. 


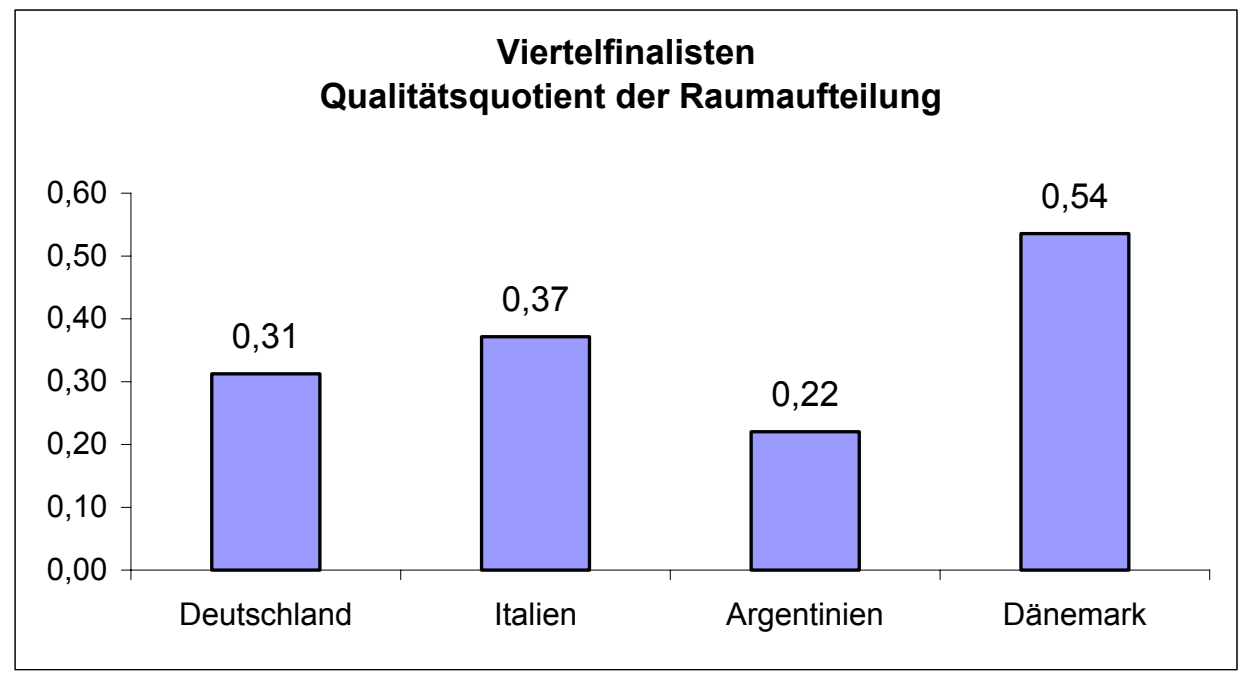

Diagramm 216 : Qualitätsquotient der Raumaufteilung bei den Viertelfinalisten.

Nur die dänische Mannschaft zeigt eine gute Raumaufteilung $(0,54)$, während die Werte der anderen Mannschaften niedriger liegen.

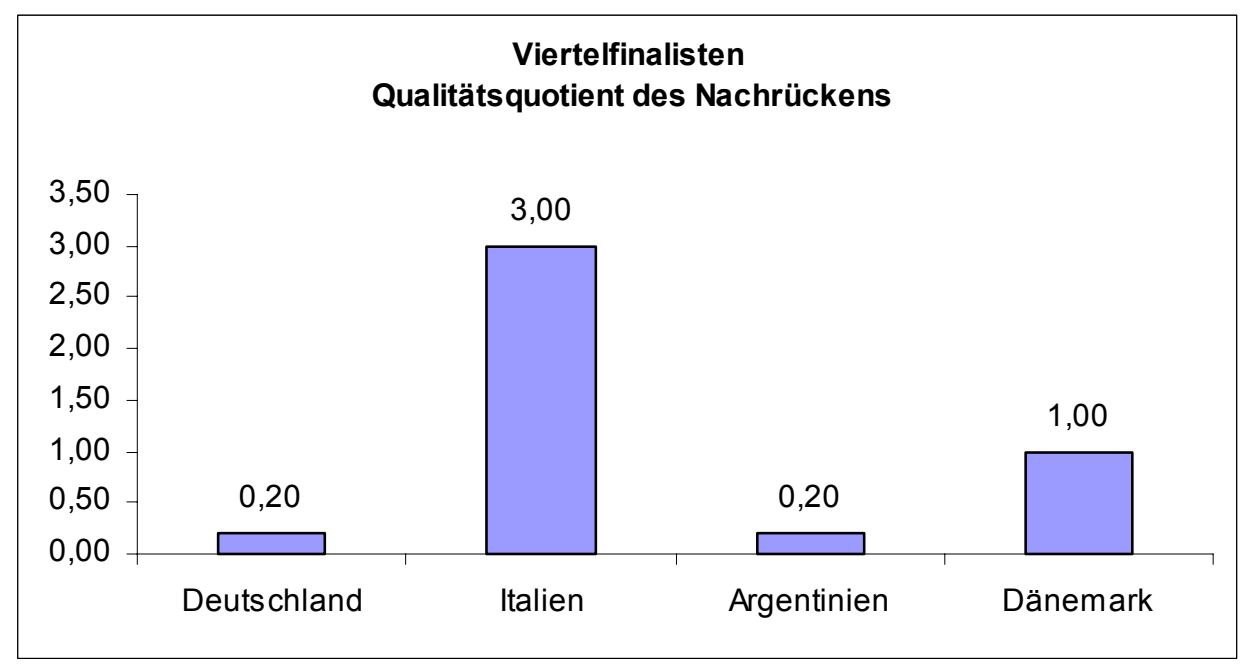

Diagramm 217 : Qualitätsquotient des Nachrückens bei den Viertelfinalisten.

Italien erreicht hier einen sehr guten Qualitätsquotienten mit dem sehr guten Wert 3, auch Dänemark ist mit 1 noch gut bewertet. 


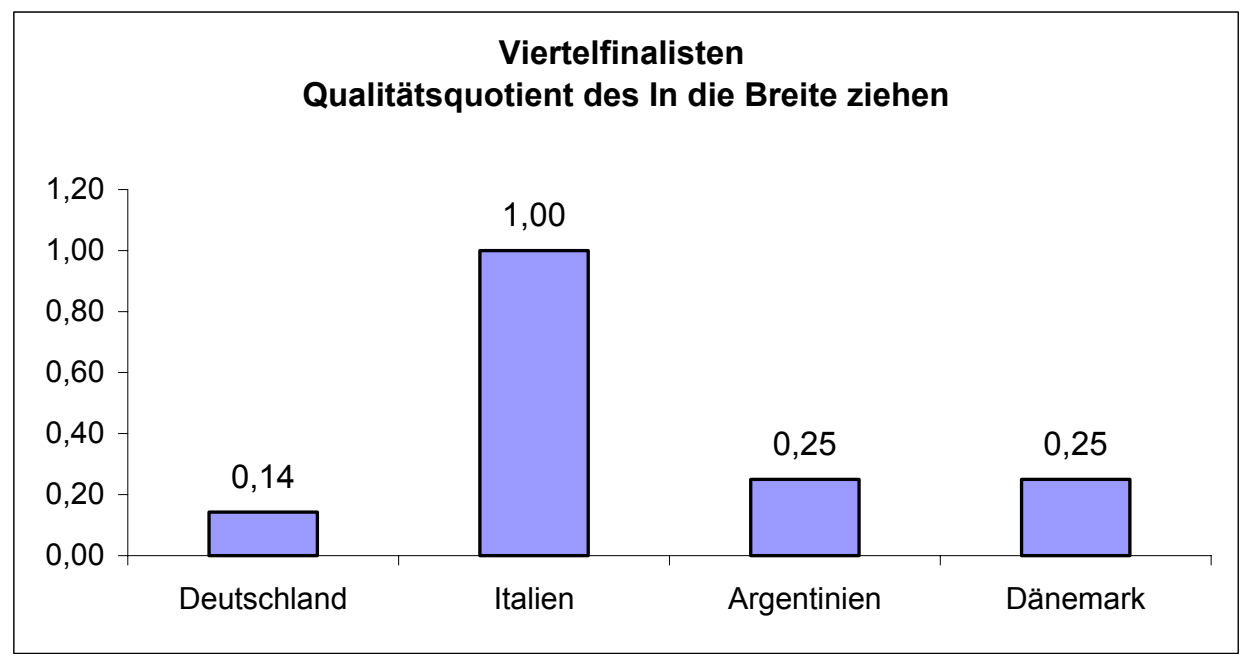

Diagramm 218: Qualitätsquotient des In die Breite ziehen bei den Viertelfinalisten.

Die italienische Mannschaft zeigt einen guten Qualitätsquotient im „Ball in die Breite ziehen“ (1,00). Die andren Mannschaften liegen stark zurück.

\subsubsection{Halbfinalisten}

Das folgende Diagramm zeigt die Qualitätsquotienten des offensiv-taktischen Elementes „Freistoß“ der Halbfinalisten.

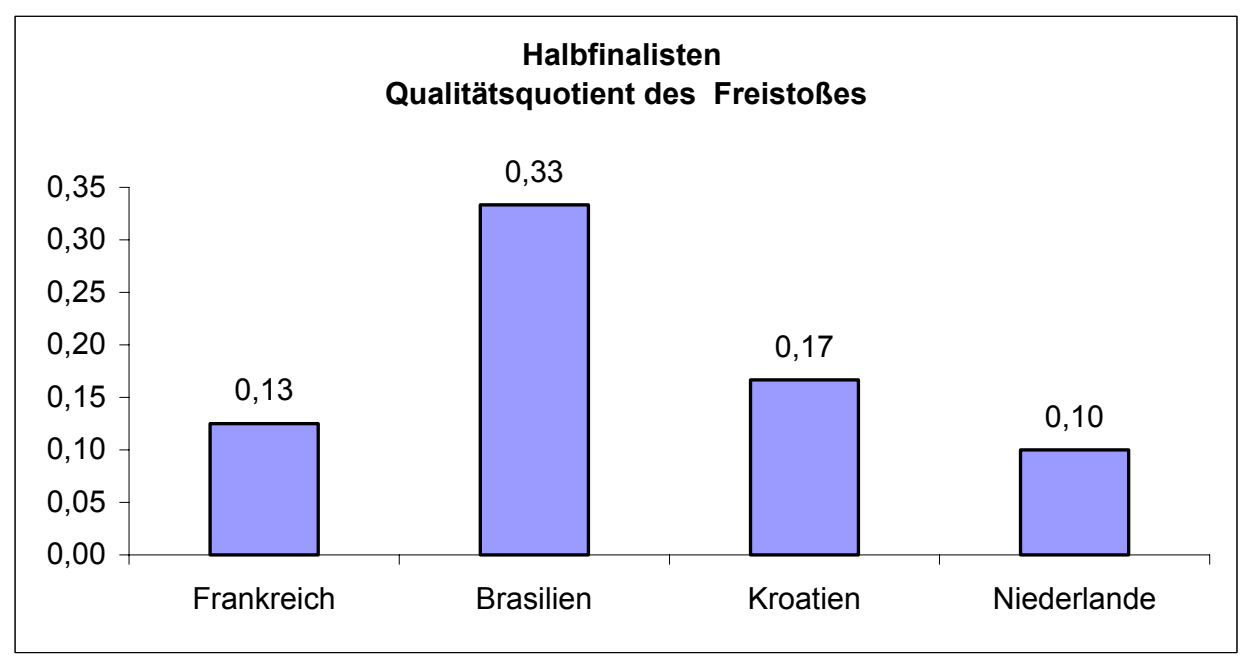

Diagramm 219 : Qualitätsquotient des Freistoßes bei den Halbfinalisten.

Die brasilianische Mannschaft zeigt den besten Qualitätsquotient im Freistoß $(0,33)$, der jedoch nicht als gut bewertet werden kann. 


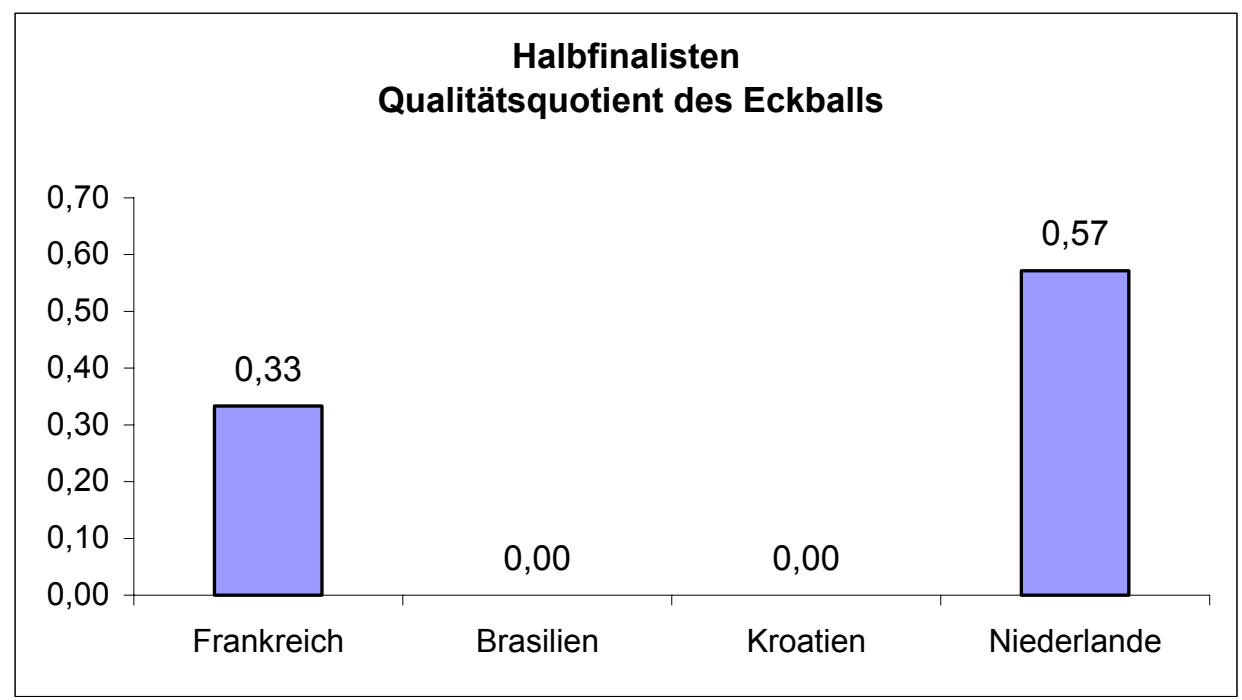

Diagramm 220 : Qualitätsquotient des Eckballs bei den Halbfinalisten.

Die Niederländer erreichen mit einem Qualitätsquotient „Eckball“ von 0,57 den höchsten Wert, der jedoch noch zu schwach erscheint.

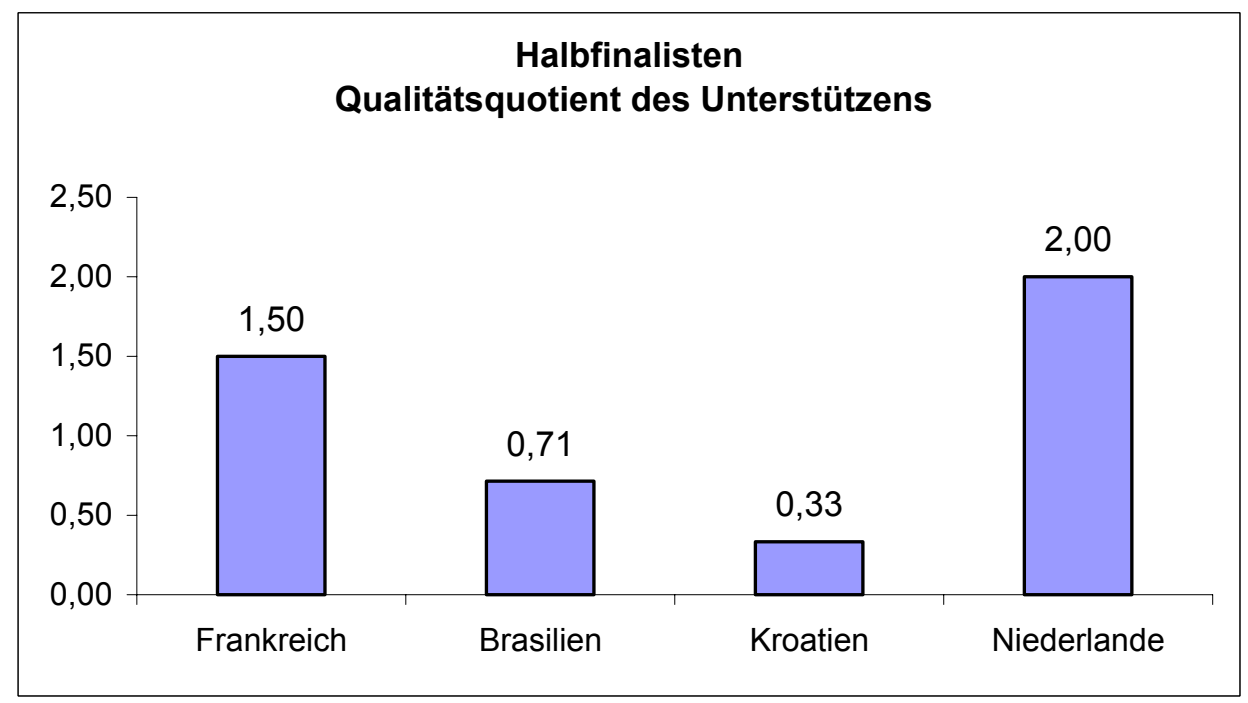

Diagramm 221 : Qualitätsquotient des Unterstützens bei den Halbfinalisten.

Den besten Qualitätsquotienten im Unterstützen zeigt sich bei den Niederländern mit einer sehr guten 2,00, unter den anderen Mannschaften hatte Frankreich mit 1,5 noch einen guten Wert. 


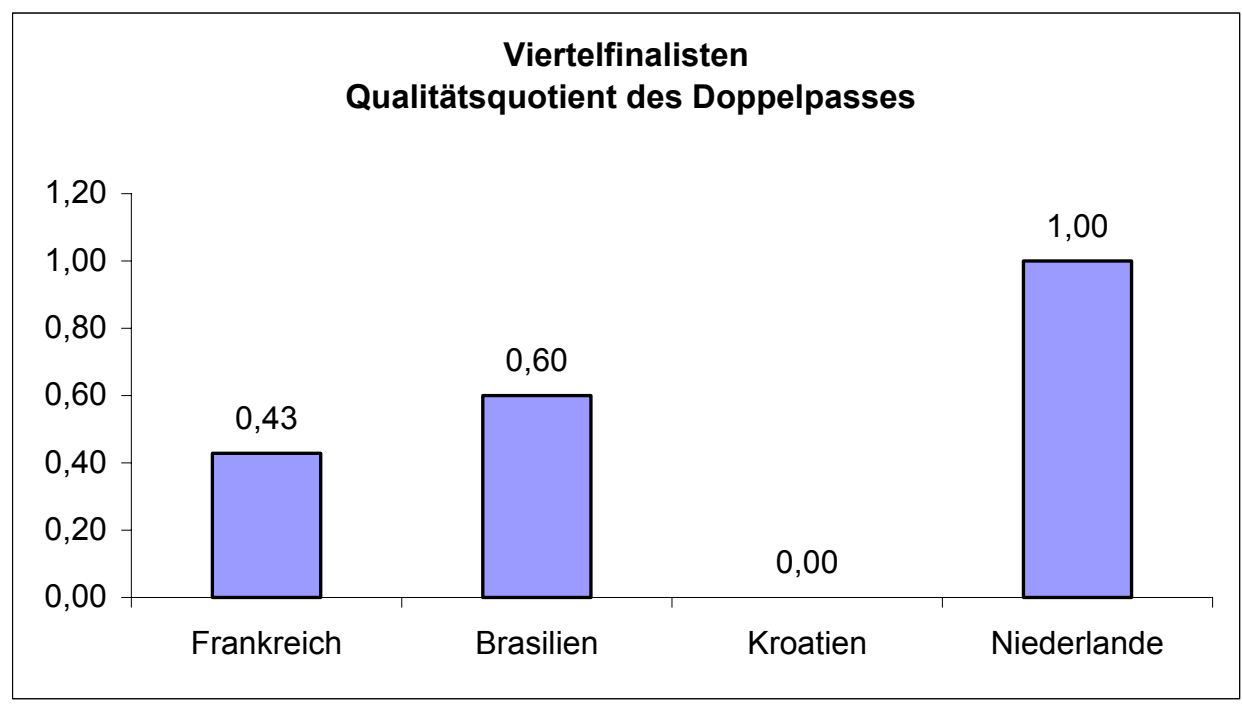

Diagramm 222 : Qualitätsquotient des Doppelpasses bei den Halbfinalisten.

Die niederländische Mannschaft erweist sich als der beste Doppelpassspieler mit einem Qualitätsquotient von 1,00.

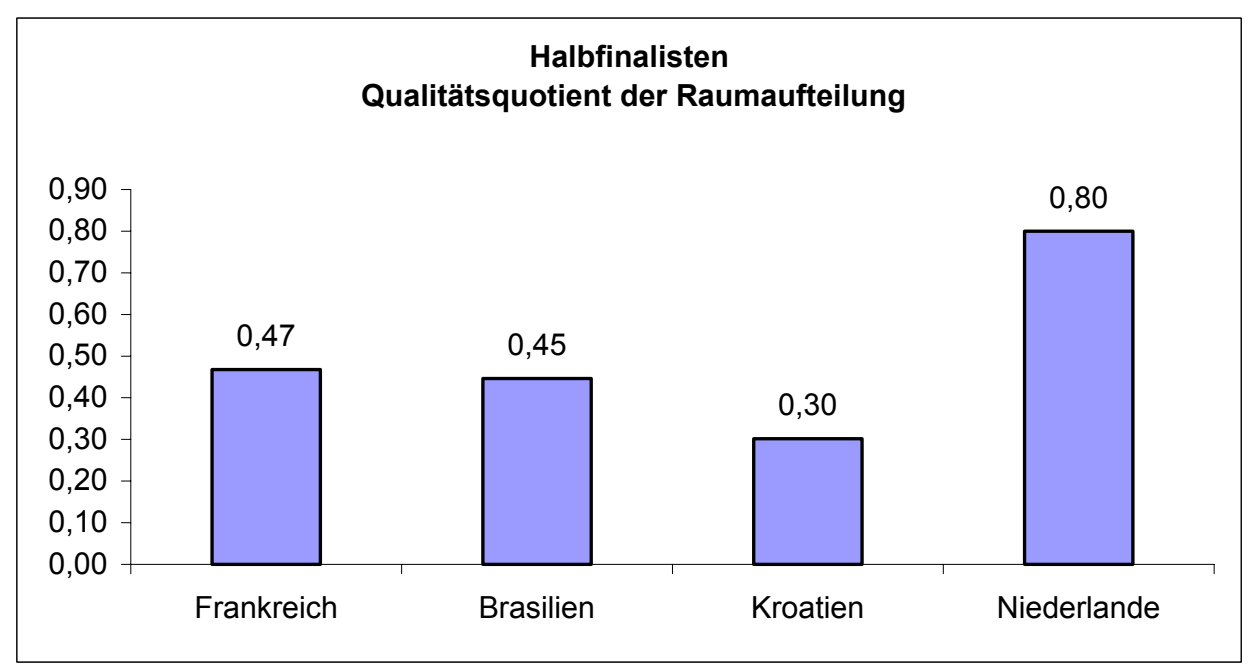

Diagramm 223 : Qualitätsquotient der Raumaufteilung bei den Halbfinalisten.

Die Niederländer zeigen sich auch in der Raumaufteilung mit dem Ergebnis 0,80 als die besten Spieler. Die Rangfolge der übrigen ist wieder ähnlich. 


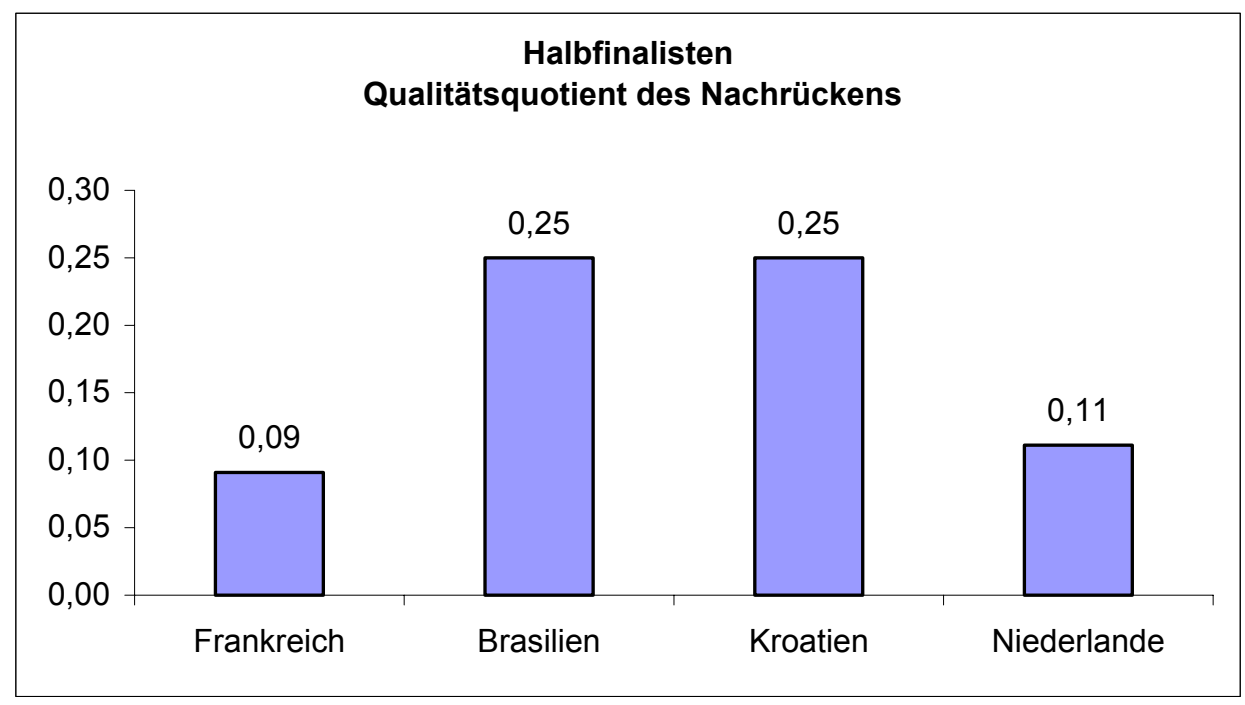

Diagramm 224 : Qualitätsquotient des Nachrückens bei den Halbfinalisten.

Hier ergibt sich eine andere Reihenfolge als in den vorhergehenden Quotienten, indem die brasilianische und kroatische Mannschaft die besseren Qualitätsquotienten im Nachrücken erlangten, ein Ergebnis von 0,25 ist jedoch recht bescheiden.

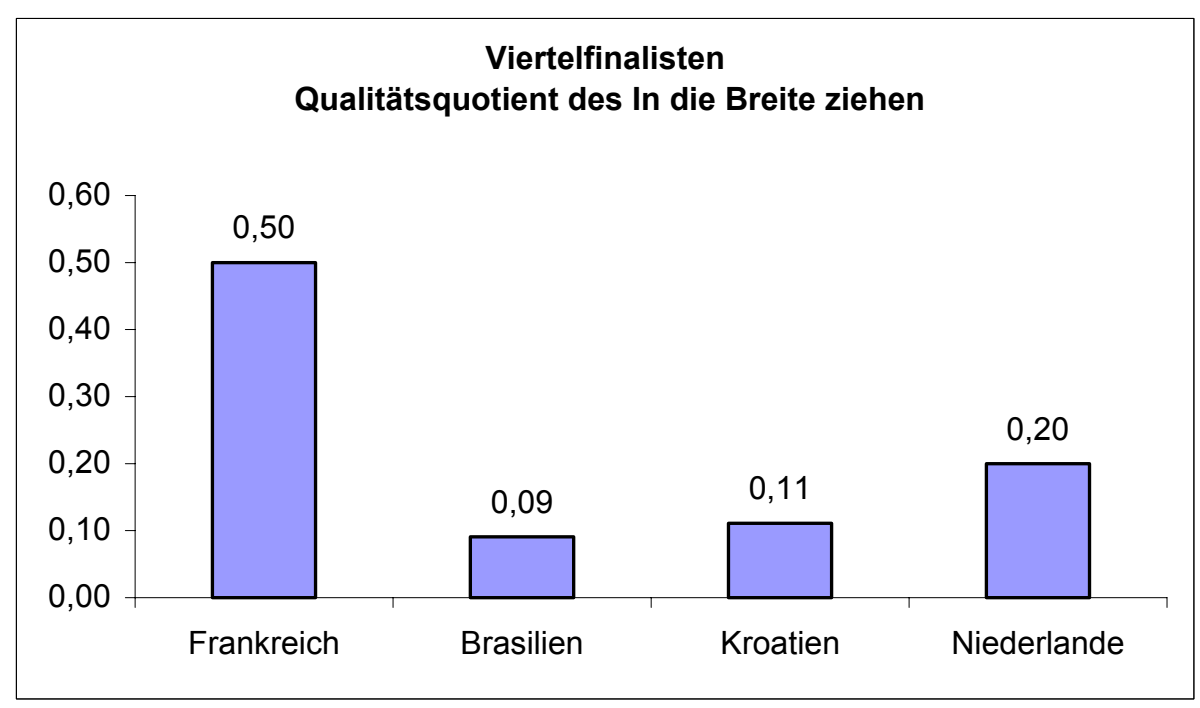

Diagramm 225: Qualitätsquotient des in die Breite ziehen bei den Halbfinalisten.

Die französische Mannschaft erhielt den höchsten Qualitätsquotienten beim „In die Breite ziehen“ mit dem Ergebnis von 0,50, einem vielleicht noch akzeptablen Wert. Die übrigen fallen jedoch stark ab. 


\subsection{Qualitätsquotienten defensiv taktischer Elemente}

\subsubsection{Achtelfinalisten}

Das folgende Diagramm zeigt den Qualitätsquotient „Pressing hinten“ der Achtelfinalisten.

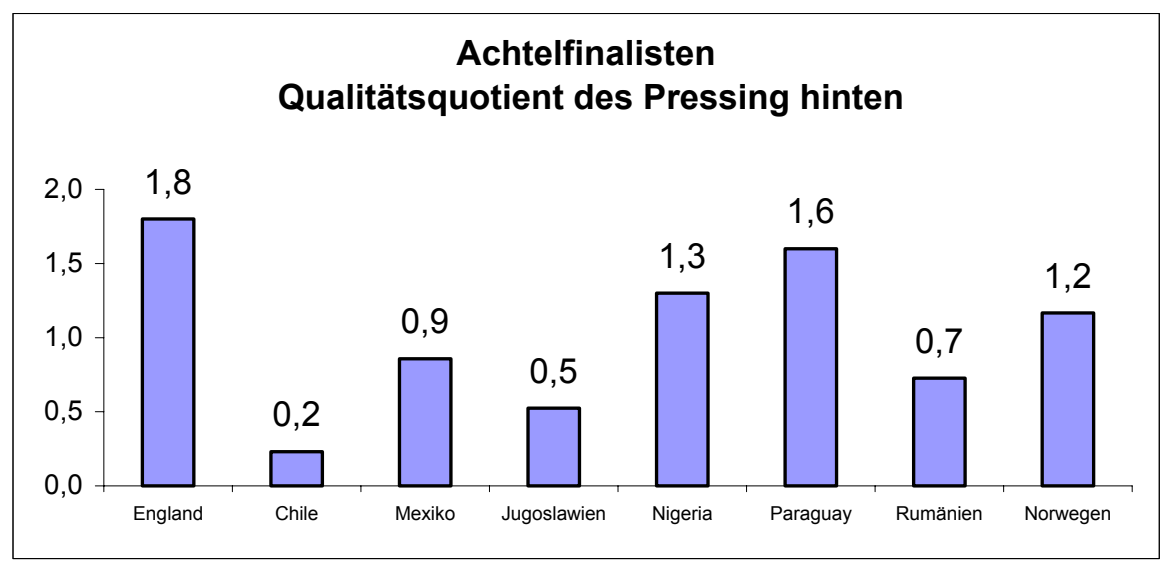

Diagramm 226: Qualitätsquotient des Pressing hinten bei den Achtelfinalisten.

Die englische Mannschaft zeigt den besten Wert beim Qualitätsquotient „Pressing hinten" mit 1,80. Aber auch andere Werte wie 1,30 für Nigeria, 1,60 für Paraguay, 1,16 für Norwegen sind gut, woraus das große Bemühen um ein starkes Abwehrspiel in der eigenen Position bei vielen Mannschaften herauszulesen ist.

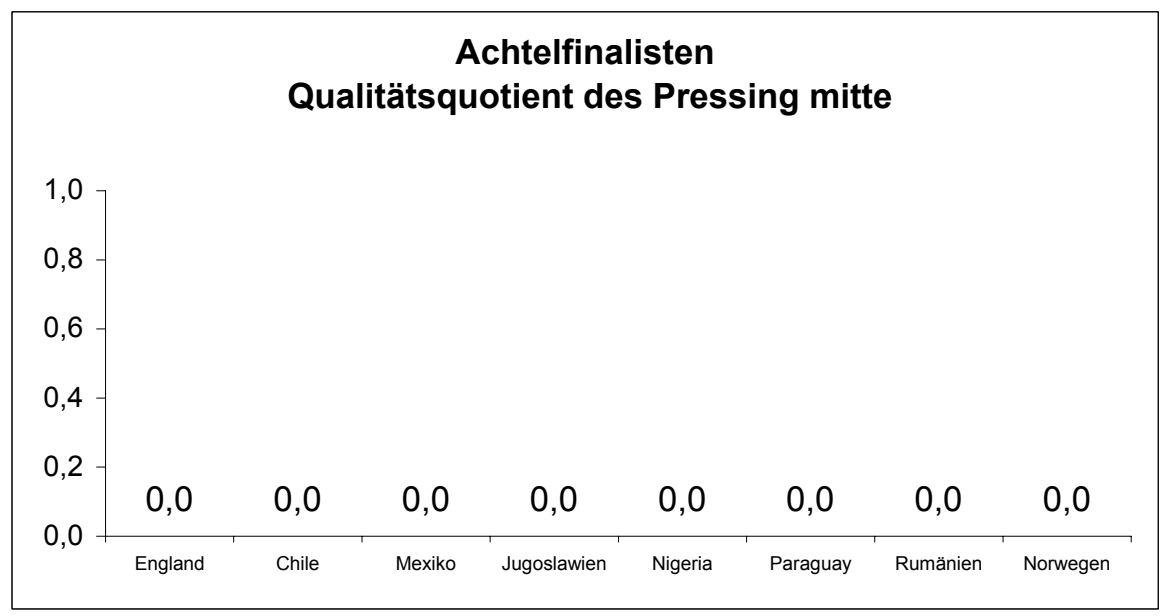

Diagramm 227: Qualitätsquotient des Pressing mitte bei der Achtelfinalisten.

Alle Mannschaften zeigen gleiche schlechte Qualitätsquotienten beim Pressing mitte mit 0. Ein sehr deutlicher Abfall zum Pressing hinten ist festzustellen, die Kräfte scheinen sich auf das Abwehrspiel im eigenen Torraum zu beschränken. Dadurch fehlt auch die Möglichkeit, dem Gegner den Ball rechtzeitig im Vorfeld abzunehmen und dadurch die eigenen Torchancen zu erhöhen. 
Das folgende Diagramm zeigt den Qualitätsquotient „Pressing vorne“ der Achtelfinalisten.

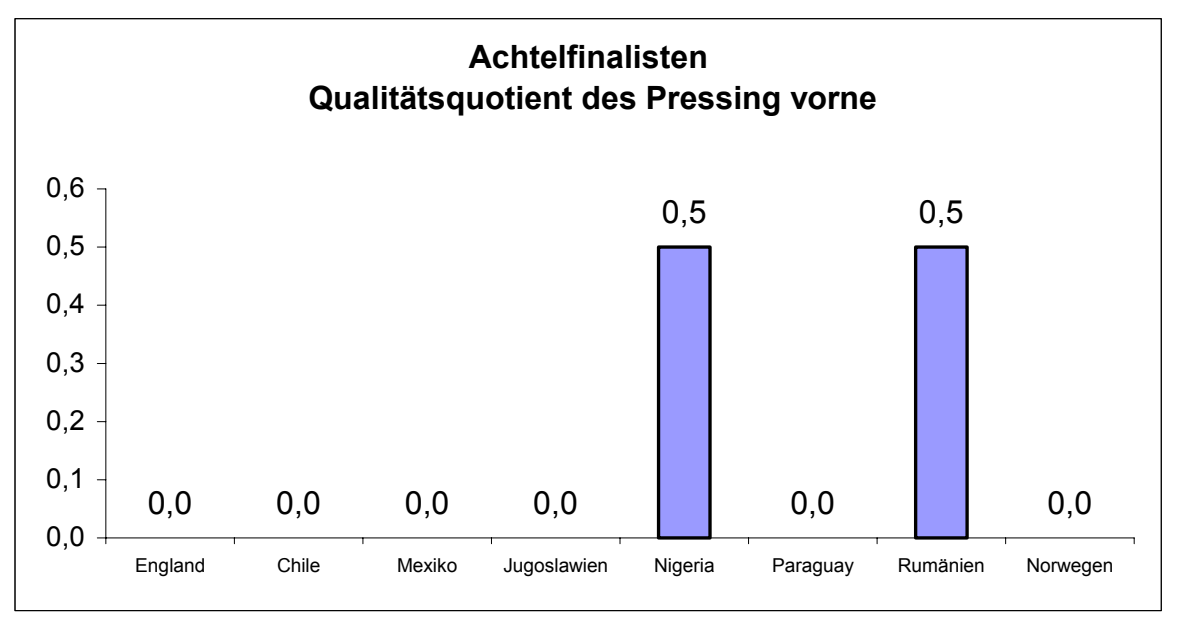

Diagramm 228: Qualitätsquotient des Pressing vorne bei den Achtelfinalisten.

Die nigerianischen und rumänischen Spieler zeigen einen halbwegs akzeptablen Qualitätsquotienten Pressing vorne mit 0,50, während die anderen Mannschaften aus Mangel an Stürmern im vorderen Bereich keine Gelegenheit zum Pressing vorne hatten.

Das folgende Diagramm zeigt den Qualitätsquotient „Raumaufteilung“ der Achtelfinalisten.

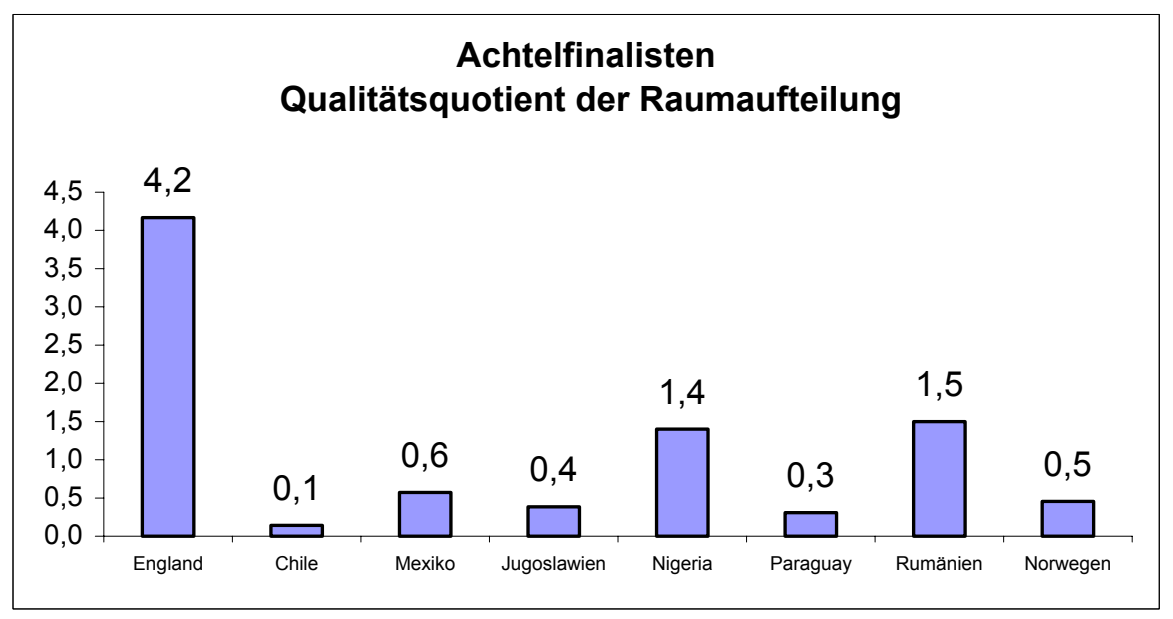

Diagramm 229: Qualitätsquotient der Raumaufteilung bei den Achtelfinalisten. 
Die englische Mannschaft hatte einen sehr guten Qualitätsquotient bei der Raumaufteilung von 4,16, von den anderen Mannschaften hatten mit 1,40 für Nigeria und 1,50 für Rumänien nur diese noch einen recht guten Wert, die anderen liegen stark zurück.

Das folgende Diagramm zeigt den Qualitätsquotient „zurückkommen“ der Achtelfinalisten.

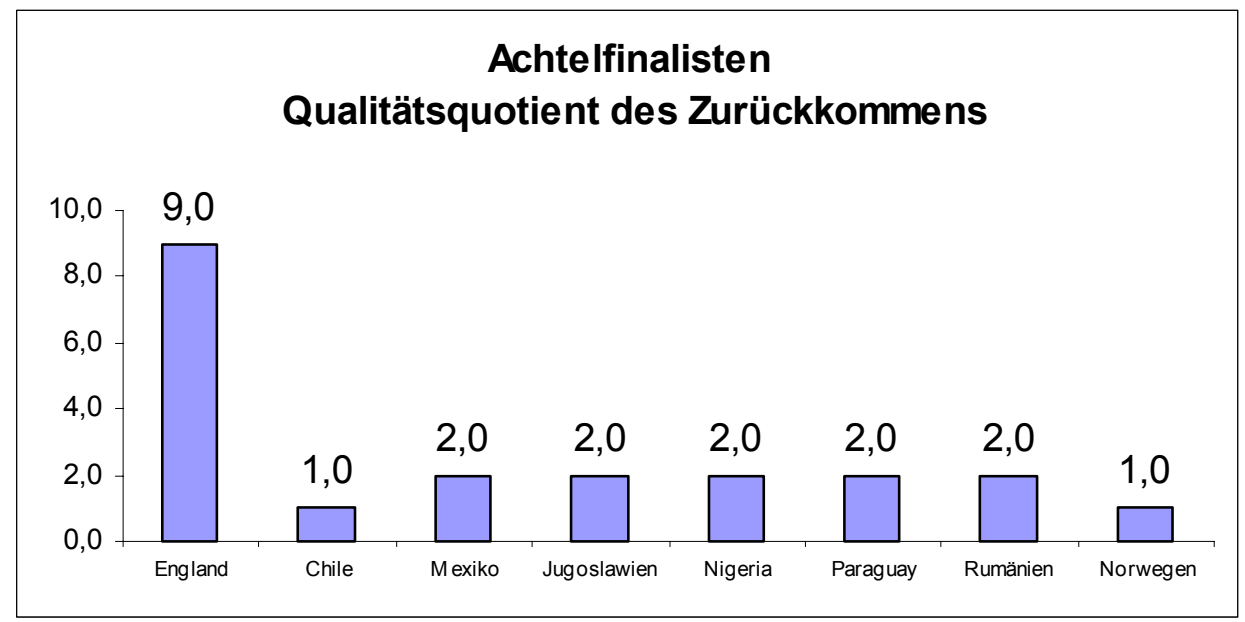

Diagramm 230: Qualitätsquotient des Zurückkommens bei den Achtelfinalisten.

Die englische Mannschaft zeigt einen außergewöhnlichen Qualitätsquotient beim Zurückkommen mit 9,00, auch bei vielen anderen Mannschaften ist der Wert 2,00 zu verzeichnen. Keine Mannschaft war richtig schlecht.

Das folgende Diagramm zeigt den Qualitätsquotient „Unterstützen“ der Achtelfinalisten.

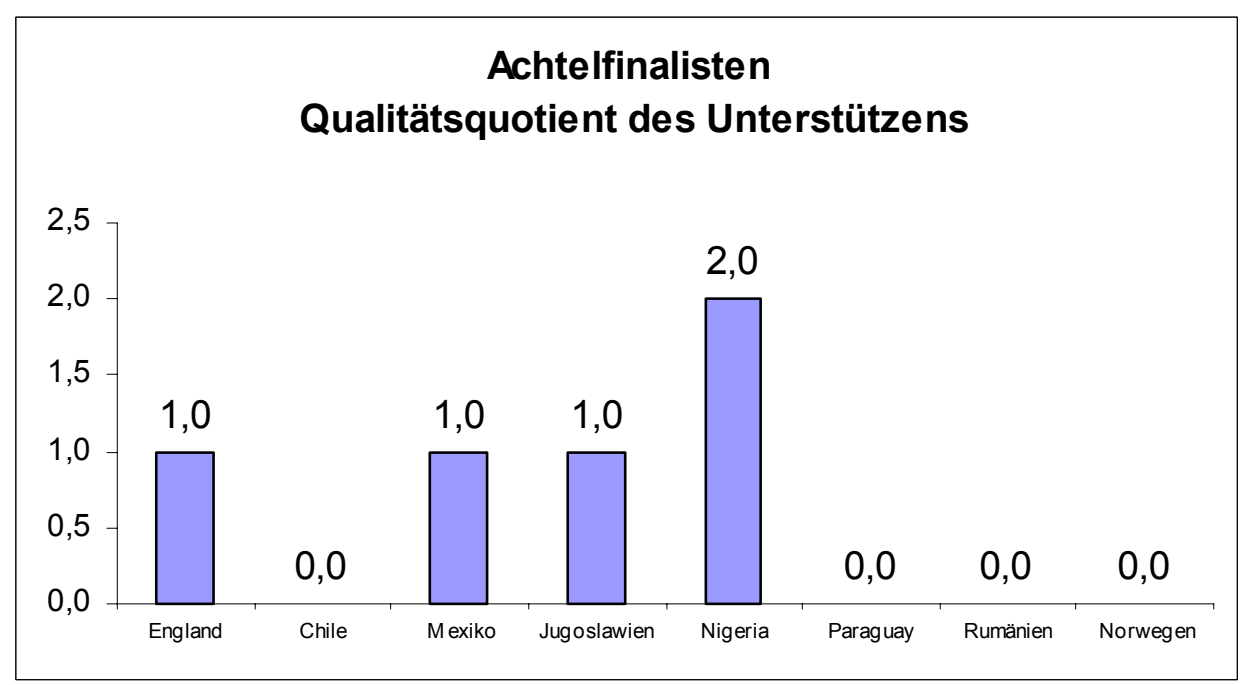

Diagramm 231: Qualitätsquotient des Unterstützens bei den Achtelfinalisten. 
Bei dem Qualitätsquotienten Unterstützen erlangt die nigerianische Mannschaft das bessere Ergebnis mit 2,0. Bei den anderen Mannschaften ergibt sich eine scharfe Unterscheidung zwischen gut und schlecht.

Das folgende Diagramm zeigt den Qualitätsquotient „Rückraum sichern“ der Achtelfinalisten.

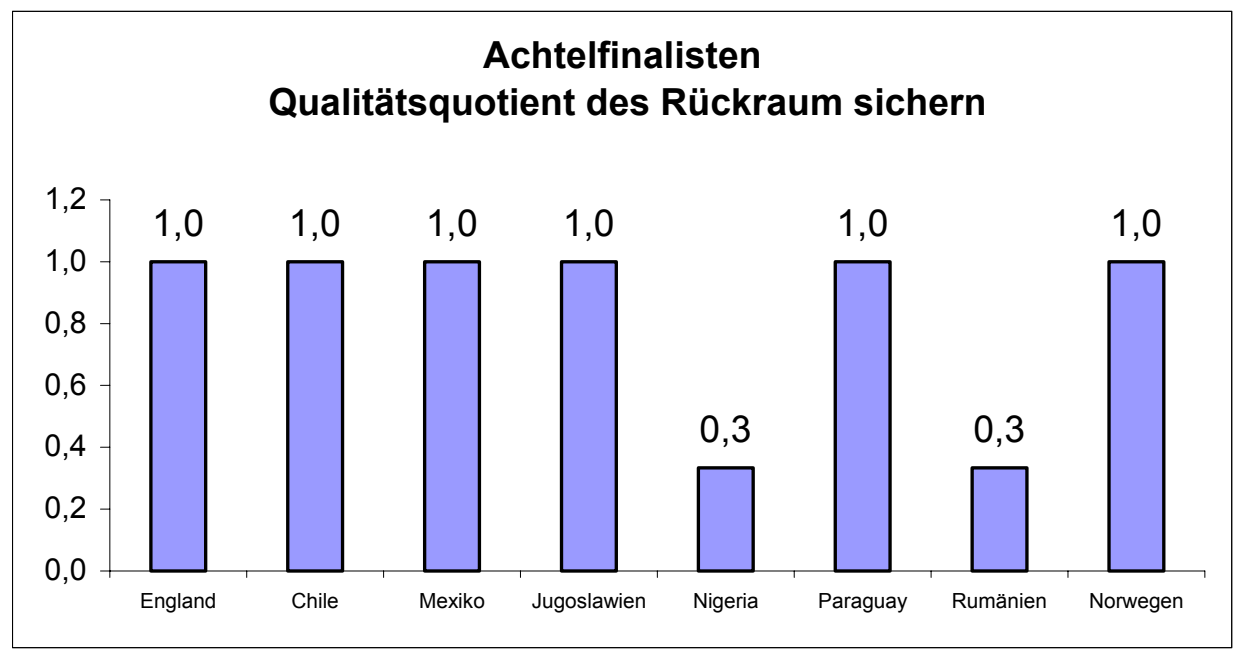

Diagramm 232: Qualitätsquotient des Rückraum sichern bei den Achtelfinalisten.

Bei Qualitätsquotienten Rückraum sichern erreichte die überwiegende Zahl aller Mannschaften den Wert 1,00, nur Nigeria und Rumänien fallen zurück.

Das folgende Diagramm zeigt den Qualitätsquotient „Abseitsfalle“ der Achtelfinalisten.

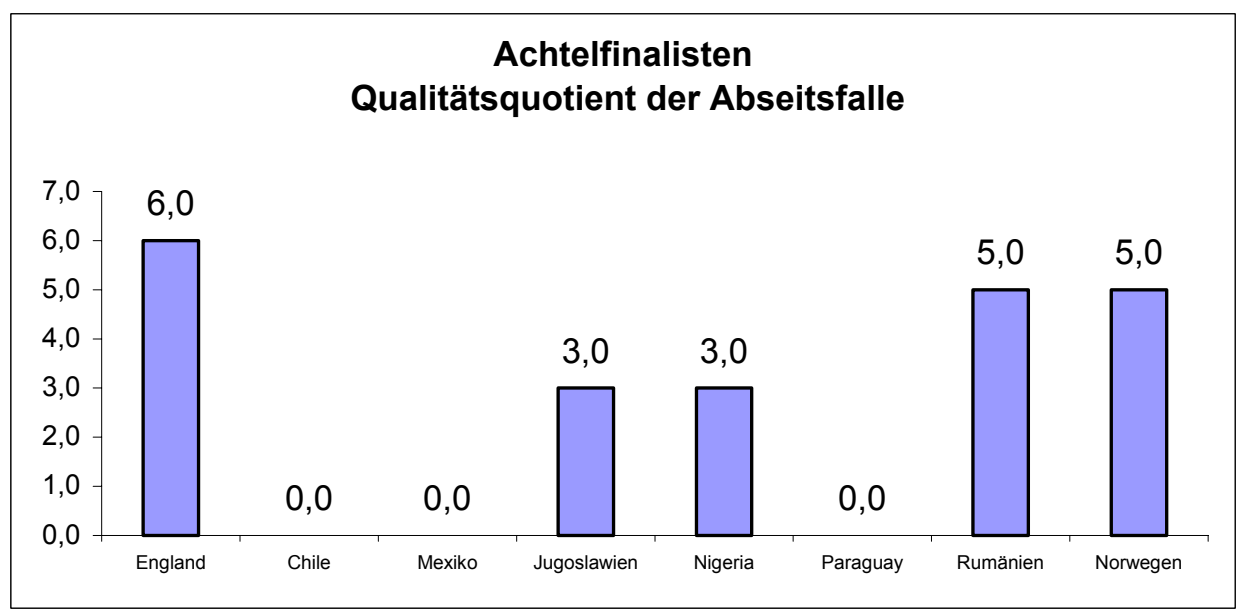

Diagramm 233: Qualitätsquotient der Abseitsfalle bei den Achtelfinalisten. 
Die englische Mannschaft erreicht auch beim Qualitätsquotienten Abseitsfalle einen außergewöhnlichen Wert von 6,00, Rumänien und Norwegen erhielten mit 5,00 ebenfalls sehr bemerkenswerten Noten. Eine 3,00 für Jugoslawien und Nigeria ist noch sehr gut, die anderen offenbaren hier deutliche spieltaktische Schwächen und wagten diese Fallen daher nicht.

Aus der Betrachtung der hochkarätigen und schwachen Werte für Abwehrtaktik in den Diagrammen geht hervor, dass die meisten über eins liegen. Dies deutet darauf hin, dass die meisten Mannschaften des Achtelfinals sich mehr auf Abwehr- als auf Angriffstaktik stützten. Ein Argument dafür ist, dass diese Mannschaften nur eine geringe Anzahl von Toren erzielten, einige Mannschaften sogar kein Tor im Wettkampf erzielten wie Norwegen, Paraguay und Rumänien.

\subsubsection{Viertelfinalisten}

Das folgende Diagramm zeigt den Qualitätsquotienten „Pressing hinten“ der Viertelfinalisten.

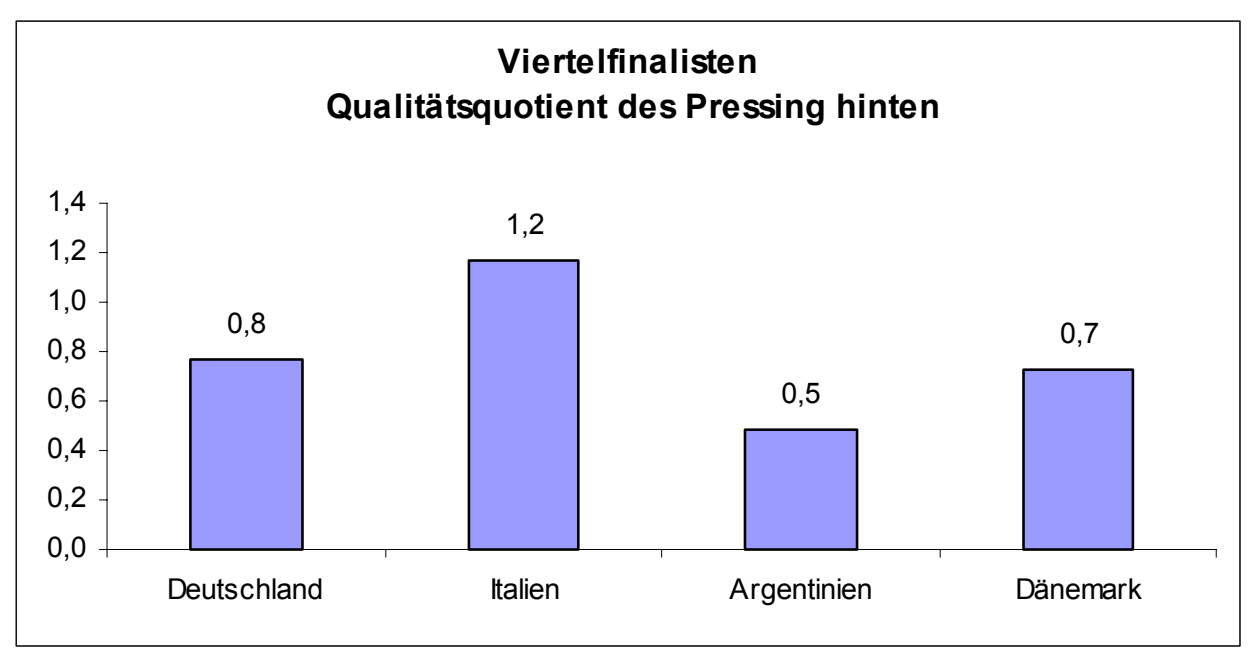

Diagramm 234: Qualitätsquotient des Pressing hinten bei den Viertelfinalisten.

Die italienische Mannschaft hatte einen guten Qualitätsquotient beim Pressing hinten $(1,2)$. Die Werte $(0,8)$ für Deutschland $(0,7)$ für Dänemark und $(0,5)$ für Argentinien scheinen schon zu schwach. 
Das folgende Diagramm zeigt den Qualitätsquotient „Pressing Mitte“ der Viertelfinalisten.

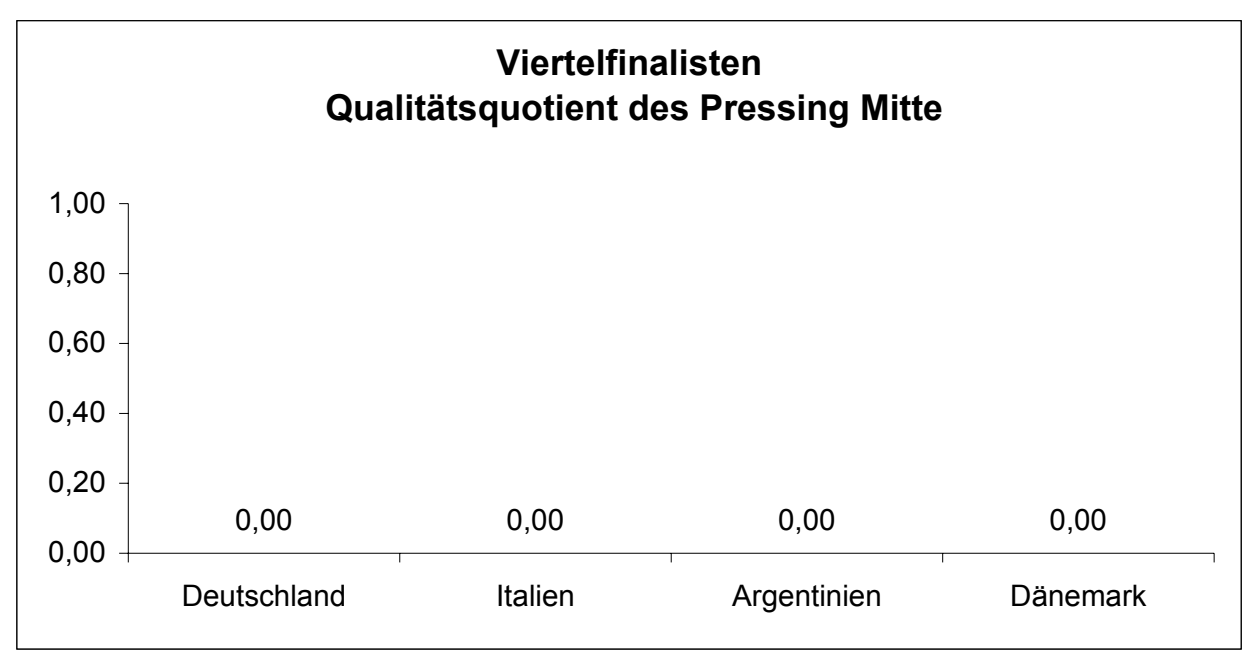

Diagramm 235: Qualitätsquotient des Pressing Mitte den Viertelfinalisten.

Alle Mannschaften zeigen wiederum den gleichen schlechten Qualitätsquotienten beim Pressing Mitte $(0,00)$.

Das folgende Diagramm zeigt den Qualitätsquotient „Pressing vorne“ der Viertelfinalisten.

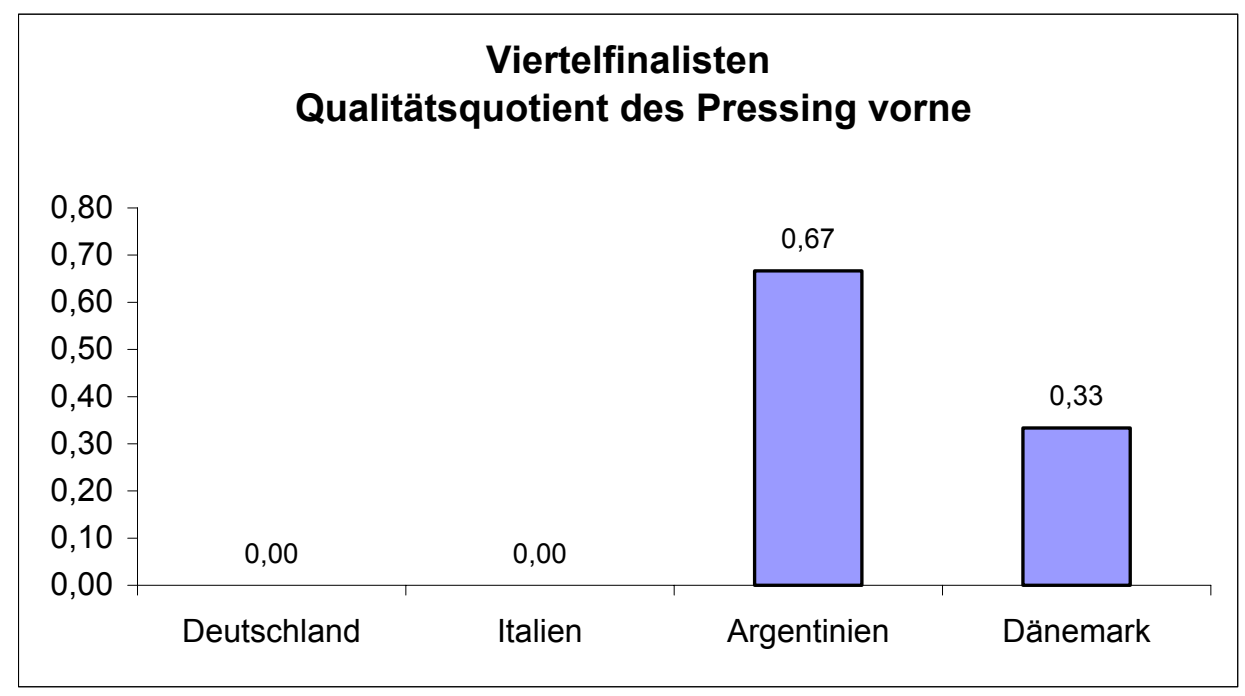

Diagramm 236: Qualitätsquotient des Pressing vorne den Viertelfinalisten.

Nur die argentinische Mannschaft zeigt ein einigermaßen positives Pressing vorne mit einem Qualitätsquotient von 0,67. 
Das folgende Diagramm zeigt den Qualitätsquotient „Raumaufteilung“ der Viertelfinalisten.

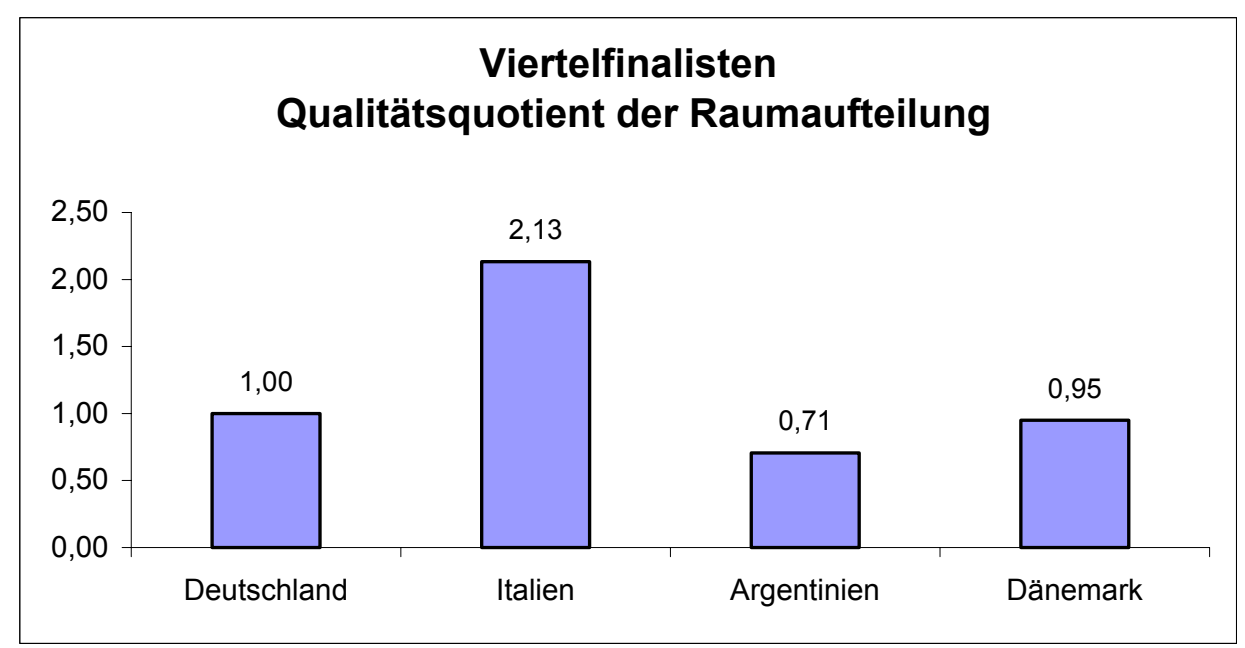

Diagramm 237: Qualitätsquotient der Raumaufteilung bei den Viertelfinalisten.

Die italienische Mannschaft zeigt sich beim Qualitätsquotient Raumaufteilung als führend $(2,13)$, von den anderen Mannschaften hat Deutschland $(1,00)$ und Dänemark $(0,95)$ einen gerade noch ausreichend erscheinenden Wert.

Das folgende Diagramm zeigt den Qualitätsquotient „Zurückkommen“ der Viertelfinalisten.

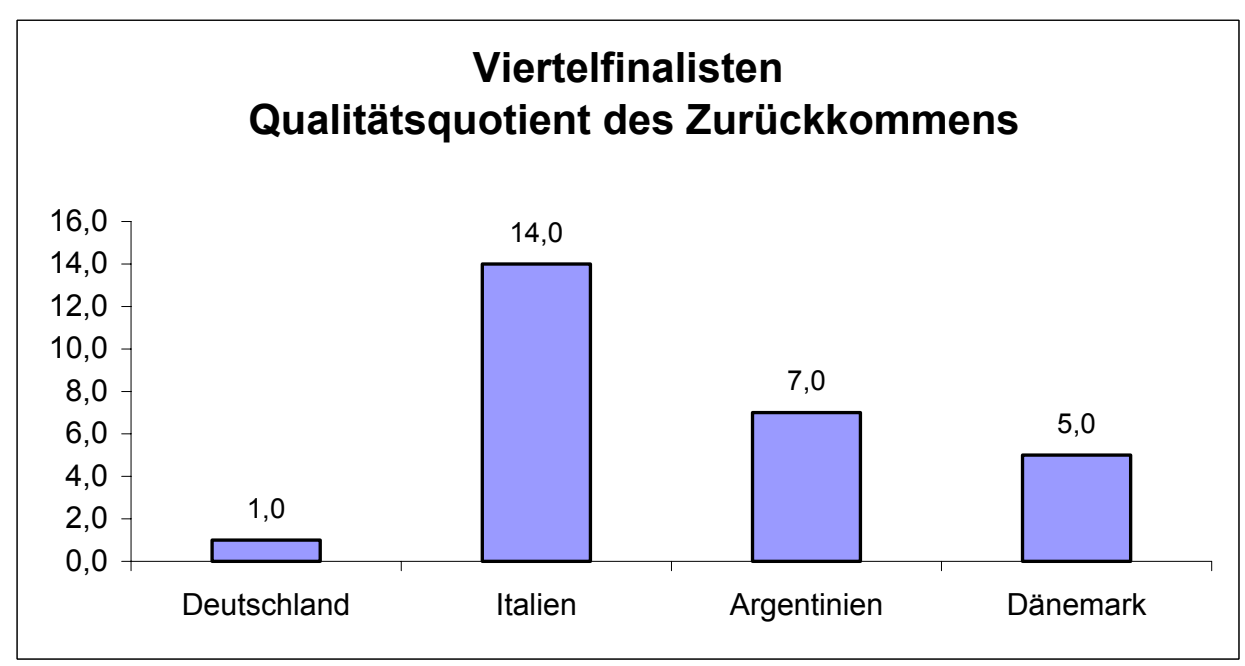

Diagramm 238: Qualitätsquotient des Zurückkommens bei den Viertelfinalisten. 
Die italienische Mannschaft erlief sich beim Qualitätsquotient Zurückkommen den überragenden Spitzenwert von 14,00, auch 7,00 für Argentinien und 5,00 für Dänemark sind noch bemerkenswerte kämpferische Bemühungen.

Das folgende Diagramm zeigt den Qualitätsquotient „Unterstützen“ der Viertelfinalisten.

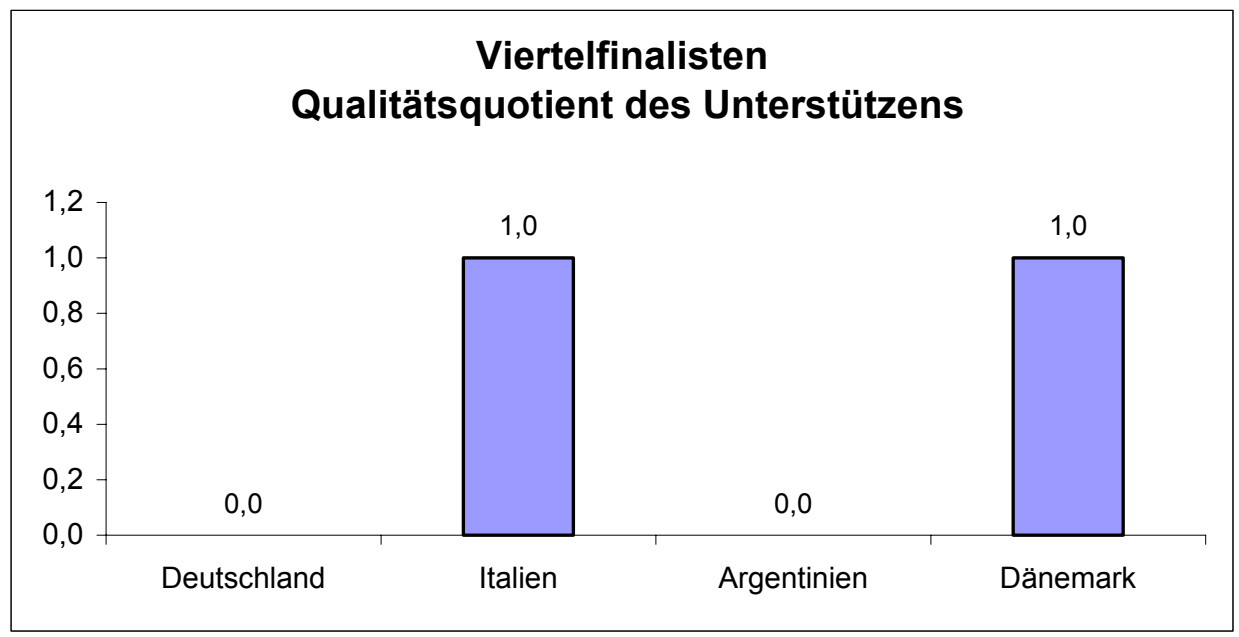

Diagramm 239: Qualitätsquotient des Unterstützens bei den Viertelfinalisten.

Nur die Italiener und Dänen leisteten wirksame Unterstützung.

Das folgende Diagramm zeigt den Qualitätsquotient „Rückraum sichern“ der Viertelfinalisten.

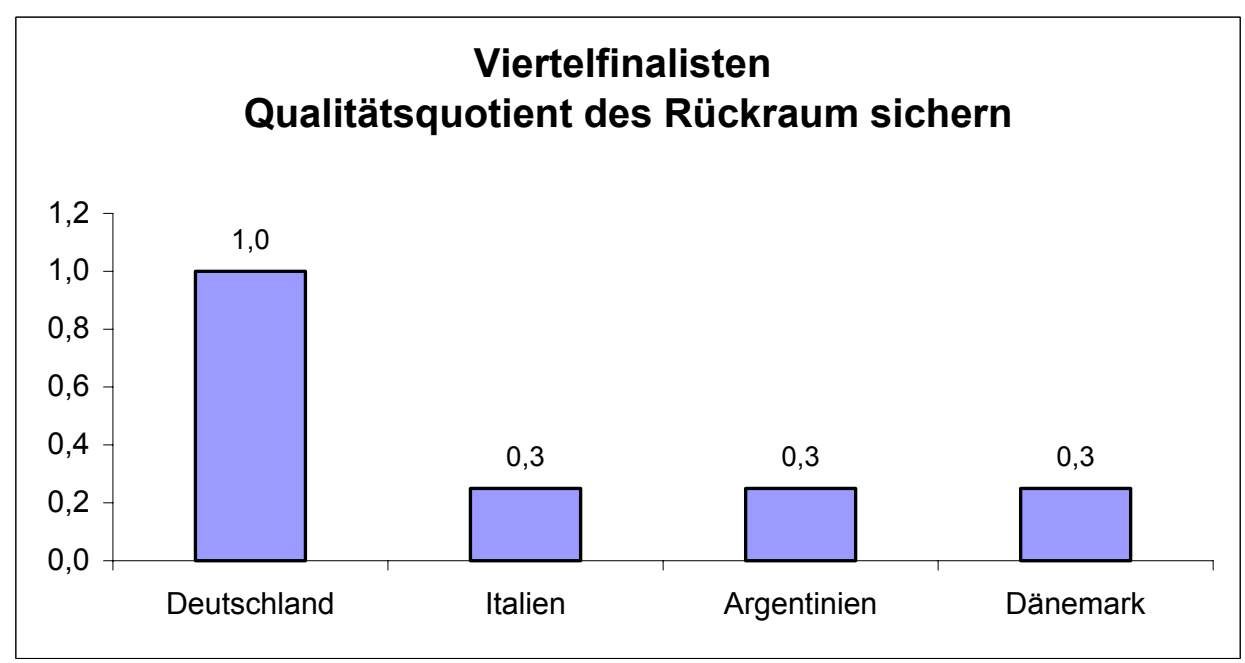

Diagramm 240: Qualitätsquotient des Rückraum sichern bei den Viertelfinalisten. 
Die deutsche Mannschaft kümmerte sich hier einzig um einen genügend gesicherten Rückraum. die anderen sind zu schwach.

Das folgende Diagramm zeigt den Qualitätsquotient „Abseitsfalle“ den Viertelfinalisten.

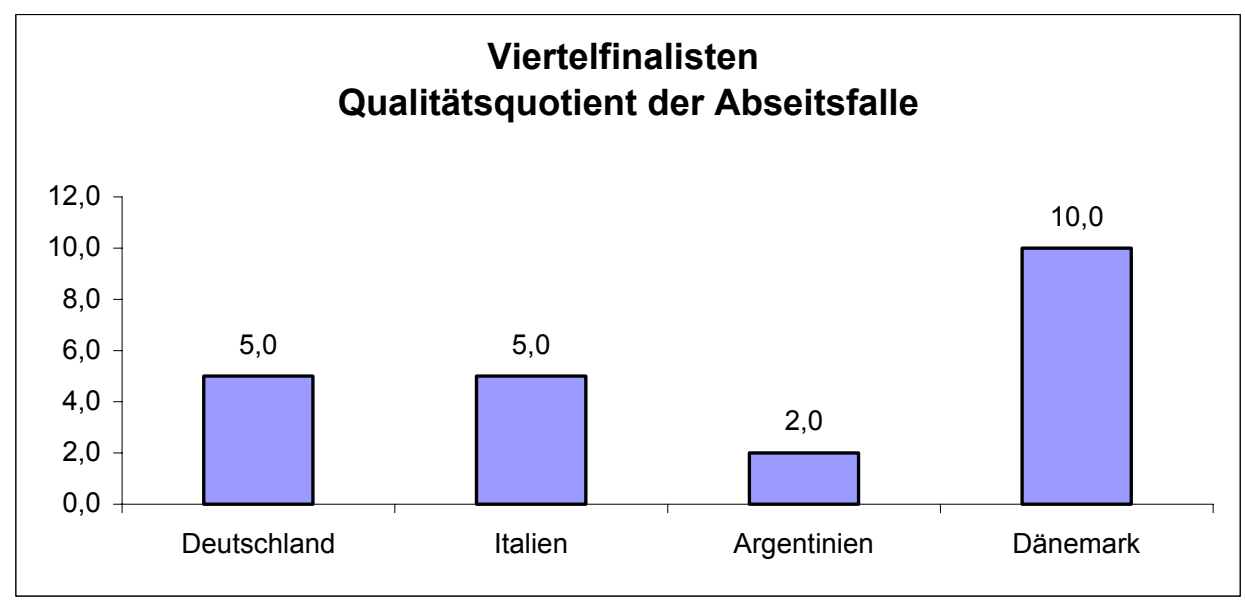

Diagramm 241: Qualitätsquotient der Abseitsfalle bei den Viertelfinalisten.

Die dänische Mannschaft erhielt einen Spitzenwert beim Qualitätsquotient Abseitsfalle $(10,00)$, auch die spieltaktischen Kenntnisse der anderen Mannschaften sind noch gut mit 5,00 für Deutschland und 5,00 für Italien sowie einem schwächeren 2,00 für Argentinien.

\subsubsection{Halbfinalisten}

Das folgende Diagramm zeigt den Qualitätsquotient „Pressing hinten“ der Halbfinalisten.

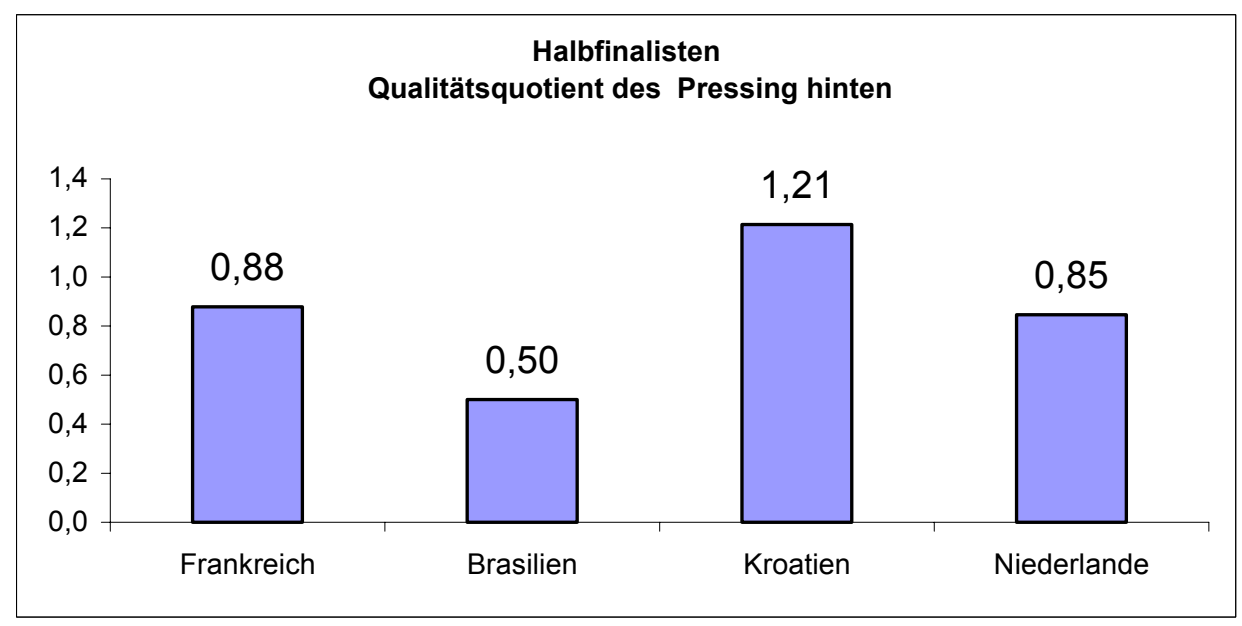

Diagramm 242: Qualitätsquotient des Pressing hinten bei den Halbfinalisten. 
Beim Pressing hinten ergeben sich Werte, die auf die defensive Qualität dieser Taktik verweisen, indem die schwache Mannschaft der Kroaten den höchsten Wert erzielte, die guten Spieler der Brasilianer jedoch—vielleicht auch aus Mangel an Gelegenheit—den schwächsten Wert.

Das folgende Diagramm zeigt den Qualitätsquotient „Pressing Mitte“ der Halbfinalisten.

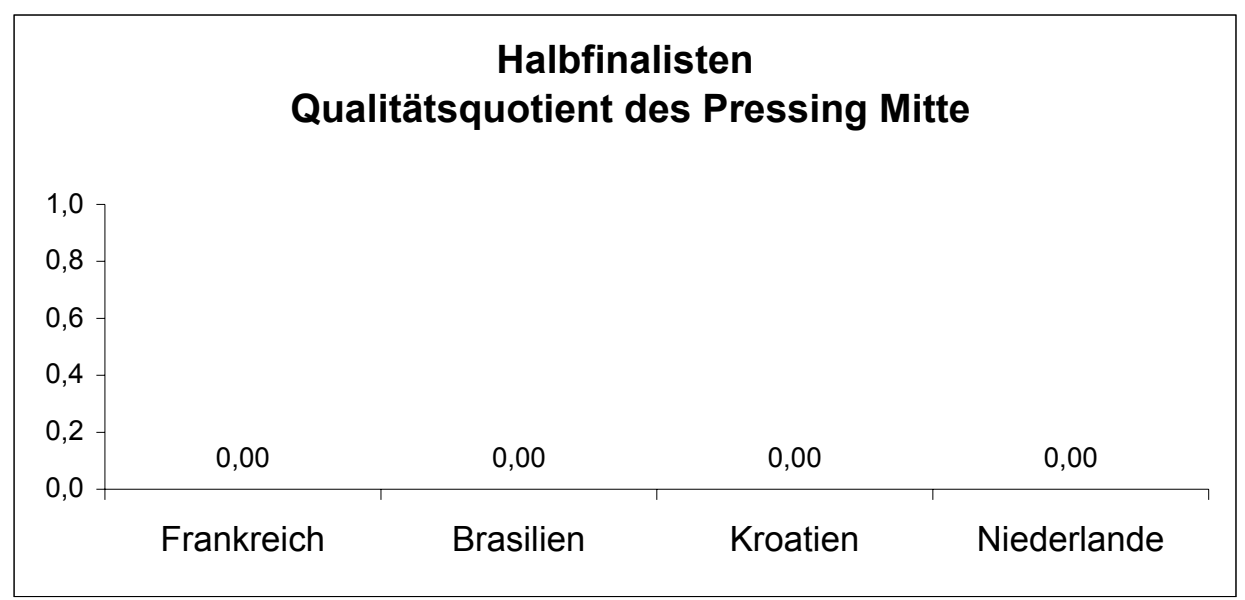

Diagramm 243: Qualitätsquotient des Pressing Mitte bei den Halbfinalisten.

Bei diesem Quotienten ist ein Pressing Mitte nicht zu erkennen, da es kein hochkarätiges sondern nur mittleres und schwaches gab.

Das folgende Diagramm zeigt den Qualitätsquotient „Pressing vorne“ der Halbfinalisten.

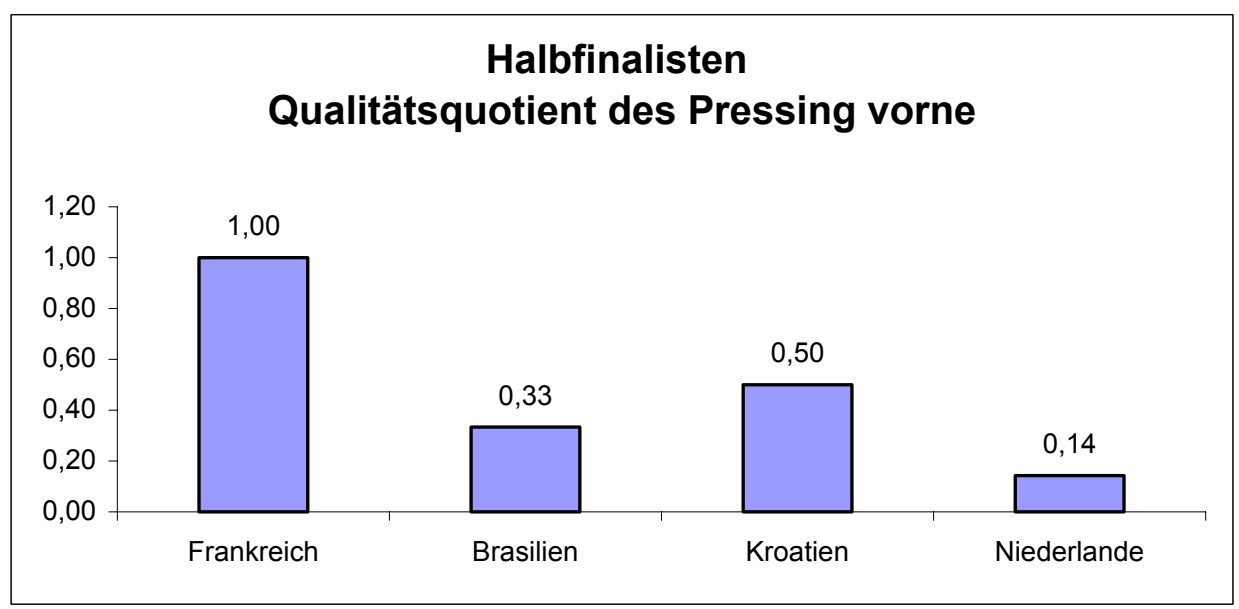

Diagramm 244: Qualitätsquotient des Pressing vorne bei den Halbfinalisten. 
Nur Frankreich führte ein gutes Pressing vorne aus, die Kroaten versuchten es, während die Brasilianer auch hier schwach erscheinen.

Das folgende Diagramm zeigt den Qualitätsquotient „Raumaufteilung“ der Halbfinalisten.

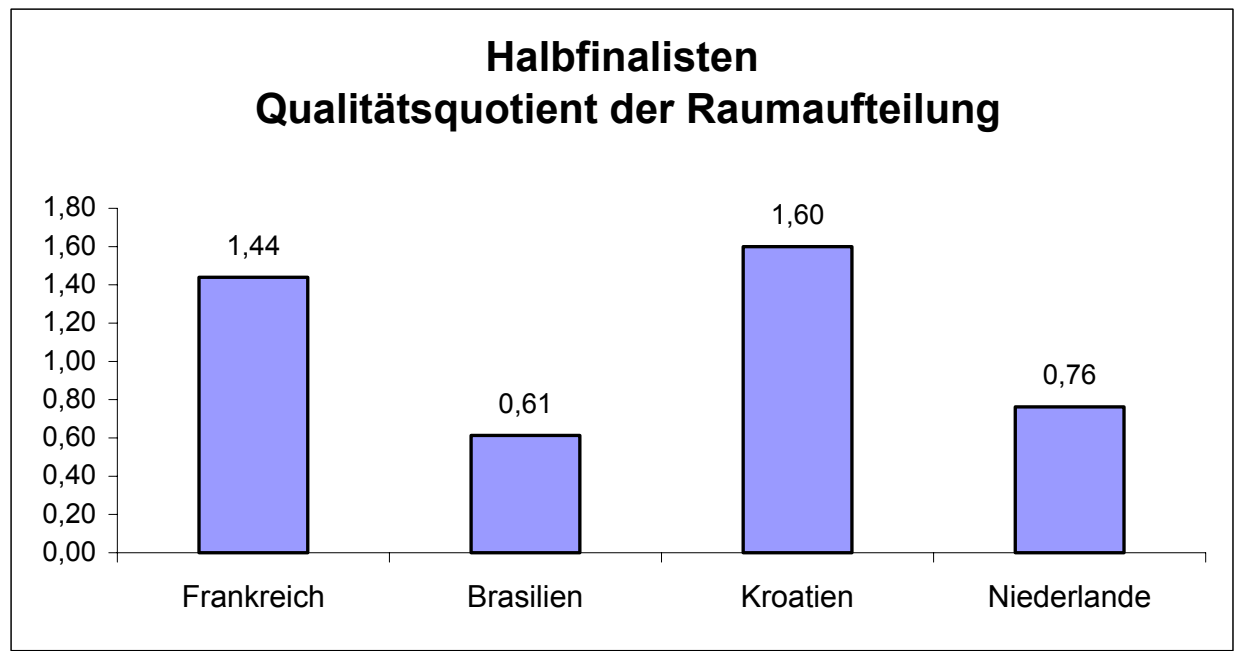

Diagramm 245: Qualitätsquotient der Raumaufteilung bei den Halbfinalisten.

Frankreich und Kroatien zeigen die besten Qualitätsquotienten für die Raumaufteilung, Brasilien und die Niederlande erscheinen schwach.

Das folgende Diagramm zeigt den Qualitätsquotient „Zurückkommen“ der Halbfinalisten.

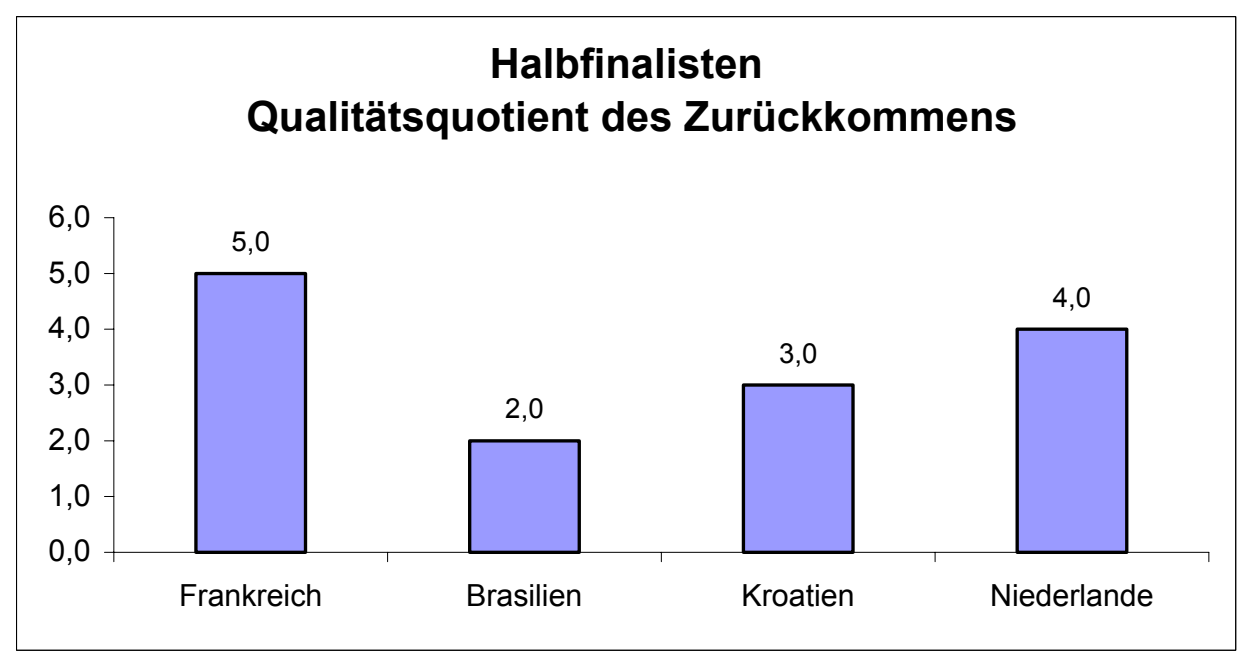

Diagramm 246: Qualitätsquotient des Zurückkommens bei den Halbfinalisten. 
Die französische und die niederländische Mannschaft zeigen sich beim Zurückkommen stark, Brasilien kümmert sich weniger um eine starke Verteidigung als das schwächere Kroatien.

Das folgende Diagramm zeigt den Qualitätsquotient „Unterstützen“ der Halbfinalisten.

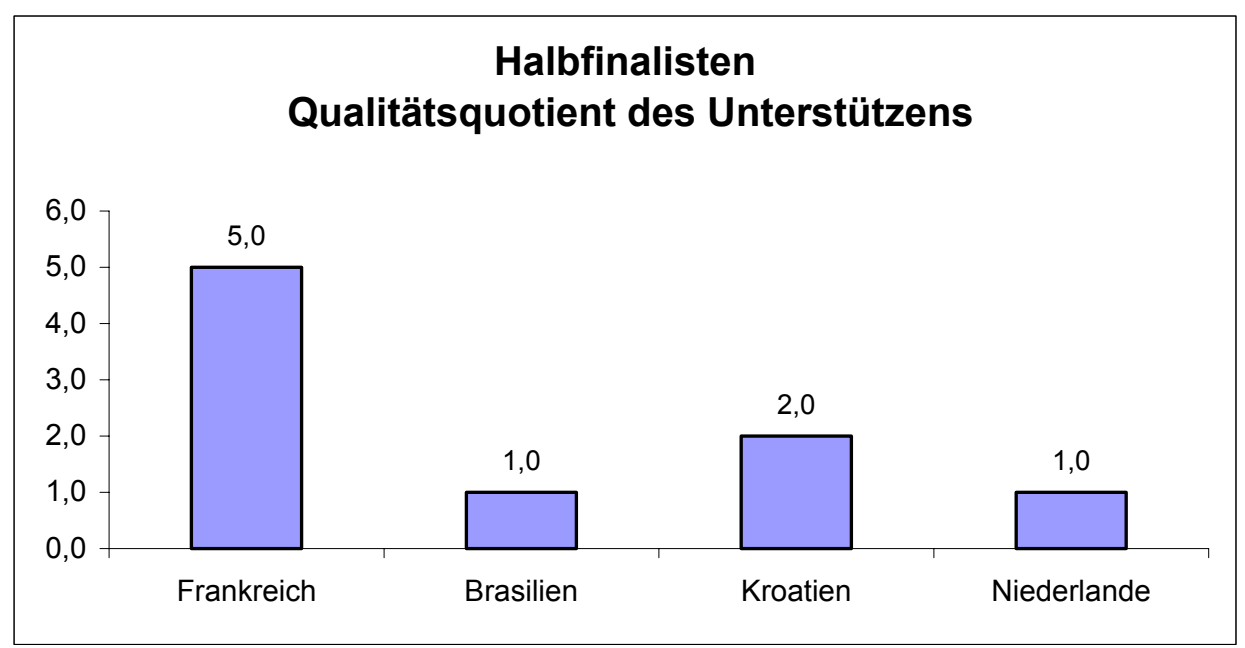

Diagramm 247: Qualitätsquotient des Unterstützens bei den Halbfinalisten.

Beim Unterstützen führt die französische Mannschaft eindeutig, Kroatien zeigt hierin noch mehr Zusammenspiel als die übrigen.

Das folgende Diagramm zeigt den Qualitätsquotient „Rückraum sichern“ der Halbfinalisten.

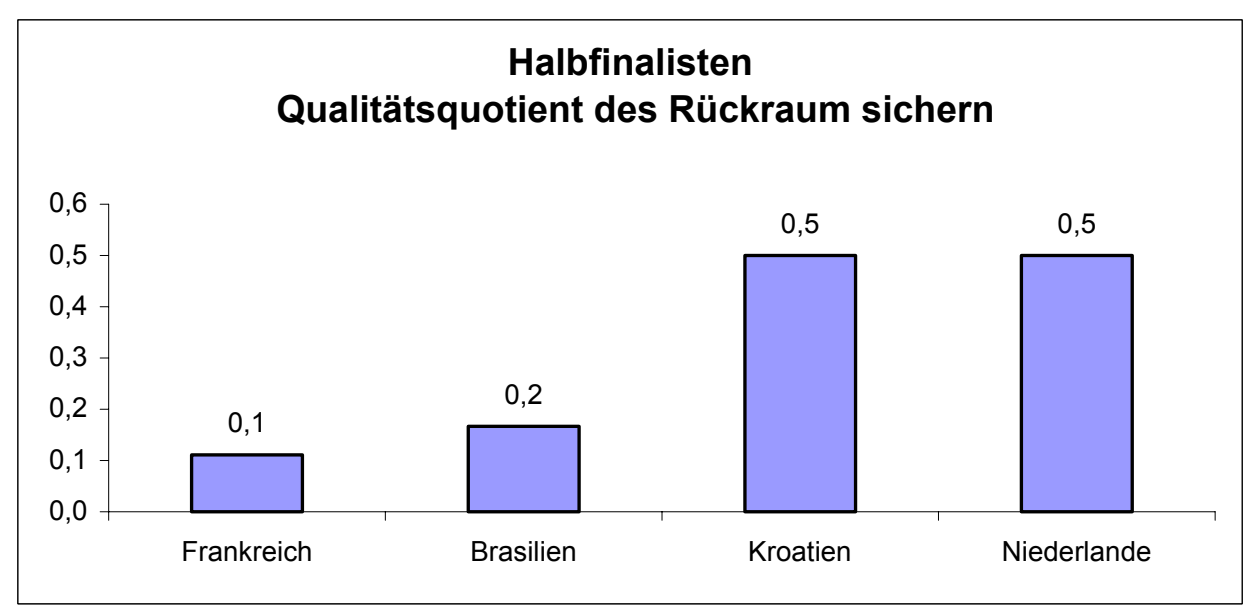

Diagramm 248: Qualitätsquotient des Rückraum sichern bei den Halbfinalisten. Alle Mannschaften zeigen sich eher schwach. 
Die brasilianische Mannschaft zeigt den besten Qualitätsquotient Abseitsfälle $(13,00)$, während die anderen Mannschaften hatten $(9,00)$ für Frankreich und $(6,00)$ für Kroatien und $(12,00)$ für die Niederlande.

Das folgende Diagramm zeigt den Qualitätsquotient „Abseitsfälle“ der Halbfinalisten.

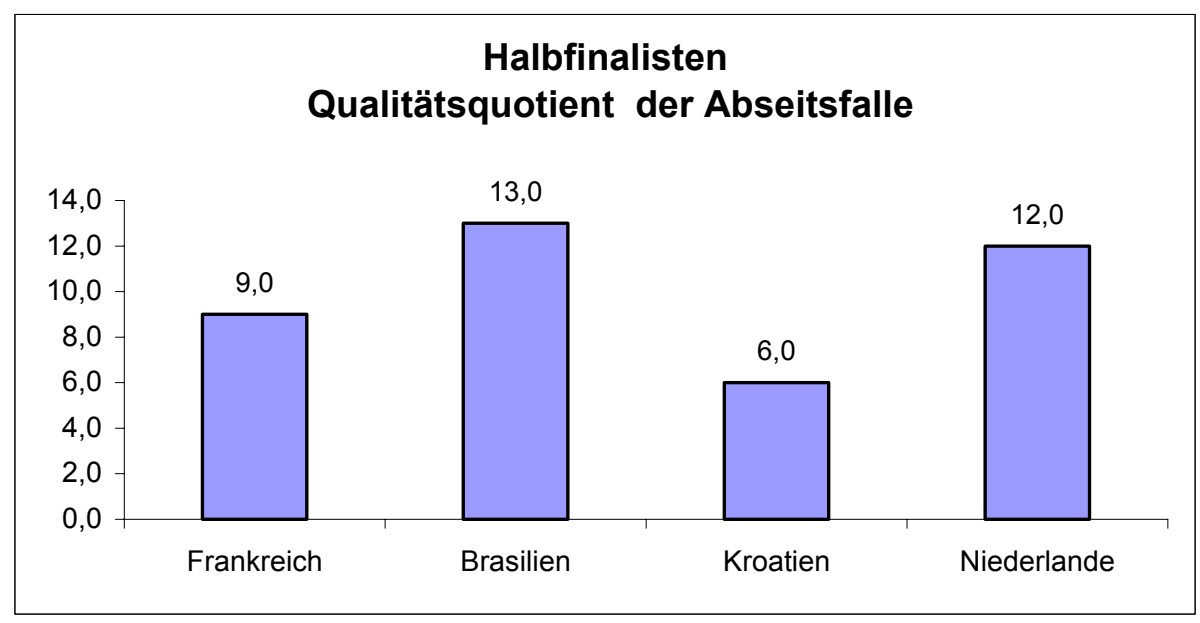

Diagramm 249: Qualitätsquotient der Abseitsfalle bei den Halbfinalisten.

Bei dieser Spieltaktik zeigen sich die Brasilianer als Meister, doch dicht von den Niederlanden gefolgt.

\subsection{Individuelle und mannschaftliche Fähigkeiten}

Im folgenden Kapitel werden durchschnittliche Qualitätsquotienten der individuellen und mannschaftlichen Fähigkeiten der sechzehn Achtelfinalteilnehmer beschrieben.

Der Qualitätsquotient einer bestimmten Mannschaft bezüglich eines einzelnen Elements ergibt sich, indem man (ungeachtet der „normalen“ Aktionen) das Verhältnis der Anzahl „guter“ und „schwacher“ Aktionen dieser Mannschaft bezüglich dieses Elements berechnet. Der Qualitätsquotient ist demnach eine Zahl, die zwischen „Null“ und „Unendlich“ liegt, wobei eine Zahl nahe Null auf eine geringe, eine hohe Zahl auf eine (sehr) hohe Qualität hindeutet.

Der Mittelwert aller Qualitätsquotienten einer Mannschaft von Elementen, die den individuellen bzw. mannschaftlichen Fähigkeiten zugeordnet sind, ergibt den durchschnittlichen Qualitätsquotienten einer bestimmten Mannschaft bezüglich dieser Fähigkeit. 
Folgende Elemente wurden den individuellen Fähigkeiten zugeordnet:

- Dribbling,

- Körpertäuschung,

- Torschuss,

- kurze Pässe,

- lange Pässe,

- Ballkontrolle,

- Freistoss,

- Unterstützen,

- Nachrücken,

- Defensives Unterstützen,

- Rückraum sichern,

Folgende Elemente wurden den mannschaftlichen Fähigkeiten zugeordnet:

- Eckball,

- Doppelpass,

- Raumaufteilung,

- in die Breite ziehen,

- Pressing hinten,

- Pressing mitte,

- Pressing vorne,

- Raumaufteilung (Defensives),

- Zurückkommen,

- Abseitsfalle.

\subsubsection{Achtelfinalisten}

Diagramm 250 zeigt die durchschnittlichen Qualitätsquotienten individueller und mannschaftlicher Fähigkeiten von England. 


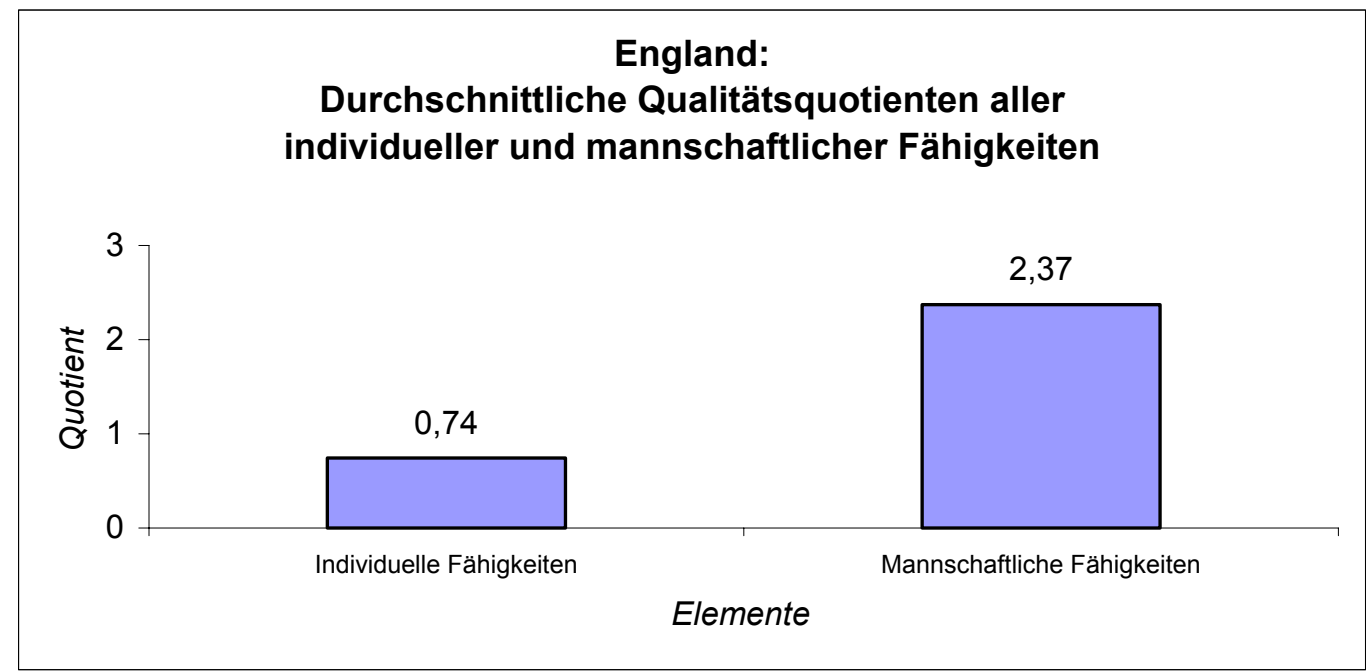

Diagramm 250 : Durchschnittliche Qualitätsquotienten individueller und mannschaftlicher Fähigkeiten der englischen Mannschaft

Aus dem Diagramm ersehen wir, dass ein wesentlicher Unterschied zwischen den individuellen und mannschaftlichen Fähigkeiten besteht, wobei Gruppentaktik offenbar besser ist und kaum Einzelspieltaktiken wie Dribbling, Täuschen und Torschiessen u.a. angewendet wurden.

Diagramm 251 zeigt die durchschnittlichen Qualitätsquotienten der individuellen und mannschaftlichen Fähigkeiten von Chile. Wie zu sehen ist, besitzt die chilenische Mannschaft bezüglich der individuellen Fähigkeiten einen durchschnittlichen Qualitätsquotienten von gerundet 0,26 , bezüglich der mannschaftlichen Fähigkeiten einen solchen von gerundet 0,37 .

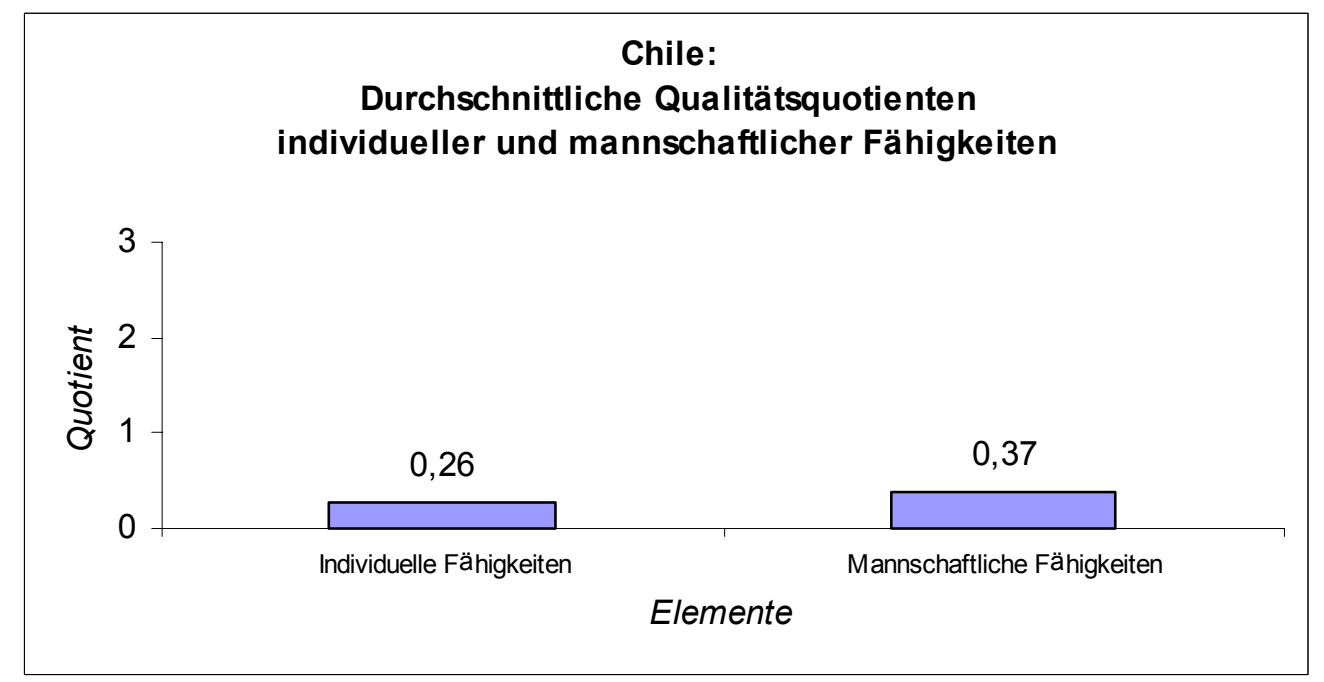

Diagramm 251 : Durchschnittliche Qualitätsquotienten individueller und mannschaftlicher Fähigkeiten von Chile. 
Aus dem Diagramm ersehen wir, dass der Unterschied zwischen den individuellen und der mannschaftlichen Fähigkeiten gering und das Niveau der beiden Spielarten verhältnismäßig niedrig ist.

Diagramm 252 zeigt die durchschnittlichen Qualitätsquotienten der individuellen und mannschaftlichen Fähigkeiten von Mexiko. Wie zu sehen ist, besitzt Mexiko bezüglich der individuellen Fähigkeiten einen durchschnittlichen Qualitätsquotienten von gerundet 0,39 , bezüglich der mannschaftlichen Fähigkeiten einen solchen von 0,43.

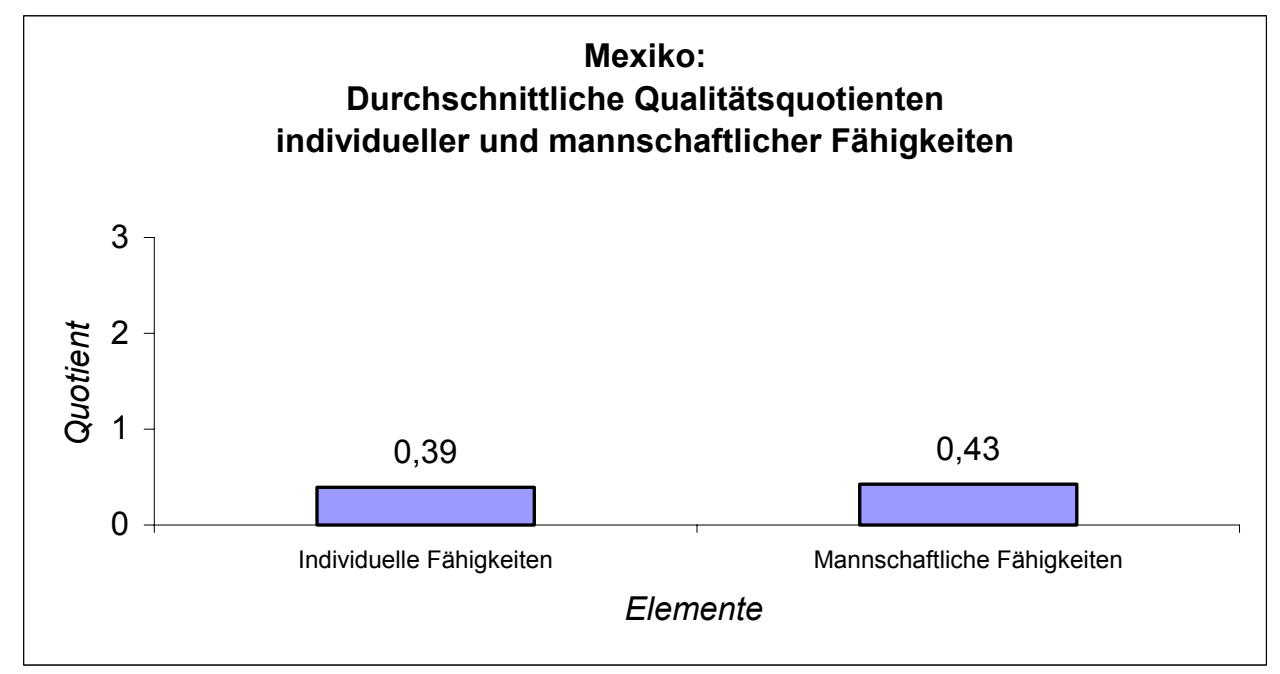

Diagramm 252 : Durchschnittliche Qualitätsquotienten individueller und mannschaftlicher Fähigkeiten von Mexiko.

Diagramm 253 zeigt die durchschnittlichen Qualitätsquotienten der individuellen und mannschaftlichen Fähigkeiten von Jugoslawien. Wie zu sehen ist, besitzt Jugoslawien bezüglich der individuellen Fähigkeiten einen durchschnittlichen Qualitätsquotienten von gerundet 0,51 , bezüglich der mannschaftlichen Fähigkeiten einen solchen von gerundet 0,64 . 


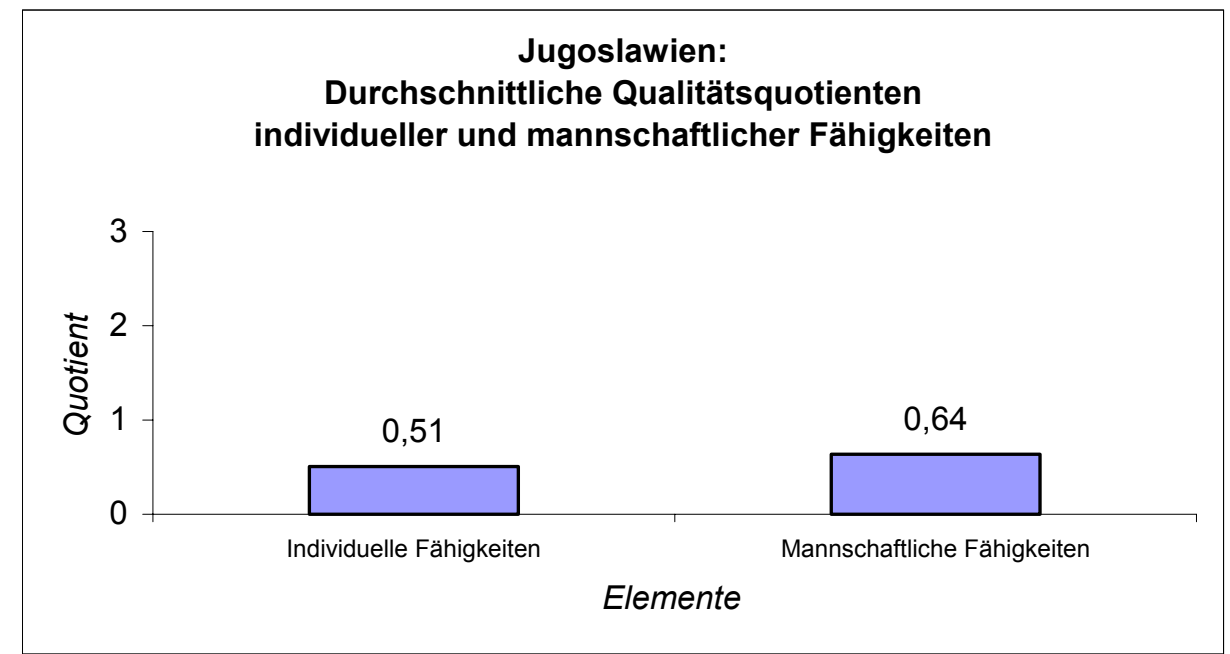

Diagramm 253 : Durchschnittliche Qualitätsquotienten individueller und mannschaftlicher Fähigkeiten von Jugoslawien.

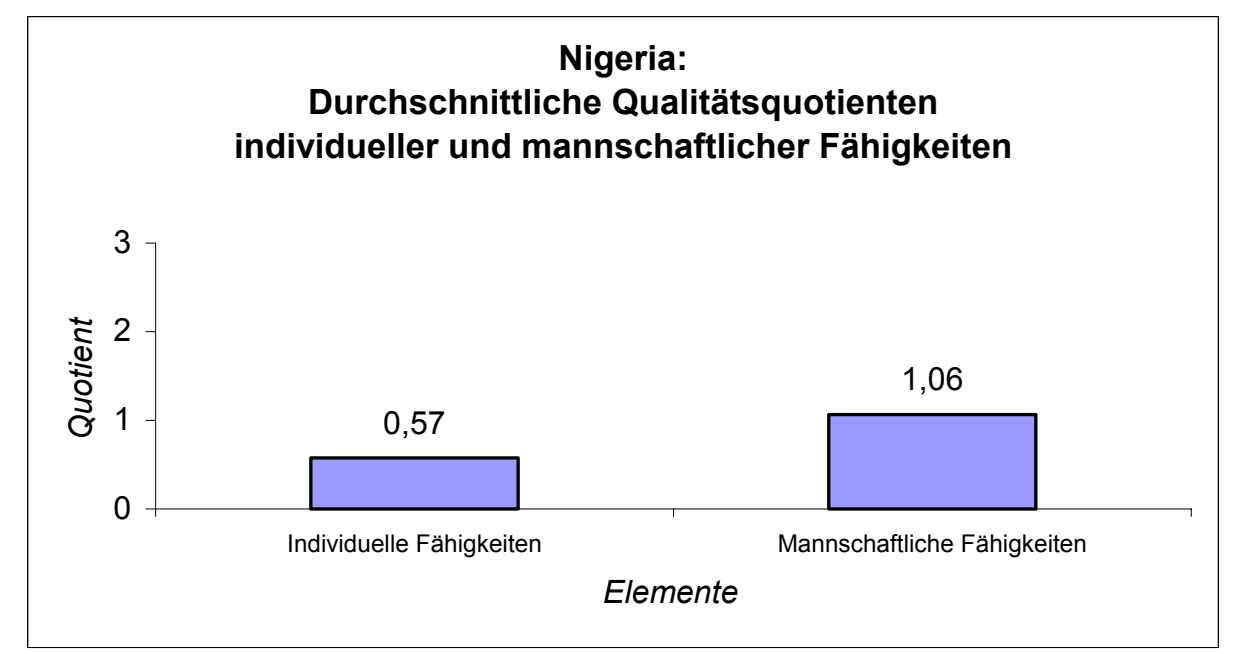

Diagramm 254 zeigt die durchschnittlichen Qualitätsquotienten der individuellen und mannschaftlichen Fähigkeiten von Nigeria.

Wie zu sehen ist, besitzt Nigeria bezüglich der individuellen Fähigkeiten einen durchschnittlichen Qualitätsquotienten von gerundet 0,57 , bezüglich der mannschaftlichen Fähigkeiten einen solchen von gerundet 1,06. 


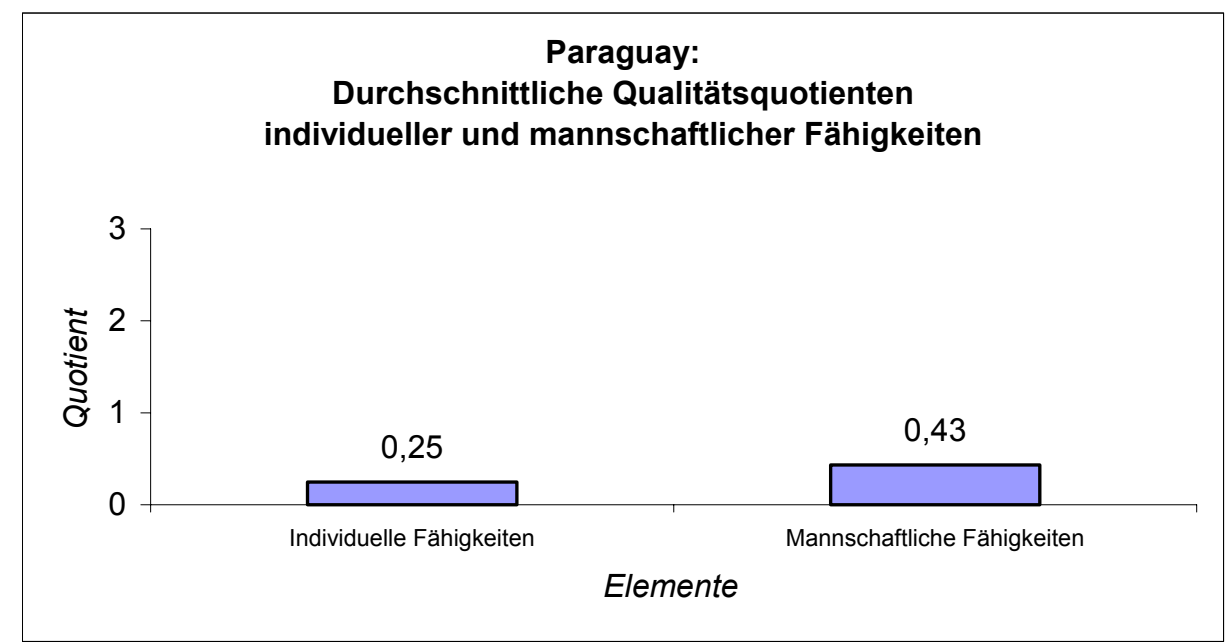

Diagramm 255 zeigt die durchschnittlichen Qualitätsquotienten der individuellen und mannschaftlichen Fähigkeiten von Paraguay.

Wie zu sehen ist, besitzt Paraguay bezüglich der individuellen Fähigkeiten einen durchschnittlichen Qualitätsquotienten von gerundet 0,25 , bezüglich der mannschaftlichen Fähigkeiten einen solchen von gerundet 0,43.

Diagramm 256 zeigt die durchschnittlichen Qualitätsquotienten der individuellen und mannschaftlichen Fähigkeiten von Rumänien. Wie zu sehen ist, besitzt Rumänien bezüglich der individuellen Fähigkeiten einen durchschnittlichen Qualitätsquotienten von gerundet 0,19 , bezüglich der mannschaftlichen Fähigkeiten einen solchen von gerundet 1,02 .

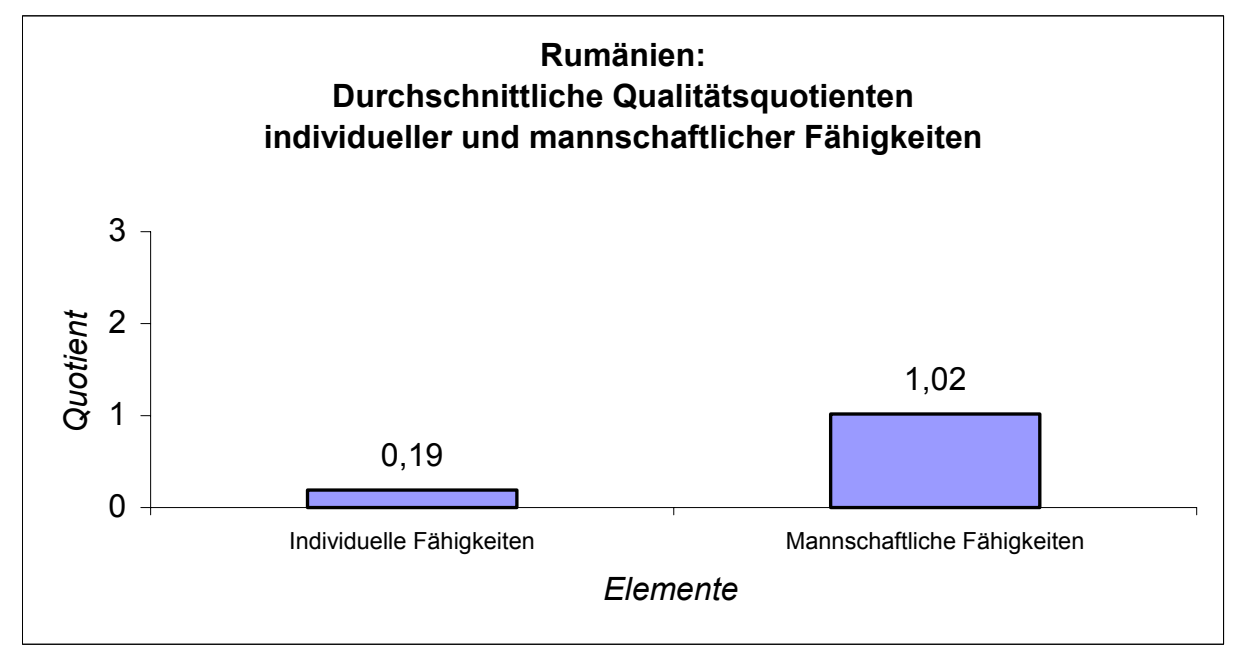

Diagramm 256: Durchschnittliche Qualitätsquotienten individueller und mannschaftlicher Fähigkeiten für Rumänien. 

Diagramm 259 zeigt die durchschnittlichen Qualitätsquotienten der individuellen und mannschaftlichen Fähigkeiten von Italien. Wie zu sehen ist, besitzt Italien bezüglich der individuellen Fähigkeiten einen durchschnittlichen Qualitätsquotienten von gerundet 0,71 , bezüglich der mannschaftlichen Fähigkeiten einen solchen von gerundet 2,50 .

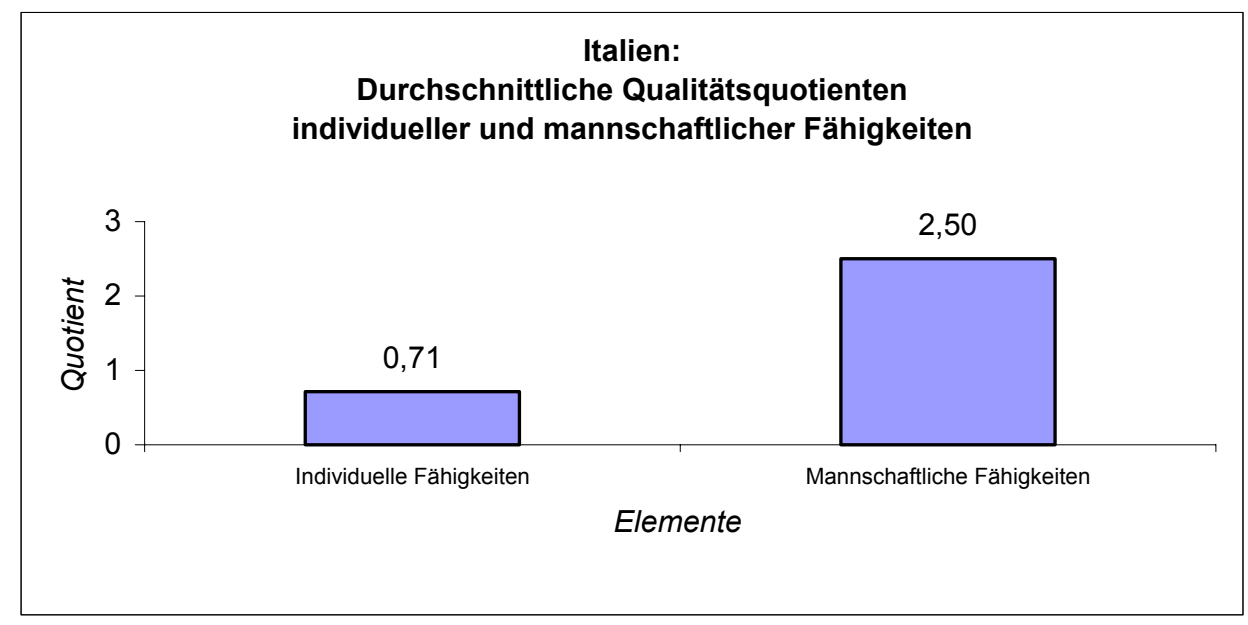

Diagramm 259 : Durchschnittliche Qualitätsquotienten individueller und mannschaftlicher Fähigkeiten für Italien.

Diagramm 260 zeigt die durchschnittlichen Qualitätsquotienten der individuellen und mannschaftlichen Fähigkeiten von Argentinien. Wie zu sehen ist, besitzt Argentinien bezüglich der individuellen Fähigkeiten einen durchschnittlichen Qualitätsquotienten von gerundet 0,33 , bezüglich der mannschaftlichen Fähigkeiten einen solchen von gerundet 1,16 .

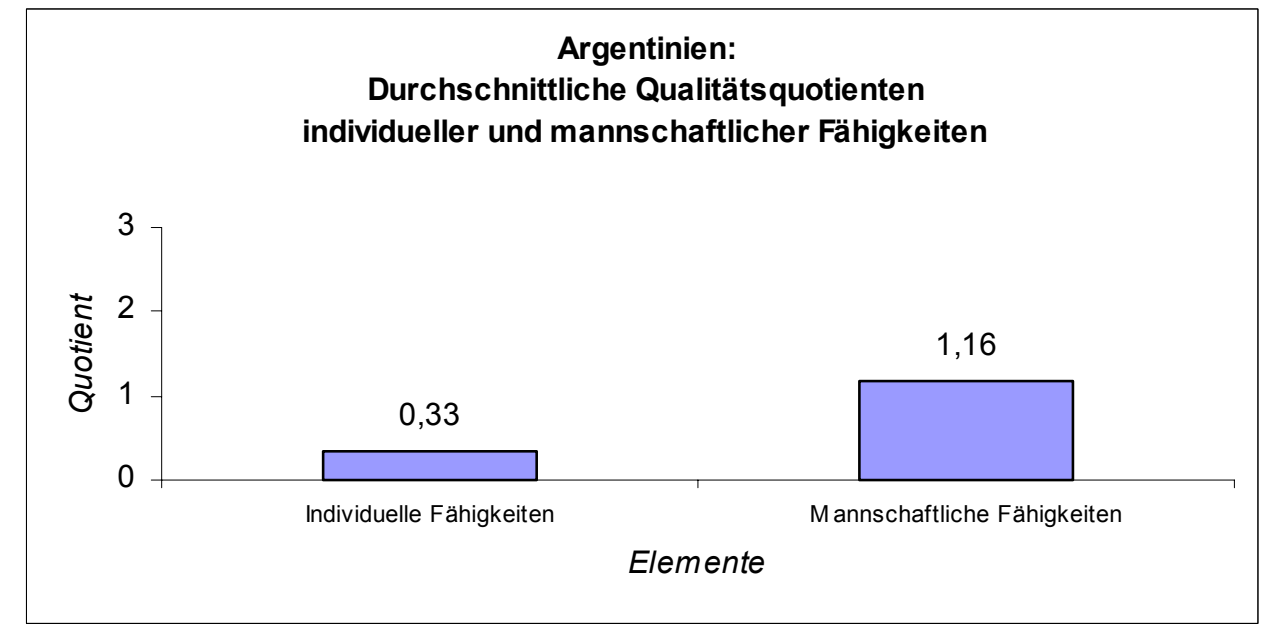

Diagramm 260 : Durchschnittliche Qualitätsquotienten individueller und mannschaftlicher Fähigkeiten für Italien. 
Diagramm 261 zeigt die durchschnittlichen Qualitätsquotienten der individuellen und mannschaftlichen Fähigkeiten von Dänemark. Wie zu sehen ist, besitzt Dänemark bezüglich der individuellen Fähigkeiten einen durchschnittlichen Qualitätsquotienten von gerundet 0,47 , bezüglich der mannschaftlichen Fähigkeiten einen solchen von gerundet 1,78 .

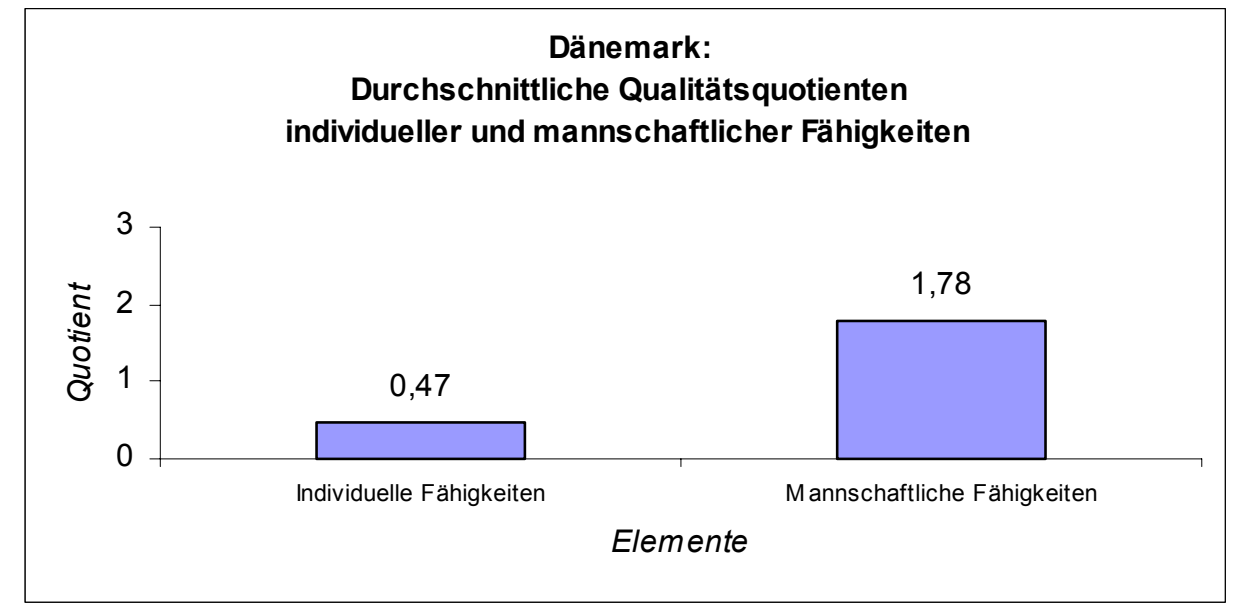

Diagramm 261 : Durchschnittliche Qualitätsquotienten individueller und mannschaftlicher Fähigkeiten für Dänemark.

\subsubsection{Halbfinalisten}

Diagramm 262 zeigt die durchschnittlichen Qualitätsquotienten individueller und mannschaftlicher Fähigkeiten von Frankreich. Wie zu sehen ist, besitzt Frankreich bezüglich der individuellen Fähigkeiten einen durchschnittlichen Qualitätsquotienten von gerundet 0,92 , bezüglich der mannschaftlichen Fähigkeiten einen solchen von gerundet 1,90 .

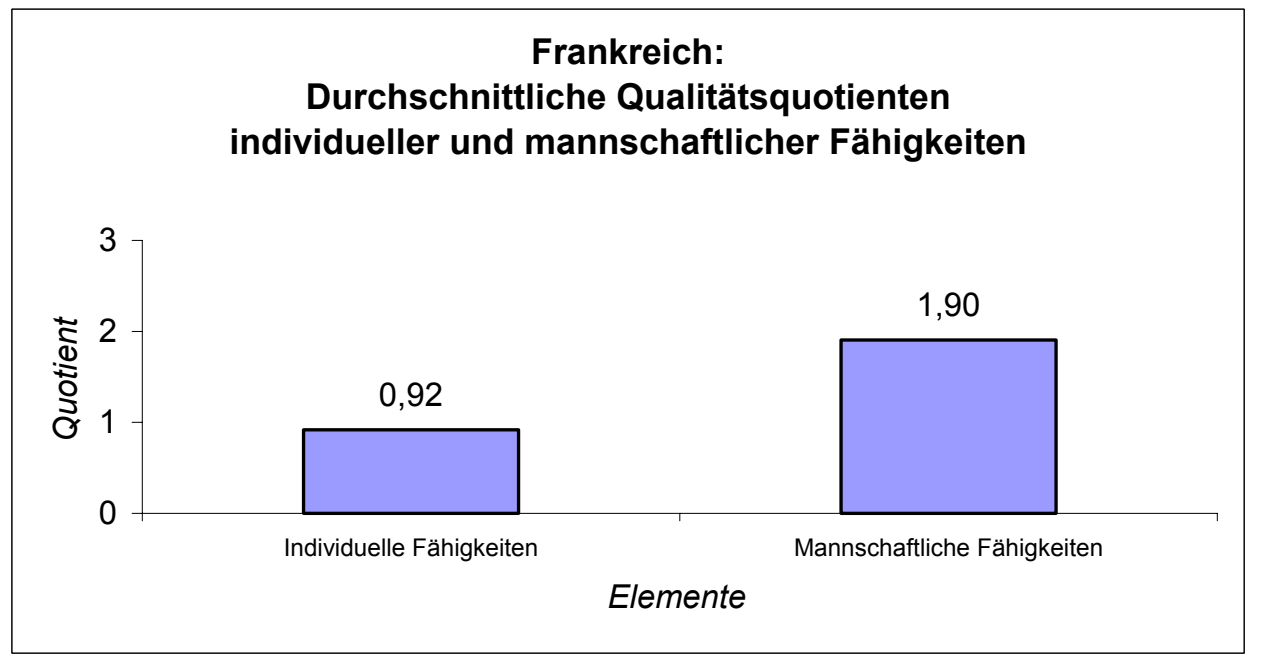

Diagramm 262 : Durchschnittliche Qualitätsquotienten individueller und mannschaftlicher Fähigkeiten für Frankreich. 
Wie zu sehen ist, besitzt Brasilien bezüglich der individuellen Fähigkeiten einen durchschnittlichen Qualitätsquotienten von gerundet 0,32, bezüglich der mannschaftlichen Fähigkeiten einen solchen von gerundet 1,76.

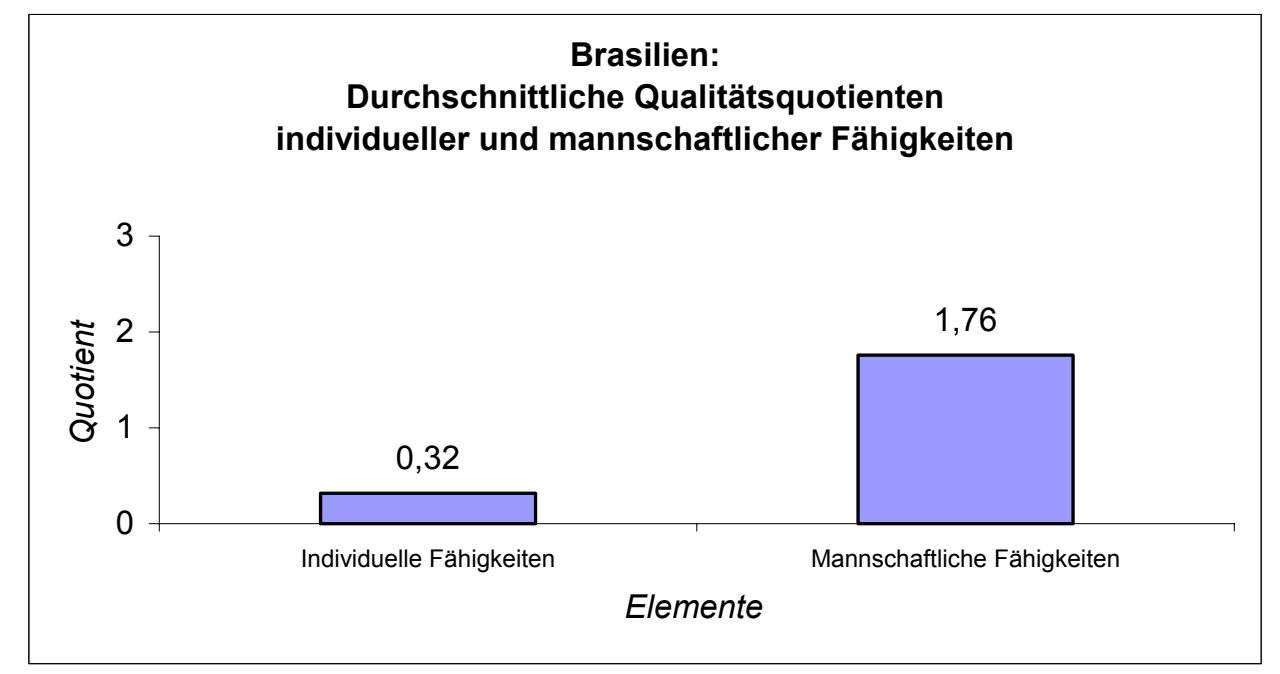

Diagramm 263 zeigt die durchschnittlichen Qualitätsquotienten der individuellen und mannschaftlichen Fähigkeiten von Brasilien.

Diagramm 264 zeigt die durchschnittlichen Qualitätsquotienten individueller und mannschaftlicher Fähigkeiten für Kroatien. Wie zu sehen ist, besitzt Kroatien bezüglich der individuellen Fähigkeiten einen durchschnittlichen Qualitätsquotienten von gerundet 0,42 , bezüglich der mannschaftlichen Fähigkeiten einen solchen von gerundet 1,27 .

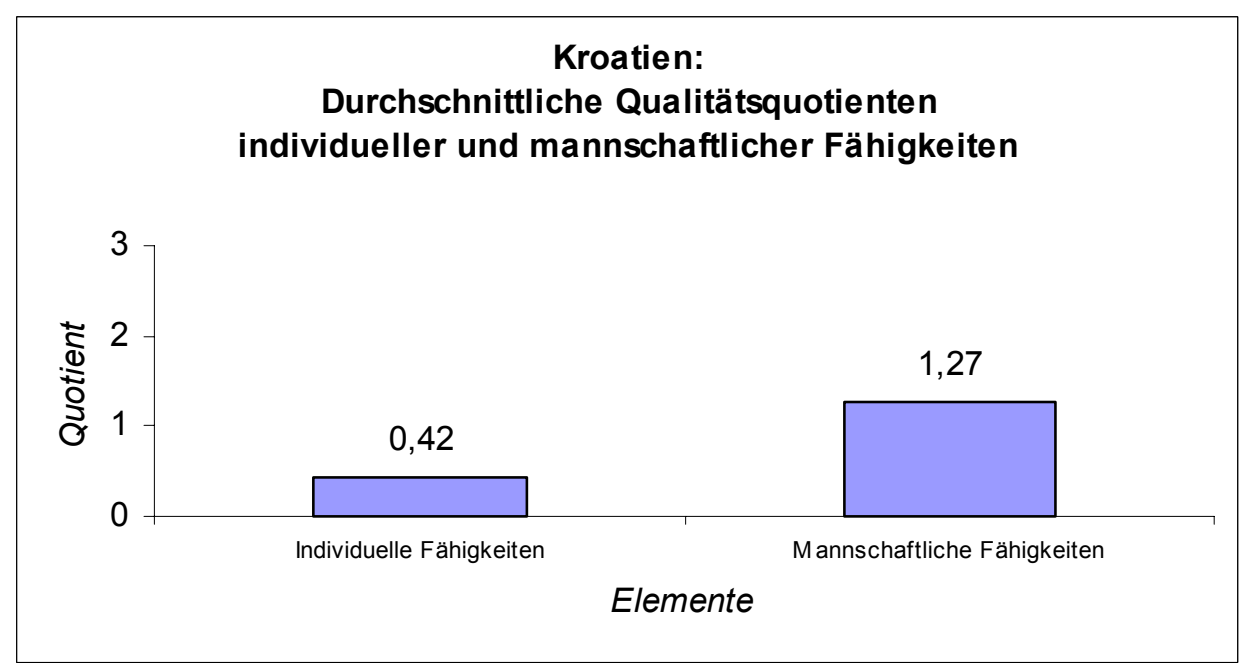

Diagramm 264 : Durchschnittliche Qualitätsquotienten individueller und mannschaftlichen Fähigkeiten für Kroatien. 
Diagramm 265 zeigt die durchschnittlichen Qualitätsquotienten der individuellen und mannschaftlichen Fähigkeiten für die Niederlande. Wie zu sehen ist, besitzen die Niederlande bezüglich der individuellen Fähigkeiten einen durchschnittlichen Qualitätsquotienten von gerundet 0,49 , bezüglich der mannschaftlichen Fähigkeiten einen solchen von gerundet 2,03.

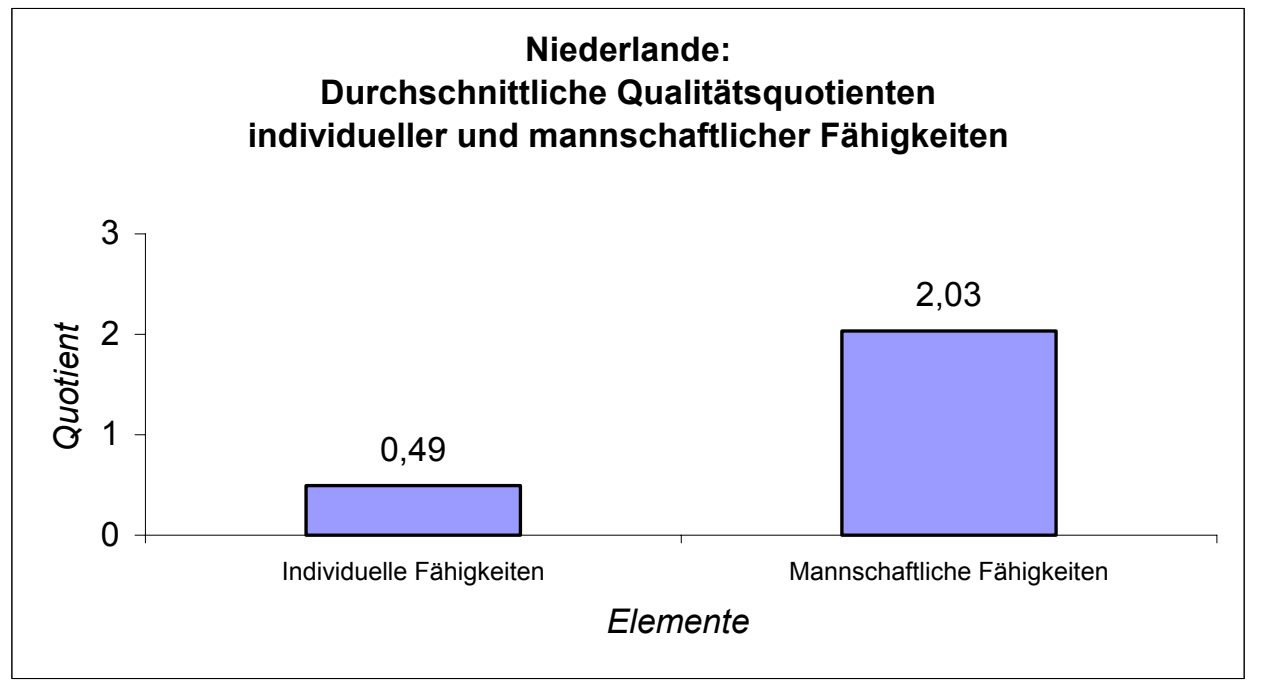

Diagramm 265 : Durchschnittliche Qualitätsquotienten individueller und mannschaftlichen Fähigkeiten für die Niederlande.

Aus dem Vergleich der vorangegangenen Diagramme können wir den Schluß ziehen, dass die mannschaftliche Fähigkeiten bei allen Mannschaften besser sind als die Individuellen Fähigkeiten, das Einzelspiel der französischen Mannschaft jedoch das Beste aller Mannschaften war.

\subsection{Technische, offensiv- und defensiv-taktische Fähigkeiten}

Im folgenden Kapitel werden durchschnittliche Qualitätsquotienten technischer, offensiv-taktischer und defensive-taktischer Fähigkeiten der sechzehn Achtelfinalteilnehmer beschrieben.

Der Qualitätsquotient einer bestimmten Mannschaft bezüglich eines einzelnen Elements ergibt sich, indem man (ungeachtet der „normalen“ Aktionen) das Verhältnis der Anzahl "guter“ und „schwacher“ Aktionen dieser Mannschaft bezüglich dieses Elements berechnet. Der Qualitätsquotient ist demnach eine Zahl, die zwischen „Null“ und „Unendlich“ liegt, wobei eine Zahl nahe „Null“ auf eine geringe, eine Zahl hohe auf eine (sehr) hohe Qualität hindeutet.

Der Mittelwert aller Qualitätsquotienten einer Mannschaft von Elementen, die den technischen, offensiv-taktischen bzw. defensiv-taktischen Fähigkeiten zugeordnet 
sind, ergibt den durchschnittlichen Qualitätsquotienten dieser Mannschaft bezüglich dieser Fähigkeit.

Folgende Elemente wurden den technischen Fähigkeiten zugeordnet:

- Dribbling

- Körpertäuschung

- Torschuss

- kurze Pässe

- lange Pässe

- Ballkontrolle

Folgende Elemente wurden den offensiven-taktischen Fähigkeiten zugeordnet:

- Freistoss

- Eckball

- Unterstützen

- Doppelpass

- Raumaufteilung

- Nachrücken

- in die Breite ziehen

Folgende Elemente wurden den defensiven-taktischen Fähigkeiten zugeordnet:

- Pressing hinten

- Pressing Mitte

- Pressing vorne

- Raumaufteilung (defensiv)

- Zurückkommen (defensiv)

- Unterstützen

- Rückraum sichern

- Abseitsfalle.

\subsubsection{Achtelfinalisten}

Diagramm 266 zeigt die durchschnittlichen Qualitätsquotienten technischen, offensiven-taktischen und defensiven-taktischen Fähigkeiten von England. Wie zu sehen ist, besitzt England bezüglich der technischen Fähigkeiten einen durchschnittlichen Qualitätsquotienten von gerundet 0,51 , bezüglich der offensiv-taktischen Fähigkeiten einen durchschnittlichen Qualitätsquotienten von gerundet 0,84 und bezüglich der defensiv-taktischen Fähigkeiten einen solchen von gerundet 2,87. 


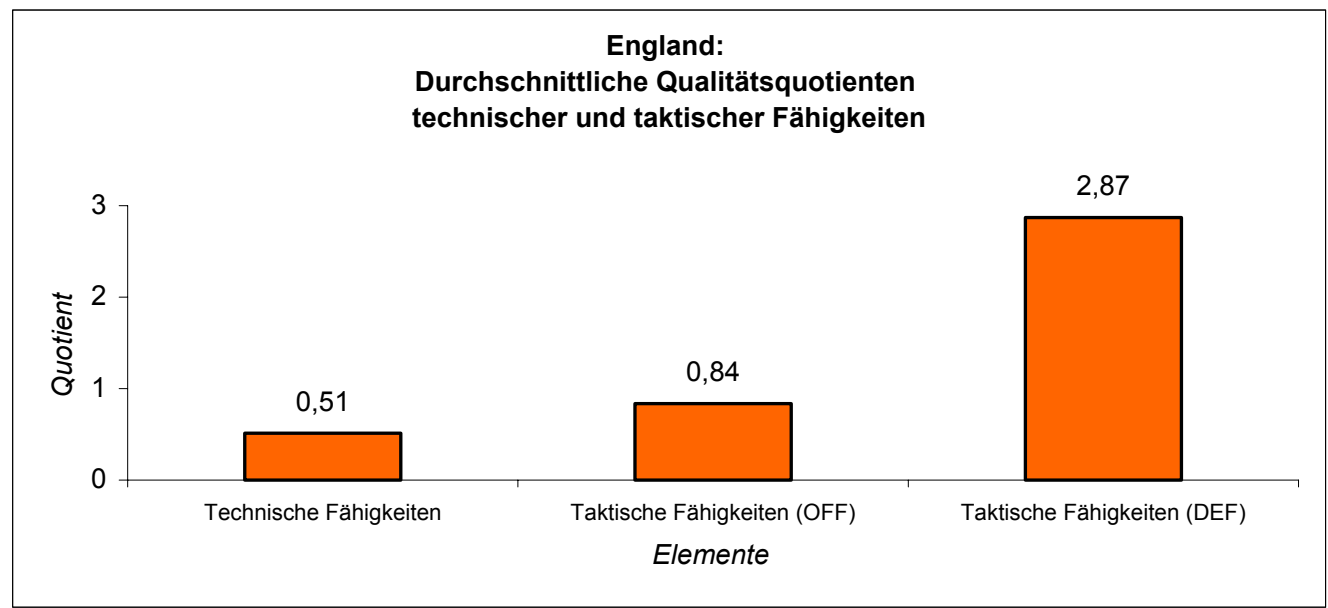

Diagramm 266: Durchschnittliche Qualitätsquotienten technischer und taktischer Fähigkeiten für England.

Aus dem Diagramm ergibt sich, dass die englische Mannschaft in den defensiven Taktiken besser ist als sowohl in den offensiven Taktik wie auch den übrigen technischen Fähigkeiten. Der Grund dürfte auch darin zu suchen sein, dass die englische Mannschaft nach einem Platzverweis ein Abwehrspiel spielte.

Diagramm 267 zeigt die durchschnittlichen Qualitätsquotienten technischen, offensiven-taktischen und defensiven-taktischen Fähigkeiten von Chile. Wie zu sehen ist, besitzt Chile bezüglich der technischen Fähigkeiten einen durchschnittlichen Qualitätsquotienten von gerundet 0,11 , bezüglich der offensiv-taktischen Fähigkeiten einen durchschnittlichen Qualitätsquotienten von gerundet 0,52 und bezüglich der defensiv-taktischen Fähigkeiten einen solchen von gerundet 0,30.

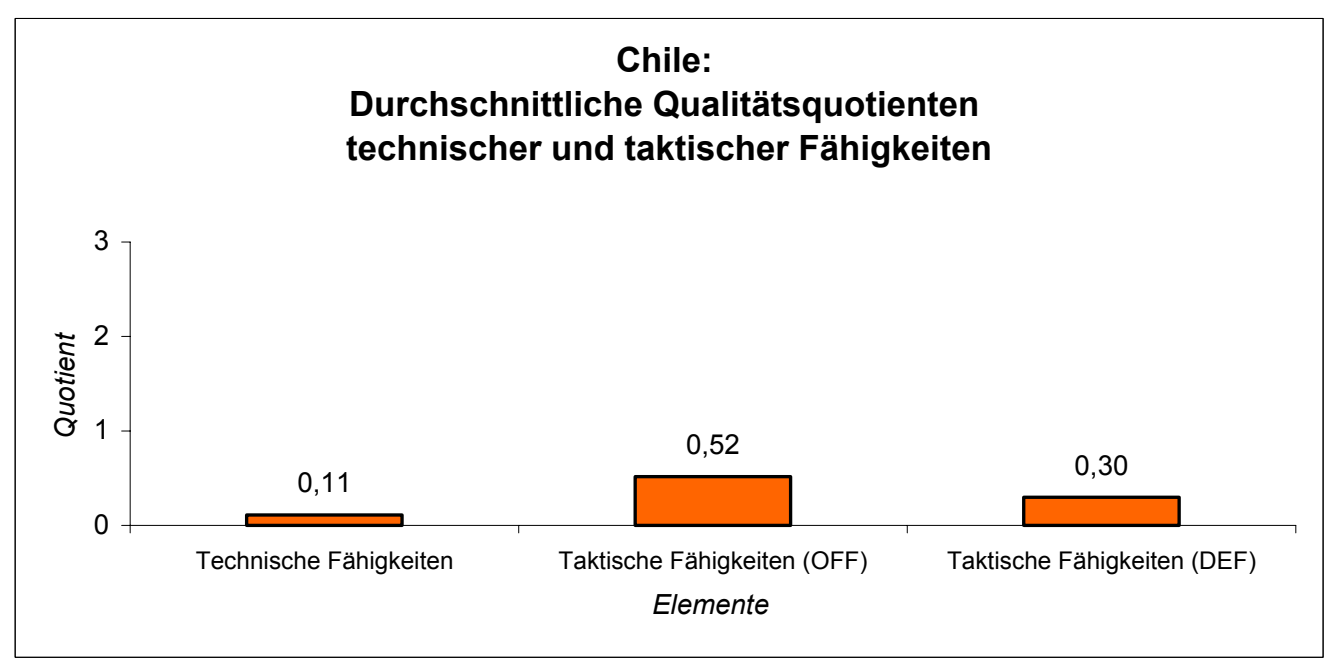

Diagramm 267: Durchschnittliche Qualitätsquotienten technischer und taktischer Fähigkeiten für Chile. 
Bei der chilenischen Mannschaft erkennt man bei der Betrachtung der Diagramme, dass ihr Angriffsspiel stärker als ihr Verteidigungsspiel und ihre spielerischen Techniken ist, was wir als vorteilhaft ansehen. Doch führt die Vernachlässigung der Verteidigung leicht zur Niederlage.

Diagramm 268 zeigt die durchschnittlichen Qualitätsquotienten technischen, offensiven-taktischen und defensiven-taktischen Fähigkeiten von Mexiko. Wie zu sehen ist, besitzt Mexiko bezüglich der technischen Fähigkeiten einen durchschnittlichen Qualitätsquotienten von gerundet 0,19 , bezüglich der offensiv-taktischen Fähigkeiten einen durchschnittlichen Qualitätsquotienten von gerundet 0,29 und bezüglich der defensiv-taktischen Fähigkeiten einen solchen von gerundet 0,68.

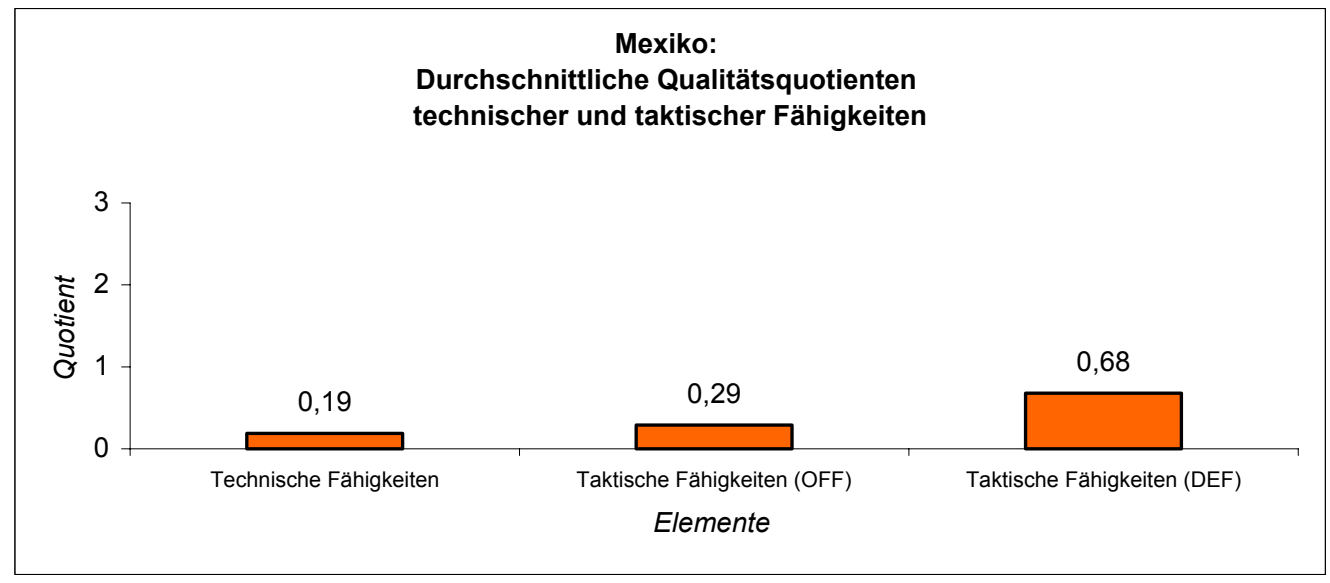

Diagramm 268: Durchschnittliche Qualitätsquotienten technischer und taktischer Fähigkeiten für Mexiko.

Aus der Betrachtung lässt sich sagen, dass die Mannschaft im Verteidigungsspiel viel engagierter als im Angriffsspiel war. Die Niederlage der mexikanischen Mannschaft im Achtelfinale war das Ergebnis mangelnden sportlichen Talents und damit einhergehender geringen fußballerischen Techniken.

Diagramm 269 zeigt die durchschnittlichen Qualitätsquotienten technischen, offensiven-taktischen und defensiven-taktischen Fähigkeiten von Jugoslawien. Wie zu sehen ist, besitzt Jugoslawien bezüglich der technischen Fähigkeiten einen durchschnittlichen Qualitätsquotienten von gerundet 0,21 , bezüglich der offensiv - taktischen Fähigkeiten einen durchschnittlichen Qualitätsquotienten von gerundet 0,40 und bezüglich der defensiv-taktischen Fähigkeiten einen solchen von gerundet 0,99. 


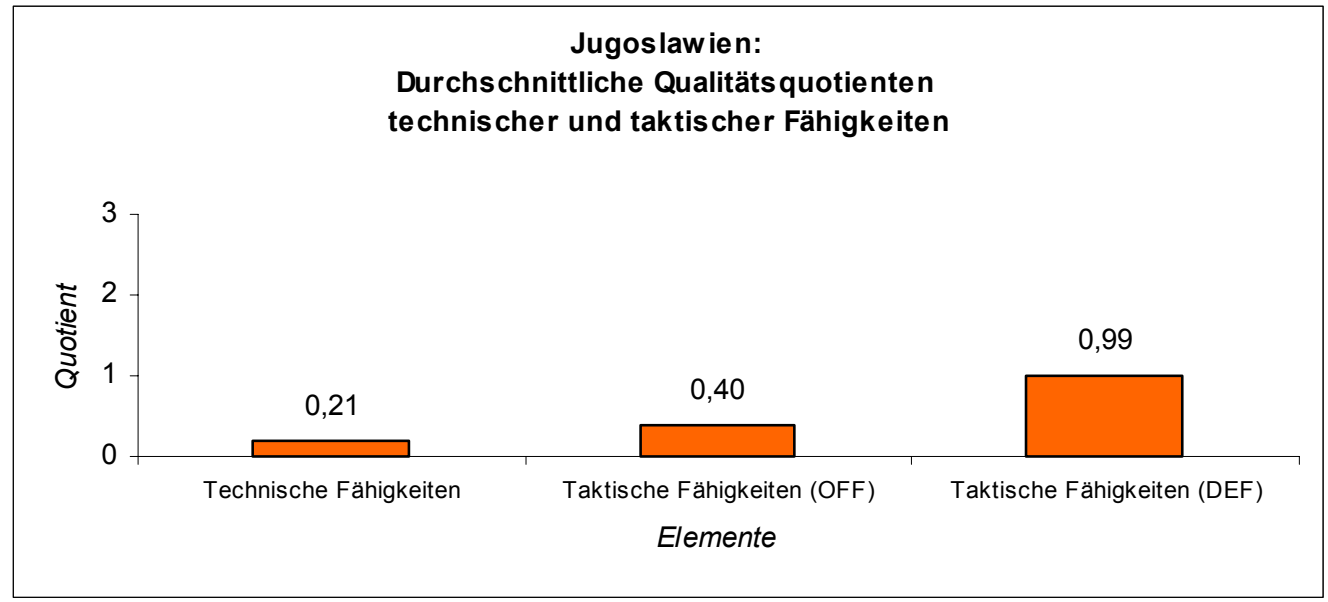

Diagramm 269: Durchschnittliche Qualitätsquotienten technischer und taktischer Fähigkeiten für Jugoslawien.

Aus dem Diagramm Nr. 269 wird deutlich, dass die Verteidigungstaktik bei der jugoslawischen Mannschaft besser ist als die Angriffstaktik und die Technik. Daraus lässt sich folgern, dass sich die jugoslawische Mannschaft mehr um die Verteidigung als um das Angriffsspiel gekümmert hat, was daran liegt, dass ihr Verteidigungsspiel stärker als ihr Angriffsspiel und ihre spielerischen Techniken ist.

Diagramm 270 zeigt die durchschnittlichen Qualitätsquotienten technischen, offensiven-taktischen und defensive-taktischen Fähigkeiten von Nigeria. Wie zu sehen ist, besitzt Nigeria bezüglich der technischen Fähigkeiten einen durchschnittlichen Qualitätsquotienten von gerundet 0,42 , bezüglich der offensiv-taktischen Fähigkeiten einen durchschnittlichen Qualitätsquotienten von gerundet 0,56 und bezüglich der defensiv-taktischen Fähigkeiten einen solchen von gerundet 1,32.

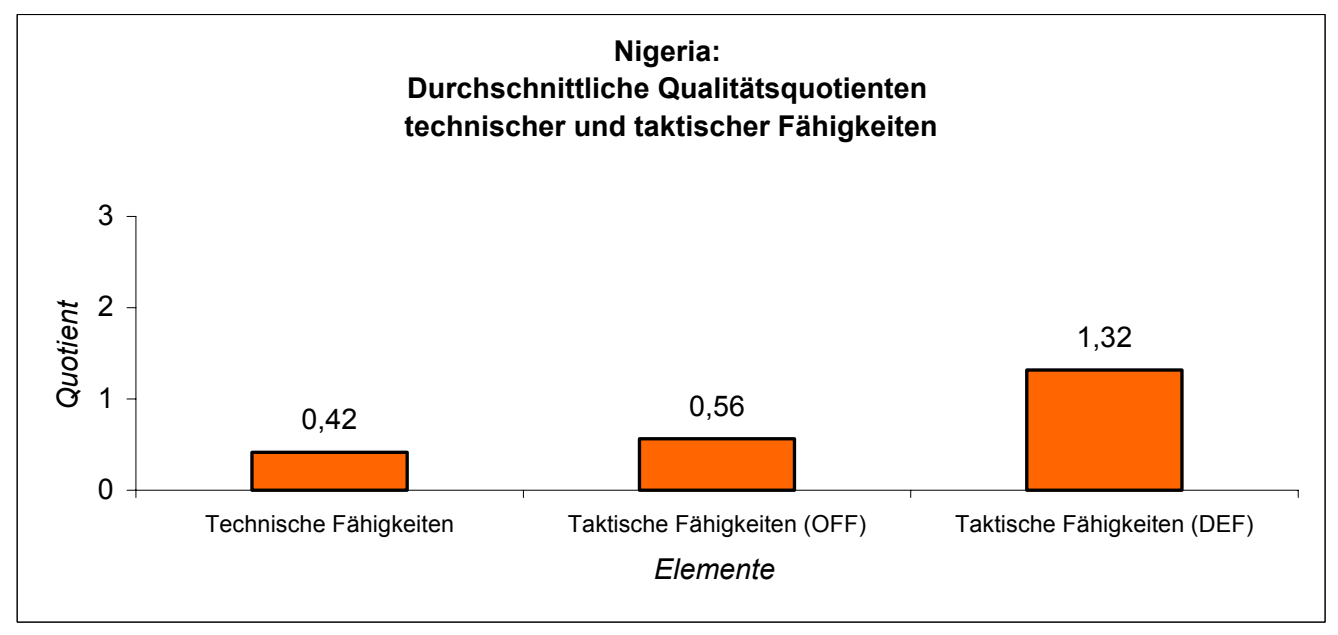

Diagramm 270: Durchschnittliche Qualitätsquotienten technischer und taktischer Fähigkeiten für Nigeria. 
Aus der Betrachtung lässt sich sagen, dass die Mannschaft im Verteidigungsspiel viel engagierter als im Angriffsspiel war. Der Grund liegt wahrscheinlich darin, dass die nigerianische Mannschaft im Achtelfinale sich überwiegend auf das Verteidigungsspiel stützte, und weniger versuchte auf Angriff zu spielen. Das führt dazu, dass weniger Angreifer vorne bleiben, so dass die offensiven taktischen Fähigkeiten seltener als hochkarätig bewertet werden konnten.

Diagramm 271 zeigt die durchschnittlichen Qualitätsquotienten technischen, offensiven - taktischen und defensiven - taktischen Fähigkeiten von Paraguay. Wie zu sehen ist, besitzt Paraguay bezüglich der technischen Fähigkeiten einen durchschnittlichen Qualitätsquotienten von gerundet 0,09, bezüglich der offensiv - taktischen Fähigkeiten einen durchschnittlichen Qualitätsquotienten von gerundet 0,23 und bezüglich der defensiv - taktischen Fähigkeiten einen solchen von gerundet 0,61.

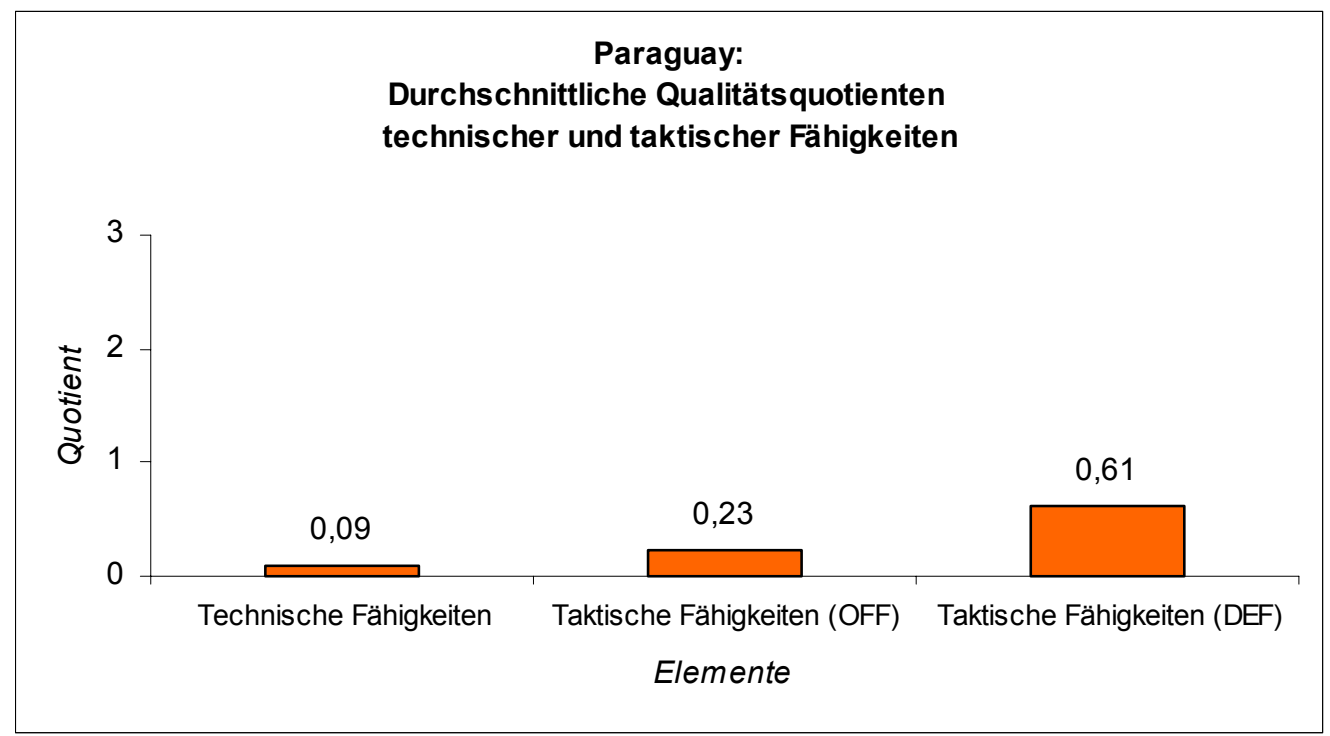

Diagramm 271: Durchschnittliche Qualitätsquotienten technischer und taktischer Fähigkeiten für Paraguay.

Aus der Betrachtung lässt sich sagen, dass der Mannschaft im Verteidigungsspiel viel engagierter als im Angriffsspiel war. Die Niederlage der paraguayischen Mannschaft im Achtelfinale war das Ergebnis mangelnden sportlichen Talents und damit einhergehender geringen fußballerischen Techniken. 
Diagramm 272 zeigt die durchschnittlichen Qualitätsquotienten technischen, offensiven - taktischen und defensiven - taktischen Fähigkeiten von Rumänien. Wie zu sehen ist, besitzt Rumänien bezüglich der technischen Fähigkeiten einen durchschnittlichen Qualitätsquotienten von gerundet 0,07, bezüglich der offensiv - taktischen Fähigkeiten einen durchschnittlichen Qualitätsquotienten von gerundet 0,25 und bezüglich der defensiv - taktischen Fähigkeiten einen solchen von gerundet 1,26.

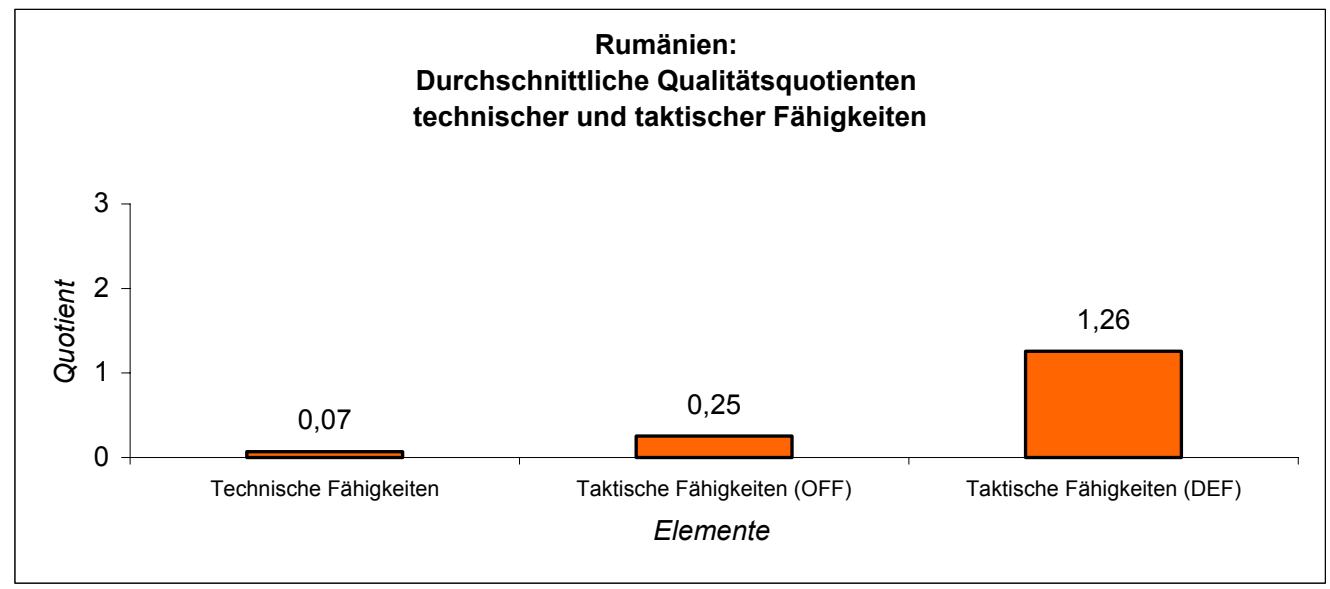

Diagramm 272: Durchschnittliche Qualitätsquotienten technischer und taktischer Fähigkeiten für Rumänien.

Bei der rumänischen Mannschaft erkennt man bei der Betrachtung der Diagramme, dass ihr Verteidigungsspiel stärker als ihr Angriffsspiel und ihre spielerischen Techniken ist, was wir als vorteilhaft ansehen. Doch führt die Vernachlässigung der technischen und offensiv-taktischen Maßnahmen leicht zur Niederlage.

Diagramm 273 zeigt die durchschnittlichen Qualitätsquotienten technischen, offensiven - taktischen und defensiven - taktischen Fähigkeiten von Norwegen. Wie zu sehen ist, besitzt Norwegen bezüglich der technischen Fähigkeiten einen durchschnittlichen Qualitätsquotienten von gerundet 0,24, bezüglich der offensiv - taktischen Fähigkeiten einen durchschnittlichen Qualitätsquotienten von gerundet 0,31 und bezüglich der defensiv - taktischen Fähigkeiten einen solchen von gerundet 1,08. 


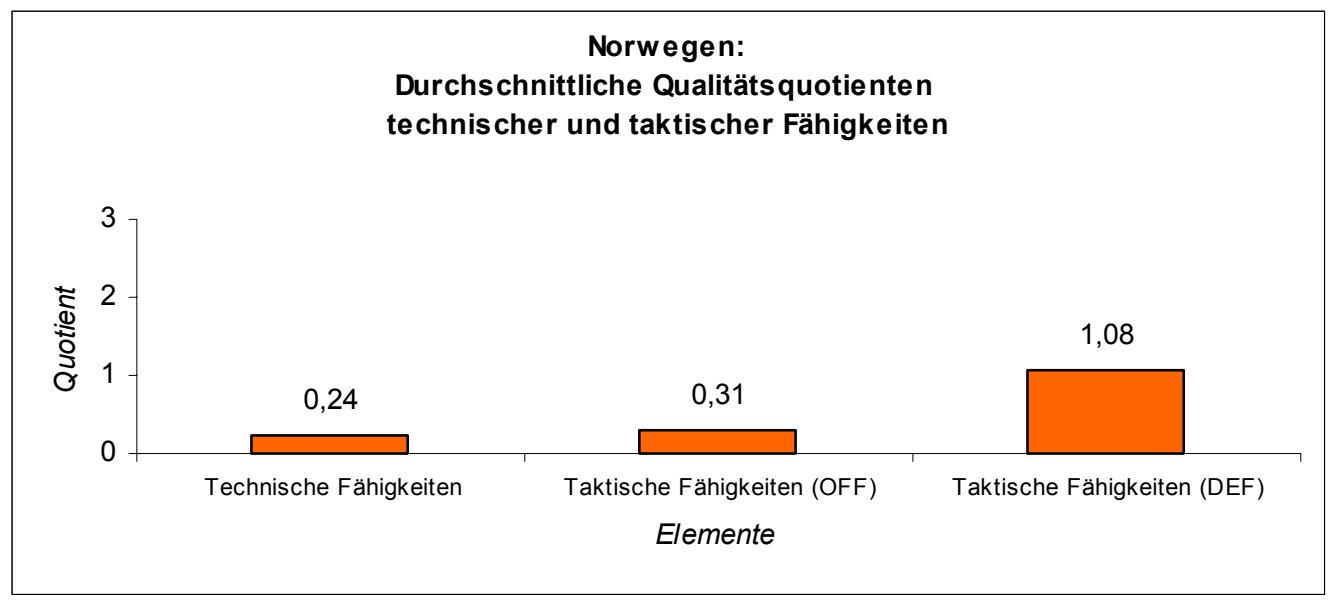

Diagramm 273: Durchschnittliche Qualitätsquotienten technischer und taktischer Fähigkeiten für Norwegen.

Aus dem Diagramm wird deutlich, dass die Verteidigungstaktik bei norwegischer Mannschaft besser ist als die Angriffstaktik und Technik. Daraus lässt sich folgern, dass sich die norwegische Mannschaft mehr um die Verteidigung als um das Angriffsspiel gekümmert hat.

Die Auswertung der Diagramme lässt den Schluss zu, dass die meisten Mannschaften im Achtelfinale bessere Verteidigungs- als Angriffs- und andere spielerischen Fähigkeiten haben. Wir nehmen an, dass die Trainer dieser Mannschaften aus Furcht vor dem Ausscheiden den Schwerpunkt auf die Verteidigung legen, besonders in den letzten Runden des Ausscheidungskampfes ab dem Achtelfinale. Ein anderer Grund könnte sein, dass diese Mannschaften keine hervorragenden Angriffs haben.

\subsubsection{Viertelfinalisten}

Diagramm 274 zeigt die durchschnittlichen Qualitätsquotienten technischen, offensiven - taktischen und defensiven - taktischen Fähigkeiten von Deutschland. Wie zu sehen ist, besitzt Deutschland bezüglich der technischen Fähigkeiten einen durchschnittlichen Qualitätsquotienten von gerundet 0,10, bezüglich der offensiv - taktischen Fähigkeiten einen durchschnittlichen Qualitätsquotienten von gerundet 0,27 und bezüglich der defensiv - taktischen Fähigkeiten einen solchen von gerundet 1,10 . 


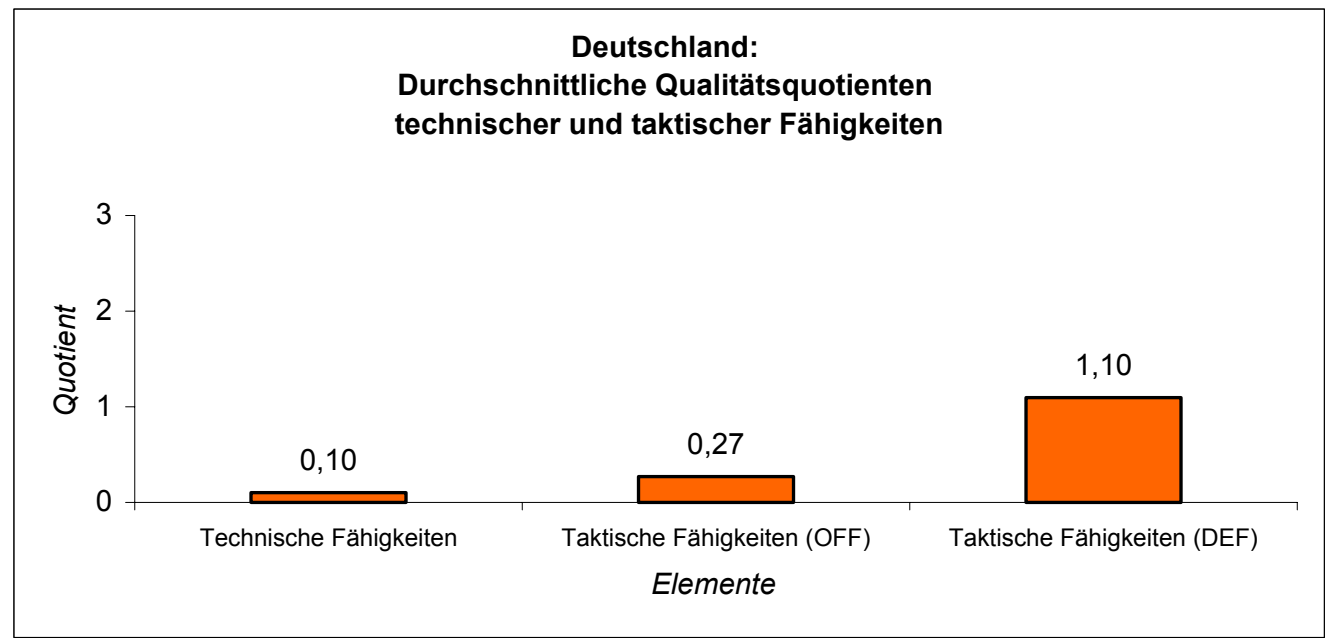

Diagramm 274: Durchschnittliche Qualitätsquotienten technischer und taktischer Fähigkeiten für Deutschland.

Aus dem Diagramm ergibt sich, dass die deutsche Mannschaft in der Defensiv-Taktik besser ist als sowohl in der Offensiv-Taktik wie auch den übrigen Technischen Fähigkeiten. Der Grund dürfte darin zu suchen sein, dass die deutsche Mannschaft nach einem Platzverweis das Abwehrspiel nicht genug gesichert hat.

Diagramm 275 zeigt die durchschnittlichen Qualitätsquotienten technischen, offensiven - taktischen und defensiven - taktischen Fähigkeiten von Italien. Wie zu sehen ist, besitzt Italien bezüglich der technischen Fähigkeiten einen durchschnittlichen Qualitätsquotienten von gerundet 0,43 , bezüglich der offensiv - taktischen Fähigkeiten einen durchschnittlichen Qualitätsquotienten von gerundet 0,96 und bezüglich der defensiv - taktischen Fähigkeiten einen solchen von gerundet 2,94.

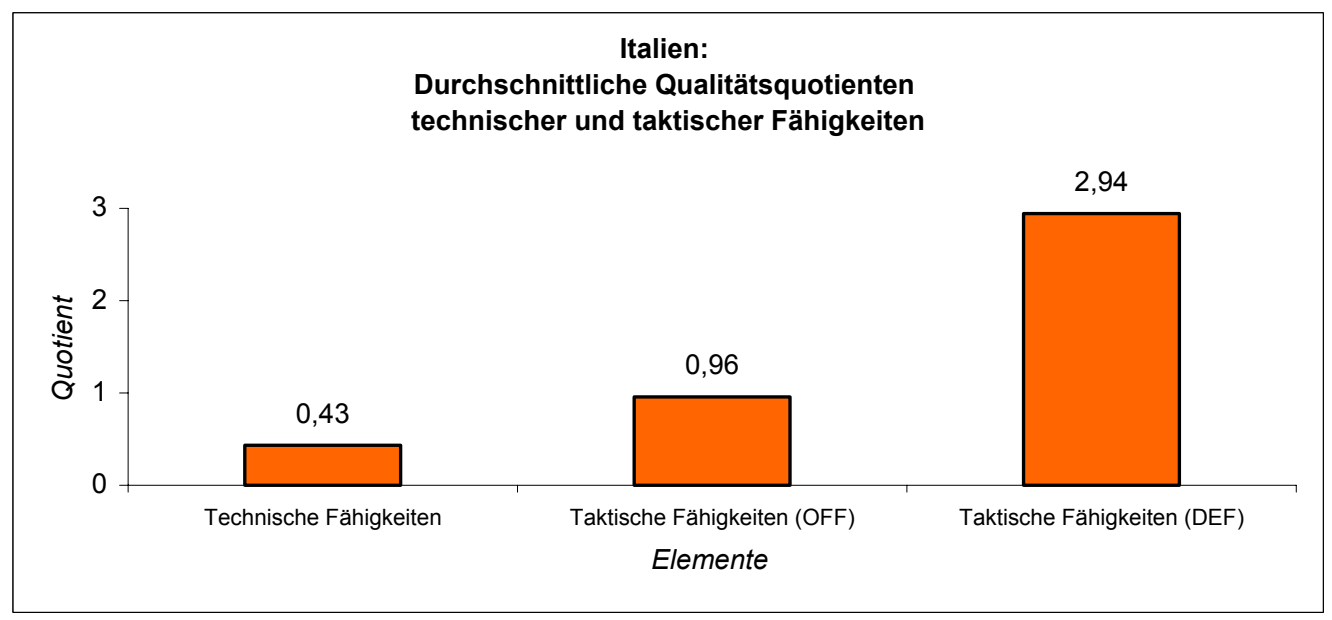

Diagramm 275: Durchschnittliche Qualitätsquotienten technischer und taktischer Fähigkeiten für Italien. 
Aus dem Diagramm ist ersichtlich, dass die Verteidigungsfähigkeit und offensivtaktischen Fähigkeiten der italienischen Mannschaft besser ist als ihre technischen Fähigkeiten. Dies deutet darauf hin, dass die Mannschaft mit dem besten Verteidigungsspiel auch mit gute offensiv-taktische Möglichkeiten besitzen kann. Allerdings konnte Italien den Wettkampf nicht gewinnen, sondern verlor im Elfmeterschiessen.

Diagramm 276 zeigt die durchschnittlichen Qualitätsquotienten technischen, offensiven - taktischen und defensiven - taktischen Fähigkeiten von Argentinien. Wie zu sehen ist, besitzt Argentinien bezüglich der technischen Fähigkeiten einen durchschnittlichen Qualitätsquotienten von gerundet 0,15 , bezüglich der offensiv - taktischen Fähigkeiten einen durchschnittlichen Qualitätsquotienten von gerundet 0,46 und bezüglich der defensiv - taktischen Fähigkeiten einen solchen von gerundet 1,39 .

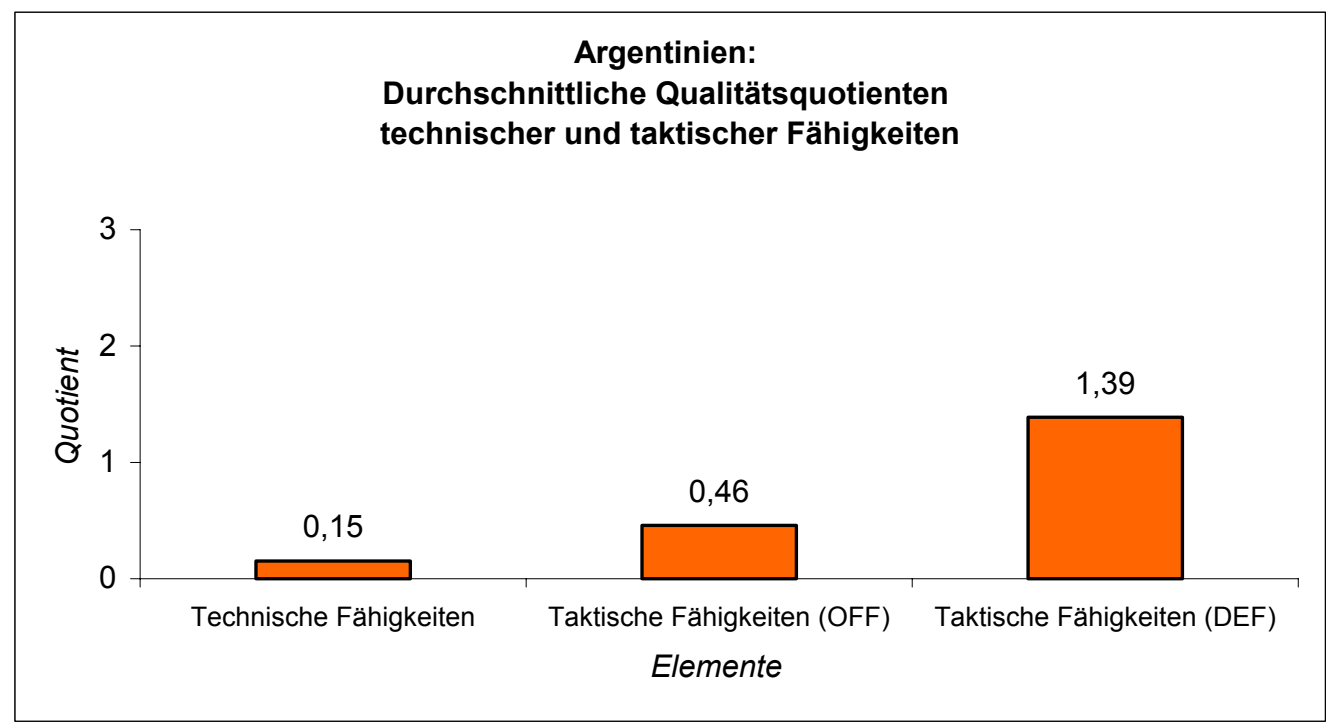

Diagramm 276: Durchschnittliche Qualitätsquotienten technischer und taktischer Fähigkeiten für Argentinien.

Aus dem Diagramm Nr. 276 wird deutlich, dass die Verteidigungstaktik bei der argentinischer Mannschaft besser ist als die Angriffstaktik und Technik. Daraus lässt sich folgern, dass sich die argentinische Mannschaft mehr um die Verteidigung als um das Angriffsspiel gekümmert hat, was daran liegt, dass ihr Verteidigungsspiel stärker als ihr Angriffsspiel und ihre spielerischen Techniken ist. 
Diagramm 277 zeigt die durchschnittlichen Qualitätsquotienten technischen, offensiv-taktischen und defensiv-taktischen Fähigkeiten von Dänemark. Wie zu sehen ist, besitzt Dänemark bezüglich der technischen Fähigkeiten einen durchschnittlichen Qualitätsquotienten von gerundet 0,23 , bezüglich der offensiv-taktischen Fähigkeiten einen durchschnittlichen Qualitätsquotienten von gerundet 0,47 und bezüglich der defensiv-taktischen Fähigkeiten einen solchen von gerundet 2,28.

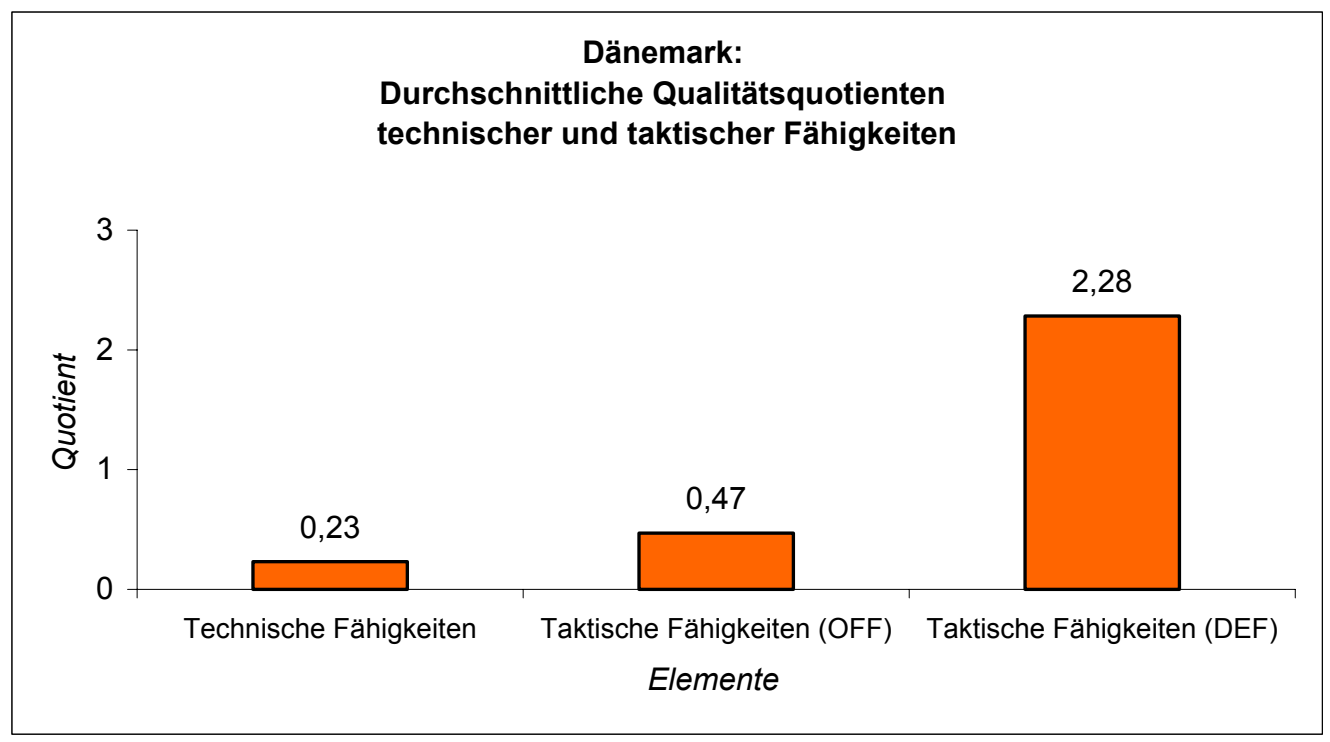

Diagramm 277: Durchschnittliche Qualitätsquotienten technischer und taktischer Fähigkeiten für Dänemark.

Aus der Betrachtung lässt sich sagen, dass die Mannschaft im Verteidigungsspiel viel engagierter als im Angriffsspiel war. Der Grund liegt wahrscheinlich darin, dass die dänische Mannschaft im Halbfinale sich überwiegend auf das Verteidigungsspiel stützte, und weniger versuchte auf Angriff zu spielen. Das führt dazu, dass weniger Angreifer vorne blieben.

\subsubsection{Halbfinalisten}

Diagramm 278 zeigt die durchschnittlichen Qualitätsquotienten technischen, offensiv-taktischen und defensiv-taktischen Fähigkeiten von Frankreich. Wie zu sehen ist, besitzt Frankreich bezüglich der technischen Fähigkeiten einen durchschnittlichen Qualitätsquotienten von gerundet 0,55, bezüglich der offensiv-taktischen Fähigkeiten einen durchschnittlichen Qualitätsquotienten von gerundet 0,49 und bezüglich der defensiv-taktischen Fähigkeiten einen solchen von gerundet 2,80. 


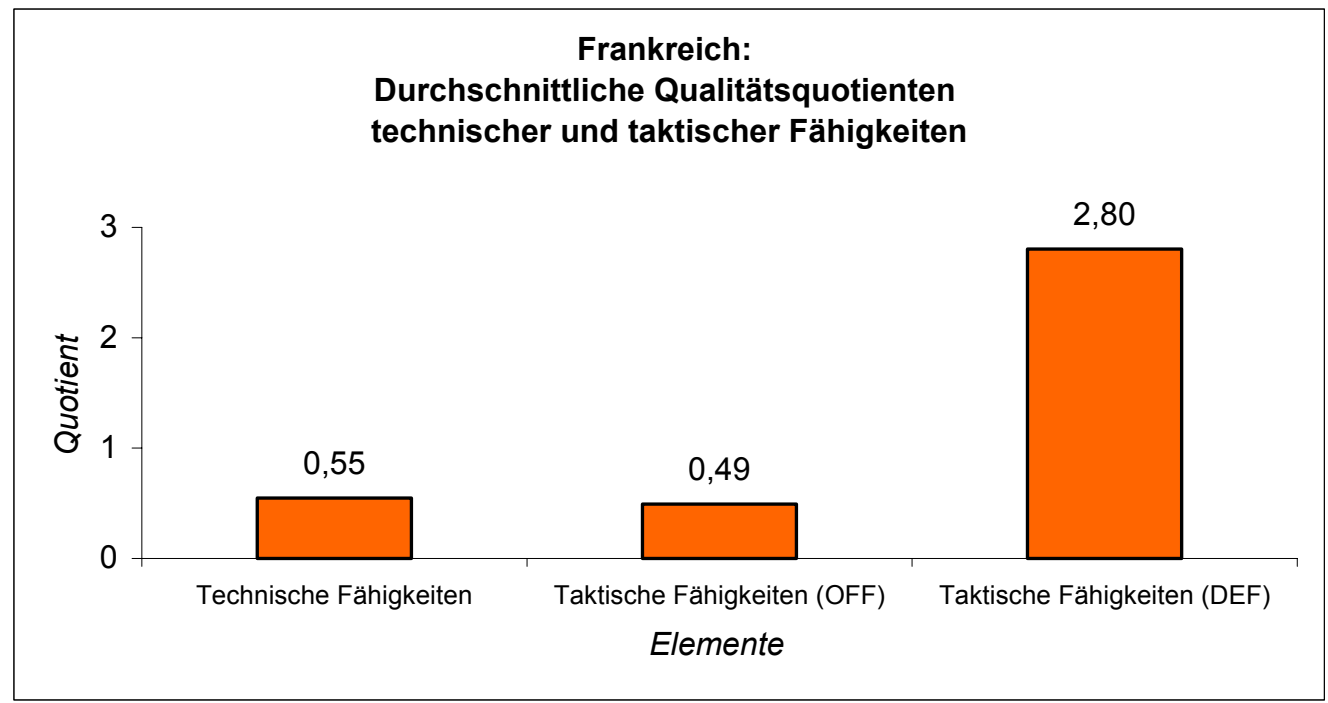

Diagramm 278: Durchschnittliche Qualitätsquotienten technischer und taktischer Fähigkeiten für Frankreich.

Aus dem Diagramm ist ersichtlich, dass die Verteidigungsfähigkeit und technischen Fähigkeiten der französischen Mannschaft besser als Offensiv- taktischen sind, was augenscheinlich besser als die der anderen Mannschaften ist. Dies deutet darauf hin, dass die Mannschaft mit dem besten Verteidigungsspiel auch die Spieler mit den besten technischen Fähigkeiten besitzt, womit sie den Wettkampf gewinnen konnte.

Diagramm 279 zeigt die durchschnittlichen Qualitätsquotienten technischen, offensiv-taktischen und defensiv-taktischen Fähigkeiten von Brasilien. Wie zu sehen ist, besitzt Brasilien bezüglich der technischen Fähigkeiten einen durchschnittlichen Qualitätsquotienten von gerundet 0,17 , bezüglich der offensiv-taktischen Fähigkeiten einen durchschnittlichen Qualitätsquotienten von gerundet 0,35 und bezüglich der defensiv-taktischen Fähigkeiten einen solchen von gerundet 2,20. 


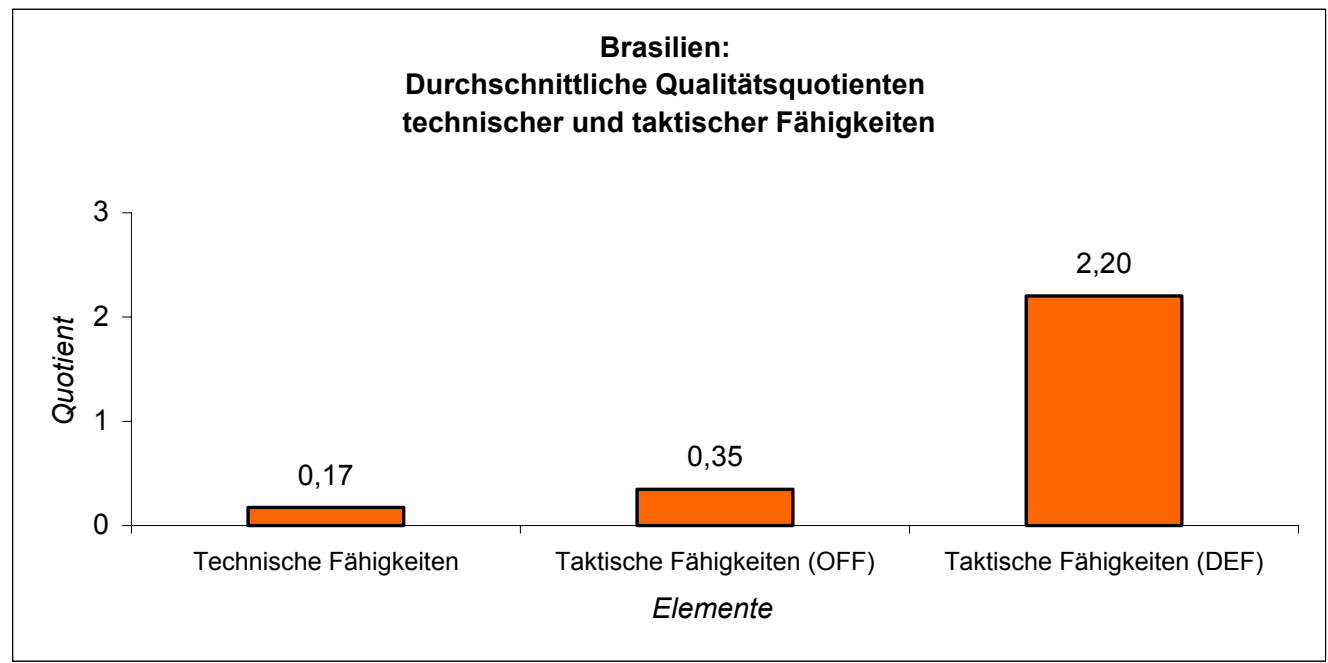

Diagramm 279: Durchschnittliche Qualitätsquotienten technischer und taktischer Fähigkeiten für Brasilien.

Aus der Betrachtung lässt sich sagen, dass die Mannschaft im Verteidigungsspiel viel engagierter als im Angriffsspiel war. Der Grund liegt wahrscheinlich darin, dass die brasilianische Mannschaft im Endspiel sich überwiegend auf das Verteidigungsspiel stützen, und weniger versuchen Angriff zu spielen. Das führt dazu, dass weniger Angreifer vorne bleiben. Im vergleichen mit der französische Mannschaft finden wir, dass die brasilianische Mannschaft schlechter ist.

Diagramm 280 zeigt die durchschnittlichen Qualitätsquotienten technischen, offensiv-taktischen und defensiv-taktischen Fähigkeiten von Kroatien. Wie zu sehen ist, besitzt Kroatien bezüglich der technischen Fähigkeiten einen durchschnittlichen Qualitätsquotienten von gerundet 0,23 , bezüglich der offensiv-taktischen Fähigkeiten einen durchschnittlichen Qualitätsquotienten von gerundet 0,17 und bezüglich der defensiv-taktischen Fähigkeiten einen solchen von gerundet 1,85. 


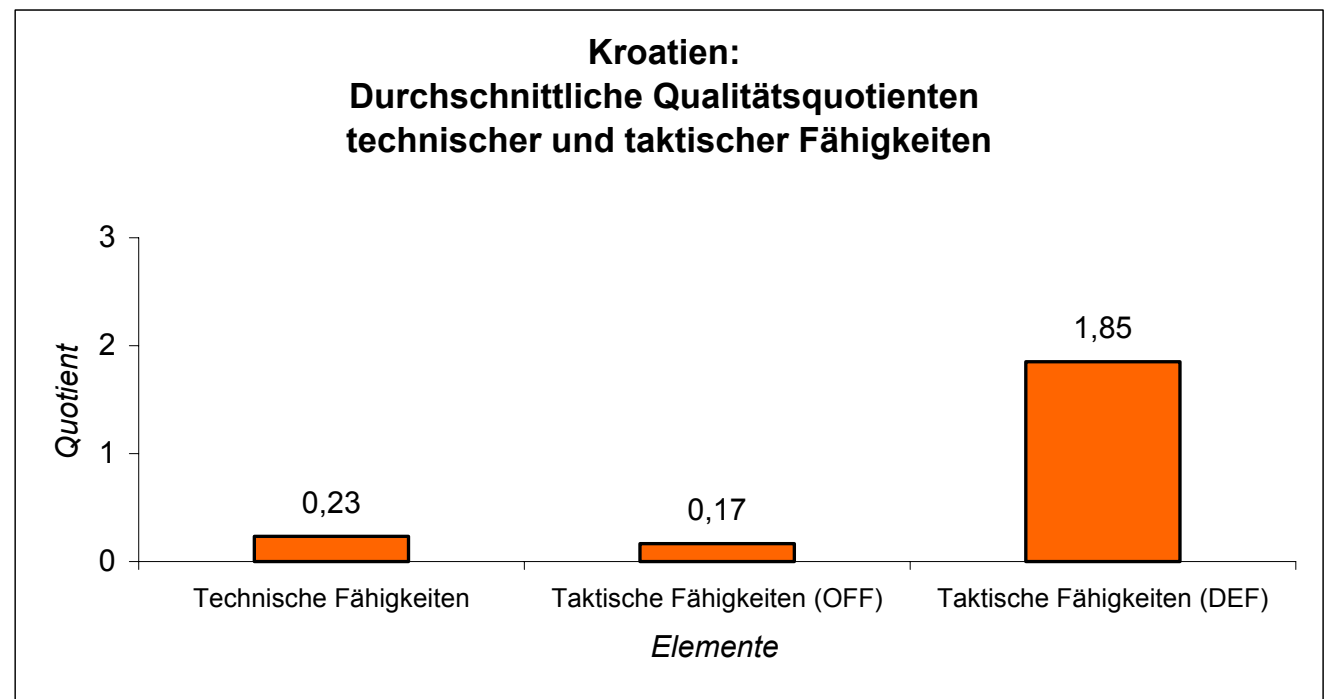

Diagramm 280: Durchschnittliche Qualitätsquotienten technischer und taktischer Fähigkeiten für Kroatien.

Aus der Betrachtung lässt sich sagen, dass die Mannschaft im Verteidigungsspiel viel engagierter als im Angriffsspiel war. Der Grund liegt wahrscheinlich darin, dass die kroatische Mannschaft im Halbfinale sich überwiegend auf das Verteidigungsspiel stützte, und weniger versuchte auf Angriff zu spielen. Das führte auf dazu, dass weniger Angreifer vorne blieben.

Diagramm 281 zeigt die durchschnittlichen Qualitätsquotienten technischen, offensiv-taktischen und defensiv-taktischen Fähigkeiten der Niederlande. Wie zu sehen ist, besitzen die Niederlande bezüglich der technischen Fähigkeiten einen durchschnittlichen Qualitätsquotienten von gerundet 0,29 , bezüglich der offensivtaktischen Fähigkeiten einen durchschnittlichen Qualitätsquotienten von gerundet 0,68 und bezüglich der defensiv-taktischen Fähigkeiten einen solchen von gerundet 2,41 . 


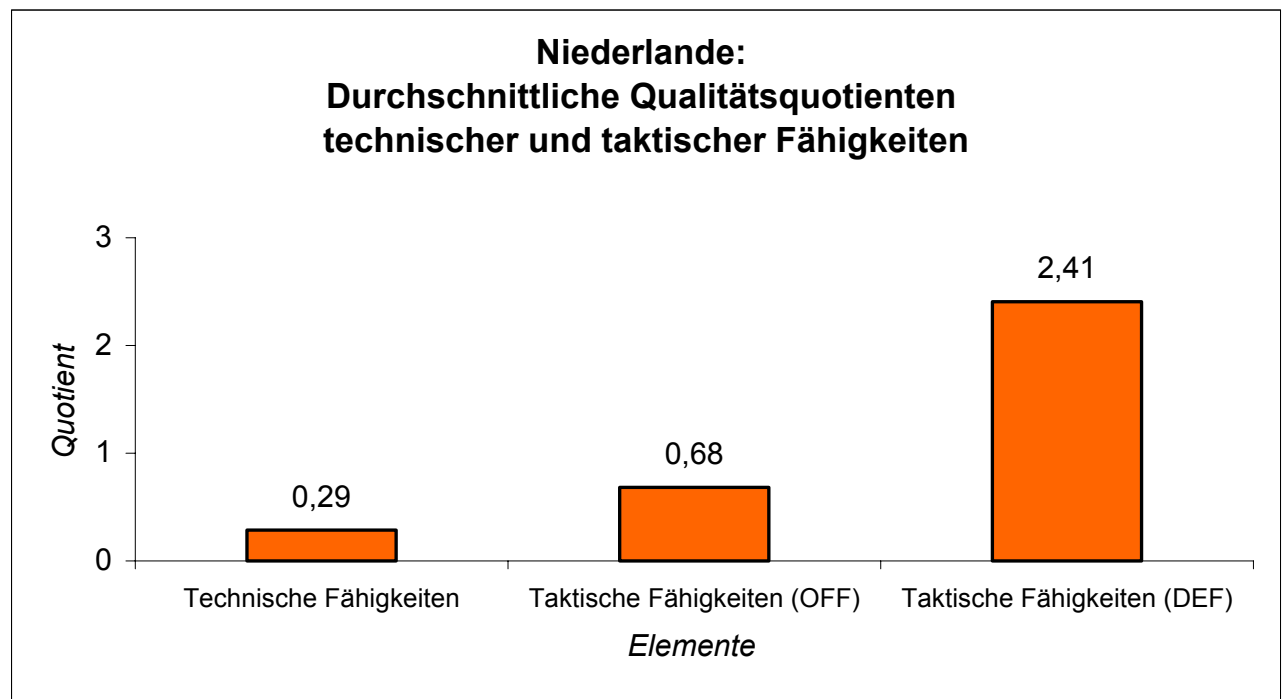

Diagramm 281: Durchschnittliche Qualitätsquotienten technischer und taktischer Fähigkeiten für die Niederlande.

Aus dem Diagramm ist ersichtlich, dass die Verteidigungsfähigkeit und offensiv- taktischen Fähigkeiten der niederländischen Mannschaft besser als die technische Fähigkeiten sind, aber augenscheinlich besser als die der anderen Mannschaften. Dies deutet darauf hin, dass die Mannschaft mit dem besten Verteidigungsspiel auch die beste offensiv- taktischen Fähigkeiten besitzt, womit sie den Wettkampf gewinnen konnte, aber sie verlor dennoch im Elfmeterschiessen gegen Brasilien.

Beim Vergleich der offensiv-taktischen Fähigkeiten zur Verteidigung und den übrigen technischen Fähigkeiten in vorgehende Diagramm fällt auf, dass die Verteidigungstaktik immer die bessere ist. Der Unterschied zwischen der Qualität des Angriffsspiels und den allgemeinen spieltechnischen Fähigkeiten sind durchwegs gering. Die meisten Mannschaften hatten ein besseres Verteidigung- als Angriffsspiel und andere spielerischen Fähigkeiten. Wir nehmen an, dass die Trainer dieser Mannschaften aus Furcht vor dem Ausscheiden den Schwerpunkt auf die Verteidigung legten, besonders in den letzten Runden des Ausscheidungskampfes ab dem Achtelfinale. Ein anderer Grund könnte sein, dass diese Mannschaften keine hervorragenden Spieler haben. 


\section{Analyse der Mannschaftsergebnisse}

In diesem Abschnitt werden die ermittelten Ergebnisse zum technischen, taktischen offensive und defensive der sechzehn Nationalmannschaften zusammengefasst und diskutiert. Anhand der einzelnen Beobachtungseinheiten wird die Struktur der technischen im Fußballspiel verdeutlicht. Mit Hilfe von Grafiken und Tabellen werden die Ergebnisse weiterverarbeitet.

Über die Möglichkeiten dieser Art von qualitativen Spielanalysen hinaus ist z.B. interessant, wie Sportwissenschaftler und Trainer die Spielstärke einer Mannschaft beurteilen. Um möglichst objektive Bewertungen abgeben zu können, sollten qualitativ und quantitativ zu erfassende Merkmale für die Spielstärke benannt werden (vgl. WINKLER 1997). hinsichtlich quantitativ zu ermittelnder Merkmale für die Spielstärke könnten u.a. folgende Merkmale analysiert worden:

- Anzahl der guten und schlechten Dribblings,

- Anzahl der guten und schlechten Körpertäuschungen,

- Anzahl der guten und schlechten kurzen Pässe,

- Anzahl die guten und schlechten langen Pässe,

- Anzahl der guten und schlechten Ballkontrollen,

- Anzahl der guten und schlechten Torschüsse,

- Anzahl der guten und schlechten Offensivtaktiken,

- Anzahl der taktischen Fehler Offensivtaktiken,

- Anzahl der guten und schlechten Freistösse,

- Anzahl der guten und schlechten Eckbälle,

- Anzahl der guten Taktiken im defensiven Spiel,

- Anzahl der taktischen Fehler im defensiven Spiel,

- Anzahl der gewonnenen Zweikämpfe in eigenen Drittel, gegnerischen Drittel und im Mittelfeldspiel.

Bezüglich der oben genanten Merkmale ist es von Belang zu untersuchen, wie oft eine Mannschaft kurze oder lange Pässen spielte, wie oft die Spieler die Mischung aus Ballführen und Balltreiben benützen und wenn der Gegner in unmittelbarer Spielnähe ist, der dribbelnde Spieler versuchte, diesen mit unterschiedlichen Finten erfolgreich abzuschütteln und den Ball besser kontrollierte; wie viel Torschüsse dabei zustande gekommen und schließlich wie viele Tore gefallen sind; wie oft eine Mann- 
schaft vom eigenen (hinteren) oder mittleren Drittel oder gegnerischen Drittel den Ball abnehmen konnte, wie viele Male die Verteidiger in richtiger Positionen waren, um den gegnerischen Angreifer abwehren zu können, wie oft eine Mannschaft Abseitsfallen benutzte (ohne Fehler), wie oft eine Mannschaft vom eigenen (hintern) oder mittleren Drittel des Spielfeldes in das gegnerische Drittel eindrang, wie oft sie davon (ohne Ballverlust) im gegnerischen Drittel weiter agieren konnte, wie viel Mal mindestens drei Spieler in richtiger Positionen waren, um den Ball behalten oder abgeben zu können; wie oft eine Mannschaft dem Gegner durch Eckbälle und Freistöße gefährlich wurde.

Eine Mannschaft, die z.B. viele Zweikämpfe in eigenen Drittel oder in Mittelfeld gewinnen und somit den gegnerische nicht Torschiessen kann, kann auf jedenfalls als stark Verteidiger eingestuft werden (vgl. die Diagramme von Achtel-, Viertel-, und Halbfinalisten S.160: 166, 175: 180 und 187: 192).

Diese Beispiele machen gleichzeitig deutlich, dass aus quantitativen Ergebnissen qualitative Bewertungen möglich sind: Eine Mannschaft, die z.B. viele kurze Pässen, Ballkontrolle und Dribbling in eigenen drittel und nur lange Pässen in das gegnerische drittel kann und somit zu wenigen Torschüssen kommt, kann keinesfalls als spielstark eingestuft werden ( $\mathrm{vgl}$. die Daten von Mannschaften in Diagramme 1 bis 24). Neben derartigen "quantitativen" Kriterien müsste für die Beurteilung der Spielstärke einer Mannschaft vor allem auch die "technische, taktische Fehlerbilanz" in Abwehr und Angriff herangezogen werden.

Je nach Zielsetzung könnten somit von fachlich gut ausgebildeten Trainern Kriterien und Merkmale benannt werden, die Grundlage qualitativer und quantitativer Spielanalysen ist. Sie wiederum könnten Trainern helfen, möglichst gute Einschätzungen/Bewertungen sowie der einzelnen Spielerleistung als auch der Mannschaftsleistung vornehmen zu können. 


\subsection{Technische, taktische- offensiv und defensiv - taktische Fähig- keiten von Halb-, Viertel- und Achtelfinalisten}

Im folgenden Kapitel werden durchschnittliche Qualitätsquotienten technischer, offensiv-taktischer und defensive-taktischer Fähigkeiten von Halb-, Viertel- und Achtelfinalisten beschrieben.

Der Qualitätsquotient einer bestimmten Mannschaft bezüglich eines einzelnen Elements ergibt sich, indem man (ungeachtet der „normalen“ Aktionen) das Verhältnis der Anzahl "guter“ und „schwacher“ Aktionen dieser Mannschaft bezüglich dieses Elements berechnet. Der Qualitätsquotient ist demnach eine Zahl, die zwischen „Null“ und „Unendlich“ liegt, wobei eine Zahl nahe „Null“ auf eine geringe, eine große Zahl auf eine (sehr) hohe Qualität hindeutet.

Der Mittelwert aller Qualitätsquotienten einer Mannschaft von spielerischen Elementen, die den technischen, offensiv-taktischen bzw. defensiv-taktischen Fähigkeiten zugeordnet sind, ergibt den durchschnittlichen Qualitätsquotienten dieser Mannschaft bezüglich dieser Fähigkeit.

Der Mittelwert aller durchschnittlichen Qualitätsquotienten von Mannschaften, die in das Halb-, Viertel- bzw. Achtelfinale eingezogen sind, ergibt den durchschnittlichen Qualitätsquotienten dieser Gruppe.

Diagramm 282 zeigt die durchschnittlichen Qualitätsquotienten technischer, offensivund defensiv- taktischer Fähigkeiten bei den Halb-, Viertel- und Achtelfinalisten.

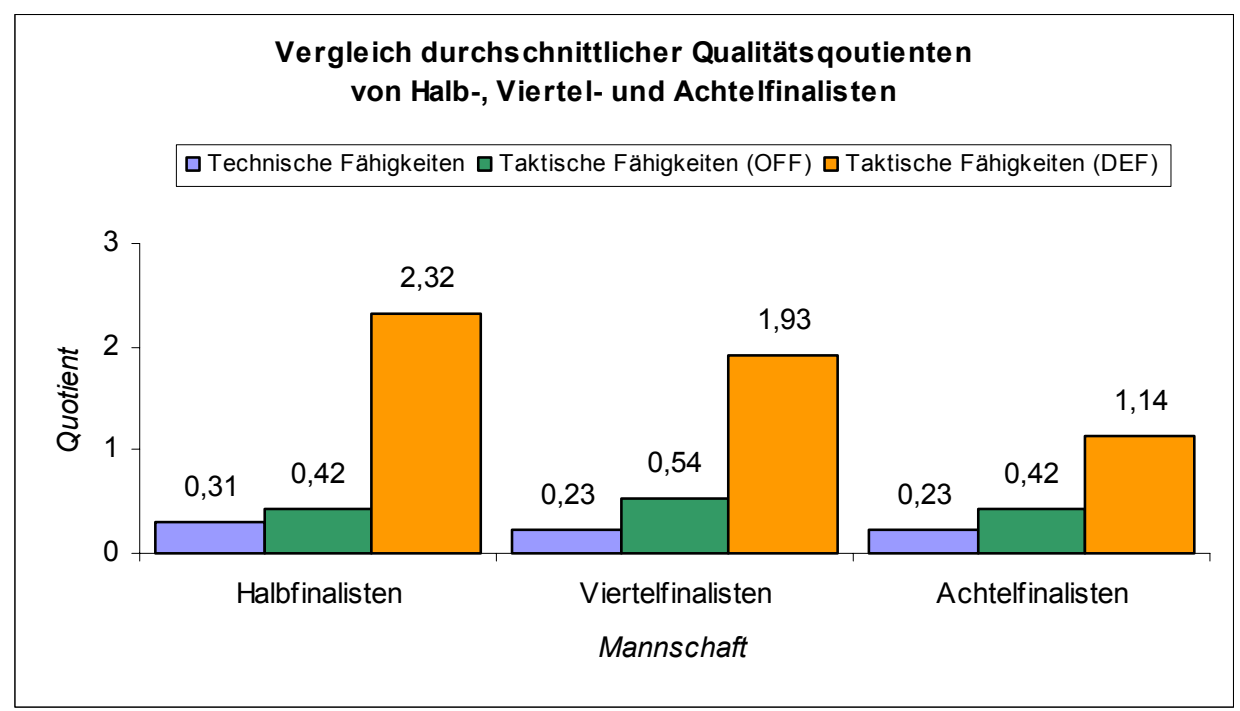

Diagramm 282: Vergleich durchschnittlicher Qualitätsquotienten von Halb-, Viertelund Achtelfinalisten. 
Wie zu sehen ist, besitzen die Halbfinalisten bezüglich der technischen Fähigkeiten einen durchschnittlichen Qualitätsquotienten von gerundet 0,31, bezüglich der offensiv-taktischen Fähigkeiten einen durchschnittlichen Qualitätsquotienten von gerundet 0,42 und bezüglich der defensiv-taktischen Fähigkeiten einen solchen von gerundet 2,32 .

Die Viertelfinalisten besitzen bezüglich der technischen Fähigkeiten einen durchschnittlichen Qualitätsquotienten von gerundet 0,23 , bezüglich der offensivtaktischen Fähigkeiten einen durchschnittlichen Qualitätsquotienten von gerundet 0,54 und bezüglich der defensiv-taktischen Fähigkeiten einen solchen von gerundet 1,93 .

Die Achtelfinalisten besitzen bezüglich der technischen Fähigkeiten einen durchschnittlichen Qualitätsquotienten von gerundet 0,23 , bezüglich der offensivtaktischen Fähigkeiten einen durchschnittlichen Qualitätsquotienten von gerundet 0,54 und bezüglich der defensiv-taktischen Fähigkeiten einen solchen von gerundet 1,14 .

Wie deutlich zu erkennen ist, waren die defensiven Fähigkeiten überall stärker als die offensiven und technischen Fähigkeiten. Dabei nahmen die defensiven Fähigkeiten von Runde zu Runde zu, wohingegen die technischen Fähigkeiten fast gleich blieben, die offensiven Fähigkeiten nur bei den Viertelfinalisten etwas stärker waren.

\subsection{Technische, offensive und defensive-taktische Fähigkeiten von} Halbfinalisten

$\mathrm{Da}$ innerhalb der Gruppe von Halbfinalisten eine Platzierungsreihenfolge existiert, werden diese Mannschaften in Diagramm 283 nochmals separat verglichen. 


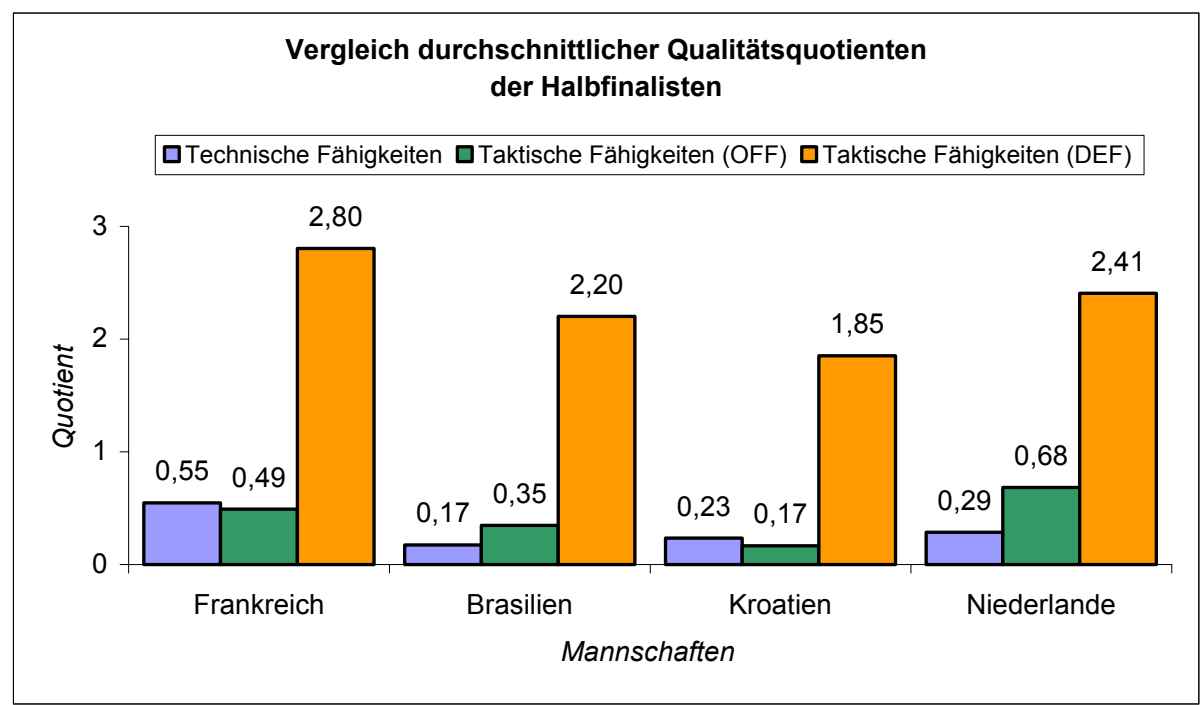

Diagramm 283: Vergleich durchschnittlicher Qualitätsquotienten der Halbfinalisten.

Wie zu sehen ist, besitzt Frankreich (Platz 1) bezüglich der technischen Fähigkeiten einen durchschnittlichen Qualitätsquotienten von gerundet 0,55 , bezüglich der offensiv-taktischen Fähigkeiten einen durchschnittlichen Qualitätsquotienten von gerundet 0,49 und bezüglich der defensiv-taktischen Fähigkeiten einen solchen von gerundet 2,32 .

Brasilien (Platz 2) besitzt bezüglich der technischen Fähigkeiten einen durchschnittlichen Qualitätsquotienten von gerundet 0,17 , bezüglich der offensiv-taktischen Fähigkeiten einen durchschnittlichen Qualitätsquotienten von gerundet 0,35 und bezüglich der defensiv-taktischen Fähigkeiten einen solchen von gerundet 2,20.

Kroatien (Platz 3) besitzt bezüglich der technischen Fähigkeiten einen durchschnittlichen Qualitätsquotienten von gerundet 0,23 , bezüglich der offensiv-taktischen Fähigkeiten einen durchschnittlichen Qualitätsquotienten von gerundet 0,17 und bezüglich der defensiv-taktischen Fähigkeiten einen solchen von gerundet 1,85.

Die Niederlande (Platz 4) besitzen bezüglich der technischen Fähigkeiten einen durchschnittlichen Qualitätsquotienten von gerundet 0,29 , bezüglich der offensivtaktischen Fähigkeiten einen durchschnittlichen Qualitätsquotienten von gerundet 0,68 und bezüglich der defensiv-taktischen Fähigkeiten einen solchen von gerundet 2,41 . 
Auch hier überwiegen die deffensiv-taktischen Fähigkeiten aller Mannschaften die anderen bei weitem, wie zu erwarten ist, sind die verschiedenen technischen Fähigkeiten einer Mannschaft in ihrer Qualität untereinander in Beziehung, eine kleine, kaum wesentliche Ausnahme zeigt sich bei den technischen Fähigkeiten, wo Brasilien den schlechtesten Rang noch vor den ansonsten schlechten Kroaten einnimmt. Die französische Mannschaft liegt bei allen Fähigkeiten deutlich vorne.

\subsection{Individuelle und mannschaftlichen Fähigkeiten von Halb-, Vier- tel- und Achtelfinalisten}

Im folgenden Kapitel werden durchschnittliche Qualitätsquotienten individuellen und mannschaftlichen Fähigkeiten von Halb-, Viertel- und Achtelfinalisten beschrieben.

Der Qualitätsquotient einer bestimmten Mannschaft bezüglich eines einzelnen Elements ergibt sich, indem man (ungeachtet der „normalen“ Aktionen) das Verhältnis der Anzahl „guter“ und „schwacher“ Aktionen dieser Mannschaft bezüglich dieses Elements berechnet. Der Qualitätsquotient ist demnach eine Zahl, die zwischen „Null“ und „Unendlich“ liegt, wobei eine Zahl nahe „Null“ auf eine geringe, eine große Zahl auf eine (sehr) hohe Qualität hindeutet. Der Mittelwert aller Qualitätsquotienten einer Mannschaft von Elementen, die den individuellen bzw. mannschaftlichen Fähigkeiten zugeordnet sind, ergibt den durchschnittlichen Qualitätsquotienten einer bestimmten Mannschaft bezüglich dieser Fähigkeit. Der Mittelwert aller durchschnittlichen Qualitätsquotienten von Mannschaften, die in das Halb-, Viertel- bzw. Achtelfinale einzogen, ergibt den durchschnittlichen Qualitätsquotienten dieser Gruppe.

Diagramm 284 zeigt die durchschnittlichen Qualitätsquotienten individueller und mannschaftlicher Fähigkeiten der Halb, Viertel- und Achtelfinalisten. 


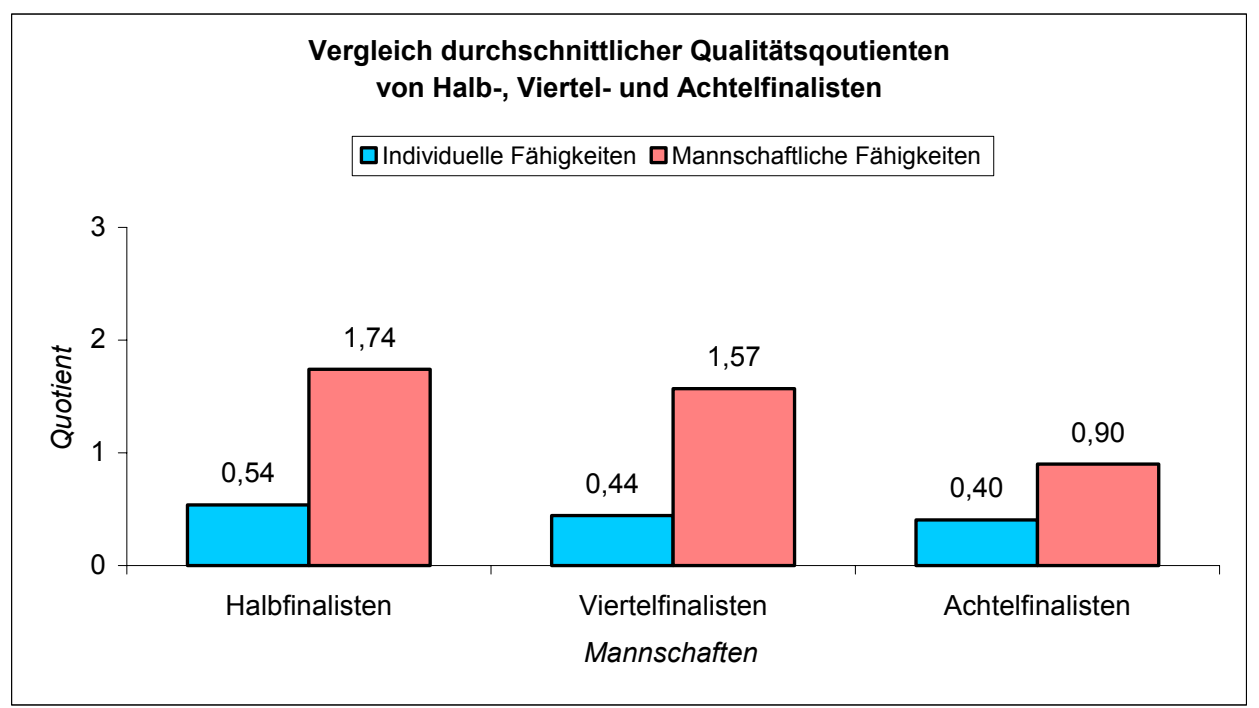

Diagramm 284: Vergleich durchschnittlicher Qualitätsquotienten von Halb-, Viertelund Achtelfinalisten.

Wie zu sehen ist, besitzen die Halbfinalisten bezüglich der individuellen Fähigkeiten einen durchschnittlichen Qualitätsquotienten von gerundet 0,54 und bezüglich der mannschaftlichen Fähigkeiten einen solchen von gerundet 1,74.

Die Viertelfinalisten besitzen bezüglich der individuellen Fähigkeiten einen durchschnittlichen Qualitätsquotienten von gerundet 0,44 und bezüglich der Mannschaftsfähigkeiten einen solchen von gerundet 1,57.

Die Achtelfinalisten besitzen bezüglich der individuellen Fähigkeiten einen durchschnittlichen Qualitätsquotienten von gerundet 0,40 und bezüglich der Mannschaftsfähigkeiten einen solchen von gerundet 0,90.

\subsection{Individuelle und mannschaftlichen Fähigkeiten von Halbfinalis- ten}

Ein wichtiger Aspekt, um die Frage Unterschiede in der Geschicklichkeit, Angriffsund Verteidigungstaktik bei den untersuchten Mannschaften im Fußballspiel bewerten zu können, ist die Darstellung der Erfolgsquoten und der Turnierplatzierung. Die Ergebnisse der Weltmeisterschaft in Frankreich machen deutlich, dass die Erfolgshäufigkeit bei den Einzel- und Mannschaftsfähigkeiten zwar ein dominanter, aber nicht der entscheidende Faktor für eine Plazierung war. Da innerhalb der Gruppe von Halbfinalisten eine Platzierungsreihenfolge existiert, werden diese Mannschaften in Diagramm 285 nochmals separat verglichen. 


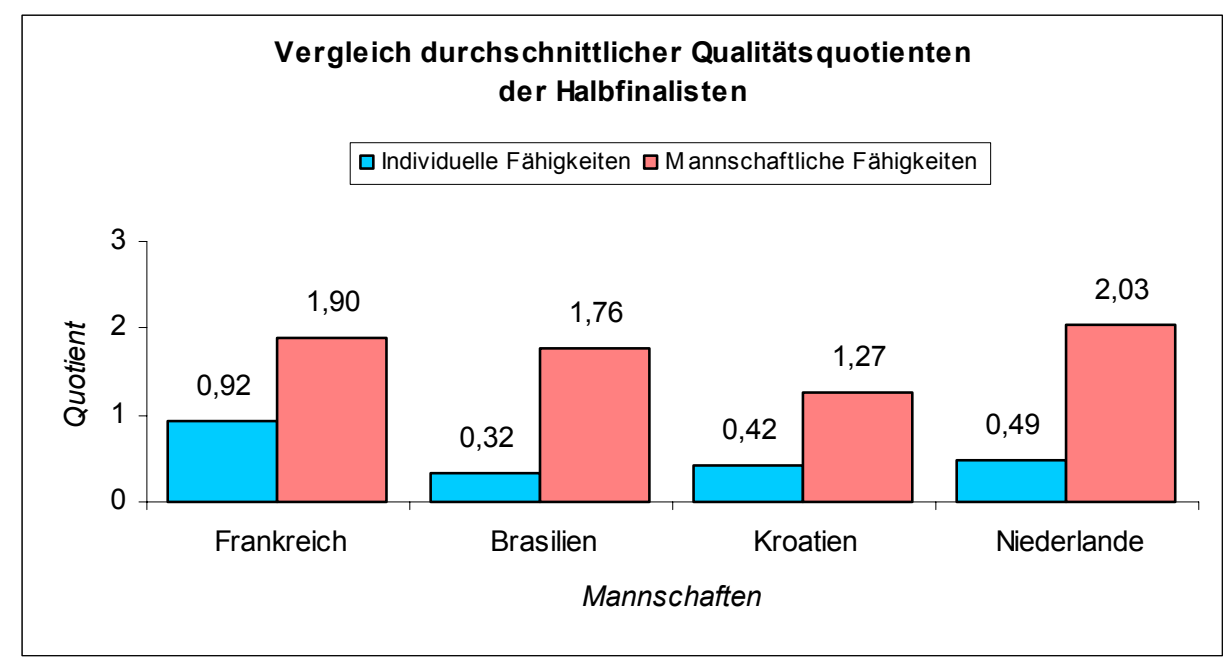

Diagramm 285: Vergleich durchschnittlicher Qualitätsquotienten der Halbfinalisten.

Wie zu sehen ist, besitzt Frankreich (Platz 1) bezüglich der individuellen Fähigkeiten einen durchschnittlichen Qualitätsquotienten von gerundet 0,92 und bezüglich der mannschaftlichen Fähigkeiten einen solchen von gerundet 1,90.

Brasilien (Platz 2) besitzt bezüglich der individuellen Fähigkeiten einen durchschnittlichen Qualitätsquotienten von gerundet 0,32 und bezüglich der mannschaftlichen Fähigkeiten einen solchen von gerundet 1,76.

Kroatien (Platz 3) besitzt bezüglich der individuellen Fähigkeiten einen durchschnittlichen Qualitätsquotienten von gerundet 0,42 und bezüglich der mannschaftlichen Fähigkeiten einen solchen von gerundet 1,27.

Die Niederlande (Platz 4) besitzen bezüglich der Einzelfähigkeiten einen durchschnittlichen Qualitätsquotienten von gerundet 0,49 und bezüglich der Mannschaftsfähigkeiten einen solchen von gerundet 2,03.

\subsection{Bewertung der Qualitätsquotienten}

Aus dem Vergleich der zusammengefassten Qualitätsquotienten der Achtel-, Viertelund Halbfinalisten wird deutlich, dass sich dieser Quotient hervorragend für die großflächige Charakteristik eines Turniers und von Teilen hieraus eignet. Im Detail kann dies jedoch anders aussehen. Auch wenn eine Mannschaft nur so gut spielen kann, wie es der Gegner zulässt, so sollen im Folgenden nicht die Qualitätsquotienten der 
Mannschaften im unmittelbaren Aufeinandertreffen verglichen werden, sondern die durchschnittlichen Qualitätsquotienten der Mannschaften insgesamt - auch wenn hierdurch bei manchen Mannschaften nur eine, bei anderen zwei und schließlich bei den Halbfinalisten der Durchschnitt von vier Spielen herangezogen werden wird. Tab. 4 zeigt die zusammengefassten Qualitätsquotienten der 16 Spiele der Achtel-, Viertel-, Halb und Finalspiele.

\begin{tabular}{|c|c|c|c|c|c|c|c|c|}
\hline \multicolumn{4}{|c|}{ Sieger Achtelfinale } & \multicolumn{4}{|c|}{ Verlierer Achtelfinale } & \multirow[t]{2}{*}{ Kommentar } \\
\hline & t.f & t.f.o & t.f.d & & t.f & t.f.o & t.f.d & \\
\hline Deutschland & 0,10 & 0,27 & 1,10 & Mexiko & 0,19 & 0,29 & 0,68 & 1 \\
\hline Argentinien & 0,15 & 0,46 & 1,39 & England & 0,51 & 0,84 & 2,87 & $\begin{array}{l}\text { Verlierer } \\
\text { besser } 2\end{array}$ \\
\hline Dänemark & 0,23 & 0,47 & 2,28 & Nigeria & 0,42 & 0,56 & 1,32 & 3 \\
\hline Italien & 0,43 & 0,96 & 2,94 & Norwegen & 0,24 & 0,31 & 1,08 & 4 \\
\hline Kroatien & 0,23 & 0,17 & 1,85 & Rumänien & 0,07 & 0,25 & 1,26 & 5 \\
\hline Niederlande & 0,29 & 0,68 & 2,41 & Jugoslawien & 0,21 & 0,40 & 0,99 & 6 \\
\hline Brasilien & 0,17 & 0,35 & 2,20 & Chile & 0,11 & 0,52 & 0,30 & 7 \\
\hline Frankreich & 0,55 & 0,49 & 2,80 & Paraguay & 0,09 & 0,23 & 0,61 & 8 \\
\hline \multicolumn{4}{|c|}{ Sieger Viertelfinale } & \multicolumn{4}{|c|}{ Verlierer Viertelfinale } & \\
\hline & t.f & t.f.o & t.f.d & & t.f & t.f.o & t.f.d & \\
\hline Kroatien & 0,23 & 0,17 & 1,85 & Deutschland & 0,10 & 0,27 & 1,10 & 9 \\
\hline Niederlande & 0,29 & 0,68 & 2,41 & Argentinien & 0,15 & 0,46 & 1,39 & 10 \\
\hline Brasilien & 0,17 & 0,35 & 2,20 & Dänemark & 0,23 & 0,47 & 2,28 & $\begin{array}{l}\text { Verlierer } \\
\text { besser } 11\end{array}$ \\
\hline Frankreich & 0,55 & 0,49 & 2,80 & Italien & 0,43 & 0,96 & 2,94 & 12 \\
\hline \multicolumn{4}{|c|}{ Sieger Halbfinale } & \multicolumn{4}{|c|}{ Verlierer Halbfinale } & \\
\hline & t.f & t.f.o & t.f.d & & t.f & t.f.o & t.f.d & \\
\hline Brasilien & 0,17 & 0,35 & 2,20 & Niederlande & 0,29 & 0,68 & 2,41 & $\begin{array}{l}\text { Verlierer } \\
\text { besser } 13\end{array}$ \\
\hline Frankreich & 0,55 & 0,49 & 2,80 & Kroatien & 0,23 & 0,17 & 1,85 & 14 \\
\hline \multicolumn{4}{|c|}{ Gewinner Spiel um Platz 3} & \multicolumn{4}{|c|}{ Verlierer Spiel um Platz 3} & \\
\hline & t.f & t.f.o & t.f.d & & t.f & t.f.o & t.f.d & \\
\hline Kroatien & 0,23 & 0,17 & 1,85 & Niederlande & 0,29 & 0,68 & 2,41 & $\begin{array}{l}\text { Verlierer } \\
\text { besser } 15\end{array}$ \\
\hline \multicolumn{4}{|c|}{ Sieger Finale } & \multicolumn{4}{|c|}{ Verlierer Finale } & \\
\hline & t.f & t.f.o & t.f.d & & t.f & t.f.o & t.f.d & \\
\hline Frankreich & 0,55 & 0,49 & 2,80 & Brasilien & 0,17 & 0,35 & 2,20 & 16 \\
\hline
\end{tabular}

Tab.4: Zusammengefasste Qualitätsquotienten der Achtel-, Viertel-, Halb- und Finalpaarungen. 


\section{Kommentare}

1- Beim Vergleich der offensiv- und der defensiv taktischen Fähigkeiten und den übrigen technischen Fähigkeiten zwischen die deutsche Mannschaft und die mexikanische Mannschaft in der vorgehenden Tabelle fällt auf, dass die Verteidigungstaktik der deutschen Mannschaft besser ist. Die Unterschiede zwischen der Qualität des Angriffsspiels und den allgemeinen spieltechnischen Fähigkeiten sind durchweg gering. Dies deutet darauf hin, dass die deutsche Mannschaft mit dem besten Verteidigungsspiel auch gute offensiv-taktische Möglichkeiten besitzen könnte. Allerdings konnte Deutschland das Spiel gewinnen. In der Summe der Qualitätsquotienten ist Deutschland der mexikanischen Mannschaft überlegen, da die defensiv- taktischen Fähigkeiten Deutschlands sehr deutlich besser waren. Die Summe der Qualitätsquotienten gibt hier den Ausschlag.

2- Entscheidung im Elfmeterschießen.

3- Aus der Betrachtung des Spiels Nr. 3 Dänemark - Nigeria lässt sich sagen, dass die Unterschiedlichkeiten bei den defensiv- taktischen Fähigkeiten zwischen den Dänen und den Nigerianern deutlich zu Gunsten der Dänen war. Die Unterschiede bei den technischen Fähigkeiten und den defensiv- taktischen Fähigkeiten waren gering. Wie bei Spiel 1 gibt die Summe der Qualitätsquotienten hier den Ausschlag.

4- Aus der Tabelle wird deutlich, dass sowohl die Verteidigungstaktik als auch die offensiv- taktische Fähigkeiten und technische Fähigkeiten der Gewinner deutlich besser als der Verlierer ist. Daraus lässt sich folgern, dass sich die siegreichen Mannschaften mehr um die Verteidigung, den Angriff und die technischen Möglichkeiten ihrer Spieler gekümmert haben als ihre Gegner. Dies bestätigt die Richtigkeit des Qualitätsquotienten

5- Aus der Tabelle ist ersichtlich, dass die defensiv- taktischen und die technischen Fähigkeiten der kroatischen Mannschaft besser als die der rumänischen Mannschaft sind, derweil die offensiv- taktischen bei der rumänischen Mannschaft besser als bei der kroatischen Mannschaft sind. Dies deutet dar- 
auf hin, dass die Mannschaft mit dem besten Verteidigungsspiel auch gute offensiv-taktische Möglichkeiten besitzen kann und somit das Spiel wahrscheinlich gewinnen kann. In der Summe der drei Qualitätsquotienten war ebenfalls Kroatien überlegen, was den Wert des Qualitätsquotienten als Messgröße bestätigt.

6- Vgl. 4.

7- Vgl. 4.

8- Vgl. 4.

9- Bei dem Spiel Deutschland - Kroatien erkennt man bei der Betrachtung der Tabelle, dass das Verteidigungsspiel und die technischen Fähigkeiten bei der kroatischen Mannschaft stärker als bei der deutschen Mannschaft sind. Trotz des Platzverweises der deutschen Mannschaft finden wir, dass die deutsche Mannschaft bessere offensiv- taktische Fähigkeiten hat. Die Vernachlässigung der Verteidigung führt jedoch leicht zur Niederlage. Der Grund dürfte darin zu suchen sein, dass die deutsche Mannschaft nach dem Platzverweis das Abwehrspiel nicht genug gesichert hat. Wie bei Spiel 1 gibt die Summe der Qualitätsquotienten den Ausschlag für Sieg oder Niederlage.

10-Vgl. 4.

11-Beim Vergleich der offensiv- und der defensiv- taktischen Fähigkeiten und den übrigen technischen Fähigkeiten zwischen der dänischen Mannschaft und der brasilianischen Mannschaft fällt auf, dass die Unterschiede zwischen den Mannschaften gering sind. Daraus lässt sich folgern, dass der Gewinner die Chancen um Tore zu schießen besser genutzt hat als der Verlierer. Allerdings ist dies das einzige Spiel, das sich nicht mit dem Qualitätsquotienten erklärt lässt.

12-Entscheidung im Elfmeterschießen.

13-Entscheidung im Elfmeterschießen.

14-Vgl. 4.

15-Spiel um Platz 3 kann wegen der unterschiedlichen Motivationslagen nicht berücksichtigt werden.

16-Vgl. 4. 
Wie deutlich zu erkennen ist, eignet sich im Einzelfall des unmittelbaren Vergleichs der Qualitätsquotient in drei Spielen nicht, wenn die Spiele durch Elfmeterschießen entschieden wurden, dies sind: Argentinien - England, Frankreich - Italien und Brasilien - Niederlande. Beim Elfmeterschießen gibt zu $50 \%$ die Leistung des Torwartes den Ausschlag. Der Torwart wurde insgesamt nicht in die Bewertung einbezogen, was sich gerade beim Elfmeterschießen als Nachteil erweist (vgl. Empfehlungen am Ende der Arbeit). Auch eignet sich der Qualitätsquotient noch in zwei anderen Spielen, nämlich Brasilien - Dänemark und im Spiel um Platz drei (Niederlande - Kroatien) nicht. Beim Spiel um Platz drei spielt die Psyche eine wichtige Rolle, besonders wenn eine Mannschaft wie die Niederlande im vorgehenden Spiel durch Elfmeterschießen ausgeschieden ist und auf Grund der Tradition der niederländischen Mannschaft mit und der Güte des Spieles eigentlich mit einem Sieg rechnen konnte. Auf der anderen Seite ging es für das junge Land Kroatien um den größten Erfolg ihrer Fußball- Geschichte. Bei so verschiedenen Motivationslagen kann man den Qualitätsquotienten nicht berücksichtigen.

Es zeigt sich, dass der Qualitätsquotient nicht in jedem Fall das Ergebnis richtig widerspiegelt. Es kommt im Fußball häufiger vor, dass die objektiv schlechtere Mannschaft trotzdem gewinnt. Immerhin lässt sich auf dem Niveau des Weltturniers schließen, dass bei 13 Spielen nur eins dabei ist, dessen Ergebnis sich nicht durch den Qualitätsquotienten erklären lässt. Wenn sich im Fußball durch eine einzelne Kennziffer alles erklären ließe, würde das Spiel an Reiz verlieren. Der Qualitätsquotient gibt aber wichtige Kennziffern für die Charakteristik des Spiels an.

Beim Vergleich der offensiv-taktischen Fähigkeiten mit den defensiv-taktischen und den technischen Fähigkeiten fällt in vorgehenden Tabelle auf, dass bei diesem Turnier die überlegene Defensiv-Taktik meist bei den Gewinnern war mit der Ausnahme von nur vier Spielen.

Aus dem Vergleich der zusammengefassten Qualitätsquotienten der Achtel-, Viertelund Halbfinalisten wird deutlich, dass sich dieser Quotient bei 11 von 12 Spielen $(91,6 \%)$ hervorragend für die Charakteristik des Turniers und von Teilen hieraus eignet. 


\section{Zusammenfassung und Ausblick}

Die Weltmeisterschaft ist der wichtigste Wettkampf im Fußball. Dabei zeigen die Spieler und Mannschaften all ihr Können in den Techniken, den Offensiv- und Defensivtaktiken. Wir finden bei der Betrachtung der Auswertung, dass die Defensivtaktik bei allen Mannschaften der wichtigste Bestandteil des Spieles war. Das vorherrschen dieser Spieltaktik erklärt auch die geringe Anzahl der Tore, die im Wettkampf erzielt wurden. Entweder weil die Mannschaften zu wenige talentierte Spieler hatten oder weil die Trainer eine Niederlage fürchteten, da sie ab dem Achtelfinale das Ausscheiden aus dem Wettkampf bedeutet hätte, richteten die Trainer das Spiel auf Defensivtaktiken aus. Zudem ließe sich auch über ein Elfmeterschießen ein Erfolg erzielen.

Das Neue an der vorliegenden Dissertation ist die Verwendung des vom Verfasser entwickelten Qualitätsquotient. Wie die Ergebnisse des Vergleichs zwischen den Achtel-, Viertel und Halbfinalisten gezeigt hat, kann man diesen Qualitätsquotienten zum Gruppenvergleich über viele Spiele problemlos mit hoher Signifikanz verwenden. Im Einzelfall des unmittelbaren Vergleichs eignet sich der Qualitätsquotient nicht, wenn das Spiel durch Elfmeterschießen entschieden wurde ("Beim Elfmeterschießen beginnt das Spiel neu"). Die zusammengefassten Qualitätsquotienten müssen in Zukunft noch weiter verfeinert werden, mit dem Ziel die Spiel entscheidenden Elemente zu identifizieren und die Anzahl der einzelnen Qualitätsquotienten ggf. zu verringern. In der vorliegenden Arbeit gab es zusammenfassend die folgenden Ergebnisse:

Beim Vergleich der Achtel- Viertel und Halbfinalisten hinsichtlich der Fähigkeit bei Dribbling, so stellen sich drei Mannschaften der Achtelfinalisten, nämlich Norwegen, Nigeria und England heraus, die den Qualitätsquotienten eins erreichten. d.h., dass der Anteil der hochkarätigen und schlechten (d.i. misslungenen) Dribblings gleich ist. Bei den Viertelfinalisten erreichte Italien den Quotienten zwei, keine andere Mannschaft erreichte auch nur den Quotienten eins. Von den Mannschaften der Halbfinalisten erreichte Frankreich den Wert 2,5, sie hatte also zweieinhalb Mal soviel gute Dribblings wie misslungene. Wir können daraus den Schluß ziehen, dass die französische Mannschaft Spieler hat, die auch unter Pressing des Gegners gut mit dem Ball dribbeln können. 
Wenn wir die Fähigkeit zum Täuschen bei allen Mannschaften betrachten, so stellen sich alle außer England, Nigeria und den Niederlanden als relativ schwach heraus. Den schwachen Mannschaften fehlt es möglicherweise an talentierten Spielern, die unter Pressing des Gegners täuschen können.

Bei der Betrachtung der Qualitätsquotienten der übrigen Techniken wie Torschuß, kurzer und langer Pässe sind überall schlechte Werte festzustellen, besonders beim Torschuß. Die Mannschaften trafen generell wenige von den versuchten Torschüssen. Den meisten Mannschaften mangelt es an Spielern, die gut Tore schießen können.

Bei der Betrachtung der Qualitätsquotienten der Offensivtaktiken aller Mannschaft des Achtel-, Viertel- und Halbfinales stellen sich alle als schlecht beim Freistoß heraus, außer Jugoslawien. Der Grund für das schlechte Abschneiden liegt vielleicht darin, dass die Spieler die Spieltaktik für einen Freistoß nicht genügend beherrschen, sich die Mitspieler möglicherweise auch nicht am geeigneten Platz zur Verwandlung des Freistoßes befinden. Auch die ausführenden Spieler haben Mängel beim Torschießen.

Beim Vergleich der Diagramme 206, 213 und 220 der Qualitätsquotienten der Eckbälle bei den Achtel-, Viertel-, und Halbfinalisten ist Italien die einzige Mannschaft, die den Wert eins erreicht hat, alle übrigen waren schlechter. Wir führen das darauf zurück, dass die Verteilung der italienischen Spieler bei der Ausführung des Eckballs gut war. Die Spieler nehmen günstige Positionen innerhalb des Strafraums ein und nutzen verschiedene Strategien bei der Ausführung eines Eckballs.

Die Werte des Qualitätsquotienten für das Unterstützen sind bei allen Mannschaften gut. Bei allen wird gute Unterstützung des ballbesitzenden Spielers geleistet, die Spieler ohne Ball bewegen sich gut mit dem Ballführenden im Angriff mit. Für die Ausführung des Doppelpasses erhielten alle Mannschaften außer der niederländischen schlechte Werte. Der Grund liegt vielleicht an der starken Manndeckung, der sich die Spieler, die einen Pass annehmen sollen, kaum entziehen können. 
Wenn wir uns in den Diagrammen 210, 217 und 224 den Qualitätsquotienten für das Nachrücken betrachten ansehen, so stellen sich die italienische und englische Mannschaften als die besten heraus. Diese beiden Mannschaften führen eine mehr defensive Spielstrategie aus. Die Mannschaft hat sehr gute Spieler, die versuchen, dem Gegner den Ball abzuschneiden und dann einen geschickten Konterangriff zu starten: mit Dribbeln und Täuschen Nachrücken sie die gegnerischen Linien.

Für die Raumaufteilung erhalten alle Mannschaften schlechte Werte. Wir finden unsere Beobachtungen bestätigt, dass die Raumaufteilung schlecht ist, was nun durch den Qualitätsquotienten ebenso ausgedrückt wird. Die Spieler nehmen nicht die günstigen Positionen auf dem Spielfeld ein, zudem spielen die Mannschaften defensiv. Der Ball wird zu langsam nach vorne gebracht, was wiederum dem Gegner die Möglichkeit gibt, einen guten Standplatz einzunehmen und den Pass abzuschneiden.

Für die Taktik des „in-die-Breite-ziehens“ können wir aus den Diagrammen 211, 218 , 225 ebenfalls die italienische und englische Mannschaft als beste der untersuchten ermitteln. Sie zeichnen sich bei der unerwarteten Ballführung von einer Seite des Spielfelds zur anderen aus, können dadurch dann eine Lücke der gegnerischen Verteidigung finden, durch die ihre Spieler einen Torschuß platzieren können.

Beim Qualitätsquotienten für Defensivstrategien der Diagramme 226, 234, 242, erkennen wir mehrere Mannschaften, die den Wert eins erreichen oder übertreffen, was auf deren gute Defensivstrategien schließen lässt. Die Spieler verfügen über große Mittel, um Druck auszuüben und den Ball zurückzuerobern.

Die Werte für den Qualitätsquotienten für das Pressing Mitte sind aus den Diagrammen 227, 235 und 243 ersichtlich. Danach haben alle Mannschaften den Wert Null, sind also sehr schwach. Wir führen das auf einen Fehler zurück: Sie sind bei dem Versuch, den Ball im Mittelfeld abzunehmen, zu langsam, besonders beim Nachrücken. Diese Mannschaften üben Pressing hauptsächlich im Verteidigungsraum aus; die Spieler ziehen sich zumeist wieder in den Strafraum zurück und können deswegen kein effektives Pressing im Mittelfeld und schnellen Kontern durchführen. 
Aus den Diagrammen 228, 236, 244 gehen die Werte für den Qualitätsquotienten für das Pressing vorne hervor. Dabei ist die französische Mannschaft die einzige sehr gute. Die Spieler dieser Mannschaft üben Pressing gleich nach dem Ballverlust im Angriffsraum aus. Die Mannschaft verfügt über viele Stürmer, die den Gegner vorne angreifen können und versuchen, ihm den Ball abzunehmen.

Beim Qualitätsquotienten für die Raumaufteilung, wie sie aus den Diagrammen $\mathrm{Nr}$. 229, 237 und 245 hervorgehen, erreicht die englische Mannschaft den Wert 4,2; Nigeria 1,4; Rumänien 1,5; Italien 2,13; Frankreich 1,44; Kroatien 1,6 und Deutschland 1,0. Diese Mannschaften zeichnen sich durch starkes Verteidigungsspiel aus. Sie verteilen ihre Angreifer gut über das Spielfeld, wodurch sie ihr Tor besser verteidigen können und den Angreifern weniger Zugangsmöglichkeiten lassen.

Für den Qualitätsquotienten für das Zurückkommen finden wir in den Diagrammen Nr. 230, 233 und 246 alle Achtelfinalisten sowie Deutschland und Italien bei den Viertelfinalisten sowie alle Mannschaften des Halbfinales mit guten „Werte“, d. h. dass die Stürmer und Mittelfeldspieler sich gut zur Verteidigung an das eigene Tor zur Unterstützung der Verteidigung zurückbewegen. Daraus wird ersichtlich, dass viele Mannschaften ihre Spieler zur Verteidigung zurückkommen lassen. Diese Strategie des Zurückkommens ist vielleicht auch ein Grund für die geringe Zahl von erzielten Toren in diesem Wettkampf, da für das oftmalige Vor- und Zurückkommen viel Kraft und Schnelligkeit benötigt wird.

Die Diagramme Nr. 231,239 und 247 bilden den Qualitätsquotienten für das Unterstützen ab; daraus ergibt sich für die Mannschaften des Achtelfinales, dass England, Mexiko, Jugoslawien und Nigeria, von den Viertelfinalisten Dänemark und Italien sowie alle Mannschaften des Halbfinales im Unterstützen gute Werte erreichen. Die Verteidiger arbeiten gut untereinander zusammen und führen gut Deckung aus.

Für das Rückraumsichern ergeben sich die Werte des Qualitätsquotienten aus den Diagrammen 232, 240 und 243; wobei alle Mannschaften des Achtelfinales außer Nigeria und Rumänien und von den Viertelfinalisten Deutschland gute Werte erhal- 
ten. Sie zeichnen sich durch gute Spieler aus, die auf von hinten sich nähernde Angreifer achten und ihre Stellung sichern.

Die Diagramme für den Qualitätsquotienten für die Abseitsfalle, Nr. 233, 241 und 249 zeigen, dass die meisten Mannschaften bei der Ausführung dieser Verteidigungsstrategie gute Werte erreichen. Das zeigt auf die besondere Bedeutung, die diese Mannschaften den Verteidigungsstrategien bei der Vorbereitung auf diesen Wettkampf einräumten, denn diese Strategie verträgt keine Fehler, wenn sie nicht misslingen und dadurch die Gefahr eines Tores bieten soll, ansonsten aber ist der Erfolg sicher. Aus dem Dargestellten wird klar, dass die Qualitätsquotienten für Verteidigungstaktiken deutlich besser sind als die für Angriffstaktiken und Techniken. Das dürften auch die Gründe für die niedrige Torbilanz der Meisterschaften sein.

Es gibt noch andere mögliche Gründe für dieses Verhalten. So kann ein Platzverweis für einen Spieler, wie dies auch bei der deutschen Mannschaft im Spiel gegen Kroatien zutraf, wo die Mannschaft fünfzig Minuten lang mit zehn Spielern auskommen musste, zu einer defensiven Spieltaktik führen. Auch die englische Mannschaft musste siebzig Minuten lang auf einen ihrer Spieler verzichten und schiede im Achtelfinale beim Elfmeterschießen aus. Die argentinische Mannschaft spielte fünfzehn Minuten gegen Holland mit einem Spieler weniger nach einer roten Karte. Die niederländischen und italienischen Mannschaften schieden ebenfalls nach dem Elfmeterschießen aus. Auch die holländische Mannschaft musste gegen Argentinien nach einer roten Karte 35 Minuten mit einem Spieler weniger auskommen. Die französische Mannschaft spielte im Finale 20 Minuten gegen Brasilien mit einem Spieler weniger nach einer roten Karte.

Wir finden, dass die Unterschiede zwischen den spielerischen Fähigkeiten der Mannschaften und den Angriffstaktiken gering sind, obgleich jede Mannschaft mit den besten ihnen zur Verfügung stehenden Spielern spielte. Es scheint daher notwendig, dass die Spieler lernen, unter dem Druck eines Wettkampfs zu spielen.

Als Schlussfolgerung der schwachen Torschussergebnisse ist zu fordern, dass die Mannschaften ihre Torschussfähigkeiten verbessern, besonders auch unter dem Druck der Verteidiger sollten sie noch bessere Ergebnisse erzielen. 
Beim Vergleich der taktischen Fähigkeiten zum Angriff und zur Verteidigung und den übrigen technischen Fähigkeiten im Diagramm 282 fällt auf, dass die Verteidigungstaktik immer die bessere ist. Der Unterschied zwischen der Qualität des Angriffsspiels und den allgemeinen spieltechnischen Fähigkeiten sind durchwegs gering.

Aus dem Diagramm 283 ist ersichtlich, dass die Verteidigungsfähigkeit und technischen Fähigkeiten der französischen Mannschaft besser als die der brasilianischen, kroatischen und niederländischen Mannschaft sind. Die Angriffsfähigkeiten der niederländischen Mannschaft sind augenscheinlich besser als die der anderen Mannschaften. Dies deutet darauf hin, dass die Mannschaft mit dem besten Verteidigungsspiel auch die Spieler mit den besten technischen Fähigkeiten besitzt, womit sie den Wettkampf gewinnen konnte.

Bei der Betrachtung des Diagramms 284 ist zu bemerken, dass die Halbfinalisten in der Verteidigungstaktik und den allgemeinen Spieltechniken besser als die Mannschaften des Viertelfinales waren, und die Mannschaften des Viertelfinales besser als die des Achtelfinales. Dafür waren die Viertelfinalisten in der Angriffstaktik besser als die Achtelfinalisten und Halbfinalisten. Beim Vergleich der Viertel- und Achtelfinalisten gleichen sich deren technische Fähigkeiten, doch sind die Halbfinalisten besser als diese beiden, da sie offenbar die talentierteren Spieler haben. Diese Spieler waren auch stark in der Verteidigung, was vielleicht ein Grund dafür ist, dass sie im Wettkampf bis zur Endrunde gelangten.

Das Diagramm 285 deutet die Überlegenheit der französischen Mannschaft bei den technischen Fähigkeiten an und die Überlegenheit der niederländischen Mannschaft im Bereich der Verteidigungsfähigkeit. Wir führen dies auf die besseren Spieler mit hohem technischem Können bei der französischen Mannschaft in diesem Wettkampf zurück. Die holländische Mannschaft erlangte wegen einer Niederlage beim Elfmeterschießen im Viertelfinale nur einen vierten Platz.

Einige andere Faktoren des Spiels konnten hier jedoch nicht untersucht werden, wie das Laufen der Spieler ohne Ball und die technischen und taktischen Fähigkeiten des Torwarts. Diese Spielbestandteile müssen mittels gesondert Videokamera aufgezeichnet werden. Die französische Mannschaft hat wahrscheinlich aufgrund ihrer ta- 
lentierten Spieler wie ZINEDINE ZIDANE und JORY D'JORKAIEW die Weltmeisterschaft gewonnen. Wir folgern daher, dass nach Talenten beständig gesucht werden muss, da diese Spieler für das Spiel entscheidend sein können.

Es wurde gezeigt, dass Spielanalysen unter verschiedensten Gesichtpunkten auch mit einem handelsüblichen Videorekorder möglich sind. Für Trainer und Sportwissenschaftler oder Leistungsdiagnostiker ist dabei interessant, dass das Verhalten und die Handlungen eines Spielers bzw. die Ketten von Spielerhandlungen sowohl unter quantitativen als auch qualitativen Gesichtpunkten systematisch erfasst, dargestellt und besprochen werden können. Falls innerhalb einer systematischen Spielanalyse nicht nur die Anzahl der verschiedenen taktischen Fehler der Spieler einer Mannschaft beobachtet, erfasst, statistisch ausgewertet und graphisch dargestellt werden (quantitativer Aspekt), sondern auch nach den Ursachen der Fehler gesucht wird mit der Intention, diese Fehler per Video zu dokumentieren und ausgewählte Sequenzen für Spielforschung einzusetzen, so handelt es sich um einen qualitativen Aspekt einer Spielanalyse.

Entsprechendes gilt für das Verhalten in den offensiven, defensive-taktischen und technischen Spielzügen. Derartige Analysen können Trainern helfen, Trainingsformen zu finden für die Planung Erfolg versprechender Angriffs- und Abwehrstrategien. Neuerungen auf dem Gebiet der Computerhardware und Software sowie der Videotechnik ermöglichen es fachkundigen Trainern, einfache und kostengünstige, qualitative und quantitative Spielanalysen durchzuführen mit Feedbackfunktion: Trainer erhalten wertvolle Anregungen für die weitere Trainingssteuerung; Spielanalysen der vorgeführten Art können dazu beitragen, sowohl Konzeptfehler bei Spielern und Trainern aufzudecken wie auch Entscheidungsfehler bei Spielern bewusst zu machen und dadurch zu verringern (vgl. WINKLER 1997; BORN 1996).

Wie gut und wie schnell Verbesserungen (vorrangig auf taktischem und technischem Gebiet) erreicht werden, hängt wesentlich ab von

- der Spielerauffassung des Trainers,

- seiner Spielanalysekonzeption,

- seinen Fähigkeiten der praktischen Umsetzung der gewonnen SpielanalyseErgebnisse im Training und nicht zuletzt

- von der Lernfähigkeit und Lernwilligkeit seiner Spieler. 


\section{Empfehlungen}

1. Die Spieler sollen sich ehe um die Qualität als die Quantität der Techniken kümmern, da sie wettkampfentscheidend sein können. Je besser die technischen Fähigkeiten der Spieler sind, desto eher sind sie den Gegnern überlegen und spielführend.

2. Die Trainer sollen Offensivtaktiken unter wettkampfähnlichen Bedingungen einüben, damit sich die Spieler an den Verteidigungsdruck gewöhnen und wie sie ihm widerstehen können.

3. Darüber dürfen natürlich die Deffensivtaktiken nicht aus dem Auge verloren werden, da sie zur Abwehr von Toren unerlässlich sind; diejenige Mannschaft aber, die die meisten Tore erzielt, ist Gewinner.

4. Es müssen Talente entdeckt und so ausgebildet werden, dass sie für die Mannschaft nützlich sind.

5. Mit Digitalisierung der Fernsehbilder lassen sich die einzelnen technischentaktischen Elemente genauer abgrenzen (z. B. kurzen / langen Pässen, Pressing hinten, mitte und vorne).

6. Auch müsste noch mehr Forschung über die Auswirkung unterschiedlicher Spielbestandteile und deren Qualität ausgeführt werden, wie z.B. der Bewegung ohne Ball, den notwendigen Grand an physischen Leistungsvorbereitung der Spieler und Forschung in die spielentscheidenden Taktiken.

7. Der Computer ist zwar schnell bei der Erstellung von Berechnungen, doch hat er Schwierigkeiten, die Qualität einer Technik oder Taktik zu bewerten. Es sollten noch weitere Untersuchungen angestellt werden, wobei Mensch mit Hilfe eines Computers einen Wettkampf beobachtet, um zu einer möglichst schnellen Auswertung der Spielzüge zu gelangen, die sofort dem Trainer zur Verfügung stellt werden können. 
8. Auch der Zusammenhang zwischen den Zuschauern und ihrem Einfluss auf Spieler und deren Spielverhalten sowie Leistung und Spielergebnis können untersucht werden.

9. Ob ein Spiel von einer Mannschaft, die ansonsten gute Leistungen zeigt, im Elfmeterschiessen verloren werden kann, sollte eine Untersuchung der dabei wirkenden Faktoren zu Tage bringen.

10. Der Einfluss einer verringerten Mannschaftsstärke auf das Verhalten der Mannschaft, den Spielverlauf und die mögliche Auswirkungen auf das Ergebnis sollten betrachtet werden.

11. Zusätzliche Studien sind auch zu den körperlichen Verfassungen, den Techniken und Strategien des Torhüters wegen seiner besonderen Bedeutung von Nöten. 


\section{Literaturverzeichnis}

- ADRIAN, M.J. / COOPER, J.M.: Biomechanics of Human Movement. 2 Aufl. Madison 1995.

- ALLENDORF, O./ BRETTSCHNEIDER, W.D. : Leistungsdatenerfassung undAuswertung im Sportspiel mit Hilfe des computergesteuerten optischen Lesestifts. In: ANDERSEN, R./ HAGEDORN,G. (Hrsg.): Beiträge zur Sportspielforschung. Berlin 1976, 106 -116.

- ALPHEIS, H.: Funktion und Grenzen der Spielbeobachtung. In : Leistungssport 14 (1984), 3, 27 - 32.

- ALPHEIS, H.: Sportspielbeobachtung im Basketball. In: Leistungssport 9 (1979), 6, 452 - 462.

- ATTESLANDER, P.: Methoden empirischer Sozialforschung. 4. Aufl, Berlin / New York 1975.

- AUGUSTIN, D.: Zur Entwicklung und Objektivierung eines qualitativen Spielbeobachtungsverfahren - Computer- und Videoeinsatz bei der Beobachterschulung als Basis objektiver Ergebnisse. In: WINKLER, W./ REUTER, A. (Hrsg.) : Computer- und Medieneinsatz im Fußball. Hamburg 2000, 77 - 94.

- BARTH, B.: Probleme einer Theorie und Methodik der Strategie und Taktik des Wettkampfs im Sport (I-III). In: Theorie und Praxis der Körperkultur, Teil I 29 (1980) 2, 127 -137.

- BARTH, B.: Strategie und Taktik im Wettkampfsport. Leistungsport 24 (1994), 3, $4-12$.

- BARTH, B.: Wettkampfvorbereitung durch komplexes strategisch- taktisches Training. In: Leistungssport 25 (1995),1, 20 -27.

- BARTLETT, R.: Sports Biomechanics: Reducing Injury and Improving Performance. London 1999.

- BAUER, G.: Fußball perfekt: Vom Anfänger zum Profi. München 1973.

- BAUER, G.: Lehrbuch Fußball: Erfolgreiches Training von Technik, Taktik und Kondition. München 1990.

- BAUER, G.: Lehrbuch Fußball: Fußball Technik Heute. München 1998.

- BAUER, G.: Lehrbuch Fußball: Erfolgreiches Training von Technik, Taktik und Kondition. München; Wien; Zürich 2001.

- BAUER, G./ ÜEBERLE, H.: Fußball: Faktoren der Leistung Spieler und Mannschaftsführung. München 1984. 
- BINDING, M.: Zur Untersuchung ausgewählter Komponenten der Erkenntnistätigkeit im Nachwuchs- und Hochleistungsbereich unter Berücksichtigung eignungsdiagnostischer Fragestellungen im Handball. Dissertation der DHFK, Leipzig 1987.

- BISANZ, G.: Meine Eindrücke von der Weltmeisterschaft in Mexiko. In: Fußballtraining 4 (1986), 8, 4 - 7.

- BISANZ, G.: Das Spielsystem ist zweitrangig. In: Fußballtraining 12 (1994), 11/12, $3-7$.

- BISANZ, G./ GERISCH, G.: Fußball. Training, Technik, Taktik. Reinbek 1980.

- BISANZ, G./ GERISCH, G.: Lehrbuch, Fußball training- Technik- Taktik. Reinbek 1997.

- BOBER, T.: Biomechanical Aspects of Sports Techniques. In: MORECKI, A. I FIDULES, K./ WIT, A. Biomechanics. Baltimore, MD: University Park Press 1981, $501-510$.

- BÖS, K.: Finte. In: RÖTHIG, P. (Ltg.): Sportwissenschaftliches Lexikon. Schorndorf 1983, 164.

- BÖS, K.: Der Heidelberger- Basketballtest. In: Leistungssport 18 (1988), 2, 17 31.

- BÖS, K.: Handbuch sportmotorischer Test. 2. Aufl. Göttingen 2001.

- BRACK, R.: Trainingswissenschaftliche Leistungsdiagnostik im Volleyball. Konditionelle technomotorische und anthropometrische Einflussgrößen. Ahrensburg 1983.

- BRETTSCHNEIDER, W./ THIRER, R.: Zum Einsatz von Mikrocomputern in der Spielbeobachtung und -Analyse. In: BÖS, K./ GAISSER, G.: Mikrocomputer an sportwissenschaftlichen Instituten. Clausthal- Zellerfeld 1984.

- BREMER, G.: Qualitative Spielbeobachtung. In: Fußballtraining 4 (1986), 2 , $13-19$.

- BUDINGER, H. / HILLMANN, W ./ STRÖDTER, W.: Hockey. Reinbek 1980.

- BUNN, J.W.: Scientific Principles of Coaching, 2 Aufl. Englewood Cliffs, NJ: Prentice- Hall 1972.

- BUTTLER, S./ KUHN, W./ MARTIN, F.: Evaluierung von Schnelligkeitsleistungen bei Amateurfußballspielern. In: WINKLER, W./ REUTER, A. (Hrsg.). Computer- und Medieneinsatz im Fußball, Hamburg 2000, 103 - 116.

- CARR, G.: Mechanics of Sport. Champaign, IL. 1997. 
- COLLINS, D.R. / HODGES, P.B: A Comprehensive Guide to Sports Skills Tests and Measurement. Springfield, III, 1978.

- CZWALINA, C.: Systematische Spielbeobachtung in den Sportspielen. Schorndorf 1976.

- CZWALINA, C.: Zur Bewertung sportspielerischer Leistung. Ein Beitrag zur Theorie des Sportspiels. In : ANDRESSEN, R./ HAGEDORN, G. (Hrsg.): Beobachten und Messen im Sportspiel. Berlin 1980, 25 - 32.

- CZWALINA, C.: Beobachtung und Test als Steuerungsinstrumente des Sportspiels in Training und Wettkampf. In: ANDRESEN, R./ HAGEDORN,G.(Ltg.) : Steuerung des Sportspiels in Training und Wettkampf. Ahrensburg 1984, 49 58.

- CZWALINA, C.: Systematische Spielbeobachtung in den Sportspielen. Ahrensburg 1988.

- CZWALINA, C.: Gütekriterien in der Beobachtenden Sportspielforschung. In: HAGEDORN, G./ HEYMEN, N. (Hrsg.): Methodologie der Sportspielforschung. Ahrensburg 1992, 61 - 72.

- DAUGS, R./ BLISCHKE,K ./ MARSCHALL, F./ MÜLLER, H.: Videotechnologien für den Spitzensport. In: Leistungssport 20 (1990), 6, 12 -17.

- DIETRICH, K.: Interaktionsanalyse des Sportspiels. In: DIETRICH, K./ LANDAU, G. (Hrsg.): Beiträge zur Didaktik der Sportspiele. Teil II: Sportspiel- Analysen, Interpretationen, Folgerungen. Schorndorf 1977, 140 -165.

- DÖBLER, H./ Monika, H./ Witt, A.: Zur Spielfähigkeit und zu Aspekten eines leistungswirksammen Spielverhalten. In: Theorie und Praxis des Leistungssports 38 (1989), 5, 323 - 333.

- DOGgART, L./ KEANE, S./ REILLY, T./ STANHOPE, J.: A Task Analysis of Gaelic Football. In: Science and Football II (edited by REILLY, T, CLARYS and STIBBE, A.) London 1993, 186-189.

- DUFOUR, W.: Computer assisted scouting in soccer. In: KUHN, W./ WERNER, S. (Hrsg.): Analyse und Beobachtung in Training und Wettkampf. Berlin 1991, 95 - 106.

- DUFOR, W. / VERLINDEN.M. Analysis of Duel- Type and Pass- Type Actions of the Match Belgium- Germany (20th November 1991). In: KUHN,W./ WERNER, S. (Hrsg.): Analyse und Beobachtung in Training und Wettkampf. Berlin 1991, 107-117. 
- EKBLOM, B.: Football (Soccer). Oxford. 1994.

- ELLIOTT, B.: Training in Sport. Chicester 1999.

- ERDMANN, R./ WILLIMCZIK, K.: Beobachtung. In: SINGER, R./ WILLIMCZIK, K.(Hrsg.): Grundkurs Datenerhebung 2. Bad Homburg 1978, 9-47.

- ESSER, H.: Qualitative und Quantitative Methoden - Eine Scheinkontroverse? In: BECKER, P. (Red): Quantitative oder qualitative Sozialforschung in der Sportsoziologie. (dvs-Protokolle, Bd 8). Clausthal- Zellerfeld 1983, 3-14.

- ERRAIS, B./ WEISZ, A.: Technique et pédagogie du tir en basket-ball. Paris, 1980.

- FASSNACHT, G.: Systematische Verhaltensbeobachtung: Einführung in die Methodologie und Praxis. München/Basel 1979.

- FERGER, K. : Die Bedeutung der Videogestützten Spielanalyse für die individuelle Trainingssteuerung im Sportspiel Fußball. In: WINKLER, W./ REUTER, A. (Hrsg.). Computer- und Medieneinsatz im Fußball, Hamburg, 2000, 143148.

- FISCHER, G.: Neuere Testtheorie. In: FERGER, H./ BREDENKAMP, J. (Hrsg.): Messen und Testen. Göttingen 1983, 604-792.

- FRIDRICHS, J.: Methoden empirischer Sozialforschung. Reinbek b. Hamburg 1973.

- FROHREICH, H.: Zur Entwicklung der Riegelabwehrsysteme im Herrenvolleyball von der EM 1983 bis zur EM 1991. In: DANNEMANN, F (Hrsg.) Volleyball inovativ.17. Symposium des Deutschen Volleyballverbandes 1991. Ahrensburg 1992, 119 - 134.

- GERISCH, G.: Leistungsbestimmung im Fußball durch Wettkampf- und Trainingsanalyse. In: GERISCH, G./ RUTEMÖLLER, E.: Leistungsfußball. KöIn 1989, 6-12.

- GLASERSFELD, E.: Einführung in den radikalen Konstruktivismus. In: WATZLAWICK, P.(Hrsg.): Die erfundene Wirklichkeit. München 1981, 16-38.

- GRAELLS, U.: Das Überzahlverhalten der Ländermannschaften im modernen Wasserballspiel : eine Analyse des Wasserballturniers der Olympischen Spiele 1992 in Barcelona mit Hilfe einer sportartspezifischen Methode der Systematischen Wettkampfbeobachtung . Dissertation. Göttingen 1996. 
- GRAUMANN, C.F.: Grundzüge der Verhaltensbeobachtung. In: GRAUMANN, C. F./ HECKHAUSEN, H. (Hrsg.) Pädagogische, Psychologische, Reader zum Funk - Kolleg Band 1: Entwicklung und Sozialisation. Frankfurt/M.1973,14-41.

- HAGEDORN, G.: Vorüberlegungen zu einer Theorie der Sportspiele. In: ANDERSEN,R./ HAGEDORN, G.(Hrsg.): Zur Sportspiel- Forschung. Berlin 1976, 18-46.

- HAGEDORN, G.: Handlungsstruktur und Didaktik der Sportspiele. In: DIETRICH, K./ LANDAU, G. (Hrsg.): Beiträge zur Didaktik der Sportspiele. Teil II: Sportspiel- Analysen, Interpretationen, Folgerungen. Schorndorf 1977, 31-50.

- HAGEDORN, G.: Braucht der Sportspiel- Trainer die Spielanalyse? In: Leistungssport 9 (1979), 1, 4-11.

- HAGEDORN, G.: Probleme des Beobachters und des Messens im Sportspiel. In: ANDRESEN, R./ HAGEDORN, G.: Beobachten und Messen im Sportspiel. Berlin 1980, 9 - 24.

- HAGEDORN, G./ LORENZ, H./ MESECK,U.: Taktik im Sportspiel. Untersuchung zur Individual-, Gruppen- und Mannschaftstaktik im Sportspiel Basketball. In: Leistungssport 12 (1982), 5, 368- 377.

- HAHN, E.: Strategische und taktische Konzepte im Sport. Leistungsport 14 (1984) 1, 13-20.

- HALL, S.: Basic Biomechanics. St. Louis 1991.

- HANSEN, G. (2003): Qualitative Spielbeobachtung (Bundesinstitut für Sportwissenschaft Bd. 2/2003). Köln.

- HARRE, D. (Ltg.): Trainingslehre. Berlin (Ost) 1971.

- HAWKINS, R.D. u.a.: The Association football Medical Research Programme: an Audit of Injuries in Professional Football. In Journal British of Sports Medicine, 35 (2001), 1, 43-47.

- HAY, J.G.: The Biomechanics of Sports Techniques. Englewood Cliffs, NJ, 1973.

- HAY, J. G. / REID, G.: Anatomy, Mechanics and Human Motion. Englewood Cliffs, NJ 1982.

- HEIN, T./ PERL, J.: Neuere konzeptionelle und technologische Ansätze in der Sportspielbeobachtung und- Analyse. In: KUHN, W./SCHMIDT, W.(Hrsg.): Analyse und Beobachtung in Training und Wettkampf. Beiträge und Analysen zum Fußballsport. Sankt Augustin, 1992, 84-94. 
- HELLER, K./ ROSEMANN, B.: Planung und Auswertung empirischer Untersuchungen. Eine Einführung für Pädagogen, Psychologen und Soziologen. Stuttgart 1974.

- HEUCHERT,R./ PIGORS,H./ THOSS, U.: Zur Charakteristik der schnellen Kombinationsangriffe im Volleyball unter zeitlichem Aspekt. In: Theorie und Praxis der Körperkultur 37 (1988), 2, 117- 123.

- HOCHMUTH, G.: Biomechanik sportlicher Bewegungen. Berlin 1974.

- HOHMANN, A.: Zur Struktur der komplexen Sportspielleistung. Ahrensburg 1985.

- HOHMANN, A./ BRACK,R.: Theoretische Aspekte der Leistungsdiagnostik im Sportspiel. In: Leistungssport 13 (1983), 2, 5-10.

- HOHMANN, A./ ROMMEL,G.: Spielbeobachtung im Fußball. In: Leistungssport 24(1994), 6, 41-46.

- HUGHES, M.: Computerised Notation Analysis in Field Games. In Ergonomics 31 (1998), 1585-1592.

- JASCHOCK, W.: Ist die Leistung im Fußball messbar? O.O.1976.

- JENTER, H./ TÜNNEMANN, H.: Zu einige aktuellen Tendenzen und Hauptstoßrichtungen in den Sportspielen (Fußball, Handball, Volleyball) aus der Sicht der internationalen Literatur. Thematische Information des Zentrums für Wissenschaftsinformation. Leipzig 1988.

- KELBER, H./ SETZEPFANDT, W.: Angriffsschwund contra Cleverness. In: Kicker Sportmagazin 1973.

- KIRKENDALL, D. / GRUBER, J. / JOHNSON, R.: Measurement and Evaluation for Physical Educators. Dubuque, lowa, 1980.

- KLEIN, G./ KOCH, T./ ELLERBROCK,V.: Die Leistungen der Torhüter bei der B-WM1983. In: Lehre und Praxis des Handballspiels 5 (1983), 4 - 5, 27- 28.

- KOLLATH, E.: Biomechanische Analysen zum Fußball- bisherige Ansätze und mögliche Untersuchungsziele. In: APEEL, H.-J./ MAURITZ, K.H.(Hrsg.): Brennpunkte der Sportwissenschaft, 1 Bd. St. Augustin 1988, 85-96.

- KOLLATH. E.: Experimentelle Analysen im Training der Sportart Fußball. In: KUHN, W.I SCHMIDT, W.(Hrsg.): Analyse und Beobachtung in Training und Wettkampf. Beiträge und Analysen zum Fußballsport IV, Berlin 1991, 56 - 66.

- KOLLATH, E.: Lehrbuch Soccer Technique \& Taktik . Aachen 1998.

- KOLLATH. E.: Lehrbuch Fußball Technik \& Taktik. Aachen 2000. 
- KOLLATH, E./ KAISER, J.: Analysen der Fußballtechnik mittels Video- Digitalisierung. In: WINKLER, W./ REUTER, A. (Hrsg.). Computer- und Medieneinsatz im Fußball, Hamburg 2000, 155 - 162.

- KOLLATH, E./ SOMMER, D.: Technikanalysen in der Sportart Fußball auf der Basis digitalisiert Videoaufnahmen. In: WEBER, K./ KOLLATH, E. (Hrsg.): Video und Computer im Leistungssport der Sportspiele. Köln 1991, 99 - 111.

- KONZAG,G.: Zur Objektivierung der Handlungsgeschwindigkeit im Fußball unter Berücksichtigung von Entscheidungsprozessen. In: SCHELLENBERGER,H. (Ltg.): Psychologie im Sportspiel. Berlin (Ost) 1981, 60-72.

- KÖTHE, TH./ STARK, G.: Stellung der sporttechnischen Ausbildung im Nachwuchstraining nach Grundsätzen des langfristigen Leistungsaufbaus. Leistungssport 26 (1996) 3, 22-28.

- KOZOSCA,I.: Basketball Lehrbuch. Methodik der Vortaktik mit Übungsformen für Schule und Verein, Bd2. Stuttgart 1982.

- KRAKE, C.: Individuelle Taktik Angriff. Eine Untersuchung zum Angriffsverhalten. In: Deutsche Volleyball-Zeitung 12 (1988), 3, 12.

- KRAUSPE, D.: Eignung im Sportspiel. In: SCHELLENBERGER, H. (Ltg.): Psychologie im Sportspiel. Berlin (Ost) 1981, 65 - 74 .

- KRÖGER, C.: Einführung in den Schwerpunkt „Beobachtung“. In: HAGEDORN, G./ HEYMEN, N: Methodologie der Sportspielforschung. Ahrensburg 1992, $58-60$.

- KRÜGER. A.: Einleitung. In KRÜGER, A./ NIEDLICH, D. (Hrsg.): 100 Ballspiel- Fertigkeitstests. Schriftenreihe zur Praxis der Leibeserziehung und des Sports; Bd.181. Schorndorf 1985, 9 - 29.

- KUGELMANN, C./ BERL, J.: Zur koedukativen Basketballausbildung für Sportstudierende. In: Sportunterricht 28 (1979), 3, 11 - 17.

- KUHN, W.: Handlungsstrategien beim Strafstoß. In: HAHN,E./ SCHOCK,K.: Beiträge zur Kognition und Motorik. Bielefeld 1985, 103 - 110.

- KUHN, W.I OTTO, R./ STEINER, J.: Computergestützte Leistungserfassung im Fußball. In: KUHN, W./ SCHMIDT, W. (Hrsg.): Analyse und Beobachtung in Training und Wettkampf. Beiträge und Analysen zum Fußballsport IV. St Augustin 1992, 118-134.

- KUHN, W./ MAIER, W./ WIEPKING, W.: Untersuchung zur Problematik der Spielzeit im Fußball. In: Leistungssport 7 (1977), 1, 22-50. 
- KUHN, W./ MAIER, M.: Zur Registrierung und Auswertung von Angriffsaktionen. In: Der Fußball- Trainer 12 (1975), 3, 22-30.

- KUHN, W.I MAIER, M.: Beiträge zur Analyse des Fußballspiels. Schorndorf,1978.

- LAMES, M.: Leistungsdiagnostik durch Computersimulation. Ein Beitrag zur Theorie der Sportspiel am Beispiel Tennis. Frankfurt a. M. 1991.

- LAMES, M.: Systematische Spielbeobachtung. Münster 1994.

- LAMNEK, S.: Qualitative Sozialforschung. Band 2: Methoden und Techniken. München 1989.

- LEHNER, R.: Beobachtung und Analyse von Angriffsaktionen im Fußball. In: Der Fußball- Trainer 6 (1976), 6-11, 24-26.

- LEES, A.: Technique Analysis in Sports: A Critical Review. In: Journal of Sports Sciences, 10 (2002), 20, 2002, 813 - 828.

- LETZELTER, M.: Trainingsgrundlagen, 3. Aufl. Reinbek 1980.

- LETZELTER, M.: Trainingsgrundlagen. Reinbek 1985.

- LIEBERMANN u, a.: Advances in the Application of Information Technology to Sport Performance. In: Journal of Sports sciences 10 (2002), 20, 755-769.

- LIENERT, G.: Testaufbau und Testanalyse. Weinheim 1969.

- LOY, R.: Systematische Spielbeobachtung im Fußball. In: Leistungssport, 25 (1995), 3, 15-20.

- LOY, R.: Computergestützte Spracherkennung- eine Datenerfassungsmethode zur systematischen Spielbeobachtung. In: Leistungssport, 26 (1996), 1, 1720.

- $\quad$ LUTTGENS, K ./ HAMILTON, N.: Kinesiology, 9 Aufl. Madison, 1997.

- MAHLO, F.: Theoretische Probleme der taktischen Ausbildung in den Sportspielen. In: Theorie und Praxis der Körperkultur, Teil I: 14 (1965), 9, 9-15.

- MARHOLD, G.: Zum Begriff der sportlichen Technik. In: Biomechanik sportlicher Bewegungen. Studientexte I der DHFK Leipzig 1987.

- MATHEWS, D.K.: Measurement in Physical Education. 5. Aufl. Philadelphia 1978, 281.

- MATWEJEW, L.: Grundlagen des sportlichen Trainings. Berlin (Ost) 1981.

- MAYERS, C.R. / BLESH, T.E.: Measurement in Physical Education. N.Y.1962.

- MAYHEW, R. / WENGER, A.: Time-motion Analysis of professional soccer. In: J. Hum. Stud.11 (1985), 3, 49 - 52. 
- MEES, U.: Verhaltensbeobachtung und Verhaltungsmodifikation. 1 Aufl. Stuttgart 1977.

- MECHLING, H./ BÖS, K.: Ein Fähigkeitsorientierter Ansatz zur Erklärung sportmotorischer Leistungen. In: Sportwissenschaft 14 (1984) 1, 32 - 49.

- MELTZOFF, A.N./ MOORE, M.K.: Imitation of Facial and manual gestures by human neonates. Science, 198 (1977), 20, 74-78.

- NEUMAIER, A.: Sportmotorische Tests im Unterricht und Training. Grundlagen der Entwicklung, Auswahl und Anwendung motorischer Testverfahren im Sport. Schorndorf 1983.

- OHASHI, J./ TOGARI, H./ ISOKAWA, M./ SUZUKI, S.: Measuring movement speeds and distances, covered during soccer match play. In: REILLY, T. I LEES, A./DAVIDS,K./MURPHY, W. (Hrsg.): Science and Football. London 1988, 329-333.

- OLSEN, E. / LARSEN, O.: Use of match analysis by coaches. In: REILLY, T. I BANGSBO, J und HUGHES, M. (Hrsg.): Science and Football III. London, 1997, 209-220.

- PATRICK, J.D./ MCKENNA,M.J.: The CABER computer system: a review of its application to the analysis of Australian Rules football. In: REILLY, T. I LEES, A. / DAVIDS, K. /MURPHY, W.J. (Hrsg.): Science and Football 1988, 267-273.

- PETROV, R.: Strategie und Taktik des Ringens. Sofia 1967.

- PÖHLMANN, R. (Hrsg.): Sportmotorik. Psychologische Grundlagen der Handlungsregulation sowie Möglichkeiten zur Effektivierung von Lernprozessen im unterrichtlichen und außerunterrichtlichen Sport (Studienmaterial zur Sportwissenschaft). PH Potsdam 1987.

- PONSONBY, D.: The World Cup: Guidelines for Excellence? In: Soccer World 10 (1974), 3, 20 - 21.

- POMMERENKE, S.: Fußball- Spielanalysen im Professionellen Bereich unterschiedliche Zielsetzungen und Methoden in der Sportwissenschaft und im Sportjournalismus, Dissertation, Georg August Universität Göttingen 1997.

- RAHNAMA, N./ REILLY, T/LEES, A.: Injury risk associated with playing actions during competitive soccer. British Journal of Sports Medicine 36 (2002), 354-359. 
- REILLY, T.: What Research Tells the Coach about Soccer. Washington, DC 1979.

- REILLY, T./ DORAN, D.: Science and Gaelic football. In: Journal of Sports Sciences 19 (2001), 181-193.

- REILLY, T./ GILBOURNE, D.: Science and Football: a review of applied research in the football codes. In : Journal of Sports sciences 21(2003) 9, 693705.

- ROTH, K.: Bewegungslehre. Reinbek 1983.

- ROTH, K.: Taktik im Sportspiel. Schorndorf 1989.

- RUTEMÖLLER, E.: Die Spielsysteme bei der WM. In: Fußballtraining 12 (1994), 11/12, 9 - 12.

- SAND, TH.: Theoretische Positionen zur Erfassung informeller Leistungsvoraussetzungen in der Sportart Ringen als eine Grundlage zur Erhöhung der Sicherheit von Eignungsbeurteilungen in dieser Sportart. Dissertation, DHFK, Leipzig 1985.

- SASS, H.: Zu einigen Problemen der Sportspielbeobachtung. In: Theorie und Praxis der Körperkultur 33 (1984), 6, 433-436.

- SCHAAL, S.: Is imitation learning the route to humanoid robots? In: Trend in Cognitive Science 3 (1999), 233 -239.

- SCHALLER, H.-J.: Untersuchung zur Struktur des Handballspiels auf funktionsanalytischer Grundlage. In: ANDERSEN, R./HAGEDORN, G.(Hrsg.): Beobachten und Messen im Sportspiel. Berlin1980, 185-203.

- SCHMIDT, G.J.: Zur Arbeit mit dem Interaktiven Video im Basketball. In: WEBER, K./ KOLLATH, E./ SCHMIDT,G.J. (Hrsg.): Video und Computer im Leistungssport der Sportspiele. Köln 1991, 177-198.

- SCHMIDT, W.: Aggression und Sport Längsschnittuntersuchung sozialpsychologischer Determinanten beim Fußball in unterschiedliche Belastungssituation. Ahrensburg 1978.

- SCHMIDT, G.: Methoden und Probleme der Erfolgskontrolle in Training und Wettkampf. In: ANDERSEN, R./ HAGEDORN,G.(Hrsg.): Zur Sportspiel- Forschung. Berlin 1976, 119-150.

- SCHMIDT, G.; Analyse und Beobachtung in Training und Wettkampf: eine methodenkritische Einführung. In: KUHN,W./ SCHMIDT,W: Analyse und Beobachtung in Training und Wettkampf, Berlin 1991, 4 - 9. 
- SCHMIDT, G./ HAGEDORN, G.: Zur Methodik und Praxis der Beobachtung und Leistungsmessung im Sportspiel. In: Leistungssport 2 (1972) 4, 259-263 und $5,331-345$.

- SCHMIDT, G./ REICHELT, M.: Video- Computer- Konfigurationen in der Sportspielbeobachtung - Ein Zwischenbericht zum Stand der Entwicklung. In: APPEL, H.J./BRÜGGEMANN,G.P. (Hrsg.): Erfassen und Messen sportlicher Leistungen. Sankt Augustin 1992, 81-105.

- SCHNABEL, G.: Beanspruchung Belastung Anpassung Lernen. Zu einem motorisch- trainingswissenschaftlichen Funktionsschema aus Sportliche Bewegung und Motorik unter Belastung. Clausthal- Zellerfeld 1991.

- SCHNABEL, G./ HARRE, D./ BORDE, A. (Hrsg.): Trainingswissenschaft. Leistung - Training - Wettkampf. Berlin 1994.

- SCHNELLER, V.: Mit ungewohnt Abwehrmaßnahmen Gegner mehr als nur irritieren. In: Handballtraining 11 (1989) 11, 7 - 12.

- SCHUBERT, R.: Analyse des Torwartverhaltens. In: Handballtraining 8(1986), 4/5, 57-61.

- SINGER, E.: Der Torwart im Hallenhandball. Böblingen1983.

- SINGER, R./ UNGERER-RÖHRIG.: Test. In: RÖTHIG, P. (Ltg.): Sportwissenschaftliches Lexikon. Schorndorf 1983, 510 - 512.

- SINGER, R./ UNGERER-RÖHRIG.: Test. In: RÖTHIG, P. (Ltg.): Sportwissenschaftliches Lexikon. Schorndorf 2003, 594 - 597.

- SÖLL, W.: Differenzierung im Sportunterricht. Erster Teilband: Pädagogische und didaktische Grundlagen. Organisatorische Differenzierung (Neigungsgruppe, Fördergruppe, Leistungsgruppe). Die Gestaltung des Unterrichts in der Liebeserziehung, Teil IV(Schriftenreihe zur Praxis der Liebeserziehung und des Sports, Bd.69). Schorndorf 1973.

- SPÄTE, D.: Kreativ, variantenreich und spielbestimmend! Analyse des Olympischen Handballturniers 1992 (I,II,III). Handballtraining 14 (1992), 9/ 10/ 12, 3-17, 13-22, 24-31.

- STACHOWIAK, H.: Allgemeine Modelltheorie. Wien 1973.

- STIEHLER, G./ KONZAG, I./ DÖBLER, H.: Sportspiele. Berlin 1988.

- STÖBER, K.: Grundsätze für die methodische Gestaltung der taktischen und der technischen Ausbildung im Sportspiel. In: Körpererziehung 35 (1985), 2/3, 68-73. 
- TALAGA, J.: Fußballtaktik. Berlin 1977.

- TEIPEL, D./ GERISCH, G./ SCHÄFER, S.: Zur Antizipation von Angriffshandlungen im Fußball. In: HAHN, E./ SCHOCK, K.: Beiträge zur Kognition und Motorik. Köln1985.

- TRAXEL, W.: Grundlagen und Methoden der Psychologie. Bern1974.

- TROSSE, H.: Handball. Reinbek 1979.

- WALDOWSKI, L.: Basketball. Reinbek 1982.

- WHITHEAD, N. Conditioning for sport. Yorkshire.1975.

- WILLIAMS, A.M./ LEE, D./ REILLY,T: A Quantitative Analysis of Matches Played in the 1991-1992 and 1997-1998 Seasons. London 1999.

- WINKLER, W.: Spielbeobachtung bei Fußballspielern. In: Leistungsfußball 21 (1983), 10, 63-68.

- WINKLER, W.: Fußball analysiert: Hamburger SV gegen Inter Mailand. In: Fußballtraining 3 (1985), 12, 19 -22.

- WINKLER, W./ FREIBICHLER, F.: Leistungsdiagnostik beim Fußballspiel. In: Leistungssport 21 (1991), 2, 25-28.

- WINKLER, W.: Computer/Video Analysis in German Soccer. In: HUGHES, M. (Hrsg.): Notational Analysis of Sport I\&II. Cardiff 1997, 21 - 30.

- WINKLER, W.: Analyse von Fußballspielen mit Video- und Computerhilfe. In: WINKLER, W./ REUTER, A. (Hrsg.).: Computer- und Medieneinsatz im Fußball. Hamburg 2000, 63 - 76.

- WITHERS, R./ MARICIE, Z. / WASILEWSKI, S. / KELLY, L: Match analyses of Australian professional soccer players. In: J. Hum. Mov. Stud.8 (1982), 4,160 170.

- YANAKA, K.I HAGA, S.I SHINDO, M./NARITA, J./ KOSEKI, S.I MATSUURA,Y./ EDA, M.: Time and motion analysis in top class soccer games. In: REILLY, T. / LEES, A. / DAVIDS, K. / MURPHY, W (Hrsg.): Science and Football. London 1988, 324 - 340.

- ZIELINISKI, S.: Zur Geschichte des Videorekorders. Berlin 1986.

- ZIMMER, W.: Zur Belastungsgestaltung in der technisch- taktischen Ausbildung in der Sportart Handball (Aufbautraining). Dissertation DHFK Leipzig 1985.

- http://www.schepart.ch/mho/Fame/Fame.htm (03.02..2003). 


\section{Arabische Literatur}

- ABD-EL-KARIM, A.: Unterrichten und Lernen in der Sportwissenschaft. Kairo 1990. (in arabischer Sprache). [eigene Übersetzung].

- ABD-EL-MONEEM, H ./ HASANEEN, S.: Methoden der Analyse des Spiels im Volleyball. Kairo 1992. (in arabischer Sprache). [eigene Übersetzung].

- ABD-EL-MAQSUD, E.: Motorischen Entwicklung bei Männern. Alexandria 1985. (in arabischer Sprache). [eigene Übersetzung].

- ABD-EL-MAKSUD, E.: Trainingslehre. Alexandria 1979. (in arabischer Sprache). [eigene Übersetzung].

- ABO-EL-MAGD, A./ SHAALAN, I.: Neue Methoden des Fußballspiels. Kairo 1997. (in arabischer Sprache). [eigene Übersetzung].

- ABO-EL-MAGD, A./ ISMAIL, T./ SCHAALAN,I.: Gruppenspiel im Fußball. Kairo 1993. (in arabischer Sprache). [eigene Übersetzung].

- ABO-EL-MAGD, A./ SHAALAN, I.: Strategie der Verteidigung im Fußballspiel. Kairo 1996. (in arabischer Sprache). [eigene Übersetzung].

- ABO-EL-ELA, A./ EL-RUBY, A.: Talentselektion im Bereich Sport. Kairo 1986. (in arabischer Sprache). [eigene Übersetzung].

- AHMED, A.: Die Verhältnisse zwischen Technik Fähigkeit und Spielergebnisse im Fußballspiel, Magisterarbeit, Sporthochschule der Universität Zakazik1991. (in arabischer Sprache). [eigene Übersetzung].

- ALAA, G.: Biomechanik der Sportbewegung. Kairo 1985. (in arabischer Sprache). [eigene Übersetzung].

- ALLAWY, M.: Die Psyche bei Training und Wettkampf, 5. Aufl.. Kairo 1993. (in arabischer Sprache). [eigene Übersetzung].

- BADAWY, E.: Studium ÜIOa.B i ÜIOa.B i ÜlOa.B i ÜIOa.B i ÜIOa.B i ÜlOa.B i. 
- EID, S. / EL-NEMR, A.: Analyse der Angriffstaktik für die Ägyptische Basketball Nationalmannschaft bei den Arabische Meisterschaft. Kairo 1994. (in arabischer Sprache). [eigene Übersetzung].

- ELKASHEF, E.: Basis für eine sportliche Selektion. Kairo 1987a (in arabischer Sprache). [eigene Übersetzung].

- ELKASHEF, E.: Talentauswahl im Turnen. Kairo 1987b (in arabischer Sprache). [eigene Übersetzung].

- ELSAYED, I.: Analyse der technischen Spielelemente und ihre Verhältnisse zu den Spielergebnissen, Dissertation, Sporthochschule der Universität Zakazik 1997. (in arabischer Sprache). [eigene Übersetzung].

- HOSNY, E.: Effektivität der Strafecke auf die Spielergebnisse im Hockeyspiel, Dissertation, Sporthochschule der Universität Helwan Kairo1974. (in arabischer Sprache). [eigene Übersetzung].

- HUSSEIN, T./ ABD EL-RAHMAN, A.: Kinesiolgie des Sports und die Grundlage der Bewegungsanalyse. Kairo 1993. (in arabischer Sprache). [eigene Übersetzung].

- IBRAHIM,M.: Angriff im Fußballspiel. Kairo 1992 (in arabische Sprache). [eigene Übersetzung].

- IBRAHIM, M.: Verteidigung zum Aufbau des Angriffs. Kairo 1994. (in arabischer Sprache). [eigene Übersetzung].

- KISCHK, M./ ELBOSATY, A.: Grundlage der Vorbereitung, Technik und Taktik im Fußballspiel. Alexandria 2000. ( in arabischer Sprache). [eigene Übersetzung].

- MAHMOOD, M.: Analyse der Technik und Taktik auf die Spielergebnisse im Fußballspiel, Dissertation, Sporthochschule der Universität Zakazik 1994. (in arabischer Sprache). [eigene Übersetzung].

- MOAWAD, H.: Basketball für alle. Kairo 1980. (in arabischer Sprache). [eigene Übersetzung].

- MOKHTAR, H.: Wissenschaftliche Grundlagen im Fußballtraining, 2. Aufl.. Kairo 1998. (in arabischer Sprache). [eigene Übersetzung].

- MORSEY, M.: Freiwürfe und ihr Verhältnisse zu Spielergebnissen, Dissertation, Sporthochschule der Universität Helwan Kairo1975. (in arabischer Sprache). [eigene Übersetzung]. 
- YASIN, T.: Bewegungsanalyse für die Kindergarten, Dissertation, Sporthochschule der Universität Alexandria 1987. (in arabischer Sprache). [eigene Übersetzung].

- YEHYA, M.: Die Verhältnisse zwischen körperlichen, technischen, psychischen Faktoren und Spielergebisse im Basketballspiel, Dissertation, Sporthochschule der Universität Helwan Kairo 1983. (in arabischer Sprache). [eigene Übersetzung]. 


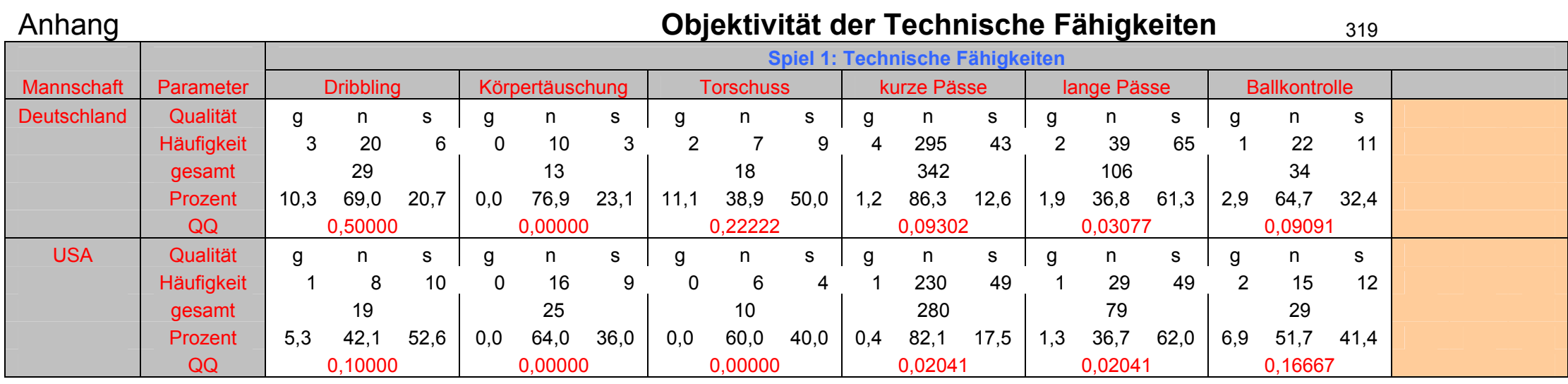

Korrelation Mannschaft A: $\quad 0,99796$

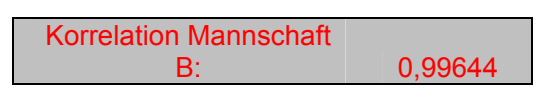

Durchschnitt:

0,99720

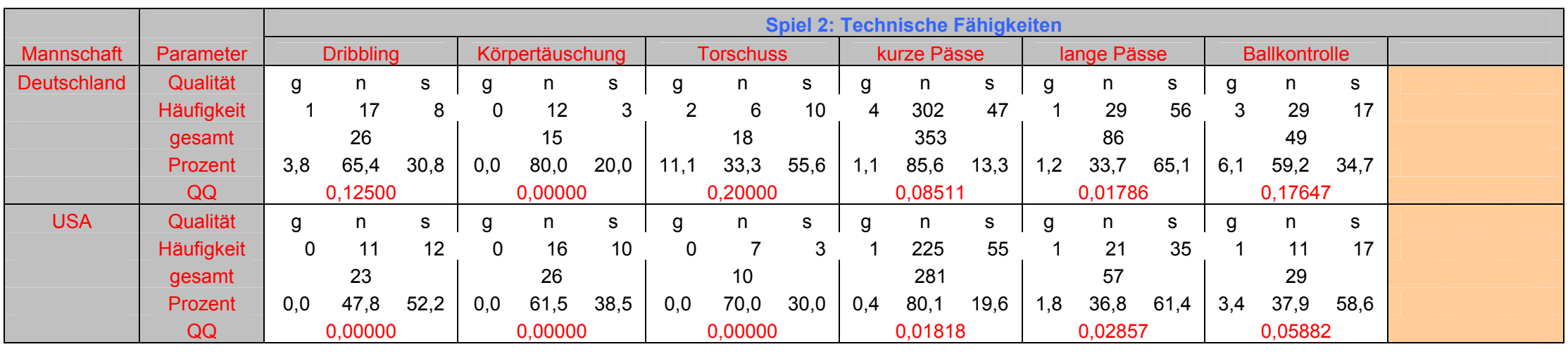


Objektivität der Offensiv-taktische Fähigkeiten

320

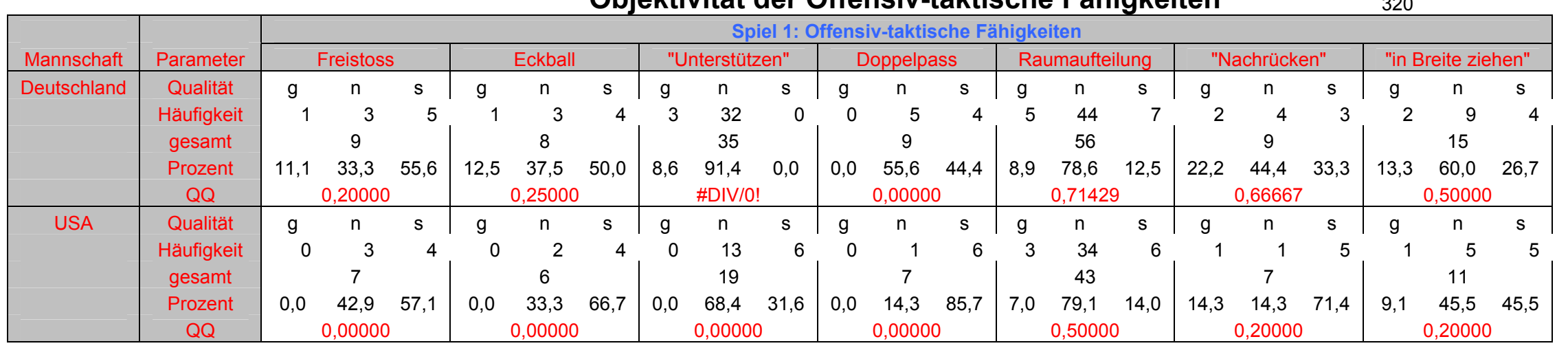

Korrelation Mannschaft A: $\quad 0,99872$

Korrelation Mannschaft

0,99792

Durchschnitt:

0,99832

\begin{tabular}{|c|c|c|c|c|c|c|c|c|c|c|c|c|c|c|c|c|c|c|c|c|c|c|}
\hline \multirow{3}{*}{$\begin{array}{l}\text { Mannschaft } \\
\text { Deutschland }\end{array}$} & \multirow[b]{2}{*}{ Parameter } & \multicolumn{21}{|c|}{ Spiel 2: Offensiv-taktische Fähigkeiten } \\
\hline & & \multicolumn{3}{|c|}{ Freistoss } & \multicolumn{3}{|c|}{ Eckball } & \multicolumn{3}{|c|}{ "Unterstützen" } & \multicolumn{3}{|c|}{ Doppelpass } & \multicolumn{3}{|c|}{ Raumaufteilung } & \multicolumn{3}{|c|}{ "Nachrücken" } & \multicolumn{3}{|c|}{ "in Breite ziehen" } \\
\hline & Qualität & $\mathrm{g}$ & $\mathrm{n}$ & $\mathrm{s}$ & $\mathrm{g}$ & $\mathrm{n}$ & $\mathrm{s}$ & $\mathrm{g}$ & $\mathrm{n}$ & $\mathrm{s}$ & $\mathrm{g}$ & $\mathrm{n}$ & $\mathrm{s}$ & g & $\mathrm{n}$ & $\mathrm{s}$ & $\mathrm{g}$ & $\mathrm{n}$ & $\mathrm{s}$ & $\mathrm{g}$ & $\mathrm{n}$ & $\mathrm{s}$ \\
\hline & Häufigkeit & 1 & 3 & 5 & 1 & 3 & 4 & 1 & 32 & 0 & 0 & 5 & 4 & 5 & 44 & 7 & 2 & 5 & 3 & 2 & 9 & 5 \\
\hline & gesamt & \multicolumn{3}{|c|}{9} & \multicolumn{3}{|c|}{8} & & 33 & & \multicolumn{3}{|c|}{9} & & 56 & & \multicolumn{3}{|c|}{10} & \multicolumn{3}{|c|}{16} \\
\hline & Prozent & 11,1 & 33,3 & 55,6 & 12,5 & 37,5 & 50,0 & 3,0 & 97,0 & 0,0 & 0,0 & 55,6 & 44,4 & 8,9 & 78,6 & 12,5 & 20,0 & 50,0 & 30,0 & 12,5 & 56,3 & 31,3 \\
\hline & $Q Q$ & \multicolumn{3}{|c|}{0,20000} & \multicolumn{3}{|c|}{0,25000} & \multicolumn{3}{|c|}{ \#DIV/0! } & \multicolumn{3}{|c|}{0,00000} & & 0,7142 & & \multicolumn{3}{|c|}{0,66667} & \multicolumn{3}{|c|}{0,40000} \\
\hline \multirow{5}{*}{ USA } & Qualität & $\mathrm{g}$ & $\mathrm{n}$ & $\mathrm{s}$ & $\mathrm{g}$ & $\mathrm{n}$ & $\mathrm{s}$ & $\mathrm{g}$ & $\mathrm{n}$ & $\mathrm{s}$ & $\mathrm{g}$ & $\mathrm{n}$ & $\mathrm{s}$ & g & $\mathrm{n}$ & $\mathrm{s}$ & $\mathrm{g}$ & $\mathrm{n}$ & $\mathrm{s}$ & $\mathrm{g}$ & $\mathrm{n}$ & $\mathrm{s}$ \\
\hline & Häufigkeit & 0 & 3 & 4 & 0 & 2 & 4 & 0 & 13 & 7 & 0 & 1 & 6 & 3 & 34 & 6 & 1 & 1 & 5 & 1 & 5 & 7 \\
\hline & gesamt & \multicolumn{3}{|c|}{7} & \multicolumn{6}{|c|}{20} & & 7 & & \multicolumn{3}{|c|}{43} & & 7 & & \multicolumn{3}{|c|}{13} \\
\hline & Prozent & 0,0 & 42,9 & 57,1 & 0,0 & 33,3 & 66,7 & 0,0 & 65,0 & 35,0 & 0,0 & 14,3 & 85,7 & 7,0 & 79,1 & 14,0 & 14,3 & 14,3 & 71,4 & 7,7 & 38,5 & 53,8 \\
\hline & QQ & \multicolumn{3}{|c|}{0,00000} & \multicolumn{3}{|c|}{0,00000} & \multicolumn{3}{|c|}{0,00000} & \multicolumn{3}{|c|}{0,00000} & \multicolumn{3}{|c|}{0,50000} & \multicolumn{3}{|c|}{0,20000} & & 0,14286 & \\
\hline
\end{tabular}


Objektivität der Defensiv-taktische Fähigkeiten

321

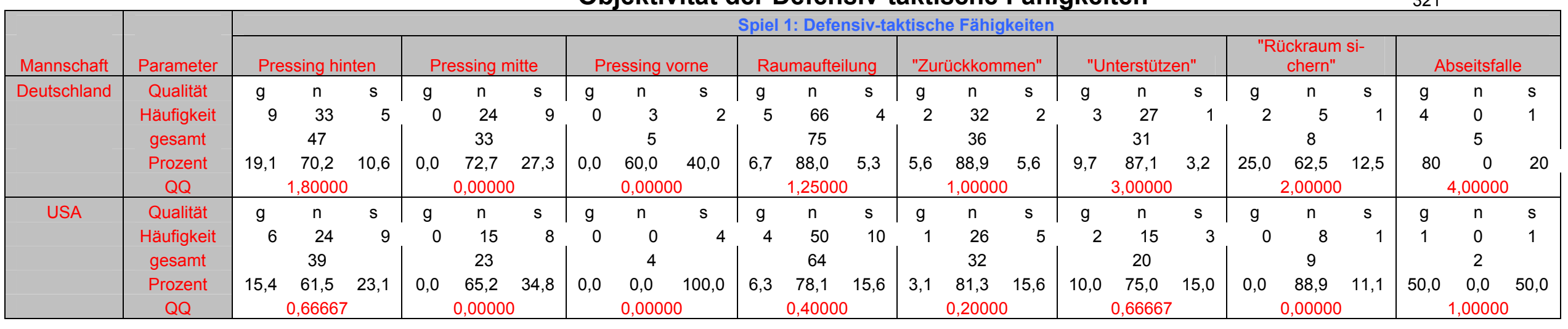

Korrelation Mannschaft A: $\quad 0,99554$

\begin{tabular}{|c|c|c|c|c|c|c|c|c|c|c|c|c|c|c|c|c|c|c|c|c|c|c|c|c|c|c|}
\hline \multirow{3}{*}{$\frac{\text { Mannschaft }}{\text { Deutschland }}$} & \multirow{6}{*}{$\begin{array}{c}\text { Parameter } \\
\text { Qualität } \\
\text { Häufigkeit } \\
\text { gesamt } \\
\text { Prozent } \\
\text { QQ }\end{array}$} & \multicolumn{25}{|c|}{ Spiel 2: Defensiv-taktische Fähigkeiten } \\
\hline & & \multicolumn{3}{|c|}{ Pressing hinten } & \multicolumn{3}{|c|}{ Pressing mitte } & \multicolumn{3}{|c|}{ Pressing vorne } & \multicolumn{3}{|c|}{ Raumaufteilung } & \multicolumn{4}{|c|}{ "Zurückkommen" } & \multicolumn{3}{|c|}{ "Unterstützen" } & \multicolumn{3}{|c|}{$\begin{array}{l}\text { "Rückraum si- } \\
\text { chern" }\end{array}$} & \multicolumn{3}{|c|}{ Abseitsfalle } \\
\hline & & $\mathrm{g}$ & $\mathrm{n}$ & $\mathrm{s}$ & $\mathrm{g}$ & $\mathrm{n}$ & $\mathrm{s}$ & $\mathrm{g}$ & $\mathrm{n}$ & $\mathrm{s}$ & $\mathrm{g}$ & $\mathrm{n}$ & $\mathrm{s}$ & & g & $\mathrm{n}$ & $\mathrm{s}$ & $\mathrm{g}$ & $\mathrm{n}$ & $\mathrm{s}$ & $\mathrm{g}$ & $\mathrm{n}$ & $\mathrm{s}$ & g & $\mathrm{n}$ & $\mathrm{s}$ \\
\hline & & 9 & 31 & 5 & 0 & 28 & 9 & 0 & 3 & 2 & 5 & 66 & 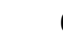 & & 2 & 28 & 1 & 3 & 30 & 2 & 2 & 5 & 1 & 4 & 0 & 1 \\
\hline & & \multicolumn{3}{|c|}{45} & & 37 & & \multicolumn{3}{|c|}{5} & & 77 & & \multicolumn{4}{|c|}{31} & & 35 & & \multicolumn{3}{|c|}{8} & \multicolumn{3}{|c|}{5} \\
\hline & & \multicolumn{3}{|c|}{1,80000} & \multicolumn{3}{|c|}{0,00000} & \multicolumn{3}{|c|}{0,00000} & 6,5 & $\begin{array}{r}85,7 \\
0,833\end{array}$ & $3^{7,8}$ & & \multicolumn{2}{|c|}{2,00000} & $0^{3,2}$ & 8,6 & $\begin{array}{c}85,7 \\
1,5000\end{array}$ & 5,7 & 25,0 & 2,00000 & 12,5 & 80,0 & 4,00000 & 20,0 \\
\hline \multirow[t]{5}{*}{ USA } & Qualität & $g$ & $\mathrm{n}$ & $\mathrm{s}$ & $\mathrm{g}$ & $\mathrm{n}$ & $\mathrm{s}$ & $\mathrm{g}$ & $\mathrm{n}$ & $\mathrm{s}$ & $g$ & $\mathrm{n}$ & $\mathrm{s}$ & & $g$ & $\mathrm{n}$ & $\mathrm{s}$ & $g$ & $\mathrm{n}$ & $\mathrm{s}$ & $\mathrm{g}$ & $\mathrm{n}$ & $\mathrm{s}$ & $g$ & $\mathrm{n}$ & $\mathrm{s}$ \\
\hline & Häufigkeit & 6 & 22 & 11 & 0 & 15 & 10 & 0 & 0 & 4 & 4 & 52 & 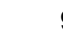 & & 1 & 24 & 6 & 2 & 17 & 2 & 0 & 8 & 1 & 1 & 0 & 1 \\
\hline & gesamt & \multicolumn{3}{|c|}{39} & & 25 & & \multicolumn{3}{|c|}{4} & & 65 & & \multicolumn{4}{|c|}{31} & & 21 & & \multicolumn{3}{|c|}{9} & \multicolumn{3}{|c|}{2} \\
\hline & Prozent & 15,4 & 56,4 & 28,2 & 0,0 & 60,0 & 40,0 & 0,0 & 0,0 & 100,0 & 6,2 & 80,0 & 13 & & 3,2 & 77,4 & 19,4 & 9,5 & 81,0 & 9,5 & 0,0 & 88,9 & 11,1 & 50,0 & 0,0 & 50,0 \\
\hline & $Q Q$ & \multicolumn{3}{|c|}{0,54545} & \multicolumn{3}{|c|}{0,00000} & & 0,000 & & & 0,444 & & & & 0,1666 & & & 1,0000 & & & 0,0000 & & & 1,0000 & \\
\hline
\end{tabular}




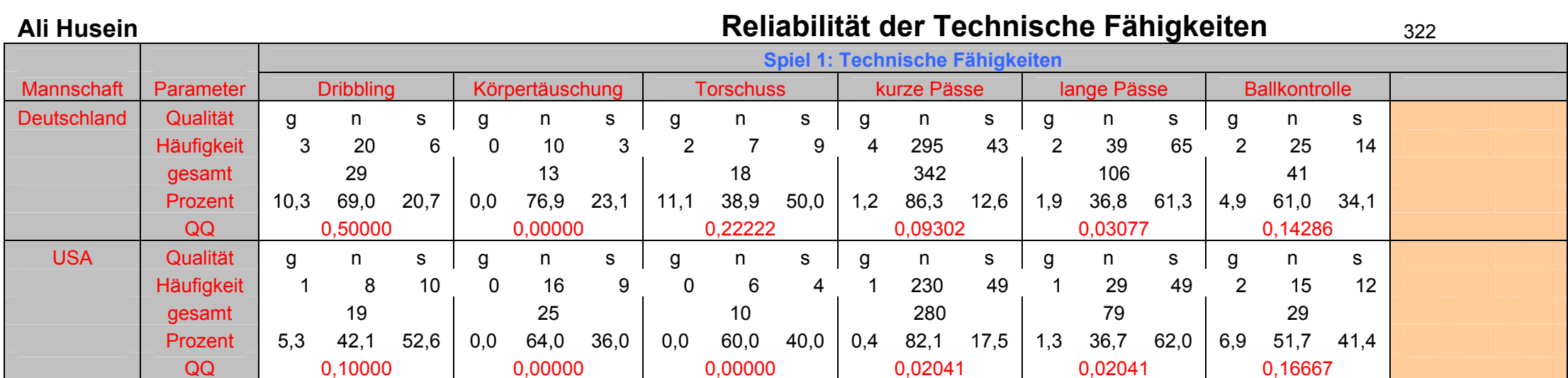

Korrelation Mannschaft A: $\quad 0,99836$

Korrelation Mannschaft

B: $\quad 0,99644$

\section{Christian Becker}

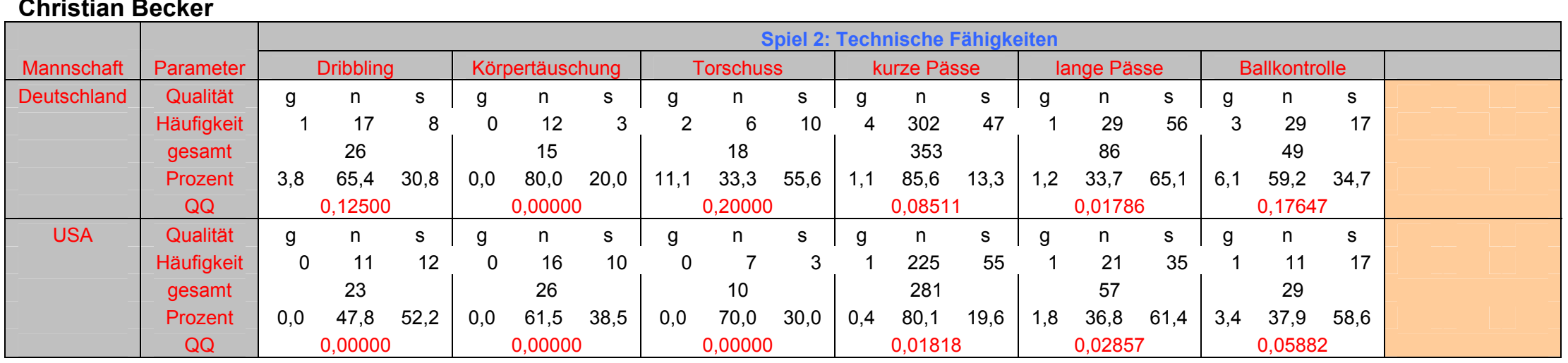




\begin{tabular}{|c|c|c|c|c|c|c|c|c|c|c|c|c|c|c|c|c|c|c|c|c|c|c|}
\hline \multicolumn{7}{|c|}{ Ali Husein } & \multicolumn{13}{|c|}{ Reliabilität der Offensiv-taktische Fähigkeiten } & \multicolumn{3}{|l|}{323} \\
\hline \multirow{3}{*}{ Mannschaft } & \multirow[b]{2}{*}{ Parameter } & \multicolumn{21}{|c|}{ Spiel 1: Offensiv-taktische Fähigkeiten } \\
\hline & & \multicolumn{3}{|c|}{ Freistoss } & \multicolumn{3}{|c|}{ Eckball } & \multicolumn{3}{|c|}{ "Unterstützen" } & \multicolumn{3}{|c|}{ Doppelpass } & \multicolumn{3}{|c|}{ Raumaufteilung } & \multicolumn{3}{|c|}{ "Nachrücken" } & \multicolumn{3}{|c|}{ "in Breite ziehen" } \\
\hline & Qualität & $\mathrm{g}$ & $\mathrm{n}$ & $\mathrm{s}$ & $\mathrm{g}$ & $\mathrm{n}$ & $\mathrm{s}$ & $\mathrm{g}$ & $\mathrm{n}$ & $\mathrm{s}$ & $\mathrm{g}$ & $\mathrm{n}$ & $\mathrm{s}$ & $\mathrm{g}$ & $\mathrm{n}$ & $\mathrm{s}$ & $\mathrm{g}$ & $\mathrm{n}$ & $\mathrm{s}$ & $\mathrm{g}$ & $\mathrm{n}$ & $\mathrm{s}$ \\
\hline & Häufigkeit & 1 & 3 & 5 & 1 & 3 & 4 & 3 & 32 & 2 & 0 & 5 & 4 & 5 & 44 & 7 & 2 & 4 & 2 & 2 & 10 & 4 \\
\hline & gesamt & \multicolumn{3}{|c|}{9} & \multicolumn{3}{|c|}{8} & & 37 & & \multicolumn{3}{|c|}{9} & & 56 & & \multicolumn{3}{|c|}{10} & \multicolumn{3}{|c|}{16} \\
\hline & Prozent & 11,1 & 33,3 & 55,6 & 12,5 & 37,5 & 50,0 & 8,1 & 86,5 & 5,4 & 0,0 & 55,6 & 44,4 & 8,9 & 78,6 & 12,5 & 20,0 & 40,0 & $40, c$ & 12,5 & 62,5 & 25,0 \\
\hline & $\mathrm{QQ}$ & \multicolumn{3}{|c|}{0,20000} & \multicolumn{3}{|c|}{0,25000} & & 1,5000 & & \multicolumn{3}{|c|}{0,00000} & & 0,7142 & & \multicolumn{3}{|c|}{0,50000} & \multicolumn{3}{|c|}{0,50000} \\
\hline \multirow[t]{5}{*}{ USA } & Qualität & g & $\mathrm{n}$ & s & g & $\mathrm{n}$ & s & g & $\mathrm{n}$ & s & g & $n$ & s & g & $\mathrm{n}$ & s & g & $\mathrm{n}$ & s & $\mathrm{g}$ & $\mathrm{n}$ & s \\
\hline & Häufigkeit & 0 & 3 & 4 & 0 & 2 & 4 & 0 & 13 & 6 & 0 & 1 & 6 & 3 & 34 & 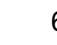 & 1 & 1 & 5 & 1 & 5 & 5 \\
\hline & gesamt & \multicolumn{3}{|c|}{7} & \multicolumn{3}{|c|}{6} & & 19 & & \multicolumn{3}{|c|}{7} & & 43 & & \multicolumn{3}{|c|}{7} & \multicolumn{3}{|c|}{11} \\
\hline & Prozent & 0,0 & 42,9 & 57,1 & 0,0 & & 66,7 & 0,0 & 68,4 & 31,6 & 0,0 & 14,3 & 85,7 & 7,0 & 79,1 & $14, \mathrm{C}$ & 14,3 & 14,3 & 71,4 & 9,1 & 45,5 & 45,5 \\
\hline & $\mathrm{QQ}$ & \multicolumn{3}{|c|}{0,00000} & \multicolumn{3}{|c|}{0,00000} & & $0,000 \mathrm{C}$ & & & 0,0000 & & & 0,500 & & &, 2000 & & & 0,2000 & \\
\hline
\end{tabular}

Korrelation Mannschaft A: $\quad 0,99546$

Korrelation Mannschaft

B: $\quad 0,99792$

Durchschnitt:

0,99669

\section{Christian Becker}

\begin{tabular}{|c|c|c|c|c|c|c|c|c|c|c|c|c|c|c|c|c|c|c|c|c|c|c|}
\hline \multirow{3}{*}{$\begin{array}{l}\text { Mannschaft } \\
\text { Deutschland }\end{array}$} & \multirow{3}{*}{$\begin{array}{c}\text { Parameter } \\
\text { Qualität }\end{array}$} & \multicolumn{21}{|c|}{ Spiel 2: Offensiv-taktische Fähigkeiten } \\
\hline & & \multicolumn{3}{|c|}{ Freistoss } & \multicolumn{3}{|c|}{ Eckball } & \multicolumn{3}{|c|}{ "Unterstützen" } & \multicolumn{3}{|c|}{ Doppelpass } & \multicolumn{3}{|c|}{ Raumaufteilung } & \multicolumn{3}{|c|}{ "Nachrücken" } & \multicolumn{3}{|c|}{ "in Breite ziehen" } \\
\hline & & g & $\mathrm{n}$ & $\mathrm{s}$ & g & $\mathrm{n}$ & $\mathrm{s}$ & $\mathrm{g}$ & $\mathrm{n}$ & $\mathrm{s}$ & g & $\mathrm{n}$ & s & $\mathrm{g}$ & $\mathrm{n}$ & s & $\mathrm{g}$ & $\mathrm{n}$ & $\mathrm{s}$ & g & $\mathrm{n}$ & $\mathrm{s}$ \\
\hline & Häufigkeit & 1 & 3 & 5 & 1 & 3 & 4 & 1 & 32 & 1 & 0 & 5 & 4 & 5 & 42 & 10 & 2 & 5 & 3 & 2 & 9 & 5 \\
\hline & gesamt & \multicolumn{3}{|c|}{9} & & 8 & & \multicolumn{3}{|c|}{34} & & 9 & & \multicolumn{3}{|c|}{57} & & 10 & & \multicolumn{3}{|c|}{16} \\
\hline & Prozent & 11,1 & 33,3 & 55,6 & 12,5 & 37,5 & 50,0 & 2,9 & 94,1 & 2,9 & 0,0 & 55,6 & 44,4 & 8,8 & 73,7 & 17,5 & 20,0 & 50,0 & 30,0 & 12,5 & 56,3 & 31,3 \\
\hline & $\mathrm{QQ}$ & \multicolumn{3}{|c|}{0,20000} & \multicolumn{3}{|c|}{0,25000} & \multicolumn{3}{|c|}{1,00000} & \multicolumn{3}{|c|}{0,00000} & \multicolumn{3}{|c|}{0,50000} & \multicolumn{3}{|c|}{0,66667} & \multicolumn{3}{|c|}{0,40000} \\
\hline USA & Qualität & g & $\mathrm{n}$ & $\mathrm{s}$ & g & $\mathrm{n}$ & $\mathrm{s}$ & $\mathrm{g}$ & $\mathrm{n}$ & $\mathrm{s}$ & g & $\mathrm{n}$ & s & g & $\mathrm{n}$ & s & g & $\mathrm{n}$ & $\mathrm{s}$ & g & $\mathrm{n}$ & s \\
\hline & Häufigkeit & 0 & 3 & 4 & 0 & 2 & 4 & 0 & 13 & 7 & 0 & 1 & 6 & 3 & 34 & 6 & 1 & 1 & 5 & 1 & 5 & 7 \\
\hline
\end{tabular}




\begin{tabular}{|c|c|c|c|c|c|c|c|c|c|c|c|c|c|c|c|c|c|c|c|c|c|c|c|c|c|}
\hline \multirow{3}{*}{$\frac{\text { Mannschaft }}{\text { Deutschland }}$} & \multirow[b]{2}{*}{ Parameter } & \multicolumn{24}{|c|}{ Spiel 1: Defensiv-taktische Fähigkeiten } \\
\hline & & \multicolumn{3}{|c|}{ Pressing hinten } & \multicolumn{3}{|c|}{ Pressing mitte } & \multicolumn{3}{|c|}{ Pressing vorne } & \multicolumn{3}{|c|}{ Raumaufteilung } & \multicolumn{3}{|c|}{ "Zurückkommen" } & \multicolumn{3}{|c|}{ "Unterstützen" } & \multicolumn{3}{|c|}{$\begin{array}{c}\text { "Rückraum si- } \\
\text { chern" }\end{array}$} & \multicolumn{3}{|c|}{ Abseitsfälle } \\
\hline & Qualität & $\mathrm{g}$ & $n$ & $\mathrm{~s}$ & $\mathrm{~g}$ & $n$ & $\mathrm{~s}$ & $g$ & $\mathrm{n}$ & $\mathrm{s}$ & $\mathrm{g}$ & $\mathrm{n}$ & $\mathrm{s}$ & g & $\mathrm{n}$ & $\mathrm{s}$ & $\mathrm{g}$ & $\mathrm{n}$ & $\mathrm{s}$ & g & $\mathrm{n}$ & $\mathrm{s}$ & g & $\mathrm{n}$ & $\mathrm{s}$ \\
\hline & Häufigkeit & 9 & 33 & 5 & 0 & 26 & 9 & 0 & 3 & 2 & 5 & 70 & 4 & 2 & 32 & 2 & 3 & 30 & 1 & 2 & 8 & 1 & 4 & 0 & 1 \\
\hline & gesamt & \multicolumn{3}{|c|}{47} & & 35 & & & 5 & & & 79 & & & 36 & & \multicolumn{3}{|c|}{34} & & 11 & & \multicolumn{3}{|c|}{5} \\
\hline & Prozent & 19,1 & 70,2 & 10,6 & 0,0 & 74,3 & 25,7 & 0,0 & 60,0 & 40,0 & 6,3 & 88,6 & 5,1 & 5,6 & 88,9 & 5,6 & 8,8 & 88,2 & 2,9 & 18,2 & 72,7 & 9,1 & 80,0 & 0,0 & 20,0 \\
\hline & $\mathrm{QQ}$ & \multicolumn{3}{|c|}{1,80000} & \multicolumn{3}{|c|}{0,00000} & \multicolumn{3}{|c|}{0,00000} & \multicolumn{3}{|c|}{1,25000} & & 1,000 & & \multicolumn{3}{|c|}{3,00000} & \multicolumn{3}{|c|}{2,00000} & \multicolumn{3}{|c|}{4,00000} \\
\hline \multirow[t]{5}{*}{ USA } & Qualität & g & $\mathrm{n}$ & $\mathrm{s}$ & g & $\mathrm{n}$ & $\mathrm{s}$ & $g$ & $\mathrm{n}$ & $\mathrm{s}$ & $\mathrm{g}$ & $\mathrm{n}$ & $\mathrm{s}$ & $\mathrm{g}$ & $\mathrm{n}$ & $\mathrm{s}$ & $g$ & $\mathrm{n}$ & $\mathrm{s}$ & $g$ & $\mathrm{n}$ & $\mathrm{s}$ & $\mathrm{g}$ & $\mathrm{n}$ & $\mathrm{s}$ \\
\hline & Häufigkeit & 6 & 24 & 9 & 0 & 15 & 8 & 0 & 0 & 4 & 4 & 50 & 10 & 1 & 26 & 5 & 2 & 15 & 3 & 0 & 8 & 1 & 1 & 0 & 1 \\
\hline & gesamt & \multicolumn{3}{|c|}{39} & & 23 & & & 4 & & \multicolumn{3}{|c|}{64} & & 32 & & \multicolumn{3}{|c|}{20} & & 9 & & \multicolumn{3}{|c|}{2} \\
\hline & Prozent & 15,4 & 61,5 & 23,1 & 0,0 & 65,2 & 34,8 & 0,0 & 0,0 & 100,0 & 6,3 & 78,1 & 15,6 & 3,1 & 81,3 & 15,6 & 10,0 & 75,0 & 15,0 & 0,0 & 88,9 & 11,1 & 50,0 & 0,0 & 50,0 \\
\hline & $\mathrm{QQ}$ & \multicolumn{3}{|c|}{0,66667} & \multicolumn{3}{|c|}{0,00000} & \multicolumn{3}{|c|}{0,00000} & \multicolumn{3}{|c|}{0,40000} & & 0,200 & & & 0,6666 & & & 0,0000 & & & 1,0000 & \\
\hline
\end{tabular}

Korrelation Mannschaft A: $\quad 0,99744$

\section{Christian Becker}

\begin{tabular}{|c|c|c|c|c|c|c|c|c|c|c|c|c|c|c|c|c|c|c|c|c|c|c|c|c|c|}
\hline \multirow{3}{*}{$\begin{array}{l}\text { Mannschaft } \\
\text { Deutschland }\end{array}$} & \multirow{7}{*}{$\begin{array}{c}\text { Parameter } \\
\text { Qualität } \\
\text { Häufigkeit } \\
\text { gesamt } \\
\text { Prozent } \\
\text { QQ }\end{array}$} & \multicolumn{24}{|c|}{ Spiel 2: Defensiv-taktische Fähigkeiten } \\
\hline & & \multicolumn{3}{|c|}{ Pressing hinten } & \multicolumn{3}{|c|}{ Pressing mitte } & \multicolumn{3}{|c|}{ Pressing vorne } & \multicolumn{3}{|c|}{ Raumaufteilung } & \multicolumn{3}{|c|}{ "Zurückkommen" } & \multicolumn{3}{|c|}{ "Unterstützen" } & \multicolumn{3}{|c|}{$\begin{array}{c}\text { "Rückraum si- } \\
\text { chern" }\end{array}$} & \multicolumn{3}{|c|}{ Abseitsfälle } \\
\hline & & g & $\mathrm{n}$ & $s$ & g & $\mathrm{n}$ & $\mathrm{s}$ & $\mathrm{g}$ & $\mathrm{n}$ & $\mathrm{s}$ & g & $\mathrm{n}$ & $\mathrm{s}$ & $\mathrm{g}$ & $\mathrm{n}$ & $\mathrm{s}$ & g & $\mathrm{n}$ & $\mathrm{s}$ & g & $\mathrm{n}$ & $\mathrm{s}$ & $\mathrm{g}$ & $\mathrm{n}$ & $\mathrm{s}$ \\
\hline & & 9 & 31 & 5 & 0 & 28 & 9 & 0 & 3 & 2 & 5 & 66 & $\epsilon$ & 2 & 28 & 1 & 3 & 30 & 2 & 2 & 6 & 2 & 4 & 0 & 1 \\
\hline & & \multicolumn{3}{|c|}{45} & \multicolumn{3}{|c|}{37} & & 5 & & \multicolumn{3}{|c|}{77} & & 31 & & \multicolumn{3}{|c|}{35} & & 10 & & \\
\hline & & 20,0 & 68,9 & 11,1 & 0,0 & 75,7 & 24,3 & 0,0 & 60,0 & 40,0 & 6,5 & 85,7 & 7,8 & 6,5 & 90,3 & 3,2 & 8,6 & 85,7 & 5,7 & 20,0 & 60,0 & 20,0 & 80,0 & 0,0 & 20,0 \\
\hline & & \multicolumn{3}{|c|}{1,80000} & \multicolumn{3}{|c|}{0,00000} & \multicolumn{3}{|c|}{0,00000} & \multicolumn{3}{|c|}{0,83333} & & 2,0000 & & \multicolumn{3}{|c|}{1,50000} & \multicolumn{3}{|c|}{1,00000} & \multicolumn{3}{|c|}{4,00000} \\
\hline \multirow{5}{*}{ USA } & Qualität & g & & $\mathrm{s}$ & g & $\mathrm{n}$ & $\mathrm{s}$ & $\mathrm{g}$ & $\mathrm{n}$ & $\mathrm{s}$ & g & $\mathrm{n}$ & $\mathrm{s}$ & g & $\mathrm{n}$ & $\mathrm{s}$ & g & $\mathrm{n}$ & $\mathrm{s}$ & g & $\mathrm{n}$ & $\mathrm{s}$ & g & $\mathrm{n}$ & $\mathrm{s}$ \\
\hline & Häufigkeit & 6 & 22 & 11 & 0 & 15 & 10 & 0 & 0 & 4 & 4 & 52 & $\varsigma$ & 1 & 25 & 6 & 2 & 17 & 2 & 0 & 8 & 1 & 1 & 0 & 1 \\
\hline & gesamt & \multicolumn{3}{|c|}{39} & & 25 & & & 4 & & \multicolumn{3}{|c|}{65} & & 32 & & \multicolumn{3}{|c|}{21} & & 9 & & \multicolumn{3}{|c|}{2} \\
\hline & Prozent & 15,4 & 56,4 & 28,2 & 0,0 & & 40,0 & 0,0 & 0,0 & 100,0 & 6,2 & 80,0 & 13,8 & 3,1 & 78,1 & 18,8 & 9,5 & 81,0 & 9,5 & 0,0 & 88,9 & 11,1 & 50,0 & 0,0 & 50,0 \\
\hline & $\mathrm{QQ}$ & \multicolumn{3}{|c|}{0,54545} & \multicolumn{3}{|c|}{0,00000} & & 0,000 & & & 0,4444 & & & 0,1666 & & & 1,0000 & & & 0,0000 & & & 0000 & \\
\hline
\end{tabular}




\section{Abbildungsverzeichnisse}

Abb. 1: Die individuellen Leistungsfaktoren und die sie beeinflussen

Rahmenbedingungen steuern das Leistungsvermögen des einzelnen

Spielers und damit der gesamten Mannschaft

(modifiziert nach BAUER 1998). 12

Abb. 2: Taktikbereiche im Sportspiel 15

(modifiziert nach BISANZ/GERISCH 1981).

Abb. 3: Gruppen von taktischen Handlungen (modifiziert nach BAUER 2001). 18

Abb. 4: Hierarchisierung der Erklärungsebenen (nach KRAUSPE 1981). 19

Abb. 5: Hierarchisierung der Erklärungsebenen (nach HOHMANN 1985). 20

Abb. 6: Bewegung ohne Ball (modifiziert nach KOLLATH 2000). 24

Abb. 7: Bewegung zum Ball (modifiziert nach MOKHTAR 1998). 25

Abb. 8: Unterteilung der Bewegungen mit Ball

(modifiziert nach MOKHTAR 1998). 26

Abb.9: Diagnostische Verfahren der Sportwissenschaft

(modifiziert nach CARL 1983). 28

Abb.10: Varianten der Beobachtung (nach LAMES 1994). 34

Abb.11: Formen und Anwendungsbereiche der systematischen

Sportspielbeobachtungen (modifiziert nach HOHMANN 1985). 40

Abb.12: Prozessmodell einer "Systematischen Spielanalyse“

(modifiziert nach WINKLER 2000). 60

Abb.13: Veranschaulichung der Modellbildung als Abbildungsform

(modifiziert nach STACHOWIAK 1973). 


\section{Tabellenverzeichnis}

Tab.1: Darstellung verschiedener Verfahren der

Sportspielbeobachtung (modifiziert nach LAMES 1994).

Tab.2: Vor- und Nachteile der quantitativen und qualitativen

Beobachtungsverfahren, (modifiziert nach ESSER 1983).

Tab.3: Weltmeisterschaftsspiel ab Achtelfinale.

Tab.4: Zusammengefasste Qualitätsquotienten der Achtel-, Viertel-, Halb- und Finalpaarungen. 


\section{Danksagung:}

An erster Stelle danke ich Herrn Prof. Dr. Arnd Krüger für seine konstruktive Begleitung der Arbeit, sein Vertrauen, seine Unterstützung und sein Rat bei der Erstellung dieser Arbeit. Besonders möchte ich noch mal Prof. Dr. Arnd Krüger, hervorheben, der mein Vorhaben von Beginn mit anregenden Herausforderungen und kritischer Sympathie begleitet und der in vielfacher Weise mein Blick für das Wesentliche geschärft hat.

Mein Dank gilt auch Herrn PD. Dr. Wolfgang Buss für seine Bereitschaft, die vorliegende Arbeit als Zweitgutachter zu beurteilen.

Ein ganz spezielles Dankeschön richte ich an zwei Personen, die nicht nur sehr positive wissenschaftliche Einflüsse auf diese Arbeit ausgeübt haben, sondern auch mich selbst positiv beeinflusst haben: Carsten Wahle und Christian Becker.

Mein besonderer Dank gilt Meiner Frau Samah Samir und meine Kinder Mahmoud und Fatma für die Unterstützung, Geduld und Toleranz während der Fertigstellung dieser Arbeit

Weiterhin bedanke ich mich bei alle Freunden, Kollegen und Helfern, ohne deren Hilfe diese Arbeit in der vorliegenden Form nicht hätte geschrieben werden können.

\section{Ali Hussein Abdelrahman}




\section{Eidesstattliche Erklärung}

Hiermit versichere ich an Eides statt, dass ich die vorliegende Arbeit selbständig und ohne Benutzung anderer als der angegebenen Hilfsmittel angefertigt habe.

Alle Stellen, die wörtlich oder sinngemäß aus Veröffentlichungen übernommen wurden, sind als solche gekennzeichnet.

Göttingen 2004 
Lebenslauf

Name:

Ali

Vorname:

Hussein Abd El-Rahman Abdallah Mohammed

Geburtsdatum:

03.08.1962

Geburtsort:

Nationalität:

Zakazik (Ägypten)

Familienstand:"

Ägypter

Schulbildung:

verheiratet, 2 Kinder

1967 - 1973 Grundschule in Hussainia (Zakazik)

1974 - 1977 Mittelschule in Hussainia (Zakazik)

Studium:

1978 - 1981 Gymnasium Zakazik

1981 - 1985 Dipl. Sportlehrer an der Sporthoch schule Zakazik mit der Note sehrgut

1993 Magisterarbeit Sporthochschule Suez Canal Universität

1995 - 1996 Aufbaustudium zur Promotion an der Sporthochschule Port Said

Berufspraxis: 1978 - 1985 Spieler in erste Liga in Ägypten 1991-- 1995 Fußball Trainer der Suez Canal Club in Port Fuad

Vorherige

Beschäftigung: 1989 -1996 Assistent, Oberassistent an der Sporthochschule der Suez Canal Universität in Port Said der Suez Kanal Universität (Ägypten) Stipendiat der Ägyptischen Regierung zur Erlangung des Dr. disc.pol. an der Georg AugustUniversität Göttingen.

Hussein Abd El-Rahman Abdallah Mohamed Ali 